
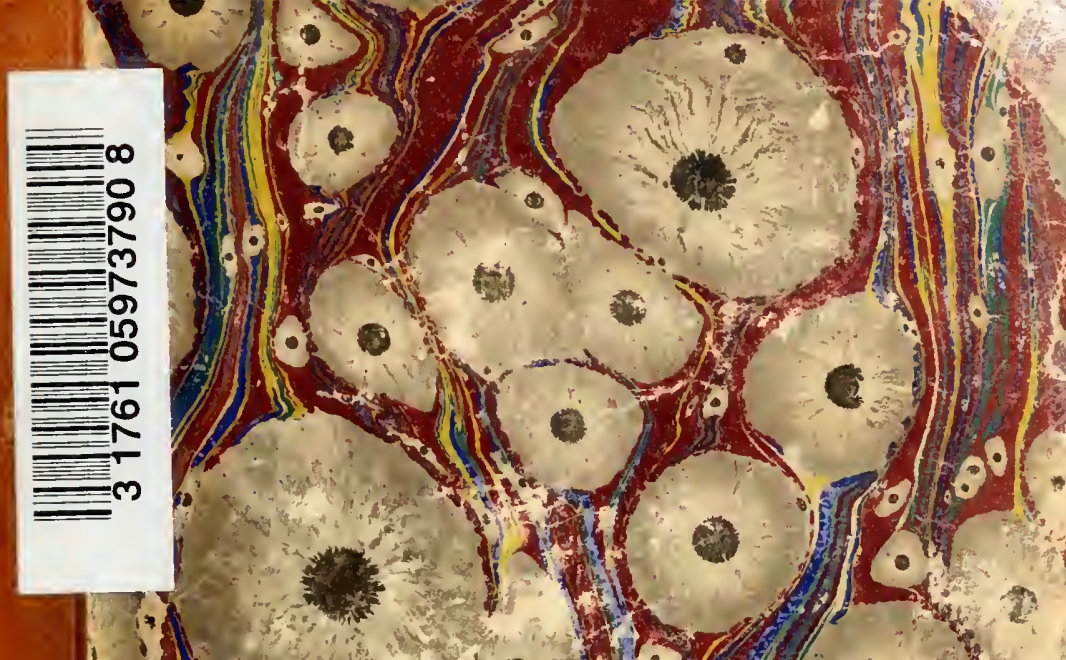

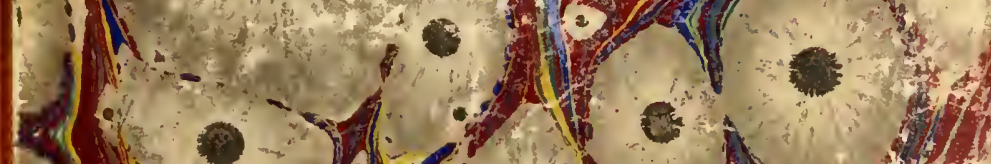

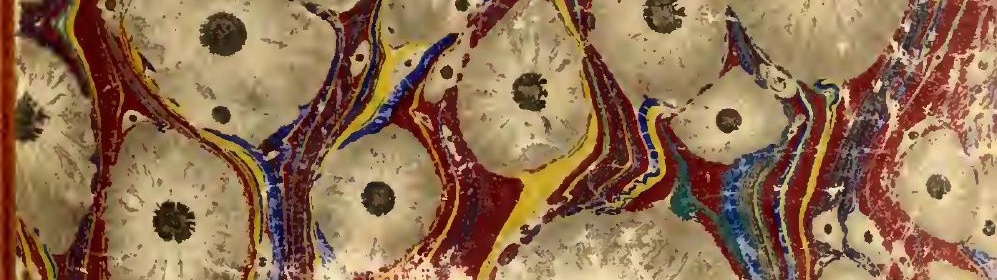
8) 1 (1)

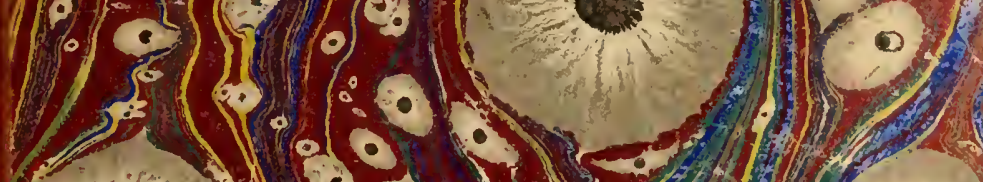

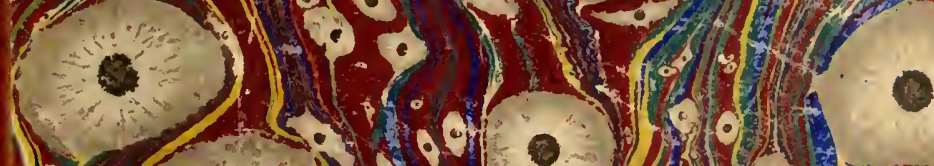

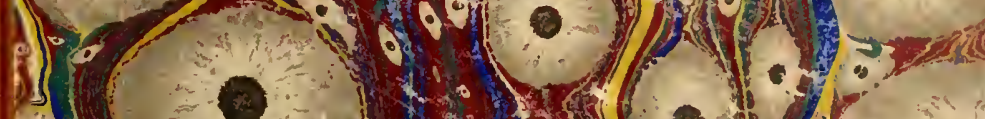

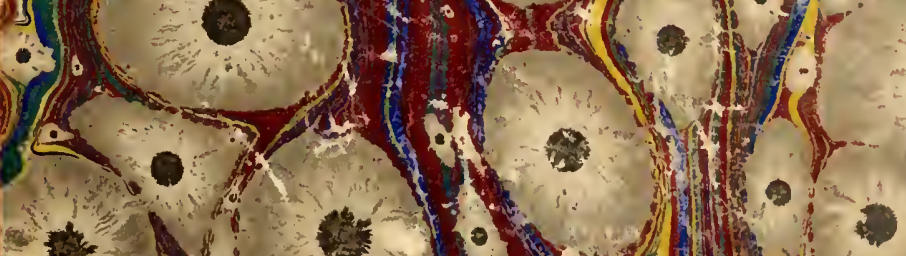

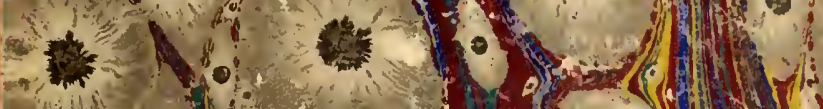

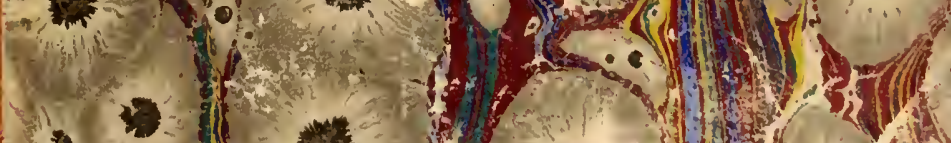









\section{LA \\ PROPRIETÉ RURALE}

EN FRANCE 


\section{OUVRAGES DU MEME AUTEUR}

Hištoire de Savoie d'après les documents originaux. 3 vol. in- 8 avec carte, 1869. (Ouvrage couronné par l'Acalémie francaise en 1871. - Prix d'histoire.)

Dix ans d'administration française en Savoie, études statistiques sur le prix de l'annexion. (Ouvrage couronné par l'Academie des sciences en 1875. - Prix de statistique.)

Statistiques de la vie humaine et de l'instruction primaire avant 1789. l'après les registres des paroisses de la ville de Chàtellerault. (Ouvrage couronné par l'Académie des sciences en 1879. - Prix de statistique.)

La crise agricole. (Hention honorable décernée par l'Académie des sciences morales el politiques en 1899. - Économie sociale.)

Les ressources de la Mutualité, études sur les associations ouvrières et patronales. (Couronné par le Musée social en 1898.)

Les assurances ouvrières et agricoles en France et à l'étranger. (Récompensé par le .Husée social en 1900.)

L'œuvre èconomique de Charles Dunoyer. (Récompensé d'un prix par l'Académie (les sciences morales et politiques en 1898.)

Le crédit territorial en France et la réforme hypothécaire, $2^{\circ}$ élition. In-8, 1889.

La sécurité du titre foncier et la publicité hypothécaire. In-S, 1890.

La commission du cadastre et les livres fonciers. In-8, 1892.

Histoire documentaire et philosophique de l'Administration des Domaines, d'après les documents inédits. 3 vol. in-8, 1900 à 1902. (Publiẻe par les Amales de l'enregistrement, $20^{\circ}$ annic.)

La Banque de France à travers le siècle, d'après les archives de la Banque, avec tableaux et diagrammes. ln- $8,1896$.

Droits de traduction et de reproduction réservés pour tous les pays, y compris la Suede, la Norvège ot la Hollande.

Coulommiers. - Imp. Pstz BRODARD. - 1121-1901. 


\title{
FLOUR DE SAINT-GENIS
}

\author{
LA
}

\section{PROPRIÉTÉ RURALE}

\section{EN FRANGE}

\section{Ouvrage couronné par l'Académie des sciences morales et politiques en 1901}

Précédé d'un rapport de M. DE FOVILLE, servant d'introduction.

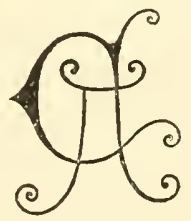

PARIS

\section{LIBRAIRIE ARMAND GOLIN}

5, RUE DE MÉZİ̀RES, "゙

1902

Tous droits réservés. 


$$
\theta
$$




\section{AVANT-PROPOS}

Ce livre a en le très grand honneur d'obtenir le prix Léon Fancher an concours de 1901 de l'Académie des sciences morales et politiques (section d'économie politique), sur le rapport de H. A. de Foville dans la séance du 3 aoùt 1901. L'éminent rapportenr a bien vonlu nous autoriser à publier les parties essentielles de ce rapport, et nous desons ajonter que l'auteur s'est empressé de se conformer aux indications qui y étaient contenues. 


\section{RAPPORT DE M. A. DE FOVILLE}

A L'ACAdénie des sciences Morales et PoLitiques DANS LA SÉANCE DU 3 AOİT 1901.

Le sujet proposé par l'Acarlémie, pour le prix Léon Faucher à décerner en 1901, était ainsi formulé :

De la situation présente et de l'avenir de la grande, de la moyenne et de la petite propriété en France.

La question pourrait paraitre oiseuse sị les ennemis de la propriété étaient, comme certains d'entre eux le croient ou le disent, à la veille de triompher. La nationalisation du sol n'épargnerait pas plus la petite propriété que la grande. Elle ne laisserait subsister qu'un propriétaire unique, le moins apte de tous à bien exploiter son domaine. Mais nous n’en sommes pas encore là. Malgré les progrès du socialisme, le droit de propriété reste fortement enraciné sur la terre française. Telle est notre conviction personnelle, et telle est celle des divers concurrents lont nous arons aujourl'hui ì apprécier le mérite comparatif.

Les mémoires présentés sont au nomlse de trois. 
L'étendue en est inégale; la valeur aussi; et le classe ment, cette fois, se fait presque de lui-mème....

Le manuscrit $n^{\circ} 1$ ne nous impose pas les mêmes réserves que les précédents. Loin de rester en deçà du but, il exagère plutòt l'ampleur et la complexité du problème posé par l'Académie. L'auteur fera bien de l'alléger un peu arant d'aller à l'impression. Mais nous pouvons nous féliciter d'avoir provoqué, sur un tel sujet, une étude si complète, si consciencieuse et parfois si pénétrante. Dans chacun des 30 chapitres dont elle se compose, on voit s'affirmer la compétence d'un spécialiste, la documentation précise et abondante d'un statisticien et d'un érudit, la dialectique d'un juriste et, quand il le faut, la hardiesse d'un réformateur. Il nous parait certain que l'auteur a beaucoup vu, beaucoup lu, beaucoup rélléchi. Il aime cordialement la terre, la terre française surtout. II se montre peut-être, çà et là, un peu optimiste; il est à coup sùr, très libéral.

Rien de toût cela n'était de nature à faire hésiter nos suffrages et la Section d'économie politique vous propose de disposer en sa faveur du prix de 3000 francs qui est à décerner.

Le développement quelquefois excessif que l'écrivain dont l'œuvre nous est soumise a donné à ses enquêtes, à ses démonstrations et à ses commentaires nous interdit de le suivre pas à pas dans son long voyage au travers des faits et des idées. Nous nous contenterons de résumer brièvement les constatations principales auxquelles l'a conduit l'observation attentive du passé et du présent, puis à dire brièvement, aussi, ce qu'il attend de l'avenir. Plus d'un trouvait téméraire, il y a quinze ou vingt ans, 
l'économiste qui, osant s'inscrire en faux contre de spécieuses prédictions, trop facilement acceptées, disait : “ Non, la Révolution française n’a pas désorganisé la propriété. Non, la terre, chez nous, ne tombe pas en poussière. Le morcellement n'y est pas chose nouvelle et, là où il dépassera le but, on le verra s'arrêter de lui-même. " Cet arrêt, qui nous semblait certain, ne s'est pas fait attendre longtemps et les statistiques fiscales, toutes abstraites qu'elles soient, suffiraient à le prouver. Jusqu'en 187\%, le nombre des cotes foncières allait progressant avec entrain : l'augmentation, depuis 1875 , ressortait à près de 50 p. 100 . Dès les premières manifestations de la crise agricole, on a vu le mouvement se ralentir, sans cesser encore. Depuis quinze ans, le recul est ininterrompu, quoique lent, et 630000 cotes environ, sur un peu plus de 14 millions, ont déjà disparu des tableaux officiels.

On étail done fondé à croire et à dirc que les causes qui tendent à grouper les biens-fonds saliraient contrebalancer, tòt ou tard, les causes qui tendent à les décomposer. Et l'on n'avail pas tort non plus de protester contre la fantaisiste théorie de Michelet, déclarant les époques de crise particulièrement favorables aux progrès de la petite propriété. C'est le contraire qui est vrai.

Mais cette vue d'ensemble, si rérélatrice qu'elle puisse ètre, laisse bien des questions sans réponse, car il serait illusoire d'admettre que la propriété sous toutes ses formes et le territoire dans toutes ses parties obéissent aux mèmes influences. Il faut distinguer, comparer, analyser. C'est à quoi s'applique, avec une extrême patience, l'auteur du mémoire $n^{\circ} 1$. Il ne se contente pas de sépa- 
rer, comme il y était convié, la grande propriété de la moyenne et la moyenne de la petite. Il rappelle et reprend les subdivisions déjà suggérées par d'autres, comme conséquence du recensement méthodique dont nos $14 \mathrm{mil}$ lions de cotes foncières ont été l'objet, pour la première fois, en 1884. Il en rapproche les statistiques des cultures et exploitations, classées par contenances, telles que les présentent les enquètes décennales du ministère de l'Agriculture, spécialement celle de 1892. Et, là mème où les deux échelles ne concordent pas, il arrive, par voie d'approximations, à en combiner judicieusement les indications respectives.

La grande propriété, par son étendue même, est celle qui appelle d'abord l'attention. Certains publicistes parlent d'elle comme si elle n'existait plus qu'à l'état de souvenir, et d'autres comme si elle continuait à absorber la majeure partie du sol national. En réalité, pour arriver à lui en attribuer la moitié, il faut abaisser la ligne de démarcation, un peu arbitraire il est vrai, qui sépare la grande propriété de la propriété moyenne; il faut en outre totaliser avec la propriété privée, non seulement les biens des communes, hospices, sociétés anonymes, etc., mais même le domaine de l'État, forestier ou autre : en tout, plus de 6 millions d'hectares. Ces éléments collectifs constituent, pour le pays, d'importantes réserves. Mais ceux-là font nécessairement fausse route qui, combinant au hasard des chiffres et des mots qu'ils ont mal compris, confondent par cela même dans de communes conclusions des éléments aussi hétérogènes qu'un réseau de chemins de fer, une forèt domaniale, un massif de montagnes incultes et l'un de ces vastes domaines individuels, avec 
chàteau et fermes, dont notre esprit évoque plus volontiers l'image quand on parle devant nous de la grande propriété. Le mémoire $n^{\circ} 1$ sait ériter ces assimilations imprudentes et les paragraphes 1 à 3 du chapitre II, et 1 du chapitre $1 v$, dans la première partie, les chapitres I, Il, et $11 \mathrm{~d}$ dans la seconde, définissent avec précision les formes diverses et les multiples missions de la grande propriélé, considérée soit au point de vue agricole, soit au point de vue social. L'auteur se plaît à voir dans la grande propriété le réservoir naturel de la petite, toujours prêt à en fournir la matière première, toujours prêt aussi à en résorber les lébris. On peut dire de la grande et de la petite propriété qu'elles s'appellent mutuellement et que, à l'une comme à l'autre, l'isolement serait funeste. La docilité des choses à la roix de l'intérèt économique s'affirme, d'ailleurs, en ce qui concerne la grande culture, par les allures contraires qu'elle affecte de nos jours dans les deux régions séparées par le cour's de la Loire. Vers le Nord, vers le Nord-Est, vers l'Est, les grandes exploitations se laissent encore entamer et le nombre des unités foncières va croissant. Au sul de la Loire, les progrès de l'élevage el la crise dont la viticulture n'arrive à triompher qu'en se transformant font rentrer peu à peu dans le cadre de la grande propriété une partie des fragments qui s'en étaient antérieurement détachés.

La petite propriété n'a pas été étudiće avec moins de soin ni avec moins de sagacité que la grande. Les paragraphes 7 et 8 du chapitre Ir et 3 du chapitre iv lui sont presque exclusivement consacrés et elle tient encore une grande place dans la seconde partie du mémoire.

Le temps n'est plus où le morcellement du sol français 
semblait principalement imputable à la Révolution et au Code civil. Il n'y a rien de paradoxal aujourd'hui à faire remonter jusqu'à l'époque féodale l'origine du phénomène et à y voir l'ouvre d'une longue suite de générations, toutes animées du mème amour pour les sillons que leur's sueurs fécondaient. Sans s'attarder longuement aux notions qui sont maintenant acquises à la science, le mémoire suit attentivement, dans ses développements et dans ses vicissitudes, la petite propriété rurale. L'auteur la connait bien et lui a rouć une vire sympathie, sachant qu'elle en est digne. Nous n'avons plus à défendre nos peasant properties contre les mépris de l'Angleterre, puisqu'après les avoir tant calomniés, depuis Arthur Young et Malthus, jusqu'à M. Caird et lady Verney, nos voisins se sont mis tout à coup à nous les envier, au point de vouloir susciter artificiellement sur le sol britannique, à coups de lois et de règlements, cette classe de petits propriétaires ruraux qui, en France, a le mérite de s'ètre créée elle-mème. Assurément, il y arait quelque chose de spécieux à dire à nos paysans que, n'ayant pour tenir tète à la grande culture perfectionnée ni le capital, ni la science, ni mème les machines, ils étaient vaincus à l'arance; mais c'était oublier ou méconnaitre les incomparables ressources, le " magique pouvoir » de la propriété. Young ne s'écriait-il pas lui-mème, saisi par l'évidence : "Donnez à un bomme la sùre possession d'un rocher; il le transformera en jardin! » Le mémoire $11^{\circ} 1$ nous dit à son tour : "Plus on décompose “ les éléments complexes de l’influence exercée par la " division du sol sur la richesse territoriale, mieux on " s'aperçoit que les accroissements de valeur " réalisés 
au cours du siècle qui vient de finir " s'appliquent surtout “ à celte petite propriété qu’on a parfois représentée " comme unc cause d'appauvrissement et de misère. La “ valeur de la grande propriété ne s'est guère accrue que " de moitié : celle de la propriété morcelée a triplé, qua" druplé et mème décuplé selon les régions. " Ainsi la plus-ralue des petits domaines serait de beaucoup la plus forte. Pour s'en convaincre, l'auteur, à défaut de constatations directes et positives, a dù procéder par roie de comparaisons un peu empiriques entre ce qu'on peut appeler la carte des plus-ralues foncières et celle du morcellement. Cette méthode ne serait pas toujours sans danger; mais nous arons ici affaire à des yeux expérimentés. Ce qu’ils ont vu nous pouvons l'admettre. Et c'est une importante constatation que celle qui montre ainsi la petite proprićté, au $\mathrm{xix}^{\mathrm{e}}$ siècle, prospérant plus que toutes les autres. Que serait-ce, si, trop sourent endettéc, elle n’arail encore contre elle la fiscalité et la procédure d'une part, de l'autre la dépopulation et l'absentéisme? Sur chacune de ces questions et sur bien d'autres, le mémoire $n^{\circ}$ i contient une foule d'olservations intéressantes et de réflexions suggestives.

La moyenne propriété, telle qu'elle se comporte aujourl'hui, semblerait laisser notre auteur plus indifférent ou plus indécis. On ne voit pas bien ce qu'on pourrail lui reprocher. Ne peut-on pas dire d'elle que, dans une certaine mesure, elle concilie les mérites, les raisons d’ètre au moins de la grande et de la petite propriété? Ici on lui refuse plutôt les uns et les autres et l'on assure que, avec les lois actuelles, " elle ne constitue pas un élément éco- 
nomique de production et de progrès ${ }^{1}$ ". On réduit son ròle, qui nous paraìt moins élroit, à servir de lien et de point d'appui commun, pour ainsi dire, aux deux types extrèmes : celui que le capital vivifie et celui que féconde le labor improbus du passan. Ce qui est remarquable, en tout cas, c'est que cette zone intermédiaire subsiste telle quelle, retenant, avec un inillion de cotes au moins, le tiers environ de notre territoire. Stagnation plus apparente que réelle d'ailleurs : la moyenne propriété n'est nullement intangible et ses deux bords voient s'accomplir, chaque année, bien des mutations; mais, d'ordinaire, les gains et les pertes se compensent. Ici, deux domaines de 30 ou 40 hectares se fondent en un seul; là un vaste héritage se décompose en deux ou trois morceaux. De l'autre côté, sur les confins de la moyenne et de la petite propriété, les petits propriétaires qui veulent et qui savent s'arrondir par d'opportunes annexions neutralisent également les efforts des dépeceurs de biens, réduits à s'attaquer à la moyenne propriété lorsque la grande a disparu ou quand elle résiste victorieusement. Voilà bien des courants contraires et tous sujets à variations; mais leur centre commun ne bouge pas : il y a là comme un point mort ${ }^{2}$.

1. Aux dernières pages du mẻmoire, la moyenne propriétė est jugćc plus favorablement qu'au chapitre 15 ; mais c'est en parlant de l'avenir et en supposant certaines réformes réalisées que l'auteur arrive à dire: - Le ròle prépondérant et civilisateur, dans l'évolution économique qui se poursuit, est réserve à la moyenne propriété. "

2. A l'impression, en ce qui concerne la moyenne propriété, l'auteur s'est efforcé de donner à sa pensée plus de clarté et de précision car, loin d'ètre indifférent ou indécis à son propos, il voit au contraire dans son développement le centre futur d'initiative et d'expansion de l'industrie agricole française. Si, avec les maurs et les lois acluelles, la moyenne propriétė ne constitue peut-ètre pas un puissant élément écomique de production et de progrès, il est certain qu'elle apparaitrait vite comme le nœud vital de la culture intensive et scientifique si les propriétaires y appliquaient leurs capitaux et surtout si, au lieu de solli- 
Grande, moyenne ou petite, les défenseurs de la propriété foncière ont deux manières différentes de concevoir ses intérêts, présents et futurs. Les uns la voudraient presque immuable; les autres aspirent, au contraire, à la mobiliser de plus en plus, sinon en fait, du moins en droit.

La question se pose d'abord au point de vue successoral. Pour l'auteur du mémoire $\mathrm{n}^{\circ} 1$ comme pour beaucoup de bons esprits, l'essentiel serait que la famille et la terre ne se séparent pas, que partout où il $\mathrm{y}$ a un toit et un domaine, quelle qu'en soit l'importance ou l'exiguïté, une petite dynastie se fixe et se perpétue. Ils attachent à cette continuité un tel prix qu'ils érigeraient volontiers en principe l'inégalité des partages pour faciliter à l'aîné la conservation du fonds paternel. Ils demandent tout au moins la liberté de tester, et le mémoire $n^{0} 1$ s'approprie en ce sens les fortes paroles de Portalis au Conseil d'Etat de 1803.

"Le droit de disposer est un droit d'arbitrage. Là où le "père est législateur dans sa famille, la société se trouve " déchargée d'une partie de cette sollicitude. Qu'on ne " lise pas que r'est là un droit aristocratique. Il est telle" ment fondé sur la raison que c’est dans les classes " inférieures que le pouvoir du père est le plus nécessaire. "Un laboureur, par exemple, a eu d'abord un fils qui, se

citer leurs fils te devenir des fonctionnaires ils avaient le bon esprit d'en faire des cultivateurs et des industriels. Et c'est alors que véritablement, par l'incidence uaturelle des choses et le retour aux champs, le mot de Boissy-rl'Anglas serait d'une application pratique : Un pays gouverné par les propriétaires du sol est dans l'étal social; formule que la démocratie française peut accepter avec d'autant plus d'empressement que l'ideal du philosophe du $\mathrm{xx}^{\circ}$ siècle doit être d'ouvrir à tous les travailleurs l'accés de la proprièté et de transformer chaque prolétaire en censitaire, afin que le magique pouvoir de la possession y change le sable en or, suivant le mot d'Arthur Young. (Note de l'auteur.) 
“ trouvant le premier élevé, est devenu le compagnon " le ses travaux. Les enfants nés depuis ćtant moins " nécessaires au père, se sont répandus dans les villes et " y ont poussé leur fortune. Lorsque le père mourra, " sera-t-il juste que l'aìné partage également le champ " amélioré par ses labeurs avec des frères qui déjà sont " plus riches que lui?"

Les Anglo-Saxons raisonnent ainsi et ne s'en trouvent pas mal. Ils éprouvent moins de répugnance à diviser les familles qu'à diviser les biens-fonds, et il y a accord pour cela entre leurs mours et leurs lois.

En France, la vie sociale a d'autres bases et les mœurs y sont levenues plus égalitaires encore que les lois. Les pères, les mères, ici, n’admettent pas aisément que les cadets aient moins de droits que les aînés. Loin de se plaindre de l'insuffisance des quotités disponibles, ils s'interdisent généralement d'en faire usage au profit d'un de leurs enfants et, par cela même, on peut croire que la réforme à laquelle Frédéric Le Play attachait tant de prix resterait assez inefficace si, par impossible, il se rencontrait chez nous un Parlement disposé à la voter.

Des mesures plus morlestes, où le Code de procédure et les tarifs fiscaux seraient seuls en cause, suffiraient, à ce qu'il semble, pour assouplir le mécanisme des partages, étant donné surtout le développement rapide et la croissante diffusion de la richesse mobilière. A certains désidérata de ce genre, souvent formulés, on ne voit même pas qu'aucune objection sérieuse ait jamais été faite. Mais nos législateurs, toujours en proie aux préoccupations électorales, ne savent écouter ni les économistes ni les jurisconsultes quand l'actualité ne les y contraint pas, 
et les textes les plus respectés, en fait, sont ceux qu’il serait le plus facile et le plus sage de reviser.

Ce que le mémoire $n^{\circ} 1$ réclame aussi énergiquement que la liberté successorale, c’est ce qu’il appelle « la sécurité du titre foncier ". Il établit que cette sécurité existe uniquement pour le riche et que la propriété naissante, celle qui mériterait le mieux la protection des lois, reste, au contraire, chez nous, exposée à mille risques. Nous ne l'aurions pas crue si précaire; mais ce sont de saisissantes démonstrations que celles qui nous sont opposées sur ce point par le $\S \breve{d n ~ c h a p i t r e ~} 11$, et le savoir professionnel est trop visible dans ce réquisitoire pour que nous puissions rester incrédules. On serait seulement tenté de demander à l'auteur comment le tableau qu'il trace des pièges tendus au petit propriétaire rural peut se concilier arec l'optimisme habitucl de ses appré ciations.

La crise agricole, il la nie presque. Les progrès de la petite propriété, il inclinerait plutôt à les exagérer qu’à les méconnaître; et, cela étant, on s'altendrait à le voir moins cmpressé à changer ce qui est. Aussi bien, il va lui-même au-derant de l'objection, comme s'il l'arait pressentie. La statistique juliciaire l'oblige à avouer que les conflits, que les litiges en matière foncière ne sont pas en France aussi nombreux qu'ils pourraient et qu’ils Jevraient l'ètre; et, pour expliquer cette contradiction, il se persuade que les hommes ralent mieux que les institutions: "Il faut qu’il y ait au cour de notre perple, s'écrie-t-il, un fonds inaltérable de franchise, de loyauté, pour qu'on essaye si rarement d'exploiter la candeur des honnêtes gens, la simplicité relative des 
paysans, en utilisant contre eux les moyens qu'offrent aux habiles les détours de la chicanc et les surprises de la procédure ! "

L'auteur, quoiqu'il en soit, appelle le tous ses vœux et la réforme hypothécaire et l'organisation des livres fonciers, tels que d'autres pays ont appris à les pratiquer.

Le gouvernement, il y a quelques années, avait mis la question à l'étude, et le mémoire dénote une connaissance parfaite des travaux de la commission extra-parlementaire du cadastre. Celui qui l’a écrit n’ignore rien de ce qu'on n'a pu dire pour et contre cette grande réforme. Les adversaires du livre foncier craignent que le jour où, gràce à lui, les mutations foncières deviendraient chez nous faciles et sùres, la spéculation n'en profite pour se faire de nos champs, de nos vignes un enjeu de plus et que l'on roie ainsi le lien existant entre la terre et l'homme se relàcher outre mesure. Le mémoire $n^{\circ} 1$ s'inspire de l'idée contraire, celle qui, dans tous les milieux, compte sur le libre jeu des intérèts pour en affermir l'équilibre. Et les raisons qu'il invoque nous paraissent concluantes.

Je n'ai fait qu'extraire d'un long exposé quelques-unes des rues qui s'y trouvent laborieusement développées. Nous pensons qu'il gagnerait à se condenser lavantage. Mais, là mème où l'enquête élargit inutilement son cadre, la richesse de la documentation et la solidité du raisonnement retiennent l'attention du lecteur et fortifient sa confiance.

La Section croit donc devoir proposer l'attribution totale du prix Léon Faucher au mémoire $n^{\circ} 1$ por- 
tant comme sous-titre Des faits, des chiffres, et comme devise cette phrase de Boissy-d'Anglas dans son rapport à la Convention nationale du 5 messidor an III : “ Un pays gouverné par les propriétaires est dans l'état social; celui où les non-propriétaires gouvernent est dans l'état de nature. "

Le Rapporteur,

A. de Foville. 


\section{A}

\section{PROPRIÉTÉ RURALE}

\section{EN FRANCE}

\section{PREMIERE PARTIE}

ORIGINES HISTORIQUES ET SOCIALES DE LA DISTRIBUTION DE LA PROPRIÉTÉ DU SOL ENTRE LES FAMILLES FRANÇAISES

\section{CHAPITRE I}

La puissance productive du sol dépend moins des proportions de sa distribution cadastrale que de son appropriation sociale.

La France est un pays agricole par excellence; l'industrie de la culture est sa source vitale, et comme la thèse de la nationalisation du sol trouve des partisans parmi les ignorants, et que les ignorants sont nombreux, il n'est jamais superflu de chercher à corriger les erreurs qui s'accréditent sur la mesure dans laquelle le sol est réparti entre les différents groupes de propriétaires.

En France, la petite propriété existe de temps immémoLA PROPRIÉTÉ RURALE EN FRANGE. 
rial. La division du sol en très petites tenures remonte aux origines des affranchissements ruraux et des accensements qui en furent la conséquence; deux fois arrêté par des séries de malheurs publics,ce mouvement agricole, associé à la conquête simultanée de la liberté civile, reprend au $\mathrm{xr}^{\mathrm{e}}$ siècle, subit un troisième temps d'arrêt pendant les guerres de religion, se relève un instant au $x^{*} u^{\mathrm{e}}$ siècle pour se ralentir encore et ne revivre quaprès la grande secousse sociale de 1790 .

Mais, à partir du $\mathrm{xv}^{\mathrm{e}}$ siècle, ce n'est plus le paysan qui profite seul de la constitution et de la sécurité du droit de propriété; à peine est-il délivré de la servitude féodale qu'il tombe sous la servitude de l'argent, l'évolution se fait à rebours. Le moreellement continue, mais un groupement parallèle reconslitue les seigneuries et les grands domaines au profit de nouveaux maitres, issus de la magistrature et du négoce. Le paysan achète ou afferme, il emprunte, et l'hypothèque, à l'heure présente, achèverait sa ruine si l'instinct de la conservation personnelle ne remédiait pas à l'impuissance des lois '.

Ce sont ees mouvements alternatifs dont il faut examiner les causes el les effets; il résultera de cette enquête la preuve que la répartition de la propriété rurale dans un très grand nombre de mains a toujours atténué les crises, et que les nations les plus orgueilleuses de leurs richesses industrielles, de leur mouvement commercial et de leur expansion coloniale, telles que l'Angleterre contemporaine et l'Allemagne depuis 1880, ont vu, dans la création artifieielle d'une classe de petits proprićtaires cullivateurs analogues à nos paysans français, l'assietle de conditions économiques et sociales plus rassurantes pour l'arenir que l'état de choses qui leur était coutumier.

L'inlérêt historique des variations de forme du droit de propriété est précisément dans ce lait que, de tous les capi-

1. M. de Saint-Genis, Mémoire sur la mropriété rurale en Bourgogne (Bulletin des sciences économiques el sociales du Comité des travaux historiques, 1896). 
taux, la terre est celui dont, malgré des fluctuations inéritables, la valeur vénale se maintient le mieux, et cela uniquement parce que eette valeur dépend du travail.

La hausse des salaires et l'emploi du crédit sont les deux caractéristiques de ce siècle. Or, la civilisation, abstraction faite du progrès moral, n'étant que la diffusion du bien-être, c'est-à-dire la satisfaction de besoins qui naissent à mesure qu'on dispose des moyens d'y pourroir, il s'ensuit que plus le prix du travail s'élève et plus il y a d'individus participant à l'accroissement et à la distribution de la fortune publique.

C'est grâce à la création de la petite propriété individuelle que le travail, qui n’était rien au début, est devenu tout et fait la loi au capital. La grève et le chòmage sont, à l'époque contemporaine, les deux termes opposés et souvent corrélatifs de ce défaut d'entente qui ne se peut coneilier que par l'arbitrage. En remontant à ses origines, on voit que le capital, à mesure qu'il s'évanouit, ne se reconstitue que par le travail. La terre fut l'élément primitif de cette exploitation; depuis, l'association ${ }^{1}$ a permis d'utiliser par la science et lindustrie toutes les autres matières premières, mais le crédit personncl restera le type du gage; c'est cet élément moral du crédit public, plus nécessaire et plus efficace dans l'industrie agricole que dans toute autre, qu'il faut protéger, exciter, mettre en relief.

Aussi est-il nécessaire, avant de proposer des remèdes, qui ne sont que des expédients tant que l'expérience n'en a pas constaté le mérite, d'examiner le problème agricole sous toutes ses faces et de peser les solulions possibles, aussi variables que les cas toujours complexes qui appellent tour à tour, au gré des érénements et au caprice des crises, l'attention des économistes et des législateurs.

L'étendue du droit de propriété, la sécurité de ee droit, quelle que soit la valeur intrinsèque de la parcelle ou du

1. On retrouve le principe de l'association aux origines les plus reculẻes de l'histoire de l'humanité. Turgot et Adam Smith redoutaient l'association professionnelle qui, d'après eux, avait pour terme fatal une conspiration contre les intérèts du Public. 
domaine, valeur considérable toujours pour l'intéressé direct, sont les premiers points à examiner puisque les règles et les procédés d'acquisition, de consolidation, d'exploitation et de transfert sont différents, suivant qu'il s'agit de la grande, de la moyenne ou de la petite proprićté, et que la possession d'un lot minuscule est ordinairement, avec notre législation imparfaite, plus difficile à établir que celle d'un vaste héritage.

La possession de la terre, ne l'oublions pas, est le but certain de lépargne : pas de ménage sans foyer, pas de famille sans héritage. Tout propriétaire foncier est libéral, parce que la conscience de son droit lui donne celle de son indépendance; il est patriote, parce quilil délient une pareelle de la Patrie ${ }^{1}$. Répétons-le. C'est ce que les pouroirs publics n'ont pas eompris. Il semble, au contraire, qu'ils aient pris à tâche d'entraver le déreloppement de la moyenne et de la petite propriété en multipliant les impòts sur la richesse acquise et les formalités con̂teuses et gênantes sur la fortune en roic de formation; cerles l'ingérence directe de l'État serail déplorable, et la meilleure protection qu'il puisse accorder à la propriété territoriale est de limiter son intervention aux cas indispensables et de se dissimuler derrière l’initiative privée. Là est son vrai ròle : rendre à l'effort en même temps raisonné el spontané des particuliers toute l'élasticité dont il est susceptible. Il le peut en réduisant des deux tiers les droits de mutation dont le taux est scandaleux ${ }^{2}$, en supprimant l'écrasant impot sur les transports à grande vitesse, en réformant les pratiques ruineuses et rexatoires de la procédure ${ }^{3}$.

Nous n’avons ni livres fonciers, ni publicité hypothécaire, ni code rural, ni liberté du crédit: la dette agraire ne cesse

1. M. de Saint-Genis, Procès-verbaux sténographiés de la Commission extra-parlementaire du cadastre (sćance du 26 novembre 1891).

2. Projet de loi déposé a la Chambre par le ministre Burdean, le 8 féirier 189\%, el qui n'a pas élé discuté.

3. La procedure est un moyen de ruiner les meilleures causes, disaiton déjà an xive siecle. (Ordonnance de mars 1336.) 
de s'aceroître, le stellionat est toléré. Certes, la France n'a pas le monopole de la pelite eulture et, dans tous les pays, le moreellement fail des progrès proporlionnels à l'amélioration de l'élal soeial, mais, nulle part, ce mouvement n'est contrarié eomme chez nous; aussi faut-il aux propriétaires ruraux franģais une singuliere énergie, une force de résistance et une puissance d'initialive ineomparables pour avoir pu se maintenir el gagner du terrain malgré la laillite de la loi. Jen citerai un seul exemple parmi des eentaines, paree qu’il se rattache directement à mon sujet. En Belgique, la loi du 21 mai 1897 a réduit de moilié les droits d'enregistrement et de transeription pour les acquisilions de petiles propriélés rurales dont le revenu cadastral n'excède pas 200 franes ${ }^{1}$. En Franee, la loi du 27 juillet 1900 sur la taxe proportionnelle hypothécaire dégrève, pour les formalités hypothécaires seulement, les très petils contrals, mais, à tilre de compensation, en reporte la charge déeuplée sur la moyenne ct la grande propriélé.

Malgré les protestations des éeonomistes et des propriétaires foneiers, le législateur n'a jamais accordé aux transferts immobiliers que des faveur's fictives, et l'intérêt fiscal, avee ses vues exclusives, a maintes fois obtenu le vole, par surprise, de surcharges fori onéreuses pour la proprićté terriloriale; nul, jusqu'iei, n’a signalé les conséquences de ces erreurs économiques. La loi du 27 juillet 1870 , en accordant un tarif de fareur aux échanges d'immeubles contigus, le subordonnait à des conditions qui en restreignaient étrangement l'application. Puis, peu après, le Trésor, reprenant d'une main ce qu'il avait si parcimonieusement aceordé de l'autre, pour des cas spéciaux et assez rares, surtaxail d'un quart ou de $20 \%$ p. 100, par roie indirecte dont l’ingéniosité passa inaperçue, l'ensemble des mutations de biens ruraux.

1. Le législateur belge est particulièrement remarquable par la méthode qu'il apporte dans la réforme raisonnée de ses lois foncières; les plus récentes ont trait à la réduction des taxes fiscales et aux facilités accordées aux sociétés de crédit rural et aux mutualités agricoles ainsi qu'au groupement des petites parcelles. 
L'article 2 de la loi du 21 juin 1873 décida que, dans tous les cas où, conformément à l'article 13 de la loi du 22 frimaire an VII (mutations par (lécès, échanges, donations), le revenu doit être multiplié par 20 et par 10 , il le serait désormais par 20 et 12 1/2, en ajoutant que celte aggravation de taxe frapperait exclusivement les immeubles ruraux.

Les mesures fiscales de ce genre attesteut combien peu il y a de méthode et de vues d'ensemble dans les actes législatifs qui touchent aux conditions économiques de la propriété rurale.

Les terreurs politiques les moins justifiées ont souvent provoqué des réactions regrettables et dont l'incidence a été fàcheuse surtout pour ceux qu'on avail la prétention de défendre en en frappant d'autres. On a prétendu ainsi que la loi libérale sur les échanges du 16 juin 1821, œurre de la Restauration, avait un but aristocratique, et qu'elle tendait à la reconstitution des grandes propriétés; voilà pourquoi l'article 16 de la loi du 21 mai 183 ' a abrogé purement et simplement la disposition exceptionmelle de la toi de 1824 .

Certes, la loi ne doil placer certaines transactions en dehors du droit commun quavec une grande réserre el en vue d'un inlérèt certain et déterminć arec précision. C'est la modicité de la valeur des parcelles rurales qui fixe le degré de protection qui peut leur être accordé; quand les immeubles sont étendus et déjà groupés, il n'y a plus qu'une convenance au lieu d'une nécessité de culture, el leur importance même les aflranchil du besoin d'invoquer un droil privilégié.

Le morcellement el la dispersion des parcelles rurales n’ont pas eu la même origine dans loules les régions; dans les paroisses d'arant 1789 , les plus nombreuses, on peut affirmer avec la certilude quapportent les textes historiques et les actes privés qui ont survécu, que la division par lanières et par écarts était le résultal du mode de distribution de la terre entre les tenanciers nouveaux dont le nombre, accru par des installations successives, nécessitait 
la création postérieure de concessions qui se juxtaposent aux cultures primitives, el cette répartition a persisté jusqu à nos jours, maintenue par la force d'inertie des paysans et leur répugnance à échanger le bien qui leur vient des aïeux. Dans d'autres localités, au contraire, le molif d'éparpillement est inverse. On retrouve des concessions étendues que l'accroissement de la famille a obligé de diviser et de répartir à nouveau ${ }^{1}$. Mais il est impossible de fixer les dates et de généraliser l'hypothèse. On peut, sans se démentir, accepter en celle matière les thèses les plus divergentes.

Ainsi, dans la région de l'Ouest, au midi de la Loire, on rencontre quantité de domaines enchevêtrés les uns dans les autres et divisés en tronçons parfois fort éloignés les uns les autres. Nul doule cependant, affirment les érudits de ee pays ${ }^{2}$, que tous n'aient été, au début, des domaines d'un seul tenant. L'explication de eet état de choses doit être cherchée dans des dédoublements de domaine, exigés par l'accroissement des familles el permis par le défrichement, la suppression des jachères et les progrès de la culture. L'éparpillement des parcelles eut pour cause, comme au moment du bail à cens féorlal, le désir d'équilibrer la valeur des terres et de les répartir avec égalité.

Nos communes, la paroisse de lancien régime, la seigneurie du moyen âge, la villa gallo-romaine de l'origine, présentent, à peu près uniformément ${ }^{3}$, le même groupement de population. Un gros village au centre, des hameaux composés de plusieurs fermes, peu d'habitations isolées, à moins qu'elles ne soient de construction récente, el sauf les moulins. Il est vraisemblable que ces hameaux furent le résultat du dédoublement ou du détriplement d'un vaste domaine primitivement aggloméré, et de l'achat en détail,

1. Voir les travaux d'érudition de MH. Marc de Ilaut, Baudrillart, Tausserat, Rémondière, P. du Maroussem, etc.

2. Les métayers en communauté du Confolentais, 1890.

3. Sauf sur les frontières, où les exigences de la défense contre l'invasion ont imposé le groupement dans des enceintes fortifiées. 
par de petits propriétaires, des débris de l'exploitation primitive ${ }^{\text {. }}$

La division de la propriété n'est pas toujours, comme dans ce cas particulier et dans bien d'autres, la conséquence de circonstances économiques contre lesquelles l'effort des lois serait impuissant et auxquelles il faut se résigner; elle est, souvent, l'efiet des moeurs.

Dans cette seconde alteruative, les lois ne sont pas moins impuissantes que dans la première; il ne faul compter, pour réagir, que sur l'initiative des individus entraînés par le caprice ou l'intérêt. L'absentéisme, par exemple, est l'une des principales causes de la répartition imparfaite de la propriété rurale, ou, pour parler arec plus de précision, des résultats incomplets que produit cette répartition telle qu'elle est à l'époque contemporaine.

L'absentéisme, ainsi que je l'expliquerai avec détail ${ }^{2}$, est plus néfaste pour le présent et l'avenir de l'industrie agricole que ne peut l'être l'émigration des ruraux vers les villes, car il est certain que le relour de la bourgeoisie opulente ou seulement aisée aux champs y ramènerait du même coup la très grande majorité des déserteurs du village.

Les relations du propriétaire avec le paysan sont d’autant meilleures que les contacts sont plus fréquents et la résidence du premier dans son domaine plus prolongée. Ou le propriétaire réside el exploite, avec un personnel dont l'eflectif varie d'après l'étendue dı domaine; on il réside et surveille ses métayers; ou, tout en résidant encore, si ses propriétés sont vastes, il confie à un homme d'affaires le souci des partages; ou, faisant de fréquentes absences, il délègue son autorité à un intendant s'il est très riche, à un régisseur s'il l'est moins, lequel, gérant en même temps

1. Ce qui le prouve, remarque II. du Maroussem, c'est le nombre incalculable de domaines géminés, construits dos à dos et formant clacun un bloc. Le même fait se reproduit, parait-il, daus plusieurs provinces du Norl el de l'Est.

2. Voir ci-après chap. $111, \mathfrak{8} 4$. 
les domaines voisins de plusieurs moyens propriétaires, reconstitue sous sa direction, par la similitude des méthodes el l'unité d'une comptabilité collective, la terre d'autrefois; ou, se désiniéressant absolument de la culture, qu'il réside pendant quelques semaines d'été ou de chasse ou qu'il ne visite jamais son héritage, eas le plus habituel, il prend un fermier el traite à forfail comme arec un banal entrepreneur de cullure.

Selon que le propriétaire a choisi l'une ou l'autre de ces combinaisons, il est évident que ses intérèts sont plus ou moins liés aux méthodes d'exploitation, el son goùt, sa sollicilude pour celte source de revenus plus ou moins vifs. S’il réside sur sa terre, le propriétaire remplit son devoir et, šil exploite à ses risques el périls, il exeree dans toute sa plénitude son rôle de patronage. S'il partage les risques avec des métayers, ou s'il s'en désintéresse en abandonnani à la chance d'un fermier, moyennaut un prix convenu, le profit et la perte, il remplit encore ses obligations dans le premier cas, mais les déserte dans le second. Que peut la loi pour l'y contraindre? Et le premier bénéfice de la liberté civile n'est-il pas de pouvoir user et abuser de son bien?

Il faudrait qu'un autre Jean-Jacques nous ramenit par persuasion vers le métier de paysan comme il y réussit pour les mères en les rapprochant de leurs enfants el en faisant une élégance du plus simple des sentimenis.

L’importance économique de la propriété territoriale ne se discute pas, mais les résultats de son mode de division et de sa répartition proportionnelle entre telle ou telle classe de la société sont si graves, leur incidence est si profonde, leurs contingences si délicales dans leur complexité, quion ne saurait apporter trop de soins à en relever les traces ni trop de prudence à les disculer.

Conclure de la siluation actuelle de la propriété rurale en France à ce qu'elle pourra devenir dans un avenir plus ou moins prochain serail bien risqué si, grâce à des enquêtes très documentées el aux savantes observations des économistes, on ne pouvait constater un courant d'opinion très 
accentué el pressentir, dans les tendances d'aujourd'hui, ce que sera l'esprit de demain.

Toutes les bonnes maximes sont dans le monde, a dit Pascal, on ne manque qu'à les appliquer. Ceux qui ont l'espril de discernement savent combien il y a de différence entre deux faits semblables, selon les lieux et les circonstances qui les accompagnent. Les mêmes paroles sont vivantes ou mortes suivant l'esprit qui les écoute, et la même semence germe ou se stérilise suivant le terrain où elle tombe.

La division de la propriété rurale dans le sens du morcellement ou dans celui du groupement ne vaut point par le fail mais par le résultal; ce résultal lui-même, dans des condilions égales, ne dérive point du système de distribution mais du parti excellent, médiocre ou pire qua su en tirer l'initiative de l'individu; tel grand proprićlaire est improductif s'il n'a ni le goût ni les moyens de la culture, tel pelit arec des ressources médiocres sait en mulliplier la fécondilé. La terre est comme un bon instrumeni de musique qui n'obéit qu'à des doigts exercés. Tant vaut l'homme, tant vaut la terre.

1. - Pourquoi la valeur intrinsèque du propriétaire rural est supérieure à celle du citadin.

Le développement de la propriété foncière, individuelle, dégagée de toute servitude autre que celle de l'impôt, est un résultat récent du progrès social. Les philosophies les plus opposées s'accordent sur le rôle prépondérant que son organisation joue dans l'évolution des socićlés humaines, el l'on ne s'étonne plus aujourd'hui de la parole qui parut fort audacieuse en 1848, que le problème de la propriéts est, après celui de la destinée humaine, le plus grand que puisse se moposer la raison, le dernicr qu'clle parviendra à risoudre'.

1. Proudhon, Contradictions économiques, II, chap. x. 
Pour bien entendre la théorie de la propriété, pour saisir les rapports qui la rallachent aux autres catégories de l'ordre économique, il est nécessairc de reconnaître que, par essence, la philosophie et l'économic politique sont identiques. Si la civilisation a pour objel d'exploiter la nature el d'organiser le travail, la raison el l'expérience enseignent qu'elle ne peut y réussir qu'en appropriant sa pralique aux exigences morales de l'humanité qui se traîne tant qu'elle ne se dégage pas des servitudes de la matière pour s'imprégner d'idéal.

Le système entier de nos connaissances repose sur le sens commun, dit-on; il n'est pas d'occasions où le sens commun ait plus l'obligation de s'affirmer et de s'employer que dans l'utilisation du droit de propriété. La passion de la terre, oblitéréc chez les citadins qui en ignorent les ressources el les joies, reste instinctive chez tous ceux qui ont vécu à la campagne. Même clans notre société fiévreuse et besoigneuse, arec l'attrait des gains faciles, l'amour de la terre a résisté à tout et reste vivace en France, non pas seulement chez les paysans qui aiment la terre avec fureur et sans mesure ${ }^{1}$, mais aussi cliez les hommes qui raisonnent mieux leurs gouts et les subordonnent davantage aux lois de l'expérience. La caractéristique de cet instinct c'est qu'il ramène vers la propriété rurale ceux qui émigrèrent dans les villes et y firent fortune; la possession du droit de propriété n’est véritablement attirante ct ses séductions ne se développent dans toute leur intensité impulsive que s'il s'agil du domaine agricole, petit ou grand.

Comment expliquer ce phénomène, sinon par la nature même de la propriété rurale et, dès lors, ne faut-il pas observer avec attention ce qui distingue la propriété agricole de la propriété urbaine et le paysan du citadin?

Dans les villes, l'exercice du droil de proprićlé n'esl qu'une spéculation; on fait bâtir ou on aclıc̀te des maisons pour les revendre avec bénéfice ou pour les louer. Le propriétaire

1. Léonce de Lavergne, L'économie rurale de la France. 1860. 
surveille de loin ses immeubles, et il peut même se délivrer de ce souci; n'a-t-il pas les compagnies d'assurances qui, pour une rederance modique, garantissen! de tous risques le capital engagé? Et n'a-t-il pas un gérant qui encaisse les loyers? La propriété rurale, au contraire, sauf le cas du fermage qui assimile en une cerlaine mesure les deux catégories de propriétaires, le rural et le citadin, oblige à une action permanente el persévérante. Le rural qui exploite n'est plus un oisif comme le citadin, lequel n'a qu'à se laisser vivre; il faut faire acte d'énergie, d'initiative, de décision, engager des capilaux, préroir à longue échéance, subir les caprices des saisons, des marchés, de la concurrence, et y résister. On s'identifie vite arec le sol, surtout quand on y inet quelque chose de soi-mème par le travail, ei ce sentiment est pour beaucoup dans le prix que nous allachons à la propriété rurale, dès qu'elle n’est pas seulement un objet de luxe et de vanité ou un asile de repos, mais qu'il s'y joint des bois, des prés, des champs, des vignes, dont il faut tirer parti pour vivre.

Le propriétare citadin est d'ordinaire un capilaliste; il faut être riclıe pour possćcler une maison dans une grande ville ou dans un centre industricl, en delıors de tout esprit de spéculation ${ }^{1}$, tandis que le propriétaire rural est liabituellement paurre ${ }^{2}$. Là est le trait en relief qui les distingue l'un de l'autre.

Il en est d'autres; et, même en lenant comple largement des inconvénients et des embarras de l'industrie agricole, dont les causes sont dans nos lois beaucoup plus que dans les contingences économiques, il faut écarter de notre esprit le tableau tragique qu'a Iracé La Bruyère des paysans du xvil ${ }^{\mathrm{e}}$ siecle.

1. A Pirris, la valeur moyenne d'une propriété bâtic varie de 120000 a 500000 francs suivant les quartiers. En 1890, on évaluait à onze milliards la valeur vénale des 83717 maisons ou usines des 80 quarticrs de Paris. (Butletin de statistique, septembre $1890,338$. )

2. Il est surtout écrasé par les trois fleaux de la population agricole : l'impôt, l'lıpothèque, la procèdure. 
Malgré les rapports des intendants de eertaines provinees où, en raison d'accidents particuliers, la misère publique atteignit un degré d'acuité extrème, pendant de courtes périodes, au lieu de généraliser la piloyable condilion des cultivateurs sous l'ancien régime, on s’approchera davantage de la vérité en la localisant, el cette vérité est déjả par elle-mème assez triste pour qu'on ne l'exagère pas ${ }^{1}$. A l'époque contemporaine, la situation a bien changé, et les progrès réalisés depuis surtout cinquante ans sont assez accentués pour satisfaire les plus exigeants ${ }^{2}$.

Une autre différence notable entre le ciladin propriélaire et le rural, c'est que le premier, perdu dans la foule des villes, n'est qu'un passant, un incomnu; l'autre, dont la maison se roit de loin, dont les cultures s'étalent et s'épanouissent, possède une individualité naturelle qu'il rève de perpétuer par ses enfants comme ces moissons qui se renouvellent sous ses yeux. Le citadin jouit de l'heure présente et a des vues courtes; le rural veut associer à son nom une trace durable du travail qu'il a accompli et du bien qu'il a fait.

Les paysans propriétaires eultivant le sol de leur's propres mains, remarque $\mathrm{M}$. Le $\mathrm{Play}^{3}$, forment, entre toutes les classes de la société, celle qui s’inspire le plus de ce sentiment el qui est le plus portée vers la transmission intégrale des biens de famille. En premier lieu, l'affection pour le domaine patrimonial n'est pas moins vive chez le paysan que chez le grand propriétaire; en second lieu, dans les conditions propres à la majeure partie de l'Europe, la transmission intégrale simpose aux familles pour les pelits domaines de paysans plus impérieusement encore que pour les grandes propriétés. Cette nécessité se présente surtout

1. Rẻmondière, Les charges du paysan avant la Révolution de 1is9, 1894. - De Saint-Genis, IIistoire économique d'une commune rurale du $X I I^{\circ}$ au $\mathrm{VIX}^{\mathrm{e}}$ siècle en Bourgogne, 1896.

2. D'Avenel, Histoire économique de la France, chap. Ix et x, 189:. De Foville, IIstoire de l'habitation en France, 2 vol., $189 ;$ et 1899.

3. La réforme sociale en France, édition de 1S66, I, : 19. 
pour ces petits domaines enclos et boisés avec habitation centrale, qui donnent tant de charme et de vie aux États scandinaves et allemands, à la Suisse, aux collines ou aux montagnes de l'Italie, de l'Espagne et de la France.

Un autre philosophe, certes bien différent d'esprit et de vues, ne dit-il pas à son tour :

La famille rustique est depuis des siècles penchée comme lc bœuf sur le sillon; elle récolte et elle ne consomme pas. Tous les arts naissent de l'agriculture el l'agriculteur reste étranger toute sa vie anx jouissances que ces arts développent. Il semble que la malédiction ait passé sur cette race condamnée aux rudes travaux, ct pourtant quelle existence au monde fut jamais plus digne d'intérêt: Dans sa famille, le paysan, bien différent de l'ouvrier des villes, est grave et hon; dans les champs, il est le compère de la nature. 11 faut le voir regarder l'heure au soleil d'un air avisé, deviner à certains signes la préscnce occulte des sources, prédire la veille si la journée du lendemain sera belle on pluvieuse. Nul n'est plus chez lui que le paysan dans l'univers. Et cependant l'état de malaise dans lequel végètent nos campagnes remonte jusqu'aux origines mèmes de notre nation ${ }^{1}$.

La propriété bàtie ou urbaine, qui se suffit à elle-ınème, est une sorte de propriété industrielle; une fois construite et aménagée, elle vit de sa vie propre jusqu'à ce yu'un accident la délruise ou qu'elle tombe de vétusté si on ne l'entretient pas. La propriété rurale, au contraire, ne vaut que par les soins incessants, le labeur de toutes les heures, la culture prévoyante et persévérante; l'habitation n'y est qu'un accessoire, elle fait partie de l'outillage agricole comme la charrue et le bétail. Dans les villes, l'ouvrier, l'artisan vivent de leur industrie, de leur salaire, ils sont mailres de leur travail el s'en approprient les fruits sitòt que l'œuvre est faite; le citadin proprićtaire, lui-mème, a pour unique instrument le temps qui accumule les fruits civils, lesquels tombent dans ses mains à des échéances certaines et il a pour garantie la présence de ses locataires. La propriété territoriale court d'autres risques que la proprićté urbaine immobilière; les fruits du trarail ne s'y récoltent qu'i des

1. Alph. Esquiros, Les paysans. 
échéances lointaines, l'exploitation en est compliquée, les profits irréguliers, la sécurité douteuse; le laboureur, le vigneron, usent leur existence ì se débattre contre les intempéries, contre leurs roisins, contre la dette et les hommes d'aflaires.

Et cependant, la propriété rurale a, dans tous les temps, conservé une valeur intrinsèque à laquelle le pouvoir de l'argent aux différentes époques de la civilisation française peut servir d'ćtalon. La mode, le gout, les habitudes se modifient, la vie des citadins se rétrécit ou s'élargit; les centres d'aclivité se déplacent; les variations économiques et la multiplicité des échanges abaissent de plus en plus le loyer de l'argent, et sa valeur nominale et la fortune mobilière est à la merci de brusques caprices et de tous les accidents. La terre, par contre, est immuable, et la généreuse nature, pour peu ru'on l'y aide, y ramène régulièrement la fécondité. Quelles que soient les différences que puissent présenter entre eux, selon leur fertilité, leur situation, et surtout le zèle et l'ingéniosilé du cultivateur, deux hectares de terre, un labour, un pré, une vigne ne cessent pas de rapporter les mèmes produits. On les cultive avec plus ou moins d'ardeur, on les exploite avec un succès variable, mais le sac de blé, la botte de foin, le tonneau de vin qu'on y récolte sont pareils aux produits d'il y a dix siècles, et comme il faut vivre, ces profits de la terre restent comparables et le prix réel n'en varie que par les oscillations de l'offre et de la demande. Aussi la prospérité de l'agriculture est-elle étroitement dépendante de la densité de la population, c'est-ìdire du voisinage des débouchés et de la certitude de la vente.

La propriété rurale, en effet, non seulement fait vivre ceux qui la cultivent, mais leur laisse en temps normal, abstraction faite, bien entendu, des cas de sinistres, d'épidémies ou de guerre, un excédent à convertir en argent, et c'est l'avantage que l'industrie agricole a sur toutes les autres où l'on est obligé de retenir le coût de la vie sur le profit brut du travail. 
Un économiste a insisté, en accumulant les preuves ${ }^{1}$, sur ce caractère spécial à la propriété foncière et qui demeure plus particulièrement encore l'apanage de la propriété rurale. Par comparaison avec les valeurs mobilières dont le numéraire est l'expression usuelle, la démonstration en a été faite maintes fois. M. d'Avenel établit que si deux capitalistes dı xil siècle araient employé chacun une somme identique (21 $750 \mathrm{fr}$. de notre monnaie équivalant, en 1201, à la valeur de 161 hectares de labour en plein rapport), I'un à des placements en prèls, l'autre à une acquisition de terre, et que leurs héritiers eussent continué, arrivés en 1790, le terrien posséderait toujours ses 161 heetares, d'une valeur rénale de 122500 franes et d'un rapport de 42000 franes, el le rentier', ruiné, n'aurait plus qu'un capital de 9500 francs à l'intérêt de $1 \mathbf{z}$ franes : à peine de quoi acheter, à cette date de 1790 , un unique hectare de labour.

J'ai moi-même rappelé, ailleurs, le fait de cette fondation de deux lampes dans une chapelle de Notre-Dame de Poitiers, en 1346, dolée I'une par 10 llorins ou franes $d$ or (valant chacun une livre tournois), lautre par un demi-setier de blé (environ $7: 3$ litres, payant à cette époque deux paires de souliers et valant 10 sols tournois); la première s'est éteinte, par l'évanouissement du capital, en 1787, la seconde brûle toujours. A valeur égale, une rente en grains offre donc infiniment plus de garanties, malgré les soubresauts des cours, qu'une rente en argent. On le vit bien, à partir du $\mathrm{xr}^{\mathrm{e}}$ siècle, par l'aflaiblissement graduel des censives féodales payables en numéraire el qui, au xrul siècle, se trouvaient réduites à rien ${ }^{2}$.

L'histoire de la propriété rurale démontre que sa plusvalue actuelle provient surtout de l'extension de la culture.

Il n'est pas douteux que la spéeulation foncière a enrichi depuis sept cents ans de nombreuses générations de paysans,

1. M. l'Avenel, dans son IIistoire économique de la propriete, des salaires, res denrées el des mix, de l'an 1200 à l'an 1800.

2. De Saint-Genis, Monographie de la commune de Chassey-en-Auxoi. (Còte-d'Or), 1897. 
devenus peu à peu propriétaires, mais cela tient moins au fait de l'acquisition en lui-mème qu'à ce que les noureaux détenteurs sont parrenus à tirer parti de tous leurs fonds, quelque mauvais ou médiocres qüils pussent ètre.

De 1790 à 1890, le territoire agricole de la France s'est agrandi de plus de 8 millions d'hectares qui procurent à leurs possesseurs un revenu de $\mathbf{4 0 0}$ millions de francs par an. Le sol des villes ne se prète pas à de si fructueuses entreprises et le prix de construction d'une maison ne peut se comparer à la faible mise de fonds qu'exige le défrichement d'un petit bois ou le desséchement d'une mare.

\begin{tabular}{|c|c|c|c|c|}
\hline \multirow{2}{*}{ APPROPRIATION DU SOL } & \multicolumn{2}{|c|}{ TOMBRE D'HECTARES } & \multicolumn{2}{|c|}{ DIFFÉREXCE POUR I $\$ 90$} \\
\hline & 1790 & 1890 & En plus & En moins \\
\hline Labours . . . . . . . . & 21000000 & 27000000 & 6000000 & \\
\hline 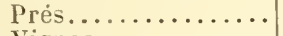 & 3750000 & $\ddot{5000000}$ & 1230000 & $\ldots \ldots \ldots$ \\
\hline Vignes............ & 1650000 & 2300000 & 650000 & $\ldots \ldots$ \\
\hline Jardins............. & 500000 & 700000 & 200000 & $\cdots \cdots \cdots$ \\
\hline 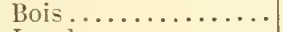 & 12500000 & 8400000 & $\ldots \ldots \ldots$ & \pm 100000 \\
\hline \multirow[t]{2}{*}{ 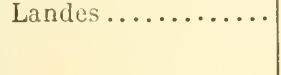 } & 10000000 & 6700000 & $\ldots \ldots \ldots$ & 3300000 \\
\hline & 49400000 & 49400000 & 8100000 & 7400000 \\
\hline
\end{tabular}

Les améliorations foncières réalisées pendant la période décennale de 1882 à 1892 ne sont pas moins instructires; elles le seraient davantage encore si on pourait avec précision faire la part relative dans ces progrès soit à la grande propriété, soit à la petite. D'après les renseignements d'ailleurs assez confus puisés dans les statistiques officielles et les présomptions que donne l'observation directe des faits, on peut admettre avee beaucoup de rraisemblance que la moyenne propriété n'y entre que pour une quantité négligeable, d'abord parce qu'elle ne dispose pas des capitaux néccssaires, ensuite parce que, à défaut de capitaux, elle n'a pas le stimulant de l'exploitation personnelle et directe ni l'encouragement du profit immédiat.

Quant à l'initiative comparée du grand et du petit propriéLA PROPRIÉTÉ RƯRALE EN FRANCE. 
taire, provoquée celle-ci par le goût de la nouveauté combiné avec lappétit de la spéculation luerative, celle-là par la nécessité de faire produire le plus possible au peu qu'on a, on peut approximativement en répartir les résultats comme suit :

AMELIORATIONS FONCIERES REALISEES DE 1882 A 1892

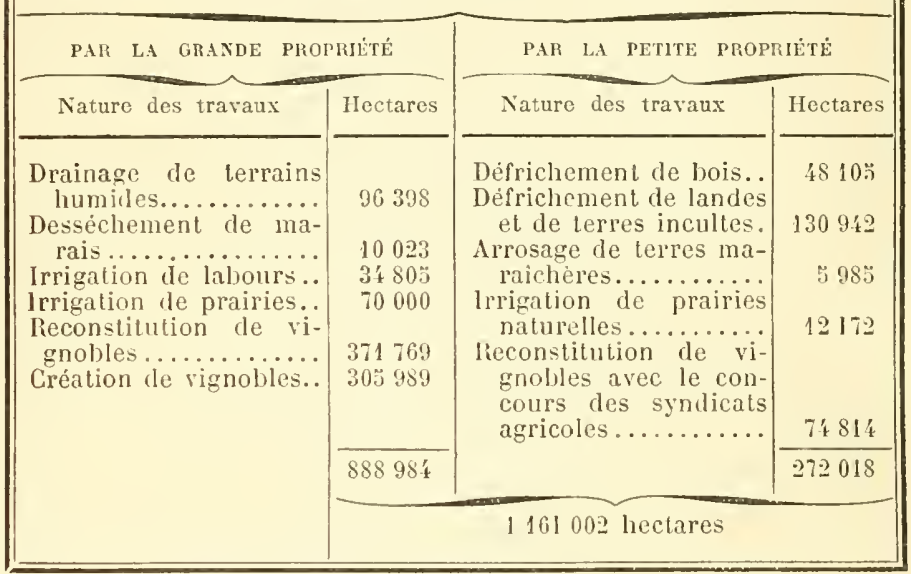

En ne tenant compte que des résultats de l'initiative privée, je déduis les $11 \% 106$ hectares reboisés par l'Élat et les Communes en exécution de la loi du 4 avril 1882 sur la conservation et la restauration des terrains en montagne.

Pour conclure, un résultat indiscutable - et, il laut l'avouer, difficile à expliquer - de l'évolution de la propriété rurale ì travers les vicissitudes qu'elle a traversées, c'est que, du жпा au $x x^{e}$ siècle, si sa valeur en capital a décuplé, son revenu n’a que triplé ${ }^{1}$. La terre a infiniment

1. Le rapport du revenu au capital, qui était, jusque vers la fin du $\mathrm{xv}^{\circ}$ siècle, de $10 \mathrm{p} .100$, ne doit plus être évalué, à partir de 1\}.76, qu'à $1 / 12(8,33$ p. 100), du denier 12 il tombe au quatorzième (7,14 p. 100) vers 1531 , et au seizième $(6,25$ p. 100) vers 1576 , enfin on ne l'estime plus aujourd'hui qu'à 3 ou 3,33 p. 100 . Un capital foncicr te 1000 francs, qui rapportait 100 francs au xu1 siecle, en vaut en 1899 au moins 10000 et ne produit que 300 francs. (Ilistoire économique, I, clap. vil, p. 360.) 
plus augmenté de prix que le pouvoir de l'argent n'a baissé, de telle sorte que l'échelle du progrès dans l'état de la civilisation contemporaine peut s'établir ainsi pour les trois grandes catégories d'industries, et c'est à l'honneur de l'humanité, puisque la rémunération du travail est proporlionnelle à son intensité directe.

Celui qui a le plus proficé des érolutions économiques successives, c'est le rendeu de travail, l'ouvrier, dont le salaire a augmenté dans les proportions que l'on sait; vient ensuite le proprićlaire rural, et surtout le propriétaire exploitant qui vit sur son hérilage et vend le superflu de sa production; mais le détenteur mobilier, le rentier, qui, ne travaillant pas, a perdu, sauf s’il a spéculé sur la misère d'autrui, cas fréquent et qui a fait de eeux qui réussirent les maitres des autres, maìtres souverains mais détestés.

L'histoire de la propiété urbaine est beaucoup plus difficile à élucider que celle de la propriété rurale. D'une valeur médiocre et longtemps stationnaire, elle a acquis des plusvalues subites à deux époques et pour des motifs qui ne se ressemblent guère. Dans les grandes villes et surtout à Paris, pendant tout le xrue siècle, elles eurent pour cause, à la fois, l'émigration de la noblesse quittant la provinee pour la cour, le luxe des financiers enrichis et anoblis, et, par voie de conséquence, le renchérissement des terrains et celui des matériaux de construction. Puis, dans tous les centres industriels, à partir de la seconde moitić du $\mathrm{xxx}^{\mathrm{e}}$ siècle, les plus-values, plus rapides encore qu'au xrri ${ }^{e}$ siècle, furent provoquées par des causes qui s'engendraient l'une l'autre: découvertes de la science appliquées à l'industrie, nombreuses entreprises industrielles et commerciales, hausse des salaires, afflux dans les villes des ouvriers des campagnes, spéculations sur les terrains, création de puissantes sociétés immobilières, mobilisation dı droit de propriété sous forme de titres d'un transfert facile el d'un prix coté. Malgré ces avantages apparents, en dépit des obstacles qu'une législation fiscale anti-économique aceumule devant les mutations de la propriété rurale, celle-ci 
conserve les préférences de tous ceux qui mettent la sécurité du placement au-dessus des promesses de gain el qui se confient plus volontiers au travail qu'au hasard.

Aussi, quels que soient les risques dont les écoles collectivistes menacent la propriété foncière, elle n'a rien à redouter; elle ne cessera point d'être la base la plus solide de la société parce que son appropriation individuelle et privée est le fondement nécessaire de l'héritage et que, sans héritage, il n'y a pas de famille.

La propriété rurale, en parliculier, sous toules ses formes, qu'elle soit grande, moyenne ou petite, deviendra de plus en plus l'objet des convoitises des capitalistes et des sacrifices des travailleurs. La propriété urbaine, à còté d'elle, n'est qu'un accessoire. Leur ròle est aussi différent que leur clientèle de propriétaires; l'urbaine n'est qu'un objet de spéculation, la rurale est un instrument de travail et de moralisation. La valeur d'une maison de ville dépend surtout du milieu où elle est située, de son affectation; son prix, à égalité d'importance et de solidité, est très variable suivant qu'elle est placée dans une ville ou dans une autre, et, dans la même ville, suivant les quartier's.

La propriété bâtie, qui n'a acquis de valeur relative sérieuse que depuis la fin du $\mathrm{xvr}^{\mathrm{e}}$ siècle, a suivi depuis lors les mêmes oscillations de prix que la propriété rurale; mais tandis que le loyer des maisons de ville subissait, selon les époques, de fortes augmentations puis de soudains reculs, celui des habitations villageoises restail à peu près stationnaire. Dans les villages, les pelits loyers de maison ont angmenté de 100 p. $100 \mathrm{du} \mathrm{xvI}^{\mathrm{e}}$ siècle au $\mathrm{xx}^{\mathrm{e}}$. Dans les villes, c'est la valeur vénale du sol qui a laaussé par l'effel de la concurrence des offres.

Les variations de prix du sol parisien nous révèlent que, si la fortune mobilière fut l'éternelle victime des révolutions économiques, si la propriété foncière rurale les a, au contraire, traversíes toutes sans encombre, et quelques-unes avec profit, la propriété des grandes cités a été la privilégiée des temps modernes. Les mortels favorisés qui ont 
hérité de leurs pères un lof de ces quelques kilomètres earrés eomposant la superficie actuelle de Paris, ont vu leur avoir non pas quintuplé ou décuplé, mais augmenté, depuis le moyen ìge, de un à deux mille ${ }^{1}$. Ce phénomène n'est pas particulier à Paris ${ }^{2}$; à Londres, le progrès de la richesse immobilière continue à suivre une lausse rapide; de 1874 à 1891, les valeurs imposables, qui servent à lassiette des taxes locales, ont passé de 20 millions de livres sterling à $34^{3}$.

Le développement inonï des valeurs mobilières, malgré le déplacement continu des capitaux, qui n'avaient autrefois d'autre emploi que de médioeres commandites et l'acquisition de la propriété foncière, a enrayé mais n'a pas arrêté le mouvement de la division des terres.

En France, de 1826 a 1891, le nombre des cotes foncières de la propriété non bàtie a passé de 10 millions à 14 el, pour la saine interprétation de ces chiffres, il ne faut pas oublier les eflorts de l'Administration des Contributions directes pour obtenir, dans toutes les communes, la réunion des cotes multiples concernant un même propriétaire.

En Belgique, le nombre des articles des ròles fonciers de 1834 à 1882 , a passé de 943 milliers à 1160 , et des augmentations analogues se relèvent dans tous les pays, indiquant, avec des temps d'arrèt ou de subites progressions suivant les périodes, la continuité du mouvement qui morcelle les anciens domaines ou défriche les terres incultes pour créer de noureaux centres d'activité agricole.

En Hollande, les statistiques de 188: dont les proportions,

1. L'hectare de terrain compris dans les 20 arrondissements actuels de Paris valait en moyenne, au $x_{1}^{\circ}{ }^{\circ}$ siècle, 652 francs de notre monnaie; il vaut aujourd'hui 1297000 francs. Autrement dit, le mètre carré est monté, dans cet intervalle de six cents ans, de 6 centimes $1 / 2$ à 130 francs. - Voir De la valeur des terrains et immeubles à l'aris, à différentes époques, par II. de Saint-Genis (Journal de la Société de statistique de Paris, 1893̈).

2. La propriété batie à Paris, avec cartes (Bulletin de statistique, septembre 1890).

3. Les valeurs immotilières à Londres (Bulletin de statistique, septembre 1891, p. 291). 
m'assure-t-on, n'ont pas sensiblement varić dans celles de 1894, marquent quel est le caractère de l'écart qui différencie la propriété urbaine el la propriété rurale. Les 337449 contribuables de la propriété bàtie paient 79 millions de florins à l'impôt foncier et les $58158 \%$ colisés de la propriété rurale seulement 46 ; mais, parmi ces derniers, 415367 possèdent des biens d'un revenu imposable de moins de 203 florins, landis que les citadins de celte catégorie ne sont qu'au nombre de 48699 .

D'après de récents traraux ', le capilal rural représente $52,40 ̈$ p. 100 el le capital urbain 47,5030 p. 100 de la fortune immobilière globale de la France. De l'aveu des économistes $^{2}$, la petite épargne est devenue aujourd'hui, dans son ensemble, le grand réservoir de la fortune mobilière française; il ne faut pas le regretler, les petits propriétaires ruraux qui savent épargner font par ces placements un apprentissage instructif et seront mieux préparés pour former entre eux des syndicats de crédit mutuel et rapporter à la terre la semence d'argent qu'ils en ont tirée.

C'est sous l'impression de craintes semblables qu'on s'efraie de l'augmentation croissante de la dette communale qui, s'inspirant fùcheusement de l'exemple de la dette publique, s'est accrue de plus de 32 millions en capital de 1890 à 1894; mais il y a une alténuation à ce péril, c'est la participation des habitants aux emprunts par le classement local des émissions. La statistique des valeurs successorales nous rassure à ce sujel; en 1898, il a élé déclaré 400 millions de franes sur le milliard de titres émis par les communes de moins de 10000 habitants ${ }^{3}$. Toul ce qui peut utiliser dans la commune même l'argent qu'clle produit, toul ce qui peut attirer le crédil vers la propriété rurale est, au point de vue de la meilleure distribution du sol, d'un excellent eflet économique.

1. Journal de la Sociélé de slatistique de Paris, décembre 1899.

2. Théry, L'économisle européen, 16 mars 1900, p. 359.

3. Bulletin bleu (septembre 1892), Annuaire statistique (1896, p. 191), Bulletin gris (1899, p. 187). 
II. - Importance comparée, à l'époque contemporaine, des propriétés urbaine et rurale, d'après la qualité de leurs détenteurs.

On est convenu, en France, de désigner sous le nom de rurale toute commune qui compte une population agglomérée inférieure à 2000 habitants, d'urbaine celle qui en compte plus de $2000^{1}$.

D’après le recensement de 1891 , le dernier dont les résultats détaillés aient été publiés au moment où jécris, les chiffres respectifs de la population urbaine et rurale sont les suivants :

$$
\text { Population }\left\{\begin{array}{lll}
\text { urbaine.... } & 14311292 \\
\text { rurale..... } & 24031900
\end{array}\right\} 38343192 \text { habitants }
$$

Depuis 1816, ces deux catégories de population ont varié en sens inverse dans les proportions ci-après :

\begin{tabular}{|c|c|c|}
\hline \multirow[b]{2}{*}{ ANNÉES } & \multicolumn{2}{|c|}{$\begin{array}{l}\text { PROPORTION POUR } 100 \\
\text { DE LA POPULATION TOTALE }\end{array}$} \\
\hline & Urbaine & Rurale \\
\hline 1846. & 2,4 & 75,6 \\
\hline $185 \% 1$. & 25,3 & 74,5 \\
\hline 18.56. & 27,3 & 72,7 \\
\hline 1861. & 28,9 & 71,1 \\
\hline 1866. & $30, \ddot{z}$ & 69,5 \\
\hline $1879 .$. & 31,1 & 68,9 \\
\hline 1876. & 32,4 & 67,6 \\
\hline $1881 .$. & $3+, 8$ & 60,2 \\
\hline i886.. & 35,9 & 64,1 \\
\hline $1891 .$. & 37,4 & 62,0 \\
\hline
\end{tabular}

L'accroissement de la population urbaine aux dépens de la population rurale n'a donc fait que s'accentuer depuis un demi-siècle, et le seul moment d'arrêt a été causé par la

1. Tout arbitraire qu'elle puisse paraitre, cette distinction, établie depuis cinquante ans par les rédacteurs de la statistique générale de la France, a l'avantage de donner des résultats parfaitement comparables entre eux, la même méthode ayant été suivie pendant 10 dénombrements consécutifs. 
guerre de 1870. La population rurale a perdu 13 p. $100 \mathrm{el}$, si le mouvement continue avec la même régularité, l'équilibre entre les deux effeclifs se réalisera vers 1920 .

La moyenne générale pour l'ensemble du territoire est de 63 p. 100 (ruraux) contre 37 p. 100 (ciladins); elle varie avec les régions, et la comparaison des départements permet de noter ceux oì elle est influencée par le voisinage des grandes villes; ainsi, dans le Nord, elle tombe à 33 p. 100, dans la Seine-Inférieure à 400 p. 100, dans les Bouches-duRhône à 17 p. 100, dans la Seine à 0,9 p. 100 .

La proportion des ruraux, qui est de 92 p. 100 dans la Haute-Savoie, de 90 p. 100 dans les Còtes du Nord et dans la Creuse, de 88 p. 100 dans la Lozère et dans les Landes, de 86 p. 100 dans la Savoie et les Deux-Sèvres, de 83 p. 100 dans la Corrèze, de 80 p. 100 dans l'Yonne, de 70 p. 100 dans la Côte-d'Or, tombe à 30 p. 100 dans le Rhòne, à 17 p. 100 dans les Bouches-du-Phòne. C'est en Bretagne, dans le Poitou, dans la région du Massif Central, dans les Alpes de Savoie, dans le Gers et les Landes, que l'élément rural subit les moindres dépressions et tend à rester stationnaire.

C'est dans la Marne (de 28 p. 100 en 1830 à $4: 0$ p. 100 en 1891), la Meurthe-et-Moselle (de 20 à 40 ), le Nord (de 403 à 63̈), le Pas-de-Calais (de 29 à 39), que les citadins se sont le plus agglomérés; dans la Dordogne, la population urbaine a doublé de 1830 à 1891 .

Le dénombrement de 1891 constale, sans l'expliquer, que l’excédent de l’immigration dans les villes a été de 18897 indiviclus, pendant que l'excédent d'émigration des campagnes s'élevait à 3803223 têtes ${ }^{1}$.

La dépopulation graduelle des campagnes frappe surtout

1. Cette différence de 66326 unités provient sans loute de ce que les statistiques annuelles classent dans la calégroric urlaine les communes de plus de 2000 lıbitanls tandis que le déuombrement ne tient compte dans ses developpements par repartition que des villes de 10000 labitants, a moins, comme certains publicistes l'ont cru, que l'écart provienne d'un fait nouveau, l'émigration à l'étranger de 66326 nationaux. 
les très petites communes, comme le montre, à quaranle ans (ie distance, ce seul rapprocliement de chiffres :

\begin{tabular}{|c|c|c|}
\hline \multirow[b]{2}{*}{ AXXÉEs } & \multicolumn{2}{|c|}{$\begin{array}{l}\text { NOMBRE TE COMMUNES AYANT UNE } \\
\text { POPULATION TOTALE DE }\end{array}$} \\
\hline & $\begin{array}{l}\text { Moins do } 500 \\
\text { habitants }\end{array}$ & $\begin{array}{l}\text { De } 500 \text { à } 1000 \\
\text { habitants }\end{array}$ \\
\hline $18 \ddot{1} 1 \ldots \ldots \ldots \ldots \ldots \ldots \ldots \ldots$ & 13684 & 11930 \\
\hline 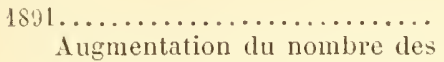 & 17390 & 10169 \\
\hline petites communes............ & 1906 & 1786 \\
\hline
\end{tabular}

La presque totalité des communes rurales (33.400 sur 36 144) voient leur population diminuer et émigrer, pour la plupart d'entre elles, au profit d'environ 400 villes de plus de 3000 habitants. On comptait déjà, en 1891, 876 communes ayant moins de 100 habitants; 3 en avaient moins de 19. C'est le département du Doubs qui renferme le plus de ees minuscules municipalités (93); vient ensuite le Jura (14).

La répartition entre les communes des deux catégories de population peut done se synthétiser sous cette forme :

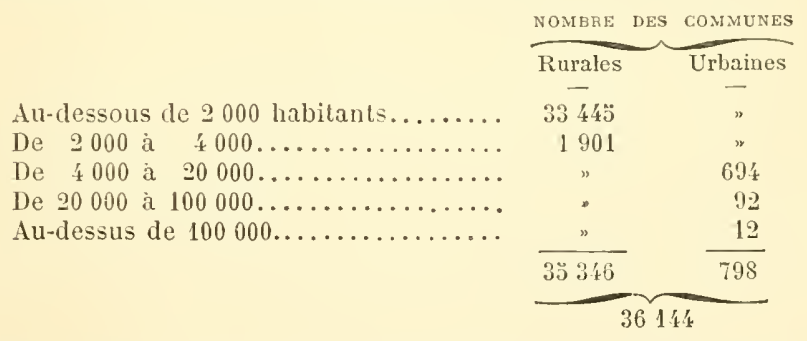

Ces données d'ensemble peuvent se compléter, se contrôler el se particulariser à l'aide de statisliques nouvelles, dont on a critiqué la précision, mais qui n'en restent pas moins de précieuses et uniques sources d'informations, je veux parler de la double enquête suivie par l'Administration des Contributions directes.

L'une, prescrite par la loi du 9 aoùt 1879, avait pour objet le recensement des propriétés non bities (propriétés rurales) et l'évaluation de leur revenu foncier; la seconde, ordonnée 
par les lois des 8 aoùt 1883 et 8 juin 1887, visait spécialement la propriété bâtie (urbaine et rurale) ${ }^{1}$.

J'utilise dans ce travail, en les contrôlant les uns par les autres, les chiffres de ces enquêtes quand ils sont comparables ${ }^{2}$, ceux du dénombrement général de 1891 publiés en 1894, et ceux de l'enquête décennale agricole de 1892 publiés en 1897. Les légères différences qu'on y peut trouver s'expliquent, sans se justifier, par le défaut de concordance des rubriques administratives, mais n’influent pas sur le sens général des conclusions qu’on en peut tirer.

Le nombre des cotes foncières pour chaque calégorie de propriété montre leur proportion relative et de combien le nombre des cotes rurales, par l'effet du morcellement et de la dispersion, est supéricur à celui des cotes supposées urbaines.

NOMBRE DES COTES FONCIĖRES D'APRÈ LES RÔLES

\begin{tabular}{|c|c|c|}
\hline MPORTANCE DES COTES & $\begin{array}{c}\text { PROPRIÉTÉ } \\
\text { NON BÀTIE } 1894\end{array}$ & $\begin{array}{l}\text { PROPUE⿱TÉ } \\
\text { BATIE } 1891\end{array}$ \\
\hline \multirow[t]{2}{*}{$\begin{array}{l}\text { Cotes au-dessous de } 20 \text { francs.... } \\
\text { Cotes au-dessus de } 20 \quad-\ldots .\end{array}$} & $\begin{array}{r}11609603 \\
2347925\end{array}$ & $\begin{array}{l}1006205 \\
3580980\end{array}$ \\
\hline & 13937528 & 6587183 \\
\hline
\end{tabular}

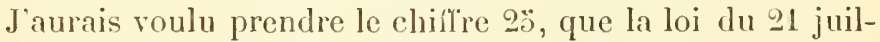
let 1897 sur le dégrèrement des petites coles foncières considère comme une limite, mais les statistiques vont de 20 ì 30 ; l'argument n'en aura que plus de force. Ce manque de symétric et l'emploi de méthodes differentes dans nos administrations publiques à propos d'éléments semblables sont

1. Le service des Contributions directes tantôt comprend la ville de Paris flans ses tableaux et tantôt l'en élimine en lui consacrant des relevés spécianx, mais sans dire toujours si elle en est ou non exclue.

2. Les résultals en ont été pullliés par le Bulletin de statistique (mai 1883, décembre 1888, novembre 189., février 189ä, etc.); malheureusement, on a négligé d'y faire le classement qui interessait le plus les études économiques, èest-à-dire la distimction entre les habilations rurales, centres ou annexes d'exploitations agricoles, et les maisons de ville, logements d'artisans et d'ouvrier's industriels, etc. 
regrettables, car elles òtent toute précision aux recherehes et contrarient les rapprochements.

En 1879 , le recensement de la propriété non bâtie avait fourni des chiffres sensiblement différents, et l'on n'a pas expliqué le pourquoi de cet écart.

PROPRIÉTÉ NON BÂTIE

Nombre des cotes

14234237 .
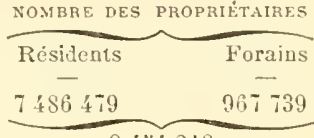

Par 1000 habitants, on trouve 23: propriétaires ruraux contre 766 membres de la famille ou ouvriers agricoles; sur 1000 feux, il y a 859 foyers de propriétaires et 131 qui ne le sont pas dans la commune.

L'enquète sur la propriété bàtie (de 1887 à 1889) a permis de recenser plus de 9 millions de maisons dont voici le détail, trop pris en bloc malheureusement :

Maisons d'une valeur locative de 20 à 2000 francs. \& $\$ 70689$

Grandes maisons et châteaux............... 4383 śt

Usines et locaux industriels............... 137019

Bàtiments affectés à des services publics....... 903039

Ensemble des propriétés bâties......... $921 \% 7181$

Si l'on retranche du nombre total des maisons d'habitation d'un revenu fiscal inférieur à 2000 franes celles recensées dans les sept départements où la population industrielle figure pour plus de 40 p. 100 de la population totale, de façon à ne conserver que les départements agricoles, l'excédent pouvant à peu près se compenser avec l'importance de la propriété urbaine de ces derniers ${ }^{1}$, il reste 7 696660 maisons quion peut appeler rurales; sur lesquelles le nombre des maisons n'ayant qu'un rez-de-chaussée ou un étage s'élève à 6648484 . Le nombre des ménages rapproché de celui des maisons ne peut pas davantage servir de contrôle, les recenseurs des trois enquêtes de 1S79, 1887

1. Les Ardennes, le Nord, le Pas-de-Calais, la Loire, le Rhône, la Seine et la Seine-Inférieure: 1174029 maisons. (Résultats statistiques du dénombrement de 1:981, graphique $n^{\circ}$ 9.) 
et 1891 ayant omis de noter le nombre des maisons rurales ou urbaines occupées par un seul ménage '.

Le revenu net imposable de la propriété non bâtie est, en 1894, de . . . . . . . . . 2523939071 franes.

Celui de la propriété batie de . . 2148976897 "

S'il est difficile, avec les éléments administratifs dont nous disposons, d'établir arec certitude l'importance en superficie, en nombre et en valeur de la propriété urbaine et de la propriété rurale, il ne l'est pas moins de préciser le nombre des individus qui vivent de l'industrie agricole, soit comme propriétaires du sol et exploitants directs, soit comme forains non résidents, soit comme membres de la famille ou collaborateurs salariés.

D'après le dénombrement de 1891, la population agricole s'élève à 17433088 individus (page 283).

La population urbaine étant de seulement 14311292 habitants, la population rurale est portće à 2:1031900 (page 379).

Enfin, la population éparse dans les hameaux et les écarts des communes, population dont le caraclère est dès lors suffisamment défini, est de 14061623 (page 37\%).

RÉPARTITION DE LA POPULATION AGRICOLE EN COMBIYNNT LES ÉLEMEXTS FOURXIS PAR LES CUIFFRES DU DEXOMBREMEXT DE 1831

\begin{tabular}{|c|c|c|c|c|c|c|c|}
\hline \multirow{2}{*}{$\begin{array}{l}\text { GROUPES } \\
\text { DE LA } \\
\text { POPULATION } \\
\text { AGRICOLE }\end{array}$} & \multicolumn{3}{|c|}{ SEXE MASCULIN } & \multicolumn{3}{|c|}{ SEXE FEMININ } & \multirow{2}{*}{$\begin{array}{c}\text { TOTAL } \\
\text { DE CHAQUE } \\
\text { GROUPE } \\
\text { AGRICOLE }\end{array}$} \\
\hline & $\begin{array}{c}\text { Adultes } \\
\text { de } \\
20 \text { a tio ans }\end{array}$ & $\begin{array}{c}\text { Infanis } \\
\text { et } \\
\text { vieillards }\end{array}$ & TOTAL & $\begin{array}{c}\begin{array}{c}\text { Adultes } \\
\text { de } \\
20 \text { à } 60 \text { ans }\end{array} \\
.\end{array}$ & $\begin{array}{c}\text { Enfants } \\
\text { et } \\
\text { vicillards }\end{array}$ & TOTAL & \\
\hline $\begin{array}{l}\text { Patrons ...... } \\
\text { Employes .... } \\
\text { Ouvriers ..... } \\
\text { Popul. active.. } \\
\text { l'amilles ..... } \\
\text { 1)omestigues... } \\
\text { lopul. inactive. } \\
\text { Population pro } \\
\text { fessionnelle.. }\end{array}$ & $\left\{\begin{array}{rrr}2 & 126 & 998 \\
& 27 & 117 \\
1 & 013 & 831 \\
3 & 197 & 2.19 \\
899 & 160 \\
161 & 130 \\
1 & 060 & 590 \\
4 & 258 & 139\end{array}\right.$ & 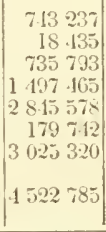 & 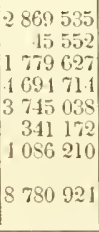 & $\begin{array}{rrr}455 & 198 \\
16 & 731 \\
598 & 931 \\
1 & 070 & 863 \\
3 & 018 & 311 \\
161 & 337 \\
3 & 179 & 678 \\
1 & 250 & 511\end{array}$ & 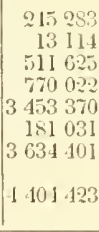 & $\begin{array}{rrr}700 & 181 \\
29 & 815 \\
1 & 110 & 5.56 \\
1 & 8.10 & 885 \\
6 & 471 & 711 \\
312 & 368 \\
6 & 81.1 & 079 \\
8 & 651 & 904\end{array}$ & 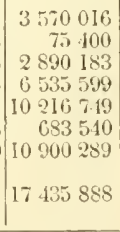 \\
\hline
\end{tabular}

1. Fait à signaler, le nombre des maisons tend à augmenter sans cesse d'un dénombrement à l'autre (379508 de plus en 1891 qu'en 18'6), mais pas dans le même rapport que celui de la population; en effet, en 18' 6 , on comptait 4,73 habitants par maison et, en 1891 , on en trouve 4,90 . 
La population industrielle n'atteint pas 10 millions, en y comprenant tous ceux qui rivent de leur travail personnel ou du salaire du chef de lamille, enfants el vieillards; la population agricole compte 8 millions $1 / 2$ d'adultes. On voit dès lors quelle est sa valeur économique et combien il est intéressant de savoir dans quelle proportion elle est proprićtaire du sol qu'elle cultive, dans quelle mesure elle exploite, par quels procédés, et quel peut ètre l'avenir d'un groupe aussi considérable qui détient dans ses mains les sources vives de la fortune publique.

Certes, l'émigration des campagnes rer's les villes a pris des proportions inquiélantes, mais il est à croire qu'elle a atteint son maximum d'intensité; deux causes générales sont de nature à ralentir ce mouvement : le déficit croissant du budget de l'État qui arrèle les grands travaux publics, l'emploi industricl de machines de plus en plus perfectionnées qui réduit le personnel des usines et ateliers. La proportion eomparée des principaux groupes professionnels, quoique défarorable à l'agriculture dont l'effectif diminue de dénombrement en dénombrement, si l'on rapproche les chiffres des enquètes précédentes, ne laisse pas que d’ètre rassurante si l'on ne s'arrète qu'à l'importance proportionnelle de chaque groupe, à l'heure actuclle, abstraction faite du passé.

Voici les chiffres de $1891^{1}$. Sur chaque groupe de population de 10000 habitants, la France compte :

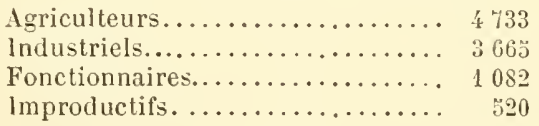

L'envahissement de la société par les employés de l'État qui, lorsqu'ils sont aussi nombreux que dans l'excessive centralisation contemporaine, ne sont plus que des parasites,

1. Je m'excuse d'employer quelquefois des moyennes, procédé qui manque de précision et conduit souvent à des erreurs graves; mais je suis obligé d'emprunter les chiffres aux publications officielles et je ne m’en sers que lorsque tonte équivoque est impossible. 
est done plus à craindre que l'abandon des campagnes dont il est, d'ailleurs, l'une des causes indirectes. Le travail et l'industrie agricoles font vivre tout le reste; les théoriciens et les idéalistes ne peurent rien contre ce fait que l'exploitation de la propriété foncière est le pivot autour duquel tournent les conditions du travail, les mours et les lois.

L'universalité territoriale de l'organisme foncier, la variété de ses applications suivant la nature du pays, les débouchés de la région, le succès de telle ou telle culture, do telle ou telle industrie agricole, empèchent qu'on puisse lui imposer des règles absolues; elles doivent se modifier selon les exigences du sol el s'accommoder aux habitudes des exploitants, sauf à les perfectionner, à les raffiner. Il n'est pas de contrée au monde mieux partagée que la France qui, situće dans la zone tempérée, entre deux mers, présente des climats et des sols très variés el se prête aux cultures les plus diverses. Cette diversité même dans le sol exige la variété des méthodes d'exploitation, et le choix de ces méthodes exerce une influence capitale sur le phénomène complexe de la division de la propriété et de la répartition des profits entre celui qui possède el celıi qui cultive.

Les indications de la statistique mettent sur la voie des solutions qui conviennent le mieux au problème rural. La moyenne de la population agricole, eu égard à la population générale, varie de 77 p. 100 dans le Lot à 20 p. 100 dans les Bouches-du-Rhone; on compte deux fois plus de propriétaires faisant valoir eux-mèmes leurs terres que de chefs d'exploitation travaillant pour le compte de propriélaires à titre de fermiers ou de métayers; enfin, le nombre des chefs d'exploitation est supérieur à celui des salariés, et c'est le côlé caractéristique de la population agricole qui diffère en cela, du tout au tout, de la population industrielle.

L'agriculture, en effel, exige des qualités personnelles, des méthodes de travail, des efforts de réllexion et de prévoyance qui développent les facultés d'initialive et de décision de l'individu. C'est une école de volonté. 
L'homme doil y compter surlout sur lui-même; la terre est son lot, le travail son instrument, il devient producteur en tant que multiplicaleur de richesses qui, sans lui, resteraient enfouies. Ce n'est plus un lieu commun de dire que la stérilité des hommes nuit à la terre el que l'une el l'autre fécondité doivent marcher de pair de même que la responsabilité, sanction de l'initiative, engendre la fierté du citoyen, el avec sa dignité son énergie.

La répartition du travail agricole le partage presque également, en nombre, entre les chefs d'exploitation et les salariés :

\section{CHEFS liEXPLOITATION}

Propriétaires travaillant exclusivement leurs terres, soit seuls, soit avec l'aide de leur famille ou d'autrui (régisseurs, maîtres-valets et ouvriers à salaire)................ 2199220

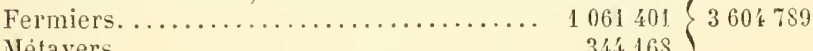

Métayers.................... 344168

AUXILIAIRES OU SALARIÉS

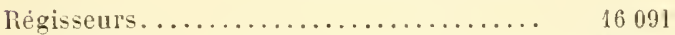

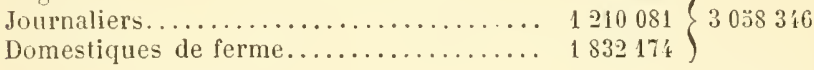

Ensemble................... 6663130

Si, au lieu de considérel les conditions du travail, on examine la division de la terre entre ceux qui la cultivent, en formant de l'ensemble des agriculleurs deux groupes, l'un composé de ceux qui exploitent uniquement le bien dont ils sont propriétaires, l'autre de ceux qui travaillent à la fois pour eux comme proprićtaires el aussi pour autrui, soit comme exploitants intermédiaires (fermiers et métayer's), soit comme salariés (journaliers), les proportions se déplacent; mais, de même qu'il y a plus de chefs d'exploitation que de collaborateurs et d'auxiliaires, on trouve également que les exploitants directs ou travailleurs agricoles propriélaires sont plus nombreux que les cultivateurs non propriélaires. 


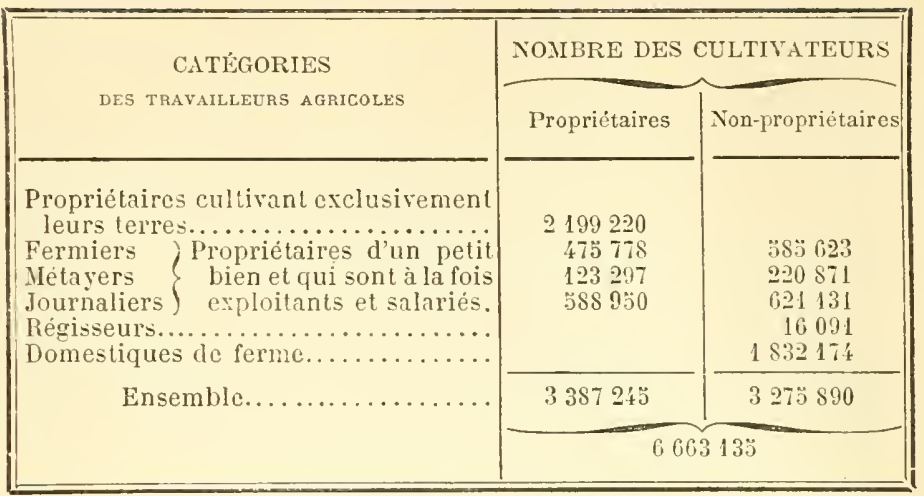

Sur l'ensemble des travailleurs agricoles, plus de la moitié, 31 p. 100, sont done propriétaires fonciers; c'est une situation économique qui est, je erois, unique dans le monde, et Ia France peut s'en montrer fière.

Les propriétaires eultivant uniquement leurs terres représentent 630 p. 100 du total, les journaliers 17 p. 100, les fermiers 14 p. 100, les métayers 4 p. 100.

L'enquête agricole de 1892 constate, comme l'aviiil déjà fait celle de 1882, que le mowrement de diminution de la population rurale affecte moins les travailleurs eux-mêmes que la famille agricole, e'est-it-dire les individus qui n'ont dans l'exploilation qu'un rôle aecessoire et subordonné, et quelquefois nul. L'intérêt agricole serait-il de retenir ces émigrants el de créer dans la famille, ou à côté de la famille, des industries dépendantes de la eulture ou connexes à l'exploitation, qui oceuperaient tous les bras disponibles en supprimant la mauvaise pratique de la dispersion? Les immigrations périodiques des Belges, des Allemands, des Italiens et des Espagnols sur nos frontières du Nord, du Sud-Est et du Mlidi, indiquent qu'il y a là, à eerlaines époques, une siluation à conserver à nos nationaux, à moins que la question de salaire (eomme il arrive partois) ne fasse au point de rue du prix de revient une économic appréciable. 
Les conditions du travail se modifient de nos jours avec une extrìme rapidité, et les éléments nouveaux qu'y apportent l'extension du ròle des syndicats et le développement des principes de la mutualité et de l'assurance ront les transformer plus profondément encore.

D’un autre côté, l’intervention dans l'ordre économique des nations de la vieille Europe de peuples jeunes, ardents, vivaces, puissants par l'immensité et la fécondité de leurs territoires, bien servis par la multiplicilé et la rapidité des moyens de transport, change tantôt insensiblement et tantôt brusquement les rapports de chaque pays, leur capacité d'échange et le prix de leurs produits. Les intérèts généraux et particuliers sont profondément atteints; aussi les procédés de l'agriculture et de l'industrie, dans la lutte à outrance que suscite une concurrence effrénée, doivent-ils se modifier sans cesse. Le législateur est impuissant à suffire à ces besoins, à ces exigences renouvelées, souvent en sens inverse, car chaque produit, naturel ou fabriqué, a ses débouchés et ses rivaux; il ne peut que protéger l'individu contre l'arbitraire et le privilège. Le mot de Montesquieu : les pays ne sont pas cultivés en raison de leur fertilité, mais de leur liberté, est accentué par les principes libéraux que Turgot inscrivait en tète des édits de $17 \%$ et de 1776. Le ròle de l'État est d'ourrir les voies et de les maintenir ouvertes, c'est à l'initiative privée de faire le reste. La tendance de tout ramener à l'État, de lui faire tout contrôler, tout inaugurer, tout diriger, tout subventionner, est détestable; elle a pour résultats les abus de l'administration et ceux de la fiscalité qui ruinent à bref délai les institutions les plus solides.

La prospérité publique a pour premier fondement la culture des terres, disait un édit royal d'il y a cent vingt-six ans ${ }^{1}$, l'abondance des denrées et leur débit avantageux, seul encouragement à la culture, seul gage de l'abondance. Ce débit avantageux ne peut naitre que de la plus entière liberté des ventes et des achats. C'est cette liberté seule qui

1. Edit du 13 septembre 17\% 
assure aux eultivateurs la juste réeompense de leurs travaux, aux propriètaires un revenu fixe, aux hommes industrieux des salaires constants et proportionnés, aux consommateurs les ohjets de leurs besoins, aux eitoyens de tous les ordres la jouissance de leurs droits.

L'agriculture a besoin de sécurité autant que de liberté; travaillant à échéance lointaine, elle a horreur du désordre aussi bien que de l'arbitraire.

C'est ce qui a fail dire à Taine : quand l'homme est misérable, il s'aigrit; mais, quand il est à la fois propriétaire et misérable, il s'aigrit davantage. Il a pu se résigner à la misère, il ne se résigne pas à la spoliation ${ }^{1}$.

De là l'extrême importance du droit de propriété en malière territoriale et, comme tout se résout en pratique, non seulement du droit considéré dans son principe, sa consolidation et sa sécurité, mais de l'appropriation de ce droit à la distribution de la rie rurale, e'est-à-dire de la mesure d'après laquelle le sol est réparii cntre les mains de ceux qui l'exploitent.

Aussi l'Académie des sciences morales a-t-elle été bien inspirée en proposant cette étude à la sagacité des économistes autant qu'à la compétence expérimentale des observaleurs qui ont la bonne fortune de vivre au milieu de paysans. Il ne suffit pas de faire de vastes enquêtes et de dresser des moyennes, comme on le fit en 1862, en 1879, en 1882, en 1887, en 1891, ni mème d'établir d'ingénieux rapprochements, comme en 1892; quand il s'agit de l'agriculture, autrement dit de la propriélé foncière rurale, de la plus grande de nos industries, de celle qui couvre la terre française de ses rastes domaines el de ses myriades de petits chantiers de travail, et qui met en oeuvre un capital de plus de cent milliards en occupant les bras de la moitié de la nation, les vues doivent être plus hautes, el c'est la direction que, par la brièveté voulue de son programme, l'Académic a voulu donner à nos recherches el à nos conclusions.

En interprétant les faits de la statistique et les exemples

1. L'Ancien Régime, liv. V, chap. I, s' i. 
tirés de l'obserration directe, il faut en dégager la philosophie et conclure des progrès accomplis à ceux que l'avenir peut et doit réaliser.

\section{III. - Statistiques de la propriété rurale en France,} d'après les chiffres combinés des publications officielles.

La vaste enquête décennale de 1892 est le document officiel le plus complet et le plus instructif que nous possédions sur l'état actuel de la propriété rurale en France.

En groupant symétriquement les valeurs eréées par l'agriculture, en éraluant en regard les charges de toute nature qui pèsent sur l'exploitation du sol, l'enquète de 1892 a établi le bilan de la richesse agricole du pays. Mais, au point de vue spécial qui m'est imposé dans cette étude, ce cadre est trop étendu, et, pour profiter des chiffres qui y sont entassés, de l'incidence des infinis détails qui y sont classés avec une méthode rigoureuse, il faut trier ce qui concerne la division de la propriété et rechercher quels sont, à l'heure actuelle, les résultats plus ou moins avantageux de chaque mode d'exploitation, selon qu'il s'agit de la grande, de la moyenne ou de la petite propriété.

Ce sera l'objet des chapitres qui vont suivre. Mais, au préalable, il convient de tracer une esquisse rapide de l'état actuel de la propriété rurale en France ${ }^{3}$.

La finale de l'enquète de 1892 (page 44) est pessimiste :

Malgré l’imperfection inévitable des évaluations de détail, dit le rédacteur de l'Introduction, on peut dérluire des considérations qui précèdent que la valeur du capital foncier s'estabaissée de 15̆ p. 100, que le produit brut de l'exploitation agricole a perdu 8 't millions, enfin que le produit net total a diminué de 329 millions, déterminant ainsi la crise agricole à laquelle le législateur s'est efforcé de remédier par le vote des mesures économiques dont la principale a été l'adoption des nouveaux tarifs de Dollane.

Malgré l'autorité qui s’attache à des appréciations officielles, je ne saurais partager cette manière de roir, et, des

1. J'ai pris les chiffres qui suivent dans les Résultats statistiques du dénomhrement de 1891 (Office du travail, 189\%), et dans la Statistique agricole de la l'rance (Ministère de l'Agriculture, 1897). 
mèmes chiffres, je crois pouvoir tirer, sans rien exagérer ni forcer, des conclusions infiniment plus rassurantes.

Il n'y a pas de crise agricole, au sens que donnent à ce mot les publicistes; il n'y a qu'un malaise, sans diagnostic particulier, variant selon les époques, les régions, les cultures ${ }^{1}$. La gène dont souflrent non pas l'universalité des agriculteurs, mais seulement quelques catégories d'industries agricoles, tantòt celles-ci, tantôt celles-là, non point simullanément mais à tour de rôle, est le résultat d'un changement un peu brusque dans l'équilibre économique auquel on s'étail habitué depuis 1860; il n'y a pas de crise agricole dans l'acception littérale du mot, il y a simplement une modification générale de la situalion économique des peuples, par l'augmentation des forces productives du monde, l'abondance de certains produits, la concurrence faite à d'autres, el le déplacement des marchés. Cette augmentation ne s'arrètera pas; la Chine, le Japon, les Indes rivalisent avee les Élats-Unis pour se suffire par leurs propres ressources et même pour inonder l'Europe de marehandises similaires, d'objets exoliques, de graines et de bétail. C'est un élal nouveau qu'il faut accepter comme définitif, qui est à son début, qui en s'accentuant viendra contrarier nos liabiludes el diminuer nos profits; ce n’est point par de vaines doléances quion se meltra en mesure de lutter avec succès et de mieux orienter ses entreprises.

Le vrai mal est cette croyance à l'omnipotence de l'État qui affaiblit notre confiance en nous-mêmes, notre énergie et notre initiative. Le remède, c'est le relèvement moral et la liberté, dont l'association syndicataire est une forme, à moins qu'elle ne tombe aux mains d'une coterie, anquel cas elle n'est plus qu'un des modes de la tyrannie collecliviste.

1. La malarlie des vers à soic a singulièrement appauvri la valléc du Rhòne depuis 1853; la culture de la garance, qui faisait la fortune du département de Vaucluse, a été anéantie par les découvertes de lit climie et l'emploi de l'alizarine artificielle depuis 1873; le Nidi, les Charentes, le Bordelais, la Bourgogne, furent dévastés par les maladies de la vigne depuis 1892, d'une manière générale; dans le Nord, le rendement de la betterave a diminué fortement par l'épuisennent du sol, etc. 
Ourrez les portes, rompez les barrières. Ne vous fiez pas à lexpédient suranné et insuffisant des tarifs de douane, ne vous emprisonnez pas dans les ealeuls étroits des vieux marchés nationaux; il faut agir plus largement, l'oreille au téléphone, et préroir les inévitables el brusques variations du marehé universel.

Mais ceci ne concerne que les grandes exploitations agricoles, instailées au prix de fortes dépenses, et qui prennent lallure industrielle, ou les syndicats qui centralisent dans leurs entrepòts les produits des petils eultivateurs, leurs adhérents. La petite culture ne peut avoir la prétention de s'occuper de combinaisons commerciales autres que celles dont elle a la tradition et l'expérience sur le marché le plus voisin; mais elle peut recevoir des indications utiles, abandonner une culture qui ne rend plus, comme la garance dans le Vaucluse ou le mùrier dans la Dròme, ou la betterave dans le Pas-de-Calais, et la remplacer par une autre d'un débit plus assuré. Les syndicats peuvent avee sucès remplir ce ròle d'indicateurs et d'éducateurs. D'autres pays sont mieux placés que nous, aujourd'hui, pour certaines productions; profitons-en au lieu de nous en plaindre, et ne nous obstinons pas dans la routine des cultures anciennes qu'on ne peut rendre rémunératrices en apparence qu'à l'aide de droits protecteurs, au grand préjudice des consommateurs.

Procurez-rous chez les autres, à bon compte, les matières premières que vous transformerez pour les revendre sous leur forme perfectionnée, ou du bétail maigre que vous céderez gras. Que le blé vienne de la vallée du Danube ou des plaines de la Beauce, l'important n'est-il pas que la farine soil saine et le pain à 20 centimes? Ne parlez pas de votre isolement. N'avez-yous pas depuis 1884 la ressource du Syndicat pour vous débarrasser des pertes de temps, des intermédiaires parasites, des courtiers équivoques, de l'exagération des prix de détail dans vos achats, et pour vous soutenir mutuellement dans les frais de transport et de vente? 
La statistique des résultats obtenus par les Syndicats ${ }^{1}$ est la preure de l'atténuation presque immédiate que les achats et les ventes solidarisés peurent apporter dans les frais généraux de la culture et des améliorations foncières, inabordables pour l'isolé, que l'association rend faciles et peu coûteuses ${ }^{2}$.

La crise agricole n'est qu'un épourantail à l'usage des sophistes ${ }^{3}$.

Le capital foncier n'a pas diminué. L'argument tiré des relerés de limpòt direct par les rédacteurs de l'enquête de 1892 ne repose que sur une hypothèse que tout contredit.

La valeur attribuce en 1882 au capital foncier agricole (91 38.4 millions de francs) n'est autre que celle relevée par le service des Contributions directes dans l'enquette (de 1879 à 1881) sur le revenu foncier des propriétés non bàties. La valeur rénale relevée de 188z à $1892^{4}$ ayant permis de constater une baisse moyenne d'environ 15 p. 100 , on a cru devoir abaisser de $15 \mathrm{p}$. 100 , en nombre rond, le chiffre de 1882 pour le ramener ì 7 i 8.47 millions, ehiffre arbitrairement adopté, après cette série d'appréciations approximatives, pour l'éraluation de 189:.

Les statistiyues que publie depuis trois ans le service de l'Enregistrement permettent de rectifier ces conclusions. En effel, si l'on consulte le mouvement des mutations immolsilières à litre onéreux, de 1820 à 188\%, d'après les comples de finances, chiffres exacts puisqu'ils sont déduits de ceux de la pereeption de l'impôt, on conslate que ce mouvement, dans ses lignes générales, n’a cessé de progresser, en nombre

1. Les associations professionnelles ouvrières en 18.99 , d'après les publications de l'office du travail (t. I, Agriculture, p. 284).

2. Annuaire du Syndical agricole de Villefranche-sur-Rhône, 1898.

3. C'est jouer sur les mots que de dire: La question des prix jour le plus grand rüle dans toutes les questions économiques. L'étude des prix de vente ne donne pas le secret d'abaisser le prix de revient pour laisser la marge à un bénéfice; c'est prendre le problème par son plus petit còté.

4. L'enquête (p. 442) n'indique pas quels sont les documents dont l'examen a permis de reconnaitre cette dépréciation dans la valeur vénale de la propriétè immobilière non bâtie. 
et en valeur ${ }^{1}$. Lorsqüil y a recul, ce recul est motivé par des crises financières qui, de la spéculation, de la banque et des entreprises industrielles, se répercutent sur l'ensemble des exploitations et des valeurs nationales, foules plus ou moins solidaires l'une de l'autre, et créent une crise économique. Les diagrammes font ressortir la régularité avec laquelle les résultats de chaque crise se manifestent, deux ans après la crise, par une année de liquidation ${ }^{2}$ où pertes et profits se règlent, et pendant laquelle il $\mathrm{y}$ a un arrêt de placements et de réalisations, sauf à reprendre ensuite la marche avec plus d'énergie, l'orage passé. Ainsi la crise industrielle et commerciale de 1826 se traduit par la liquidation de 1828; celle de 1830 (révolution) en 1832, celle de 1836 en 1838, celle de 1817-18\%8 (crise industrielle en 1817 et révolution en 1848 ) de 1849 à $185 \% 2$, celle de 1837 en 1859 , celle de 1863 (influence des traités de 1860) en 1863, se liquident dans des conditions qui permettent, l'équilibre une fois rétabli par le nouveau classement des contre-parties, de faire succéder à chaque recul une reprise.

Pour les époques les plus récentes, la crise de 1870 (guerre ct révolution) se manifeste par un recul de 742 millions de francs en 1870 et de 204 millions en 1871 sur les sommes (2 424 millions de francs) employées à des acquisitions foncières en 1869. Mais, preuve indiscutable de la vitalité de ce pays et de l'étendue de ses ressources, le niveau du mouvement d'achat remonte, dès 1872 , à 2400 millions, malgré les emprunts pour l'indemnité de guerre et les pertes sèches subies par les particuliers, de la frontière jusqu'à la Loire.

Le point culminant est atteint en 1880 ; la crise financière de $188 \%$, le Krach Baring de 1890, l'effondrement des valeurs des mines d'or anglaises en 189\%, abaissent l'échelle à 2031 cn 1887, à 2011 en 1889 (Exposition universelle), à 2078 en 1893, à 2009 en 1897.

1. Bulletin de statistique de l'administration de l'Enregistrement, 1898, I, p. 157 à 167.

2. C. Juglar, Des crises commerciales et de leur retour póriodique en France, en Angleterre et aux Ètats-Unis, 1897. 
En étudiant la répartition par département, on s'aperçoit que ces reculs, dans les années de liquidation qui suivent les crises, affectent surtout les grandes villes comme Paris, Lyon, Bordeaux, et les départements industriels du Nord et de l'Est ${ }^{1}$; il est difficile de dégager exactement la part de la propriété r'urale dans le ralentissement du mouvement d'acquisition, ralentissement qui, par voie de conséquence, produit la baisse des prix, les offres étant plus nombreuses que les demandes; cependant, il est certain, d'abord, que le mouvement des mutations foncières à titre onéreux est infiniment plus considérable et plus actif sur la propriété rurale que sur la propriété urbaine ${ }^{2}$, ensuite que ce mourement porte surtout sur des immeubles d'un prix minime.

STATISTIQLE DE $189 \%-189 \%$

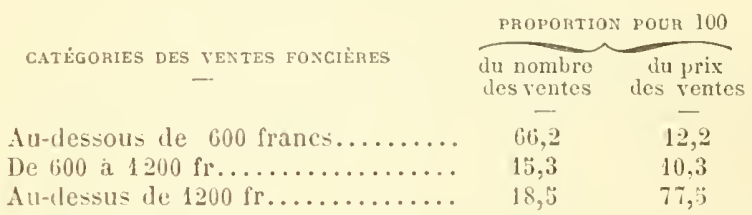

Ce rapprochement fail saisir pourquoi la baisse des prix n'a qu'une légère répercussion sur une masse de petites opérations de 82 p. 100 en nombre et de seulement 22 p. 100 en valeur, tandis qu'elle influence avec une réelle intensité les opérations qui, même étendues à des acquisitions médiocres (de 1200 à $̋ 000$ fr. par exemple), représentent inversement à peine 18 p. 100 en nombre et, au contraire, environ 78 p. 100 en valeur.

Pour l'année 189', en écartant les moyennes, on trouve

1. Les flroits de vente perçus sur les mutations immobiliires à Paris, de 1877 a 1897 , l'établissent avee préeision. Le prorluit de ces droils, de 25 millions et demi qu'il était en 1879 , de 36 millions en 1881, est tombé à 13 millions et demi en 1586 , pour remonter à 24 millions en $18 \% 1$, et retomber à $20 \mathrm{en} \mathrm{189 \% ,} \mathrm{a} 17 \mathrm{et}$ demi en 1856. (Bullelin de slatistique de l'Enregistrement, 1897, p. 211.)

2. La répartition des ventes immobilières d'apres l'importance des prix, en 189i. (llid., p. 213.) 
en chifres absolus que les prix d'acquisition des immeubles classés d’après leur imporlance se répartissent comme suit :

Immeulles d'une valeur vẻnale de moins de $5000 \mathrm{fr}$., e'est-à-dire pouvant ètre considérés conme représentant des immeubles ruraux dans la proportion de 98 p. 100.

$\begin{array}{cc}\text { Nombre } & \text { valeur } \\ 647603 & 593232600 \mathrm{fr} .\end{array}$

Immeubles d'une valeur vénale supérieure ou égale à $5000 \mathrm{fr}$. et parmi lesquels se trouvent nécessairement des biens ruraux :

$$
\text { Ensemble... } \frac{63999}{713602} \quad \frac{1369073500}{189 \div 326100 \mathrm{fr}}
$$

Si, de 1878 à $189 \%$, le nombre des mutations s'est, par un mouvement continu, abaissé dans une proportion considérable, leur importance en valeur vénale a augmenté, toutes compensations faites, d'au moins 8 p. 100 '. L’appréciation d'une perte de $10 ّ$ p. 100 sur le capital foncier n'est donc pas justifiée.

La plus-value croissante de la fortune territoriale de la France est, au contraire, démontrée par les statistiques, non plus uniquement fiscales mais économiques, que le service de l'Enregistrement commence à publier et dont les informations serviront à rectifier beaucoup d'opinions inexactes. Ses relevés n'indiquent plus, comme autrefois, seulement les valeurs taxées, mais ioutes les valeurs qui ont apparu dans les déclarations de mutations par déè̀s, de telle sorte qu'on peut désormais déterminer la valeur exacte des patrimoines et la répartition des biens de différentes catégories qui composent ces patrimoines.

Pour l'année 1898, le nombre minimum des immeubles transmis par décès et leur réelle importance, d'après leur

1. L'abaissement en nombre exige à lui seul un examen attentif, car la baisse n'a pas été uniforme sur toutes les catégories de transmissions foncières. Les licitations et soultes de partage que les crises influencent peu ont diminué de 8 p. 100 , les échanges de $23 \mathrm{p} .100$, les ventes de 18 p. t00. (Bulletin de l'Enregistrement, 1898, I, 162 et 163.) 
capitalisation légale, se résument, pour tout le territoire continental (y compris la Corse) par les chifïres suivants ' :

$\begin{array}{cc}\text { CatÉGories } & \text { Nombre } \\ \text { des immeubles } & \text { des déclarations } \\ \text { de successions }\end{array}$

Immeubles urbains...... 96819

Immeubles ruraux...... $24655 \mathbf{4}$

313366
CAPITAL

des valeurs

funcières déclarées

1570351359 fr.

1519810899

$3090162288 \mathrm{fr}$.

La démonstration en est faite, pour la continuité de longues périodes, par la progression constante de ce que les spécialistes appellent l'annuité successorale, c'est-ì-dire le chiffre annuel variable des valcurs comprises dans les déclarations de successions et taxées pour la perception de limpôt de mutation. Le fait caractéristique est la régularité arec laquelle se poursuit l'évolution de la richesse successorale; la courbe générale de l'annuité ne présente ni dépressions inattendues, ni brusques sursauts; elle se développe sous l'action constante et graduelle rles forces économiques ? Je ne parle ici, pour ne point sortir du eadre limité de mon sujet, que de l'annuité immobilière; mais il faut remarquer que le merveilleux développement, de $18: 0$ à $\mathbf{1 8 6 9}$ surtout, de la richesse mobilière, dù à l'essor de l'esprit d'entreprise, à la plus grande excitation commerciale et industrielle, à l'attrait plus vif des spéculations financières, a exercé une influence notable el inévitable sur l'activité du marché foneier. Les capilaux étaient attirés par les valeurs industrielles et les fonds d'Élat que popularisaient et classaient les emprunts publics et les émissions de sociétés,

\section{Bullelin de "Envegistrement, 189., 1) is9.}

2. Les majorations artifiriellement procurées par des dispositions législatives d'ordre fiscal n'ont eu qu'une portee secondaire quant aux immcubles, mais très apprécialle quant aux valeurs mohilières; far l'efret ale ha loi le $18: 30$, trois nouvelles sources de richesses sout livrées au fise (rente française, fonds pullies élrangers, titres de sociétés étrangères); les lois de 1863, de 1871 et de 1875 taxent ou surtaxent de nouvelles valeurs et leur ensemble tendra ì rapprocher de plus en jus l'annuité successorale mobilière, si faible en 1826 ( 157 conlre 880 ), le l'annuité immobiliere (2 863 contre 2886 en 189 '), qu'elle dépasse en 1896 (2 798 contre $270 \ddot{3}$, en millions de francs). 
mais une partie du gain se reportait sur la sécurité du placement foncier.

Lamnuité successorale immobilière suit la progression que voici (en millions de franes $\left.{ }^{1}\right)$ :

\begin{tabular}{|c|c|c|c|}
\hline $1826 \ldots \ldots \ldots \ldots$ & 880.3 & $1863 \ldots \ldots \ldots \ldots$ & 1982.3 \\
\hline $1830 \ldots \ldots \ldots$ & $9 \div 3.0$ & $1872 \ldots \ldots \ldots$. & 2148,3 \\
\hline $1840 \ldots \ldots \ldots$ & $999, \ddot{3}$ & $1873 \ldots \ldots \ldots$ & 2216,6 \\
\hline $1848 \ldots \ldots \ldots$ & 1244.7 & $1880 \ldots \ldots \ldots$ & 2787.9 \\
\hline $185 \%$.......... & 1428,9 & $1890 \ldots \ldots \ldots \ldots$ & 2922,1 \\
\hline $1860 \ldots \ldots \ldots \ldots$ & 1543,9 & $1892 \ldots \ldots \ldots \ldots$ & 3029,6 \\
\hline $1867 \ldots \ldots \ldots$ & 1767,2 & $1893 \ldots \ldots \ldots \ldots$ & 3043,0 \\
\hline
\end{tabular}

Depuis les vingt-einq dernières années, les valeurs sont comparables, la législation fiscale est restée la mème, le milieu économique n'a point subi de perturbations radicales, el l'on troure qu'entre les chiffres de 1873 et ceux de 1896, le capital foncier successoral s'est accru de 72.1 millions.

En 1826, les valeurs mobilières n'entrent que pour 34 p. 100 dans l'ensemble des valeurs taxées; la part des immeubles étant de 66 p. 100; en 1849, la proportion ne s'est pas nolablement modifiée, de 39 p. 100 pour les unes à 61 p. 100 pour les autres; en 1869, ces cotes deviennent respectivement $4 \dddot{3}$ p. 100 et $\breve{3}$ p. 100 ; encore quelques étapes, et cette légère supériorité de l'annuité immobilière va s'évanouir définitivement; cela ne signifie pas que le capital foncier s'effondre, mais uniquement qu'il s'élève à côté de lui une richesse nouvelle, créée par le génie de la spéculation, commanditée par le erédit el dont le gage, quoi qu'on fasse, sera toujours la terre ${ }^{2}$. Depuis 1891, l'équilibre est rompu en faveur des valeurs de papier; certes, il n'est pas douteux que le mouvement foncier ait souffert de cet accaparement des capitaux, mais on ne sait trop

1. Journal de la Société de statistique de Paris (mai 1899, 143).

2. Les valeurs de Bourse sont frappées de toutes parts. Cette richesse, qui, au début du siècle, était réputée négligeable, constitue de nos jours une des branches les plus fructueuses du revenu fiscal et supporte une part d'impôt au moins égale à celle des immeubles. (E. Besson, La progression des valeurs successorales au XII. $\mathrm{I}^{\mathrm{e}}$ siècle, 1899.) 
sïl faut le regretter, car la liberté économique sait à propos réagir sur ses propres exagérations, et l'intérêt est sa mesure et son frein. Ce qui, dans tous les cas, est une singulière atténuation au préjudice discutable qu'elle a pu causer à la richesse territoriale, c'est que celle nouvelle forme de la fortune publique a fait dériver vers elle toutes les surtaxes d'impòt, allégeant d'autant la condition fiscale de la terre ${ }^{1}$.

Le revenu foncier était de 2 milliards $1 / 2$ en 18301 ; il s'est avancé, en 1862, au chiffre net de 3096 millions. La valeur vénale a suivi une progression parallèle; le prix moyen de l'hectare, qui de 1789 à 1821 a varié de 300 à 800 franes, atleint $127 \%$ franes en $18 \% 1$, el $1830 \mathrm{fr}$. 39 en $1879^{2}$; au vrai, et en se dégageant de lobsession des moyennes, il vaut

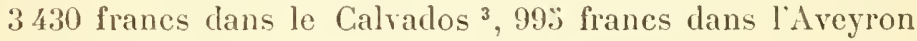
el 422 franes en Corse.

Quil y ait un temps d'arrêt dans le courant qui porte vers l'acquisition foncirre, éest indubilable; mais de là à conclure a une diminulion intrinsèque du capital foncier rural, il y aurait peut-c̀tre imprudence, ear la propriété urbaine, sauf quelques cas particuliers, paraît êlre la seule atteinte, ct, surtout en malière agricole, le rapport du revenu net au capital est trop rariable el ce revenu lui-mème est d'une détermination trop délicate pour qu'on puisse faire d'une appréciation arbitraire un argument déeisif.

L'écarl de 81 ' millions entre 1882 el 1892, dans le produit brut de l'exploitation du sol, eomme élément parliculièrement significatif de l'intensilé de la crise agricole, n'est pas mieux justifié que le soi-disant aflaissement de la valeur vénale du capital ioneier rural. En effet, on procide dans ces conclusions par liypothèses à larges moyeunes, basées

1. En 1890, dit $\mathbf{1 1 . ~ N e y m a r c k , ~ n o u s ~ p o s s e d o n s ~ e n ~ F r a n c e ~} 28$ milliaris de titres en plus de ceux que nous avions en 1870. (Ine nouvellévaluation du revenu les valeurs mobilières, 1893.)

2. Bulletin de statislique du ministere des Finances (mai 1883).

3. Et, dans ce mèrne département, 3340 francs tlans l'arrondissement de Bayeux et 2232 dans celui de Falaise. (Ibid., flecembre 1888.) Preuve de la diseretion qu'il faut apporter dans l'emploi des moyennes, fiction qu'il faut proscrire. 
sur des à peu près, et l'on ne s'explique pas l'intérêt qu'eurent les ministres de 1897 à faire croire qu'il existait une crise agricole el que les paysans étaient en perte de 329 millions de francs, comme l'ont proclamé tous les organes de la presse politique parisienne.

La comparaison de la valeur des produits agricoles de 1882 à 1892 se traduit par de nombreux mourements en sens opposés, tantòt de hausse el tantòt de baisse ${ }^{1}$. Les céréales ont donné, pour la période de 1876 à 1883,292 millions d'hectolitres par an, pour celle de 1886 à $1895,2 \% 9$ millions; il y a done bénéfice pour le rendement, mais il y a perte de ¿46 millions sur le produit, paree que le prix de vente à l'hectolitre est tombé de 13,71 à 12,17 . La production en fourrages, inférieure de 6 millions de lonnes en 1892 sur celle de 1882, a été payée $ّ$ millions de franes de plus ${ }^{2}$.

Le déficit porte sur les vignes. L'envahissement général du vignoble français par le phylloxera coïncide précisément avec la période décennale de 1882 à 1892. Les fléaux se multipliaient, le mildew s'étendail parlout, le black-rool commençait à paraître; la superficie prorluetive s'amoindrissait rapidement:

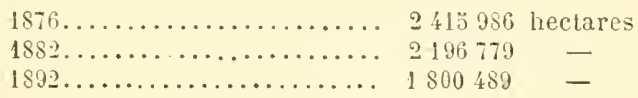

L'énergie du vitieulteur français ne se démentit pas el la reconstitution se fit avec une rapidité étonnante ${ }^{3}$. Le dépar-

1. Toutes ces comparaisons, dans les tableaux récapitulatifs de l'enquête, sont faites à l'aide de moyennes.

2. La quantité et la valeur de la production des prairies, nalurelles et artificielles, ont suivi une progression constante. De 1840 à 1892 , cette catégorie d'exploitation accuse une plus-value de 1 milliard et demi de francs.

3. C'est seulement dans des circonstances de cette nature que l'État peut intervenir efficacement en ne se substituant pas aux particuliers, mais en encourageant leur initiative. Les lois des 45 juillet 1878 et 2 aoùt 1879 favorisèrent la défense des vignes; celle du 10 décembre $\mathbf{1} 888$ autorisa l'organisation de syndicats obligatoires; celle du $1^{\text {er }}$ decembre 1887 exonėa de l'impôt foncier les terrains replantés en vignes; enfin, celle du 3 août 1891 provoqua les reconstitutions rapides. 
tement de l'Hérault possédait 180000 hectares plantés en vignes; en 1888, il ne lui restait que 3330 hectares d'anciennes vignes; en $189 \%$, son vignoble était remonté à plus de 160000 hectares.

Le désastre viticole est donc un accident, un hasard; il a ruiné quantité de familles, il a modifié l'état économique de plusieurs régions, surlout dans l'Ouest (les Charentes), mais on ne peut pas le considérer comme un facteur universel de malaise, comme l'élément prépondérant d'une crise agricole. Il a joué le rôle d'un vaste incendie, d'une inondation, d'un cyclone, et la perte qu'il a causée est considérable si l'on compare la valeur marchande de la production aux trois époques convenues :

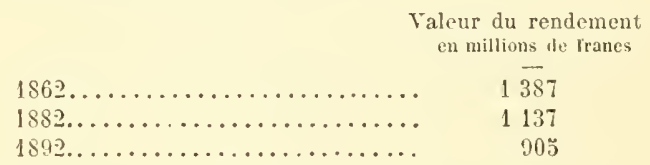

Mais il serait imprudent de prendre pour bases du revenu sur lequel doivent compter les propriétaires de vignes les résultats incessamment variables d'une culture dont le rendement peut passer du simple au double d'une année à l'autre :

PRODCCTION EN HECTOLITRES

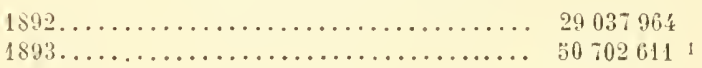

Il faut espérer que la France ne perdra pas l'une des plus précieuses de ses richesses agricoles, celle qui lıi assurait un monopole à l'étranger et pour laquelle elle n'avait aucune concurrence à redonter. L'insuffisance des récoltes est un malheur contre lequel il n'y a point de remède sauf', pentctre, cehui des cullures intercalaires qui permet de suppléer au déficit de l'une par l'abondance de l'autre; mais cette

1. En $189 \%$, la récolte baisse à 27 millions l'hectolilres; elle se releve

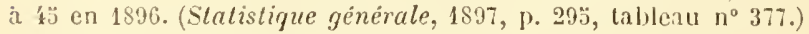


méthode d'exploitation présente aussi des inconvénients et n'est pas possible partout '.

Une des considérations qui, en négligeant les chiffres arbitraires et les cas fortuits, comme ceux que je viens de signaler, lendent à diminuer singulièrement la portée effective de la soi-disant crise agricole, c’est l'atténuation dans une forte proportion des charges générales de la culture.

L'enquête de 1892 estime colte économie à 982 millions de francs, sans toutefois justificr ce calcul avec beaucoup de précision.

Labaissement du taux de fermage réduit de 10 p. 100 le loyer de la terre; ce résultat n'est qu'une moyenne, c'est-àdire une équiroque.

Le taux moyen de fermage d'un hectare de labour ne varie-t-il pas de 92 francs ( ${ }^{\text {re }}$ classe) à 28 franes ( ${ }^{e}$ classe), et celui d'un hectare de vigne de 133 à 33 , c'est-à-dire de 3 à 1? Dans la première classe, le prix cle fermage de l'hectare de vigne ne s'abaisse-t-il pas lui-même, selon les régions, de 338 francs (Seine) et 331 francs (Hérault) à 70 franes (Haute-Saône)?

Ce qui est plus sérieux, c'est la baisse des salaires agricoles. L'enquête l'évalue à 183 millions de moins à payer par les chefs d'exploitation, mais l'explication qu'elle en donne est discutable. La diminution sur les salaires est plutôt le fait d'une diminution de l'effectif des salariés que du salaire luimême; quiconque vit à la campagne sait combien on a de peine à trouver des auxiliaires, hommes ou femmes, domestiques ou manœuvres, et quel prix il faut les payer ${ }^{2}$. C'est l'attrait des villes, où l'on croit, et l'on se trompe, que les salaires étant plus élevés le profit net est supérieur, qui

1. Il existe actuellement à peine $1 / 10$ de la superficie des vignobles avee cultures intercalaires ( 108197 heetares en 1892).

2. Dans le Morvan, pays pauvre, en mars 1900 , on ne trouve d'ourriers agricoles, même parmi les jeunes gens de vingt ans, qu'au prix de 2 franes par jour et nourris. On paie de simples laveuses de $1 \mathrm{fr}$. 203 a 1 fr. 50 , en les nourrissant. 
cause l'émigration à l'intérieur; mais la culture n'en souffre pas, à cause de l'afflux périodique des travailleurs pour la fenaison, la moisson et la vendangre. Les méthodes d'exploitation y gagnent, parce qu'on prend de plus en plus l'habitude de remplacer les bras de l'homme par des machines ${ }^{1}$.

Bref, l'ensemble des crises locales ou momentanées qui ont pu affecter tantôt une région, lantôt une culture, nont pas eu sur l'industrie agricole de notre pays une action déprimante. Les profits ont pu être diminués sur certains points, relevés sur d'autres, il s'est produit des déplacements de force, l'équilibre général n’a pas été rompu. Bien loin de justifier la légende d'une vaste crise agricole, les résultats de l'enquète de 1892 , corroborés par les statistiques économiques ou fiscales les plus récentes, établissent, au contraire, que la condition matérielle des populations agricoles, en France, n’a cessé de s’améliorer, et leur condition morale de se relever.

Le sujet est curieux et peu connu, il prèterait à des développements étendus; mais je dois me horner, ne voulant donner ici qu'une esquisse rapide mais fidèle de l'étal actuel de lagricullure.

Il n'est pas inutile de considérer à des points de vue différents la répartition par condition de la population agricole de la France, parce qu'on peut apprécier ensuite plus sùrement les effets, dans ses mains, de la distribution de la propriété rurale, distribution qui exerce une influence capitale sur le choix et la possibilité des diverses méthodes d'exploitation du sol.

Le recensement de 1891 la divise en catégories professionnelles comprenant, non seulement les chefs dexploitations (dont j’ai donné le détail ci-dessus) mais leurs familles, leurs

1. De 1882 à 1892, tandis que le nombre des charrues simples n'a augmenté que de 10 p. 100 , celui des bisocs ou polysocs s'est aceru de 25 p. 100 . Il y a un accroissement te 88 p. 100 sur les faneuses et rateaux à cheval, de 99 p. 100 sur les semoirs mécaniqnes, de 103 p. 100 sur les faucheuses. Les machines à battre ont doublé. 
ourricrs et auxiliaires, en un mot tous ceux qui vivent de chaque classe d'exploitations.

Nombre de têtes

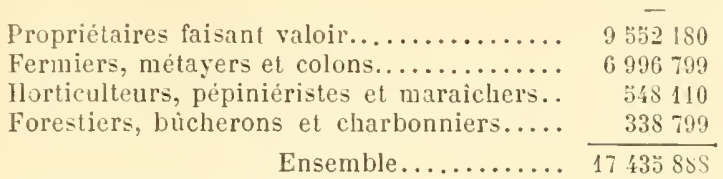

La population agricole représente les 46/100 de la population générale, les 47/100 de la population classée par prolession. On compte deux fois plus de propriétaires faisant valoir eux-mèmes leurs terres que de chefs d'exploitation travaillant pour le compte de propriétaires à titre de fermiers ou de métayers; signe de virilité et promesse de fécondité, car il est dans notre nature de mettre infiniment plus de zèle dans les travaux dont nous ne partageons le gain avec personne.

Sur 100 propriétaires cultivant eux-mèmes leurs terres, on en compte 62 qui ont recours à l'aide douvriers; il faul voir là une conséquence du morcellement du sol, car un très grand nombre de petits domaines suffisent à faire virre leurs propriétaires avec leurs familles. Les exploitations agricoles dirigées pour le comple d'autrui occupent beaucoup plus d'ouvricers et le nombre des auxiliaires est plus considérable que celui de l'ensemble des fermiers, métayers et colons.

Pour 100 personnes vivant de la profession, il y a, dans l'agriculture, 20 p. 100 de patrons et 17 p. 100 de salariés; dans l'industrie, 10 p. 100 de patrons contre 37 p. 100 d'ouvricrs, le reste se composant des familles. L'avantage, ici encore, reste donc à l'agriculture, tout au moins aux yeux de ceux qui pensent quil est plus profitable, pour le gain du travail et pour la dignité de l'homme, de travailler pour soi que pour autrui et chez soi que chez le voisin ".

1. Les conditions analogues de la population industrielle, à quelques exceptions près, sont en proportion inverse de celles de la population agricole, ce qui s'explique aisément (?) si l'on considère que ces deux groupes réunis représentent près des $3 / 4$ de la population totale de la France. (Bulletin de statistique du ministère des Finances, novembre 189', p. 507.) 
La répartition géographique de la population vivant de lagriculture n'est pas sans importance sur le régime de la propriété foncière. La proportion varie peu dans le centre de la France, entre la Seine et la Dordogne; l'effectif agricole, très dense dans les Alpes, dans les Cévennes (Ardèche, Lozère, Cantal, Lot) el dans les Côtes-du-Nord, se raréfie au Nord et it l'Est et tombe au-dessous de 24 p. 100 dans les départements du Nord, du Rhòne, de la Seine-Inférieure ${ }^{1}$. Mieux encore que les détails que je viens d'indiquer, trois ordres de faits constatent les progrès incessants de la population rurale et réduisent à néant les objections de ceux qui, dans un but de dénigrement ou avec des projets de spéculation, font grand bruit de la crise agricole et réclament pour l'atténuer une intervention de l'État, laquelle, si la crise existait en réalité, n'aurait d'autre résultal que de l'aggraver.

Ce sont les progrès de l'habitation, de l'alimentation, de l'acquisition.

L'habitation, ce vêtement à distance, est le plus caractéristique des éléments par lesquels se manifeste le bien-ètre des familles; c'est le plus apparent et c'est aussi celui qui se modifie avec le plus de lenteur, à cause de sa nature qui l’immobilise et de la dépense très lourde que nécessite sa moindre transformation.

L'habitation, depuis surtoul soixante ans, a fait sur tout le territoire des progrès considérables; toutefois, quantité de nos rillages offrent encore un aspect misérable, beaucoups de maisons sont couvertes en chaume, bèles et gens vivent souvent dans une promiscuité désolante, et des paysans mème riches méconnaissent les plus simples notions de l'hygiène.

Le nombre des maisons rurales d'habitation n'a fait que croître depuis 18306 , date du premier recensement de cette nature $^{2}$. Il y avait, en $18 \ddot{36}, 6418962$ maisons ayant un

1. Recensement de 1891, graphique $n^{\circ} 48$.

2. Je considere comme habitations rurales les maisons ayant moins de deux étages. Le nombre total (moins le département de la Seine) des maisons en France (d'un toyer inférieur à 2000 francs et sans y com- 
rez-de-chausséé ou un rez-de-chaussée et un étage; depuis lors, on en a construit 1938168 . Le nombre des maisons n’ayant qu'un rez-de-chaussíc a diminué sensiblement et celui des maisons à un élage augmenté de 33 p. 100. C'est dans la région centrale qu'il reste le plus d'anciennes maisons basses. La preuve de l'amélioration continue des maisons d'habitation est dans la progression du produit de l'impôt des portes et fenètres qui passe, de 30 millions de francs en 1838, à 40 en $18 \% ̈ 3$, à 38 en 1870, à 89 en $1893^{\circ}$. On comptait cependant en 1891 plus de 346000 maisons vacantes, 2 p. 100 dans le Centre el le Nord, jusqu'à 8 et 10 p. 100 dans le Midi, précisément là où l'on constate le plus de constructions neuves.

Le progrès de l'habitation varie de région à région et même, dans un département, de commune à commune.

Chaque contrée a son type caractéristique, mille fois répété, sans altérations sensibles. Mais, autant les maisons rurales se ressemblent dans chaque région considérée isolément, aulant elles diffèrent comme physionomic extéricure et comme aménagement, quand on passe d'une partie de la France à une autre ${ }^{2}$.

Le mode de groupement des maisons rurales est également varié; tantòt elles sont agglomérées comme si elles s'attiraient l'une l'autre, tantòt elles sont dispersées et disséminées; il faut en voir la cause dans les traditions historiques plutôt que dans des raisons économiques. L'industrie initiale ayant été partout l'exploitation du sol, il semblait naturel qu'après le lotissement ou l'oceupation chaque

prendre les locaux industriels, les bâtiments de l'État et les chàteaux, était, en 18ä6, de 7360408 ; en 1891, il était, d’après le dénombrement, de 7842033 , et, d'après le service des Contributions directes, de 8870689 . Cette erreur de 1 million en nombre est du fait des agents recenseurs (Rapport officiel, p. 93) et doit mettre en garde contre la précision relative des statistiques établies par les municipalités.

1. Plusieurs monographies ont été publiées dans cet ordre d'idées avec plans et croquis : Monographie de la commune de Chassey-en-Auxois (Cóte-d'Or), par 11. de Saint-Genis (1897-1901); - De l'habitation dans le departement de l'Oise, par M. Baudran, 1901, etc.

2. Statistique générale de la France, 1896, tableau 557. 
famille allàt vivre sur son domaine, au centre de son bien, surtout à l'époque où la culture extensive rayonnait largement et où la circulation était malaisée; mais l'isolement était un danger, et les groupes primitifs se sont coneentrés ou dispersés selon que l'exigeaient les circonstances de temps el de lieu. La question de l'habitation a d'ailleurs été traitée récemment arec une ampleur et une science qui me dispensent d'y insister davantage ${ }^{1}$.

Les progrès de l'alimentation paysanne, en quantité et en qualité, sont encore plus accentués. Je ne parlerai, pour abréger", que du pain et de la viande.

Les céréales ont de tout temps été en France la base de la nourriture de la population et par conséquent la culture principale du pays ${ }^{2}$. Dans les villes, on fait un usage presque exclusif de pain de froment; dans les campagnes, on consomme en outre, suivant les régions, une certaine quantité de seigle, de sarrasin et de maïs; mais, à mesure que l’aisance se répand, les grains inférieurs sont délaissés et remplacés par le blé. La quantité de froment nécessaire à la consommation du pays n'a cessé de croître, en voici la série progressive, en millions d'heetolitres :

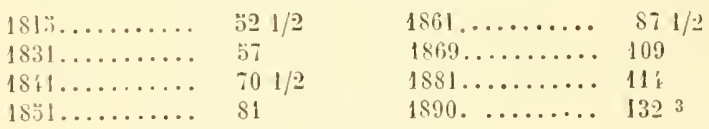

Depuis 1878, il n'y a plus dans le commerce des blés d'excédent des exportalions sur les importations qui ont passé, de 4399 hectolitres en 1833 , à 27 millions $1 / 2$ en 1891 .

La France est un pays importateur de blés; elle l'est de plus

1. Enquêle sur les condilions de l'habilation en France, provoquée par le Comité des travaux historiques et dirigée par M. de Foville, 2 vol., $1899_{t}^{\prime}-1899$.

2. On pourrait dire inversement que la réussile de celít culture sur notre sol en a provoqué la consommation directe. (Revue ales Deux Mondes, 1880, 1, 613.)

3. Les statistiques donnent en bloc la quantité des grains et des farines; la farine yest convertie en grains sur le pied de 70 kilogrammes de farine pour un quintal de grains, le poirls de l'hectolitre de blé étant évalué à 75 kilogrammes. (Enquête de 1892, p. 113 de l'Introduction.) 
en plus, et, depuis soixante ans, les besoins de la consommation saccroissent plus rapidement que la production. Celle-ci n'a pas diminué du fait du rendement (très variable d'ailleurs suivant les régions) qui a sensiblement augmenté, mais la superficie consacrée à cette culture a perdu 193690 hectares depuis trente ans (1862), soit 1,61 p. 100.

Le mouvement n’a pas été uniforme; en 1892, les superficies ensemencées en froment étaient restées stationnaires dans 50 départements, araient augmenté dans 18 et diminué dans 19. Il y a un rapport étroit, sur lequel je reviendrai dans les chapitres qui suivent, entre ces transformations de cultures et la division de la prepriété.

L'augmentation dans plusieurs départements a élé le résultat des ravages causés par les parasites de la vigne (Lot, Dordogne, Charente-Inférieure, Saòne-et-Loire, HauteGaronne, Aude, Tarn, Vienne, etc.); on a dù remplacer les vignes détruites par des cultures diverses dont la plus naturelle parut être le froment. Ce fut un tort, au lieu de remplacer il fallait reconstituer ${ }^{1}$. Pour d'autres (Creuse, Corrèze, elc.) elle est le signe d'un progrès cultural qui a permis la substitution progressive du froment au seigle et

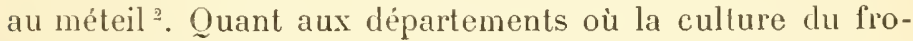
ment a diminué, ce sont ceux de l'ancienne Normandie qui ont étendu leurs herbages à son détriment ${ }^{3}$, ceux qui ont créé des prairies (Doubs, etc.), ceux qui ont reconstitué

1. En 1830 , il y avait plus de 20000 hectares de vignes en Seine-et-Oise; en 1899 , il n'en restait que 6700 , du fait de la substitution de céréales ou de plantes légumières. $0 \mathrm{r}$, dans cette région, le produit net de l'bectare de vigne est de 1400 francs. Quelle est la culture courante, blé, avoine, betteraves, qui peut donner un revenu équivalent, alors que l'hectare planté en vignes n'a pas, en général dans ce département, une valeur vénale supérieure à 8000 francs. (Société nationale d'agriculture, Journal officiel du 3 avril 1900, p. 2093.)

2. Ce sont les céréales des pays paurres (Limousin, Auvergne, Sologne, Bretagne); sur 2422 milliers d'hectares, depuis 1862 , ces cultures en ont perdu 613 .

3. Depuis dix ans, dit l'enquête de 1892, les prairies artificielles ont gagné 92585 hectares, les prairies naturelles 277403 hectares, les herbages pâturés 930423 hectares. Chaque année, depuis 1893, l'accroisscment est d'environ 7 p. 100. 
leurs vignobles en empiétant sur les céréales (Hérault, Gard, Vaucluse, Var, etc.)

En résumé, depuis soixante ans, la consommation du froment, en France, s'est accrue de $\breve{3} 4$ millions d'hectolitres et la production indigène de 39 millions; la différence vient de l'étranger. La quantité de froment à réserver pour la semence est de 2,07 hectolitres à l'hectare, celle qui est affectée aux transformations industrielles (pàtes alimentaires, ete.) peut šéraluer à ö p. 100 de la production totale (récolte et importation); le reste va dircctement à la consommation humaine.

En 1831, chaque individu consommait par an 1,64 hectolitre de froment; en 1891, il en absorbait 2,69; en 1898, 2,72. C'est unc augmentation de 108 litres par bouche.

L’accroisscment considérable du capital des cheptels, en quantité et en qualité, a eu pour conséquence immédiate l'augmentation dans la consommation de la viande.

La quantité de viande fraiche produite par les animaux abattus en France a suivi la progression suivante :

En milliers de $\mathrm{kg}$.

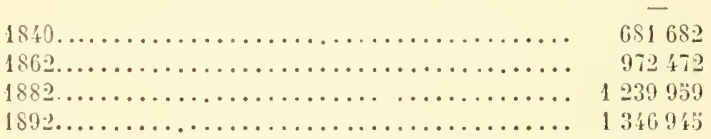

Si lion admet que les viandes de eheval, de mulet et d'âne sont consommées à peu près exclusivement par les centres industriels et les villes importantes, on peut calculer dans quelles proportions les viandes fraîclıes participent à l'alimentation des populations urbaines el rurales.

\begin{tabular}{|c|c|c|c|}
\hline $\begin{array}{l}\text { QUANTITE } \\
\text { DE VIANDE }\end{array}$ & $\begin{array}{l}\text { TOTALE } \\
\text { COXSOYYÉE }\end{array}$ & \multicolumn{2}{|c|}{$\begin{array}{l}\text { RĹPARTITION PROPORTIONNELLE } \\
\text { DE LA VIANDE CONSOMNEE }\end{array}$} \\
\hline $\begin{array}{c}\text { Dans les communes } \\
\text { do plus } \\
\text { de } 10000 \text { halitants }\end{array}$ & $\begin{array}{c}\text { Dans les communes } \\
\text { de moins } \\
\text { de } 10000 \text { habitants }\end{array}$ & $\begin{array}{l}\text { Par } \\
\text { la population } \\
\text { urbaine }\end{array}$ & $\begin{array}{l}\text { Par } \\
\text { la population } \\
\text { ruralo }\end{array}$ \\
\hline $\begin{array}{l}\text { Kilogrammes } \\
632767315\end{array}$ & $\begin{array}{l}\text { kilogrammes } \\
711858258\end{array}$ & 47.83 p. 100 & 32,17 p. 100 \\
\hline
\end{tabular}


L’importation apporte un fort contingent qui s’ajoute à la production indigène. Le mouvement des viandes fraiches dépecées se traduit depuis trente-sept ans par des excédents d importation continus, sauf pour l'année 1893, et il en est de mème, de 1870 à 1896 , pour les viandes salées et fumées ${ }^{1}$.

On a signalé une tendance des grandes villes à diminuer leur consommation en viandes fraîches tandis que celle des viandes salées ou apprêtées (charcuterie, etc.) y augmente. On l'attribue à l'exode de plus en plus général des familles aisées à la campagne pendant l'été, et surtout à l'usage croissant et excessif des boissons alcooliques et des bières étrangères qui déshabitue de manger, pour boire. Ce fléau, jusqu’ici enfermé dans les grandes villes, commence à altaquer les populations rurales ${ }^{2}$ du fait de la licence des cabarets, qui s'installent dans les plus petits villages ${ }^{3}$.

On a calculé les progrès faits depuis trente ans dans la consommation de la viande fraîche, par tête et par an, à la ville ${ }^{4}$ et à la campagne :

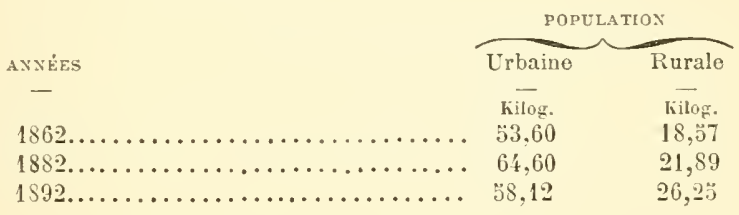

1. Enquête de 1892, Introduction, p. 343.

2. La robuste santé des paysans est atteinte par le tabac et l'alcool. Celle des enfants elle-même est menacée; plus de joues fraîches et roses, des mines pâles et amaigries et des maux d'estomac, surtout cliez les jeunes filles. Les médecins en voient la cause dans les séances trop longues de l'école et dans la suppression trop brusque de la vie en plein air. ll faut aussi faire la part de l'hérédité. Les enfants portent la peine des excès, alcooliques ou autres, de leurs parents.

3. En 187' (sans compter Paris), il y avait 342980 débitants; en 1893 , on en compte 42\%575. (Statistique générale, 1897, tableau $\mathrm{n}^{\circ} 401$. .) La quantitẻ d'alcool pur consommé par tête et par an a passẻ de 1 litre 46 en 1830 à 4 litres 19 en 1896. (Ibid., tableau $n^{\circ} 392$.)

4. Depuis 1872, les viandes salées remplacent avec excès les viandes fraîches dans l'alimentation des classes laborieuses et surtout des ourriers industriels. 
La consommation des viandes fraîches de 1882 à 1892 a donc diminué de 6,48 dans les villes et augmenté de 4.36 dans les campagnes; les deux facleurs de ce mourement en sens inverse sont incontestablement, à la ville, l'usage de plus en plus immodéré du tabac ${ }^{1}$ et des boissons alcooliques, à la campagne la diffusion du bien-être.

L'alimentation rurale s'est augmentée et améliorée, depuis 1870 surtout, dans une très forte proportion, par l'utilisation des animaux de basse-cour dont l'élevage a fait de grands progrès ${ }^{2}$. La poule au pot du dimanche n'est plus un rêve, le paysan ne se prive ni de poulets, ni de canards, ni d'oies, et le vou d'Henri IV est largement rempli.

Le troisième fait démonstratif de la plus-value eontinue clu capital foncier et du rerenu agricole aboutissant à un bénéfice est la progression soutenue dans l'accumulation de l'épargne. Cette accumulation de capitaux entre les mains de la population rurale s'alteste par les dépòts à la Caisse d'épargne, l'achat de titres par petites coupures, et l'acquisition de terres.

Le nombre des caisses d'épargne privées, de 21:k qu'il élait en $183 \%$, s'est élevé à 1676 en 1893 (y compris les succursales); aux mèmes dates, le nombre des livrets a passé de 121527 i 6328947 , et le solde dù aux déposants le 31 décembre, de 62 millions à 3 milliards 286 millions de francs. La Caisse nationale d'épargne ne fournit pas de statistique dont les chiffres soient comparables à ceux des caisses privées; mais, en 1893, l'excédent des versements sur les remboursements était de 69 millions $1 / 2^{3}$. Il serait intéres-

1. De 1830 à $189 \%$, le poids des taluacs livrés aux débitants pour être vendus a sauté de 11 à 36 millions de kilogrammes. Les sommes déboursees par les consommateurs en $1890 \%$ ont dépassé $\$ 13$ millions de franes, soit 10 fr. 85 par tête sur l'ensemble de la population el 18 franes si on répartit la dépense seulement sur les adultes de quinze a soixante ans.

2. L'enquète agricole $(1897,317)$ effeure ce revenu des fermes qu'on évalue à 6 fr. 2.3 a l'hectare et qui a exporté, en 1892 , pour 64 millions de francs. La part de la basse-cour dans l'alimentation paysanne est devenue considérable; c'est un progris rẻel.

3. Il faut aller chercher les détails dans les rapports annuels adressés au président de la liépublique. En 1895, il y avait 7391 bureaux de 
sant, au point de rue de l'évaluation de la fortune mobilière de la population rurale, de rechercher le ròle des caisses privíes el celui de la caisse nationale selon les régions, ce sont des questions qui influent directement sur le problème du crédit agricole et sur celui de l'acquisition et de la division de la propriété; mais je ne puis ici qu'en signaler les lignnes générales.

Les paysans ne changent pas volontiers d'habitudes et confient de préférence leur épargne aux caisses locales, connues d'anciemne date, administrées de façon non seulement à conserver mais à faire fructifier les dépôts qui leur sont confiés ${ }^{1}$; ils se méfient de l'État, cet être anonyme qui peut tout, attire tout à lui et rend difficilement ee qu’il a pris ${ }^{2}$; les hommes d'affaires écartent leur clientèle de ce gouffre qu est devenue la Caisse des Dépòts et Consignalions, transformée, par les lois récentes sur l'assistance el la mutualité, en courtier du Trésor.

Si l'épargne rurale va de préférence aux caisses privées, qu'on a sous la main, où le dépôt est facile et le retrait rapide, elle se porte aussi, depuis les grands emprunts nationaux et les trop nombreuses émissions de valeurs à lots, sur les petites coupures du Crédit foncier et de la Ville de Paris, et mème sur la rente 3 p. 100 el les obligations de chemins de fer ${ }^{3}$. On a calculé que la province, abstraction

poste faisant concurrence aux 1676 caisses d'épargne privées; le nombre de comptes en cours était de 2488073 et le solde dû aux déposants dépassait 753 millions. La moyenne des livrets avait passé de 22 f fr. 97 en 1882 à 303 francs en 1893. (Journal officiel du 27 décembre 1896.)

1. Article $1^{\text {er }}$ des statuts de la Caisse d'épargne de Châteaurenault (Indre-et-Loire) publiés au Journal officiel du 3 avril 1900, p. $208 \ddot{.}$. Le nombre des livrets au-dessous de 500 francs entre pour 79 p. 100 clans le nombre total; la proportion de ceux au-dessous de 100 francs est de $5 \check{5}$ p. 100, au-dessous de 20 francs de 330 p. 100. (Caisse nationale, 1895.)

2. On n'a pas oublié une affaire de prévoyance qui passionna trois générations dle nos pères, la Tontine ou Caisse Lafarge, crée le 2 mars 1791, qui ruina les souscripteurs et fit tomber 70 millions dans la caisse du Trésor par les décrets des 4 nivôse an Vl et $1^{\text {er }}$ avril 1809.

3. II. Neymarck a publiẻ un travail très curieux et très documenté sur le Morcellement des valeurs mobilières. 
faite de la clientèle des grandes villes, absorbait depuis 1880 environ 27 p. 100 des titres émis.

Il est hors de doute que s'il y arail une véritable crise agricole et qu'elle sévîl avec l'intensilé et la continuité qu'on lui suppose, le bien-être et les facultés d'épargne du cultivateur en auraient souffert et que les effets de cette gène seraient visibles.

Mais e'est dans le mouvement foncier que se manifeste avec le plus d'évidence la vitalité rurale. Si l'on dressait le bilan complet de tout ce que les propriétaires et exploitants agricoles tirent de leur poche pour les frais de la culture, l'impòt, et toutes les formes de la prévoyance (assurances contre lincendie et les risques agricoles, assurances sur la vic, cotisations pour les sociétés de secours mutuels, les syndicals agricoles, etc.), en tenant compte de ce que leur coûtent l'alimentation, l'entretien et l'éducation de leurs familles, on serait éfonné du chiffre de revenu médiocre accusé par les statistiques ${ }^{1}$.

En eflet, qu'on me permette de revenir un instant sur les résultats généraux tcls que les publie la statistique officielle, et, à son imitation, rappelons comme point de départ l'évaluation du produit brut de l'exploitation du sol en 1882 :

Résultats en millions de fr.

Production végétale......................... 11502

Procluction animale....................... 7183

Ensemble...................... $\overline{18683}$

$\Lambda$ déduire comme faisant partie du capital d'exploitation qui se reconstitue chaque annee (semences, pailles, engrais, nourriture du bétail).......... 5 르:

Rieste........................... $\overline{\mathbf{1 3 4 6 1}}$

Dont il convient de retrancher :

$1^{\circ}$ La dépréciation sur le produil brut qu'établit l'enquête de 1892 , savoir........... $2^{\circ}$ Les frais généraux à $40 \mathrm{fr}$. par heetare cultive et 8 fr. par heetare tle bois.......

$3^{\circ}$ Les charges principales de la culture (impôts, salaires, etc.) que l'enquête diminue de 582 millions sur celles tle 1802 , reste. 1023 i

lieste pour le produit net de $1892 . . . . . . . . . . .$.

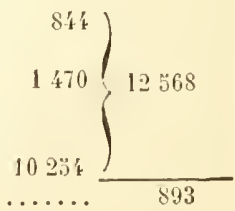

1. Le produit brut agricole, en 1882, était évalué à 13461 millions de 
Cetle évaluation paraît invraisemblable. Il ne semble pas rationnel que nos 3604789 chefs d'exploitation n'aient à se partager que 893 millions de francs, soit $242 \mathrm{fr}$. 23 par tête au lieu de 330 franes en 1882, alors qu'ils payent à leurs 6663130 salariés plus de quatre milliards de franes de salaires.

C'est la preuve du danger des moyennes et de l'impossibilité, avec notre outillage administratif actuel et nos routines bureaucratiques, d'élablir avec une précision absolue des résultats qu'on ne samait avancer sans les faire suivre d'un point d'interrogation, suivant la spirituclle expression de M. de Foville ${ }^{1}$.

Depuis 1878 seulement, les relevés d'actes et de mutations établis par l'administration des Finances contiennent les classements et les détails qui permettent de transformer les comptes de la pereeption de l'impòt, de statistiques purement fiscales, c'est-à-dire d'une portée très étroite et très limitée, en statistiques économiques dont l'utilisation est quasi universelle. Depuis 1890, le service de l'Enregistrement, qui constate sur ses registres tous les mouvements de la fortune publique et peut en mesurer les plus légères oscillations, a multiplié par des combinaisons ingénieuses les cadres de ses relevés de façon à saisir el à fixer toutes les manifestations de l'initiative sociale. C'est à ces tableaux $^{2}$ que la science économique pourra désormais demander ses informations les plus sûres; l'exaclitude de leurs chiffres ne pourra pas être contestée puisqu'ils sont

francs et le produit net à 1153 millions, soit 387 francs par hectare cultivè pour le produit brut et 330 francs par tête d'exploitant pour le produit net. (Bulletin de statistique du Ministère, 1888, février, p. 158.) Or, en 1892, on estime que le produit brut a subi une dépréciation de S4'́ millions; la quote-part derrait donc être diminuée d'autant.

1. Le morcellement, p. 77 et 79 . Les enquêtes décennales, ajoute-t-il, n'ont qu'une valeur relative; toutefois, les résultats peuvent en itre acceptés à titre d'approximations, toutes les fois qu'il n'y a pas de raison pour que les erreurs commises se portent d'un côté plutôt que de l'autre. On ne peut pas avoir l'ironie plus courtoise.

2. Quatre volumes ont déjà été publiés en $1897,1898,1899$ et 1901 , et il serait à souhaiter qu'on allouât à cette publication un crédit suffisant pour la renılre aussi complète que possible. 
l'expression de la pereeption des droits, après un triple contrôle, et qu'on en écarte avec soin les moyennes et les ealculs arbitraires.

Un diagramme (1898, page 166) montre le mouvement ascensionnel continu des mutations immobilières à titre onéreux, de 1826 à 1895 , avee les reculs et les interruptions que eausèrent les erises politiques, financières, commerciales et industrielles; le nombre des ventes ordinaires, pour l'ensemble, est en décroissance, mais cet affaissement frappe les acquisitions d'un gros prix et non les petites.

Malheureusement, la répartition par catégories de prix des aequisitions d'immeubles n'a été encore faite que deux fois, en 1841 et en $189 \ddot{3}$, et les elassements étant différents, leurs ehiffres ne sont pas comparables '. Toiei les indications que révèle le mouvement foncier de lannée 1894.

\begin{tabular}{|c|c|c|c|c|c|c|}
\hline \multirow{2}{*}{$\begin{array}{l}\text { CATEGGORlli } \\
\text { DES veNTES } \\
\text { d'apres leur prix }\end{array}$} & \multicolumn{2}{|c|}{$\begin{array}{c}\text { 1MMEUBLES } \\
\text { URBAIXS }\end{array}$} & \multicolumn{2}{|c|}{$\begin{array}{l}\text { IMMEUBLES } \\
\text { RURAUX }\end{array}$} & \multicolumn{2}{|c|}{$\begin{array}{l}\text { IMMEUBLES } \\
\text { MIXTES }(\Lambda)\end{array}$} \\
\hline & Nombre & $\begin{array}{c}\text { Total } \\
\text { des prix }\end{array}$ & Sombre & $\begin{array}{c}\text { Total } \\
\text { des pirix }\end{array}$ & Nombre & $\begin{array}{c}\text { Total } \\
\text { des prix }\end{array}$ \\
\hline $\begin{array}{l}\text { Au-dessous de } 50 \text { fr... } \\
\text { De } 500 \text { ir. a } 10010 \mathrm{fr} . \\
\text { De } 1000 \mathrm{fr} \text {. } 55000 \mathrm{fr} \text {. }\end{array}$ & $\begin{array}{l}23905 \\
17166 \\
10583\end{array}$ & $\begin{array}{r}5509100 \\
12422900 \\
98125200\end{array}$ & $\begin{array}{ll}325 & 162 \\
100 & 763 \\
118 & 977\end{array}$ & $\begin{array}{r}62492600 \\
69626900 \\
245233100\end{array}$ & $\begin{array}{r}5076 \\
5158 \\
10815\end{array}$ & $\begin{array}{r}1192800 \\
3777300 \\
26552700\end{array}$ \\
\hline Totaux...... & $8165 \hat{k}$ & 116057200 & 541902 & 377352600 & 21019 & 31822800 \\
\hline $\begin{array}{l}\text { De } 5000 \text { à } 10000 \mathrm{fr} \ldots \\
\text { Lu-dessus de } 10000 \mathrm{fr} \text {. }\end{array}$ & $\begin{array}{l}13598 \\
17261\end{array}$ & $\begin{array}{r}93.148500 \\
737507600\end{array}$ & $\begin{array}{l}17985 \\
11288\end{array}$ & $\begin{array}{l}155932 \\
290900 \\
2909\end{array}$ & $\begin{array}{l}2851 \\
3016\end{array}$ & $\begin{array}{r}19791600 \\
103200700 \\
\end{array}$ \\
\hline T'OTALX GÉNÉRALX... & 112513 & 917013300 & 571175 & $792 \quad 191700$ & 26916 & 151818100 \\
\hline
\end{tabular}

1. L'enquéte de 18 't ne distinguait pas la propriété arbaine et la propriété rurale et n'avait consiléré que trois catégories : de 600 franes et au-llessous, de 600 à 1200 francs, et au-dessus de 1200 francs. L'enquête de $18 \% 3$, qui reste permanente et dont les éléments continueront à ètre releves chaque année, sépare le mouvement urbain du mouvement rural el développe 29 tranches ou catégories classées par l’importance des prix de vente. 
Sur les 713604 contrats enregistrés ${ }^{1}$ en 1894, pour un prix approchant de 2 milliards de franes, les immeubles ruraux figurent en nombre pour 574173 , ayant coûté 792 millions $1 / 2$.

Dans celte dernière catégorie, 314902 acquisitions ont élé faites pour des prix inférieurs à $\not 000$ francs, 17983 pour des prix variant de $\ddot{3} 000$ à 10000 franes, el 11288 seulement, $1 / 3$ à peine, ont coùté plus de 10000 franes.

Parmi les grosses rentes, les plus nombreuses sont celles (pour les biens ruraux toujours) de 10000 à 20000 franes (6933) et de 20000 à 00000 franes (3 288); les autres sont négligeables. Parmi les petites ventes, les plus nombreuses sont celles au-dessous de $\check{500}$ franes qui forment 56 p. 100 de l'ensemble.

Il serait à désirer que, dans les prochaines statistiques, on descendit aux prix intermédiaires, de 100 à 000 francs, et qu'on fìt, par contenance, un classement analogue à celui par importance de prix, en indiquant les parcelles qui comportent une habitation.

Dans la réalité, le nombre des petites acquisitions rurales est infiniment plus considérable, el, dans certaines régions, notamment dans l'Est et dans l'extrème Sud-Ouest, pour des causes diverses, il arrive qu'un immeuble passe successivement dans sept à huit mains sans qu’il soit établi de titre régulier. Cette situation fàcheuse se maintient à cause de la proportionnalité à rebours des taxes fiscales; elle n'a cessé de s'aggraver, au cours du siècle, par suite de l'augmentation successive des droits de timbre. Actuellement, les charges fiscales frappant les petits contrats suivent la proportion décroissante ci-après ${ }^{2}$ :

1. Les imperfections de nos lois foncières, l'abus des sous-scings privés, l'exagération des droits de mutation et des honoraires des notrires font que $30 \mathrm{p}$. 100 environ des ventes se dérobent à l'Enregistrement au grand préjudice de la sécurité du droit de propriété.

2. Il n'y a qu'un remède : création de livres fonciers, réorganisation du service des mutations cadastrales qui n'existe qu'au budget, réduction des tarifs (la moins-value serait compensée par la suppression de la fraude). 


\begin{tabular}{|c|c|c|c|}
\hline $\begin{array}{l}\text { Impor } \\
\text { des acqui }\end{array}$ & $\begin{array}{l}\text { ance } \\
\text { sitions }\end{array}$ & & $\begin{array}{l}\text { aux des taxes } \\
\text { r chaque lou fr. } \\
\text { du prix. }\end{array}$ \\
\hline 100 & franc & 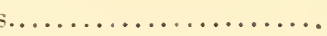 & 17,37 \\
\hline 200 & - & $\ldots \ldots \ldots \ldots \ldots \ldots \ldots$ & 12,12 \\
\hline 300 & 一 & $\ldots \ldots \ldots \ldots \ldots \ldots \ldots \ldots$ & 10,37 \\
\hline 400 & 一 & $\ldots \ldots \ldots \ldots \ldots \ldots \ldots \ldots \ldots$ & $9, \mathbf{k} 9$ \\
\hline วั00 & - & $\ldots \ldots \ldots \ldots \ldots \ldots \ldots \ldots$ & 8,97 \\
\hline 1000 & - & $\ldots \ldots \ldots \ldots \ldots \ldots \ldots \ldots \ldots$ & 8,49 \\
\hline 5000 & -- & $\ldots \ldots \ldots \ldots \ldots \ldots \ldots \ldots \ldots$ & $i, 20$ \\
\hline
\end{tabular}

Sur les différents modes d'exploilation du sol, la culture directe ${ }^{1}$ comptant dans l'ensemble pour 71 p. 100 à clle seule, ce courant continu vers l'aequisition de la petite et surtout de la très pelite propriélé, n’a rien qui puisse surprendre. Il est à la fois une cause et un effet. L'enquète signale une augmentation, de 1882 à 1892 , de 1,16 p. 100 dans le nombre des chefs d'exploitation et une diminution de 11,43 p. 100 dans celui des salariés: la population rurale ayant baissé de

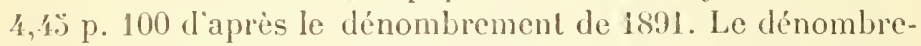
ment de 189:3 constate également l'accroissement des grandes aģ̨lomérations au détriment des campagnes par un déplacement des ruraux vers les centres industriels et commerciaux; toutefois, ce mouvement est moins accentué que dans les périodes précédentes, il y a temps d’arrêt, et cerlains départements agricoles (Finistère, Gironde, Héranlt, Marne, Morbihan, Oise, Seine-et-Marne, Seine-ct-Oise, Var, Ilaute-Vienne, Vosges) se signalent par des augmentations.

Ce symptôme est ì relenir comme une marque de reprise de soi-meme par la population agricole à qui les déceptions de la ville ont fait payer cher l'expérience acquise depuis 1862 . Il a une imporlance d'autant plus grande que le rapprochement des chifres du mourement foncier et de ceux du déplacement des travailleurs agricoles, passant d'une classe à l'autre, permet d'arriver, sans hypothèse arbitraire, par le seul constat des faits, à des conclusions quelque peu différentes de relles de l'enquête de 1892.

1. Soit $33872 \%$ cxploitants directs contre $140 \% 569$ fermiers ct métayers. (Enquête de 1892, p. 369 de l'Introduction.) 
Adoptant le mème classement en catégories, mais laissant les moyemnes pour ne prendre que les chiffres absolus (ceux-ci supposís exacts), on trouve, de 1882 à 1892 , les diflérences qui suivent:

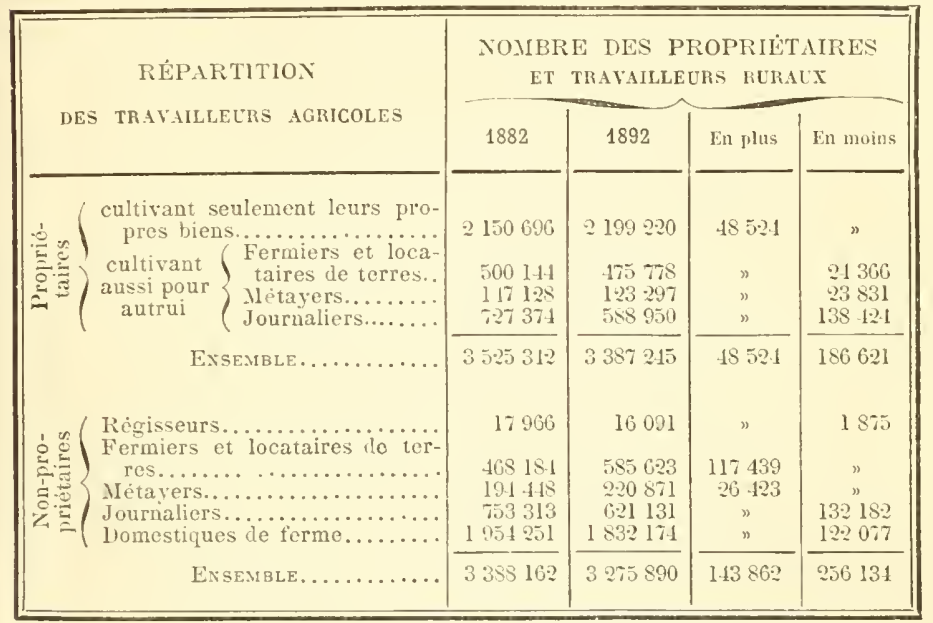

La population agricole a done perdu 250369 cultivateurs, dont 3.91 p. 100 sur les propriétaires et 3,31 p. 100 pour les salariés, dit l'enquête; pour les autres, il n'y a qu'un déclassement, ils ont passé d'une catégorie dans l'autre, les uns montant d'un échelon, les autres descendant. C'est la condition normale de l'humanité; je ferai seulement remarquer que l'agriculture est la seule industrie, en France, où le nombre des patrons reste, malgré les crises, supérieur à celui des salariés.

Deux faits dominent ce tableau en ce qui touche la grande catégorie des propriétaires-exploitauts, cette base essentielle de la population des travailleurs agricoles : l'augmentation du nombre des propriétaires se livrant exclusivement à la culture de leur propre héritage, la très forte diminution de la classe des journaliers, c'est-à-dire des très petits propriétaires. 
L'enquêle suppose que les 138000 journaliers qui manquent à l'appel de 1892, se sont haussés d'un cran et sont devenus à leur tour, jusqu'à concurrence de 90000 , propriétaires ne travaillant plus pour autrui, tant en remplacement de la perte probable sur la première classe en dix ans qu'en augmentation de celle classe; les autres (48000) auraient vendu leurs biens, soit pour émigrer vers les villes, soil pour devenir de journaliers locataires. Mais, en calculant sur une diminution supposée normale du nombre des propriétaires, les rédacteurs de l'enquête ont oublié de tenir comple du jeu des mutations par décès, qui comblent les vides, et du résultal des partages qui mettent deux ou trois proprićlaires sur un mème domaine, lì où il n’y en avait qu'un. Dans la seule année 1898, il y a cu 246534 mutations par décès d'immeubles ruraux, d'une valeur en capilal de un milliard et demi de francs; sur 430810 déclarations relatives à des valeurs diverses (dont 313360 concernant des biens fonciers), 281353 ćtaient dévolues en ligne directe et, dans cette ligne, 62.992 à des familles comptant de 4 à 7 enfants, c'est-à-dire rraisemblablement it des ruraux ${ }^{1}$. Si l'on rapproche ce mourement successoral considérable du grand nombre d'acquisitions de propriétés rurales (\$74.173 en 189\%) constatées annucllement, en dehors de celles qui se dérobent à l’impôt et échappent à la statistique, on ne peut pas atre surpris de l'augmentation dn nombre des proprictaires exploitant uniquement leur bien. Mais, comme il y a infiniment moins de proprićtaires de celte catégorie que d'acquisitions, il faut en conclure à une sorte de sélection produite par le jeu naturel des contradictions économiques dans les rangs des trois autres classes de propriétaires: locataires de terre, mélayers el journaliers. Parmi ceux-là, cenx qui ont réussi, ont agrandi leur domaine, acheté des parcelles contiguës ou des enclaves, ćlargi leur exploitation; les autres, soit par le désir du changement, soit par l'effet de la maladie, des mauraises récoltes, de

1. Bulletin de stalistique de l'Enregistrement, 1899, p. 195. 
l'obligation de vendre l'héritage au lieu de le partager ${ }^{1}$, ont disparu de ce compartiment de la statistique; bien peu ont pu se hausser d'un échelon et se suffire en abandonnant les ressourees du salaire d'autrui, puisque l'ensemble des propriétaires ne fournit qu'une augmentation de 48524 contre une perte de 186621 unités.

Quant aux eultirateurs non propriétaires, en diminution de 236134 , le défieit apparent s'explique jusqu'à coneurrence de 143862 par l'augmentation du nombre des fermiers et des métayers ${ }^{2}$. Il s'est produit dans la catégorie des salariés un mouvement ascensionnel comparable à celui que jai signalé dans les propriétaires, se déplaçant d'une classe à l'autre; ici, le progrès est plus sensible. Des journaliers, et surtout des domestiques de ferme ${ }^{3}$, se sont faits métayers et locataires de terre, les premiers dans le Midi et le Centre, les autres principalement dans le Nord et le Nord-Ouest.

La première étape de l'ouvrier agricole pauvre, dès qu'il a pu se dégager par son épargne de l'obligation de travailler à gages, e'est la loeation; il a son initiative, suit son idée, se dépense à la peine et se loue lui-mème volontiers de temps à autre à la journée, mais il a conquis une demi-indépendance et s'efforee d'arriver à la seconde étape, celle de la propriété.

L'exploitation de ees petites loeatures, de ces chétifs domaines, a dit l'un de ceux qui ont le mieux expliqué les ressources du moreellement, rend la vie plus douce, plus saine, à quantité de familles. Le paysan français aime à dormir sous un toit qui lui appartienne; et quand le terrain

1. Conformément aux articles 826 et 827 du Code civil.

2. Les fermiers de la région du Nord représentent à eux seuls les 53 p. 100 de l'ensemble. Les métayers, au contraire, sont très nombreux dans le Midi, 49 p. 100 de leur classe : puis, dans le Centre. La reconstitution des vignobles a donné un nouveau relief au contrat de métayage.

3. Qui, nourris, logés et bien payés (de 100 à 500 francs au moins, selon l'àge et l'aptitude), car le salaire a partout augmenté de 8 à 10 p. 100, sauf pour les servantes (moins-value de 10 à 14 p. 100), sont mieux en situation que les journaliers chargés de famille de réaliser des économies et de travailler à leur compte, leur rêve, dès qu'ils ontamassé un pécule suffisant.

la pRopriété rurale en france. 
sur lequel cet humble toit projette son ombre lui appartient aussi, sa bèche et son arrosoir en savent tirer plus de profit que ne ferait la charrue d'un riche fermier. Voilà done un homme qui, si peu propriétaire qu'il soil, jouit réellement de l'ètre : et s'il est plus heureux ainsi, il y a aussi chance que, par surcroît, il devienne nieilleur ${ }^{1}$.

On m'excusera d'avoir insisté sur ces menus détails, ils mont paru nécessaires pour déblayer le terrain devant l'étude qui va suivre et jeter le plus de lumière possible sur le problème foncier de l'exploitation agricole qui se résume dans cette simple formule : Quelle est, actuellement, en France, la division de la propriété rurale? Serait-il à souhaiter qu'elle füt autrement?

Il serait présomptueux de trop affirmer dans une matière aussi délicate où, quand les éléments d'appréciation ne manquent pas, ils sont arbitraires, contradictoires ou équivoques. Les chiffres sur lesquels on raisonne ne sont que des résultantes, c’est-à-dire des différences finales dont les mourements de détail, en sens contraire, se manifestant par des écarts inattendus, nous restent le plus souvent inconnus par leur infinic variélé el leur complication mìme. Aussi convient-il de ne point trop fouller l'analyse et de n'apprécier qu'arec réserve.

Cependant, quand il s'aggil d'une impression générale sur l'état actuel de la propriété rurale, en France, el sur les résultats de son exploitation, il est permis de reconnaître que de grands efforts ont été faits depuis trente ans par les pouvoirs publics et les sociétés agricoles en faveur de l'agriculture. Mais l'intervention officielle, quelque éclairée qu’elle puisse etre, ne suppléera jamais à l'inilialive individuelle, à l'énergie privée. Là est la vraie force, l'action libre.

Aussi, est-il plus nécessaire que jamais de multiplier les informations, de fournir aux intéressés des documents répétés et précis. On sail que l'enseignement agricole a élé considérablement développé, que les principes scientiliques 
ont pénétré dans les fermes, que les syndicats ont mis en pratique les bienfaits de l'association; mais l'éducation économique du pays est presque tout entière à faire, et l'Académie fait œuvre utile et patriotique en multipliant les enquètes afin de dissiper les erreurs et les préjugés qui obscurcissent si souvent, pour la plupart des intéressés, les vérités les plus incontestables.

Rien n'est plus topique, à ce large et universel point de vue, que l'étude de la répartition de la propriété dans les mains de ceux qui la possèdent ou la cultivent. L'économie sociale exige autre chose que des chiffres présentés avec un art plus ou moins administratif ou plus ou moins ingénieux.

Il faut des chiffres, mais il les faut exacts; et arec eux, il faut surtout la réflexion qui les éclaire les uns par les autres et l'esprit philosophique qui leur donne la vie. Il convient aussi d'écarter les expédients équivoques et les théories malsaines, les uns répandant une fausse sécurité, les autres déprimant les caractères et brisant les ressorts.

Les prix ont baissé, il y a eu des pertes effectives, des souffrances réelles, et, à tout prendre, on pourrait dire que la crise agricole est permanente, parce qu'elle se compose, quelle que soit la période pendant laquelle on l'étudie, de quantité de petites crises localisées, spéciales tantòt à une région et tantôt à une culture ou à une industrie rurale; mais, cette permanence mème, co perpéluel déplacement des oscillations économiques n'en fait plus un mal subit, unique, auquel on peut porter remède par une action immédiate, mais un état normal, quil faut subir en s'accommodant du mieux possible à ses exigenees.

Pour connaître cet état et chercher son équilibre dans les manifestations de son instabilité, il est nécessaire de multiplier les comparaisons, de fixer la mesure des eluangements survenus dans la constitution et la répartition de la propriété, dans l'exploitation du sol, la distribution des eultures, les conditions du travail et du crédit, afin d'examiner s'il y a lieu ou de eréer des institutions nouvelles ou de laisser 
l'intérêt privé manifester son initiative dans une absolue liberté.

Ce qu'il faut dire, dès à présent, c'est que la France agricole ne cesse pas de progresser. Une population où l'on compte autant de patrons que de salariés et où le nombre des propriétaires augmente d'année en année, où le bien-être se prouve par des progrès continus et sensibles dans l'habitation, l'alimentation et l'épargne, où les cheptels morts el vifs s'accroissent en quantité et en qualité, où l'outillage industriel se substitue à la force musculaire pour l'épargner, où l'instruction devient universelle ${ }^{1}$, où le sentiment de la mutualité s'affirme, cette population ne souffre pas, et son arenir est assuré.

1. De 1890 à 1895 , le nombre des conserits illettrés est tombé de 26 à 18: dans les départements du Nord et de l'Est, la proportion des illettrés sur la population totale n'est plus que de 2 1. 100 pour les hommes, de 1 p. 100 pour les femmes. 


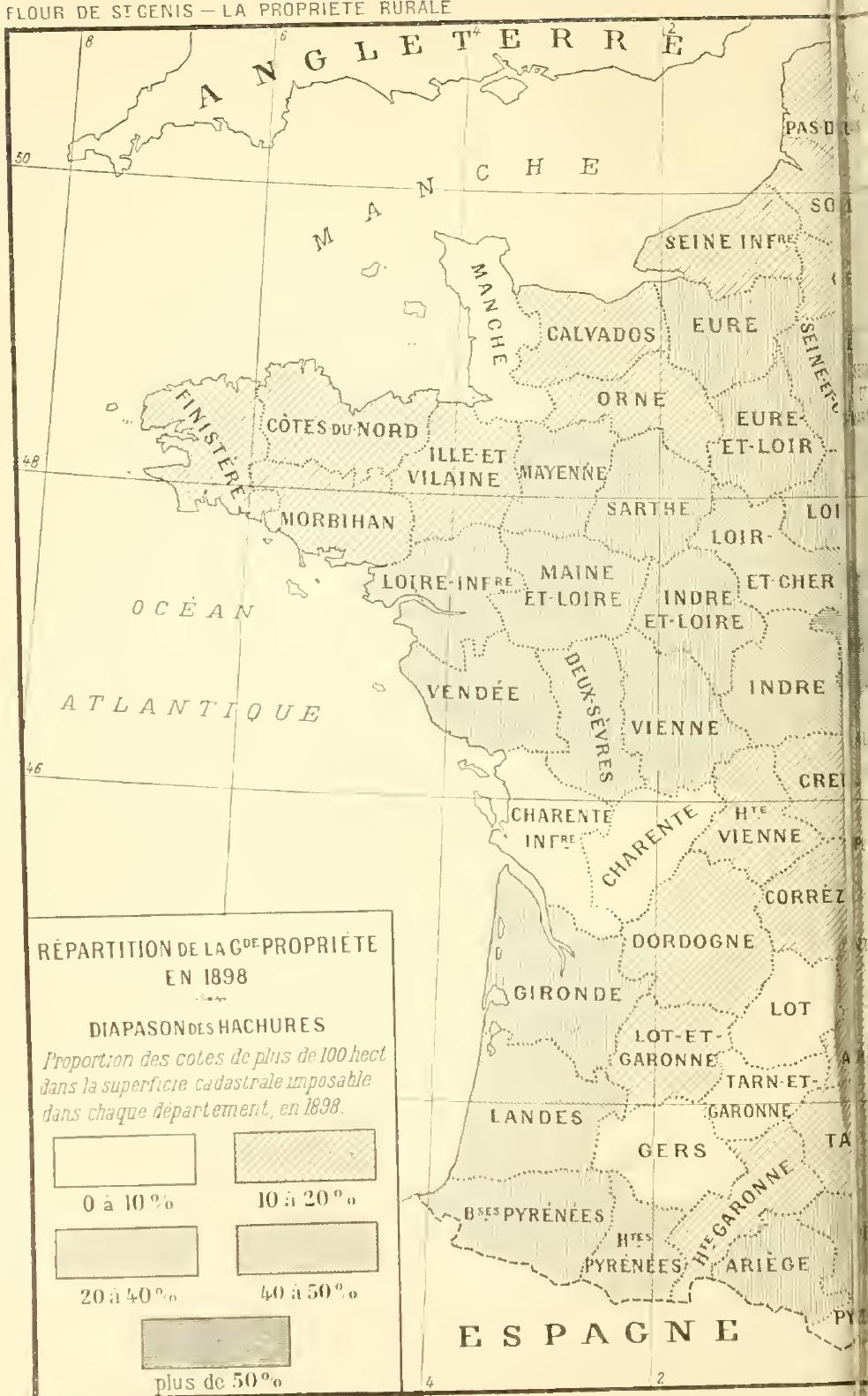




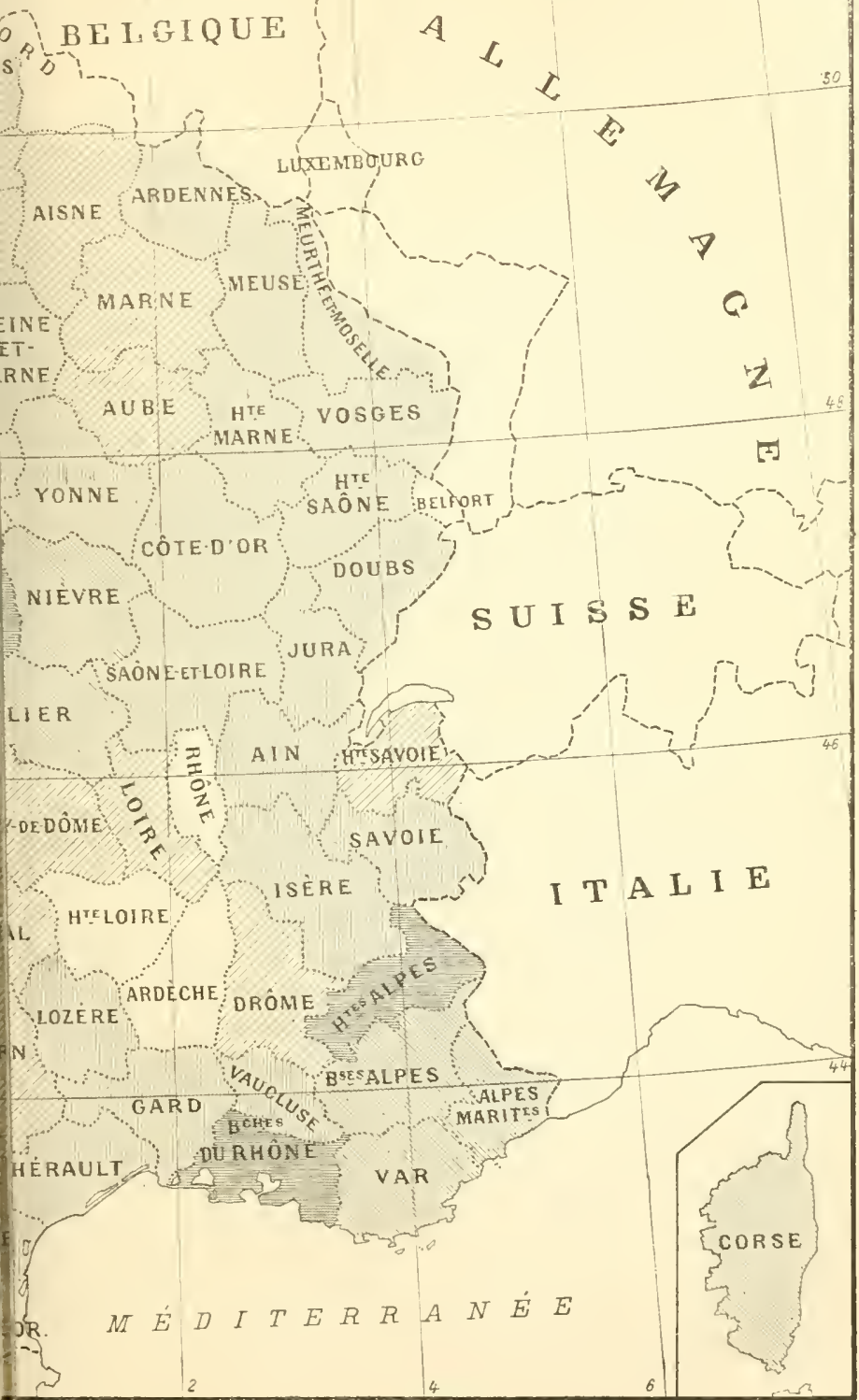





\section{CHAPITRE II}

Classement du territoire agricole de la France, à la date de 1898, en grande, moyenne et petite propriété.

La moyenne propriété a jusqu'ici été quelque peu dédaignée par les statisticicns et les économistes, et c'est en elle pourtant que réside l'avenir de l'industrie agricole.

A cntendre la plupart des spécialistes, il n'y aurail que deux facteurs véritables de l'exploitation scientifique du sol : la grande propriété et la petite. La moyenne ne serait qu'un élément accessoire, plutòt neutre qu'actif, et dont le ròle économique est presque négligeable si on le compare à celui des deux autres catégories, dont l'une représente un capital énorme el l'autre une activité laborieuse plus précieuse encore. Les statistiques et l'expérience, assure-t-on, prouvent également cet état d'infériorité de la moyenne propriété qui en fait un bloc inerte et quasi stérile en le livrant au métayage et au fermage dans les pires conditions de ces deux systèmes d'exploitation. Le dessein de ce livre est de réagir contre ce parti pris et de rectifier cette opinion en montrant précisément que ce bloc n'est inerte que parce qu'il est abandonné, qu'il n'est stérile que parce qu'il est inexploité, et que, mise en valeur, la moyenne propriété, en raison même de la qualité de ses possesseurs, peut devenir une mine plus productive que celles de l'Alaska ou du Cap.

La petite et la grande propriété existent en tout pays et se 
côtoient; c'est une de leurs conditions de progrès; elles se prêtent l'une à l'autre un concours précieux en affectant une forme imprévue de la mutualité. Un économiste en a défini le caraclère en disant : Tout grand propriétaire prévoyant devrait apporter un soin minutieux à maintenir ou à créer autour de lui un large anneau de petits propriétaires ${ }^{1}$. La politique des petits États intermédiaires n'est qu'une application de ce principe, tant il est vrai que l'humanité n'obéit qu'à quelques lois générales fort simples et qui s'engendrent logiquement par le contact des intérèts. Mais la grandc et la petite propriété ne se peuvent conceroir sans la moyenne.

On ne peut, aujourd'hui, traiter le grave sujet de la division de la propriélé, de sa répartition qui influe si directement sur le mode d'exploitation et sur le succès de la culture, sans citer le livre où M. de Foville a fixé la théorie du morcellement ${ }^{2}$ avec la délicatesse el la sûreté d'analyse qui sont indispensables pour arriver, non pas peut-être à la vérité absolue, mais à une approximation très voisine de la vérilé.

Le morcellement se présente sous trois formes différentes qu’il faut distinguer arec soin si l’on veut éviter les équiroques, si aisées en pareille matière; les statistiques rurales sont relativement récentes et les chiffres, relevés à des points de vue qui ont varié, ne sont pas toujours comparables; pour les utiliser, il faut rechercher leur origine et leur portée réelle. Ainsi, le Lableau des coles foncjères comprises dans les rôles des contributions directes constituent l'élément principal des étndes sur la division de la propriété immobilière, et cependant c'est une base bien incertaine el bien fragile.

Le nombre des cotes, seule indication des relevés publiés de 1832 à 1882 3, n’offrail aux économistes et aux statisti-

1. Paul Leroy-lieaulicu, sur la propriéte foncière (Revue ctes Deux Mondes, 1886, I, 831).

2. Le morcellement, Paris, 1886.

3. Vingt de ces dépouillements annuels, comprenant ceux de 1871 a 1883 et ceux antérieurs à l'incendie des archives du ministere des 
ciens que des à peu près, puisqu'on ignorait leur chiffre et par conséquent la proportion de leur répartition en telle ou telle catégorie. L'administration fit établir des relevés rétrospectifs, basés sur le montanl des cotes ${ }^{1}$; ce fut un premier progrès, mais on s'apercut bientôl qu'il était insuffisant. Ces opérations, en effet, ne lournissaient, au point de vue des manifestations du moreellement du sol, qu'une source d'information défectueuse, à cause du défaut de fixilé du terme choisi pour le classement des cotes, lesquelles, comprenant des centimes additionnels, variables de commune à commune et d'année en année, étaient affectées d'une instabilité qui ôlait aux rapprochements toute précision. On y pourvut en établissant la statistique des cotes de chaque département par catégories de comlenances, et e'est avec ces chiffres qu'il est possible, aujourd'hui, de elasser les 87 départements d'après l'état pour chacun d'eux de la division de la propriété et de déterminer les tendances et les variations du morcellement. J'ai emprunté à ces statistiques la plupart des chiffres de cette éturle ${ }^{2}$.

La division de la propriété s'entend done de l'étendue de chaque domaine, petit ou grand, puis du fractionnement parcellaire à l'intérieur d'une propriété et enfin de la dispersion des parcelles qui constituent une propriété. Ce qu’on appelle à proprement parler le moreellement atteint son point culminant là où non seulement il $\mathrm{y}$ a beaucoup de propriétaires, mais où l'héritage de ces nombreux propriétaires se compose de quantité de parcelles distinctes et où, par sureroît, toutes ces parcelles d'une propriété déjà petite sont éparses et disséminées. Ce phénomène n'est pas particulier à notre société rurale contemporaine, il se retrouve à toutes les époques de notre histoire économique.

Finances, ont étẻ publiés au Bulletin de statistique et de législation comparée du ministère des Finances, octobre 1882, p. 322, et novembre 1883, p. 537.

1. Pour les années $1816,1826,1836,1842$ et 1858 (Bulletin, octobre 1882, p. 321).

2. Bulletin de statistique el de tégislation comparée du ministère, décembre 1883 , p. 645 ; aout et octobre 1884, p. 156 et 388. 
Il est d'usage de distinguer dans la propriété foncière rurale trois catégories, dont voici la détermination, l'importance relative et la proportion comparée ${ }^{1}$ :

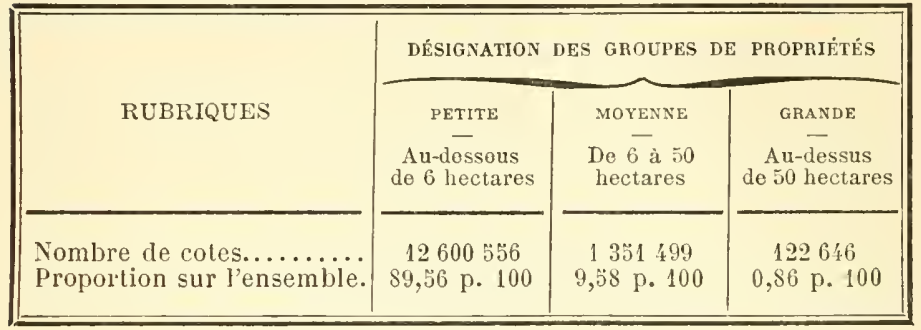

Il semble donc qu'il y ait une énorme disproportion entre la petite et la grande et que celle-ci se trouve, au point de vue tout au moins de la suprématie d!u nombre, dans un incontestable état d'infériorité vis-ì-vis de la première; mais si, au lieu de considérer le seul nombre des cotes, on examine la contenance qu'elles représentent, l'équilibre se reforme.

La très petite propriété ne possède que 1/10 du sol national, la très grande en détient encore $1 / 6$, et, à mesure qu'on entre dans le détail, on s'aperçoil qu'il ne faut pas plus s'effrayer d'une prétendue pulvérisation du sol que de ce soidisant mourement irrésistible de concentration qui ferait, à bref délai, absorber la petite propriété par la grande. Voici un tableau qui, par le rapprochement comparé du nombre et de la contenance, remet les choses au point.

1. II. de Foville explique, avec sa discrètc courtoisie, pourquoi il ne faut accepter qu'arec une certaine réserve les chiffres et les moyennes des Contributions directes. (Loco citato, p. 92.) 


\begin{tabular}{|c|c|c|c|c|}
\hline \multirow{2}{*}{$\begin{array}{c}\text { DÉSIGNATION } \\
\text { DES GROUPES DE PROPRIÉTÉS }\end{array}$} & \multicolumn{2}{|c|}{ NOMBRE DE COTES } & \multicolumn{2}{|c|}{ CONTENANCES IMPOSABLES } \\
\hline & $\begin{array}{c}\text { Parts proport. } \\
\text { pour } 100\end{array}$ & $\begin{array}{l}\text { Nombres } \\
\text { absolus }\end{array}$ & $\begin{array}{c}\text { Parts proport. } \\
\text { pour } 100\end{array}$ & $\begin{array}{l}\text { Nombre } \\
\text { d'hectires }\end{array}$ \\
\hline \multirow{6}{*}{ 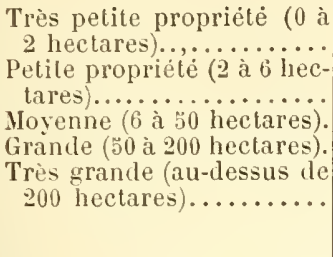 } & 74,09 & $10 \div 26368$ & 10,53 & 5211436 \\
\hline & 15,47 & $217 \div 188$ & 15,26 & 7543347 \\
\hline & 9,58 & 1351499 & 38,94 & 19217902 \\
\hline & 0,74 & 1050070 & 19,04 & 9398057 \\
\hline & 0,12 & 17676 & 16,23 & $80175 \%$ \\
\hline & 100,00 & 1407 \& 801 & 100,00 & 49388304 \\
\hline
\end{tabular}

La très petite propriété est à la grande, en nombre, comme 74,09 est à 0,12 et en contenance comme 10,3̈3 est à 16,23. L'écart du nombre est prodigieux, 10 millions contre 17000 ; mais le chiffre de la contenance n'est-il pas plus étonnant encore? Ces einq millions d'hectares émiettés sous la forme de douze millions d'héritages minuscules ne sont pas des non-valeurs, et il serait intéressant de savoir au juste ce que rapportent à leurs modestes el laborieux propriétaires ces lots de terre hachés si menu.

Le second échelon, la petite propriété (de 2 à 6 hectares), conserve sa proportion, de 180,47 p. 100 en nombre à 15,26 p. 100 en contenance. Cette équivalence mériterait une explication que les documents administratifs ne donnent pas et que les économistes n'ont pas eherchée.

La moyenne propriété fournit matière à des rapprochements dont les conclusions sont à l'inverse de celles de la très petite propriété. Tandis qu'en nombre elle ne clonne que 9,58 p. 100, en contenanee elle est de 38,94 p. 100. Cette catégorie de propriétaires, dix fois plus nombreuse que celle de l'aristocratie terrienne ( 1334499 contre 122 746), possède un territoire d'une étendue à peu près égale, l'écart entre les deux proportions n'étant que de 3,67 p. 100 en sa faveur.

Ce n'est pas, comme on l'a souvent écrit, dans les départements les plus pauvres que s'est maintenue la très grande 
propriété; ainsi les 3 départements où sa contenance absorbe plus de 50 p. 100 de la superficie imposable totale sont : les Hautes-Alpes ( 59,8 p. 100), le Cher $(53,8$ p. 100) et les Bouches-du-Rhône (̋̋3,5 p. 100); dans ces mêmes départements les cotes minuscules de moins de 10 ares, avec une moyenne d'environ 2 à 4 ares, se répartissent comme suit :

\begin{tabular}{|c|c|c|}
\hline & $\begin{array}{l}\text { Nombre } \\
\text { de cotes. }\end{array}$ & $\begin{array}{c}\text { Hectares } \\
\text { à partager. }\end{array}$ \\
\hline Hautes-Alpes.................. & 9133 & 368 \\
\hline 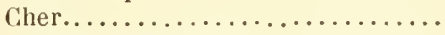 & 25292 & 1209 \\
\hline Bouches-du-Rhône............. & 39624 & 903 \\
\hline
\end{tabular}

On voit avec quelle prudence on doit se garder de conclusions trop absolues qui seraient démenties par les faits.

Bref, si l'on considère uniquement la superficie de chacune des trois catégories, on trouve les proportions suivantes:

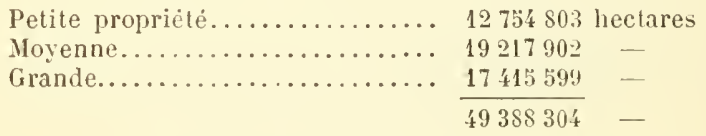

La moyenne propriété, dont le rôle économique semble très effacé et quasi négligeable, paraît donner par son étendue un démenti à l'opinion qui assigne à la grande propriété l'influence éducatrice et patronale, en quelque sorte, et à la petile propriélé l'impulsion sociale de bas en haut, du travail au capital, en ne lui laissant à elle qu'une condition intermédiaire et sacrifiée.

La vérité se trouve entre ce double point de vue; d'après les stalistiques des mutations foncières relevées par l'Enregistrement, e'est dans celle catéğorie moyenne que les transferts sont les plus fréquents, on dirait une zone neutre placée entre la grande el la petite propriété pour empêcher celle-ci de trop mordre et de trop rongrer et l'autre d'allirer of d'absorber les menues parcelles; c'est l'analogue des Marclies géographiques de notre ancienne histoire; c’est en mème temps une école pratique, car la moyenne propriélé oecupe 
le plus de salariés et forme les métayers et les fermiers qui deviendront à leur tour propriétaires et exploitants directs. Ceròle secondaire ne laisse donc pas que d'être fort utile et peut-ètre indispensable à la marche générale de l'exploitation agricole.

Pour apprécier avec sùreté la fonction de chaque catégorie de propriété dans les progrès des industries rurales, il faudrait connaître l'origine des nouveaux propriétaires, leur étal social avant qu'ils ne soient devenus acquéreurs de terre; le problème de la moyenne propriété serait résolu ou à peu près, si l'on savait oì se recrutent ses détenteurs, et dans quelle proportion : capitalistes, spéculateurs, forains en quête de placements stables, petits propriétaires arrondissant leur héritage par le groupement de parcelles. Les statistiques sont muettes sur tous ces points.

Mais on est d'accord pour reconnaître, ce qui vient à l'appui de notre thèse, que les mouvements de division n'ont rien de régulier ni d'uniforme; au risque de me répéter, j'y insisterai fréquemment; ils sont alternatifs. Souvent, sous l'influence de causes locales, ils se contrarient dans la mème région, ils s'enchevêtrent les uns dans les autres, et l'on ne peut, quant à leurs résultats immédiats, rien affirmer arec précision.

Les cartes et les diagrammes, si je ne craignais d'en abuser ${ }^{1}$ et de substituer à l'esprit économique et philosophique qui doit me guider dans cette étude un caractère trop scientifique et technique, sont très suggestifs, et traduisent par des impressions claires le chaos inévitable des chiffres accumulés. Plus on décompose les éléments complexes de lïnfluence exercée par la division du sol sur la richesse territoriale, mieux on aperçoit que les accroissements de valeur s'appliquent surtout à la petite propriété qu'on a quelquefois représentée comme une cause d'appauvrissement et de misère; tandis que la valeur de la grande propriété ne s'est guère accrue que de moitié, celle de la

1. Voir les cartes annexées à ce travail, $n^{\text {os }} 1$ à 3. 
propriété divisée, morcelée, a triplé, quadruplé et même décuplé, selon les régions et les localités. La grande proprićté s'est appauvrie plutôt qu'augmentée dans le Nord, le Nord-Est et l'Est, elle n'a fait quelques progrès réguliers que dans les départements du sud de la Loire; les petites exploitations s'y multiplient, et surtout les très petites acquisitions que néglige trop la statistique administrative, et celles-ci mordent principalement sur la moyenne propriété ${ }^{1}$ qui, à certaines époques, el sous l'influence dc crises temporaires ou locales, se recompose à son tour', parce que c'est celle qui compte le plus de propriétaires capitalistes qui restent, par conséquent, en dehors de l'impression directe de ces accidents, et les exploitent à leur profit ${ }^{2}$, toutesles fois qu'ils n'en sont pas trop meurtris eux-mêmes.

Chacune des formes de la division du sol, - la grande, la moyenne, la petite propriété, - conserve à travers les âges sa raison d'être; elles développent mieux leurs avantages relatifs à mesure que chacune accuse son type distinet, type qui ne se modifie guère, tout en s'accommodant, s'il le faut, à l'état économique ambiant.

C'est ainsi que la grande propriété, féodale au début, puis aristocratique et financière arant 1789, s'est elle-même démocratiséc en tombant aux mains de sociétés de spéculateurs. Dès 1836, on préroyait, au profit de la classe bourgeoise ${ }^{3}$, celte mobilisation du sol par des titres gagés sur la terre, représcntant les capitaux mis cn circulation soit pour diviser le droit de propriété sans morceler les grands domaines, soit pour grouper ee droit dans quelques mains sans troubler la condition légale des détenteurs de la moyenne propriété. C'est l'idée que la société du Crédit foncier devait réaliser, à partir de 18ə̃2, par des émissions dont le monopole

1. Discours de M. Méline à la Chambre des députés, à propos de l'interpellation sur la crise agricole (seance du 13 novembre 1897).

2. On en aurait la preuve formelle si l'on possédait depuis dix ans le classement des mutations foncières rurales par contenance et par prix complétant l'essai public̉ par l'Enregistrement pour 18930.

3. British and Foreign lieview, $1^{\text {er }}$ novembre 1836. 
et la dispersion paraissent à quelques bons esprits plus dangereux que ne sont efficaces les prèts officiels à la terre', commandités par la spéculation avec le privilège de l'État.

Les progrès apparents del'exploitation agricole ne suffisent pas, en effet, pour marquer la mesure de l'avancement d'un peuple. Il faut qu'il y ait harmonie dans l'organisation du mécanisme économique el souplesse dans le jeu de ses rouages, en les laissant se combiner d'eux-mêmes en toute liberté, sans recourir aux artifices de la loi civile ou aux expédients plus fàcheux encore des taxes fiscales.

Les trois formes de propriété dont nous voudrions, dans cette étude, déterminer l'état actuel, apprécier l'importance relative et calculer le ròle futur, ne sont pas destinées à s'anéantir l’une par l'autre, comme certaines écoles le redoutent ou le désirent. Leur avenir est de se compléter au moyen d'une division rationnelle du travail agricole, dont les méthodes et les procédés naîtront de la nature mème des choses parce qu'ils seront logiquement appropriés, si l'arbitraire et l'utopie n'en contrarient pas le développement, au mode même de division du sol. L'unité du principe de liberté, loin d'entraìner l'uniformité des conditions du travail, les diversifie à leur grand avantage. Jamais le mythe grec du lit de Procuste ne trouve de plus nombreuses applications que dans l'exercice raisonné de l'exploitation rurale, qui paraît toujours la mème et qui n'est jamais semblable.

La grande propriété pourra, dans certains cas, s'agrandir aux dépens de la petile, elle ne l'absorbera pas, et ce phénomène d'attraction serait vite corrigé par son excès mème au moment où il deviendrait nuisible. Elle se mobilisera par le crédit, utilisera l'accroissement du capital et l'énergique

1. Sans tomber dans les exagérations de banquiers concurrents évincés, on sait par les statistiques les mieux contrôlées que, du fait d'annuités trop fortes, les prêts soi-disant à long terme se liquident à brève śchéance, et que les emprunteurs, dans la plupart des cas, se trouvent dans l'alternative ou de la dépossession par la vente, ou du délaissement fui crée au profit de la Compagnie un colossal domaine de main-morte. J'ajoute que l'émission continue des valeurs à lots substitue au produit l'efforts honnêtes et laborieux l'immoralité du gain facile. 
auxiliaire des machines, en substituant de plus en plus le travail intelligent qui fait penser au labeur purement musculaire qui abrutit.

La petite propriété continuera à féconder le sol et à propager le goût du chez-soi et de l'épargne. Il est de nombreux produits que l'aceès facile du marché multiplie, et que la petite culture intensive peut seule créer avec avantage en défiant le coûteux outillage des grandes exploitations, tout comme il est des métiers qui résistent à l'accaparement de la grande iudustrie ${ }^{1}$. Le travail personnel, excité par la certitude d'un gain qu'on ne partage avec personne, a produil de merveilleux résultats, tirant beaucoup de rien. La très petite propriété est une caisse d'épargne en nature, suivant la très juste expression de Sismondi, toujours prête à recevoir tous les petits profits, négligés ailleurs, el à utiliser tous les moments de loisir du proprictaire et de sa famille. Dans l'ordre économique, l'étude de la propriété minuscule a ouvert des horizons nouveaux, de même qu'en astronomie la découverte des nébuleuses a élargi l'univers.

Entre la petite propriété qui cxerce l'initiative personnelle et la grande qui emploie les ressourees du crédit et de la science, la moyenne proprićté joue un rôle intermédiaire que son étendue superficielle, dépassant de près de 2 millions d'hectares celle de la grande et de 6 millions el demi celle de la petite, promet à un rôle plus actif. La classe moyenne des propriétaires se recrute, en effet, parmi des gens instruits qui, lorsqu'ils achètent, le font moins dans un bul de lucre que pour jouir de l'aisance conquise par le travail 2, el leurs conseils, leur direction, leurs commandites, pourraient améliorer les conditions du travail agricole s'ils se désintéressaient moins de leur rôle social et s'associaient

1. Wolowsli, He la division du sol el de son avenir, 1837.

2. La moyenne propriété occupe près de 39 p. 100 de la superficic totale du territoire; et il faut tenir comple de la propriété bâtic, si considérablement aceruc par le goût de la villégiature et qui ne laisse pas que d'exercer une action indirccte sur la mise en valcur de la propriété ruralc. (Le morcellement, p. 91 ct 96.) 
à l'existence et aux progrès de leurs fermiers ou métayers. Il y a, lans ce groupe de délenteurs du sol, des ressourees négligées et des activités qui sommeillent; c'est au milieu de la propriété moyenne que, par l'association et l'exemple, l'intelligence des procédés agricoles et la pratique des améliorations foncieres peuvent le plus efficacement se communiquer de proche en proche à l'ensemble de la eulture.

Mais l'aceroissemenl du capital foncier, aree ses variations inévilables, et l'augmentation du produit brut ne constituent pas les uniques éléments du véritable progrès économique. Quand on reul discemer ce qui est utile ou nuisible à l'avancement intégral d'un peuple, il ne faut pas se borner à mesurer la quantité de la richesse générale, il faut s'inquiéter de sa répartition entre les différents groupes et peser le bien-ètre des individus. Certes, le bien-ètre, en matière agricole, est une des conséquences immédiates de l'augmentation du produit brut, mais les petits ménages, les familles de travailleurs, n'entrent lout à fait dans l'indépendance relative que par le gain d'un produit net, si mince soit-il, paree que, avec l'épargne, il accumule lentement les éléments de la véritable aisance, celle qui se traduit par un relèvement moral. Le produit net, entre les mains des propriélaires qui cultivent leur propre héritage, au lieu de s'émieller entre les mains des auxiliaires, des intermédiaires et des bailleurs de fonds, profite exclusivement à la famille et crée pour son chef le crédit personnel, source et garantie de tous les autres.

Là est l'intérêt social des recherches qui ont pour objet l'étude de la division du sol el du ròle plus ou moins aclif, plus ou moins prépondérant que joue ou peut jouer, selon les circonstances, chaque groupe de propriétaires, en admeltant le classement généralement adopté. La division de la propriété n'est pas un simple problème de production; le côté purement matériel est accessoire quand il s'agit d'encourager la perpétuité de la famille, la dignité de l'homme, l'esprit de prẻroyance, les éléments de liberté et d'ordre qui sont liés à la possession de la terre. 


\section{I. - Répartition proportionnelle de la grande pro- priété dans les différentes régions de la France.}

La grande propriété, d'après les statistiques officielles, n'absorbe pas tout à fait la moitié de la superficie du territoire ${ }^{1}$ et, sur cette étendue, soit $46 \mathrm{p}$. 100 , il faut compter 63 p. 100 de terrains non cultivés; aussi n'est-on pas surpris de roir que les 138671 exploitations supérieures à 40 hectares $^{2}$, malgré leur étendue, n’aient qu'une valeur vénale à peine équivalente au tiers de celle des 5563581 domaines de la petite et de la moyenne propriété.

On admet l'arbitraire de ces évaluations qui ne sont que très approximatives, et s'il esł aussi malaisé de recenser les rastes domaines, on doit en conclure qu'à part quclques détails dont la précision peut être contrôlée, les statistiques de la petite propriété foncière ne doivent être acceptées qu'arce beaucoup de réscrve ${ }^{3}$.

Ce qu'il serait intéressant de connaitre, en ce qui touche particulièrement la grande propriété, c'est la manière dont les diverses exploitations se subdivisent, soit au point de vue de la nature et de l'utilisation des terrains, soit surtout au point de vue de la condition des propriétaires. Ce dernier élément aurait une importance extrème au point de vue économique car, dans les allures générales d'un pays comme la France, la grande propriété individuelle et la grande pro-

1. Le territoire agricole est calculé dans les statistiques déduction faite des bois de l'Etat, considérés comme une exploitation unique et d'une nature spéciale; les forêts domaniales sont d'une contenance de 1089096 heetares dont 18 p. 100 à l'état de surfaces improductives.

2. De bons esprits regrettent que le elassement des trois groupes dans la division de la propriété n'ait pas étendu jusqu'à 50 hectares la limite de la moyenne propriété; le nombre des exploitations de cette calégorie est de $533 \nmid 3$ en 1892 pour $56 \$ 66$ en 1882.

3. Le travail de recensement, dit $\mathrm{H}$. de Foville, a élé fait commune par commune sans reetification ultérienre, de sorte que tout domaine sitné sur deux communes se trouve dédonblé; telle exploitation de t50 hectares est comptée pour cinq lots au lieu d'un, par la statistique officielle, parce qu'elle est imposée dans einq communes. 
priété collective jouent un rôle fort différent. Les biens de main-morte, comprenant ceux des communes qui vont s'émieltant peu à peu par les ventes de parcelles, ceux des chemins de fer, des hospices, des congrégations religieuses, des sociétés de spéculation foncière qui s'accroissent et se reconstituent par un mouvement continu, dépassent déjà la dixième partie du territoire imposable ${ }^{1}$.

Il en est de la grande propriété comme de ces vieilles forèts dont on déplore aujourd'hui le gaspillage; elle est nécessaire, on ne pourrait la supprimer sans inconvénient, et il faut laisser au temps le soin de la diminuer au fur et à mesure que la culture intensive mordra sur ces vastes espaces pour en tirer un meilleur parti par l'emploi d'une main-d'cuvre passionnée et minutieuse. Quant à ceux qui redoutent sa reconstitution trop rapide entre les mains de collectivités dont la perpétuité deviendrail dangereuse en ralentissant le mouvement des mutations, c'est une éventualité problématique; il faudrait d'ailleurs distinguer entre la vraie main-morte d'autrefois, et l'histoire nous apprend quels sont les remèdes révolutionnaires qu'on y apporte, - et la concentration de la propriété aux mains de sociétés qu'il faut encourager si elles ont en vue le progrès scientifique et industriel de la culture, qu'il faut brider si elles se bornent à spéculer sur la crédulité publique.

Le progrès agricole n'exige pas, comme le progrès commercial et industriel, l'apport de gros capitaux et la substitution absolue des grandes exploitations aux petites; c'est question de mesure et d'opportunité. Les crises, et il en est d'inévitables, ont une répercussion d’autant plus fìcheuse qu'elles se concentrent sur des groupes solidaires dont la gêne ou l'arrèt ont autour d'eux des incidences immédiates

1.

En milliers d'hectares.

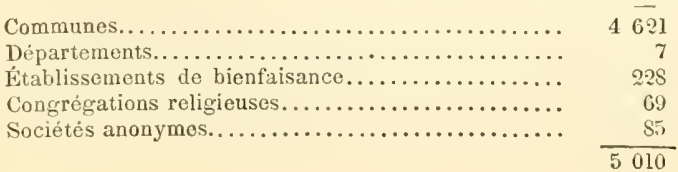

LA PROPRIÉté RURALE EN France. 
et multipliées. Si tout le monde souffre, le petit propriétaire qui cultive lui-même son bien et qui n'est pas à la merei, comme dans l'industrie ou la grande culture, du respect d'autrui pour les échéances, souffre certainement moins que les autres, ce qui, en matière économique, est déjà une marque certaine de supériorité; enfin, ses déboires ne tendent pas, comme ceux des grands propriétaires, à se reporter brusquement sur le consommateur, par le renchérissement artificiel des produits de première nécessité ${ }^{1}$.

En supposant que la conversion des grandes propriétés territoriales en papiers de crédit, comme on l'a fait pour la propriété urbaine, discrédite eet te mobilisation en diminuant la sécurité, la fixité, qui sont l'élément primitif de la richesse immobilière, il y aura toujours contre-poids par lineontestable solidité de la masse des petits domaines, et la vitalité de l'effort individuel rétablira constamment l'équilibre entre la propriété privée et la propriété collective.

Jai essayé de traduire par des cartes la répartition des groupes de propriétés en Franee, en y résumant, d'une manière expressive sinon tout à fait préeise, les grandes lignes de la division territoriale ${ }^{2}$.

Le territoire oceupé par la grande propriété se réparlit comme suit par nature de cultures :

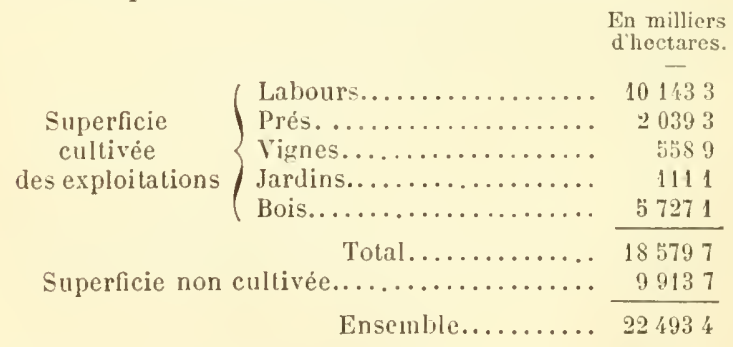

1. Paul Leroy-Beaulieu, Journal des Débats du 230 novembre 1884.

2. Les documents oủ j'ai puisé les chiffres que je traduis par ces graphiques sont : le Bulletin de statistique du ministère des Finances (mai 1883, p. 576: août et octobre 188', p. 162 et 388) et les tableaux de l'enquête agricole de 1892. Ces chiffres varient souvent l'un document à l'autre. 
Pour 100 hectares, la grande propriété en cultive 10 p. 100 et en laisse stériles ou improductifs 60 p. 100. Aussi, par comparaison aux deux autres groupes de propriétés, les genres de culture se distribuent proportionnellement ainsi pour 100 hectares :

\begin{tabular}{|c|c|c|}
\hline $\begin{array}{l}\text { Catégories } \\
\text { do culture. }\end{array}$ & $\begin{array}{c}\text { Grande } \\
\text { propriété. }\end{array}$ & $\begin{array}{l}\text { Petito et moyenne } \\
\text { propriété. }\end{array}$ \\
\hline Labours............ & 39,18 p. 100 & 60,82 p. 100 \\
\hline Prés $\ldots \ldots \ldots \ldots \ldots$ & $31,10 \ldots$ & $68,90^{\circ}-$ \\
\hline Vignes............ & $31,0 \div$ & 68,96 \\
\hline Jarılins. ........... & 23,27 & 76,73 \\
\hline Bois............ & 67,92 & 32,08 \\
\hline
\end{tabular}

La grande propriété se développe surtout dans les régions accidentées oì la petite propriété se raréfie, sauf autour des agglomérations industrielles; la zone où elle s'aceuse avec toute son ampleur suit la frontière, du nord au sud, s'accentuant dans les départements des Alpes et des Pyrénées, atteignant son maximum d'intensité dans les Landes et le Cher. Le groupe du Centre, à domaines très étendus, comme l'Allier qui compte des propriétés de plus de 1000 hectares, a sa statistique faussée, à ce point de vue spécial, par la méthode qui recense par métairies et par fermes, subdivisant ainsi arbitrairement le bloc appartenant au même propriétaire et ne formant souvent qu'un seul tenant ${ }^{\perp}$, sans établir un relevé parallèle.

Quel que soit le groupe de propriétés, les mouvements généraux d'augmentation et de diminution, résumés par des moyennes pour l'ensemble du territoire, répondent à une série de mouvements intérieurs de sens contraires et d'intensités différentes, d'où la nécessité de relever, département par département, les différences les plus notables, sans avoir la prétention de conclure du particulier au général.

1. Sous le nom d'exploitation, qui est l'unité à recenser, il faut comprendre l'ensemble des terres cultivées par un seul individu (propriétaire, agriculteur, fermier, métayer ou même journalier) cultivant un bien rural comme propriétaire ou comme locataire, que ces terres forment un tout compact ou soient composées de parcelles éparses. (Instruction du 18 avril 1891.) 
C'est ainsi qu'il faudrait plusieur's statistiques superposées pour se rendre un compte aussi approximatif que possible de la répartition des grandes propriétés sur l'ensemble du territoire. Les tableaux de l'administration ne sont malheureusement pas comparables, n’étant pas établis sur les mêmes cadres ${ }^{1}$, et, mème en serrant de très près les chiffres, on est forcé de s'arrêter à des hypothèses.

Pour atténuer autani que possible cet inconvénient qu'arouent d'ailleurs avec une parfaite bonne foi ${ }^{2}$ les rédacteurs de l'enquête, j’ai classé les 86 départements en trois catégories : $1^{\circ}$ départements comptant plus de 100 exploitations d'une contenance chacune de plus de 200 liectares; $2^{\circ}$ départements comptant plus de 300 milliers d'hectares affectés à la propriété de plus de 40 hectares à l'unité; $3^{\circ}$ départements où l'on constate une plus-value sur la valeur vénale d'au moins 66 p. 100. Ces indications n'auront d'ailleurs, dans l'état actuel de nos statistiques, qu'une valeur très générale, la petite, la moyenne el la grande propriété, comme la petite, la moyenne el la grande culture, affectant des allures très diverses dlans les différentes parties d'un même département.

De 1882 à 1892, on constate un accroissement, en nombre et en étendue, de la grande culture au-dessus de 100 heetares, mais de la très pelite proprićté dans une proportion infiniment plus forte: ce double accroissement s'est réalisé au détriment de la culture moyenne de 10 a 40 hectares, catégorie d'étendue habituellement la plus atteinte par les mouvements de dépression. Nais il serait téméraire de généraliser, les oscillations constatées dans l'Ouest se compensant par des variations à peu près équivalentes dans le Centre ou le Nidi, et les causes de morcellement ou de

1. Dans l'enquête de 1892 , le nombre el l'étendue des exploitations de la grande culture (p. 223 des résultats numériques) sont relevés en bloc tandis que les tableaux de détail (p. 227) les repartissent par catégories d'étendue, ce qu'il aurait fallu faire partout avec un cadre idenlique permettant les rapprochements.

2. 1'. 3 '19 de l'Introduction. 
groupement n'étant pas les mèmes dans le Languedoc que dans la Champagne.

Ce qui ressort avec le plus de relief, pour les trois groupes de propriétés, aux trois élapes de 1862, 1882 et 1892, e’est la régularité décroissante du nombre des exploitations, au fur et à mesure que s'accroil leur superficie ef surtout leur répartition superficielle. De 1882 à 1892 , l'ensemble de la grande culture est affecté d'une légère diminution, 0,09 p. 100, correspondant à deux mouvements en sens contraire, lun de diminution pour le nombre des exploitations de 40 à 100 hectares, l'autre d'augmentation, pour celles de plus de 100 hectares. D'où la nécessité, si l'on veut raisonner juste, de multiplier les compartiments el de créer pour ce quion appelle administrativement la grande culture autant de catégories que pour la petite. Chacune de ces tranches a sa raison d'ètre, son rôle, sa fonction; celles de 100 heclares ne ressemblent en rien à celles de 500 ou de 600 , et un domaine de 400 hectares dans les Landes n'a ni la mème valeur, ni les mèmes méthodes de culture, ni les mèmes tendances à l'absorption des parcelles voisines qu'un domaine de 400 hectares dans le Cher.

La France peut ètre partagée en deux régions où la grande culture prend des allures très différentes. Au nord de la Loire, sa superficie diminue et le nombre des exploitants augmente; au sud, c'est l'inverse, la superficie s'accroît avee un nombre plus restreint de propriélaires ${ }^{1}$.

Au Nord, à la suite de la crise sucrière, de l'épuisement des terres à betteraves, de la pénurie des fermiers, des exploitations à superficie étendue se sont divisées et réparties soit entre des propriétaires qui sont parvenus par le groupement à cultiver plus de 40 hectares, soil entre des détenteurs de plus pelits domaines. La diminution des emblavures de céréales ${ }^{2}$ a eu pour contre-partie l'exlension

1. Voir aux pièces justificatives la carte $n^{\circ} 1$, d'après les proportions mathematiques tirées des chilfres que donnent les tableaux statistiques récapitulatifs de l'enquête de 1892.

2. Les départements qui ont augmenté leur superficie cultivée en blẻ 
des cultures fourragères qui, depuis dix ans, ont augmenté de 7 p. 100 en étendue et de 14 p. 100 en produit. Au sud de la Loire, l'abaissement de la valeur vénale à la suite de la perte des vignobles a fait passer dans les mains de grands propriélaires les domaines que leurs exploitants ne pouvaient reconstituer à l'état productif, faute de capitaux, et qu'ils ne pouvaient conserver, faute de produit pour vivre. La vente s'imposait; d'où l'apport au groupe de la grande propriété d'un nombre assez considérable d'exploitations de moyenne importance réunies entre les mains de peu d'exploitants sous la forme d'agglomérés d'une étendue plus élargie ${ }^{1}$.

Dans le Midi, la reconstitution des vignobles détruits par les maladies a surtout profité à la grande culture qui a même envahi les labours et restreint les céréales pour élargir les plantations ${ }^{2}$; l'Hérault, le Gard, les Bouches-du-Rhòne, le Vaucluse, le Var, sont dans cetle catégorie. Ailleurs, ce sont d'autres raisons : les cinq départements de l'ancienne Normandic ont étendu leurs herbages ${ }^{3}$; la Loire-Inférieure et le Doubs ont créé des prairies. La progression de l'élevage est très marquée, el bien qu'il soit acquis aujourd'hui à l'encontre des opinions d'il y a trente ans, que l'extrême morcellement n'est pas incompatible avec la multiplication du bétail ${ }^{4}$, il est permis d'admettre que la grande culture est pour une part considérable dans ce progrès. La carte de la production en bétail comparée à celle de la prédominance

sont précisément ceux où il y a le moins de très grands domaines, Charente-Inférieure, Dordogne, Lot (`ui met en blé 25533 hectares de plus), Saône-cl-Loire. (Enquête de 1892, tableaux de la p. 93 de l'Introduction.)

1. Mouvement de concentration favorisé par les alternatives de la lutte contre le pluylloxéra et la eréation de syndicats de spéculation sur la propriété rurale.

2. Dans le département de l'Ilérault, de 1882 à 1892 , plus de 34000 lıectares de terres à froment ont été convertis en vignobles; dans les Bouches-du-Rhône, 16000.

3. Le seul département de la Manche a transformé en herbages, depuis dix ans, plus de 20000 hectares de labours, le Calvados, 14000.

4. Voir les faits et les chillres énoncés par M. Hipp. Passy (Essai sur les systcmes de culture) et $\mathbf{M}$. de Foville (Le morcellement). 
de la propriété à superficie étendue fournit à ce sujet des éléments de comparaison curieux et que je n'ai trouvés nulle part'. Le cheptel vif de l'agriculture française est estimé, pour 1892, à plus de cinq milliards de franes; la machinerie agricole a suivi un accroissement continu et si le nombre des moteurs a diminué de $\breve{~ p . ~} 100$ depuis 1882, leur puissance s'est accrue de 12 p. 100; quant aux outils perfectionnés (semoirs, faucheuses et moissonneuses) d'un prix élevé, ils sont devenus très nombreux dans le Nord et le Centre, où le capital roulant des grandes exploitations permet leur achat.

Pour résumer, les pays à grande propriété diviséc sont l'Aisne, l'Areyron, la Còte-d'Or, la Gironde et les Landes; ceux à grande propriété étendue, l'Allier, le Cher, les HautesAlpes, le Jura, etc., mais avec des inégalités aussi variées que les régions.

NOMBRE DES EXPLOITATIONS DE LA GRANDE PROPRIÉTÉ PAR CATÉGORIE DE SUPERFICIE

\begin{tabular}{||c|c|c|c|c|}
\hline $\begin{array}{c}\text { Etendue } \\
\text { de } 40 \text { à 50 } \\
\text { hectares }\end{array}$ & $\begin{array}{c}\text { Étendue } \\
\text { de } 50 \text { à } 100 \\
\text { hectares }\end{array}$ & $\begin{array}{c}\text { Étendue } \\
\text { de } 100 \text { à 200 } \\
\text { hectarcs }\end{array}$ & $\begin{array}{c}\text { Étendue } \\
\text { de 200 à 300 } \\
\text { hectares }\end{array}$ & $\begin{array}{c}\text { Étendue } \\
\text { de plus de 300 } \\
\text { hectares }\end{array}$ \\
\hline 53343 & 52048 & 22777 & 6223 & 4280 \\
\hline
\end{tabular}

Si l'on compare ces chiffres à ceux de 1882, on s'aperçoit que le résultat n'en est pas tout à fait conforme aux conclusions admises, c'est que, à mesure qu'on serre de près le sujet et qu'on entre dans le détail, les déductions lirées des moyennes s'évanouissent en fumée.

1. Voir les cartes $n^{0 s} 1$ à 3. 


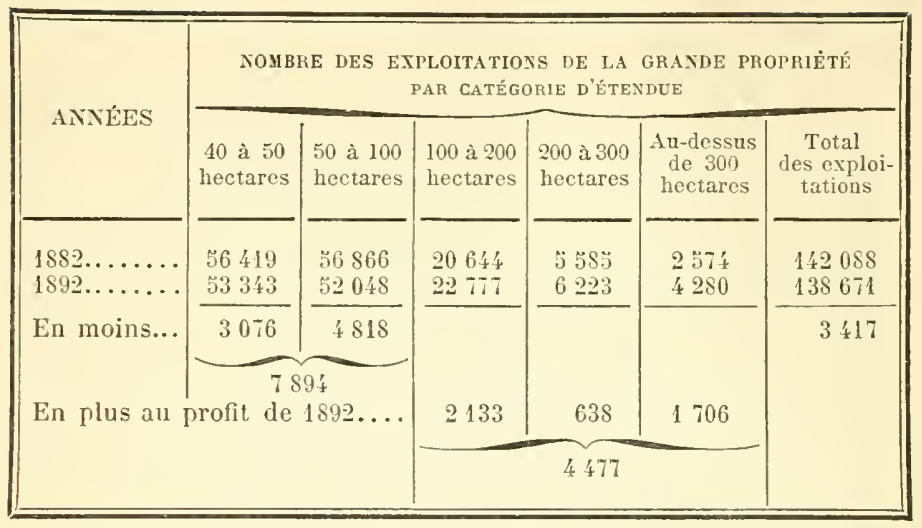

Le nombre total des exploitations de la grande culture a done diminué de 3417 en dix ans, mais ee n'est qu'une apparence et l'on se ferait une idće fausse de la situation si l'on s'arrêtait à ce ehiffre sans en décomposer les éléments.

En premier lieu, la grande propriété n'a point perdu de terrain; l'étendue totale de ses exploitations est, en 1892, de 22493.400 hectares; elle étail, en 1882, de 22296100 ; gain : 197300 .

En second lieu, ee n’est pas la grande propriété proprement dite, celle de plus de 100 heetares, qui a diminué en nombre, e'est la eatégorie inférieure, dont la statistique aurait profit à faire une elasse à part el très étudiće paree qu'elle est infiniment plus sensible à l'influenee des erises et plus mobile que l'autre. La elasse qui se rapproche le plus de la moyenne propriété (de 40 à 0 o hectares) a diminué de 3076 unités, et la suivante (de 30 à 100 heetares) de $4818^{1}$.

Par contre, les trois eatégories qui constituent la véritable grande propriété se sont augmentées de 447 exploitations,

1. L'enquête de 1892 constate, aux p. 224 et 223 des relevés numériques, que la petite el la moyenne propriété ont perdu ensemble 653800 hectares et ne donne pour contre-partie qu'une augmentation de 197300 hectares à la grande propriété; que sont devenus les 456500 hectares formant la différence? 
et ce nest évidemment pas par voie de concentration, de déplacements inlérieurs, puisque le nombre el l'étendue se sont accrus simultanément, mais par absorption, par acquisition, en toul ou partiellement, des 789 4 domaines détachés des classes intermédiaires (exploitations de 40 à 50 et $ð 0$ à 100 hectares).

On roil dies lors combien il faut apporter de mesure dans les appréciations de notre état économique et avec quelle discrélion il faut faire usage des chiffres qui paraissent offrir le plus de garanties et qui n'ont qu'une valeur de relation ${ }^{1}$.

Les départements qui renferment des exploitations agricoles d'une étendue supérieure à 300 hectares ne sont quau nombre de 12 (Aisne, Bouches-du-Rhòne, Cher, Corse, Còte-d'Or, Gironde, Indre, Landes, Loir-et-Cher, Loiret, Nière, Var). Le Var tient la tète arec les Bouches-du-Phône, quant au nombre. Pour la superficie, les Landes tiennent le premier rang avec 567 millier's d'heetares, la Corse avec 533 , puis le Cher avec 46'.

Le progrès de la valeur vénale ne laisse pas que d'ètre un précieux élément d'appréciation, mais il serait imprudent d'en tirer des conséquences trop absolues, car les départements à rastes domaines sont aussi ceux où l'on constate le plus de résultats contradictoires. L'Allier, l'Aude, les Landes offrent des plus-values variant de 100 à 200 p. 100 suivant les localités, le Gard et les Hautes-Alpes perdent de 10 à 20 p. $100^{2}$. Il est à peu près certain que les mutations de la

1. Le contròle de la statistique municipale (enquêtes administratives) serail difficile à faire, mème avec le concours de la statistique fiscale, la seule qui offrirait quelque précision si elle était plus symétrique. En 183\%, il y avait 13361 cotes foncières de plus de 1000 francs; ce chiffre s'élevait à 163 \$6 en 1842 , retombait à 13870 en 18308 , et n'a pas été donné depuis, d'apres l'aveu du Bulletin bleu (1884, p. 156). En 188't, l'disne possédait 178 cotes au-dessus de 200 hectares, l'Ain 199, l'Allier 505, l'Aveyron 218. etc., comment faire concorder ces données avec le nombre réel des exploitations ou avec celui des domaines?

2. C'est ici surtout qu'il faut tenir compte du milieu, des circonstances et des modalités. En 1892, la valeur à l'hcctare de la terre à froment a varié de 1280 francs dans les Landes à 4821 francs dans le Nord, à 
grande propriété n'ont sur la cote des prix qu'une influence médiocre, à moins qu'il ne s'agisse d'acquisitions de convenance faites à tout prix par des capitalistes, el que les cours du marché foncier dépendent surtout de l'offre et de la demande de la petite et de la moyenne propriété.

\section{II. - Origines historiques de la grande propriété territoriale.}

La très grande propriété, en France, n'est pas le résultat de la conquête comme dans les autres pays des deux mondes; elle est la conséquence du contrat de fief lorsque, à partir du $\mathrm{IX}^{\mathrm{e}}$ siècle, le besoin de sécurité obligea les détenteurs du sol à abdiquer leurs droits en échange de la protection de leurs personnes et de leurs biens. Déjà très morcelé avant le $1 \mathrm{x}^{\mathrm{e}}$ siècle dans tous les pays de culture, en dehors des vastes forèts qui couvraient les deux tiers du territoire, si bien qu'un écureuil pouvait aller des sources de la Meuse aux rivages de l'Océan de Bretagne en sautant de branche en branche, le sol français le fut plus encore à partir du $\mathrm{x}^{\mathrm{e}}$ siècle, si l'on remplace l'expression de propriété par celle de culture. Avant la féodalité, la propriété individuelle était peu de chose, la propriété collective absorbait presque tout, que ce fût celle de la commune ou celle de la famille. En dehors, il se forma deux types de grands domaines : le domaine fiscal et le domaine ecclésiastique. Ces trois groupes de propriétés eurent des destinées différentes et qui, cependant, abontirent à un résultat semblable, la reconstitution de la très petite propriété individuelle. Le domaine fiscal de l'ancien empire romain s'émietta à partir du v viècle et servit a possessionner les tribus germaines; les terres collectives des municipes ${ }^{1}$, auxquelles se substituèrent les com-

4962 francs dans la Savoie; celle de l'hectare de pré, de 1622 francs (Landes) à 6000 francs (Lot); celle de l'hectare de vigne, de 1400 francs (Ardèche) à 7000 francs (Hérault).

1. Origine des grandes propriétés foncières en France (Bulletin du Comité des travaux historiques, section d'économie sociale, 1892, I, p.94). 
munautés rurales ${ }^{1}$ se groupèrent et s'accumulèrent entre les mains des chefs militaires en vertu du contrat de sauvegarde $^{2}$; les biens ecclésiastiques seuls ne cessèrent de croitre et ne furent démembrés que par accident. A partir du $\mathrm{xi}^{\mathrm{e}}$ et du xul siècle, la nécessité de vivre de la terre et d'attacher l'homme à la culture produit cette évolution économique qui, par le bail à cens, fait le paysan propriétaire du sol qưil cultive jusqu’à ce que le bourgeois, enrichi par le commerce, la banque et la justice, le dépossède en le rejetant dans le prolétariat rural.

Intervient alors la Rérolution française qui liquide la dette féodale et ourre à tous le libre accès à la propriété, tout en laissant imparfaite une œuvre que la société contemporaine, arec ses audaces mêlées d'insouciance, n'ose achever ${ }^{3}$.

L'histoire peut ici renir efficacement en aicle à l'économie sociale. On a prétendu que l'histoire n’a rien de scientifique, que n'étant qu'un ensemble de faits et d'accidents particuliers dont linventaire sera toujours incomplet et qui se peuvent distraire ou multiplier arbitrairement, elle est impuissante à fournir des enseignements pratiques pour la conduite de la vie et ne peut donner utilement de leçons ni aux individus ni aux peuples. C'est une erreur manifeste. L'histoire, telle qu'elle est comprise aujourd'hui, n'est plus un simple instrument de culture intellectuelle et offre autre chose au penseur qu'un banal attrait de curiosité. Il est rare que les faits historiques n'aient pas été la conséquence toujours directe sinon toujours immédiate des faits économiques, et la véritable histoire ne se peut comprendre que par l'union de ces deux ordres de faits et la recherche de leurs incidences réciproques. Rien n'est isolé dans la nature, tout se combine, se répereute et s'harmonise dans le monde physique; il en est de même dans l'univers moral.

1. Bulletin du Comité des travaux historiques, 1888, p. 112; 1893, p. 294.

2. Histoire documentaire et philosophique de l'administration des Domaines, 1900, t. I, chap. vi, vi et Xxi.

3. Voir Laferrière, Histoire du Droit francais. 
Les statistiques, si nous en avions de rétrospectives, nous permettraient de remédier sur nombre de points à la négligence des historiens et de corriger leurs erreurs de vision; rapprochées avec discernement des accidents si variés de la vie des peuples, elles feraient apprécier quand, comment, pourquoi el dans quelle mesure il y a eu relation de cause à effet, et quels sont les faits économiques qui impressionnent le plus l'équilibre de ce monde politique, toujours en mouvement, toujours instable, parce qu'il ne s'appartient pas et qu'il est à la discrélion de tous les accidents extérieurs ${ }^{1}$.

La statistique n'est sùre qu'en multipliant les divisions, les subdivisions, les définitions el les distinclions, surtout en matière rurale.

Ainsi, dès que l'on parle des origines de la grande propriété, il faut distinguer entre la propriété, possession d'un individu, et l'exploilation (unité de notre statistique administrative), expression qui autrefois signifiait un ensemble divisé entre une infinilé de tenanciers et qui, aujourd'hui, n’est prise qu“isolément pour chaque chef de culture. Les ville gallo-romaines étaient de vastes domaines qui conservaient leur persomnalité malgré les partages el la division ${ }^{2}$, assez élendus pour avoir formé les paroisses de l'Aneien Régime, c'est-à-dire nos communes acluelles, ruoique réparties en de nombreuses tenures libres ou serviles. Ces domaines ont probablement été disloqués pour la plupart entre le $\mathrm{V}^{\mathrm{e}}$ el le $\mathrm{x}^{\mathrm{e}}$ siècle; ils l'élaient tous, réserve laite des biens de l'Église el du domaine personnel de cerlains chefs militaires, entre le $\mathrm{x}^{\mathrm{e}}$ el le $\mathrm{xI}^{\mathrm{e}}$ sićcle, éporque de la transformation quasi générale des masures serviles de la glèbe en tenures féodales, en censives. Il paraît impossible, dans l'élat actuel de la science, el malgré les découvertes de l'érudition contemporaine, de constater si parmi les vastes

1. Voir MI. Juglar, te róle de la stutistique au point de vue historique el au point de vue éeonomique, novembre 1898.

2. M. de Saint-Genis, Histoire doeumentaire et philosophique te l'administration des Domaines, 1900. 
agglomérations lerriennes d'à présent ', dont quelques-unes, dit-on, groupent plusieurs milliers d'heetares ${ }^{2}$, il en est qui puissent reporter leur origine jusqu'aux premiers temps des institutions féodales el si, pour les autres, on retrouverait les étapes de leur aecroissement continu par des acquisitions suceessives. Ce lravail de patience a été essayé pour quelques localités ${ }^{3}$; s’il élait exécuté avee minutie et sûreté sur plusieurs points de la France, on s'apercerrait peut-être que la plupart des très grandes propriétés actuelles n'ont pas une origine plus reeulée que le xrrie siècle, et qu'elles nappartiennent plus aux familles qui en constituèrent la souche primitive; et que les autres, de date plus reeulée, furent démembrées arant 1789 .

Si l'on envisage le domaine direet ${ }^{4}$, il y avait, du $x^{e}$ siècle au $\mathrm{Xr}^{\mathrm{e}}$, de rastes fiefs, agrandis ou diminués par les modifications qu'y apportaient les mariages, les substitutions, les héritages; si l'on s'en tient au domaine utile, on se trouve en présence de millions de tenures, héréditaires et libres si l'on déchiffre les minutes des notaires et si l'on dépouille les registres des cours de justice au lieu de lire les historiens modernes, avee une médiocre réserve resserrée au pied des tours féodales. Sans le double fléau de la chasse et de la

1. Nos statistiques, très insuffisantes, avouons-le, ne donnent que le nombre global des exploitations de plus de 200 hectares (Bulletin de statistique du ministère, août 1854) ou le 300 hectares (Enquéte de 1822, p. 229 des relevés numériques).

2. Ainsi les Contributions directes publient, en 1884, que le Cher contient 627 cotes (de plus de 200 hectares) pour une superficie de 251760 heetares et les Landes le même nombre (621) pour 307523 heclares. I] faudrait savoir le nombre de celles au-dessus de 600 et de 1000 hectares. Pour la même eatégorie, l'enquête de 189.2 accuse 336 exploitations pour le Cher et 302 pour les Landes. Les comparaisons ne peuvent conduire qu'à des hypothèses, procédés à répudier en statistique.

3. M. Tausserat, Mouvement de la propriété de la seigneurie de Chevilly de 1496 à 1663, Bourges, 1878.

4. 11 faut surtout, en parlant de l'époque féodale, éviter ccrtaines confusions de mots, telles que celle de domaine direct et de domaine utile, accidents qui peuvent arriver aux érudits les mieux documentés sur d'autres points. (Bulletin du Comité des travaux historiques, 1892, 1, 100 : Conséquences des chartes de franchises.) 
vaine pâture ${ }^{1}$ et sans l'empiétement des bois sur la terre à charrue, le paysan aurait pu se eroire le maître de sa terre comme le bourgeois dans sa ville, l'artisan dans sa corporation se sentaient chacun chez soi. Les seigneurs très entichés de leur directe, étaient au contraire toujours prèt à l'abandon de l'utile, ne dût-il rapporter qu'un denier à l'arpent.

De concessions en coneessions, le sol des fiefs s'émietta ne laissant plus que l'apparenee d'une grandeur d'opinion qui devint banale dès que nobles et roturiers purent également en faire l'acquisition, et les grands domaines que représentaient les seigneuries d'autrefois ont disparu sous la dent des rats de roture, selon le mot du président Bouhier ${ }^{2}$.

Mais, ee qui est vrai pour une période ne l'est pas pour une autre, il ne faut pas se lasser de procéder par comparaisons de temps aussi rapprochés que possible afin, non pas de eonelure à des moyennes qui napprennent rien, mais à des chiffres résumant le sens et l'étendue de chaque mouvement alternatif.

Des documents commus, on peut conclure à deux faits généraux, au moins pour les parties du territoire qui s'étendent au nord et à l'est de la Loire jusqu'à la vallée du Rhòne, en empiétant sur le Plateau Central : $1^{\circ}$ Les seigneurs féodaux conservent la directe et rendent l'utile ${ }^{3}$; leurs réserves constituant un faire valoir personnel sont négligeables tant elles sont restreintes, en dehors des forêts et des ćlangs;

1. Au moyen âge, la chasse est libre pour tout le mondle; dans certains pays, elle est obligatoire pour le seigneur tenu a défendre le paysan conlre le mauvais gilier; à partir $\mathrm{lu} \mathrm{xv}^{\mathrm{e}}$ siècle, le gentilhomme fait un plaisir de ce qui n'était qu'un devoir, s'en réserve le privilège et le rend intolérable.

2. Dans une paroisse (le l'Auxois (ancienne Bourgogne), dont quatre seigneuries se partageaient les 7938 journanx (à 34 ares 28 c.), il ne reste en 1900 que trois grands domaines, deux variant de 40 à $\$ 0$ hectares, et un de 500 ; sur les 2646 hectares, les paysans, déjà largement propriélaires au $\mathrm{XV}^{\circ}$ siècle, en possèdlent all $\mathrm{xIx}^{\mathrm{\theta}} 2056$ (Mémoire sur la propriélé rurale en Bourgogne, 1836), c’est-à-dire près des $4 / 0$.

3. L'examen les terriers seigneuriaux de la province de Bourgogne permet de constater au xve siècle l'extrême livision du sol; les domaines s'y composent de lanières étroites el disséminées. (Bulletin du Comité des (ravaux historigues, 1896, M. (le Saint-Genis.) 
$2^{\circ}$ A partir du $\mathrm{xv}^{\mathrm{e}}$ siècle, les grands domaines se reconstituent par le groupement, la concentration des parcelles; comme arant le $\mathrm{xu}^{\mathrm{e}}$ siècle, la propriété était aux mains de seigneurs qui ne cultivaient pas eux-mêmes, faisant la guerre; ainsi, au $x^{\prime}{ }^{\prime}{ }^{e}$, elle tombera aux mains de seigneurs d'un autre genre, qui uc eultiveront pas davantage parce qu'ils lont le commerce ou rendent la justice ${ }^{\text {. }}$.

Dans ce nouveau mouvement de concentration, la propriété seule se reconstitue, la culture reste morcelée et divisée; il y a peut-être sur place autant d'exploitants du sol, mais le nombre des propriétaires foneier's a diminué, ceux rque la mauraise fortune a évincés tombant, s'ils n'émigrent pas vers les villes, à la condition de métayers ou de locataires.

C'est quen effet l'évolution sociale qui s'est produite du $x^{e}$ au $x_{1}{ }^{e}$ siècle par l'affranchissement des serfs et l'accensement des terres, - la libération de la terre se trouvant étroitement et fatalement liée à la libération de l'homme, - avail eu des conséquences, d'abord rémunératrices et fécondantes, ensuite gênantes et ruincuses.

Depuis le $\mathrm{xi}^{\mathrm{e}}$ siècle, le morcellement territorial des grandes seigneuries entre les mains des serfs suivit son cours inévitable par le partage des propriétés censitaires entre les héritiers des premiers affranchis. Grâce au bienêtre que produit l'aisance, qu'engendre le travail excilé par la liberté, les familles se multipliaient et la culture intensive n'en devenait que plus féconde; mais il vint un moment où, après deux ou trois générations, l'unité primitive de la manse affranchie se trouva divisée entre quinze ou vingt chefs de l'amille et où chaque lot devint trop exigu pour nourrir les nouveaux venus. Il fallut s'expatrier. La crise, on le conçoit, dut ĉtre à peu près générale.

1. Le nombre des propriétaires forains, en Bourgogne, a toujours été, avant et depuis 1r89, supérieur à celui des résidants. En 1895, à Vicde-Chassenay, sur 419 cotes foncières, 163 appartiennent à des propriétaires habitant la commune, et 256 à des forains. (Loco citato.) A Beurizot, en 1899 , sur 310 cotes, 179 sont foraines. 
Il y eut, d'après ecrtaines coïneidences, un exode à peu près qénéral, daus les régions que j’ai signalées; il se manifeste largement dès le $x \mathrm{v}^{\mathrm{e}}$ sièele et les événements du $x \mathrm{v}^{\mathrm{e}}$ ne purent que l'accélérer. Les héritiers qui trouraient leurs parts trop réduites les cédaient à leurs parents ${ }^{1}$ ou les vendaient à des tiers, ils allaient grossir ensuite la population des villes où le nombre des artisans s'accroit précisément à eelte époque ${ }^{2}$. Les aequéreurs des parcelles délaissées ne furent pas seulement des cohéritiers, ear tous n étaient pas assez riches pour reconstituer l'hérilage paternel; beaucoup de seigneurs profitèrent ${ }^{3}$ de loccasion pour agrandir les faibles réserves qu'ils avaient eonservées en rachetant les parcelles eensitaires, et les bourgeois des villes, désireux de s installer parmi les paysans dans l'intérêt du commeree de grains, de bétail et d'argent qu'eux-mêmes monopolisaient, leur firent une sérieuse coneurrenee ${ }^{4}$ en eréant une classe intermédiaire, et que jusque-lił on ne connaissait pas, de

1. Vingt et une familles des environs de Gien (Loiret) ont eté suivies, par un patient observatenr, penclant deux siècles, de 1 t50 à 1650. $\Lambda$ u debut. c'est l'aisance et presinue la richesse. besoins tres restreints el facilité très grande d'y pourvoir. Aussi est-ce merveille comme on pullule, de chaque foyer sort une tribu. En 1530, les 21 souches ont fourni 200 branches. Puis vient le mouvement inverse: on est trop nombreux, on se gène; un s'arrache des inietles de pré el de labour. En 1650, sur les 200 familles, il n'en restait plus que 6, loutes les autres avaient été remplacées par des étrangers. (D'Avenel, Histoire économique.)

2. Celte ipoque est celle ou l'on inscrit dans les statuts des corporations de nouveaux arlicles réglementant l'apprentissage et s'effurgant à restreindre l'invasion des étrangers, vilains et manants du plat pays. (Arehives municipales des villes de l'bst.)

3. H. Tausserat a reconstilué la série tles acquisitions de certains d'entre eux, au vu des actes. Ia seigneurie de lury ne possédait pas, en 1 \$00. un domaine utile de 20 luectares; en 1700 , elle en affermait 600 . En 1380. la superficie moyenne de chacun des 14 fiefs dépendant de la chitellenie de Lury n'alteint pas 13 liectares; en 1700, par suite d'achats ininterrompus. leur élendlue dépassait pour chacun 000 hectares. Ne peut-on y voir l'origine des grandes exploitations actuelles du Cher?

i. Jusquä la fin du $x^{\circ}$ siècle, les Juifs avaient eu le inonopole du change et les prèts d'argent; les cliretiens leur firent coneurrence. Lo numeraire itait rare, on commerçait surtout par le moyen des ichanges en nature et e'cst ce qui explique la rapide fortune des paysans qui se firent courtiers et des hourgeois qui devinrent marchands, entrepositaires, prèteurs sur nantissement dans les villages. 
propriétaires fonciers roturiers, n'exploitant pas directement. Cet ensemble de circonslances el de faits explique à la fois l'extrème division du sol et son extrème mobilité par la multiplicité des transferts à une époque où il semble que la culture ne fùt qu'un servage et la terre une valeur dépréciée.

Du milieu du $\mathrm{xrr}^{e}$ siècle à la fin du xrrı la grande propriété se reconstitue par l'acquisition de petites parcelles ${ }^{\text {; }}$ l'exemple est donné de haut, les parvenus ont tous à cœur de vieillir leurs fortunes récentes en s’installant dans de vastes domaines, Fouquet et Colbert ont rivalisé de luxe foncier ${ }^{2}$. Le progrès agricole se dessine, le lotissement de biens communaux, des défrichements, augmentent la surface productive, et ce peuple gêné de métayers et de fermiers qui a remplacé dans une forte proportion, surtout dans la plaine et près des villes, les anciens petits propriétaires dépossédés, cherche à modifier les procédés d'assolement et d'élevage pour rendre plus rémunératrice l'exploitation de ces terres de la bourgeoisie ${ }^{3}$ dont le produit net lui échappe ${ }^{4}$.

1. Les grandes fermes de Beance et de Brie datent du xvi ${ }^{e}$ siècle; de même que les vastes herbages de la Normandie, du Berry, et les domaines répartis en petites métairies du Morvan. Les capitalistes de ce temps comprirent que le morcellement excessif du moyen âge, nécessaire pour défricher et peupler, constituait une entrave au développement raisonné de la production agricole, et que le groupement pouvait faire de l'exploitation rurale une vẻritable industrie.

2. On sait les sommes folles (1S millions de livres) que dépensa Fouquet pour acheter le terrain et bâtir le château de Vaux-le-Vicomte, près de Melun. En 1670, Colbert achète au duc de Tresmes la baronnie de Sceaux avec 120 arpents; en 1682, le parc de Sceaux, entourẻ de murs, en englobait 709. (Gazette des Beaux-Arts, jer novembre 1899.) Le domaine royal de Versailles, devenu résidence, ne s'agrandit point par l'adjonc. tion de grandes ètendues de terrain mais par l'achat d'une infinité de menus lopins appartenant à de petites gens, fermiers, paysans, comme l'attestent les milliers de dossiers des Archives. En 1789, le domaine comptait près de 9000 hectares. (Ph. Gille, Versuilles et les deux Trianons, 1899.)

3. Expression typique d'un rapport de l'Intendant de la généralité d'Alençon, en 1700 , et qui marque bien le déclassement qui s'étajt opèré, les cultures étendues éparses aux mains des paysans s'étant concentrées et agglomérées dans celles des bourgeois.

4. En 1789, les rédacteurs des caliers de doléances des paroisses de la généralité de Toulouse déclarent qu'une des causes les plus gènérales 
A la veille de la Rérolution, landis que les voyageurs et les humoristes signalent, surtout dans la banlieue des villes et dans certaines régions à cultures intensives, un excessif morcellement, les administrateurs et les économistes remarquent que les fermes augmentent d'importance à mesure qu'on s'éloigne de Paris, de Lyon, de Bordeaux, de Toulouse, et que l'évolution s'est produite dans un double sens: diminution du nombre des propriétaires par le groupement de plusieurs petits héritages dans les mèmes mains ${ }^{1}$; diminution du nombre des parcelles par la transformation de minuscules cultures en labours ou en herbages d'un seul tenant ou la suppression de jardins de paysans englobés dans un pare ${ }^{2}$.

La grande propriété, telle qu'elle existait en 1789, toujours abstraction faite des biens ecclésiastiques el du Domaine du Roi, n’arait donc pas la féodalité pour origine; clte étail le produit de la réaction commencée à la fin du $x v^{e}$ siècle par la réfeclion générale de la plupart des terriers seigneuriaux ${ }^{3}$, l'ourre lente el continue d'une concentration aux mains d'une nonvelle classe de propriétaires fonciers beaucoup plus que dune reconstitution (ce lint la très rare exception) de l'étendue des tiefs et des héritages primitils créés par le contral de sauvegarde el l'éranonissement momentané de la petite propriélé individuelle. Yoilà le fail économique; le fait politique, c'est que la reconstitution de la grande propriété eut licu, non pas au profit de la vieille aristocratie militaire

de la misere publique est l'absenee de propriétés personnelles entre les mains des cullivateurs, obliges de travaller pour un salaire modique insuffisant a l'entretien de leurs familles.

1. M. Tausserat cite la piéce des Vaflées, de 30 hectares, proche le chateau de Chevilly (Cher), ou l'on planta l'avenue le noyers qui conduit au château, qui elait divisce entre 39 propriétaires, el yue la famille de Courauld acquit par morceaux suivant des contrats échelonnés pendant quatre siccles.

2. \$1. "l'Avenel cite le pare le Blaru (Seine-et-Oise) qui, de 3 hectares et demi en $15 \% 0$, s'élargit en 28 hectares en 1677, sans que l'ensemble du domaine ent angunenté.

3. Lettres patentes lu roi Louis XII, de février 1499 , renouvelant et confirmant celles de Charles VIll. 
el féodale, dont bien peu de représentants figuraient encore parmi la noblesse de cour, mais à l'avantage d'une aristocratic nouvelle, issue du liers élat, et qui, s'affublant des anciens titres, n'est plus reconnue dans l'histoire sous ses noms palronymiques que par les érudits '.

J'eslime que, pour ne point exagérer certains éléments de celte étude au détriment des autres, quel que soit l'intérèt majeur du sujet, ces brèves inlicalions suffisent pour fixer les grandes lignes des origines, en France, de la propriété étendue et de ses ricissitudes. Je laisse de còté, à dessein, la question des biens communaux, l'une des plus controversées de notre histoire; le problème est différent ${ }^{2}$.

Ce qu'il importe de mettre en lumière, c'est le passage de la société gallo-romaine à la société barbare, ou plutôt la lente et continue pénétration de ces deux sociétés l'une par l'autre, aboutissant à un élal économique nouveau, à un modus vivendi paraissant équilibré par de mutuelles concessions et que viennent soudain bouleverser les invasions du $\mathrm{IX}^{\mathrm{e}}$ siècle. La ruine qui frappa les curiales gallo-romains a

1. Colbert, fils d'un marchand de Troyes, l'ut fait marquis de Seignelay et laissa ce titre à son fils aîné; il fit de son frère un marquis de Croissy et de son second fils un archevêque de Rouen, de son petitfils un comte d'Estouteville et de son neveu un marquis de Torcy.

Le Tellier fut marquis de Louvois et pourvut de benux titres ses sept fils, l'un évêque de Clermont, l'autre marquis de Barbesieux, l'autre marquis de Souvré, etc.

Fouquet maria ses filles au due de Charost et au duc d'Uzès, ses fils furent comte de Vaux et marquis de Belle-lsle. L'Armorial de d'llozier n'est plus qu'une savonnelte à museaux de greffiers, disait Bussy-Rabutin.

2. L'Académie l'a posé en $185 \%$. Jusque-là, au lieu d'étudier la question en elle-mème, on en subordonnait la solution à l'idée qu'on se faisait de la féodalité et de son rôle. Tantôt on n'a reconnu aux communes qu'un simple droit d'usage, originairement concédé par la bienveillance tu seigneur; tantôt, au contraire, on leur a attribué la propriété primitive et l'on n'a vu dans les seigneurs que des usurpateurs abusant de leur juridiction pour s'emparer de droits qui ne leur avaient jamais appartenı. La première opinion est visible dans la grande ordonnance des Eaux et Forêts de 1669, qui reconnait aux seigneurs le droit dle triage, c'est-à-dire le droit de prendre en toute propriété le tier's des communaux; la seconde opinion est le principe de toute la législation domaniale de la Révolution. (Comptes rendus de l'Académie des sciences morales et politiques, $1855-1836$. 
son équivalent dans la misère qui étreignil les paroisses rurales sous Louis XIV ${ }^{1}$. Mais, ce que les populations apeurées du moyen âge liquidèrent par l'abdication universelle, s’abaissant par leur libre volonté de l'échelon de propriétaire libre à celui de fermier perpétuel, la Nation de 1789 le liquida d'office par la Révolution.

Quelle a done été la part de la Révolution dans la division et la répartition du sol; quelle inflrence immédiatc a-t-clle exercée sur la condition des populations rurales en France et quel sort fit-elle à celte grande propriélé qui a élé si décriée el qui le mérite si pen?

La France est dans l'indivision, avaient répété les Encyclopédistes, et quiconque possède par indivis a le droil de demander le partaģe. C'était exagérer la thèse de ceux qui prétendaient que, sous la triple influence des droits féodaux, de l'arbitraire royal et de la surcharge d'impôts, la France n'était plus qu’un pays de main-morte. La preuve du contraire est faite. En attribuant à la Révolution de 1789 l'honneur et le mérite d'avoir créé le paysan-propriétaire, on a mis en circulation une légende que détruit l'examen impartial des faits, et une errenr historique aussi coupable que celle qui, au siècle précédent, voulait voir dans la nation deux classes ennemies, des vainqueurs et des vaincus.

Un spécialiste ${ }^{2}$ a résumé, quant au régime économique de la proprićté rurale sous l'ancien régime, dans une formule expressive el qu'on ne vulgarisera jamais trop, les résultats obtenus par l’érudition la plus consciencieuse. En voici le texte :

Mon enquête à travers les róles des vingtiemes m'a fait loucher du doigt deux faits essentiels d'où découlent maintes conséquences : le premier, qu'il y avait, avant 1789, une multitude de propriétaires fonciers; le second, que ces proprictaires ne payaient que des cotes minimes, d'une exiguité que je n'aurais jamais soupconnèe si je ne l'avais saisie sur tes rôles mèmes. L'impression qui reste de ces éludes, c'est que,

1. Les alleux des municipes, c'est-it-lire les biens communaux, furent a leur tour inféodés comme l'avaient élé les biens libres des particuliers. (Le Berquier, Revue des Deux Mondes, 1859, I, 384.)

2. M. Gimel (Bulletin du comilé des travanx historiques, 1890, I, 114). 
sous l'ancien régime, ce qui dominait c'était la très petite propriétẻ et la très grande, la première considérable par le nombre, la seconde par l'étendue.

La liquidation foncière engagée par la Constiluante et qui ne se termina que sous la Restauration, mit aux enchères, d'après les calculs les plus modérés, 1/10 du territoire national; or, à notre époque, malgré l'activité du mouvement foncier, les acquisitions ne portent guère, année moyenne, que sur $1 / 50$ du capital foncier; le brusque apport sur le marehé de tant de valeurs à la fois amena pour la valenr rénale des immeubles une dépréciation de 30 p. 100, et la crise des assignats vint compliquer, ralentir et contrarier l'opération.

Lincendie du ministère des Finances pendant la Commune de 1871 a fait disparaitre les archives de l'administration des Domaines relatives au séquestre, à la restitution et à la vente des biens du clergé et des biens des émigrés. C'est une perte irréparable ${ }^{1}$, et ce point capital de notre histoire économique ne sera jamais éclairci. On peut toutefois, par les documents qui restent et les commentaires qu'on en fit, esquisser les caractéristiques de ce morcellement inattendu et qui n'a d'équivalent que dans le Days Book britannique.

Ce qui est hors de doute, c'est que la grande propriété de main-morte fut brisée, disloquée, morcelée; c'était le vœu de la loi. La rente des biens des émigrés se fit avec d'autres vues, plus étroitement fiscales; mais le résultat parut identique, et il ne pouvait guère en ètre autrement.

Les biens ecelésiastiques furent vendus en bloc et non en détail, c'est-à-dire tels qu'on les trouvait, tels que l'Église elle-mème les avait reçus, a-t-on remarqué, l'un après l'autre, des pieux donateurs auxquels elle avait succédé, ici une ferme, là un moulin, ailleurs une prairie ou un bois.

1. Le regretté M. Paul Chalvet, chef alors de la section domaniate, y perdit le manuscrit de l'histoire de la liquidation des domaines nationaux. Les doubles de ces archives sont dispersès dans les directions de l'Enregistrement de chaque chef-lieu et aussi dans les Archives départementales. Quelques rares statistiques partielles tirées des comptes de finances ont èté publiées, mais elles restent incomplètes. 
La Convention procéda autrement pour les biens des émigrés considérés comme la représentation effective de ces seigneuries féodales dont tant de récits avaient perpétué l'exécration. On ne voulut pas que, même au jour de l'enchère, ces châteaux et ces terres cussent pu conserver un semblant de personnalité; on les fractionna. On les subdivisa en lots, non pas, comme on l'a supposé ${ }^{1}$, en vue de faciliter les petiles acquisitions et de créer une classe de propriétaires qui existait déjà, mais uniquement pour briser le prestige qu'on pouvait y attacher encore et pulvériser à tout jamais l'esprit féodal el aristocratique.

Le dépècement des 30000 plus grands domaines de France, effectué dans un si court espace de temps d'abord par la spéculation, car le classement définitif ne s'en acheva que bien des années plus tard, était l'alteinte la plus profonde que la grande propriété pût subir; on croyait qu'elle serait mortelle, mais, comme son ròle économique est une nécessité, clle ne tarda pas beaucoup à reparaitre.

On éraluait les biens dı clergé à 3 milliards en capilal, dont un milliard en propriétés urbaines, un milliard en forêts, un milliard en terres; on ne vendit guère que ce dernier tiers, et à vil prix ${ }^{2}$. Les détails des rentes seraient fort difficiles it retrouver, la statistique ne peut en être qu'hypothétique.

Les documents sont plus précis pour les biens des émigrés dont on ne put vendre que tout au plus une moitié; l'Empire el la Restauration restituèrent en nature ce qui restait ${ }^{3}$.

1. Il suffit. pour s'en assurer, de lire les rapports pen connus du Comité de législation et du Comité des Donaines (Arehives Nationales).

2. Comme on disait à Mirabean : Vous ne trouverez pás d’acquéreurs pour tant de terres à la fois, il répondit : Mienx vaut les donner four une apparence de prix que de les laisser slériles; lans un an elles produiront des fruits qui augmenteront la masse publique.

3. NI. de Fovilte a résumé lo télıat avec infiniment de clarté, et je lui emprunte la plupart des chifres qui suivent. (Le morcellement, chapitrev.) 
L'expropriation de la noblesse émigrée ou suspecte se fit en deux séries, avant et après le 12 prairial an III.

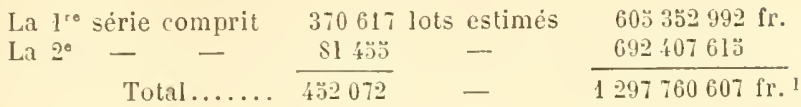

La prophétie de Uirabeau s'était réalisée; l'opération foncière sur les profits de laquelle on avait gagé les émissions de papier-monnaie, c'est-à-dire le crédit public, avait avorté, mais les immeubles restaient entre les mains de détenteurs qui sauraient en tirer parti; en réalité, c'était un cadeau à la Nation, comme l'insinua Mr. de Martignac en présentant la loi d'indemnité.

La grande propriété était mise en miettes; à qui allèrent ces miettes? MIM. Léonce de Lavergne, de Molinari, Marc de Haut, Taine, affirment qu'elles n'étaient pas à dédaigner, ces miettes, et qu'elles servirent à constituer cette catégorie intermédiaire, la moyenne propriété, dont nul ne parle avant 1789 et dont H. Gimel lui-mème n’a pas trouvé les traces.

Cela peut être vrai pour les biens ecclésiastiques qui furent les premiers vendus et sous leur forme normale. ce qui les maintenait à l'état d'exploitation au lieu de les déprécier par une division mathématique et brutale. Vrai encore, peutêtre, pour les $8145 \%$ fonds aliénés postérieurement au 12 prairial an III, et dont le revenu moyen avant 1790 s'élevait, pour chacun, à environ 400 livres.

Mais, MM. Gimel, Paul Leroy-Beaulieu et de Foville ont certainement raison contre II. Taine lorsqu'ils soutiennent que ce n'est pas à la moyenne propriété mais à la petite, et

1. On s'étonnera peut-être de voir une valeur presque égale attribuée à des immeubles dont le nombre présentait un écart si considérable; mais les évaluations n'en furent faites qu'à titre approximatif lors de la discussion qui précéda la loi du 27 avril 18203 sur le milliard d'indemnité, et comme les prix avaient été stipulés payables en assignats et que ces papiers en l'an $\mathrm{Y}$ perdaient le même jour 73 p. 100 à Bordeaux et 27 p. 100 à Mende (exécution de la loi du 5 messidor an V), le Trésor n’a jamais pu établir avec précision quel avait été le produit net de celte gigantesque opération. 
même à la très petite, que sont allés les 370617 lots vendus aux enchères avant le 12 prairial an III, et dont le prix d’acquisition varia de 700 à 2000 francs, c'est-à-dire en assignats, suivant la date, peut-être seulement de ə̈0 à 120 francs.

En France. dit un ćconomiste dont les opinions paraîtront sans doute bien vieillies ${ }^{1}$, les terres qui dépassent une certaine étendue perdent toute valeur vénale, faute d'acquéreurs; pour en trouver, il faut les diviser et solliciter ainsi les petits capitaux.

La période rérolutionnaire, continue-t-il, n'a pas été autre chose que l'invasion, la conquète et le partage du territoire entre les rainqueurs. Le tiers état a fait main basse sur les biens du clergé, de la noblesse et des corporations; les propriétés frappées de main-morte ou grevées de substitutions sont rentrées dans la circulation où elles ont versé un capital de plus de 2 milliards de franes; mais l'opération dura plus de trente ans. La passion connue du paysan pour la terre a donné lieu à de sauvages mais lucratives spéculations. Les premiers qui s'en avisèrent lurent des artisans enrichis à qui l'instinct plébéien, instinct de destruction el de nivellement tant qu'il n’est pas éclairé, révéla promptement cette source de profits. La première lande se composait de chaudronniers et de revendeurs de ferraille experts il calculer le prix des débris; ils s’abatlirent comme une rolée de corbeaux sur les grands domaines et sur les vieux châteaux, achetant ces ruines à vil prix pour les débiter au poids de l’or. La terre fut disséquée par lols d'un ou de deux arpents, les châteaux furent démolis et les matériaux vendus, la pierre pour de la pierre, le bois pour du bois, le fer pour du fer. C'est ainsi que les derniers vestiges de l'art et du régime féodal disparurent de la France.

Après ces ravageur's de villages, apparut la bande noire, plus méthodique et plus rusée. Aujourd'hui qu'il n'y a plus de chateaux à détruire, la spéculation se porte sur les

1. Anonyme, dans la Revue des Deux Mondes, 1836, IV, page 303. 
moyennes propriétés; elle les décompose partout où elle peut les atteindre, et les distribue par petits lots.

Ce sont ces subdivisions successives de lots en d'autres lots de plus en plus petits qui ont permis à M. Gimel d'alfirmer, au vu des cotes fonciùres, que la grande propriété vendue révolutionnairement en $4 \$ 2000$ lots primitifs s'est émiettée après deux générations en 1222000 parcelles, et à M. de Foville de dire que la Révolution a fait sortir de terre un demi-million de propriétaires nouveaux parmi lesquels, comme partout, les petits étaient certainement en majorité ${ }^{\text {. }}$

La Rérolution a délivré la terre de toutes les servitudes, sauf de celle de l'argent, ce qui n'était pas en son pouroir"; elle a simplifié, fortifié la propriété individuelle, et la réforme qu'elle accomplit servit puissamment les intérèts de l'agriculture et par suite ceux de tous les détenteurs du sol, quils fussent petits, moyens ou grands.

La grande propriété se reconstitua vite, non seulement entre les mains des particuliers, par la restitution aux anciens propriétaires des biens mis sous séquestre et non aliénés, par la constitution des majorats, le rétablissement partiel des substitutions, les libéralités de la cour, l'emploi du milliard d'indemnité, et le jeu de la spéculation, mais encore sous la forme particulière de la main-morte après le Concordat. Cette grande propriété nouvelle avait perdu les défauts le l'ancienne; devenue désormais accessible à tous, elle n'est plus que le privilège de la fortune aequise et chacun peut y prétendre, en y mettant le prix.

L’histoire de la propriété nous en montre la mobilité continuelle; le passé tout entier nous ofire le spectacle des forces économiques se jouant des combinaisons législatives qui ont pour but de maintenir ou d'empècher certaines inégalités des conditions; le niveau, un instant dérangé, se réta-

1. M. de Foville a raconté l'amusante histoire des mutations successives d'un lot domanial, la Censelette d'Eringhem, près de Dunkerque, vendue aux enchères en 179', sur la mise à prix de 2000 lirres, plus de 21000 , et revendue en $1793 \mathrm{plus}$ de 65000 livies, mais en assignats. (Journal de la Société de Statistique de Paris, juillet 1883.) 
blit de lui-mème, et ce niveau n'est pas celui d'une égalité chimérique mais l'équilibre incessamment variable d'inégalités qui se còtoient, se déplacent, se pénètrent et se combinent par la pratique de la liberlé.

\section{III. - Rôle économique de la grande propriété par son morcellement limité.}

La grande propricté, comme loules les institutions dont le ròle social est considérable, a eu des détracteurs obslinés et des partisans fanatiques. Malthus a prédit que vers l'an 1910, gràce à l'inconséquente et infinie division du sol, il n’y aurait plus guère d'autres personnes riches, en France, que celles qui recerraient un salaire du Gouvernement. Nous semblons nous approcher de ce pileux résultal, si l'on ne considère que l'attrail qu'exercent les fonctions publiques sur le paresscux égoïsme de la jeunesse contemporaine, mais la faute n'en est pas à l'extrème moreellement de la propriété, et notre pays posside d'autres ressourees que les salaires que le Gouvernement emprunte à l'impòt.

Lorsque la Révolution crut avoir détruit la grande propriété el que les événements eurent amené la réaction, la politique s'empara de ces questions el les agita avec une vivacité passionnée. On se refusait à accepter l'évidence : l'acroissement du nombre des propriélaires, la création de nouveaux produits et de nouvelles richesses, l'essor du erédit, lout eela sortait des orages de la Rérolulion. On s'atlaquail ì l'inlluence de nos lois civiles el à la nowrelle constilution du aroil de propriété.

Le morecllement des propriétés était dénoncé comme une calamité nationale; on disait que le sol le la France élait pulvérisć el (ju'au lieu de compter par arpent on ne complail plus qu'à la toise. En 182:; , les plaintes prirent un caractère plus vif, et un discours parlementaire se termine par celle prosopopée lamentable :

Pourquoi tant de misires dlans nos campagnes? Pourquoi uos denrées sans acheteurs, notre bétail invendu, nos villes désertes el, pendant 
que les consommateurs soufrent, pourquoi nos producteurs s'épuisentils en vains eflorts pour trouver des gens qui achètent des grains, des meubles, des étofTes, et même les vêtements et des souliers? Ouvrez le Code, là se trouve la solution. La propriété est réduite en poussière, la loi française proscrit virtuellement la charrue!

A soixante-quinze ans de distance, on peut roir combien était grande l'erreur qui dictait ces paroles. Notre loi des partages, mème en admettant qu’il soit désirable de la corriger quelque peu, n’a point produit les conséquences désolantes que lui imputait l'esprit de parti.

On a pu dire mème que e'est à peine si le principe du partage effectif des successions suffit à contrebalancer l'active et énergique influence qu'exerce la force de concentration de la richesse et des possessions territoriales ${ }^{\text {. }}$

C'est chose naturelle, nécessaire, que la propriété se morcèle dans certaines régions, tandis qu'elle se recompose et s'agglomère dans d'autres. Il n'y a rien de eapricieux ni d'indifférent dans ce double mouvement, dominé par les exigences du milieu qui poussent les individus à faire ceci et cela et à le faire tantôt ainsi et tantôt autrement suirant que leur intérèt, bien ou mal compris, le leur conseille. Il n'est ni rationnel ni pratique de vouloir donner une solution unique à des questions complexes, et il serait aussi ridicule de vouloir empècher la grande proprićté de s'accroìtre que la petite de se diviser. L'intérêt privé est juge de ses convenances, et l'histoire nous enseigne ce qu'ont toujoul's coûté les lois du Maximum.

Le ròle de la grande propriété n’est pas contestable; il varic d'intensité ou d'utilité suiranl les pays et, dans chaque pays, suivant les époques; considérable au début des civilisations, il paraît l'ètre un peu moins à leur déclin, mais comme le déclin d'un peuple n'est qu'une transformation, une étape de l'humanité, il ne faut pas croire que le ròle diminue parce qu'il a changé de forme. Si l'équilibre est momentanément rompu d'un côté ou de l'autre, le plus sùr

1. M. Molowski, sur la division du sol (Revue des Veux Mondes. 1857, IV, page 647). 
agent de rétablissement est la concurrence, c'est-à-dire l'exercice de la liberlé. C'est dans ce sens que la grande propriélé est l'un des rouages essentiels du mécanisme social.

Elle agil d'abord comme réservoir de la petite propriété individuellc; en France, là où la culture intensive n'a pu se dérelopper sous forme de petits hérilages, faute de grands domaines à morecler, il a fallu s'attaquer à la proprićté collective et diviser les biens communaux; en Anglelerre, où la loi rend la grande propriété intangible, on en est réduit à créer fietivement des lots à distribuer. Dans ce pays, à l'heure actuclle ${ }^{1}$, la grande propriété fait faillite à l'une de ses fonctions les plus nalurelles, celle d'alimenter le besoin de posséder, source du travail et ferment de l'épargne, et l'on est obligé d'y suppléer artificicllement.

Si l'on s'exagère, dans des sens divers, tantôt les effets de la concentration de la proprićté, tantòt l'influenec de la grande propriété sur le dércloppement de l'agriculture, c'est qu'il y a, suivant les circonstances, une mesure à l'une et des limites à l'autre. Qui dit grande propriété, ne dit pas toujours grande culture. Les plus grandes propriétés peurent se diviser en petites cultures et il importe peu, ì ce point de vue parliculier, que 10000 hectares soient possédés par un seul s'ils se parlagent en 200 fermes de $\$ 0$ hectares chacune. On tombe alors dans le systeme de la ferme en bloc qui ruina l'agriculture française an xvin ${ }^{e}$ siecle ${ }^{2}$ comme il ruine eneore l'lilande.

On fait souvent confusion entre des choses que l'on croil semblables et qui ne le sont pas, de mème qu'il s'élève d'inu-

\section{Lois récentes sur les Allotments et les Small holdings.}

2. La plupart des grandes terres, à la fin de l'ancien régime, et surtout depuis $17 \% 0$, etaient louees en bloe à des fermiers-géniraux, gens l'affaires de la ville voisine, fui faisaient exploiler par des sous-fermiers, comme un entrepreneur divise ses travaux entre dee sous-entrepreneurs, et qui firent de cette combinaison un commerce très lucratif. La terre nourrit ainsi deux maitres au lieu d'un, au détriment de l'exploitant qui travaille pour iux.

Des le $x v 11^{\circ}$ siècle, sourllis conseillait à Richelieu de donner á quelque notaire les 20 petites fermes de son duelé pour les tenir en ferme générale dont il paierait le fermage deux fois l'an. 
tiles polémiques entre gens qui sont du même avis au fond mais qui ont négligé de s'entendre, au préalable, sur la valeur d'un mot. Ce n'est pas l'étendıe senle qui conslitue la grande propriété, il y faut joindre l'unité d'exploitation.

Ce qui importe à la culture, ce n’est pas que la propriété soit grande, mais quielle soit riche. La richesse est relative; on peut être paurre avec une grande propriété, riche avec une petite. Le propriétaire de mille hectares qui les laisse en bois et en friches et se contente d'y renir chasser tous les ans n'est que d'un mauvais exemple, tandis que le propriétaire de cent hectares, qui réside, qui défriche, qui fait avec succìs des essais de fumure el d'élevage, exerce une action véritable et crée autour de lui une atmosphère de nouveautés intelligentes. La meilleure organisation de la propriété rurale est celle qui attire rers le sol le plus de capitaux, que les détenteurs de la terre soient assez riches relativement à l'étendue qu’ils possèlent ou qu'ils aient naturellement le goût d'y dépenser en partie leurs revenus.

C'est généralement le ròle qu'on altribue aux grands propriétaires, ce n'est pas toujour's celui qu'ils remplissent en réalité. Il n'y a pas en France les colossales fortunes de l'aristocratie anglaise; on ne peut done demander qu'ì l'État l'exécution d'entreprises analogues ì celles dont le duc de Bedfort, le duc de Portland, lord Leicester, lord Spencer et beaucoup d'autres ont doté, par calcul autant que par générosité, l’agriculture de leur pays.

Si la grande propriété n'est qu'un moyen de lucre, et qu'elle se subdivise elle-même, en vue uniquement de gagner davantage et sans aucun souci d'expériences utiles ou de participation directe à la vie économique du pays et à son progrès, quel inconvénient trouverait-on à ce qu'elle fût divisée? C'est une fiction qui la maintient dans celte calégorie. En lait, elle n’appartient ni à la grande, puisqu'elle s'émiette de ses propres mains, ni à la petite, puisque les exploitants n'y apportent qu'un intérêt mixte et transitoire. Elle tombe dans la banalité de ces terres qu'un spécu- 
lateur traile en atelier industriel et qu'un économiste d'il y a cinquante ans décrivait en ces termes dédaigneux :

Les grands domaines qui ont échappé à la dissection légale sont morcelés par la culture. On distribue la terre par petits lots pour l'afermer aussi bien que pour la vendre, et le possesseur en retire le mème avantage dans les deux cas. Le paysan, quand il ne peut pas devenir propriétaire. veut du moins posséder en qualité de fermier. Là oì un fer. mier de profession, connu, expérimenté, qui a des capitaux et qui présente des garanties de compétence et de solvabilité, offrira 30 francs de rente par arpent, le petit eultivateur en donne 40 sans hésiter, pour un maigre lot, et sera préféré. Le maitre du sol, le son côté, ne considere plus dans quel état la terre lui sera rendue, nourrie ou épuisée, il ne voit que l'accrue du fermage en bloc et la valeur faclice qu'en recev'ra son bien. Les notaires favorisent ces arrangements, parce que au lieu d'un client ils en auront vingt.

Ainsi les grandes fermes sont aujourd'hui encore plus clairsemées sur le territoire que les grands domaines; la Beauce elle-même, cette vaste plaine de blé aux portes de la capitale. oi la charrue du gros fermier pouvait tracer le sillon à travers 200 arpents sans se heurter aux limites de l'exploitation, qui figurait un champ d'expériences oủ toute découverte à peine connue était mise à l'essai, se hẻrisse de cultivatenrs au détail, race besoigneuse, ignorante et nolitique comme les paysans d'Irlande. D'une grosse ferme on en fail trente, oủ la production n'aura pour exeitant que la misère et ne pourra l'apaiser.

Ce fait est encore actuel, et il s'est aussi souvent produit en Flandre, en Champagne, en Languedoc que dans Ia Beauce, de 1837 à 1899.

On attribue habituellement à l'aristocratie anglaise un rôle exclusif dans le progrès de l'agriculture, mais on oublie trop que ces propriétés géantes ${ }^{1}$ ne sont pas les seules et que la Gentry, celle classe intermédiaire, beaucoup plus riche que notre bourgeoisie rurale mais qui en est l'équivalent, a rendu de réels services ${ }^{2}$. Au point de vue de l'étendue

1. En 1878 , plus de ij millions d'hectares (1/6 du Koyaume-Uni) appartenaient à 91 individus seulentent; 17 d'entre eux possédaient des proprietés variant en superficic de 2'i à 40000 hectares et 19 en détenaient chacun plus de 60000 . (Financial lieform Almanack.) En 1536, on comptait 1011 propriétaires fonciers possédant clıacur plus de 375000 francs de revenu agricole. (New Domesday Book.)

2. En Angleterre, une movenne de 2000 franes de rente, dans les Comtes. correspond à ce que sont, en France, ż à 6000 livres de revenu, en province. 
restreinte deses domaines à côté de ceux des Landlords, et à celui de la richesse, la Gentry représente ce que sont chez nous la plupart de nos grands propriétaires. Beaucoup de proprićtés se divisent en Angleterre, un très grand nombre de petits propriétaires vendent leurs héritages pour devenir fermiers, prolession qui a la réputation là-bas d'enrichir vite, tous les jours de nouvelles résidences se construisent pour de nouveaux Country gentlemen, mouvements complexes rendus plus faciles par l'immense quantité de valeurs mobilières en circulation el par la rapidité avec laquelle se forment des fortunes dans le commerce et l'industrie; un autre puissant facteur de la richesse publique outre Manehe, c'est la précocité de la jeunesse, qui s'établit et se trouve en pleine possession d'un état ou d'un métier à l'àge où nos fils sont encore sur les banes d'écoles où ils n'apprennent qu’à douter de tout excepté d'eux-mèmes.

En France, le rôle de la grande propriété, après celui passif de réservoir dont le trop-plein sert aux irrigalions du voisin, peut être, avec des proportions plus modestes qu’en Angleterre, celui, relativement actif, de ferme-modèle.

En France, la grande propriété n'est plus que rarement l'apanage héréditaire d'anciennes familles, comme en Angleterre, en Autriche, en Allemagne; mais elle est devenue le luxe intelligent des millionnaires.

Le grand propriétaire de nos jours s'éprend volontiers des méthodes scientifiques, des machines perfectionnées, des croisements de race, de toute eette besogne dispendieuse et aléatoire, indispensable aux progrès agricoles si l'on veut lutter avec les pays voisins, que l'Etat n'aborde que chichement et maladroitement, et qui rapporte plus d'honneur que de profit. La grande propriété fait des expériences qui servent à la petite infiniment plus qu'à elle-même.

Le paysan propriétaire est là à une école gratuite, a-t-on dit excellemment ${ }^{1}$, à une leçon de choses. Il regarde avec intérêt

1. Paul Leroy-Beaulieu, sur la propriété foncière (Revue des Deux Mondes, 1886, I, page \$32). 
et scepticisme son entreprenant roisin et si, entre 10 ou 100, une de ces nouveautés tourne à bien, quand le succès a élé vingt fois démontré, le petit propriétaire imite sans risques. Il adopte le cépage nouveau, la greffe nouvelle, l'assolement récent, le procédé qui mettait le sourire à ses lèrres quand il en faisait l'essai comme salarié du riche.

La grande propriété instruit la petite, car de plus qu'elle elle a les capitaux qui permettent l'initiative et, simon la science, tout au moins ce reflet de science qui fait oser.

Enfin. troisième terme de l'utilité éeonomique de la grande propriété, aux heures de erise, lorsque les petits propriétaires ont épuisé leurs réserves et qu'ils sont sans ressources soit pour lutter plus longlemps contre l'épreuve et attendre des jours meilleurs, soit pour reconstituer leurs eultures ou leur en substituer d'autres, les gros propriétaires achètent ees pareelles, ees modestes héritages appauvis dont nul ne voudrait, puisqu'ils ne rapportent plus rien et que, seuls, ils sont en mesure de remettre en valeur. La crise du phylloxéra a montré quel préeicux concours les propriétaires eapitalistes apportèrent à l'énergie des populations du Midi et de l'Ouest par l'aequisilion de terres derenues stériles à des gens ruinés qui en tirèrent un double profit, diabord par le prix de vente, ensuite par le salaire du travail de reconstitution ". Dans le Beaujolais, de propriétaires devenus mélayer's, beaucoup de viguerons, au lieu d'émigrer vers les villes et de s'y perdre ${ }^{2}$ ont élé sauvés, eux et leurs familles, par l’intervention, à la fois généreuse el intéressée, de la grande propriété. Il se fait ainsi, depuis que le monde est monde, et il continuera à se faire entre la propriété du capitaliste et celle du paysan un échange également profitable aux inté-

1. Hans l'Hérault, il y avait en 1862 plus de 70000 exploitations de vignes et terres d'une superficie inférieure à 1 lectare; en 1892, il en reste a peine 40000 et la grande propriété détient à elle seule la moilić du territoire cultivable (28: millions d'heetares, dont 168 en vignobles sur un ensemble de 574 ).

2. I)es statistiques récemment produites à l'Aeadémie des sciences ont démontré que les familles d'ouvriers agricoles immigrées à l'aris y disparaissent à la troisième génération. 
rèts généraux, soit que la grande propriété s'aecroisse, soit quielle se morcèle.

Ce qui, d'ailleurs, est extrèmement remarquable, ce qui corrobore la réalité expérimentale de eette thèse qu'on ne taxe plus de paradoxale, c'est, dans tous les pays du monde, le parallélisme universel de la grande et de la petite proprété, eelle-ei eôtoyant l'autre. L’étroile association éeonomique de ees deux eatégories d'exploitation de la terre se manifeste parlout par des faits qu'on ne peut méconnaître.

Sans comparer les éléments dont les earactères sont différents, puisque l'étendue des pareelles et leur revenu n'ont pas dans les différents pays la mème valeur proportionnelle qu'en France, l’importanee des groupes est une indication topique.

La Grande-Bretagne passe pour un pays de grandes fermes et, eependant, e'est dans la moyenne proprićté, de 100 à 300 acres, qu'on trouve le plus grand nombre d'exploitations, plus de 18 millions eontre 9 millions de plus grandes et $\check{5}$ millions de plus petites; mais les fermes de plus de 300 acres (l'aere équivaut à 40 ares $1 / 2$ ) oceupent, suivant la région (Angleterre, Pays de Galles on Éeosse), un tiers ou un quart de la surface eultivée.

L'Irlande, dont la superficie n'est que le quart de celle de la Grande-Bretagne ${ }^{1}$, compte un plus grand nombre d'exploitations, ce qui signifie que la eulture y est infiniment plus divisée, mais non la propriété ${ }^{2}$.

\begin{tabular}{|c|c|c|}
\hline $\begin{array}{c}\text { NOMBRE } \\
\text { DES EXPLOITATIONS RURALES }\end{array}$ & $\begin{array}{l}\text { GRANDE- } \\
\text { BRETAGNE }\end{array}$ & IRLANDE \\
\hline $\begin{array}{l}\text { Pour l'ensemble du territoire cultivé.. } \\
\text { Fermes de plus de } 100 \text { acres.......... } \\
\text { Fermes de plus de } 1000 \text { acres........ } \\
\text { Exploitation de } 1 / 4 \text { d'acre à } 1 \text { acre... }\end{array}$ & $\begin{array}{r}3935 \text { \$ร5 } \\
98937 \\
663 \\
23512\end{array}$ & $\begin{array}{r}563254 \\
32233 \\
17 \\
49805\end{array}$ \\
\hline
\end{tabular}

1. La Grande-Bretagne a une superficie de 314951 kilomètres carrés, l'Irlande de 8't2 2 2.

2. Bulletin de statistique du ministère des Finances, octobre $1886,402$. 
A comparer la misère de l'Irlande à l'opulence de la Grande-Bretagne, on y voit la conséquence immédiate du rôle différent que jouent dans ces deux pays, non pas la grande propriété, mais les grands propriétaires. La passion des Anglais pour la campagne, la vie au grand air, la chasse, les sports de tous genres, les y attire, les y fait vivre; au contraire de nous, ils considèrent les villes comme leur atelier, leur bureau, mais leur résidence, le home, est aux champs.

Il est probable que s'il $y$ avait en Irlande autant de châteaux qu'en Écosse ou dans les comtés de l'Ouest, les populations qui y végètent verraient leur condition saméliorer, ne serait-ce que par la pratique dont Taine formulait la philosophie égoïste par une image énergique : En Angleterre, on balaie les paurres comme des ordures, pour les mettre en tas dans un coin. On soigne la campagne lorsqu'on I'habite, et, dans des conditions semblables, l'absentéisme est le fléan de la grande propriété pour ceux qui l'entourent autant que pour cenx qui en vivent.

Ilais il ne faut pas perdre de rne que l'Angleterre rurale est forte par son peuple de fermiers, et que la tlı́orie de la grande propriété, à part des cas assez rares, y loit rester distincte de celle de la grande culture. L'Angleterre est pleine de fortunes firites dans la culture; ces exemples font de cette carrière une des plus recherchées pour le profit en mème temps qu'elle est une des plus agréables, des plus honorées, des plus saines pour l'esprit et le corps. La classe des gentemen furmers correspond ì notre bourgeoisie; elle est instruite, active, et son importance sociale l'associe anssi étroitement que le proprićlaire à la solidité de la terre. Aueun d'eux ne songe à devenir propriétaire, remarque un économiste', leur condition est lien meilleure; pour aroir 3000 franes de revenu net comme propriétaire, un capital de 100000 franes ne suffil plus, Iandis qu'il n'est besoin que de 30000 franes pour les aroir comme fermier.

1. Léonce de Lavergne, lievue des Deux Hondes, 1833, I (L'éeonomie rurale en Anglelerre). 
Le ròle de la grande propriété, en Angleterre, est donc suriout de fournir à l'industrie de la culture la terre qu'elle ne pourrait se procurer autrement que par le bail à ferme, faute de capitaux, qu’en se créant des charges intolérables. Cette industrie est protégée, excitée, souvent commanditée par la présence fréquente du propriétaire, résidant une partie de l'année au milieu de ses fermes et y dépensant volontiers, par ostentation ou par goùt, les revenus que lui apportent ses entreprises industrielles ou commerciales. Mais tout cela n'est pas applicable à la France où le droit successoral, la législation foncière et les habitudes sociales ne s'accommoderaient pas de certaines contradictions qui sont, de l'autre côté du détroit, une cause de cohésion puissante et qui, cliez nous, deviendraient vite un ferment de dissolution.

En Hongrie, qui a comme l'Angleterre la réputation d'être le pays des vastes domaines, pour une population d'environ 14 millions d'habitants, on compte 2 millions et demi de propriétaires fonciers qui se répartissent par catégories dans des proportions significatives :

Catégories.

Nombre

des propriétés.

Petite propriété comprenant les exploitations d'une superficie moindre que 17 hectares 20 ares.... $23 \$ 8000$

Moyenne propriété de 17 h. 20 à 113 hectares... $11898 \mathrm{l}$

Grande proprieté :

De 115 à 575 hectares.... 13748

De 5730 à 5730 hectares... 4693

De plus de 5700 hectares. $\quad 231$

18674

Ensemble.......... 2485635

En Autriche, 4116216 propriétaires se partagent inégalement 52 millions de parcelles dont la moyenne est de $\dddot{37}$ ares. En 1883, les grands propriétaires terriens, ainsi classés comme payant chacun plus de 2500 franes d'impòt foncier, étaient au nombre de 1133 ; on n'en trouve plus que 1012 en 1893.

Les États-Unis eux-mêmes n'échappent pas à la loi générale qui veut que, là où la terre est libre, elle se morcelle à 
mesure que la culture se perfectionne. Malgré les énormes Latifundia qui se sont constitués depuis quelques amées au Minnesota, au Dakota, au Texas, en Californie, l'étendue moyenne des exploitations dans la grande république américaine n'a pas cessé de se restreindre. Elle était de 82 hectares en 1850 , de 80 en 1860 , elle tombe à 62 hectares en 1870 , puis à 59 en $1880^{1}$. Plus de la moitié des terres cultivées appartient à des fermes d'une étendue de moins de 40 hectares, dit M. Levasseur ${ }^{2}$; les fermes de plus de 400 hectares sont une très rare exception. L'étendue moyenne pour l'ensemble des États-Unis ne dépassait pas $5 . ;$ hectares en 1890.

Le ròle économique de la grande propriété est donc aussi variable que les conditions du milieu dans lequel elle se développe ou se maintient; il n’exerce une influence véritablement active et efficace que lorsque la grande propriété ne se sépare pas de la grande culture en lui substituani la spéculation par division el par intermédiaire.

Son rôle moral peut devenir plus efficace encore quand il est compris avec largeur el appliqué avec persévérance, discrétion el sympathie. Mais, là eneore, les lois ne peuvent rien et tout dépend des initiatives individuelles donnant l'exemple et créant un courant d'opinion. C'est une des bonnes pratiques que nous aurions profit à cmprunter à l'Angleterre. Par sa présence aux champs, le propriétaire anglais maintient, en effet, le prestige de ses droits, il donne autour de lui l'exemple du travail intellectuel, en mème temps que, sur sa réserve (Home-Farm), il se livre à d'utiles essais qui tendent à perfectionner la culture du domaine el à transformer les procédés agricoles de lout le pays. Le Countri! genlleman el le Landlord, grâce à la résidence, à la préoccupation des intérêts locaux et ì leur délense, entretiennent arec les petits propriétaires voisins et avec les

1. P. Leroy-Beaulieu (Revue des Deux Mondes, 1886, I, 838).

2. L'Agricullure aux Elats-Unis, édition de 189k, page 49. Les Farms s'entendent d'exploitations rurales et non de fermes à bail, qui sont relativement pell nombreuses. 
laboureurs el éleveurs du Comté des rapports constants qui toument à l'arantage commun. En France, la bourgeoisic propriétaire, plus encore que la noblesse ou la classe qui se qualifie encore ainsi, s’éloigne de plus en plus du paysan; un maladroit amour-propre, les prétentions mal déguisées les séparent, ôtent toute confiance mutuelle, leur enlèvent jusqu’à celte participation aux affaires locales si nécessaire à la véritable représentation du pays, à la diffusion des idées saines et à leur défense contre les empiètements des ambitieux et des sectaires. La franche aeceptation de la vie rurale, arec ses responsabilités, ses charges et ses profits, pourrait aroir pour notre pays d'autres conséquences que de simples améliorations dans les méthodes de culture.

Il y avait en 1862, disent nos statistiques, 154167 exploitations de plus de 40 hectares, ce nombre est réduit, en 1892, à 1386 i. Les lacunes des statistiques ${ }^{1}$ ne permettent pas de trouver dans quels groupes se sont reclassées les 13496 unités qui ont disparu; mais on le peut, de 1882 à 1892; l'écart pour cette période est de 3417 en bloc; mais, dans le détail, la moyenne propriété économique (de 40 à 100 liectares) perd 7894 unités, et la grande (au-dessus de 100$)$ en gagne $44 \bar{i}$, sans que la différence en nombre lui fasse perdre de son étendue, au contraire. N'y a-t-il pas dans ce fait un avertissement, une indication?

\section{IV. - Répartition régionale}

de la moyenne propriété.

Le ròle accessoire attribué par les économistes et par le plus grand nombre des statisticiens à la moyenne propriété, l'indécision de sa définition puisque la mesure de sa contenance varie, suivant les auteurs et les sources d'information, de 10 à 40 hectares, rendent assez difficiles l'exacle détermination de son état présent, et c'est le cas de dire à son

1. Enquête agricole de $1892 ;-$ Tableaux, pages 220 et 229. 
propos que notre impuissance de prouver est égale à notre impatience de connaître. Ici surtout il eùl été nécessaire de diviser, de subdiviser, de multiplier les distinctions, de rechercher à quelles catégories sociales appartenaient les propriétaires des 711118 exploitations rurales que les relevés officiels classent dans ce groupe, de les répartir par profession, de savoir le nombre de leurs cnfants, le chiffre de leur fortune mobilière, le degré de leur culture intellectuelle, tous éléments de nature à fixer l'observateur sur les causes qui déterminent ces terriens à demeurer dans unc inaction relative tandis quils ont à leur disposition une source de profit dont ils ignorent le rendement. C'est à ces lacunes que j'essaie de pourvoir, afin de peser la valeur probable de cette portion inexploitée de la fortune nationale.

La moyenne propriété, c'est-à-dire cette catégoric d'exploitations rurales que nos statistiques classent comme variant en élenduc de 10 à 40 hectares, occupe sur la carte une superficie totale d'un peu plus de 1.t millions d'hectares. Pour se rendre compte de son importance absolue, il faut la considérer au triple point de vue du nombre des exploitations ${ }^{1}$, de leur étendue, de la nature de leur culture.

Quant à son importance relative, clle se dégagera naturellement du rapprochement des chiffres qui vont suirre et de la condition mixte qui lui est faite, au point de vue agricole pur, par les conditions de la grande et de la pelite cullure, au point de vue économique par les variations en sens divers de la grande et de la petite propriélé.

I. Voir, à la page 336 re l'Introduction de l'enquête de 1892 , les motifs qui obligent à prendre les exploitalions comme équivalentes aux propriètés, malgré l'écart très apjréciable qui existe entre leur nombre rẻel. 



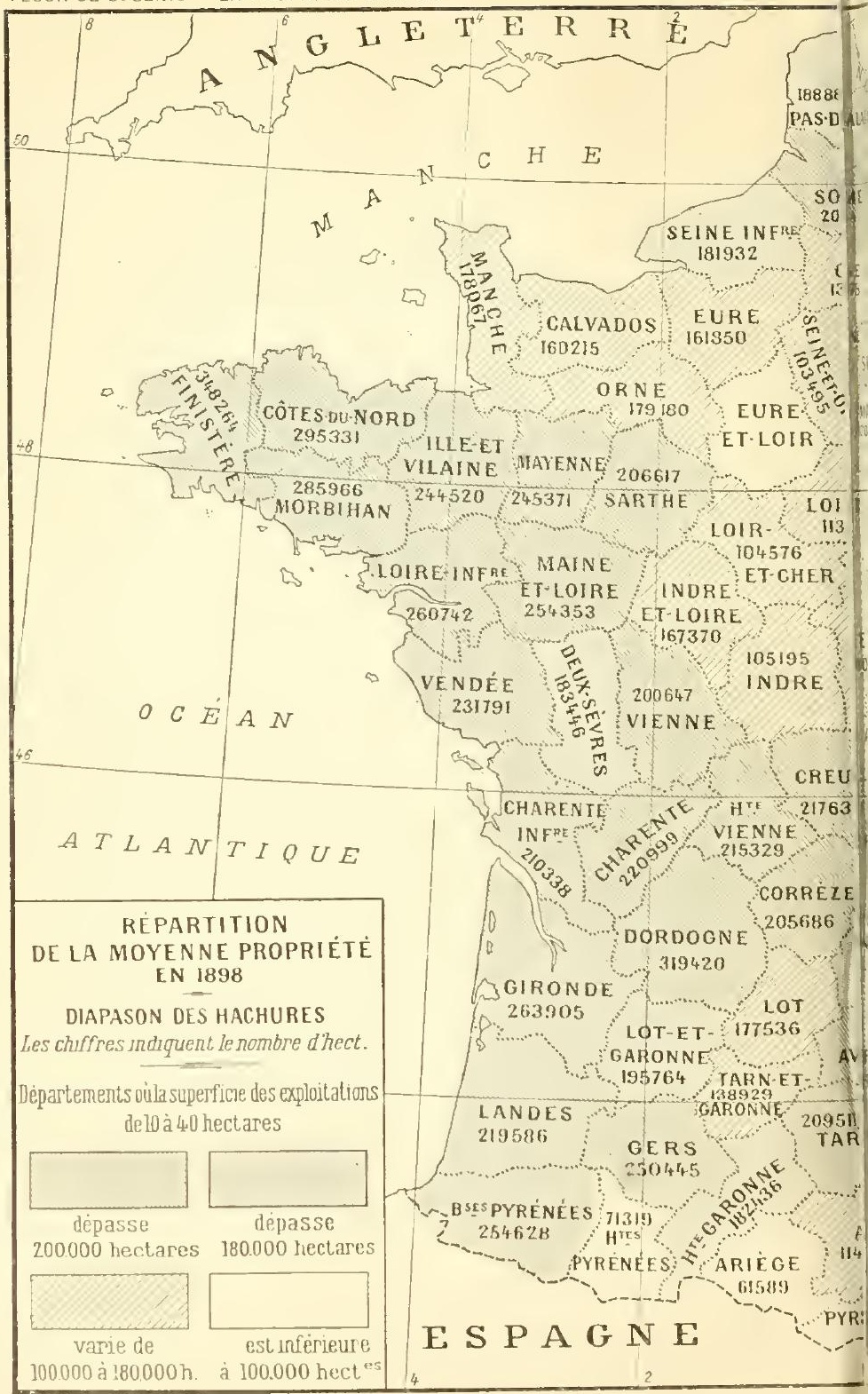


AIs L GIQUE 180516

EINE:

MARNE MEUSE

151232

AISNE

-ET217909

ARNE:

Di56... LUिसEMBOURG AUBE HTE VOSGES
I77631

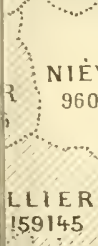

NIE
$\quad 960$

LLIER
I59145

180388

6079

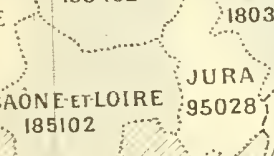

SAONEETLOIRE 95028
185102

$=0 \quad 0$

A I N

H'NÉSAVOIE'

S U I S S E

JY-DEDÔME

\section{8}

9 HELOIRE

TAL 207621

ARDECHE
LOZERE 162463

124253

O

39

(1)

\section{$191208 \quad \cdots \quad 56164$}

80519 

EXPLOITATIONS HURALES EN 1892.

Catégories

des exploitations.
Nombre des exploitations.

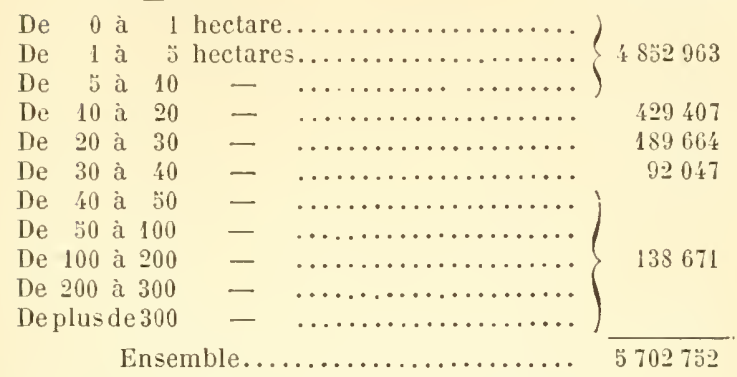

La propriété dite moyenne, de 10 à 40 hectares, comprend done 711118 unités, tandis que les exploitations au-dessous de 10 hectares la dépassent, en nombre, de plus de 4 millions $1 / 10$, et que la grande propriété, celle au-dessus de 40 hectares, d'après la terminologie administrative, lui est inférieure de plus de 572000 articles. Il y a donc là un groupe important, dont la formation et le ròle sont également intéressants à étudier.

EXPloitations DE 10 A 40 inectares. Catėgories.

Nombre.

De 10 à 20 hectares................... 429407

De 20 à $30 \quad$ - ..................... $18966^{\prime}$

De 30 à $40-\ldots \ldots \ldots \ldots \ldots \ldots \ldots \ldots . .62017$

Ensemble........ 711118

Par comparaison avec 1862 et 1882 , on trouve les écarts dont voici le relevé pour le nombre de chaque subdivision d'exploitations.

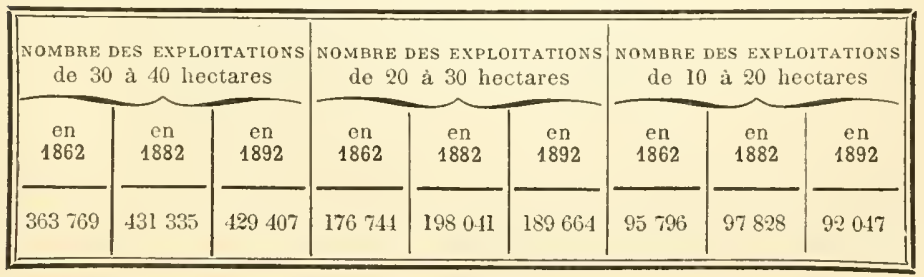


La période décennale de 1862 à 1882 se caractérise par une augmentation de $9089 \%$ exploitations el celle de 1882 à 1892 par une perte de 16086 , d'où résulte cependant un gain de 74809 unités depuis trente ans. Les trois catégories sont en diminution depuis dix ans, et le déficit se répartit comme suit entre elles :

\section{Perte en nombre.}

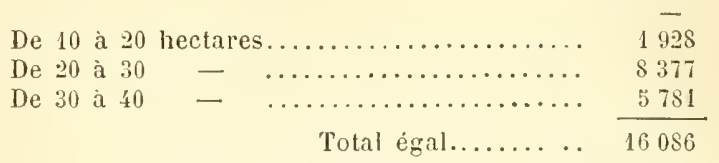

De même que c'est la propriété moyenne qui, pendant les crises, est le plus habituellement affectée, ainsi parmi les trois classes entre lesquelles on la subdivise c'est l'intermédiaire qui offre le moins de résistance et paraît normalement le plus influencée par l'incidence des événements extérieurs. Les causes de cette particularité sont évidemment très complexes et très obscures; peut-ètre pourrait-on en déterminer quelques unes, en les localisant, en étudiant par département, et dans chaque département par nature de culture, comment et pourquoi les écarts se sont produits ou s'ils ne résultent que de eoïncidences fortuites.

Les 711118 exploilalions de 10 à 40 hectares absorbent 14313417 hectares du territoire agricole ${ }^{1}$, et sont réparties comme suil par nature de cultures:

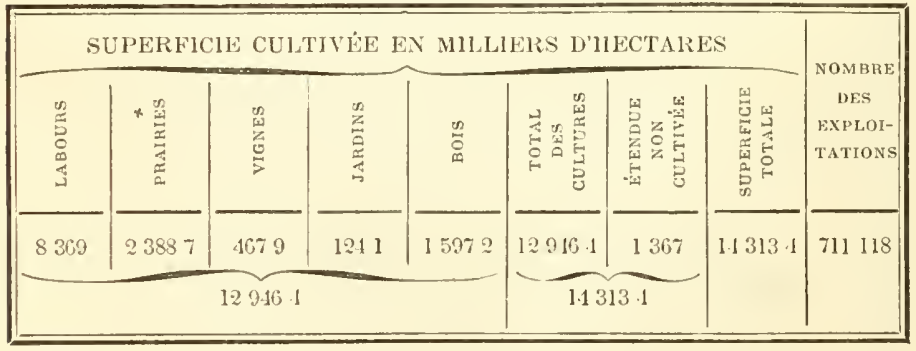

1. Calculé déduction faite des bois de l'litat; la superficic non cultivée (Landes, etc.), d'unc contenance le 6226189 hectares en 1892, y est comprise dans la plupart des relevés. 
Le nombre des exploitations varie beaucoup par département el, selon les départements, leur étendue n'est pas proportionnelle à leur nombre; en voici quelques exemples:

\begin{tabular}{|c|c|c|}
\hline DÉPARTEMENTS. & $\begin{array}{c}\text { Nombro } \\
\text { des } \\
\text { exploitations } \\
\text { lo } 10 \text { à } 40 \text { hectares. }\end{array}$ & $\begin{array}{l}\text { Superficie } \\
\text { de ces domaines } \\
\text { en milliers } \\
\text { d'hectares. }\end{array}$ \\
\hline Averron.$\ldots \ldots \ldots \ldots \ldots \ldots \ldots$ & ... 11886 & 257 \\
\hline Gers. $\ldots \ldots \ldots \ldots \ldots \ldots \ldots \ldots \ldots$ & 13492 & 231 \\
\hline Gironde $\ldots \ldots \ldots \ldots \ldots \ldots \ldots$ & 14209 & 264 \\
\hline Loire-infërieure. . . . . . . . . . . & 12503 & 261 \\
\hline Maine-et-Loire $\ldots \ldots \ldots \ldots \ldots \ldots$ & 12291 & 253 \\
\hline Basses-Pyrénées.............. & 13056 & $25 \%$ \\
\hline Vaucluse.................... & 3777 & 71 \\
\hline Hautes-Pyrénėes................ & $3 \$ 31$ & 2 \\
\hline Loiret..................... & 6170 & 114 \\
\hline Eure-et-Loir................. & 7330 & $1 \ddot{2} 2$ \\
\hline
\end{tabular}

Il est évident qu'en prenant les moyennes on se ferait une très fausse idée de la condition des Hautes-Pyrénées eomparées aux Basses, ou du Vaueluse à eôté de la Gironde.

Les départements où l'on trouve le plus de domaines de 10 à 40 hectares sont : la Dordogne avec 18644 exploitations, le Finistère avec 16988 , les Côles-du-Nord avec 14998, le Iorbihan avec 14113. Ceux où il y en a le moins sont: Vaucluse, Savoie, Hautes-Pyrénées, Ariège, AlpesMaritimes, qui n'arrivent pas à 4000 ; les Bouches-du-Rhòne et la Haute-Savoie (à ehacun 4130) et Meurthe-et-Moselle $(4800)^{1}$.

Si l'on pénètre plus avant eneore dans le détail, on s'aperçoit que e'est la catégorie de 10 à 20 hectares rqui est la plus nombreuse.

Nombre Nombre d'exploitations total. de 10 à 20 hectares.

\begin{tabular}{|c|c|c|}
\hline & & \\
\hline Dordogne................. & 18644 & 11098 \\
\hline Finistere... & 16988 & 10149 \\
\hline es-du-Nord........ & $1+998$ & 9854 \\
\hline orbihan .......... & $1 \div 113$ & 9716 \\
\hline
\end{tabular}

1. Le département de la Seine, bien entendu, n'entre pas dans mes caleuls; on ne lui attribue que 219 exploitations de cette classe, formant environ 5000 hectares dont plus des $4 / 5$ en labours. 
Dans les trois catégories qui forment la classe de la moycnne propriété, c'est celle dont l'unité est la plus restreinte qui reste la plus nombreuse, sans doule parce que sa mesure se rapproche le plus de la petile propriété et que c'est colle-ci qui attire et retient la clientèle la plus àpre au travail el à l’épargne. Ce fait, qui se généralise dans tous les départements, même dans celui de la Seine, est toutefois atténué dans $2 \%$ d'entre eux par cette particularité que les trois catégories ont entre clles des écarts moins accentués et que, notamment, la réunion des deux dermières (de 20 à 30 el de 30 à 40 hectares) équivaut presque au total de la première (de 10 à 20 ), contrairement à ce qui se passe en Saroie, par exemple, où la première catégorie est de 2217 el le total des deux autres de 676 seulement, et dans le Jura, où ces chiffres sont respectivement de 4331 et de 1117 ; dans l'Allier, elle les dépasse.

\begin{tabular}{|c|c|c|c|c|}
\hline \multirow{3}{*}{ DEPARTENLNTS } & \multicolumn{4}{|c|}{ NOMBRE DES EXPLOITATIONS MOYENNES } \\
\hline & \multirow[b]{2}{*}{$\begin{array}{l}\text { Do } 10 \text { ì } 20 \\
\text { hectares }\end{array}$} & \multicolumn{3}{|c|}{ DES DECX AUTRES CATÉGORIES } \\
\hline & & $1)$ e 90 à 30 & De 30 à 40 & $\begin{array}{c}\text { Total } \\
\text { deces deux } \\
\text { catégories }\end{array}$ \\
\hline Allier............. & 3318 & 2187 & 1443 & 3630 \\
\hline liasses-AIpes........... & 3 ifl & 1927 & 1143 & 3070 \\
\hline Arrleche............. & \& $\$ 83$ & $24: 7$ & 1310 & 3757 \\
\hline Calvarlos ............. & $4 \ddot{3 i i}$ i & 2163 & 1273 & $3+36$ \\
\hline Cantal................ & 3070 & 2917 & 1764 & 6881 \\
\hline Cher................ & 2707 & 1401 & 852 & 2253 \\
\hline Corrèze............... & $37 \cdot 34$ & 3232 & 1050 & 1282 \\
\hline 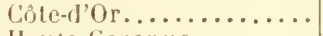 & $\because 127$ & 2554 & 1349 & 3903 \\
\hline Jaute-Garonue. ........ & 3878 & 2950 & 1137 & 4087 \\
\hline Hérault............... & 2879 & 1587 & 783 & 2370 \\
\hline 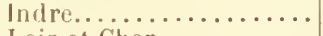 & 2933 & 1493 & 874 & 2) 369 \\
\hline Loir-et-Cher . ........... & 2846 & 1282 & 958 & 2240 \\
\hline 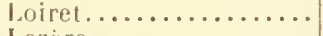 & 4049 & 1467 & 630 i & 2121 \\
\hline 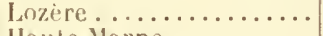 & 3578 & 2122 & 1219 & 3341 \\
\hline Jante-Mlarne............ & \& 548 & 2495 & 1220 & 3715 \\
\hline Meurthe-et-Xloselle...... & 2707 & 1376 & 717 & 2093 \\
\hline 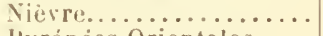 & 2782 & 1036 & 600 & 1636 \\
\hline J'yrénées-Orientales..... & 1713 & 644 & 432 & 1076 \\
\hline Deux-sevres............ & 36,23 & 1972 & 1577 & 3549 \\
\hline 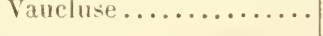 & $204: 3$ & 1278 & 454 & 1732 \\
\hline
\end{tabular}


Ces variations ne sont pas particulières à une région, ni lans un sens ni dans lautre; elles affectent parfois des lépartements fort éloignés l'un de l'autre el dans des proortions semblables bien qu'il soit téméraire de leur assigner es mèmes causes; il est donc difficile de dresser une carte idèle de la répartition de la moyenne propriélé sur létendue lu territoire. On n'y pourrait arriver que par des cartes uperposées traduisant les rapports réciproques de la ;rande, de la moyenne et de la pelite culture.

Si l'on compare les résultats d'ensemble pour la période le 1882 à 1892, la diminution se répartit ainsi :

NOMBHE DES EXPLOITATIONS

\begin{tabular}{|c|c|}
\hline 1882 & 1892 \\
\hline- & - \\
\hline 431 & 429 \\
\hline 198 & 190 \\
\hline 98 & 92 \\
\hline$\pi 2 \pi$ & 711 \\
\hline
\end{tabular}

La diminution se localise dans le Centre de la France, de 'Est à l'Ouest, et dans le Sud (au Centre et à l'Est), aussi jien en ce qui concerne le nombre que l'étendue totale des xploitations de 10 à 40 hectares. La perte pour l'ensemble le 49 départements atteint 424000 hectares, soit 79 p. 100 le la diminution superficielle totale. La région du Nord à l'exception du Nord et du Pas-de-Calais) a vu la moyenne sulture s'y augmenter légèrement en nombre, un peu plus sn superficie.

Dans les trois régions du Nord-Ouest, du Nord-Est et du Šud-Ouest, le mouvement de la moyenne propriété s'effectue sn sens inverse suivant qu'il s'agit du nombre ou de 'étendue. En Bretagne, surtout dans la Manche el dans 'Orne, le nombre s'est accru d'un millier, avec perte de ;0 000 hectares. Dans le Nord-Est, on compte 600 unités de noins arec 6000 hectares de plus; dans le Sud-Ouest, ;000 exploitations de plus avee 23000 heetares de moins.

Ce sont la très grande et la très pelite propriété qui se sont 
emparées des terres devenues disponibles par le résultal final de ces complexités de groupement el de morcellement.

\section{V. - Origines historiques de la moyenne propriété.}

La moyenne propriété est d'origine récente; clle ne procède ni de la féodalité comme la grande propriété, quion pourrait à ses débuls comparer arec quelques explications à nos pays de protectorat, ni du trarail rural comme la pelite propriété.

Elle n'est point issue de la nature mème des choses, c'est le produit de la spéculation et, aujourd'hui, elle s'est si fortement implantée dans l'ensemble économique qu'elle en fait partie intégrante el paraît désormais indispensable à son éruilibre.

Elle date de la constitution de la propriété rurale, non plus directement aux mains des cultivateurs exploitant par cux el leurs familles, mais entre celles de paysans enrichis devenus marchands et de bourgreois des villes empressés à derenir propriélaires fonciers el confiant la cullure à des censiers, des métayers ou des fermiers. On trouve dès le $\mathrm{xv}^{\mathrm{e}}$ sicele les traces de celle concentration de parcelles par les forains ${ }^{1}$; les paysans et les seigneur's étaient ruinés, tous les citadins qui avaient quelque argent profiterent de l'occasion qui s'ollrail d'acheler à vil prix des terres laissées en friches et des châteaux ahandonnés. Le mouvement se généralisa et s’accentua ì mesure que, la bourgeoisie se déreloppant par l'industrie ef le négoce, les lıommes du tiers élat acquirent des charges de magistrature et des offices publics et firent de l'acquisition de la propriété foncière le complément et la preuve de leur avènement social.

Ciest dans ce déclassement de la propriété, dans celle

1. M. de Saint-Genis, Mémoire sur la propriété rurale en Bourgorne (Bulletin du Comité des lravaux historiques, 1896). 
évolution qui, renouvelant sous une autre forme el dans d'autres conditions l'érolution de la sauregarde au $\mathrm{x}^{\mathrm{e}}$ siècle, refit du paysan-propriétaire un simple prolétaire rural. qu il faut voir la cause économique sous l'influence de laquelle le bail à ferme se substitua au bail à cens.

C'est dans les coutumes, dans les lirres des domanistes et des commentateurs qu'il faudrai avoir la patience de chercher les véritables et solides éléments de l'histoire de la propriété depuis le $\mathrm{xr}^{\mathrm{e}}$ siècle. Les formes rariées de location, d'appropriation de la culture à la terre el du salaire qui la rémunère marquent les étapes de la division du sol et fixent l'époque où se constitua cette moyenne propriété qu'on pourrait appeler la propriété foraine.

Le bail à cens constituait l'abaudon irrévocable de la lerre à la condition quoon la cultivat, puisque la rente n'était garantie que par le produit de la culture; vint ensuite l'emphytéose, c'est-à-clire le bail à cens limité à cent ans; et le colonage antique vit sa durée se rétrécir de plus en plus, à mesure que la terre perdait un peu de sa fixité immuable pour devenir un objet d'échange dont la valeur vénałe subissait des dépréciations ou des plus-values appréciables. Il faut étudier clans Estienne Pasquier, Dumoulin, Loyseau, d'Argentré, Fonmaur, Pothier, les formes intermédiaires de tenures qui s'intercalent entre les types extrèmes, le cens el le bail à court terme : le domaine congéable en Bretagne, le droit de marché en Picardie, le WValdrecht en Alsace, le métayage dans les provinces du Centre et du Midi, les dérivés de l'emphytéose, le bail à vie et mème le bail à trois vies consenti à trois laboureurs qui héritent les uns des autres ${ }^{1}$.

Le propriétaire forain, obligé de déléguuer sa surveillance, imagina mille moyens de contròle et de gestion à distance;

1. La Lorraine avoisinait le Lnxembourg avec ses Schilling-Güter, les provinces rhẻnanes leurs Schafft et leurs Vogtegüter, biens affermés à titre quasi perpétuel, tandis que le Waldrecht d'Alsace permet le trans. fert du bail aux héritiers directs du preneur mais n'en autorise ni la cession ni la vente. 
il y réussit d'autant mieux que ce nouvel ordre de propriétaires-fonciers, mâtiné de négoce et de judicature, excellait dans la chicane. Le contral d'emphytéose fut du $x^{\mathrm{e}}$ a $\mathrm{xrI}^{\mathrm{e}}$ siècle l'une des sourees de la constitution des souches de la moyenne propriété, car ce mode légal du bail à long terme s'appliquait généralement à des domaines complets, formant un corps d'exploitation, plutôt qu'à des parcelles isolées. En effet, à l'expiration des quatre-vingt-dix-neuf ans, le bail prenait fin el un nouveau contral devenait nécessaire. Il se présentail alors deux éventualités. Ou le maitre du fonds y rentrait en indemnisant le détenteur de ses débours, ou celui-ci conservait le domaine à de nouvelles conditions ou par un autre mode de location. Mais il arrivait le plus souvent qu'au cours du siècle le tenancier s'était enrichi et le propriétaire appauvri ; ce dernier, sans attendre l'échéance, vendail son droit de rachat ì l'usager qui devenait plein propriélaire.

La période du $x v^{-e}$ siècle au $x x^{-e}$ fut également favorable à la petite et à la moyenne propriélé. Le hourgeois prit les gros morceaux, le paysan ne négrligea pas les mieltes.

Tandis que les nouveanx riches, de noblesse récente ${ }^{1}$ on d'extraction roturière, rachètent parcelle ì pareelle tout ce qu'avaient aliéné les détenteurs du sol depuis saint Louis, une masse danciens chàteaux, non habités pendant des demi-siècles, tombaient cn ruines ${ }^{2}$.

Les laboureurs riverains mordent à belles dents sur leurs dépendances; là oì le maître ne réside pas, el c'est le cas de beaucoup de belles demeures vidées par l'absentéisme, le séjour aux armées ou à la cour, les lambris seigneuriaux n'abritent plus que les mélayers, leur bétail el leurs fourrages. Faute de réparation, les fermes el les mélairies se sont effondrées, el les familles paysannes se sont logées au plus

1. L'èdit de 1696 autorisa qui le voulut à choisir et à porter des armoiries, sauf a les faire enregistrer en payant une taxe de 20 livres.

2. Une seigneurie de 800 hectares, en bauphiné, ne rapportait plus en 1720 que $2 \mathrm{fr}$. 40 à l'hectare de droits feodaux (cité par M. d'Avenel, Ilist. économ., 1, 781). 
proche. On signale sans cesse, au xvil $^{\mathrm{e}}$ siècle, comme on le faisait déjà au $\mathrm{x}^{\mathrm{e}}$, dans les terriers, les dénombrements, les enquètes, ce qu'on appelle une masure de chateau, une cour aree des tours où les paysans ront se fournir de pierre.

Nombre de villages, en Bourgogne, en Dauphiné, en Languedoc, ont été bâtis de neuf, de 1710 à 1850 , avec des lailles et des moellons arrachés aux portes, aux cheminées, aux murailles des vieilles habitations seigneuriales du $\mathrm{xvI}^{\ominus}$ et du $\mathrm{x}^{\mathrm{e}} \mathrm{I}^{\mathrm{e}}$ siècle.

La Piérolution vint à son tour fournir, dans un très court espace de temps, un contingent considérable au groupe intermédiaire des propriétaires fonciers inauguré de 1600 à 1789 par la spéculation bourgeoise. C'est ce groupe d'opportunistes qui profita, ainsi qu'il résulte de l'ensemble des documents, de la vente non morcelée des biens du clergé en ne prenant qu'une part limitée aux criées en petils lols des biens des énigrés.

Depuis cette seconde poussée de la petite bourgeoisie vers l'acquisition brusque de la terre, le groupe de la moyenne propriété est resté stationnaire.

S’il s’accroìt d'un còté, il s'échancre de l'autre, pareil à ces rivages de l'Ouest de la France où les dunes gagnent insensiblement sur l'Océan tandis que les còtes de Normandie et les falaises du pays de Caux s'effritent sous le choc des flots.

Aujourd'hui qưil n'y a plus de châteaux à détruire, la bande noire, avee des allures doucereuses, s'attaque ì la moyenne propriété et la bat en brèche partout où elle peut pénétrer par les fissures de la dette hypothécaire. Les banquiers de petites villes, les escompteurs de villages, les huissiers, les notaires et les avoués sont venus, armés de leurs procédures et de leurs tarifs. La spéculation ne s'arrête que lorsque les bourses sont épuisées et qu'il n'y a plus profit pour les intermédiaires à émietter par la saisie la moyenne propriété pour en faire acheter les débris par la petite ${ }^{2}$.

1. Mémoire sur la propriété rurale en Bourgogne (loco citato).

2. Du droit de saisie en France (M. de Saint-Genis). 
Toutefois, la moyenne propriété ne se laisse entamer que sur les bords, le noyau résiste, le bloc se maintient et, sil se désagrège de quelque eôté, ne tarde pas à se recomposer d'un aulre. C'est un phénomène qui persiste, de 1829 à 1899 . A quelques centaines d'unités près, la catégorie de 20 à 40 hectares (et le fait s'aceuserait avec plus de préeision si l'on allait jusqu’à ö0) est encore ce qu'elle était à la date des premières constatations eadastrales ${ }^{1}$.

La très petite propriété a pullulé, la grande s'est raréfiée, et la moyenne est restée stationnaire. Il y a là, entre 20 et ¿0 heetares, un bloc neutre, un bloe qui ne perd ni ne gagne, ou du moins, pour être tout à fait exact, qui ressaisit d'un eôté ce qu'il peut perdre de l'autre. C'est ee que M. Gimel, dans ses comparaisons statistiques, nomme le point mort de la bielle, ce que M. de Foville appelle le point fixe de la courbe. Il semble que la petite propriélé s'élargisse aux dépens de la grande en passant par-dessus la moyenne quielle ébrèche à peine dans ses oscillations ${ }^{2}$. Les chiffres confirment les prérisions que M. de Forille énoncait en $188{ }^{\circ}$.

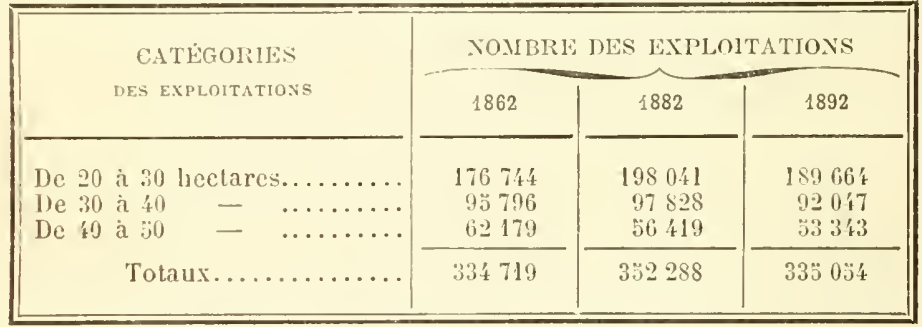

1. 11. de Foville, Le morcellement, 113.

2. Mettez votre montre à trois heures cinquante, dit spirituellement I. de Foville (Le morcellement), et regardez-la mareher pendant cinq minutes. A gauche, l'aiguille des minutes monte rapidenent, c'est la petite proprietè qui progresse; à droite, l'aiguille des henres s'alıaisse lentement. c'est la grande propriété qui fléchit; l'axe immoljile qui sert de pivot a ces deux rotations contraires et inégales, cest la moyenne propriété dont l'effectif ne change pas.

- Il n'est pas d'image plus saisissante et plus exacte. 
A trente ans de distance, après un mouvement accentué en arant, le groupe de 1892 est retombé, avec un écart insignifiant de 333 unités, au chiffre de 1862. Les lacunes des statistiques ne permettent malheureusement pas de faire les mèmes rapprochements pour l'étendue des exploitations à ces dates successives.

On pourrait expliquer, peut-être, la fixité relative de ce groupe en supposant que c'est sur des fortunes de cette catégorie que la loi des partages exerce le plus directement son action dissolvante, défaisant ainsi par le jeu forcé de la division de l'héritage l'accroissement qu'apporteraient au bloc les acquisitions annuelles de l'épargne. En 1833̈, il y avait 39871.4 cotes foncières de 100 à 00 francs; on en trouve 440104 en 1842 et 448473 en 18.58 .

\section{VI. - Rôle économique de la moyenne propriété par sa fixité relative.}

L'agriculture semble ètre dans une période de clifficultés dont on n'apercoit pas encore le terme, mais il n'y a point de raison pour désespérer de son avenir. L'agriculture se transforme, et toutes les périodes de transition sont pénibles; elle prend le caractère d'une industrie dans l'acception modeme du mot, et son développement, qui exige le concours des capitaux et l'emploi des machines, oblige le propriétaire foncier à sortir de son isolement traditionnel et à se mèler activement au mouvement économique. Son objectif, en faisant appel au crédit, doit être d'obtenir une rapide diminution du coùt de production même en face d'une demande considérablement accrue.

La grande propriété, avec ses 138000 possesseurs sur une population de 36 millions d'individus, peut-elle suffire aux exigences croissantes de la consommation, et, même en mettant en valeur les 4 millions d'hectares non cultivés qu'elle détient, multiplier ses ateliers agrricoles de façon à créer pour l'agriculture ce progrès incessant qu'a réalisé la 
grande industric? c'est peu probable. Des tentatives dans ce sens ont été faites par des propriétaires intelligents, riches et désintéressés; ils ont souvent réussi, mais leur œurre a disparu avec eux. On a essayé de créer des sociétés agricoles à gros capitaux pour traiter la culture comme l'industrie el il n'apparaît pas que ces associations de spéculateurs plutôt que dagronomes aient tenu toutes leurs promesses. C'est que la nature ne s'accommode pas de notre réglementation et que ce que nous appelons ses caprices déroute nos prévisions; on ne procède pas avec le climat, le terrain, la variété des semences et des produits comme on le fait dans une usine métallurgique arec le fer et le charbon; la nature vit, et ses éléments ne sont pas aussi malléables qu'on sc l'imagine: ils n'obéissent pas à l'homme et c'est l'homme qui doit observer ce qui leur plaît et se plier à leurs besoins au lieu de prétendre leur imposer ses théories. Autant, dans l'industrie, le maniement des capitaux, leur emploi, la conduite de la fabrication, réclament la rigidité de la règle et l'unité de direction, autant lagriculture reut, au contraire, de souplesse ei d'opportunisme.

La minutie des soins, la particulière attention quil faut apporter an choix des méthodes pour chaque espèce de culture, la nécessité de confier à des spécialistes expérimentés le labour, l'élevage, le repeuplement, l'irrigation, le vignoble, le jardin, obligent ì créer dans une grante exploitation aulant d'exploitations indépendantes, autant de services appropriés, quil y a de cultures principales on d'industries agricoles. Force est d'avoir recours ì des sala iés; si instruils et si dévoués qüïls soient, ils ne peuvent suppléer à l'oeil du maître, à son initiative; tout est vile compromis quand, au lieu de décisions promptes, il faut en référer à un directeur ou à un comité. Là est le grand écueil des exploitations agricoles menées industriellement, qu'elles soient dirigées par le propriétaire lui-mème, qui a des régisseurs et des chefs de culture, ou conduites au nom d'une sociélé financière par l'intermédiaire d'un personnel hiérar- 
chisé. L'association implique un cercle d'action trop étendu pour yue l'énergie d'un seul homme y suffise, et, à part l'élevage, le labourage ou la viticulture en grand et spécialisés, la variété des produits et la complexité des soins obligent à diviser la surveillance et la responsabilité. Dans de pareilles conditions, il semble qu'il y ait profit à morceler l'exploitation, à diviser le domaine trop vaste, à créer des fermes indépendantes dont l'étendue ne dépasse pas les forces d'un seul homme et les ressources combinées qu'y peut apporter le zèle intéressé d'une famille. L'enscmble des fermes profiterait de leur réunion dans les mains d'un mème propriétaire qui, individu ou sotiété, les aiderait de son crédit, de ses capitaux, de ses expériences, et leur fournirait à tour de ròle ce puissant auxiliaire de la mécanique agricole qui coùte si cher d'achat et d'entretien.

Pour d'autres raisons, la petite propriété est impuissante à réaliser certaines améliorations et l'association mème, car le ròle des syndicats est une arme à deux tranchants, ne peut lui apporter quelques-unes des ressources qui lui manquent qu'en compromettant son indépendance et sa vivacité dinitiative.

Pourquoi le groupe de la moyenne propriété, tel qu'il est, et en raison mème des défauts et des qualités qui peuvent utilement s'amalgamer et se corriger l'un l'autre, ne remplirait-il pas ce ròle important auquel la petite propriété ne peut prétendre efficacement qu'en transformant les conditions qui la caractérisent?

La plus grande partie de la diminution relative de la richesse agricole, disait récemment un économiste américain ${ }^{1}$, est une conséquence incontestable du progrès de lindustrie et du bien-ètre social.

C'est qu'en effet les succès industriels et les gros salaires attirent les ouvriers agricoles vers les villes, leur émigration limite la concurrence et fait hausser la main-d'œure,

1. MI. Bemis, de I'Ĺniversité de Chicago, en 1893, cité par II. Levasseur (L'Agriculture aux Etats-Unis, 421). 
d'où une augmentation du prix de revient et une diminution de produit net. Bras et capitaux se retirant à la fois, il y a crise. Force est done, pour remplacer les bras, d'avoir reeours aux machines et, pour acheler les machines, de faire appel au crédil, nouveau problème.

La moyenne propriété, limilée dans son développement par la complexité de ces diverses causes, est comme enfermée dans le cercle étroit qu'elle ne peut briser sans se condamner elle-mème. Cependant, elle possède un vaste champ d'exploitation et de travail, environ 19 millions d'hectares dont les deux dixièmes sont encore en friche, et plus de 700000 exploitations appartenant sclon les probabilités à plus de 600000 propriélaires ${ }^{1}$, instruits, alertes, el plus ou moins pourvus de ressources personnelles dont ils pourraient décupler l'effet en se syndiquant.

En agriculture comme en toules choses, désormais l'intelligence doit prendre la place de l'énergie purement mécanique, et le sol doit ètre traité comme un instrument de production qui rapporlera proportionnellement à la mesure de l'habileté avec laquelle il sera traité. On peul diversifier les cultures, el l'esprit de mélhode excilé et vivifié par l'esprit d'entreprise doit modifier sans mises de fonds excessives la culture superficielle el de rendement faible. Les moyens propriétaires que leur origine, leurs goùts, et, la plupart du temps, le milieu où ils continuent à vivre, enlèvent à l'isolement rural et à la routine traditionnelle qui en est la conséquence, ne sont-ils pas mieux préparés que tous autres à él er leurs enfants de façon à leur enseigner que l'agriculture peut être une carrière plus saine, plus indépendante, plus honorable que beaucoup d'antres, et qui doit devenir aussi luerative que celles qui dérivent de l'industrie proprement dite ou se greffent sur elle?

L'Angleterre n'est-elle pas, it juste titre, aussi frìre de sa double catégorie de country gentlemen el de gentlemen far-

1. Ce sont les cliffres de $\lambda$. de Foville, qui appelle judicieusement moyenne propriété les domaines de 6 a 50 hectares, tandis que la statistique officielle les classe de 10 a 40. 
mer's qui, ensemble, réunissent les qualités sociales dont nous roudrions voir, chez nous, les possesseurs de la moyenne propriété sc préraloir, que des rastes domaines de ses Landlords?

L'un des arguments de ceux qui traitent la moyenne propriété de négligeable, c'est que la grande propriété, étant essenticllement farorable à l'extension de la grande culture, excrce une action prépondérante sur le progris agricole.

La conclusion est contestable et l'exemple de I'Angleterre la contredit quelque peu.

II n'y arait guère jusquici, dans le Royaume-Uni, aux yeux des économistes, que deux sortes de propriétés: les grandes et les moyennes. Les grandes ne s'étendent que sur un tiers du sol et, une portion de ce ticrs étant divisée en petites fermes, il s’ensuit que l'action de la grande propriété ne se fait scntir que sur un quart tout au plus. Et il est douteux que ce quart soit le mieux cultivé ${ }^{1}$. Le comté de Lancaster, le plus riche au point de vue agricole, est composé de moyennes propriétés et de fermes où l'on rivalise de soins et de méthodc.

La Grande-Bretagne comptait, en 1886, 229437 propriétés de 30 à 300 acres, c'est-à-dire de 20 à 120 hectares, parmi lesquclles celles de 101 à 300 acres (de 40 à 120 hectares), au nombre de 79573 , étaient les plus vastes, embrassant une superficie de près de 14 millions d'acres sur un territoire d'environ 33. L’importance de cette catégorie intermédiaire, dont les économistes ont jusqu'ici fort négligé le ròle, se révèle à mesure qu'on en étudie de plus près les détails et les affinités.

1. Le plus grand propriẻtaire foncier de la Grande-Bretagne, le duc de Sutherland, possède d'un seul bloc plus de 300000 hectares dans le nord de l'Écosse, mais ces terres valent 50 francs l'hectare. Les domaines immenses du duc de Northumberland dans le comté de ce nom, ceux du duc de Devonshire dans le Derby, situés dans des régions accidentées et peu productives, ne servent guère à la diffusion des méthodes nouvelles et des exemples profitables, tandis que dans le Leicester, le Warwick, le Lincoln, l'exemple immédiat des proprietaires de moyenne fortune (domaines de 80 hectares) se propage et se vulgarise par le voisinage et les relations. 
La moyenne propriété tient une place dans le système économique de la plupart des pays d'Europe, et, à tout considérer, les proportions entre les trois grandes divisions de la statistique sont à peu près les mèmes partout, pour peu qu'on ait le soin de ne point appliquer à tous les pays, et même dans chaque pays à toutes ses régions indifféremment, l'étalon admis pour la France; la mesure, en effet, ne peut en être la même en Belgique ou en Hongrie, pas plus qu'elle ne peut être pareille en Sicile ou en Lombardie.

En Hongrie, la moyenne propriété (de 17 à 110 ŏ hectares) compte 118981 domaines contre 1867 pour la grande et 2 millions et demi pour la petite; en Autriche, sur 4116216 propriétaires se partageant 52 millions de parcelles, il n’y a que 1133 grands terriens contre 122139 propriétaires payant plus de 500 florins d'impôt foncier rural.

En Hollande et en Belgique, pays dont la culture intensive est connue el où la pelite propriété révèle des conditions sociales excellentes, la grande propriété est très rare et la moyenne y forme un groupe compact el prépondérant ${ }^{1}$.

En Allemagne, à part dans cerłaines régions industrielles el commerçantes, il y a une tendance marquée à la diminution de la moyenne propriélé, au profit de la très pelite el de la grande.

Exploitations en nombre.

1866

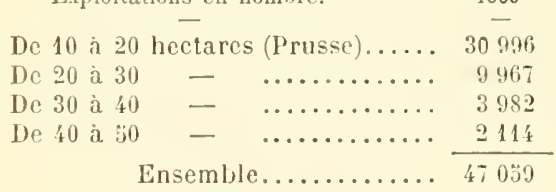

1880

$$
\begin{array}{r}
25983 \\
774 ! \\
3023 \\
1414 \\
\hline 38169
\end{array}
$$

La statistique de l'Émpire ne peut ètre éludiée qu'en détail à cause des différences profondes qui existent entre

1. Les petites exploitations, qui avaient augmenté en nombre de 1866 à 1880, sont en décroissance: il y a, all contraire, accroissement continu, depuis 1890 , dans les domaines de 10 a 50 hectares; les grands propriétaires du Brabant se concentrent eux-mêmes tle plus en plus. (Joumal de la Sociélé de statistique de Paris, juillet 1900, p. 239 : Statistique agricole de la Belgique.) 
les provinces rhénanes, par exemple, dont la tenure foncière se rapproche beaucoup de la France du Nord-Est, du Luxembourg et de la Belgique, et les districts maritimes de la Ballique el de la mer du Nord qui sont répartis en vastes domaines. Un rapprochement suffira pour marquer la difficulté quon éprouve ì conclure avec précision, en pareille malière, du particulier au général.

\begin{tabular}{|c|c|c|c|}
\hline DISTRICTS & $\begin{array}{c}\text { Superficic } \\
\text { des terres eultivées } \\
\text { en heetares. }\end{array}$ & $\begin{array}{l}\text { Nombre } \\
\text { des propriétés } \\
\text { rurales. }\end{array}$ & $\begin{array}{l}\text { Moyenne } \\
\text { de chaquo } \\
\text { proprieté. }\end{array}$ \\
\hline & $\ldots \quad 197380$ & 4232 & $4 h .5$ \\
\hline antzig.. & 382268 & 21150 & 275 \\
\hline
\end{tabular}

Le distriet d'Aix-la-Chapelle comple plus de 22000 exploitations rurales inférieures à g hectares, tandis qu'on n’en trouve pas 6000 dans les environs de Dantzig '.

Aux Élats-Uniseux-mèmes, où la liberté de l'espace semble encourager les très grandes propriétés et les très grandes cultures, les fermes ou exploitalions rurales n'occupent que le tiers du territoire, 33 p. 100, et la superficie cultivée n'en est à peine que les 22 p. 100 ; la tendance des exploitations à réduire leur élendue et à concentrer leurs ressources et leurs efforts sur un terrain plus resserré est manifeste. La grandeur moyenne des fermes ne cesse de décroître :

\begin{tabular}{|c|c|c|c|}
\hline $\begin{array}{c}\text { ANNÉES } \\
-\end{array}$ & $\begin{array}{l}\text { Nombre } \\
\text { des fermes } \\
\text { par milliers. }\end{array}$ & $\begin{array}{l}\text { Superficie } \\
\text { en millions } \\
\text { d'hectares. }\end{array}$ & $\begin{array}{c}\text { Superficie moyenne } \\
\text { des fermes } \\
\text { en hectares. }\end{array}$ \\
\hline $18 \check{0} 0 \ldots \ldots \ldots \ldots \ldots$ & . 1449 & $\overline{118}$ & 82 \\
\hline $1860 \ldots \ldots \ldots \ldots \ldots$ & 2044 & 165 & 79 \\
\hline $1870 \ldots \ldots \ldots \ldots \ldots$ & 2659 & 168 & 61 \\
\hline $1880 \ldots \ldots \ldots \ldots \ldots$ & 4009 & 217 & 53 \\
\hline $1890 \ldots \ldots \ldots \ldots \ldots$ & 456 千 & 252 & ธ̋วั \\
\hline
\end{tabular}

Le tolal des terres cultivées a passé de 450 millions d'hectares à 144, et, détail caractérislique qui montre la part qu’il

1. Ces chiffres sont dẻjà anciens, mais les résultats des recensements récents effectués depuis 1893 pour l'exécution des lois sur l'assurance obligatoire des onvriers agricoles confirment la tendance des exploitations moyennes à diminuer légèrement, dans une proportion inférieure à celle de 1860 à 1880. 
faut faire à l'outillage dans l'industrie agricole, tandis que la valeur vénale des fermes va de 17 millions de francs à 68,4 , l'estimation du matériel d'exploitation s'est haussée de $773 ̈$ millions a $20340^{1}$. Le nombre des petites fermes diminue dans les États de l'Est, remarque M. Levasseur, à qui j'emprunte ces chiffres, el celui des fermes de moyenne étendue augmente ${ }^{2}$. L'étendue générale des fermes double de 1850 à 1890 , celle des cultures triple, la valeur vénale quadruple et le matériel d'exploitation triple. En quarante ans, le nombre des héritages que nous appellerions en France la moyenne propriété est devenue le 14,4 p. 100 du nombre total des domaines ruraux. II est vrai que le gouvernement des États-Unis possède les ressources des terres domaniales, que nous n'a vons plus en France, et qui permettent de distribuer, chaque année, des terres libres a plus de cent mille familles d'immigrants; dans la vieille Europe, la spéculation foncière ne peut se jouer à l'aise comme dans les prairies du FarWest ou les solitudes de la Californie; il faut mordre sur des droits acquis, il faut entamer des héritages qui se défendent, et e'est uniquement l'équilibre des intérêts en contact qui détermine les déclassements dans tel ou tel sens.

En France, le groupe de la moyenne propriété rurale a trois caractéristiques : en lui-même, considéré comme élément économique, il est d'une fixité relative; et, sil se démembre d'un côté, il se reconstitue presque aussitôt de l'autre; envisagé an point de vue de ses détenteurs, on voit que ceux-ci, dans la très grande majorité des cas, ne rési-

1. Aux États-Ünis, et cela peut aussi bien se faire en France quoique dans de moindres proportions, un capitaliste intelligent peut réaliser une bonne opération en achetant des terres pour les louer. M. Levasseur (L'agriculture aux Etats-Unis, 291) en cite des exemples, entre autres celui d'une ferme de 160 acres ayant coûté 5944 francs et dont le prix fut payé par la part du propriétaire dans la récolte de deux années.

9. 11 faut distinguer entre le Nord et le Sud. II y a d'énormes écarts d'un État à l'autre; une ferme de 100 à 200 acres (40 à 80 hectares) ne représente dans ce pays que de la moyenne eulture. La moyenne des fermes est, en étendue, de 86 acres dans le Michigan, de 190 dans le Nebraska. 
dent pas et n'exploitent pas directement; en d'autres termes, ce sont des forains et ils exploitent leur propriété par des intermédiaires, fermiers ou mélayers.

Il est regrettable que les statistiques ne relèvent pas des délails qui auraient une importance capitale pour l'étude de l'économie sociale; car il serait intéressant de savoir quelle est la nature de propriété que cultivent les fermiers et les métayers dont on fixe le nombre à une unité près, quelle est l'étendue des domaines qu'ils exploitent, et quelle est la condition de leurs proprictaires. Les recherches et les rapprochements quion pourrait tenter n'aboutiraient qu'à des hyppothèses, je ne m'y attarderai pas ${ }^{1}$.

L'enquète sur l'évaluation du revenu foncier des propriétés non bàties effectuée en exécution de la loi du 9 aoùt 1879 fournit quelques évaluations, malheureusement trop sommaires, sur le nombre des propriétaires forains.

Le nombre des cotes foncières rurales comprises dans les ròles de 1879 est de 14234237 à répartir entre 8434218 propriétaires, soit une moyenne de 394 propriétaires pour 1000 cotes.

Parmi ces cotes, on en trouve $\ddot{3} 382733$ afférentes à des propriétaires forains, c'est-à-dire n'habitant pas la commune où les biens ruraux recensés sont situés, et, dans un relevé spécial, figurent 296276 individus désignés par celte rubrique : Habitants ne possédant point de propriétés foncières dans la commune où ils résident mais notoirement connus

1. Qu'on me permette encore une observation sur la méthode des statistiques officielles, tout en reconnaissant que la matière est délicate et qu'il est plus aisé de critiquer que de réussir. On pourrait détacher les départements industriels des départements absolument agricoles et sėparer leurs relevés. Ainsi, dans le département du Nord, le nombre des cotes foncières a passé de 223306 en 1829 à 320094 en 1884́, et la superficie des cotes aux dépens desquelles se sont créées les 96 iss nouvelles n'est pas sensiblement réduite. C'est qu'il ne s'agit point ici d'exploitations rurales au sens du mot, mais des abris bàtis pour les 500000 ouvriers de l'industrie (mines, tissages, nétallurgie) dont s'est accrue la population du département. De la tous ces petits lots qui ont surgi en foule dans la banlieue des villes et dans les villages; si on les compte, et il est difficile de faire autrement, les moyennes générales sont faussées. 
pour être propriétaires ailleurs ${ }^{1}$. En prenant ces chiffres pour base du calcul, on pourrait admettre qu'il y a, en France, près de $\mathbf{1} 400000$ proprićtaires ruraux forains, ainsi que je l'établirai ci-après.

De 1882 à 1892 , le nombre des travailleurs agricoles exploitant pour autrui a diminué de 171131 (fermiers, métayers et journaliers) et celui des cxploitants directs cultivant exclusivement leur propre bien a augmenté de $485 \% 14$. L'écart final de 122617 a été expliqué de plusieurs façons et l'on y a vu la conséquence d'un mouvement de concentration, l'ensemble des fermiers et colons, propriétaires ou non propriétaires accusant une augmentation de 93663 ; mais il serait malaisé de vouloir fixer avec précision le nombre de ceux qui se sont simplement déclassés et le nombre de ceux qui ont définitivement abandonné la vie rurale. Il ne l'est pas moins de rechercher si l'augmentation du nombre des fermiers non propriétaires, qui s'est surtout accentuée dans le Nord et le Centre, et plus légèrement dans le Midi, correspond aux mourements de dilatation ou de compression de la moyenne propriété, soit en nombre, soit en étendue ${ }^{2}$.

Leflectif des cultivateurs travaillant pour autrui d'me manière permanente, abstraction faite des locations verluales qui ne sont que de courte durée et ne portent d'habitude que sur de très petits lots ${ }^{3}$, sanf dans les villes, et des journaliers, peut, par induction, servir, à défaut d'éléments plus directs, à déterminer approximativement l'imporlance du groupe des propriétaires forains.

1. Iiulletin de slatistipue du ministère des Finances, mai 1883, p. 596.

2. Voir aux Pièces justificatives les cartes $n^{\circ} 1$ (Travailleurs agricoles propriptaires), $\mathrm{n}^{\circ}$ II (Valeur vénale de la propriété vurale en France) et $n^{\circ}$ III (Modes d'exploitation du sol).

3. D’après les statistiques, il y a eu en 1896 plus de 1467790 Laux ou locations verbales taxés (Bulletin de statistique de l'Enregistrement, 1898, I, 126); mais les relevés ne distinguant pas les locations rurales les locations urbaines, on peut seulement noter le nombre consilérahle de ces locations dans les lépartements industriels : Seine (17'48'), Nord (6' 285), Rihône ( 4852 k), Seine-Inférieure (54252), Bouches-du-Rlıône $(43722)$. 
Fermiers :

$\left.\begin{array}{l}\text { Propriétaires.............. } 475778 \\ \text { Non-propriétaires...... } 585623\end{array}\right\} 1061401$

Métayers :

Propriétaires......... 123997

Non-propriétaires...... 220 871$\} \quad 344168$

14050569

D'autre part, l'enquête de 1892 établit ainsi le nombre des exploitations de la culture indirecte, qui est celle qu'adoptent forcément les propriétaires forains :

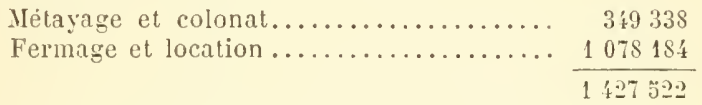

Mais un propriétaire forain peut posséder plusieurs fermes et plusieurs métairies, dans quelle mesure? on l'ignore, et les relerés des Contributions directes, avec leur apparence de précision, ne donnent pas la solution du problème.

Nous sommes donc en présence de deux chiffres dont l'écart est considérable, mème en tenant compte des corrections admises. D'une part, le nombre des labitants notoirement connus pour être propriétaires des domaines ruraux (propriétés non bâties) hors de la commune : 296276 ; d'autre part le nombre des cotes de 1879 afférentes à des forains : əั 82 7ั3. Le nombre des cotes fiscales est supérieur au nombre des propriétaires, même dans la même commune où l'unité de cote est de droit "; des calculs minutieux ont été entrepris à deux reprises, en 18.31 et 1879, pour déterminer le nombre de propriétaires correspondant en moyenne à 100 coles; d'après ces calculs, le rapport serait tombé de 63 p. 100 en 18301 à 60 p. 100 en 1879; mais le grand nombre des cotes parasites que l'observation directe des rôles permet de constater autorise à compter 4 cotes par propriétaire. La mobilité croissante de l'existence, la facilité des communications, la rapidité des transports, le goût des déplacements

1. M. de Foville a expliqué comment l'exactitude des statistiques était faussée par l'intérêt personnel des percepteur's à multiplier les cotes. (Le morcellement, 67.) 
périodiques même chez les gens les plus occupés, nous engagent à être à la fois propriétaires sur des points éloignés l'un de l'autre, à l'Est ou à l'Ouest, au Nord ou au Midi, ce que nauraient pas compris nos pères et ce qui, d'ailleurs, leur eût été plus difficile qu'à nous.

Le calcul par les cotes donne 1393688 forains, chiffre qui se rapproche, avec une approximation qu'on ne peut exiger plus serrée, des deux autres éléments d'appréciation :

Nombre des forains d'après les cotes........ 1395688
Nombre des cultivateurs exploitant à titre per-
manent pour le compte d'autrui.......... 1403569
Nombre des fermes et métairies exploitées
pour le compte d'autrui par la culture indi-
recte.......................... 1427522

L'écart entre les deux chifires extrèmes s'explique aisément (31 834) par l'approximation du calcul el par la vraisemblance qu'il y aurait à altribuer aux capitalistes qui possèdent des immeubles ruraux la propriété de plusieurs domaines en même temps.

Arrètons-nous au chiffre global de 1400000 pour une population de 2231313 propriétaires agricoles vivant de la rente de la terre, et de 936729 individus vivant exclusivement de leurs revenus, soil à titre de propriétaires d'immeubles ou de valeurs mobilières, soit à titre de pensionnaires et de retraités, plus 420133 personnes vivant de professions libérales ${ }^{1}$.

C'est done un tolal de 3608373 personnes, sans compler les industriels et les commercants dont le contingent est énorme, où peul se recruter le personuel des propriétaires forains. Si l'on dressait, lors du prochain recensement, leur statistique par condition, on puiserail certainement dans ces chiffres de précieuses indications sur les origines et les ressources du groupe de la moyenne propriété et sur le rôle qu'il peut jouer dans l'harmonie générale de notre économirque.

1. Dénombrement de 1891, pages 3030 , 311 et 763 . 
Il n'est pas sans intérèt de voir quelle est la répartition territoriale des propriélaires forains, car, fait bizarre, il n'y a pas de corrélation apparente entre le nombre des cotes foraines et celui des forains notoires ${ }^{1}$. Voici le relevé des 13 départements où le nombre des cotes foraines dépasse cent mille, avee leur nombre d'habitants propriétaires au dehors sans l'ètre dans la commune de leur résidence.

\begin{tabular}{|c|c|c|}
\hline \multirow[b]{2}{*}{ DÉPARTEMENTS } & \multicolumn{2}{|c|}{ NOMBRE DES } \\
\hline & $\begin{array}{l}\text { Cotes } \\
\text { foraines. }\end{array}$ & $\begin{array}{l}\text { Habitants connus } \\
\text { pour forains. }\end{array}$ \\
\hline 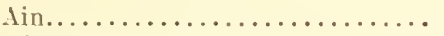 & 100575 & $197 t$ \\
\hline 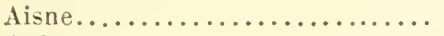 & 128831 & 2336 \\
\hline 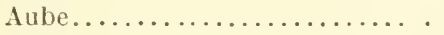 & 114088 & $1 ! 69$ \\
\hline Charente-Inférieure........... & $1 \div 8782$ & 2451 \\
\hline 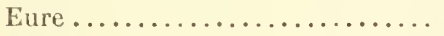 & 103351 & 3543 \\
\hline 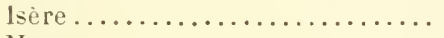 & 116060 & 3713 \\
\hline 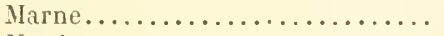 & 113857 & 1821 \\
\hline Nord $\ldots \ldots \ldots \ldots \ldots \ldots \ldots \ldots$ & 117809 & 4770 \\
\hline 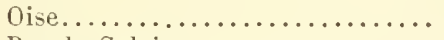 & 134842 & 3142 \\
\hline 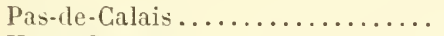 & 138408 & 4135 \\
\hline Haute-Saone ............... & $10 \div \div 87$ & 1630 \\
\hline 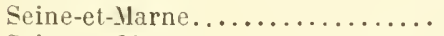 & 127383 & 2057 \\
\hline 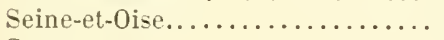 & 161084 & 2110 \\
\hline 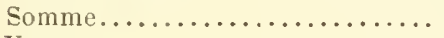 & 142585 & 2286 \\
\hline 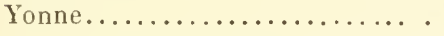 & 152609 & 1226 \\
\hline
\end{tabular}

Parmi les départements où le nombre des forains notoires dépasse 4000, le Rhòne tient la tête avec 30936 propriétaires ruraux tandis que le département de la Seine n'en accuse que 16103 . Viennent ensuite la Seine-Inférjeure, l'Ille-etVilaine, le Morbihan et les Côtes-du-Nord; ces régions présentent une faiblesse relative du nombre des cotes foraines alors que le nombre des résidants propriétaires au dehors est très élevé; il y a là l'indice de situations locales particulières qui semblent de nature à provoquer une étude spéciale et minutieuse qui pourrait devenir féconde en révélations.

Voici les 19 départements où se présente celte anomalic et qui comptent plus de 4000 résidants forains.

1. Bulletin de statistique du ministère des Finances (mai 1883); résultats du recensement des propriétés non bâties de 1879 . 
DÉPARTEMENTS -

Bouches-du-Rhône.............

Côtes-du-Nord...............

Haute-Garonne.............

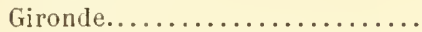

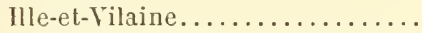

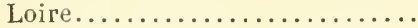

Loire-Inférieure..............

Lot-et-Garonne..............

Maine-et-Loire...............

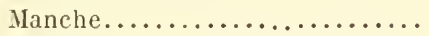

Morbihan.................

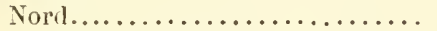

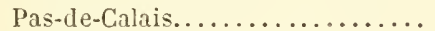

Puy̌-de-Dòme...............

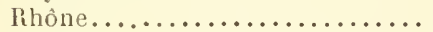

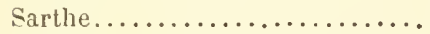

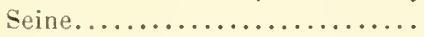

Seine-lnfërieure.............

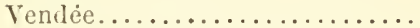

\begin{tabular}{|c|c|}
\hline $\begin{array}{l}\text { Cotes } \\
\text { foraines. }\end{array}$ & $\begin{array}{l}\text { Résidants } \\
\text { exclusivement forains. }\end{array}$ \\
\hline $266 \div 3$ & 4676 \\
\hline 60547 & 6272 \\
\hline 601950 & 5570 \\
\hline 830143 & 6840 \\
\hline 68943 & 10331 \\
\hline 44827 & 5298 \\
\hline วั8 761 & 5310 \\
\hline 56665 & 5865 \\
\hline 72111 & 4824 \\
\hline 79017 & 7896 \\
\hline 34231 & 6128 \\
\hline 117809 & +770 \\
\hline 138408 & 4135 \\
\hline 99200 & 433 ' \\
\hline 50652 & 30936 \\
\hline 65120 & 6900 \\
\hline $51330 ّ$ & 1610 \\
\hline 72806 & 13303 \\
\hline 76177 & 6532 \\
\hline
\end{tabular}

Les rares départements qui comptent moins de 1000 résidants propriétaires exclusivement forains sont: Hautes et Basses-Alpes (442) et 706), Haute-Loire (833), Lozère (531), Hautes-Pyrénées (983) et le territoire de Belfort (321) dont la petitesse justifie le chiffre; quant aux autres, on remarquera que ce sont les plus pauvres de France.

Le classement des cotes foncières par catégories de contenance, d'après l'enquête administralive de 1884 (moins Paris et 36.4 communes rurales non cadastrées) fournit des chiffres de beaucoup inférieurs aux autres termes de comparaison, mais que je crois utile de signaler parce qu'ils indiquent la force de colésion de ce groupe intermédiaire dont lavenir sera certainement, malgré le délaissement actuel, aussi utile que brillant ${ }^{1}$.

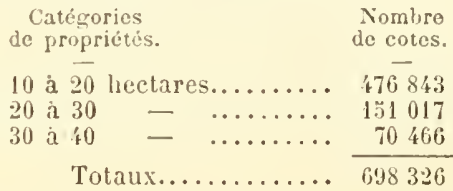

Contenance imposable.

6629491

3652024

2418572

12700087

1. Bulletin de statistique du ministère des Finances, août 188', p. 156. 
La répartition par quotité d'impòt des cotes rurales afférentes à la moyenne propriété ne donne pas de résultals plus concluants, à causc de l'impossibilité de faire entrer dans un cadre rigide des variétés aussi mobiles el aussi changeantes d'après les régions ${ }^{1}$.

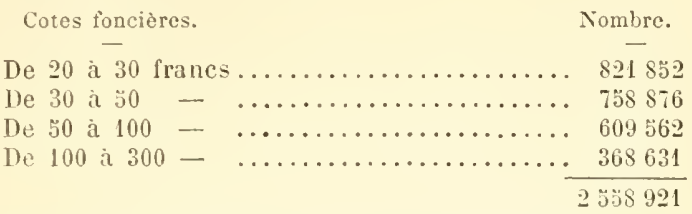

Les modes d'exploitation agricole sont au nombre de deux : l'exploitation directe, dans laquelle le propriélaire exploite seul arec sa famille ou arec l'aide d'autrui (journaliers, régisseur, maitre-ralet) et l'exploitation indirecte qui revèt deux formes, le fermage à bail ou la location verbale, et le métayage, partage proportionnel et souvent à moitié des produits ou de certains produits, le propriétaire fournissant la terre arcc ou sans un capital et un matéricl.

La culture directe et le fermage se partagent à peu près la superficie du territoire cultivé; $\dddot{3} 3$ p. 100 à la première, 57 p. 100 au second, le métayage intervient pour 11 p. 100.

La répartition n'est pas indiquée pour la moyenne propriété; si lon défalque les forains, que reste-t-il formant le groupe particulièrement intéressant des propriétaires exploitant personnellement les héritages de 10 à 40 hectares? Et dans quelle proporlion ce groupe figure-t-il dans la masse des 33872400 travailleurs agricoles? Il ne faut pas oublier, en effet, qu'on fixe à 711000 en chiffres ronds le nombre des exploitations de 10 à 40 hectares ${ }^{2}$ avec unc superficie de 14 millions d'hectares $1 / 3$. De quelle nature est la population de ces domaines?

L'idéal serait qu'ils fussent tous livrés à la culture directe.

1. Cette statistique est celle de 1858 reproduite en 1884 . Je connais dans la Côte-d'Or un domaine de 39 hectares, affermé 1600 francs, qui paie avec les centimes additionnels 220 francs d'impôt foncier en 1899 .

2. Enquête de 1892, p. 36 ' de l'Introduction. 
Et quel concours actif à la production nationale, quels germes de fécondité el de richesse jetés sur ce sol, si la plupart des fils de la bourgeoisie, au lieu de poursuirre la chimère des diplòmes et la quiétude somnolente et déprimante des fonclions publiques que leur nombre même et leur irresponsabilité discréditent, remplaçaient sur celte lerre qui reut êlre maniée avec sollicitude des fermiers négligents ou des mélayers ignorants!

Les domaines appartenant à des forains pourraient être offerts avec arantage à la culture direcle; de nos jours, à mesure que la propriété est devenue plus exigeante, l'exploitation a été moins profitable. L'une a gagné et l'autre risque de perdre; pourquoi ne pas les mettre d'aceord en les fusionnant? Toules les terres françaises susceptibles de culture ne sont pas mises en valeur; il reste sur notre territoire 6226189 hectares en landes el friches, dont 4603 166 appartiennent à des particuliers. Sur ce lot, 1307000 hectares sont détenus par la moyenne propriété. N'y-a-t-il pas de ce còté un bénéfice à réaliser?

Lindustrie agricole comporle toujours deux associés souvent réunis dans la même personne : le propriétaire et l'exploitant. Le premier foumit le champ d'expérienees, la matière première, le second la met en œuvre. Quand il s'agit de transformer un sol inculte, la part de l'ouvrier, du pionnier, est prépondérante, et ce n'est que justice. Celle part diminue au profit du propriélaire, à mesure que la terre mise en valeur n'a plus besoin que d'entretien et n'offre plus les risques du début. $\Lambda$ notre époque, la terre défrichable ne fait à la terre productrice qu'une médiocre concurrence ${ }^{1}$, mais cette concurrence ne serait point négligeable si elle se produisait armée des capilaux et de l'outillage intellectuel que possède la moyenne propriété et qu’elle n'ulilise qu’à demi lorsqu'elle a l'idée de s'en servir.

Autrefois, la disproportion de l'étendue du sol avec le

1. De 1882 à 1892, on n'a défriché que 1309 '2 lıcctares de landes et terres incultes (Morbihan, Côtes-du-Nord, Finistèrc, Haute-Vicnne, Creuse) et 48105 hectares de bois (Gironde et Orne). 
nombre des habitants était telle que la valeur de la terre dépendait uniquement des bras qui pouvaient la eultiver; aujourd'hui la plupart des héritages ruraux ressemblent à des comptoir's placés au milieu d'un marché et qui seraient abandonnés; pour y attirer les clients il faut y apporter des marchandises. La moyenne propriété se trouve dans les conditions les plus favorables à une exploitation raisonnée et rémunératrice; elle détient la terre : qu’elle y applique une part des capitaux que ses détenteurs éparpillent un peu partout; qu'elle y utilise la jeunesse et l'initiative que ses enfants gaspillent à des riens et, des trois groupes de la propriété foncière, ee sera celui-ci dont le ròle deviendra rapidement entraìnant et décisif.

\section{VII. - Répartition régionale de la petite propriété.}

La question de la pelite propriété estl'une des plus importantes de l'économie rurale; bien comprise, elle est la clé du problème social. On peut l'envisager à un double point de rue : eelui de la propriété proprement dite et celui de l'exploitation eulturale. Les deux problèmes se côtoient et se pénètrent, de telle sorte que, ici plus qu'ailleurs, la sùreté des statisliques se heurte à des diffieultés d'un ordre très particulier. On ne sait trop, en effet, sil vaut mieux substituer dans les relerés la notion d'étendue à la notion de valeur, ni comment il est possible de toujours distinguer le fait de la propriété du fait de la culture; or, ce qu'il importe de savoir, au point de vue spécial de cette étude, c'est à la fois le nombre des petits propriétaires, l'étendue de leur propriété, et leur mode d'exploitation.

Les rédacteurs de l'enquête agricole de 1892 ont fait, à cet égard, l'areu de leur embarras. Le ministère de l'Agriculture, dans la statistique agricole décennale de 1882, tout en publiant par eatégories d'étendue, dans les tableaux numériques, le nombre et l'étendue de cotes foncières spéciales, cotes dites agraires, c'est-à-dire ayant un caractère 
exclusivement agricole, ainsi que leur étendue moyenne par catégoric, admettait simplement la valeur relative du nombre et de l'étendue tolale de ces cotes, comparées dans les mèmes conditions à deux époques différentes pour en tirer l'indice d'un mouvement d'augmentation ou de diminution de la petite propriété, mais ne considérait pas l'étendue moyenne des cotes agraires comme la mesure de l'étendue moyenne des propriétés agricoles. Pour arriver à des conclusions pratiques, aussi certaines qu'clles puissent l'ètre dans une matière encore mal observée, il faudrait multiplier les distinctions, les catégories et les groupements; on obscurcil ce sujet en le généralisant.

C'est surtout en ce qui concerne la petile propriété que les déductions tirées du nombre des cotes peuvent induire en erreur, car le nombre des cotes, d'abord en raison de la nature mème des choses, ensuite par la faute de certains procédés administratils, est infiniment supérieur à celui des proprićtaires et, par conséquent, an nombre des propriétés.

Une pelite propriété, située sur les confins de deux ou de trois communes, peut être constituée par de nombreuses et petites parcelles éparpillées sur ces communes et se subdiviser en plusicurs coles quoique n'appartenant qu'à un seul propriétaire. Ici, plus encore que dans les chapitres précédents, la division de la propriété ne se peut apprécier que par la minutie du détail, en évitant les généralités, la petite propriété el la culture affectant des allures très diverses non seulement selon les régions mais encore dans les différentes parties d'un même département.

Les relevés officiels entendent par pelite culture les exploitations de moins de 10 liectares ${ }^{1}$ et par très petite celles de moins de 1 hectare; pour éviter les redites et abréger des explications inutiles ici, je confondrai le plus souvent,

1. 11. de Foville, qu'il faut tonjours citer, semble prendre parti pour les anciens classements do 1833 i 18 \$1, limitant la très petite propriété au maximum de 2 hectares, ce qui est peut-être trop, et la petite a 6 hectares, chiffre plus rationnel que les 10 hectares des relevés officiels d'à présent. 
comme il arrive d'ailleurs dans la réalité, la petite culture et la petite propriété. En 1892, le nombre des exploitations sur le territoire agricole de la France se répartissait ainsi :

\section{PETITE PHOPRIE்TÉ}

Exploitations d'une étendue inférieure à 10 hect. 4852963

HOYEXNE ET GRAX̃DE PROPRIÉTÉ

Exploitations d'une étendue supérieure à 10 hect. $\frac{849789}{\text { Ensemble........ }} \quad 5702752$

L'importance, en nombre, est donc considérable et, si chaque unité représentait un électeur, on voit quelles conclusions sociales pourraient se déduire de celte répartition inégale de la propriété rurale. La proportion se renverse évidemment dès qu'ils s’agit de l'étendue; la part de cette catégorie de propriétaires n’est, en effet, que de 12 millions $1 / 2$ d'hectares sur ö0.

Pour serrer de plus près les recherches, on peut, avec les statistiques de 1892, subdiviser en trois catégories les exploitations de la petite propriété : $1^{\circ}$ cultures de parcelles d'une superficie inférieure à un hectare : ce sont les plus nombreuses; $2 \circ$ d'une étendue de 1 à 5 hectares, supérieures elles-mèmes d'un million d'unités au bloc que forment ensemble la grande et la moyenne propriété; $3^{\circ}$ enfin, celles de ò à 10 hectares, formant l'aristocratie de la petite propriété, servant de transition aux héritages d'une valeur vénale plus grande et dont le nombre se rapproche sensiblement de celui du bloc riche ou présumé tel.

Catégories d'exploitations. $\begin{gathered}\text { Nombredes } \\ \text { exploitations. }\end{gathered} \begin{gathered}\text { Superficie en } \\ \text { milliers d'liectares. }\end{gathered}$

\begin{tabular}{|c|c|c|}
\hline - & - & - \\
\hline $\begin{array}{l}\text { Très petite culture au-dessous } \\
\text { de } 1 \text { hectare..................... }\end{array}$ & 2235405 & 13273 \\
\hline Cultures de 1 à 5 hectares.... & 1829259 & $54 \$ 92$ \\
\hline Cultures de है à 10 hectares... & 788299 & $5753 ี 5$ \\
\hline & 4852963 & 125720 \\
\hline
\end{tabular}

La première observation qui découle de la comparaison de ces chiffres, c'est que plus l'étendue de la propriété 
augmente et plus le nombre des propriétaires de la catégorie diminue.

Il est difficile de savoir quelle est pour cet ensemble de petites exploitations la proportion exacte des propriétaires; dans la banliene des grandes villes, où les cultures maraîchères sont très nombreuses et le terrain très cher, il n'est pas douteux qu'il y ait plus de locataires que de propriétaires; dans les communes rurales, le phénomène inverse se produit, mais je crois à peu près impossible, dans l'état actucl de nos statistiques, de fixer les ćcarts ou les proportions avec une précision scientifique. Il faudra s'en tenir à des hypothèses, à des approximations arbitraires qu'on peut croire aussi rapprochées que possible de la réalité, tant que les statistiques municipales centralisées par le ministère de l'Agriculture et celles du service des Contributions directes ne seront pas établies et combinées de façon à se contrôler mutuellement et à donner avee sûreté le nombre des propriétaires et leur condition sociale.

Ces renseignements, indispensables pour apprécier les incidenees économiques et philosophiques de la division de la propriété el de sa répartilion entre les différentes classes de travailleurs ou de capitalistes, seront facilement obtenus et avee plus d'exactitude lorsque les statistiques du service de l'Enregistrement se feront plus détaillées en ce qui concerne le mouvement des mutations foncières, et surtout lorsque l'institution de livres fonciers simplifiés ${ }^{1}$ permettra de reconstituer le terier général de la France, en dégageant le plus possible le crédit territorial des servitudes de la procédure et de limpôt.

Il n'est pas moins instructif de rechercher les formes d'exploitation de la petite propriété et quelles sont les cultures qu'elle affectionne de préférence. En voici le tableau d'ensemble.

1. Rapport général sur l'institution des livres foneiers et leur fonetionnement en ce qui concerne les droits réels immobiliers autres que les privileges et les hyjotheques, publić par la Commission extra-parlementaire du cadastre, le 14 décembre 1899. 


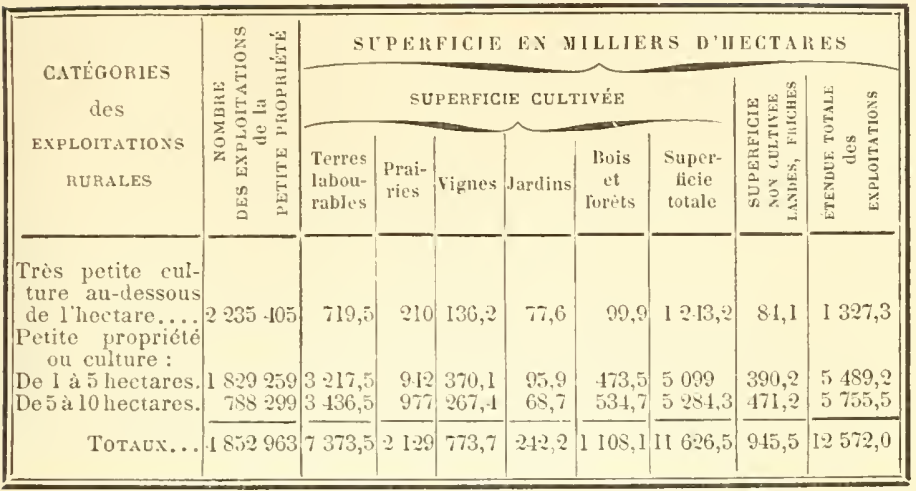

Du premier coup d'ceil, on apercoit que la très petite culture s'adonne aux exploitations qui exigent des soins minutieux et assidus, le jardinage et la vigne; et cette impression s'accentue si l'on remarque que les labours attribués a la petite propriété par les statistiques ${ }^{1}$ ne sont pas ensemencés en grains mais fournissent les produits maraìchers de grande culture tels que les pommes de terre, et les racines alimentaires, carottes, navets, panais, raves, turneps, choux, artichauts, ete.

Par comparaison aree la surface moyenne des autres catégories d'exploitations rurales, on trouve, en 1893, les proportions suivantes :

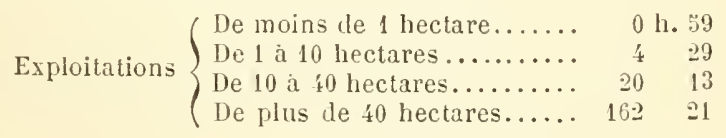

C'est-à-dire que la moyenne de l'étendue d'un héritage appartenant à la très petite propriété ne dépasserait pas $\check{9} 9$ ares, et celle d'un domaine de la petite 4 heetares 29 ares.

Le contrôle de ces données par les autres sources d'informations permet de supposer que eette dernière moyenne

1. Statistique agricole de 1892 , p. 137 de l'Introduction, et Tableaux numériques à la suite. 
est trop élevée et qu’il est prudent de limiter les comparaisons et de les restreindre, dans un même département, tout au plus à un arrondissement ou à un groupe de communes voisines.

Si la proportion des terres labourables alleint son maximum dans la grande propriété (39,18 p. 100), ce maximum apparlient pour les prés et les vergers à la moyenne culture $(36,43$ p. 100) et pour les vignes et les jardins à la petile culture $(3 \Xi, 42$ p. 100 et 34,48 p. 100). La très petite culture, en raison de l'exiguïté du terriloire qu'elle occupe, ne présente de proportion superficielle appréciable que pour les vignes el les jardins, dont la valeur exceptionnelle de production compense le peu d'élendue.

L'élendue moyenne des très petiles exploitations (de 0 à 1 hectare) varie, par département, de 40 ares dans les BassesAlpes, à 70 dans la Haute-Vienne, 73 dans l'Hle-et-Vilaine, 74 dans la Mayenne. Pour la petite propriété, les écarts vont du simple au double; l'étendue moyenne des domaines de 1 à 5 hectares va de 1 h. 96 dans les Basses-Alpes, à 3 h. 43 dans les Hautes-Alpes, 3 h. 68 dans les Landes, 3 h. 69 dans la Maute-Narne et 3 h. 76 dans l'Orne, en passant par tous les degrés intermédiaires. Pour ceux de $\ddot{j}$ à $10 \mathrm{~h}$., la moyenne, de 4 h. 93 dans les Basses-Alpes, saule à 8 h. 08 dans l'Eure, 8 h. 1 ; dans le Pas-de-Calais, et 8 h. 68 dans les Pyrénées-Orientales.

Le nombre des exploitalions de la petite propriété n'offre pas moins de variélé que leur étendue, suivant les régions, el, comme ici il s'agit de chiffres absolus, la comparaison n'en est que plus instruclive.

Les tres pelites exploitations, dit la publication administrative de 1892-189', se rencontrent au nombre moyen d'environ 26000 par département. Celte asserlion, si clle élait prise à la lettre, donnerait une notion inexacte de la réalité puisque, sil s'en trouve, en effet, 20293 dans l'Oise, on en compte 7676 dans les Hautes-Alpes et $\$ 9062$ dans le Puyde-Dòme. Pour la calégorie de 1 à : hectares, l'échelle varie de 493.37 dans le Puy-de-Dôme el de 40789 dans l'Isère, à 
8000 dans les Hautes-Alpes et la Mayenne, à 9000 dans la

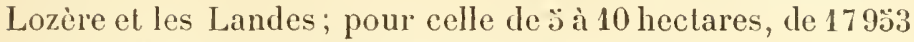
dans la Manche à 1810 dans les Basses-Alpes ${ }^{1}$.

Si, au lieu du nombre, on considère la superficie, la très petite propriété ne couvre pas 10000 hectares dans 12 départements (Mayenne, Sarthe, Vendée, Eure-et-Loir, à l'Ouest; Indre-et-Loire, Loiret et Cantal, au Centre; llauteSaòne, à l'Est; Landes, Lozère, Ilautes et Basses-Alpes, au Sud); par contre, l'étendue de cette catégorie d'exploitations dépasse 20000 hectares dans $\mathbf{S}$ départements (Manche, Pasde-Calais, Aisne, Vosges, Aveyron, Puy-de-Dôme, Dordogne et Gironde).

La petite propriété (de 1 à 10 hectares), peu étendue dans les Pyrénées-Orientales et les trois départements des Alpes, couvre plus de 200000 hectares dans chacun de ces 7 départements : Còtes-du-Nord, Charente-Inférieure, Puy-deDòme, Saône-et-Loire, Dordogne, Gironde et Isìre, passant de 52097 hectares dans les Pyrénées-Orientales à 264702 dans le Puy-de-Dôme.

L'examen des cartes ci-jointes montrera le peu de symétrie el de régularité régionales de ces mouvements. Il convient de noter ici que, de 1882 à 1892, la très pelite propriété s'est accrue, en nombre et en étendue, landis que la petite a diminué dans les deux sens.

\begin{tabular}{|c|c|c|c|c|}
\hline \multirow[t]{2}{*}{ ES } & \multicolumn{2}{|c|}{$\begin{array}{l}\text { Exploitations } \\
\text { de moins de } 1 \text { hectare }\end{array}$} & \multicolumn{2}{|c|}{$\begin{array}{l}\text { Exploitations } \\
\text { de } 1 \text { à } 10 \text { hectares }\end{array}$} \\
\hline & $\begin{array}{c}\text { Nombre. } \\
-\end{array}$ & $\begin{array}{c}\text { Contenance en heet. } \\
-\end{array}$ & $\begin{array}{l}\text { Nombre. } \\
-\end{array}$ & Contenance en hect. \\
\hline $18 \delta 2 \ldots$. & 2167667 & 1083833 & 2633030 & 11366274 \\
\hline $1892 \ldots$. & 22330403 & 1327253 & 2617558 & $1124+750$ \\
\hline
\end{tabular}

Le fail caractéristique de la situation présente de la petite propriété telle qu'elle est définie administrativement, e'est son accroissement continu, en nombre et en étendue. Mais,

1. II faut excepter le département de la Seine et la circonscription de Belfort que leur territoire forcément restreint met en dehors de toute comparaison. La Seine compte 13517 exploitations rurales de notre catégorie, dont 11031 n'ayant qu'une contenance moyenne de 24 ares. 
pour pénétrer au fond des choses et se mettre en présence de l'exacte réalité, il faut distinguer dans celte catégorie et considérer isolément la très petite propriété ${ }^{1}$ et la petite; on apercevra alors un double mouvement en sens inverse qui se compliquerait éridemment d'autres oscillations en sens variés à mesure qu'on multiplierait, si les statistiques s'y prêtaient, les divisions et les subdivisions, afin de serrer de plus près le phénomène économique qui fait de la culture intensive el de l'amour du foyer domestique les corollaires immédiats dı fractionnement de la possession du sol.

D’après l'enquète de 1892 , la majorité des départements (60 sur 87, y compris Belfort) présentent une augmentation des très petites exploitations au-dessous de 1 hectare. Ces augmentations se rencontrent dans toutes les régions, el de préférence dans la région du Nord (Pas-de-Calais, Somme, Aisne, Oise, Seine-Inférieure, Eure et Eure-et-Loir), puis dans le Centre-Ouest (Loire-Inférieure, Maine-et-Loire, Vendée, les deux Charentes, Deux-Sèvres, Vienne et HauteVienne). Les diminutions se présentent au Centre, dlans un groupe compact formé de \& déparlements (Loir-et-Cher, Loiret, Yonne, Indre, Cher, Nièvre, Creuse, Allier et Puyde-Dôme), et dans le Sud-Est où 4 départements seulement sont en augmentation contre 7 en diminution.

L'étendue de ces pelites propriétés a une tendance à s'accroître, surtout dans les régions où leur nombre a diminué, fait qui pourrait donner lieu, en l'étıdiant de près, à les observations suggestives; ainsi celte catégorie a perdu ¡000 hectares dans le Nord ${ }^{2}, 2300$ dans le Loirel, et en a

1. Il serait à désirer qu'au lieu de considérer les parcelles d'un hectare comme constituant le minimum officiel de la division de la terre, on poussât le constat jusqu'aux limites extrêmes des exploitations minuscules. Ainsi, en Angleterre, les statistiques descendent jusqu'à 1/4 d'acre, ou 10 ares 12 centiares (l'acre, anglaise équivalant à 40 ares 467). On comptait, dans ce pays privilégié de la grande propriéte, en 1885, plus de 73320 exploitations de moins de 40 acres.

2. Dans cette région, il serait possible que les pertes de salaires et l'endettement résultant des grèves de mineurs et de métallurgistes, aient obligé beaucoup d'ouvriers à aliéner leurs modestes liéritages, de 1882 à 1892. 
gagné de 4000 à 16000 dans l'Aisne, la Haute-1larne, les Vosges, la Dròme, elc.

Par contre, les exploitations de 1 à 10 hectares ont diminué de nombre et d'étendue, donnant ainsi le branle au mourement analogue que j’ai indiqué plus haut ${ }^{1}$ pour la moyenne propriété (de 10 ì 40 hectares). Mais ce mouvement r’a pas été uniforme et, quoique son incidence finale se traduise par une perte, cependant il a été atténué par des oscillations en sens contraire. Le nombre de ces exploilations a diminué dans 39 départements et augmenté dans 48; leur étendue a varié 41 fois en moins et 43 en plus. Le Nord et le Centre ont des plus-values, l'Ouest et l'Est des déficils. Un calcul très serré de rapprochements établirait que ces mouvements inverses sont en quelque sorte parallèles et que les très petits propriétaires ont acquis au moins les $2 / 3$ de ce que perdaient leurs voisins à domaines plus étendus.

L'enquète agricole, dans ses développements sur la répartition de la population rurale, ne s'explique point sur la part qui revient à la petite propriété dans le groupe des exploitants dircets, au nombre de 3387 눙, qui représentent près de 71 p. 100 des travailleurs de la terre. Il est malaisé de le déterminer.

Toutefois, comme il est certain que tous les héritages de la petite propriété, à part de très rares exceptions dans la troisième catégorie (de : à 10 hectares), sont exploités par la culture directe, en déduisant du total de l'étendue appartenant à ce mode d'utilisation.

183244 les milliers d'hectares cultivés ${ }^{2}$ détenus par les petits propriétaires. . . . . . . . . $11626 \%$

Il resterait à la culture directe de la grande et de la moyenne propriété.

66979

Et l'on aurait toujours à résoudre ce problème : pour cul-

1. Au \& IV ci-dessus.

2. Les statistiques déduisent de la surface totale, tantôt seulement les landes et friches, tantôt aussi les bois et forêts. 
tiver 48329963 héritages, d'une superficie exploitée de près de 12 millions d'hectares, nous n'avons que les $2 / 3$ des exploilants directs accusés par les statistiques, soit environ 2238162 chefs de culture. D'oì la conclusion forcée que pour 2 millions $1 / 2$ de ces propriétaires la possession et la mise en valeur de leur petit domaine ne sont que l'accessoire d'un travail plus important.

Le travail de recensement des cotes foncières exécuté en 1884 par le service des Contributions directes ${ }^{1}$ fait ressortir des chiffres curieux mais dont la concordance avec les résultats des statistiques agricoles ne peut s'établir. A cette date, le nombre des cotes rurales foncières d'une eontenance de moins de 10 hectares, c'est-à-dire rentrant dans la catégorie de propriétés qui fait l'objet de ce chapitre, se subdivisait comme suit :

\begin{tabular}{|c|c|c|c|c|}
\hline \multirow{2}{*}{$\begin{array}{c}\text { CATÉGOHES } \\
\text { DES COTES RUHALES FONCIÈRES } \\
\text { par contenance }\end{array}$} & \multirow{2}{*}{$\begin{array}{l}\text { NOMBRE } \\
\text { DE } \\
\text { COTES }\end{array}$} & \multirow{2}{*}{$\begin{array}{l}\text { CONTENANCE } \\
\text { IMPOSABLE } \\
\text { en } \\
\text { hectares }\end{array}$} & \multicolumn{2}{|c|}{ PROPORTION PAR 100} \\
\hline & & & $\begin{array}{c}\text { pour } \\
\text { le } \\
\text { nombre }\end{array}$ & $\left|\begin{array}{c}\text { pour } \\
\text { la } \\
\text { eontenanee }\end{array}\right|$ \\
\hline De 0 à 10 ares inclusivement. & 2670512 & 108231 & 18,98 & $0,2-2$ \\
\hline De 10 ares à 20 ares....... & 14449.1 & 213789 & 10,28 & 0,43 \\
\hline De $20-$ a $50-\ldots \ldots \ldots$ & 2482380 & 825784 & 17,61 & 1,66 \\
\hline De 50 - à 1 hectare...... & 1987480 & 1426785 & 14,12 & 2,88 \\
\hline De 1 à 2 hectares........ & 1841045 & 2636867 & 13,07 & 5,34 \\
\hline De 2 a $3 \quad-\quad \ldots \ldots \ldots$ & 932444 & 2286670 & $6,6,3$ & 4,62 \\
\hline De 3 à & 5730533 & 1986474 & 4,08 & 4,02 \\
\hline De 4 a & 388631 & 1737703 & 2,76 & 3,52 \\
\hline De 5 a 6 & 280060 & 1532500 & 2,00 & 3,10 \\
\hline De 6 a 7 & 210560 & 1364150 & $\overrightarrow{1}, 50$ & 2,77 \\
\hline De 7 a $s$ & 164390 & 1230180 & 1,17 & 2,50 \\
\hline De 8 a 9 & $1308: 1$ & 1111027 & 0,93 & 2,25 \\
\hline De 9 a $10 \quad-$ & 107020 & 1016285 & 0,76 & 2,05 \\
\hline Total pour la petile propriété. & 13213383 & 17476413 & 93,92 & 35,36 \\
\hline 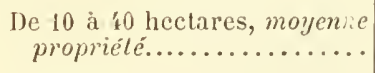 & 698326 & 12700087 & 4,93 & $2 \ddot{3}, 73$ \\
\hline $\begin{array}{l}\text { De plus de } 40 \text { hectares, } \\
\text { grande propriété.......... }\end{array}$ & 163092 & 19211772 & 1,13 & 38,91 \\
\hline Totaux.... & $\overline{14074801}$ & $\overline{4938830 \mathrm{i}}$ & 100,00 & 100,00 \\
\hline
\end{tabular}

1. Bullelin de stalistique du minislère des Finances, aoùt 1884, p. 180 Les cotes de moins de 3 francs, dont la proportion sur l'ensemble était de $\mathbf{6} 7$ p. 100 en 1830̈, figuraicnt en $18: 38$ pour près de 31 p.100.(Itid., p. 1:66.) 
C'est avec un cadre analogue et des subdivisions aussi détaillées, correspondant avec des relerés semblables pour les mutations entre vifs et par décès inserites à l'Enregistrement, quion pourrait écrire l'histoire de la France rurale.

D'après le recensement de 1891 , les 47 centièmes de la population classée vivent de lagriculture, à quelque titre que ce soit, et 25 vivent de l'industrie, 10 du commerce, 3 des transports; l'importance sociale de l'agriculture est done si considérable que son rôle aclif reste prépondérant el que tout ce qui la touche doit être étudié avec une extrême attention.

La population rurale est tout entière composée de travailleurs, elle vit de son labeur beaucoup plus que de ses revenus puisque le ralentissement ou l'abandon de la culture suffisent à stériliser le sol, et la nation est solidaire de son ardeur ou de sa négligence, puisqu'elle dépend avant tout des produits qu'elle récolte, crée ou transforme.

La population rurale doit donc ètre considérée sous tous ses aspects si l'on veut tirer des éléments variés de sa complexité le sens de son rôle naturel et les règles libérales qui seules peuvent exciter ses initiatives el protéger ses évolutions. Elle est d'autant plus intéressante qu'elle se compose, dans la proportion d'au moins $7 \ddot{\mathrm{p}}$ p. 100 , d'individus qui n'ont d'autre capital qu'un modeste héritage et qui vivent, eux et leurs familles, en le faisant valoir de leur mieux ${ }^{1}$.

La masse agricole se répartit ainsi :

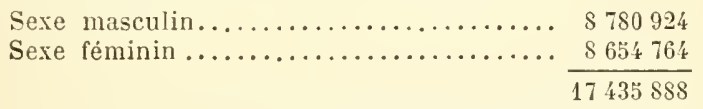

Ce qui donne, pour l'ensemble du territoire français, 4733 agriculteurs pour 1000 habitants ${ }^{2}$. Elle se classe, par groupe d’àge, les deux sexes réunis, comme suit:

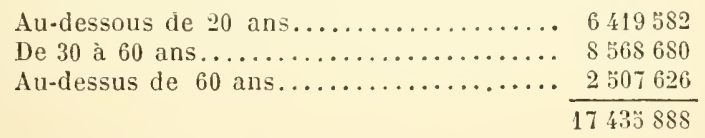

1. Cartes I à III (pages 68, 118, 192.)

2. Ces chiffres sont tirés des Résullats statistiques du dénombrement cte 1891, p. $27 \mathrm{I}$ et suiv. 
Si l'on considère la condilion des personnes, la répartition est celle-ci :

Patrons..........................

Familles des deux catégories...

Domestiques attachés à la personne, des deux catégories ${ }^{1} .$.

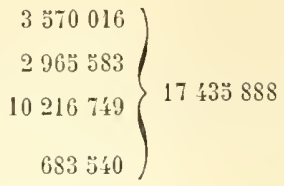

Les travailleurs agricoles se décomposent à leur tour en quatre groupes :

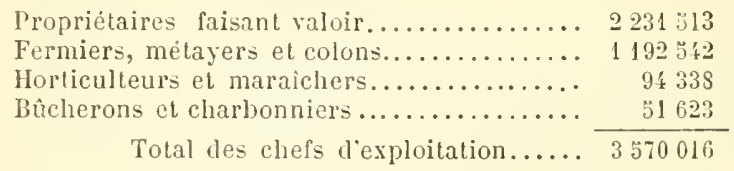

La proportion de la population agricole à la population totale variail, en 1891, de 20 p. 100 dans les Bouches-duRhône à 77 p. 100 dans le Lot el 74 p. 100 dans le Cantal. Les propriétaires ou exploitants de la petite culture varient, eux-mèmes, sur ce nombre, de 66 à $7 \%$ p. 100 suivant les départements.

La stalistique agricole ne donne pas les mêmes chiffres que le dénombrement :

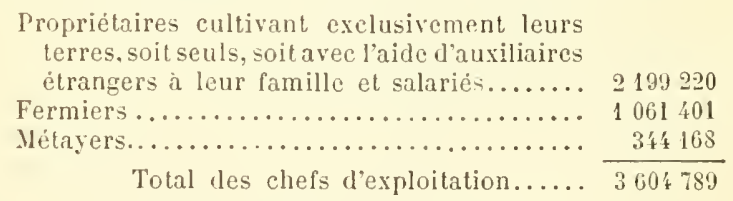

Cet écart de 34773 individus n'est pas expliqué. Il faut d'ailleurs ajouter au nombre des chefs dexploitation celui des journaliers ("58 900) propriétaires d'un petit bien qui, à ce titre, sont à la fois exploitants et salariés, d'où un chiffre global de 4193739 exploitants directs.

1. On est surpris de voir attribuer à la classe des bûcherons 4869 domestiques attaclıés à la personne (Résultats statistiques du dénombrement de 1891, 283); je n'en ai jamais vu dans les huttes de charbonniers, ni dans le Morvan, ni clans les Mlpes ou les Vosges. 
Si, d'autre part, on envisage le groupe des exploitants au point de vue exclusif de leur qualité de propriétaires, on trouve la classification ci-après dont les divergences avec les relevés similaires troublent quelque peu le raisonnement des amateurs de précision ${ }^{1}$.

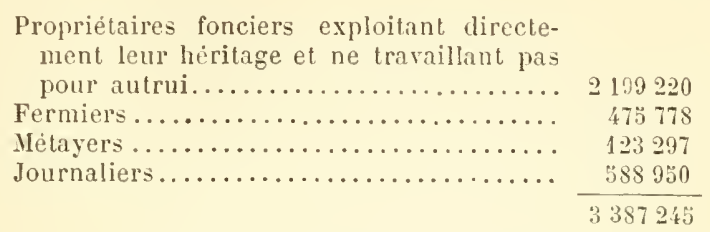

Sur ce nombre, quels sont les propriétaires détenteurs de la petite propriété, de celle dont l'étendue n'atteint pas 19 hectares? Le problème reste à résoudre.

Sa solution est d'autant plus difficile que ces petits propriétaires, s̋ils englobent tous les journaliers et métayers de cette catégorie, empiètent aussi sur le groupe des fermiers et même sur celui des exploitants de leur propre hérilage à l'exclusion de celui d'autrui.

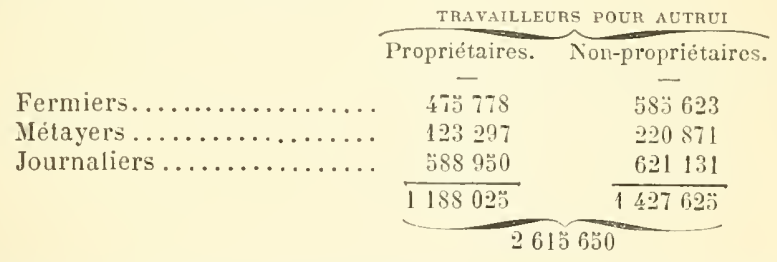

En 1892, le nombre des fermiers-propriétaires s'élevait à 238000 dans la région du Nord, descendait à 173000 dans le Centre et tombait à 63000 dans le Midi. Pour les métayerspropriétaires c'est l'inverse; de 600000 dans le Midi, ils passent à $\mathbf{4 8 0 0 0}$ dans le Centre et à seulement 9000 dans la région du Nord. Quant aux journaliers-propriétaires, leur répartition prend une autre allure ; c'est le Centre, de l'Atlantique à la frontière suisse qui en possède le moins (168000)

1. Ces derniers chiffres sont tirés de l'enquête agricole de 1892 (p. 386 de l'Introduction). 
tandis qu'ils sont plus nombreux dans le Midi (211000) et dans le Nord (207000). Ces lignes générales, à l'encontre de ce qui arrive d'ordinaire, ne sont pas sensiblement déformées si on serre la question de plus près en localisant les recherches par département ${ }^{1}$.

Le nombre des fermiers-propriétaires varie de 132 dans les Landes à 20963 dans la Somme; celui des métayers-propriétaires, de 12 dans les Ardennes, 18 dans la Seine-Inférieure, 33 dans la Meuse, 41 dans l'Aube à 4815 dans la Haute-Garonne, 4786 dans la Dordogne, 4468 dans le Lotet-Garonne, $437 \%$ dans les Basses-Pyrénées; celui des journaliers-propriétaires, de 600 dans les Haules-Alpes et de 647 dans la Mayenne à 2833 I dans l'Hlérault, 14324 dans l'Aisne, 17746 dans le Pas-de-Calais, $1439 \%$ dans la Somme. C'est dans cette classe de travailleurs salariés qu'on trouve le plus de petits propriétaires fonciers.

Les récentes stalistiques de l'Enregistrement, élaborées sur un plan nouveau avec une parfaite intelligence de la précieuse contribution qu'elles peuvent apporter à la science économique ${ }^{2}$, permettent de serrer de plus près encore la question et de se rapprocher autant que faire se peut de la réalité, sinon pour fixer le chiffre approximatif du groupe de la petite propriété, au moins pour apprécier son importance, sa virilité, l'énergie de ses initiatives.

En premier lieu, la consistance de la petite propriété à l'état de domaine patrimonial peut nous ètre révélée par le mouvement des valeur's successorales dont l'actil' ne cesse de croître. Les droits de succession sont, par excellence, un impôl de stalistique. La courbe de l'annuilé successorale est, en quelque sorte, le tracé graphique de la richesse générale du pays. On peut la comparer à un instrument de pré-

1. Voir la carte ${ }^{\circ}$ III (Modes d'exploitation et leur répartition géographique en France).

2. Quatre volumes du Bulletin de statistique de l'administration de l'Enregistrement ont déjà paru (1897, 1\$98, 1899 et 1901), sous l'impulsion de .I. Fernand Faure et par les soins de son collaborateur, M. Salefranque. Je leur emprunte les chiffres que je vais citer. 
cision qui saisit au passage, dans leurs manifestations les plus délicates, les valeurs que le jeu de la dévolution héréditaire amène successivement dans le champ de son objectif. Par la juxtaposition de ces épreuves particlles, on recompose l'image totale, sans doute plus ou moins déformée, mais suffisamment véridique pour autoriser des conclusions raisonnées sur l'ensemble de la situation.

Notons d'abord que la progression réalisée par l'annuité suceessorale, de 1826 à 1830 , ne tient pas uniquement à des causes d'ordre économique, mais aussi à des mesures fiscales qui ont élargi le champ sur lequel s'exeree le prélèvement de l’impôt. A partir de 18:il, la loi taxe les valeurs mobilières, les poursuit et les saisit à mesure que les entreprises industrielles et les emprunts publics se multiplient sous les formes les plus variées ${ }^{1}$.

La matière imposable, non pas accrue mais constalée par l'effel des lois fiscales de $18 \% 0,1863,1871$ et $187 \%$, grossit et s'enfle de plus en plus. Il faut remarquer que le développement survenu de 1830 à 1869 dans l'annuité des successions mobilières est le produit incontestable de causes économiques, de l'intensité de la vie commerciale et industrielle du pays, et l'on admet que les majorations artificiellement procurées par les mesures d'ordre fiscal n'ont eu qu'une portée secondaire; il en est tout autrement de 1870 à 1900 ; il s'est créé pendant cette période 28 milliards de titres nouveaux sur lesquels l'impôt a prélevé une large part ${ }^{2}$.

Ce qui ressort de cette comparaison, e'est la tendance croissante de l'annuité successorale mobilière à s'équilibrer avec l'annuité immobilière qui était, en 1826 , de 66 p. 100 contre 34 p. 100. En 1830 , elle tombe à 61 p. 100 contre 39 , et lécart s'atténue progressivement; en 1869, la part des

1. II. Neymarck, Une nouvelle évaluation du capital et du revenu des valeurs mobilières en France (1893); - Le morcellement des valeurs mobilières (1896).

2. On peut affirmer, sans crainte d'exagérer, que la fortune mobilière a supporté pour une très large part, depuis 1870 , le poids de nos désastres et que son élasticité a permis notre rapide relèvement national. (Journal de la Société de statistigue de Paris, 1899, p. 15ّ2.) 
valeurs mobilières s'élève à $4 \ddot{\mathrm{p}} \mathrm{p}$. 100, celle des biens fonciers s'abaisse à ăĕ p. 100; en 1891, l’équilibre est rompu, la proportion est renversée, le rapport pour 100 qui est de 68 pour les meubles, tombe à 36 pour les immeubles, exactement le contraire de ce qu'il était soixante-dix ans plus tôt, dans le premier quart du siècle!

Il ne faut pas croire cependant que celte forme nouvelle de la fortune privée soit une source originale, créée de toutes pièces par un afflux de richesses inédites; les valeurs mobilières ne sont que la représentation de biens réels, exploités par le travail agricole ou industriel, el majorés par le crédit.

La terre reste toujours le fonds productif et rémunérateur par exeellence; sans glisser dans l'exagération des physiocrates, ne pent-on pas dire que les titres des sociétés les plus variées ne sont en définitive qu'une hypothéque sur la propriété territoriale, de même qu'un emprunt d'État est une lettre de change tirée sur le crédit de la nation? La terre est ainsi mobilisée par les valeurs de papier, car son exploitation se retrouve à l'origine de toutes les entreprises; les porteurs de titres sont les commanditaires (actions) ou les créanciers (obligalions) du travail, et les spéculations de Bourse elles-mêmes, l'agio sur le papier de crédit, ont pour gage et pour objet la propriété foncière.

En 1898, le nombre des successions dans lesquelles se trouvent compris des immeubles ruraux a été de $246.5 \%$ et la valeur en capital de ces biens a dépassé 1 milliard 1/2 de francs ${ }^{2}$. En multipliant cette annuité spéciale par le coefficient qu'admel la science de la statistique ${ }^{3}$, c'est-à-dire par

1. Dans la seule année 1899, les six grandes compagnies de chemins de fer ont émis 446096 olıligations 3 p. 100 et $21 / 2$ p. 100 pour un capital versé de près de 192 millions de franes. Depuis 1883 , elles on dispersé lans l'épargne française 7 millions de titres pour un capital de 3 milliards en eliifres ronds.

2. Exactement 1519810898 franes sur un total de 6 milliards 621 millions pour toutes valeurs déclarées. (Bulletin de statistique de l'Enregistrement, 1899, p. 189.)

3. Journal de la Société de statistique de Paris, juin 1899, p. 193. 
43, on obtient comme évaluation en capital de la propriété rurale en France la somme de 68382 millions de francs, assez rapprochéc de celle de 77847 millions acceptée par les conclusions de l'enquète agricole de 1892. Il laut remarquer à ce propos, pour aller au-devant des objections, que pour ces calculs de très grande approximation, il est admis par les professionnels compétents que la fraude en matière de dissimulation ou d'atténuation de revenu se compense avec la non déduction du passif ${ }^{1}$.

Quelle est la part de la petite proprićté dans cet ensemble colossal? Les cadres actuels de la statistique ne permettent pas de le préciser; on ne peut que rapprocher certains éléments pour en tirer un peu de lumière.

On a essayé de déterminer le chiffre de la fortune privée par département en utilisant les données fournies par les successions et les donations, mais on n'obtient ainsi que des moyennes générales d'un pur intérèt didactique; le problème que je me pose ne sera résolu que lorsque les statistiques de l'Enregistrement permettront d'étudier les mouvements de la propriété, soit entre vifs, soit par décès, en les classant dans des cadres très multipliés afin de subdiviser les catégories par nature, par contenance, par valeur et par la condition des propriétaires. En prenant pour base les chiffres du dénombrement de 1896, on trouve que la fortune privée par tête, qui est de 18780 francs à Paris (où tous les ans des malheureux meurent de faim) et de 11563 francs dans Seineet-Oise, tombe à 1833 francs dans les Hautes-Alpes, à 1706 francs dans l'Ariège, et même à 319 francs en Corse, où il y a aussi de la misère, mais on n'en meurt pas comme dans les villes.

D'après les relevés de l'Enregistrement, le capital de la fortune immobilière de la France dépasse 107247 millions de francs dont presque le tiers $(32$ p. 100) centralisé dans le seul département de la Seine.

1. Le passif varie de 10 à 20 p. 100 de la valeur des biens déclarés. (Rapport de M. Cordelet au Sénat, du 9 juillet $1896, n^{\circ} 171$, p. 4 I et 47.) 
Comment se répartit cette valeur entre la propriété urbaine et la propriété rurale? L'enquête de 1898 le détermine :

\begin{tabular}{|c|c|c|}
\hline Propriété urbaine ......... & 509993 & $\begin{array}{l}4753 \text { p. } 100 \\
5245 \text { p. } 100\end{array}$ \\
\hline $\begin{array}{l}\text { Propriété rurale............ } \\
\text { En millions de francs. }\end{array}$ & $\frac{002401}{1072474}$ & $\frac{5 Z 45 \mathrm{p} \cdot 100}{10000 \mathrm{p} \cdot 100}$ \\
\hline
\end{tabular}

La valeur vénale de la propriété rurale est done supérieure à celle de la propriété urbaine; pour celle-ci, on ne constate de valeurs supérieures an milliard que dans 7 départements, y compris la Seine; pour la propriété rurale, nous en rencontrons 16 où ce chiffre est dépassé; en tète viennent la Seine-Inférieure (1 688 millions) et le Nord (1 942); 32 départements dépassent 300 millions; les Hautes-Alpes et la Corse (13 millions 4) sont les plus paurres. La statistique hypothécaire, quand on aura pris la peine de la faire, viendra corriger ces chiffres et en fixer la valeur absolue; mais nous n'y trouverons pas encore l'état réel de la petite propriété dont le ròle économique tend à prendre une prépondérance marquée dans les conditions de progrès de la sociélé contemporaine et dont, par conséquent, il est urgent d'étudier les mouvements alternatifs jusque dans les oscillations minuscules qui ont paru jusqu'ici les plus négligeables.

$\mathrm{Si}$, après aroir recherché quelle élait la consistance de la propriélé rurale, on essaic de déterminer l'intensité de ses mouvements, la mobilité el la périodicité de ses transferts, on devine dans quels documents on pourra plus tard trouver des éléments de certitude, mais, quant à présent, ces documents restent muets sur le problème qui nous oceupe.

Depuis 1826 jusqu'en 1896 , le capilal engagé par les acquisitions foncières a presque doublé, de 1004 millions de francs en $18 \geq 6$, a 1840 millions en $189 \%$. Le nombre de ces mutations (acquisitions d'immeubles, urbains et ruraux, à titre onéreux) a diminué depuis 1878 , spécialement pour les biens urbains ${ }^{1}$.

1. De 873355 contrats en 1878 , le nombre en est tombé à 59966 en 1890, pour se relever à 713913 en 1893 . (Bulletin statistique de l'Enregistrement, 1898, p. 166.) 
Le seul document qui permette de conclure est le relevé des ventes immobilières de l'année 1894, classées par nature (urbainc ou rurale) et par catégories de prix, publié par l'Enregistrement en 1897. J'en tire les chiffres qui se rapportent à la présente étude, c'est-à-dire aux mouvements de la très petite el de la petite propriété.

Mais, au préalable, l'Enregistrement n̈indiquant pas les contenances, il faut $\mathrm{y}$ suppléer en calculant l'équivalence des prix par les données de l'enquète agricole de 1892. La valeur vénale de l'hectare de terre étant au maximun ( $1^{\text {re }}$ classe de chaque culture) et au minimum ( ${ }^{\circ}$ classe) celle du tableau ci-après, en additionnant le quotient du total des deux extrêmes divisé par $\ddot{3}$, puis en prenant la moitié du résultat, on a la moyenne ${ }^{1}$ de la valeur d'un hectare de terre, chiffre éridemment exagéré puisqu'il peut varier pour la seule nature en labour dans le même département (Ain) de 801 francs à $327 \%$ francs, et que je ne prends que comme base conventionnelle d'un calcul approximatif.

NATURE DES CULTURES

\begin{tabular}{|c|c|c|}
\hline \multirow{3}{*}{ 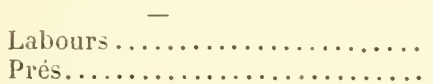 } & - & - \\
\hline & $2 \$ 66 \mathrm{fr}$. & $668 \mathrm{fr}$. \\
\hline & 3730 & 1008 \\
\hline 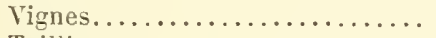 & 3359 & 1004 \\
\hline 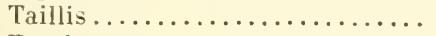 & 1350 & 421 \\
\hline 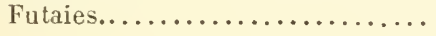 & 2093 & 701 \\
\hline & $\mathrm{fl}$. & \\
\hline
\end{tabular}

D'où il ressort que si l'hectare coûte 1720 francs, les כ0 0 ares reviennent à 860 francs el les $2 \ddot{3}$ ares à 430 francs.

La très petite propriété (au-dessous de 1 hectare) paiera

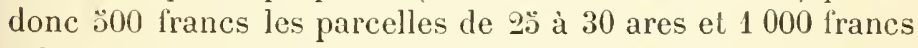
celles de 40 à 60 ares, la contenance moyenne par parcelle variant dans le même département (Ain) de 11 ares à Tré-

1. On m'excusera, malgré mon peu de goût pour les moyennes, de les employer ici, mais ce n'est que pour la facilité de la démonstration.

$\frac{13400}{\ddot{\jmath}}=2680$ et $\frac{3802}{\ddot{\jmath}}=760 ; \frac{2680+760}{2}=1720$ francs à l'hectare moyen. 
voux à 87 ares à Nantua; et la petite propriété (de 1 à 10 hectares) paiera $\$ 600$ franes le petit domaine de $\ddot{z}$ hectares à cultures variées ${ }^{1}$.

Or, pendant l'année 1894, il a été enregistré 586787 acquisitions de biens ruraux dans les conditions qui suivent :

\begin{tabular}{|c|c|c|c|c|c|}
\hline \multirow{2}{*}{$\begin{array}{l}\text { CATÉgORIES } \\
\text { de } \\
\text { PBIX }\end{array}$} & \multicolumn{2}{|c|}{$\begin{array}{c}\text { VENTES DIMMEUBLES } \\
\text { RURAUX }\end{array}$} & \multicolumn{2}{|c|}{$\begin{array}{l}\text { VENTES D'INMEUBLES } \\
\text { URBAIN ET RURAX } \\
\text { pour un prix unique }\end{array}$} & \multirow{2}{*}{$\begin{array}{c}\text { TOTAL } \\
\text { DES VENTES } \\
\text { DES DEUX } \\
\text { CATÉGORIES }\end{array}$} \\
\hline & $\begin{array}{l}\text { Nombre } \\
\text { des ventes }\end{array}$ & $\begin{array}{c}\text { Total } \\
\text { des prix }\end{array}$ & $\begin{array}{l}\text { Nombre } \\
\text { des ventes }\end{array}$ & $\begin{array}{c}\text { Total } \\
\text { des prix }\end{array}$ & \\
\hline \multirow{6}{*}{$\begin{array}{l}\text { Au-dessous de } 500 \mathrm{fr} \text {. } \\
\text { De } 500 \text { à } 1000 \text { fr.... } \\
\text { De } 1000 \text { à } 5000 \text { fr... } \\
\text { De } 5000 \text { a } 10000 \mathrm{fr} .\end{array}$} & & millions & & millions & \\
\hline & 325162 & 62.3 & 5076 & 1,5 & 330238 \\
\hline & 100763 & 70 & 5158 & 3,8 & 105911 \\
\hline & 118977 & 2 占, 2 & 10813 & 26,6 & 129792 \\
\hline & 17983 & 116 & 2851 & 20 & 20836 \\
\hline & 362887 & 493,7 & 23900 & 51,9 & 586787 \\
\hline
\end{tabular}

La petite propriété a done dépensé, dans la seule année 189., plus de $\$ 30$ millions $1 / 2$ de franes pour 686787 acquisitions.

L'absence de toute stalislique hypothécaire sérieuse publiće jusqu'à ce jour ne permel pas de savoir quels furent, sur cette somme considérable, les prix payés comptant et ceux qui sont renus grossir la dette agraire. Toutefois, me statistique de l'année 1898 accuse pour cetle année-là 293786 inscriptions d'office prises pour conserver le privilege du vendeur, conformément à l'article 2108 du Corle civil. On peut en dédnire, et les professionmels s'accordent sur ce point, que la moitié au moins des acquisitions de la petile propriélé se l'ail à crédit, à des échéances qui varient de trois à ciuy ans.

Telle est, en France, la condition actuelle de la pelite propriété. Quant à la portée morale de cet élat de choses, je ne puis mieux faire que de citer l'opinion de M. de Foville.

1. Bulletin de slalistique du ministère des Finances, décembre 1883, page 63̈1. 
Le paysan français aime à dormir sous un toit qui lui appartienne; et quand le terrain sur lequel eet humble toit projette son ombre lui appartient aussi, sa bêclıe et son arrosoir en savent tirer plus de profit que ne ferait la charrue d'un habile fermier. Voila un homme qui, si peu propriétaire qu'il soit, jouit l'éllement de l'être; et, s'il est plus heureux comme cela, il y a aussi chance qu'il soit meilleur.

VIIl. - Origines historiques des mouvements alternatifs de dislocation, de concentration et de morcellement qui ont, tour à tour, favorisé ou entravé la constitution de la petite propriété.

La petite propriété individuelle est un produit de la liberté; la très petite propriété est une conséquence directe de la paix publique et de l'esprit d'indépendance des travailleurs.

En France, la petite propriété foncière est aussi ancienne que la nation; elle disparut au $\mathrm{r}^{\mathrm{e}}$ siècle dans le cataclysme social qui obligea les habitants à abdiquer leurs droils et à céder leurs biens pour acheter à ce prix la sécurité de leurs familles. C'est une évolution à la fois économique et politique, qui se retrouve à des époques différentes dans la vicille histoire de certains peuples et dont l'un des exemples les plus curieux et les moins connus date de l'ancienne Égypte ${ }^{1}$.

Je ne me mêlerai pas au débat qui dure encore sur ce que furent les formes primitives de la propriété; c’est un

1. Lorsque le juif Joseph eut expliqué au Pharaon le songe des sept vaches grasses et des sept vaches maigres, et qu'il eut élé investi du pouvoir, il leva un impòt de 20 p. 100 en nature sur tous les prodnits de la terre et les entassa dans les greniers publies; lorsque la famine fut venue, Joseph vendit le blé aux Egyptiens; la seconde année, l'argent épuisé, ils échangèrent leur bètail contre du blé pour vivre; la troisième année, n'ayant plus que les corps et la terre, ils s'offrirent comme esclaves pour ne point mourir de faim. Toute l'Égypte, excepté les biens des prêtres, appartenant ainsi au roi, Joseph distribua des semences aux anciens propriétaires du sol pour qu'ils pussent cultiver et leur imposa une redevance de 1/5. (La Genese, chap. xuvil.) La famine produisit ainsi en Egypte le même résultat qu'en France, au $1 \mathrm{x}^{\circ}$ siècle de notre ère, la terreur des Northmans. 
problème qui exige une étude spéciale. M. Sumner-Maine et M. de Laveleye ont soutenu que chez tous les peuples, à l'origine, lindividu avait droit à unc part dans le fonds productif mis en commun et possédé collectivement par des tribus, des communaulés ou des groupes.

M. Fustel de Coulanges et M. Belol ont contredit celte thèse el démontré que si la propriété collcctive se rencontre chez certains peuples à un point de Jeur histoire, il en est d'autres, telles les populations de la Grèce ct de l'Italie, qui dès l'antiquité la plus reculée ont toujours connu el pratiqué la propriété privée et où il n'est resté aucun souvenir d'un temps où la terre ait été commune. II. Julian Corbctt a même établi récemment, en šinspirant de faits et d’aperçus nouveaux, qu’il n’est pas certain que les communautés des Gaules aient cu généralement une origine germanique, el il soutiendrait volontiers l'opinion contraire.

Les Germains, dit-il, en empruntant aux Celtes le régime de la proprièté collective, l'ètablirent même sur des terres que les Celtes n'avaient pas cultivées avant l'invasion et qui furent occupées par les immigrants lorsque, leur population ayant augmenté, les terres mises en valeur par Ies Gallo-Romains ne leur suffirent plus.

Ce régime de propriété fut seul pratiqué et maintenu jusqu’à l'époque où de nouvelles générations s'étant élevées et ayant acquis des connaissances agricoles qui faisaient défaut aux Barbares primitifs, la forme de la propriété privéc prit naissanec et se développa comme plus en harmonie que la propriété collective avee le carnetère et les muturs des Germains si fortement imprégnés du sentiment individualiste ${ }^{3}$

D'après les partisans de cefte these, ce qui pourrait faire penser que lelle a élé l'évolution réaliséc par les formes primitives de la propriété grermanique, d'abord individuelle comme la propriété gréco-latine, puis collective par un retour en arrière, apres avoir pris contact avec les Celles, c'est que le signe caracléristique des pays ou le régime de la propriété collective a toujours été en vigueur, c'est-à-dire l'existence des villages, ne se rencont l’c pas dans les contrées

1. Bullelin du Comilé des travaux historigues et scientifiques, 1888 , p. 11 i: : Economie sociale. 
qui ne furent habitées, à l'origine, que par les Giermains seuls, sans mélange avee d'autres races.

En ces matières obscures, si ingénieuses que soient les hypothèses et si vraisemblables qu'elles paraissent, il ne faut pas s'aventurer dans des conclusions absolues ni affirmer que la propriété collective des terres est d'origine purement celtique. L'argument tiré des villages est singulièrement atténué par le fait incontestable, dans les Gaules, de la dislocation de la grande propriété romaine et de la transformation des ville où le groupe des exploitants, artisans, gens de métier, devint un centre d'habitation ordinaire, une fois son affectation spéciale disparue, de mème que le territoire du domaine héréditaire, l'alleu, devint la paroisse de l'ancien régime, la commune d'aujourd'hui.

La propriété collective du sol, quelle que soit la place qui lui appartienne dans la ehronologie des institutions humaines, n'a jamais conslitué qu'un régime inférieur, imposé par les circonstances, et destiné à disparaître tôt ou tard, à mesure que les conditions de l'existence s'améliorent et que la notion de la dignité du citoyen, le sentiment de I'individualité prennent le dessus sur la nécessité du groupement primitif en vue de la défense commune.

L'histoire locale ne constate-t-elle pas, dans certaines régions, que les communautés d'habitants dont notre vieille France a conservé les traces ', ne constituaient qu'une dérivation, une forme plus compliquée de l'exploitation de l'héritage familial, de l'unité primitive, par le maintien de l'indivision? Il ne faut pas confondre, en effet, deux états bien différents, l'un économique et l'autre politique, auxquels on applique souvent le mème nom. On peut entendre par communautés d'habitants ou communautés de villages, ces municipalités de formation rudimentaire qui fonctionnent d'elles-mèmes avant d'être organisées par des contrats ou des privilèges. Les habitants réunis en communauté

1. On trouve une bibliographie complète des travaux publiés sur ce sujet aux p. $29 \dot{x}$ et 293 du Bulletin du Comité des travaux historiques de l'année 1893 : Économie sociale. 
l'administrent eux-mêmes directement, tant qu'ils sont en petit nombre ou qu'une autorité supérieure a limité leur indépendance en leur imposant des chefs chargés, dans une mesure plus ou moins étendue, de la gestion des intérêts communs; ils n'ont pas de magistrats, mais seulement des délégués, des agents d'exécution, des commis à ceci ou à cela, suivant la vieille expression, de simples mandataires, nommés sans être investis d'une autorité normale et permanente; et les chefs de famille restent toujours prêts à se réunir pour délibérer sur des résolutions nouvelles ${ }^{1}$.

Tout autres sont les communautés d'habitants qui possèdent en commun et dont l'héritage primitif, accru par des acquisitions, des dots, des apports, constitue à mesure que la famille-souche a produit des rejetons plus nombreux, une sorte d'exploitation en commandite qui se cultive et se conserre au mieux des intérêts de la collectivité. A mesure que l'érudition locale fouillera cette mine encore peu cxplorée ${ }^{2}$, on s'apercerra que les exemples n'en sont pas aussi rares qu'on le pense. Il ne s'agit point ici d'une loi civile ou d'une tradition organisant la propriété collective, encore moins d'étrangers renant s'associer en vue de défrichements ou d'entreprises agricoles, comme le firent les Ordres monastiques, mais simplement d'une famille dont les branches, se multipliant arec le temps, couvrent le sol et l'exploitent à fond; de fils, de petits-fils qui, durant plusieurs générations, restent unis, groupés, associés pour continuer l'oeuvre des ancêtres, el ne se séparent que lorsque l'héritage ne sulfit plus à les nourrir ${ }^{3}$. Il y a, dans cette fixité plus ou moins durable du domaine paysan, consenlic d'accord, une ressemblance typique arec l'inaliénabilité du domaine seigneurial pour maintenir la puissance du chef contre ses

1. Bulletin du Comité des travaux historiques, 1894, p. 2l et 309 : Des institutions municipales dans l'organisation de l'ancienne France.

2. Ilide, année 1886, p. 2'.

3. Notice sur le domaine des Tocquart dans les Vosges (Bulletin du Comile des travaux historiques, année 1886, p. 41). 
propres faiblesses, précaution devenue l'un des principes de la monarehie française ${ }^{1}$.

Dans tous les cas, il faut écarter des faits observés toute idée communiste et n'y voir que la mise en pratique de traditions dont l'origine historique remonte bien au delà des temps féodaux, et dont l'existence juridique ${ }^{2}$ dans la législation allemande contemporaine doit servir à éclairer le passé. En Allemagne, et surtout dans l'Allemagne du Sud, un régime spécial détermine la dévolution successorale des biens ruraux.

La pensée qui le fit établiı législativement pour consacrer la tradition et le fait est pure de toute iđée de féodalité et vise uniquement l'intérèt de l'exploitation agricole el la perpétuité de la famille rurale. C'est le Hoferecht. Le Bauerhof ou IIof est un logis de ferme arec lous les champs qui en dépendent; il est considéré comme indivisible et cette inviolabilité résulte tantòt de la coutume, tantòt de la volonté du propriétaire qui peut le créer comme l'Américain du Nord peut instituer le Home-stead exemption au profit de son héritage ${ }^{3}$. Ces mesures, et celles analogues qu'on pourrait trouver dans d'autres pays ne sont pas un retour à la collectivité des civilisations à leur début; elles marquent, au contraire, le respect de la propriété individuelle, de l'héritage, et le vif désir d'en maintenir l'intégrité.

Les communautés d'habitants du xirl ${ }^{\mathrm{e}}$ et du xiv ${ }^{\mathrm{e}}$ siècle sont en germe dans les communautés agricoles du $\mathrm{Ix}^{\mathrm{e}}$ siècle, mentionnées dans le Polyplyque d'Irminon, et il n'est pas douteux que les institutions municipales ne soient sorties après un long travail de ces intérêts communs groupés avec une cohésion plus ou moins étroite, suivant l'exigence des

1. Édit de René de Lorraine sur l'inaliénabilitè du domaine ducal, en date du 29 décembre 1449.

2. Les coutumes ont été traduites en lois, qui n'ont entre elles que de légères diffẻrences, dans le Schaumbourg-Lippe en 1870, l'Oldenbourg en 1874, le Hanovre en 1880, le Lauenbourg en 1881, la Westphalie en 1882, le Brandebourg en 1883, la Silésie en 1884, le Schleswig-Holstein en 1886, la Hesse en 1887, le grand-duché de Bade en 1888, etc.

3. M. Levasseur, L'agriculture aux États-Unis, 1894, p. 467. 
temps et des lieux. Mais l'unité parcellaire de ces groupements, la cellule pour ainsi dire de ces corps organisés, a toujours été l'héritage privé, la propriété individuelle, d'autant plus respectable qu'elle était plus petite et sans défense.

L'origine des communaux se rattache à toutes les transformations du régime primitif de la propriété rurale, et, lorsqu'on aborde cette question particulière si étroitement rattachée par la force des choses à celle de la petite propriété individuelle, il ne faut pas perdre de vue les efforts de notre ancienne législation française pour provoquer la constitution el les initiatives de la propriété individuelle tout en sauregardant les intérèts communs du groupement rural, source des familles nouvelles, souche de rejetons sans nombre '.

Lédit de juin 1769 sur le parlage des terres communales en Lorraine, celui de 1774 sur le même objet pour la province de Bourgogne, et d'autres encore, sont particulièrement instructifs parce qu ils affirment l'intention du gouvernement de favoriser les progrès de l'agriculture en développant la propriélé privée. Ils visent la mise en valeur de marais ou de lerres ineultes au moyen de partages de jouissance entre les habitants chefs de famille, ì la condition expresse que les parts qui tomberont en ligne collatérale ou qui deviendront vacantes, de quelque manière que ce soit, feront retour à la communauté el seront adjugées anx ménages les plus anciens non encore pourvus 2 .

La petite propriété gallo-romaine disparut sous le coup des terreurs et des désastres que répandirent des Alpes à l'Océan el de la Meuse aux Pyrénées les invasions incessam-

1. N. Rameau, Les masures de la glèbe el les charles de franchises (Bulletin du Comilé, 1892, I, p. 93).

2. L'arrêt du Conseil du 25 février 1779 réglemente l'attribution des parts de marais lans la province d'Artois et montre quelle fuc la portée économique de ces sages mesures. Les lois révolutionnaires sulstituèrent, pour un moment, le partage a titre de propriété alu partage à titre de jouissance et la livision par tête à la division par feu; l'arrêté des consuls du 9 fructidor an X reınit en vigueur l'arrêt de 1779. 
ment répétées des pirates du Nord, au cours du $\mathbf{I x}^{\mathrm{e}}$ siècle.

L'histoire des Carolingiens, aussi bien que eelle des Valois pendant la guerre de Cent Ans, racontent les erises les plus cruelles et les plus persistantes qu'ait subies le peuple français.

Puis la paix revenue, un calme relatif rétabli, il fallut réglementer le nouvel ordre économique, et les vastes domaines créés par la peur se démembrèrent de fait par un nouvel aspeet de ce problème permanent de la lutte pour la vie qui, depuis l'origine des choses, domine l'humanité.

Depuis deux siècles, tout le monde était pauvre et vivait de misère; comme après toutes les grandes secousses, on se plut à respirer; comme après les rudes épreuves on voulut bien vivre; et les chef's militaires, les seigneurs, pour utiliser les vastes étendues dont le malheur des peuples les avait fait propriétaires, les restituèrent à leurs anciens possesseurs à la condition de participer au produit de leur culture et de leur élevage. Les chartes de franchise, du xil ${ }^{\circ}$ au $x^{\prime} I^{\circ}$ siècle, vinrent détruire l'érlifice hâtivement construit, du $\mathrm{IX}^{\mathrm{e}}$ au $\mathrm{XI}^{\mathrm{e}}$ par les chartes de sauvegarde. La petite propriété couvrit de nouveau la France, et les procédés en usage à l'époque féodale la firent encore plus moreelée, plus menue qu'elle ne l'était avant la prodigieuse débâcle sociale dont Charlemagne mourant avait pressenti l'approche.

Le bail à cens ${ }^{1}$ et la charte de franchises marchèrent de pair, quoiqu'on se préoccupât davantage de libérer la terre que l'homme, et la période des Croisades permit aux laboureurs d'accroître leur patrimoine récent par l'acquisition de parcelles détenues par les soldats, les pèlerins et même les seigneurs appauvris par les frais des expéditions lointaines.

L'histoire seule peut éclairer ces époques obseures dont l'étude des contrats privés et des terriers ecclésiastiques permet de deviner avec quelque certitude l'étonnant état économique.

1. L'analogue de l'Enzel des pays musulmans, notamment en Tunisie. (Revue algérienne, 1899, décembre.) 
Les villx, les seigneuries, les paroisses et enfin les seigneuries se substituant aux paroisses, ne sont que les formes successives d'une même exploitation agricole où le droit du propriétaire, le procédé de travail et le partage des produits de culture ont varié arec les temps. La démocratie rurale n'est point une invention moderne; elle a existé à toutes les époques et, depuis les origines les plus reculées, il y a eu lutte entre celui qui possède le sol et celui qui le met en valeur. Ce dernier l'a presque toujours emporté. Ne faut-il pas en conclure que si, dans les pays de petite culture tout au moins, la terre appartenait aux familles qui l'exploitent directement, tout antagonisme de classe y disparaitrait?

La très pelite propriété sortit triomphante de ces quatre siècles de désorganisation sociale pendant lesquels toute règle étant rompue, toute hiérarchie brisée, tout droit aboli sauf celui de la force, les individus sans ressources et sans défense se donnaient à merci à qui voulait ou pouvait les nourrir ${ }^{1}$. Pendant cette période confuse, où la diversité des situations se complique du manque d'unité dans les vues, l’isolement élant universel, les princes, les évêques, les paysans, les citadins, trouvent des expédients différents, des moyens variés de corriger les périls et la misère par la solidarité des intérèts. Jamais on ne pratiqua davantage la participation et la mutualité, car contrairement à ce qu'on a cru longtemps, du fail des errenrs propagées par les encyclopédistes du xrmin siècle, lorsque la féodalité mit de l'ordre dans ce chaos, ce ne ful pas aux dépens mais au profit de la liberté.

Le maître du sol, n'en tirant qu'un méchant parti par le travail servile, eul recours aux plus sûrs excitants de l'initialive et de l'effort, il mit l'homme aux enchères et le paya d'autant plus cher que son concours lui était plus indispensable ${ }^{2}$.

1. Voir ci-dessus les faits analogues que rapporle la Genese vers l'an 1840 avant l'ère chrétienne : Nil novi sub sole.

2. M. de Saint-Genis, Histoire économique d'une commune rurale en Bourgogne du $X I^{\circ}$ au $X I X^{\circ}$ siecle (Réforme sociale lu $1^{\text {er }}$ juillet 1896). 
En effet, vers le xir siècle, l'homme est devenu plus précieux que la terre, plus précieux que l’or, car il représente le travail permanent et produetif. Pour l'attirer, on lui offre la liberté; pour le retenir, on lui promet la sécurité; pour exciter son initiative, on lui coneède le droit d'héritage. Aussi, quelle poussée d'énergies et de labeurs du xrre au xIV ${ }^{\mathrm{e}}$ siècle!

Rien de plus ingénieux que les méthodes variées qui, suivant les régions, sur les bords de la Meuse et du Rhin, dans les vallées de la Seine, de la Loire, du Rhòne, dans les Cérennes, les Alpes ou les Pyrénées, sur les eoteaux arides du Languedoe et de la Provenee comme dans les herbages de Normandie, les chàtaigneraies du Limousin, les hauts plateaux de l'Auvergne et du Larzae, approprièrent la règle à la eulture et l'homme à la règle. Peu à peu, la coutume se substitua à l’arbitraire et une idée se dégagea de ees compromis, de ces transactions, un principe surgit de ees calculs et de ees contrats: l'idée du droit de propriété dérivant du devoir de eulture; le prineipe de l'abonnement équitable remplaçant le prélèvement eaprieieux sur les fruits du travail.

Le moreellement du sol, eonséquence immédiate de l'affianchissement et de l'accensement, a donc constitué définitivement la très petite propriété individuelle et, par là, formé le peuple laborieux des paysans de France; mais, ici encore, il y a des distinctions à faire et des faits à noter qui ne sont pas contradietoires mais se complètent, s'expliquant les uns par les autres ${ }^{1}$.

L'affranehissement des tenaneiers serviles issus de la panique du $\mathrm{x}^{\mathrm{e}}$ siècle n'a pas été le seul facteur de la création d'une elasse très nombreuse de laboureurs, vignerons et forestiers libres; la constitution rurale dans ses assises inférieures a eu aussi une autre origine que la transtormation graduelle du serf en tenancier féodal, puis en abonné

1. Mémoire sur la propriêté rurale en Bourgogne (Bulletin du Comité des travaux historiques, annėe 1896). 
libre. De même que certaines seigneuries sont demeurées tout entières serves jusqu'à la Révolution ${ }^{1}$, de même il y eut, au XIII siècle, des paysans engagés libres.

Des familles, étrangères à la seigneurie ou au domaine primitif, attirées ou transplantées, y firent souche, à certaines époques, dans une plus forte proporlion peut-être que les habitants d'origine. Le serf affranchi pouvait se déplacer en faisant l'abandon de sa tenure; quant an tenancier servile, sil demeurait assujetti au droit de poursuite, il pouvait s'en dégager par la faculté de l'entrecours ou rompre le lien qui l'attachait à la glèbe en payant le droit de forfuyance, de même que le débiteur d'une rente foncière pouvait user du droit de díguerpissement en renonçant judiciairement à la propriété de l'immeuble grevé. Ces déracinés, quittant par accorl ou par fraude une seigneurie pour s'instalter dans une autre, ne le faisaient qu'à bon escient, sans abdiquer leur liberté civile, et devenaient tenanciers à prix débattu².

Bref, du $x_{1}^{e}$ au $x^{*} v^{-e}$ siècle, abstraction faile des différences qui séparent l'exploitation par le serf' censitaire ${ }^{3}$ de celle du paysan affranchi devenu propriétaire par le bail à cens, il se produisit un phénomène qui ne se verra plus; l'abandon de la terre en toute proprićté à quiconque voulut en prendre, moyennant un très faible intérèt annuel à payer aux anciens détenteurs. Le paysan, affranchi ou libre, aequiert le fonds sans rien débourser el profitera des plusvalues. Il nétait pas de conditions plus favorables pour provoquer l'émiettement des grands clomaines et pousser à la

1. Monographie de la commune de Chassey-en-Auxois (Côte-d'Or). (Bulletin de la Société des sciences historiques et naturelles de Semur, 1897.)

2. On trouve peu de contrats relatifs i ces accords parce que la plupart des coutumes ratiliaiont les conventions verbales par le fait de la résidlence de l'an et jour, mais on y fait de fréquentes allusions dans les procès civils, et les archives des Parlements et des Cours des comptes abondent en indications sur cette pratique.

3. Le serf censitaire, qui n'est pas maitre de son corps, ne peut l'ètre de sa tenure : il est taillable et corvéable à inerci. 
culture intensive dans la mesure où le permettait l'ignorance d'un temps oì les biens ecclésiastiques seuls étaient exploités arec méthode et intelligence ${ }^{1}$. La misère du $\mathrm{xv}^{\mathrm{e}}$ siecle, les guerres du $\mathrm{xvI}^{\mathrm{e}}$ et de la fin duxvir ${ }^{\mathrm{e}}$ siècle ralentirent ce mourement, car dans toutes les crises les capilalistes ont profité de l'appaurrissement des miséreux, mais il n’en resta pas moins assez fort pour que la législation se soit efforcée, jusquà la moilié du xvir ${ }^{\mathrm{e}}$ siècle, sans succès d'ailleurs, d'empècher les te:res nobles de passer aux mains des roturiers.

Parallèlement à la constilution de la très petile propriété par les censives, il se forma des héritages plus élendus par les groupements de parcelles au moyen d'aequisitions. L'émiettement eut pour contre-partic, dès le xive siècle, la reconstitution d'exploitations moyennes. Une famille laborieuse et économe achetait peu à peu, accumulant ses ressources et attendant les occasions, des parcelles souvent fort éloignées les unes des autres et dont elle réussissait rarement, par voie d'échanges, à corriger la dispersion. On a cherché à expliquer pourquoi, dans certaines régions où la constitution cadastrale des domaines a peu changé, on rencontre des champs divisés en lanières ${ }^{2}$ et enchevêtrés comme à plaisir dans d'autres propriélés. La cause en est, peutètre, notamment en Lorraine et en Bourgogne, dans les traditions de l'ancienne science agronomique. Les terres cultivables étaient distribuées en trois saisons ou années alternativement consacrées au blé, à l'avoine, au repos (jachères) ; les contenances des parcelles devaient être également réparties entre les trois eatégories de la rotation culturale, et il importait peu que les pièces fussent réunies puisqu'elles n'étaient ni labourées, ni ensemencées, ni moissonnées simultanément. Cela peut expliquer le maintien de la division, mais la cause

1. C'est aux moines de Champagne, de Bourgogne et de Languedoc que la France doit la création de ses vignobles les plus renommés.

2. Voir les plans annexés au Mémoire de M. de Saint-Genis sur la propriété rurale en Bourgogne, documents XIV et XV (Bulletin du Comité des travaux historiques, 1896). 
première doit en être cherchée dans le principe d'égalité qui présida au lolissement des tenures primitives ${ }^{1}$.

Vers la fin du $x v^{e}$ siècle, dès que la sécurité relative de l'exploitation agricole eut été établie, on s'efforça de consolider les droits apparents dont les détenteurs du sol étaient en possession après une crise très longue pendant laquelle les lamilles avaient été dispersées, les fortunes compromises et les titres perdus. Les seigneurs et les particuliers s'y trouvaient également entraînés; ceux-ci, pour se défendre contre des revendications toujours possibles et se créer une protection avec le propre intérèt de celui dont ils se déclaraient débiteurs; ceux-là, pour rétablir leur crédit et recenser les hommes, cadastrer les propriétés, en vue des taxes futures. Les différents droits féodaux s'unifiant el s'absorbant dans le prix primitif de la terre, on trouvail déjà légitime de laxer le sol, dans ses produits et dans ses transferts, au profit d'une entité nouvelle, le prince, c'est-à-dire l'État.

Le mourement général d'enquêtes, qui passionna les populations rurales de France de 1492 à 1508 , eul pour conséquence le renouvellement des terriers seigneuriaux et pour résultal le constal du droit des hommes libres ou des censitaires à posséder les hérilages dont ils étaient possesseurs. Ce qui est caractéristique, ce qui prouve le nombre et la force sociale des paysans propriétaires, ce sont les termes par lesquels les édits justifient l'ordre d'enquêter.

Charles VIll preserit la recherche des droits du prince, à cause de l'usurpation et violence des seigneurs voisins sur la franchise des hommes qui, en les molestant, les forçaient à déserter leurs herritages ou ì relomber bénévolement dans la main-morte pour ne point délaisser leur's meix, familles et cultures. Lonis XII ordonne de refaire le terrier de la chattellenie de Semur, pour la défense des droits de nos hommes, villes el villages molestés par les usurpations des seigneurs voisins (Leltres patentes du 19 février 1499). Charles VIII et

1. Loco cilato, à propos du cadastre de la commune de Vic-de-Chassenay en Auxois. 
Louis XII, à l'extrème limite du $\mathrm{xr}^{\mathrm{e}}$ siècle, renouvelaient pour les villages ce que Louis VI arait fait pour les communes au $\mathrm{xII}^{\mathrm{e}}$ siècle. La royauté prenait la défense de la propriété paysanne comme trois cents ans plutòt elle avait soutenu les libertés bourgeoises contre l'arbitraire féolal.

Pour abréger, je m'en tiendrai à cette région de la Bourgogne; les conclusions qu'on peut tirer de l'examen des terriers et de leur comparaison avec les ròles des vingtièmes et de la taille, suivant les cas, ainsi que des archives du Parlement de Dijon, sont applicables, pour cette époque, avec de légères nuances, à la plupart des régions de la France, sauf dans l'Ile-de-France et l'Artois.

Cinq faits principaux ressortent de l'étude des textes : l'extrème division du sol, la mobilité de la propriété, la modicité des redevances censitaires, la quantité de villages et d'exploitations isolées ruinés par les Routiers et les Écoreheurs, la tendance des bourgeois à acheter des biens ruraux. En voici sommairement les conséquences au point de vue des conditions de la petite propriété.

A Forléans, où Bussy-Rabutin eut plus tard un rendezvous de chasse, Jean Pertuisot, bourgeois de Semur, possède une exploitation dont les 27 pièces, d'une contenance de 6 h. 73 d'aujourd'hui, soil 25 ares en moyenne à la parcelle, affermées à plusieurs cultivateurs, ne sont pas agglomérées mais éparses ou enclavées dans les héritages voisins; il les a acquises dans le cours de trois années de sept propriétaires qui ont quitté le pays. (Titre de 1494.)

Le domaine de Jean Turreau, à Cernois, est un exemple de la façon dont s'évanouissait un lhéritage lorsque, par le malheur des temps, la culture y devenait impossible. Le domaine, acheté par un homme serf, à une époque indéterminée, au prix d'un boisseau d'argent, est divisé, à la mort du premier propriétaire affranchi, entre ses héritiers dont le nombre et la qualité sont inconnus. Une portion de cet héritage passe à deux frères communs en biens et, à leur mort, à leurs deux fils qui en firent entre cux le partage : cette portion du domaine primitif, un instant divisée du vivant des 
deux copartageants, se réunit de nouveau par la mort de l'un qui fit tout passer sur la tête de l'autre. Celui-ci, le Jean Turreau du Terrier (rédigé de 1484 à 1504), dissipa l'héritage, qui fut vendu en justice ${ }^{1}$; en 1503 , celte fraction du meix primitif se trouvait subdivisée entre les mains de 15 nouveaux acquéreurs ${ }^{2}$.

Le nombre considérable des mutations par vente, accensement ou partage est constaté dans les contrats du $\mathrm{xv}^{\mathrm{e}}$ siècle et l'on en parle comme d'un fait habituel et normal, affectant les fiefs aussi bien que les terres de roture. Pour la petite seigneurie de Chassey, je trouve dans les minutes notariales, pour une population de 36 teux, de 1529 à 13049 , 44 contrals d'acquisition et 9 partages.

Dans la mème paroisse, le produit du cens, qui était de 106 écus en 1566 , était tombé à 4 livres en 1766 ; l'ensemble des droits seigneuriaux, estimés 600 livres an $\mathrm{xvI}^{\mathrm{e}}$ siècle, s'abaissail à 170 en 1780 et la taille s'était réduite de gö livres a 30 (ceci, remarque $u n$ inventaire de 1782 , a propos de la taille, parce que le seigneur a rachelć plusieurs héritages qui en étaient ci-devant chargés, el surtout par la désuélude). M. d'Avenel cile nombre de faits analogues; senles, les redevances en nature conservaient une valeur relative; mais les droits féodaux, de conflits en transactions d'où la part du seigneur sortit toujours atténuće, ne cessèrent d'aller en diminuant depuis le lendemain de l'abolition du servage jusqu'an jon de la Révolulion; l'héritier dn noble de vieille date perd tout ce que gagnent les héritiers dı ser".

Les terrier's abondent en indicationsqui altestentl'ancienne richesse du pays de Bourgogne el l'état de détresse oin il étail tombé. Quantité de tenures désignées dans les anciens titres comme ćlant d'un lon revenu, sont colćes, au xvi siècle,

1. Comme de nos jours, les frais de procédure dévoraient l'héritage. Le domaine fut vendu 20 franes, qui passerent anx frais du decret et à l'arriéré des 21 gros de cens dus au roi a la Saint-liemy.

2. L'un des adjudicataires, Jean Lambert, revendit son lot, l'année suivante, à Gérard Reuillon, homme libre et proprictaire à Cernois, „jui en accrut son propre bien; ce lieuillon figure comme princijal témoin à l'enquête de 1488. 
sous cetle formule : un meix où il y avail courlil aujourdhui en uléserl...; un lieu où était chüleau aujourdhui en masure...; un moulin où ne sont plus que las de pierres; ctc. ${ }^{1}$.

La crise rurale du $\mathrm{xv}^{\mathrm{e}}$ siècle fut fatale aux petits propriétaires de cette région. Tous furent pillés, beaucoup incendiés et égorgés 2 . Les terriers mentionnent des cliaumes, des pàtis, de nouveaux bois avec des meix ruinés, indiquant d'anciens défrichements que la végétatiou forestière ressaisit après leur abandon, et d'anciens centres de culture d'où les habitants ont déguerpi.

La dépopulation, la perte du bétail et des semences réduisirent la population rurale au dénûment le plus complet et firent tomber la terre à vil prix. Les bourgeois des petites villes voisines en profiterent pour acheter tout ce qu'on voulut vendre, et la plupart des anciens propriétaires, n'ayant assez de ressources ni pour cultiver ni pour émigrer, furent trop heureux de devenir les fermiers des acquéreurs de leurs biens : c'est à cette date, en effet, que le métayage et le fermage se substituent à la culture directe.

A partir du $\mathrm{xvI}^{\mathrm{e}}$ siècle, le bourgeois fait donc concurrence aux gens d'Église ct aux paysans, seuls véritables possesseurs de la terre depuis que les seigneurs s'étaient dessaisis du domaine utile pour quelques redevances, ne conservant que de médiocres réserves ${ }^{3}$. Il se produit une évolution économique capitale, une transformation radicale dans la répartition de la propriété foncière; l'invasion du tiers état bour-

1. C'est à la période de 1438 (pestes et famines) à 14 'ł2 (passage des Routiers incendiaires) qu'il faut, semble-t-il, reporter le point culminant de cette crise rurale en Bourgogne.

2. Les possesseur's de fiefs préféraient la rente censive, si minime qu'elle fût (depuis un denier jusqu'à un sol par arpent) à l'exploitation directe qui leur était onéreuse et qu'ils dirigeaient sans gon̂t ni aptitudes. (Bulletin du Comité des travaux historiques, 1892, 1, p. 100 et 101.)

3. L'an 14\{2, les Écorcheurs mirent à sac les paroisses de l'Auxois; à Vic-de-Chassenay, le nombre des feux tomba de 70 à 23 dont 18 mendiants. (Monographie de la commune de Vic-de-Chassenay par M. de Saint-Genis; - Journal de la Société de statistique de Paris, mai 1884: Essai sur l'aisance du paysan lorrain à partir du XV siècle, par II. Guyot, Nancy, 1888.) 
geois dépossède le tiers état rural. Ce ne sont pas seulement les magistrats, les financiers, les innombrables privilégiés titulaires de charges et d'offices qui achètent les seignemries et les fiefs et s'affublent de la peau des nobles d'épée, selon le mot de Renauldon, ce sont aussi les procureurs, les notaires de village, les petits marchands, les artisans riches, qui veulent avoir un bien rural.

Dès 1503 , sur les 13 acquéreurs d'une portion du domaine Turreau dont je viens de parler, 7 sont des habitants de Semur. De 1494 à 1504, en relevant le nom des censitaires du roi propriétaire, à Forléans, Cessey, Arnay (en Auxois), on trouve quantité de forains, marchands, magistrats et même seigneurs, devenus acquéremrs de parcelles qưils lowent à des paysans.

L'envahissement des gens de robe, des bourgeois titulaires de charges et d'offices, ne se ralentit jamais. En 1789, les rôles d’impôt conslatent que, sur les 30 propriélaires cotisés au terroir du village de Sauvigny-lès-Bourbilly (Auxois), on comple 12 forains, dont 3 nobles et 9 avocals, procureurs et magistrats.

Malgré la direction donnée par l'Académie des sciences morales, on n'a pas encore accordé au xvil siecle et surtout au xrine la place considérable que cette époque de transilion cloit occuper dans l'histoire du régime foncier de la France avant 1789 , et, à part les ourrages de MM. L. Delisle, Levassemr et G. d'Avenel, c'est à des élrangers qu'il faut recomrir pour se guider dans les grandes lignes du mouvement moderne qui pousse vers l'acquisilion de la terre ${ }^{1}$.

$\Lambda u x v^{e}$ siècle, la division du sol était dans son plein épanouissement; elle avait commencé dès la fin du xi siècle par la répartilion des grandes propriétés féodales entre les mains des serfs, et, par I'incidence logique, elle suivit son cours

1. Parmi ceux qui nous ont apporté la contribution la plus précieuse, il faut mettre au premier rang $\mathbf{H}$. Karéien, professeur d'histoire à l'Université de Saint-Pètersbourg, avec son livre sur Les paysans el la question paysanne en France dans le dernier quart du XVIII' siecle (1899). 
dans la subdivision des petites propriétés censitaires entre les enfants des premiers affranchis, sauf les cas où l'héritage demeura indivis; les familles, en effet, s'étant fort multipliées sous laction bienfaisante des chartes de franchise ${ }^{1}$, après deux ou trois générations, lunité primitive de la manse servile se troura partagée de droit et souvent de fait entre 10 à 12 cohéritiers. La trop grande subdivision du sol provoqua des liquidations coûteuses et nombre d'héritiers, ayant leur part trop réduite pour bàtir une maison, acheter un cheptel el continuer dans d'étroites limites une exploitation sans avenir, la vendirent et émigrèrent dans les villes où ils peuplèrent les faubourgs ${ }^{2}$.

Les désastres du $\mathrm{xv}^{\mathrm{e}}$ siècle complétèrent l'œeuvre de dislocation, et l'érolution économique qui suivit les guerres de religion du $\mathrm{xrI}^{\mathrm{e}}$ siècle et les discordes civiles du commencement du xvir acheva de déposséder le paysan au profit du bourgeois qui, se reerutant lui-même dans la population rurale, revenait d'instinct au sol natal, attiré par une sorte d'atavisme.

La reconstitution des seigneuries par le rachat des censives $^{3}$ est un fait acquis, mais moins fréquent que ne le fut la constitution, à partir du $x_{v i}{ }^{e}$ siècle surtout, non point de domaines agglomérés formant une ou plusieurs exploitations se suffisant à elles-mêmes, mais de fortunes territoriales bourgeoises composées d'une infinité de parcelles affermées, sans la moindre idée de concentration culturale, à de très nombreux cultivateurs.

1. Au moyen âge et dans quelques provinces le mot de franchise devint synonyme d'alleu et désigna la terre ou le village qui, clégagé de toute serviturle territoriale et de tout droit seigneurial, était libre comme son possesseur et ne dépendait que du roi, ajoutèrent les ingénieux légistes du $\mathrm{XIv}^{\ominus}$ siècle.

2. Il y aurait une étude curieuse à faire en rapprochant de la période de peuplement des villes et de la création des cultures maraîchères dans leurs faubourgs la revision des statuts des corporations de métiers cherchant à se défendre contre l'intrusion de nouveaux membres; ces deux ordres de faits sont concomitants de l'émigration des campagnes dans les villes et de l'acquisition, sur toute l'étendue du territoire, de milliers de parcelles rurales par des forains.

3. Tausserat, Chronique de la châtellenie de Lury, Bourges, 1878. 
D'une part, la noblesse de robe el de finance reconstitue les anciens fiefs démembrés par le bail à cens et s'y substilue à la noblesse d'épée que dévorent les detles de cour, au témoignage de Ime de Sévigné, de Bussy-Rabutin, de Saint-Simon; d'autre part, la bourgeoisie prend possession du sol rural au moyen d'acyuisitions incessantes et l'exploite par le métayage et le fermage ${ }^{1}$.

Le morcellement et la mobilité du sol, qui remontent aux temps les plus reculés, ont pu se ralentir quelquefois sous l'influence de circonstances accidentelles et déprimantes, mais ces reculs n'ont pas duré el les mêmes tendances se sont perpétuées avec une intensité croissante jusqu'à la veille de 1789; les villes elles-mèmes se laissaient entrainer par le courant et achetaient des biens ruraux ${ }^{2}$. La jurisprudence arait vainement essayé de protéger l'intégrité des fiefs et des héritages ${ }^{3}$; tant il est vrai que les lois ne peuvent arrêter le progrès économique el que les mœurs sont plus fortes que la fiction des règlements. L'histoire de la propriété nous la montre mobile et fuyante comme l'onde malgré l'apparente fixité de ses droits. De mème qu'elle ne vaut que par l'homme qui l'exploile, ainsi il sera toujours impossible, en essayant de la retenir dans les mains de tel ou tel grroupe, d'empêcher les riches de se ruiner, les pauvres de s'enrichir, et les pauvres qui se sont à moitié enrichis de retomber dans le dénuement plus vite qu'ils n'en étaient sortis.

1. On a quelquefois expliqué par le désil de rétablir la suprématie territoriale des anciennes seigneuries le mouvement de concentration

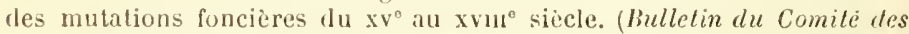
travaux historiques, 1884 et $1892,1,102$.) Je crois qu'il faut surtout faire la part tres large aux placements des capitaux bourgeois en biens fonds pendant la même période; c'ést un apercu neuf sur la question.

2. L'arrèt du Conseil du 2-2 avril 1673 permet aux villes, paroisses, communautés ct gens le main-morte d'acquẻrir de petits domaines ruraux pour les posséder au même titre que les autres acquéreurs. (BBosquet, Dictionnaire des domaines et droits domaniaur. 1775, 1, 290)

3. Tous fiels nobles sont impartables es intlividus, sauf s'il n'y a que (les filles héritières; - nul ne peut lisposer de son héritage en biens inmeubles par testament pas plus que leur usufruit, sauf pour la valeur dans ce cas d'une année de revenu et en vue de legs pitoyables. (Articles 336 et 427-428 de la Coutume de Normandie.) 


\section{LA TERRE AUX BOURGEOIS}

Comme la coutume de Bourgogne, la coutume de Normandie s'efforçait de maintenir intact le bien de famille, le Meix de l'Est, la Manse du Midi, le Manoir de l'Ouest, l'héritage de l'ancienne France, qu'on veut rétablir aujourd'hui sous le nom prétentieux de Home-stead, comme si nous avions besoin d'emprunter à l'étranger une expression pour une institution qui fut française dès l'origine de la nation.

Tout héritage est noble, roturier ou tenu en franc-alleu. En principe, l'ainé avait les deux tiers de la succession et les puìnés un tiers; de plus il avait, s'il était noble, un fief à son choix, s’il était de roture, le manoir ou résidence familiale.

S'il n'y a qu'un manoir roturier aux champs, dit la Coutume 1, anciennement appelé hébergement, chef d'héritage, honneur en propre du mari ou de la femme, en toute la succession, l'aîné peut, avant que de faire les lots et partages, léclarer en justice qu'il le tient, avec la cour, clos et jardin, en baillant récompense à ses puinés des héritages de la même succession.

Mais si la dévolution successorale était soumise à des règles restrictives, la vente était permise, et la saisie ne venait que trop souvent évincer les familles du toit paternel.

Charles Loyseau au XviI ${ }^{\mathrm{e}}$ siècle $^{2}$, Renauldon au $\mathrm{XVIII}^{\mathrm{e}}{ }^{3} \mathrm{ont}$ énuméré, l'un les abus des justices de villages, l'autre ceux de l'arbitraire en matière de procédure et de fiscalité. La condition des petits propriétaires fonciers était devenue déplorable et, il ne faut pas hésiter à le reconnaître, si la pelite propriété a continué à vivre, grâce à la patiente ténacité du paysan, elle a vécu dans la misère. Sans glisser dans les exagération de La Bruyère, il y a dans les écrits de Vauban et dans les rapports des intendants de Louis XIV des preuves suffisantes du malheureux état des populations rurales. On a énuméré les charges du paysan avant $1789^{4}$. En 1694, le curé d'Époisses forçait $\mathrm{II}^{\mathrm{me}}$ de Sérigné à donner

1. Coutume de Normandie, article 35 b.

2. Discours sur l'abus des Justices de villages, 162 千.

3. Traité historique et pratique des troits seigneuriaux, 1765.

4. Rémondière, Les charges du paysan avant et depuis 1789 . 
chaque mois 20 boisseaux de blé el de seigle aux habitants de ses villages pour les empêcher de mourir de faim; un siècle plus tôt, de 1598 à 1601 , au même château de Bourbilly en Auxois, $\mathrm{I}^{\mathrm{me}}$ de Chantal faisait construire le four des pauvres, et l'on y venait quêter, même parmi les bons laboureurs, de six lieues à la ronde. En 17000 , les Encyelopédistes s'écriaient : Ne serait-il pas possible de simplifier les possessions de façon qu'un seul héritage n’ait plus une multitude de maîtres qui semblent se relayer pour affliger celui qui le cultive?

Mais ceux qui disaient avant 1789 : Les quatre éléments sont asservis à la puissance capricieuse du seigneur : pour respirer dans la seigneurie, pour y tenir feu, pour y cuire son pain, pour y puiser l'eau, il faut payer; la terre, qu'on la eultive ou qu'on la laisse en friche, doit payer; les hommes, leur vie, leur mort, leur liberté, leurs contrats, leurs héritages, leurs troupeaux, leur commerce, leurs moindres actions, tout est devenu l'objet d'un droit seigneurial, ceux-là, ennemis systématiques de la féodalité, oubliaient que la taille, les vingtièmes, la gabelle, les privilèges étaient choses plus arbitraires el plus oppressives que ees droits amoindris et discutés.

Ils ne voulaient pas avouer que les Fermes générales étaient plus intraitables que les seigneurs, mème de noblesse récente; et ils seraient bien étonnés s'ils revenaient au monde, de voir, eent dix ans après la Révolution, la procédure plus coûteuse, la fiscalité plus inquisitoriale, la jurisprudence plus arbitraire qu'en 1790 , el tous les droits seigneuriaux rélablis, superposés l'un à l'autre et raffinés avee la plus inexorable ingéniosité sous le nom d'impòts.

Tout cela proure avee quelle liberté d'esprit il faut appréeier les opinions divergentes et avee quelle réserve il convient de tirer des conclusions des faits observés; car ce qui, à la mème époque, était vrai en Bourgogne pouvait ne pas l'être en Bretagne ou en Provence. S'il est établi qu'avant le xvir siècle quantité de très petits domaines et même des lopins de terre étaient possédés en pleine propriété par des 


\section{LA TERRE AUX BOURGEOIS}

paysans ou de petits bourgeois et affranchis de redevances foncières léodales ${ }^{1}$, il est également prouvé que des seigneuries el mème des paroisses tout entières sont demeurées volontairement serves, de droit et de fait, jusqu'en $1789^{2}$, et que, à la veille de la Révolution, des grens à l'aise, des gens instruits, avaient parfois intérèt à revendiquer la qualité de scrf et à se faire déclarer tels par arrèt de justice ${ }^{3}$.

On épuiserait des volumes à commenter ces contradictions morales et économiques; j’abrège à regret et je résume.

Depuis le moyen àge, la valeur vénale de la terre a augmenté dans une proportion infiniment plus forte que le revenu foncier; sous l'administration de Colbert, la terre se vend avee une plus-value des deux tiers, mais le blé baisse d'un cinquième; on se rappelle les cris de détresse de $\mathrm{M}^{\mathrm{me}}$ de Sévigné. Si vous n'aviez du blé, je vous en offrirais du mien; jen ai vingt mille boissean $x$ à vendre, je crie famine sur un tas de blé (octobre 1673); Vendez nos grains dès que les intendants le permettront, écrit-elle à $\mathbf{M}^{\mathrm{me}}$ de Guitaut, ou si vous voulez que je me moque des rats, faites-en farine (1693).

La terre produisait donc, et si le paysan crevait de misère c'est qu'il vendait à perte pour payer ses fermages ou ne renclait pas et, vu l'interdit de déplacer les grains, ne pouvait même pas échanger contre du bétail le superflu de sa récolte.

Un fermier ne gagne quasi rien, disait encore $M^{\text {me }}$ de Sévigné, et si on le force à payer, on le met à bas. Après huit ans de bail, le fermier de Bourbilly reste débiteur de 14000 livres sur 32000 .

Tous les fermiers ne mouraient pas de faim; $M^{\text {me }}$ de Guitaut le lui fait entendre en souriant, et Mre de Sévigné l'apprit en Bretagne, à ses dépens, de la jolie petite fermière de Bodégat. (Lettre du Iö juin 1680.)

Le prix de la terre tombe de 1692 à 1720 et ne reprend son

1. M. Loutchisky, La petite propriété en France avant la Révolution et la vente des biens nationaux, 1897.

2. Monographie de la commune de Chassey-en-Auxois, Semur, 1897.

3. Arrêt du Parlement de Dijon du ǰ̉ août 1768. 
mouvement ascensionnel qu'en 1750 , atteignant son apogée sous Louis XVI. La spéculation en avait fait une marchandise et, pendant cette période, quand le paysan pouvait acheter, il le faisait pour s'arrondir et cultiver, tandis que le bourgeois s'efforçait de payer pour revendre aussitôt plis cher; n'exploitant pas directement, il ne s'attachait pas à ses acquisitions et n'y cherchait qu'un profit immédiat.

Ce mouvement économique, en faisant passer une très grande partie du sol, la moitić peut-être, des mains des petits propriétaires exploitants dans celles des propriétaires forains, provoqua la rapide extension du mélayage puis du fermage.

Je parlerai du métayagre plus loin ${ }^{1}$, je ne veux ici qu'indiquer son caractère. En fait, le métayer est un fermier qui n’a pas d'avances et sur lequel le maître se paie en nature; à la veille de la Révolution, Turgot se représente le colon partiaire comme un simple ouvrier agricole, un valet auqucl le proprićtaire abandonne une partie des fruits à titre de gages. Notre conception du métayage n'est plus la mème, puisque nous y voyons une société de gains et de perles, une mutuelle participation.

D’une manière générale, on peut dire que, sous lancienne législation, il n'y avait pas de nuance juridique tranchée qui séparât les différents modes d'cxploitation de la terre, les diverses combinaisons de tenures aussi variées dans chaque région que le caprice des individus.

Les expressions de mélayage ou fermage servaient indis_ tinctement à désigner, au rapport d'Antoinc Loisel ${ }^{2}$, la mise des terres en gagnage, c'est-à-dire en produit. Toutefois, le Théalre d'agriculture et mesnage des champs d'Olivier de Serres ${ }^{3}$ donne la préférence au fermagre, seul usité dans les terres du roi, des princes et de tous ceux dont la condition

1. Bouisson, Le métayage ou bail à colonat partiaire, 1897. - Code rural, loi du 18 juillet 1889 .

2. Institutes coutumières, 1607 : Des rentes, IV, titre 1.

3. Qui parut de 1599 ả 1600 et eut lutit éditions en moins de dix-huit ans. 
et les goùts ne pouvaient s’assujeltir aux minuties de la surveillance des cultures, du partage et de la vente des fruits. Le métayage n'était acceptable que pour les propriétaires assez rapprochés de leurs terres pour les visiter souvent; les forains éloignnés choisissaient le fermage.

En Bourgogne, le mélayagge n'était pas réglementé. On ne trouve pas une seule fois le mot métayage ou son équivalent dans les sept volumes des Commentaires sur le droit civil à l'usage du duché de Bourgogne que publia le professcur Jean Bannelier, à Dijon, en 1776. Mème en matière de cheptel, la coutume de Bourgogne est muette. L'habitude s'en vulgarisa à partir du $x^{\prime} I^{e}$ siècle, mais le nombre en diminua de moitié au $\mathrm{xv}^{\ominus}{ }^{\ominus}$, tant à cause des mortalités du bétail qui affligèrent les campagnes bourguignonnes en 1714 et en 1743, qu'en raison de l'àpreté des bailleurs dont l'usure dévorail les paysans. Pour les protéger, un vieil avocat au parlement de Dijon, Henri Colas, publia en 1763 un traité des cheptels dont il emprunta les règles aux coutumes du Nivernais, du Bourbonnais et du Berry. Le bail à cheptel était l'accessoire habituel du bail à métairie et, sauf dans les pays de vignes, l'élevage étant présumé plus rémunérateur que la culture en labour, le cheptel devint le principal et absorba l'exploitation. La chose existait moins le nom, comme il résulte de nombreux actes notariés ${ }^{1}$, et l'on a montré comment et pourquoi s'effectua la substitution d'un régime à l'autre ${ }^{2}$, et, surtout dans les grands domaines, le remplacement des métayers par un fermier à rente fixe.

Dans un domaine rural de Bourgogne dont l'histoire économique a été étudiée de très près ${ }^{3}$, l'exploitation directe par le propriétaire se maintint de 1527 à 1602 ; elle fit place

1. Analyse et inventaire des Minutes notariales de l'Auxois, depuis le $\mathrm{XvI}^{\mathrm{Q}}$ siècle jusqu'à l'an XI, publiés en 4896 par la Société des sciences historiques et naturelles de Semur (Còte-d'Or).

2. Némoire de M. de Saint-Genis sur le métayage en Bourgogne (Bulletin du Comilé des travaux historiques de l'année 1897).

3. Journal de la Société de statistique de Paris, mai 1884. - Bulletin du Comilé des travaux historiques, rapport de M. H. Baudrillart, 188̈̈, p. 182. 
au mélayage de 1613 à 1640 , et au fermage de 1611 jusqu'à l'époque actuelle ${ }^{1}$. C'est ainsi que les choses se passèrent dans cette province, lout au moins pour la grande et la moyenne propriété.

Le fermage est très ancien pour la petite propriété acquise par des forains, on en voit de nombreux exemples dans les terriers et enquètes de la fin du $\mathrm{xv}^{\mathrm{e}}$ siècle; quantité de parcelles minuscules, éparses ou enclavées, sont affermées par les propriétaires limitrophes exploitant leur propre héritage et mème par des paysans qui, ayant cessé d'ètre propriétaires, sont devenus fermiers de profession. Les prés étaient affermés à prix d'argent, les autres cultures en nature, de là cette confusion explicable qui a longtemps, surtout à cause de la combinaison prédominante dı cheptel, rejeté dans l'ombre le fait du métayage. En général, dit un rapport officiel du commencement de ce siècle ${ }^{2}$, il y a peu de métayers en Bourgogne, et, au contraire du Berry el du Nivernais, les fermes elles-mêmes y sont de peu d'élendue. Mais, ce qui était rrai en 1811 ne l'était pas encore avant 1789 et surtout avant 1700 , et les économistes qui se préoccupaient surtout de la grande propriété tenaient peu de compte des parcelles lisséminées qui demeuraient, à leurs yeux, des quantités négligeables. C'est cette catégorie qui, au contraire, devient l'objet de notre curiosité la plus attentive et aussi la plus justifiée.

L’étude des minutes notariales ménage bien des surprises et modifiera plusieurs des opinions reçues. Voiei quelques indications tirées des centaines de faits semblables que j’ai observés.

Le 2'x février 1วّ̋2, Jean Durey prend à mélayage de Jean Jacob, procureur, pour six ans, un mcix à Nenétoy près Semur, contenant 4 ouvrées de vigne en une pièce, 6 soitures et demie de pré en 7 parcelles et

1. Ce domaine, dont la consistance n'a pas varié depuis le xvo siècle, comprend 45 liectares divisés en 150 parcelles éparses, dont la plupart en forme de lanières, ce qui fait une moyenne de 30 ares à la parcelle.

2. Peuchet, Slatistique officielle de la Cóte-d'Or, publiée à Dijon en 1811 par l'ordre du ministre de l'Intérienr. 
fournaux et demi de labours en 32 parcelles. (Minutaire de $\mathrm{M}^{\mathrm{e}}$ Godol, nolaire à Semur.)

Le 3 janvier 153 , bail, pour la moitié des fruits, du meix Mazoyer à Menéloy. (Ibid.)

Le 103 janvier 1562, hail a croît-cheptel, selon Ia Coutume de Bourgogne, d'un fort troupeau de lietes chevalines, aumailles, laine portant, pourceaux, oies, poules, chapons.

Le 13 février 172t, hail à culture et grangeage pour quatre ans, du domaine le Champforgueil en Beaujolais, moyennant le tiers des gerbes, moitié du vin et 36 livres de fermage pour 12 soilures de pré. (Minutaire de $M^{\circ}$ Bruère, notaire à Givry-Cortiambles.)

En 1729, la métairie de Turley (Auxois) est exploitée ì moitie fruits par Hubert Marsot et sa femme, qui ont reconnu par billet tenir en cheptel une pouliche, 3 mères vaches et 40 brebis à partager.

Je lis dans un aveu du 14 février 1449, que bien avant l'année 1389, une pièce de 00 journaux en terre et pré à Chasserot (en Auxois) avait été amodiée à André Forneron par le seigneur de Chassey.

La plupart des baux étaient consentis à la charge de payer moitié en argent, moitié en nature ou en corvées. En examinant la série successive des baux, on aperçoit une tendance manifeste et constante des métayers à diminuer de plus en plus la somme des redevances en nature el des services personnels, s'efforçant ainsi de réduire ou lout au moins de rendre fixe le prélèvement du propriétaire sur le produit de la récolte. C'est le mème sentiment qui fit substituer, en matière d’impòt, la taille abonnée à la taille arbitraire; on s'habitue à payer une taxe qui ne varie pas, mais rien n'est plus insupportable pour le débiteur que la mobilité d'une dette dont la nature est fatalement de croître plutôt que de diminuer.

L'exploitant du sol, fermier ou métayer, a toujours été le moins exact des payeurs; criant misère à toute occasion, il affecte de n'avoir pas le sentiment des échéances. Tout lui est sujet à retardement, écrivait $\mathrm{M}^{\mathrm{me}}$ de Sévigné; la pluie, le soleil, le trop ou le pas de blé. Et Bussy-Rabutin, grand propriétaire comme elle au pays d'Auxois, lui donnait des conseils pratiques sur la dureté qu’il faut avoir pour ne point 
dissiper son bien en bonnes grâces à des finauds. Les seigneurs qui avaient des fermiers déléguaient leurs fermages à leurs propres créanciers ou chargeaient de leurs affaires un marchand ou un procureur du voisinage. J'estime, disait Sourdis à Richelieu, que votre duché consistant en vingt petites fermes il faut les réunir à les bailler à un gros fermier; vous ne serez volé qu'une fois. Ce système des régisseurs de biens, très onéreux pour les cultivateurs, commode mais coûteux pour les propriétaires, ne profita qu'aux intermédiaires; ce fut avec l'impòt et la procédure, pendant le $\mathrm{xvi}^{\mathrm{e}}$ siècle et le $x_{\text {vir }}{ }^{\mathrm{e}}$, la troisième plaie de l'agriculture et l'une des causes de ruine du petit propriétaire foncier qui ne trouvait de capitaux ou d'auxiliaires qu'à des taux usuraires.

En Bretagne, la situation paraît avoir été plus mauvaise encore qu'en Bourgogne, en Lorraine et en Franche-Comté.

Au milieu du dernier siècle, les commissaires des États de Bretagne s'exprimaient ainsi :

La misère des temps présents fait que l'on trouve peu de fermiers si le propriétaire ne leur fournit des bestiaux, pailles, marnis el engrais, même des grains de semenee, ee quion nomme cheptels 1.

En Bourgogne, les pelils châlelains renoncèrent à la culture directe de leurs réserves et l'on voit, dis la seconde moitié du xvir siècle, un bail général remplacer les baux isolés et les contrats de mélayage; par contre, et ce délail indique l'intérêt économique qu'il y a à noter l'élat des personnes, les notaires, procureurs et marehands qui loment leurs biens ruraux font dominer le bail à cheptel dans la combinaison; les premiers ne cherchent 'ju'un revenu normal de leur propriélé, les seconds spéculent surtout sur les besoins d'argent du paysan.

Il est nécessaire de noter loules ces particularités afin de reconstiluer pièce à pièce la condition réelle de la petile propriélé aux approches de 1789.

1. Mémoire du 26 novembre 1752 . - On retrouve les mêmes exigences dans un bail à ferme du 13 février 1724 par Edme de Thésut a Claude Boinon. (Minutaire de $\mathbf{M}^{\circ}$ Bruère, notaire à Givry en Beaujolais.) 
Dès le début du xvır ${ }^{\mathrm{e}}$ siècle, on rencontre des syndieats de marchands associés pour l'exploitation en bloc des terres d'une scigneurie dont ils tirent un gros bénéfice en les sousaffermant à leur tour aux paysans de la paroisse ${ }^{1}$. Plus tard, les paysans s'apercoivent du préjudice que leur font les forains et ils s'associent entre eux pour tenter l'entreprise ${ }^{2}$ à leur propre compte. On trouve mème au xrir siècle quelques exemples d'associalions de ce genre, soit entre parents ${ }^{3}$, soit entre étrangers ${ }^{4}$.

Dans le livre de raison (inédit) du seigneur de Chassey à la date de 1779 , je copie la mention ci-après qui révèle, d'une part, la tendance manifeste des paysans à s'associer, à se syndiquer comme on dirait aujourd'hui, à mutualiser leurs efforts, de l'autre, leur intérêt évident à se coaliser contre les lorains et à s'approprier le monopole de la culture à l'exclusion des spéculateurs du dehors, mème par un retour pur el simple au métayage :

Nles 68 journaux $3 / 4$ de bonnes terres à tierce allure doivent rapporter à la vente du grain $6821.10 \mathrm{~s}$. Plusieurs de mes habitants laboureurs m'ont offert de labourer ces terres en se chargeant de tous les frais de culture, semailles, moissons, rentrées, a charge par les laboureurs de rendre dans mes granges moitié des gerbes de chaque graine qui se trouveront dans mes héritages.

If reste 322 journaux de seconde qualité dont ils m'ofrent le tiers des gerbes, soit 2 gerbes sur 5 , et moi je les estime rapporter 5 mesures et demie le journal. Ceux qui m’ont fait la proposition sont Jean Garnier, Jacques Camusat, etc.

1. Bail de la seigneurie de Chassey (18 mars 1697) à un syndicat formé de trois laboureurs et de leurs femmes; bail à trois marchands de Semur (9 avril 1723); bail à deux marchands de Semur associès pour celte anodiation (25 août 1733); bail à un marchand d'Espréaux (26 juin 1752).

2. Le 23 avril 1762 , huit laboureurs de Chassey afferment solidairement les terres de la seigneurie à raison de 8 boisseaux de blẻ ou avoine par journal, sec ou trempé. Chacun d'eux afferme isolément les parcelles en pré, moyennant un prix en argent calculé d'après leur produit.

Le 25 avril 1764 , les anciens baux étant écluus, bail de tout ce qui reste des terres à dix-sept autres laboureurs et vignerons, engagés solidaires pour six ou neuf ans.

3. En 1708 , renouvellement d'un bail de 1675 , prorogé en 1684 au profit de Claude Legoux et de ses beaux-frères.

4. En 1640 , bail du domaine de la Rochetle (Vic-de-Chassenay) à deux laboureurs associés. 
Depuis 1780 , écrit-il à une autre date, ils m'ont offert de tout amodier à moitié: mais je suis plus tranquille arec les fermiers Grosse-Tête et Panétrat qui sont de bons payeurs.

Le mourement des mutations foneières, soit par rachat des censives pour reconstituer la réserve seigneuriale, soit par acquisition des forains aux habitants, soit par ventes des paysans entre eux, ne eesse pas depuis le xvi $^{\circ}$ siècle jusqu'en 1789, et porte sur des parcelles minuscules. En voici un exemple tiré du même pays d'Auxois.

En 13991, Philibert Bouhot vend pour un écu de 3 livres un petit quarron de jardin sis au village de Chassey, contenant la semence d'une chapelée de chénevis. En 1646, le seigneur achète d'un serf 3 journaux $1 / 4$ de terre et 1 ouvrée de vigne situés en différents lieux et formant 5 pièces, ce qui met la moyenne de chacune d'elles à 23 ares 130 centiares; en 1652, il achète à part $1 / 3$ de journal (11 ares 42 ), $3 / 4$ de journal (25 ares 71 ) et $1 / 2$ journal (17 ares 14 ), le tout pour 55 livres, prix exceptionnellement élevé. En 1i8., un vigneron vend à un maçon, pour 100 livres et les vins, 2 pièees séparées formant $3 / 4$ de labour et une parcelle de 1/2 journal; à cent trente ans de distance, le prix est doublé mais la ténuité des parcelles est restée la même !.

Le 1/t mai 1600, Verpinet vend à la dame d'Allerey la moitié d'une grange couverte en paille dont elle a déjà le tiers de l'autre moitié, et les deux tiers d'un quart de sée de pré, l'autre liers et les deux tiers de ce qu'elle ne possède pas encore du bâtiment élant à Crespy et aux hoirs Guiot ${ }^{2}$.

Le mourement naturel de réaction qui suivit la crise économique de 1690 à 1715 s'accentua à partir de 1730 , ot jusque vers 1790 la hausse préralut avec une intensité qui rappelle les plus-values subites du $x r^{e}$ siècle; la valeur vénale des biens ruraux tripla en moins de quatre-vingl ans.

1. Ln 1573, Gaspard Farnier vend a un marchand de Semur pour 17 livres et une peau de hique une haste de chenevière contenant la semence d'un moiton (1/2 boisseau ou 12 litres) de chènevis, ensemble les saules y étant, qui est chargée de 3 boisseaux d'avoine de cens envers le seigneur de Souhey.

2. Minutaire de $\mathbf{I}^{\circ}$ Quignard, notaire royal à Semur. Lin 1705, le manoir de la Lézardière (Sarthe) appartenait à plusieurs propriétaires, l'escalier de la tour était commun jusqu'i la onzième marche, l'un avait le rez-rle-chaussée, l'autre l'étage, le troisième les greniers. (Bullel. du Comilé des trav. hist., 1883̈, 181.) 



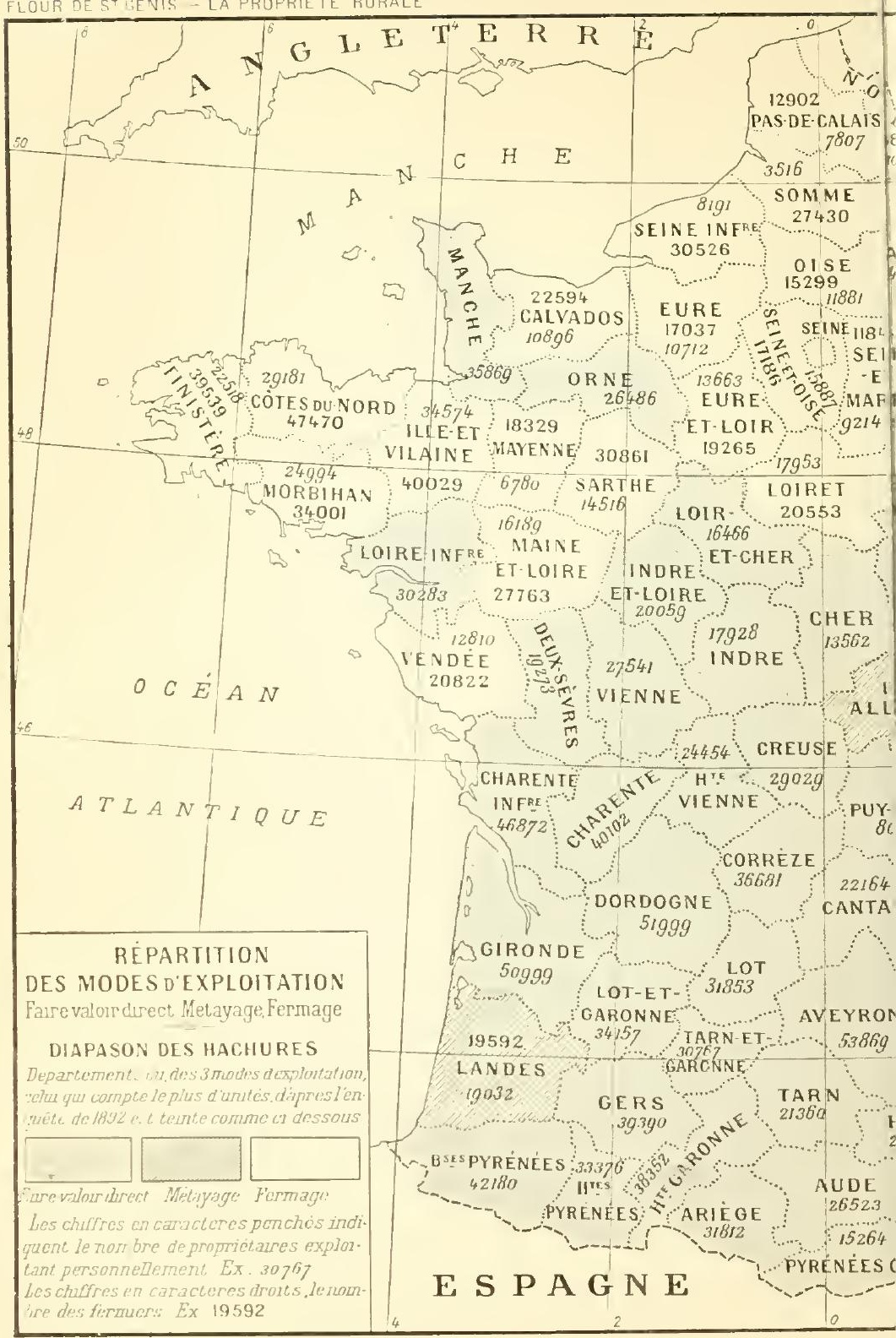


BELGIQUE

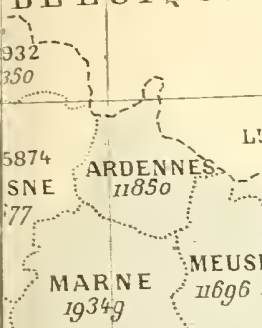

JNNE

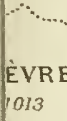
COTIE.D OR 16434 DOUBS 1013

\section{JURA}

SAONE-ETLOIRE 26047 35145

\section{AIN :HÉSAVOIEV

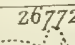 \\ 35180} 0 .

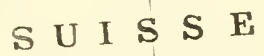

S U I S S E

\section{SAVOIE}

ÔME:<smiles></smiles>

HELOIRE :

41412

ARDECHE 39844

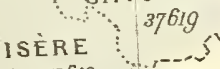

Ż

DROME

\section{0}

$25 \quad 23108$ 39158

$\mathrm{C}_{2}$
$\mathrm{CO}_{2} \mathrm{C}$ BSESALPES

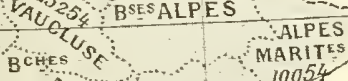
DU RHÔNE AU:
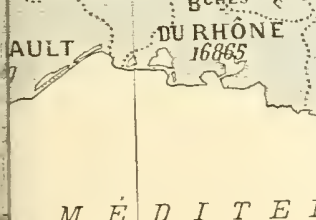

(25882 है

$M \quad E \quad D I T E R \quad R \quad A \quad N \quad \dot{E} E$

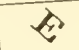

17

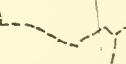

H $^{\text {te-Garonne }} 10.780$

Loire-Infèr.

10.263

Lot-et-Gar. 11.091

B.-Pyrénées 11.125

Saône-et-L. 12.199

Tarn

12,310

Vendée

11.448

$\mathrm{H}^{\mathrm{te}}$-Vienne

12.868

Dans les Landes et dans l'Allier, le nombre des métayers dépasse celui des propriétaires exploitant et celui des fermiers. 

En faut-il conclure ì une concurrence plus active pour l'aequisition de la terre entre bourgeois et paysans? Mais alors comment concilier celle thèse avec l'opinion accréditée - et poul-être fausse - que le paysan, dévoré par la chicane et la taille, pouvait à peine récolter assez de grains pour faire cuire son pain et ne mangeait de viande qu'aux fétes carillonnées. Si l'on en croit les mémoires du temps, le paysan se faisait paurre pour n’être pas écrasé par les répartiteurs de la capitation, de la taille, des ringtièmes et de toutes les crues qui rejetaient sur les paysans et les bourgeois non privilégiés le fardeau de taxes fort lourdes et dont le trésor public ne profitait guère que jusqu'à concurrence des deux cinquièmes.

Rien ne peut mieux peindre l'état du paysan français au xviI $^{\mathrm{e}}$ siècle que le récit que fait Jean-Jacques Rousseau de son arenture de 1732 , dans un pays qüil ne nomme pas, entre Dijon et Lyon.

J'entrai chez un paysan dont la maison n'avait pas belle apparence, mais c’était la seule que je vis. Il m’offrit du lait écrémé et de gros pain d'orge en me disant que c'était tout ce qu'il avait. Jugeant de la vérité de mon histoire par celle de mon appétit, et pensant que je n'étais pas lá pour le vendre, il ouvrit une petite trappe à còté de sa cuisine et revint un moment après arec un bon pain bis de pur froment, un jambon très appétissant quoique entamé et une bouteille de vin dont l'aspect me réjouit le cœur. Je ne savais de quoi il avait peur.

Enfin, il prononça en frémissant ces mots terribles de commis et de rats de cave. 11 me fit entendre qu'il cachait son vin à cause des aides, qu'il cachait son pain à cause de la taille, et qu'il serait un homme perdu si l'on pouvait se douter qu'il ne mourût pas de faim. Je sortis indigné et attendri, déplorant le sort de ces belles contrées à qui la nature n'a prodigué ses dons que pour en faire la proie des barbares publicains ${ }^{1}$.

Vingt ans plus tard, le marquis d'Argenson disait de même, en racontant le soin que les petits propriétaires du Poitou et de la Touraine prenaient à paraître misérables, tout en se traitant comme gens à l'aise, porte close et volets fermés.

En 1697, Le Pesant de Boisguilbert, qui confessa la vérité comme Vauban, aroue que dans la généralité de Rouen, les

1. Confessions, partie $1^{\mathrm{re}}$, livre $\mathrm{IV}$.

LA PROPRIÉté RURALE EN FRANCE. 
paysans furent obligés, pour avoir de l'eau et du pain, de vendre à vil prix tout ce que leurs peres avaient acquis pendant les deux siècles précédents; en $1707^{1}$, il oppose à ce tableau celui des plaines du Languedoc, qui ont moins souffert des maux de la guerre et que les traditions de la province ont défendues contre l'abus d'une fiscalité impitoyable.

Près de Montauban, dit-il, il est impossib]e de trouver un pied de terre qui ne rapporte tout ce qu'il peut produire. Il n'y a point d'lomme, quelque paurre qu'il soit, qui ne soit couvert d'un habit de laine l'une manière honnète, qui ne mange du pain et ne boive de la boisson autant qu'il lui en faut, et presque tous mangent de la viande: tous ont des maisons couvertes de tuiles, et qu'on répare dès qu'il en est besoin.

Les petits propriétaires ruraux que plaignaii Rousseau en 1732, d'Argenson en $174 \overline{7}$, se trouraient, vis-à-vis des exigences et de linquisition des commis des Fermes et de l'arbitraire des Élus, dans la situation de tous les travailleurs que des administrateurs impréroyants exploitent au jour le jour. Les mauvaises lois produisent partout les mêmes effets.

C'est ce qui se roit encore de nos jour's en Irlande. Aussitôt que le cultivateur a tiré du sol de quoi vivre, il évite toute amélioration, parce qu'il croit que les fruits de son industrie lui seraient enlevés par le propriétaire, toujours attentif, simagine-l-il, ì tirer de l'apparence d'une production en progrès la preure que la rente peut être augmentée. Il en est mème, en Égyple, du fellah, qui simule la pauvreté de peur que le moindre signe d'une aisance accrue ne l'expose à de plus lourles extorsions.

En France, avant 1789, le paysan français, métayer ou fermier, enterrait son argent plutòt que de le mettre dans sa culture; propriétaire, il attendait les oceasions et achetait de la terre, tout en eriant misère. Ce sont ces apparences et ces faits contradictoires qui faussent l'idre qu'on a pu se laire de la France rurale du $x^{\prime} \mathrm{ri}^{\mathrm{e}}$ siecle, et l'on a quelque peine à concilier, à moins de se livrer à des études approfondies el localisées, les notes de Turgol, Necker, Arthur Young,

1. Détail de la France, $1^{\text {ro }}$ partie, chap. xxr. 
Rigby, parlant de l'immensité des petites propriétés rurales, s'émerveillant des résultats de la culture intensive sur des héritages d'un demi-arpent, et s'écriant : le magique pouroir de la propriété, quand sa sécurité est certaine, change le sable en or, - it côté des plaintes officielles et de l'accroissement des mendiants.

Je lis dans les cahiers de doléances de la paroisse de Saint-Beury en Bourgogne ${ }^{1}$ les déclarations suivantes :

Cette paroisse de 9 ' feux paie plus de 4000 livres d'impôts directs: elle ne possède pas le quart de son territoire qui est à 7 forains ${ }^{2}$, il n'y a que 11 laboureurs et 2 proprietaires (M. de Brachet et M. de Fresne sont les seigneurs (lu lieu); 33 habitants (sur 470) peuvent à peine subvenir à leurs pressants besoins et 50 sont réduits à l'aumône; en 1788 , on a saisi 11 partieuliers ne pouvant payer leur eote, et les exploits de perquisition n'ont rien trouvé chez eux qui pût être vendu pour payer les frais; s'il ne survient un nowvel ordre des choses on ne peut douter que la misère n'arrive à son comble.

C'est de l'hypocrisie devant le maître el devant le Fise que la Révolution a délivré le paysan; elle ne l'a point promu propriétaire, il l'était depuis des siècles, mais elle lui a rendu sa dignité d'homme en en faisant un citoyen. Les lois de la Constituante, de la Législative et de la Convention ont en même temps libéré la terre et l'homme en décrétant la suppression des servitudes seigneuriales et l'unité du droit de propriété ${ }^{3}$.

Le jeu du morcellement et les progrès de la petile propriété en ont-ils été accélérés? e'est indubitable, mais il faut éviter l'exagération dans l'un ou l'autre sens; aussi faut-il excuser Michelet, qui a décrit avec enthousiasme les travaux merveilleux et féconds du petit propriélaire, de

1. Document inédit; l'original de ee eahier primaire, daté du 13 mars 1789, est aux archives de la eommune de Beurizot (Côte-d'Or).

2. Ces forains étaient des privilégiés dont les biens furent moreelés ear, en 1899, sur 310 cotes foneières, 131 seulement concernent des résidants, 179 sont des eotes foraines.

3. Ce point est d'une importance eapitale, en ce que la réforme supprimait la dualité des droits en réunissant le domaine direet qui appartenait à un nombre restreint de propriètaires au domaine utile qui était dans les mains de millions de tenaneiers. 
s'être trompé, par une généralisation trop absolue, sur l'heure économique la plus propice aux acquisilions de la petite épargne rurale.

Aux temps les plus mauvais, dit-il, aux moments de pauvreté universelle où le riche même est pauvre et vend par force, alors le pauvre est en élat d'acheler; nul acquéreur ne se présentant, le paysan en guenilles arrive avec sa pièce d'or, et il acquiert.

Il y en eut des exemples, surtout à l'époque de la vente des biens nationaux. Mais M. de Foville fait remarquer que, si l'exception confirme la règle, e'est surtout dans les périodes de prospérité que les petits propriélaires rognent ou dépècent les grands domaines; alors les goujons dévorent le brochet; mais, pendant les crises, celui-ci prend sa revanche. Certes, la pelile épargne ne pourra jamais luller contre les capitalistes dont les sources de revenu ne faiblissent pas loules à la fois, el qui onl des réserres, cependant elle a des ressources cachées qui ménagent bien des surprises.

Si, aux heures calamiteuses, le paysan n'a pas les moyens de profiter de la vilité des prix pour s'attaquer aux grands domaines, celuiqui est économe el prévoyant peul s'agrandir aux dépens de son voisin, pelil comme lui, et qui, gêné par des accidents agricoles, ne peut plus payer les intérèts de ses prix d'acquisilion ou, après avoir élé forcé de vendre son bélail, vend ensuile pour virre une terre quil ne peut plus cultiver.

La Révolulion n’a pas accru sensiblement, par le fait seul des ventes révolutionnaires, le domaine de la très petite propriélé. J'ai expliqué comment, it mon sens, ce grand marché, subitement ouvert, avail surtoul profité à la moxenne propriété et aux syndicals de la bande noire. Ce n'est que pelil à petil, et par l'eflet du lemps, que le classement s'est opéré el que les propriétés achetées par lots pour être revendues ont ju, à leur tour, être subdivisées de nouveau el réparties entre des acquéreurs qui surgissenl un à un, au gré des occasions et des circonstances, et rarement 
en groupe compact et empressé comme ceux de la pure spéeulation. Les cas ou les habilants du voisinage se sont adjugé aux enchères la propriété divisée de leur ancien seigneur sont relativenent assez rares; il $y$ en a des exemples en Bourgogne, à Chassey, à Saint-Beury, ete.; et ailleurs, mais, en général, les immeubles de cette catégorie n'ont acquis leur assiette définitive qu'après avoir passé par plusieur's mains et souvent par toute une sćrie d'intermédiaires absolument élrangers à la culture '.

Dans certains départements, partout où les cultivateurs s'enrichissent par l’industrie ou l'émigration, les paysans vont d'eux-mèmes au-devant de la spéculation; ils tentent les propriétaires forains en oflfont d'une parcelle deux et trois fois ce qu'elle vaut. Par l'effet de cette concurrence, le prix des terres s'est élevé au point que la proportion du revenu au capital n'est plus, dans quelques localités, que d'un chiffre insignifiant; mais qu'importe aux petits cultivateurs laborieux que le loyer du capital diminue? Il leur suffi que la terre produise en raison de leurs efforts, de leur passion du travail.

Dans la Creuse, le Cantal, le Puy-de-Dòme, où le sol, coupé de vallées étroites, profondes et peu fertiles ${ }^{2}$, semble réservé aux pàturages et à la grande culture, la petite propriélé, depuis 1850 , ne cesse de faire des progrès considé-. rables. Chaque année, le cinquième des ouvriers adultes quittent leurs foyers au printemps ${ }^{3}$ et vont louer leurs bras à Paris, comme macons, tailleurs de pierre, charpentiers; ils reviennent dans leurs montagnes au mois de décembre avec des millions de franes épargnés sur leurs salaires et qu'ils

1. L'étude de la vente des biens nationaux dans les archives des directions départementales de l'Enregistrement n'a pas été faite; elle offrirait un puissant intérêt historique et économique.

2. Il faut excepter la Limagne d'Auvergne et les hauts pâturages du Larzac sur les confins de l'Aveyron qui sont d'une richesse proverbiale.

3. D’après le dénombrement de 1891 , la proportion des originaires de clıaque département ayant immigré dans la Seine, par rapport à la population respective de chaque département est de 13 p. 100 pour le Cantal, de 11 p. 100 pour la Greuse, de 12 p. 100 pour la Nièvre. 
emploient immédiatement à des achats de terres. La multitude des acquéreurs est telle qu'un sol médiocre, exposé à une perpétuelle enchère, en tire une plus-value incessante ${ }^{\prime}$.

D'après l'Enquête agricole de 1892, le nombre des très petites exploitations était de :

\begin{tabular}{|c|c|c|}
\hline & $\begin{array}{l}\text { Au-dessous } \\
\text { de I hectare. }\end{array}$ & $\begin{array}{l}\text { De } 1 \text { à } 5 \\
\text { hectares. }\end{array}$ \\
\hline Cantal. & 13720 & 12819 \\
\hline Ches & 23 วับ4 & 16148 \\
\hline Creuse. & 20773 & 19891 \\
\hline Puy-de- & 59952 & 49337 \\
\hline
\end{tabular}

Et, si l'on considère les valeurs comprises dans les déclarations de suecession, on s'apercoit que, toutes proportions gardées, eu égard à la superficie du département et à sa population, les régions où les capitaux fonciers dominent et où l'on trouve le moins de numéraire el de valeurs mobilières, sont précisément celles où le courant des émigrations intérieures est le plus prononcé et le plus régulier.

Dans les départements où l'industrie el le commerce sont en pleine prospérité, la bourgeoisie des villes a longtemps converti ses épargnes en fonds de lerre; elle achetait également à mesure qu'elle réalisait les profits, e'est-à-dire par petites sommes el par petils lols; celte pralique a duré de 1816 a 1866 ; pris l'essor des valeurs mobilieres et des titres industriels a changé le courant, el les bénéfices de l'activité bourgeoise se sont portés surles fonds d'État et les valeurs de portefeuille; en voici la répartition pour 1898 d’après l'aclif brul successoral ${ }^{2}$.

1. Pour lanne $189 \%$, le produit des seuls droits d'enregistrement a dépassé 1650 millions dans la Creuse, 1902 dans le Canlal, 467 dans le Puy-le-Dòme; dans ces :" départements, le capilal sur lequel est peru le droit de mutation à titre onéreux n'a cessé de croître depuis 1850 .

2. Bulletin de statistique de l'Enregistrement, 1899, p. 186 à 189. 


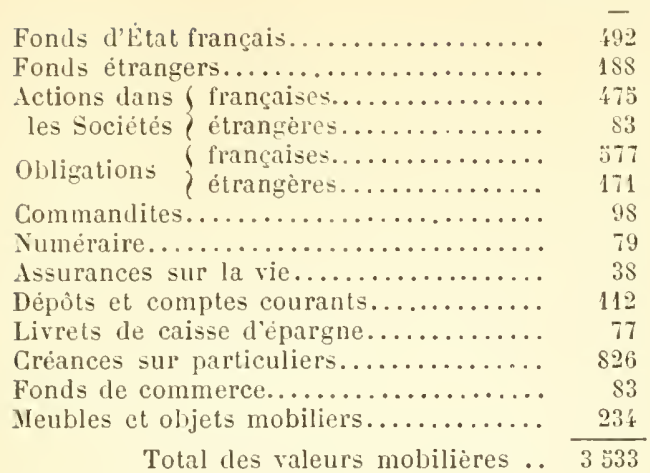

Valeur estimative des immeubles :

Ruraux....................... 1520

Urbains ........................ $\frac{1570}{3090}$

Voilà done à quelle concurrence formidable se heurte, à l'époque contemporaine, le goût de l'acquisition de la terre chez les détenteurs de la petite épargne. La propriélé foncière a désormais deux rivales, mieux armées qu'elle-même pour la lutte économique dont l'objet est l'emploi, dans cette catégorie d'acquisitions minimes, du superflu du salaire : la caisse d'épargne qui se rapproche de plus en plus de la clientèle, la coupure de titre avec l'attrait de la loterie ${ }^{1}$.

Cependant, en 1894, on a enregistré en France 477230 ventes immobilières, urbaines ou rurales, dont le prix ne dépassait pas 1000 francs et 682039 de moins de 10000 francs d'une valeur vénale de 734,3 millions de francs, sans compter les milliers d'acquisitions par actes sous seingsprivés que l'exagération des taxes fait se dérober à l'impòt.

Il existe donc de ce côté un courant d'une intensité que les obstacles juridiques ou fiscaux, la concurrence et les

1. Étudier à ce point de vue les recherehes si précises de M. A. Neymarck sur le morcellement des valeurs mobilieres (1896) et la part du capital et du travail dans les salaires. 
crises n'arrêtent point et qui révèle d'énormes ressources ou une force de résislance incomparable.

La loi des partages, qui offre des inconrénients dans certains cas (articles $810 \%$, 820 et 827 du Code civil) exerce, on ne peut le nier, une influence décisive sur l'activité des mutations foncières. Lorsque les propriétés se divisent par l'héritage, les compensations se font rarement à prix d'argent entre les enfants ${ }^{1}$, chacun réclame sa parl de chaque lot; autant de pièces de terre, autant de partages, ainsi que le veut la loi d'égalité quand clle est interprétéc par l'ignorance et l'égoïsme ${ }^{2}$. Il est facile, d'ailleurs, de se rendre compte de la portée de la loi des partages en réparlissant les successions d'après le nombre des enfants à qui elles sont échues ${ }^{3}$.

En 1898, il y eut 430810 déclarations de mulations par décés; sur ce nombre, 343366 contenaient des immeubles, se distinguant en urbains ou ruraux dans cette proportion :

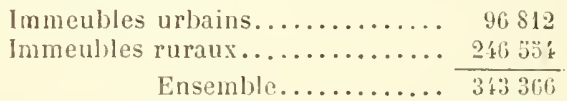

Sur ce nombre, 281333 concernent des hériliers en ligne directe descendante, ainsi réparties par nombre d'enfants :

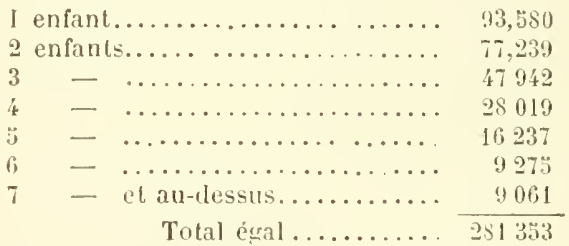

1. En 1896, les statistiques de l'Enregistrement enregistrent $8 \$ 552$ licitations et soultes de partage entre cohéritiers et copropriétaires pour une valeur estimative de 230 millions de francs, ce qui fait une moyenne Ie 2720 francs par opération sur lesquels le lisc et les officiers ministériès prélèvent de 20 à 40 p. 100.

2. En 1896, il y a eu 52300 actes judiciaires concernant les partages : jugements frononcant l'homologation de liquidation ou de parlages; sentences arbitrales ayant le mème objet; jugements et procès-verlaux portant adjudieation d'immeubles, soit devant un tribunal, soit devant un notaire commis. Ce chiffre varie peu d'une année à l'autre; en 1895 , il avait èté de $5 \% 2 \%$ '.

3. Bulletin de stalistique de l'Enregislrement, 1899, p. 181 et suivantes. 
Les valeurs transmises par ces mutations s'élevaient au apital de 3 1,70 millions de francs.

Les mariages recomposent les fortunes, il est vrai, mais non les domaines ${ }^{1}$. Lor'sque le cohéritier a pris possession de son lot, il ne tarde pas à le vendre, avec l'espoir de tirer meilleur parti d'une valeur de convenance payée fort cher par un roisin que d'une soulte formant créance et loujours suspecte d'estimation faible dans les arrangements de famille. Les parcelles qui n'avaient pas une valeur échangeable arant d'ètre détachées de l'ensemble et qui ont acquis une valeur positive du fail seul de leur isolement qui les met à la portée d'un plus grand nombre, s'élèvent à une valeur de convention el sans mesure marchande quand on prétend les réunir de nouveau. Un hectare de terre valait 1000 francs dans une propriété de 200 hectares, il en raut 3000 pour le paysan qui n’en possède qu'un. Qu'un propriétaire voisin fasse des ofires pour l'acquérir, il ne l'obtiendra qu'à la condition de le payer deux et trois fois sa valeur. Ciest ce qu'on exprime par cette formule spirituelle : Si vous ne possédez rien, vous pouvez acheter dans de bonnes conditions; dès que rous avez quelques toises au soleil, si vous voulez vous étendre, on vous tient la dragée haute et les obstacles se multiplient de tous còtés.

Telles sont les causes qui, tour à tour, excitent ou ralentissent, sans l'arrêter jamais, le mourement incessant qui divise la propriété ou qui en groupe les parcelles. La science de la statistique possède aujourd'hui mille moyens d'observer ces phénomènes avec précision, et lorsque les matériaux d'étude que les services publics et les recherches privées constituent avec une émulation louable se seront accumulés, on pourra en tirer des conclusions neuves et de nature à modifier bien des opinions.

1. En 1898, on a rédigé 82310 contrats de mariage; les apports des époux $y$ sont constatés pour un milliard 1/10 et les donations faites par des tiers à cette occasion pour près de 500 millions, d'où rẻsulte, pour les noureaux conjoints, un avoir total qui dépasse $1620 \ddot{~ m i l l i o n s ~ d e ~}$ francs. (Bullet. de statist. de l'Enregistrement, 1899, 150.) 
L'histoire de la très petite propriété au cours du xix siècle, quand elle pourra ètre fouillée dans ses moindres détails, jettera une grande lumière sur les nombreuses questions sociales qui nous agitent el que le $\mathrm{xx}^{\mathrm{e}}$ siècle voudra résoudre.

Ce qui est certain, e'est que la petite propriété, et surtout la très petite, ce premier échelon du salarié vers l'indépendance, est très vivace. Elle s'accroît sans cesse, et se fixe, se consolide avec beaucoup plus de permanence et de stabilité que ne pourraient le faire croire la modicité de ses ressources. L'essentiel, en effel, au point de vue social, est moins de la eréer que de la faire durer.

L'émiettement du sol n'a rien qui puisse effrayer les économistes, il s'arrêtera de lui-mème à la limite où il cesserait d'être utile. On peut s'en convaincre en lisant dans les Petites Affiches ou les annonees judiciaires des journaux de province l'avis des biens à vendre. On a signalé depuis longtemps la minutie des désignations; comme au $\mathrm{XvI}^{\mathrm{e}}$ siècle on comptait les saules d'un pré, ainsi en 1900 on numérote les arbres d'un jardin; plus la parcelle est petite, plus on détaille ce qui la caractérise. On s'étonnait, il y a soixante ans, que les 1300 hectares de la commune d'Argenteuil, dans la banlieue de Paris, fussent divisés en $3883 \ddot{~ p a r c e l l e s, ~}$ qu’il n'y eût pas une charrue sur ce territoire et que tout le travail s'y fît à la bèche. On n'est plus surpris, aujourd'hui, de rencontrer des conditions pareilles autour de toutes nos grandes villes, tans le Vaucluse, dans le Var, et, chaque fois que s'ourre une ligne nourelle de chemin de fer, l'étude du tracé révéle pour l'expropriation, sur lout le pareours, en rase campagne, loin des villages, un si grand nombre de parcelles par kilomètre que la mesure de leur traversée varie de 40 à 13 mitres ${ }^{1}$.

Le rêve, c'est d'arrèter l'émigration des campagnes vers

1. Les résultats d॥ dénombrement de 1896 établissent que, sauf 3 exeeptions, la population de toutes les villes ayant plus de 30000 habitants a augmenté par l'immigration de l'intérieur'; 2 't lépartements selllement sur 86 ont augmenté, dont 17 par la natalilé, notamuent les Bouches-du-lihóne, Je Norl et Seine-et-Oise (la Seine mise à part). 
les villes en faisant de tout ouvrier agricole un propriétaire.

Rien ne relève davantage l'homme à ses propres yeux que le sentiment de sa responsabilité. Les affranchis sont arrogants et ranileux, dira-t-on; mais cette arrogance des débuts se transforme peu à peu en dignité. On l’a vu de 1789 à 1792 , le paysan citoyen participa à l'aecroissement moral de la France; ce fut comme si, revenant en arriere, on l'eùt élevé au rang de seigneur. Il a senti naître en lui un homme et a compris ee qu'il assumait de devoirs ${ }^{1}$. Le salarié devenu propriétaire fera de même.

1. En 1788, đit Taine (L'Ancien Régime, 433), en beaucoup d'endroits, le seigneur n’a plus que ses droits f́́odaux, tout le reste du sol est au paysan.

Aussi l'effort de la Révolution se porta moins sur la dislocation des propriétés que sur leur afranchissement de tous les droits demeurés entre les mains de tiers étrangers à la culture. Ce fut un mouvement analogue à celui qui se produirait si les propriétaires ruraux d'aujourd'hui roulaient supprimer d'un trait de plume le dette agraire, c'est-àdire l'hypothèque de vingt milliards que les capitalistes ont sur la terre

Mais Taine n'a pris qu'un les còtés cle la question quand il a dit (La Révolution, 35̃1) : La crainte de manquer de pain n'est que la forme aiguë d'une passion plus générale, qui est l'envie de posséder et la volon té de ne pas se dessaisir; la Révolution est par essence une translation de la propriété, une expropriation immense el brutale. (Ibid., 386.) 


\section{CHAPITRE III}

\section{L'effort constant des populations rurales pour arriver à l'acquisition de la terre}

atteste l'importance sociale du droit de propriété.

La première forme de la propriété est d'être libre et individuelle; elle prend ensuite la forme collective avec les tâtonnements d'une civilisation enbryonnaire laquelle, à mesure qu'elle se consolide, qu'clle s'élargit et se raffine elle-mème, restilue à l'exercice du droit d’aequérir el de posséder l'individualité qui est son slimulant, la liberté qui est sa vie.

La propriété se manifeste par une oceupation souveraine, effective, qui exclut toute idée de participation et de communaute, a dit un profond philosophe ${ }^{1}$; celte occupalion, dans sa forme légilime et authentique, n'est autre que le travail.

C'est le molif qui contraint la société, laquelle ne vil que du travail, à concéder à l'individu le droil de propriété et à lui en garantir la sécurité. C'est par son origine, le travail, que la propriété est particulièrement respectable; elle l'est aussi par son objet qui est l'héritage, e'est-ì-dire l'existence de la famille, son bien-être el son avenir. La sociélé, pour ces deux raisons, l'une cause première, l'autre conséquence directe, a conslitué la propriété comme sa base indispen-

1. Proudhon, Système des contradictions économiques, II, ch. xI, \& 2. 
sable, après la religion, et tous les eflorts de la législation, dans tous les pays, à loutes les époques, ont convergé rers sa stabilité et sa prédominance.

C'est ainsi que le fait est devenu le droit. La propriété, produit naturel de la combinaison de l'occupation et du travail, aurait pu paraitre $u$ principe d"anlicipation et d'envahissement, car on ne possède pour soi qu'en excluant autrui, si clle n'avail étó reconnue, consacrée et légitimée par le contrat social. Thomas Reid et Emmanuel Kiant admeltent par surcroit que l'acquisition permise à l'individu, par le consentement de la société, peut indéfiniment et logiquement s'accroitre sous la protection sociale, ce qui ne saurait arriver dans lélat de nature et d'isolement oì lindividu seul défend sa propriélé contre les convoitises el les entreprises d'autrui.

Tout est provisoirc dans l'évolution économique des peuples, tout y est variable el contingent, sauf le droit de propriété dont la fixité peut être considérée comme absolue, abstraction faite des nuances de détail qui le caractérisent dans les différentes civilisations. Aussi, l'un des phénomènes permanents du monde économique est-il l'effort conslant des populations rurales pour arriver à l'acquisition de la terre.

C'est la propriété du sol qui fixe l'individu et multiplie les familles. Les plus anciens écrits de l'humanité, les Védas, les livres hébraïques, l'attestent ${ }^{1}$; en anéantir l'individualisme en rétablissant la communauté primitive, ce serait détruire le stimulant du travail, la conscience de la responsabilité personnelle, la notion de ces devoirs envers soi et envers les autres qui se perpétuc dans la famille, ct Larir ainsi la source la plus féconde de l'activité humaine d'où découle la richesse sociale.

Il faut à l'homme la sécurité du travail et, dans la mesure où elle est possible, la certitude du lendemain; il les trouve dans la possession de la terre mieux que partout ailleurs.

1. Dans Juda et Israël, le peuple était innombrable comme le sable de la mer et il vivait dans l'abondance et dans la joie, parce que tout homme demeurait sans crainte, chacun sous sa vigne et son figuier. (Les Rois, 11l.) 
La maison disparaît dans un incendie, le bétail dans une razzia, la récolte dans un orage, la terre seule ne meurt pas, régulièrement féconde et d'autant plus généreuse qu’elle a été plus violentée par la bêche ou la charrue.

Si l'on étudie dans leurs détails les systèmes d'organisation sociale que l'opinion contemporaine place au premier rang, on constate que ce sont en résumé ceux qui réalisent avec le plus de simplicité le problème de la propriété. L'État n'intervient guère que dans les cas d'utilité publique, et les formes de l'expropriation sont corrélatives à la liberté politique de fait ${ }^{1}$; mais il s'applique, par contre, à renfermer dans des limites étroites les biens de main morte afin de conserver à la propriété le caractère individuel et d'éviter les abus auxquels a donné licu dans le passé la possession perpétuelle à titre collectif par des groupes, des associations ou des syndicats.

A la liberté de possession qui ne se discule plus que dans les deux cas que je viens de citer, expropriation et mainmorte, il faudrait ajouter la liberté de transmission qui n'est complètement acquise qu'aux deux peuples dont le sens pratique a devancé tous les autres daus les roies de la liberté civile, les Anglais et les Américains du Nord. Ils pensent, à l'encontre de nos idées latines, que la libre possession des biens entraîne comme conséquence naturelle le pouvoir de les transmettre à qui bon semble. Les autres nations dites civilisées, tout en se piquant de libératisme et en intervenant de moins en moins - sauf en France dans les conditions de jonissance de la propriélé, revendi. quent le privilège de réglementer les transferts et la dévolution successorale. C'est, en effet, le moyen le plus sùr, avec la direction de l'enseignement public, pour pousser les peuples insensiblement dans tel out tel sens et peser sur les sentiments et les intérêts.

1. Quand la liberté civile est livrée à l'arbitraire administratif, le pouvoir exécutif déclare l'utilité publique; chez les peuples les plus libres, c'est le pouvoir législatif. Comparer les articles 19, 20 et 21 de la loi du 13 avril 1900 à l'art. 2 de la loi du 3 mai 1811. 
Mais la masse des individus, trop ignorante pour aperceroir la portée de eertaines institulions, de ecrtaines entraves, de eertaines mesures fiseales d’inquisition ou de dépression, se préoceupe moins des embarras qui peuvent, à un moment donné, eontrarier ses vues que de la possibilité immédiate qui s’ofire à elle de s'élever d'un degré, par la propriété, dlans la hiérarehie sociale.

Le désir d'arriver à la possession d'une parcelle de eette terre qui produit tout el qui est le signe de la riehesse est instinetif chez l'homme le plus inculte; il semble qu'il comprenne, par une sorte d'intuition alavique, que possesseur autrefois, par ses ancêtres, de ces biens qu'il eonvoite, dépossédé par les hasards de l'existence, il ne fait, s`il parvient à derenir propriétaire, que rentrer en possession de son patrimoine perdu. Il a comme la notion eonfuse d'un droit primitif qu'il ne peut revendiquer, ses titres ayant disparu, mais que son labeur, son zèle, sa patience, lui méritent à nouveau, et qu’en somme il aura payé deux fois.

L'existenee d'une elasse nombreuse privée de loute propriété et rivant en quelque sorte dans un état de dénûment héréditaire est un fait nouveau et aceidentel ". La propriété est le régime normal de l'humanité; et partout, eependant, il y a des populations entières qui n'ont plus de foyer ${ }^{2}$, qui vivent d'un salaire eapricieux, exposées à toutes les conséquences des ehômagres qu'elles subissent ${ }^{3}$ ou des grèves

1. Depuis l'état de paix de 181 à à 18\%0, l'extension subite du régime manufacturier a changé les conditions de la vie rurale. Enlevant les populations au lieu natal, il les priva du patronage des voisins, des riches, les isola, les accumula dans des localités dépourvues d'institutions propres à conjurer les effets de la pauvreté, du vice et de l'imprévoyance. (Le Play, La Réforme sociale en France, 1866, 11, \& XLIX.)

2. D'après le dénombrement de 189l, plus de 650000 individus ont quitté leur commune d'origine; par rapport à la population totale, l'èmigration à l'étranger n'est que de 1,7i p. 1000 ; mais l'émigration des campagnes dans les villes atteint 24 p. 1000.

3. Les clròmages varient suivant les industries et les régions. Ainsi, pour les cordonniers de Fougères, la journèe est réduite de 1/4 et souvent de $1 / 2$, de mars à novembre; les tisseurs de Fresnoy-le-Grand 
qu'elles provoquent'; c'est la preuve d'une désorganisation sociale dont les racines sont profondes, qui n'a pas surgi spontanément, dont les conséquences dissolvantes s'accroissent arec le temps et à laquelle on ne pourra remédier par le procédé impuissant de la spoliation des riches mais par le relèrement continu, au moral et au matériel, des classes laborieuses. Ce relèvement ne peut aroir pour levier que l’attrait de la propriété, le retour à la vie de famille, à la fixilé des entreprises; pour ramener l'ouvrier industriel, ce déraciné, vers l'existence régulière qu'il abandonne en désertanl les villages pour les villes, il faut tirer parti de celle passion de la terre qui sommeille en lui, mais qui ne meurt ni ne s'éteint, et dont la vivacité peut renaître soudain pour peu que l'occasion s'en présente et que l'intérèt dans son esprit s'accorde arec le sentiment.

C'est dans la vie rurale que lintérèt privé de chacun s’identifie le mieux avec l’intérèt général; il semble que les harmonies de la nalure y préparent, sans effort, un équilibre dont les conditions sont faites de besoins semblables et de services mutuels oì la réciprocité est plus souvent oflerte que réclaméc.

La médiocrité de la vie rurale n’est pas un obstacle à laisance, quoi que prólende une École qui a le niveau pour idéal, el qui soulaite pour aului la somnolence des abdications el la triste habitude des partages. Les crises économiques sont fréquentes dans les pays riches, faisait remarquer, en 1892, la liéforme économique, el e'est dans les pays pauves fue règne le plus habituellement, par une sorle d'ironie sociale, une prospérité relative.

Un économiste disait récemment: Nous regorgeons de mélaux précioux, de vivres, de bétail, de marchandises de

(Aisne) choment du L $^{\text {or }}$ octobre au 31 decemlre; les mégrissiers d'Annonay cloòment de septembre a fin mars, ctc. (Office du travail : Salaires et clurep du travail dans l'industrie franşrise, II, 189t.)

1. En 1898, il y a en 368 grèves comprenant 82065 grévistes qui ont cessé le Iravail pendant 1216306 journces, y compris 15393 t journces perdues par 5900 ouvriers non grévistes, contraints au chỏmage par la Erève. (O/fice du travail, statistique des greves, 1899.) 
toutes sortes et nous sommes, paraît-il, plus pauvres que Job. Cet or, ees virres, sont plus saerés que le voile de Tanit auquel nul ne pouvait toucher.

Les marchés le l'étranger se ferment pour nous et notre marché s'ouvre pour l'étranger seul. Les industriels se plaignent, les commerçants se plaignent, et aussi les producteurs, et aussi les consommateurs. Qui a tort et qui a raison? Qui expliquera ees contradictions? Les blés russes arrivent à Marseille avec une prime de cent sous parquintal, les vins d'Espagne et d'Italie inondent nos entrepôts, et nos chaix sont pleins. J'ai lu quelque part qu'à Lille, à Roubaix, une ouvrière fabrique à elle seule, par jour, 78 paires de bas au métier, et qu'elle marche pieds nus. Laboureur el vigneron trouvent la ruine dans l'abondanee, de mème qu'en 1692 les paysans de Bourgogne, dont les greniers eraquaient sous le poids du blé, n'avaient pas deux pistoles pour acheter du drap.

Quelle est la cause de la crise agraire en Sicile, avec ce feu qui coure sous la cendre et, de temps à autre, comme l'Etna, se révèle par de subites secousses? La suppression des petits propriétaires; les syndicats de travailleurs et de révoltés (Fasci dei lavoratori) n'y sont qu'un expédient dangereux. Le régime féodal, aboli de droit en 1812, n'a pas cessé d'y subsister de fait. Pour le Barone, la terre n'est qu'un fief, le paysan qu'un instrument de gain. Pareil au fellah d'Égypte, le rural sicilien, pressuré par d'àpres fermiers, s'épuise pour les propriétaires dont les vastes domaines demeurent en partie incultes et gagne à peine du pain. Et cependant, la Sicile a nourri des millions d'hommes, le sol y garde sa fécondité proverbiale; les céréales, la vigne, les arbres fruitiers, les mines de soufre, devraient garantir la richesse et le bonheur à celte île privilégiée. On y meurt. de misère, et le meurtre n'y est plus traité comme un crime mais comme une revanche un peu brutale '.

1. L'Italie, depuis les lois agraires cle l'ancienne Rome, peut être considérée comme l'un des champs d'activité où se sont le plus accentués, et avec le moins de succès, les efforts des populations rurales pour par- 
L'Irlande avee ses tenanciers, l'Allemagne du Nord avee ses paysans cantonnés par les hobereaux ' sont-elles, à ce point de rue particulier, beaucoup plus favorisées que la malheurcuse Sicile? Les habitants se hâtent d'émigrer aussitôt qu'ils ont réuni assez d'argent pour payer leur passage outre mer et s'expatrier ${ }^{2}$. Le paysan français émigre peu; il se sent à l'aise; il se sait chez lui, et celui qui n'est pas encore propriétaire attend avec patience de pouvoir l'ètre ${ }^{3}$.

Dans nos traditions francaises, et il faut les mainienir, la propriété c'est l'héritage, e’est-à-dire le domaine de la famille; celui qui le possède n’en est que le cépositaire, même šil l'a créé, à plus forte raison s'il lui vient de ses ascendants; il le doit à ses enfants. Notre vieux droit disait: Le mort saisit le vif. En matière d'héritage foncier, le mort

venir à la possersion de la terre. les actes publics, pour ne parter que de l'époque contemporaine, se sont multiplićs pour distribuer le sol aux mains de prolétaires laborieux. Mais, déclare déja, en 1812, un document napolitain ofticiel, il arrive 9 fois sur 10 que les lots provenant de la division des propriétés communales tombent en des mains si misérables, si dépourvues de ressonrces nu de patience, que sans en tirer nul profit clles ont fini par les abandonner. En 189\%, on constatait, à Rome, que la bonne volonté des paysans se heurtait à l'indliflèrence des riches proprietaires et au défaut de capitaux et de débouchés. Le salarié agricole, y dit-on, pent quelquefois manger un morceau de pain blane, mais le petit proprietaire rural meurt de faim.

Voir. sur l'état de la grande proprieté dans les Calabres, un article de M. G. Goyau lans la Revue des Deux Mondes (1898, I, 89).

1. God. Cavaignac, L'évolution agraire en Prusse au XIT' sircle (Revue' des Deux Mondes. $1^{\text {er }}$ novembre 1892).

2. D’après les dernières enquêtes en Prusse, on ỹ compte, pour 26 millions d'hectares, 3 millions d'exploitations rurates, dont $1 / 3$ seulement ( 232168 avec 2 k millions d"hectares) constituent lin profession unique des propriétaires et suffisent a leur existence; les autres, au nombre de $1 s 08028$ cultirant seulement 2 millions $1 / 2$ d'hectares, sont l'accessoirc et l'appoint d'autres professions.

3. Les chiffres officiels des émigrants français à l'étranger, d'après les statistiques du ministère de l'Intérieur sont les suivants:

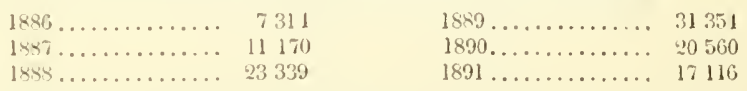

La population rurale ne figure dans ces chiffres que pour une moyenne de 17 p. 100. 
ne disparait pas tout entier, il se continue, il revit dans ses enfants. Mais la famille d'aujourd'hui, et e'est un facteur important de l'état social noureau, n'est plus la famille élendue d'avant 1789, alors quion se déplaçait peu el que le cousinage ramifiait les branches de la gens latine et en faisait comme une collectivité dans la paroisse et ses cuvirons, ee n'est mème plus la famille dı Code civil dont l'article 733 élend la vocation héréditaire jusqu'an 120 degré; elle so réduit actuellement au père, à la mère et aux enfants. Aussi la loi de $189:$ l'a-t-elle resserrée légalement en écartant les collatéraux pour faire une place à l'époux survivant et, depuis, on a reconnu à l'enfant naturel les droits que l'équité lui accorlait.

En mesurant, depuis plusieurs siècles, la volatilisation du capital, sous l'action combinée de la diminution de valeur de l'or et de l'argent, de la dépréciation de la monnaic, de la baisse continue du taux de l'intérêt, on s'aperçoit que la richesse la moins variable, la moins aléatoire est la terre, paree que ses produits vaudront toujours leur prix proportionnellement aux besoins de la consommation, et qu'on peut en vivre, mème sans les vendre.

Toutes les fois qu'on a parlé de réforme agraire, à quels expédients s'est-on arrêté? Aux seuls qui sont connus pour ètre dune appropriation immédiate : ou clonner de la terre aux prolétaires sans travail, on donner du crédil aux propriétaires obérés. Le premier n'est pas d'une exécution facile. Faudrait-il partager entre les ouvriers qui ne sont pas du métier et dont l'apprentissage serait onéreux, en admettant qu'ils y prissent goût, les friches et terres incultes dı Domaine ${ }^{1}$ ou exproprier les terrains stériles appartenant à des particuliers qui ne les utilisent pas², pour les allotir à

1. Les terres domaniales cultivées, y compris les bois et forêts, sont d'une superficie de 1116708 hectares; ics landes, friches, ete., de 103730 hectares. Les terres stériles ou incultes appartenant à des collectivités (départements, communes et hospices) ont une superficic de 1463616 hectares.

2. Les particuliers possédaient, en 1892, \&605166 hectares qui n'étaient pas mis en valeur. 
des journaliers agricoles? On a pu l'essayer, dans de très petites proportions, en Sicile ${ }^{1}$; mais les conditions économiques ne sont pas les mêmes, heureusement, dans notre pays, el mieux raul se borner à ne pas entraver le mouvement incessant qui, par le trarail et l'épargne, crée, chez nous, de nouveaux propriétaires d'autant plus altachés à leur entreprise qu'elle est une œurre d'énergie el de rolonté.

Le crédil se mérite, a-t-on dit, el la caution type, le réritable gage de n̈importe quelle espèce de crédit, e’est le crédit personnel; il en est de mème de la propriété, elle se mérite autant quiclle se paie. La est le secret de sa force.

Le siècle qui a précédé la Révolution ful, au dire des économistes et des historiens du temps ${ }^{2}$, l'un des plus misérables de notre histoire rurale. Tout était en surface et en facade; si l'on ereusait, si l'on entrait, ruine et misère. Et cependant la France a récu, et durant cette période, elle a accumulé les énergies qui lui permirent de faire 1789 et de conduire les grandes guerres de 1792 à $181 \%$. L'auraitelle pu si, comme en Irlande, comme en Italie, comme dans les provinces baltiques entre le Niémen et l'Oder, elle n'avait eu pour eitoyens que des cultivateurs faméliques, des déracinés ayant perdu jusqu’aux attaches morales qui lient le cœur au sol natal?

Depuis les $\mathrm{xIl}^{\mathrm{e}}$ el $\mathrm{x}_{111^{\mathrm{e}}}$ siceles où l'accensement el l'affranchissement reconstituèrent la très petite propriété, l'effort du paysan propriétaire fut de s'agrandir, l'effort de l'ouvrier agricole de posséder. Nais le groupe des prolétaires ruraux, à celte époque, étail quasi négligeable, el celui qui écrira l'llistoire de la propriété en s'aidant des terriers, des encuêtes, des minutes notariales et des registres paroissiaux, pourra pronver que jusqu'au $x v^{0}$ siècle il n’élait pas un cultivateur, en France, qui ne fùt proprićtaire.

1. En 189:3. M. Crispi a partagé a titre provisoire les terres domaniales de Calatabiano (Sicile) entre 279 peres de famille, moyennant une très modique redevance pour les dix premières années.

2. Taine en a reproduit et résumé les appréciations. (I'Ancien Régime, livre $\mathrm{V}$, ch. r.) 
Le prolétariat rural est né des destructions el des pillages du $\mathrm{Xv}^{\mathrm{e}}$ siècle, des guerres civiles du $\mathrm{xvI}^{\mathrm{e}}$, de la crise économique du xrir ${ }^{\mathrm{e}}$, enfin du développement industriel du xix provofuant l'émigration des eampagnes et la dislocation des familles agricoles. Mais toujours, daus la bonne fortune comme dans la mauraise, appaurri ou à l'aise, le paysan a conservé le groùt de la terre, la passion de posséder el, quand il possède, d'acquérir encore.

Du $\mathrm{x}^{\mathrm{e}}$ au $\mathrm{x}^{\mathrm{r}} \mathrm{l}^{\mathrm{e}}$ siecle, le paysan a beaucoup acquis par achat de pareclles et surtout par empiélement et par usurpation. Les géomètres connaissent encore le plénomène des haies qui marchent et des forèts qui s'échancrent; mais une circonstance était particulièrement favorable à ce que la terre se dégageât par désuétude, et l'homme avec elle, de ses derniers liens avec le propriétaire primitif. Beaucoup de norcellements datant des temps scigneuriaux, les tènements, divisés et subdivisés par les partages, les ventes, les échanges, devaient se reconstituer une fois l'an pour le paicment du cens et des redevances; peu à peu, la trace de ces divisions s'était effacée, le souvenir de la dette s'était perdu. On ne savait où retrouver le débiteur, souvent mème il se montrait récalcitrant, demandait la production des titres ${ }^{1}$, et, de guerre lasse, cens et redevances s'évanouissaient sans que le cultivateur pût arguer d'un autre titre que de celui de la possession.

An $\mathrm{xry}^{\mathrm{e}}$ siècle surtout, les grands domaines furent désertés et le paysan s'empara de tout ce qui n'élait ni gardé ni défendu. Puis, tandis que les nouveaux riches, nobles ou rolurier's, se créent, de 1600 à 1789 , de vastes domaincs, cn rachetant parcelle à parcelle tout ce qu'avaient aliéné les délenteurs du sol au temps de saint Louis, quantité de châteaux s'écroulent, faute d'argent pour les réparer, et les laboureur's riverains mordent sur les dépendances, les jardins, les ehènevières, les courtils abandonnés. La rente de la

1. Tel le procès interminable que firent les paysans de Toutry et autres paroisses au maréchal de Bourdillon, marquis d'Époisses. (Archives du Parlement de Dijon.) 
terre, les droits féodaux, nétaient plus qu'un reste de taille sur les gens, selon l'expression de Forbonnais, plutot que le produit direct des choses. Dis 1730 , nombre d'anciens nobles et même d'anoblis se trouvèrent réduits à la pautvreté arec des titres de propriété immenses; ils n'avaient que la nue propriété stérile de terres dont le paysan exploitait la pleine jouissance; de là ces nombreuses acquisitions que les paysans, si misćrables qu'ils nous apparaissent, firent auprès de gens ruinés ${ }^{1}$ et qui n'avaient pas, comme eux, la ressource de la culture direcle. Toutes les épargnes des basses classes, dit Turgot, qui ailleurs sont placées sur des particulier's et dans les fonds publics, sont destinées en France à l'aehat des terres. Entre mille preuves, on peut rappeler la déclaration de lintendant de Caen trourant que, sur l'ensemble des cotes foncières du vingtième, $1 / 3$ n'excède pas cinq sous et un autre tiers vingt sous ${ }^{2}$.

Wichelet a vu de mème; mais, en généralisant trop, il prète à l'équivoque; ce qu il dit du propriétaire, trop aisé ì décourager, el quiil fallait ramener vers la propriété, pour qu'il se reprît à l'aimer, à la culliver, à la laire produire ${ }^{3}$, ne s'applique pas, dans sa penséc, au paysan, au petil propriétaire, mais au noble, au bourgeois, au capitaliste, an forain, qui ne résidait pas et n'avait, sauf des cas très rares ${ }^{4}$, ni vues économiques, ni théories humanilaires, ni même le souci de ce qui se passait ehez lui.

La terre ne voulait plus produire, dil-il, on semait le moins possible. Le sol pesuit à ses propriétaires, la terre leur était odiense. On la ionnait presque pour pien; déja un quart du sol de France était aux mains les lahouremrs: circonstance heurense, ce semble, pour la production; eb bien, on ne prorluisait pas. L'école ćconomiıne hâta le unouvement qui dans cette France encore agricole, oil la manufacture ctait fort secondaire, devait à tout prix défricher, algmenter la culture du seul aliment de la population d'alors.

1. Taine, L'Ancien Rérgime, liv. V, chap. I. - G. l'Avenel, Histoire économique, I, liv. II, cliap. 111.

2. Letlre de II. de Fontette du 16 noveml)re 1772 (Archwes nationales, I1, $\left.\mathrm{n}^{\circ} 1 \mathrm{i} 63\right)$.

3. llistoire de France (Louis XV et Louis XVI), t. IVII, chap. xu.

4. Montesquieu, le due l'Harcourt, Buffon, l'Argenson, le bailli de Nirabeau, etc. 
La nécessité de multiplier le nombre des propriétaires décidés à l'exploitation directe et comprenant que l'industrie agricole vaut les autres, n'est pas une noureauté dans l'esprit des économistes; on en trouve déjà la préoccupation dans Olivier de Serres, el cerlains écrits du $\mathrm{xvI}^{\mathrm{e}}$ siècle nous apprennent que le goût de la propriété, en Normandie par exemple, conduisait facilement à l'appropriation illégitime. C'étaient de petits propriétaires qui en donnaient l'exemple, empiétant les uns sur les autres et sur le domaine commun, labourant d'andace la terre du voisin, déplaçant les bornes ou en posant à leur gré ${ }^{1}$, plantant des poiriers dans un chemin communal ${ }^{2}$.

Le marquis de Mirabeau, dans sa Philosophie rurale, en 1764, ripostait à Quesnay, faisant de la terre la source unique de toute richesse, que c'était l'homme qui fécondait la terre par le travail et que la stérilité de l'un causail la sécheresse de l'autre puisque leurs fécondités étaient solidaires. Quant au moyen d'associer le travail et la terre pour leur profit commun, M. de Montyon en donnait la formule.

Puisque l'abondance des produits nécessaires à la vie influe sur la densité de la population, disait-il, il faut que chaque individu ait la possibilité de les obtenir par la propriété du sol ou par le travail.

Il y a trop de grandes propriétés, ajoutait-il; il est essentiel que la propriété des terres soit distribuée de telle sorle qu'il n'y ait presque aucun habitant qui n'en possède quelque porlion.

Ce retour à l'idéal qu’avait réalisé le xum ${ }^{\mathrm{e}}$ siècle sous la pression de l'intérêt n'a cessé d'être l'objectif principal des populations agricoles.

Le paysan, qu'il fût de Bretagne, de Bourgogne, de Lorraine, de Languedoc ou de Provence, tel qu'il sorlit du

1. La roture ne faisait qu'imiter les procédés de certains nobles; avant que le sire de Gouberville, en Normandie, vers 1553, eût à modérer l'ardeur de paysans trop entreprenants, un arrêt des commissaires de la Chambre des comptes de Dijon, condamne le sire de Thoisy (18 mai 1502) à arracher six bornes qu'il a plantées sans droit sur lés chenins publics du village de Cernois.

2. II. Baudrillart, Gentilshommes ruraux de la France, I, 40. 
régime féodal, forma deux groupes. Les uns, plıs hardis ou plus ambilieux, cherchèrent à s'agrandir, à transformer leurs censives originelles en pelits fiefs; le changement de condition nest pas toujours un élément de durée, et bien peu de ceux qui firent souche de seigneurs ont vu leur lignée se prolonger ${ }^{1}$. Les autres, salisfails d'ètre devenus propriétaires et maitres d'eux-mémes, nayant pour prétention que de perpétuer d'honnêtes familles travaillant l'héritage des anciens, se groupant en communautés d'liabitants pour résister aux entreprises des scigneurs voisins, cherchant uniquement à libérer leur terre de toute charge, à diminuer ou à racheter leur rente, à payer le moins d'impòts possible, ceux-là ont duré. 11 suffit, pour s'en assurer, de comparer les contrats, les rôles d'impòts, les registres des paroisses; on $y$ retrourera, parmi les notables de nos communes rurales, les rieilles familles d'il y a quatre siècles. Cette noblesse là ne vaut-elle pas les autres?

Par l’abolition des droits féodaux, la Révolution porta un coup décisif à ce qui restait de propriété collective; elle incorpora à la propriété individuelle, au domaine privé, une masse de territoires qui jusque-là y étaient réfractaires et, sans avoir eréé le moreellement, l'étendit. C'est moins la vente des biens nationaux qui a grossi le nombre des pelits propriétaires que l'extension de la eulture, laugrmentation de la surface cultivée, l’immensité des landes, pâtures el forêts indivises qui lurent envalies par les habitants des villages roisins el vinpent accroître les lots que possédait déjà la petite propriélé paysanne 2 .

Ricardo el Stuart Mill font du propriétaire foncier un être

1. M. de Saint-Genis, IIstoire du fief rural de li Rochelle (Bullelin du Comité des travaur historiques, 1883, 70; 1892, 70 et 182).

2. A partir du 10 aout 1793 . chaque commune ou particulier se croit le Aroit de s'approprier ce qui lui convient des propriétés publiques, produit ou sol. Les biens communaux ne sont pas plus respectés que les biens nationaux. Il suffit que le liers des habitants demande le partage pour gu'il s'exécute, en petits lots et par lête (Décrets des lí août 1792 et 10 juin 1793). En moins de deux ans $90 \mathrm{p} .100$ des communes dilapiderent ainsi (en delıors des bois) leur réserve territoriale. 
privilégié, en ce sens que tous les progrès de la civilisation lui profitent, une sorte de parasite des sociétés avancées, qui tire à lui le profit net du travail collectil et des améliorations sociales, sans qu'il ait besoin de s'y associer par son aclivité personnelle, par son industrie, ni mème par cette abstinence d'une genre parliculier que l'on appelle l'épargne. Lobservation des économistes anglais, vraie dans le passé, le deviendra de moins en moins, à mesure que la concurrence étrangère annulera les ressources naturelles de notre sol et que l'impòt exercera sur ses produits un prélèvement de plus en plus lourd.

Ce n'est plus à la nature seule que la proprićté rurale devra désormais les préférences qui lui élaient acquises jusqu’ici; il faudra qu’au travail le propriétaire sache associer l'industrie, la prévoyance el le caleul. La hausse des salaires, la baisse des prix de rente, peuvent déprécier la propriété rurale et ramener des crises semblables à celles du $\mathrm{xv}^{\mathrm{e}}$ sic̀cle $(1330$ à 1480$)$, du xvil ${ }^{\mathrm{e}}(1633$ à $163 \ddot{\text { el }} 1690$ à 1710$)$; mais, en France, le but certain de l'épargne est l'acquisition de la terre et rien ne prévaudra contre un sentiment qui est dans le sang de notre race ganloise.

I. - Pourquoi il faut distinguer, en matière économique, entre le morcellement, le fractionnement et la dispersion de la terre.

La division de la propriété se présente sous trois formes diverses que l'on a souvent confondues, ce qui conduit à des équivoques et à des erreurs. Si l'on entend par propriété l'ensemble d'un hérilage ou d'un domaine, fùt-il divisé en plusieurs exploitations (fermes, métairies ou réserves), on peut l'envisager au point de vue du morcellement du sol, éestà-dire de son étendue globale, soit qu'on la compare à la superficie du territoire de la commune, soil qu'on la rapproche des autres propriétés similaires pour la classer dans une des catégories de la grande, de la moyenne ou de la 
petile propriété. On peut ensuite l'étudier à deux autres points de vue, le fraclionnement et la dispersion. Le fraclionnement, si au lieu d'ètre d'ine seule pièce, la propriété se compose de parcelles agglomérées formant une ou plusieurs exploitations; la dispersion si, el ciest le eas le plus fréquent, la propriété, au lieu d'être d'un seul tenant, est iormée de plusieurs parcelles éparses, sourent éloignées les unes des autres ou formant enclave au milieu de propriétés voisines.

Il semblerait que la statistique des propriétés et par conséquent leur classement dussent ètre des plus simples, si l'on s'en référe au cadastre et à l'impôt foncier, éléments apparents de toute recherche. Malheureusement il n'en est rien, et les différents services publics qui ont pour mission principale de constituer et de consolider le droit de propriété, pour mission accessoire d'en suivre les mouvements et de les laxer pour l'impôt, ne font s'accorder ni leurs définitions ni leurs procédés, de telle sorte que la plupart des chiffres ne sont pas comparables, el que la cole foncière, le domaine, l'héritage, l'exploitation, ne figurent que les formes arbitraires ct fuyantes d'une entité qui se transforme en Protée à mesure qu'on croit en saisir et en fixer l'unité logique.

Si chaque propriété élail comprise tout entière dans lo périmètre d’une seule et même commune, le nombre des cotes foncières donnerait le nombre exact des propriétés foncières avec leur étendue, puisqu'une cole est la part de l'impôt foncier que chaque propriété doit acquilter dans la commune, à raison de son revenu. Mais les proprićtés ne s'arrêtent pas toutes aux limites de la commune; leur superficie s'étend 'juelquefois sur plusieurs communes limitrophes, soit par la simple juxtaposition des parcelles, soit par leur fractionnement ou leur dispersion, de sorte que tel propriétaire, qui n’a qu'une propriété sur les confins de la commune, peut avoir une portion de sa terre sur la commune qu'il habite el l'aulre sur une ou plusieurs communes voisines. Si la propriété se compose de nombreuses parcelles, elle peut avoir des parcelles éparpillées sur plusieurs 
communes; il y a dans ce cas, pour celle propriélé, aulant de cotes qu'il y a de communes sur lesquelles elle s'ítend '.

En d'autres termes, le fractionnement et le chevauchement ou la dispersion d’un certain nombre de propriétés sur une ou plusieurs communes, bien que se compensant dans une certaine mesure pour une circonseription plus grande, comme le département, par exemple, constituent, pour les statistiques, le principal obstacle en ce qui concerne le relevé du nombre et de l'étendue des propriétés.

La difficulté devient plus grande cncore si l'on calcule par exploitation, comme dans l'enquête de $1892^{2}$, au lieu de calculer par propriété, parce qu'alors il peut se rencontrer plusicurs modes de culture (culture direcle ou réserve, fermage, métayage) dans une mème proprićté et que la statistique établie sur ces principes compte 7 ou 8 cxploilations, c'est-à-dire 7 à 8 unités de culture, là où il n'y a en réalilé qu'une seule propriété au sens de l'économie sociale. Ces divergenees d'apprécialion ct de classement sont de nature à vicier toutes les conjectures qu'on peut tirer de la classification officiellc en grande, moyenne el petite propriété.

Les mèmes inconvénients se reproduisent dans les statistiques antérieures oủ l'on se base, tantôt sur la notion de valeur, tantòt sur la notion d'étendue, de telle sorte que le contrôle de ces chiflres par les statistiques de l'Enregistrement devient impossible; ces dernières, en effet, ne subdivisent pas arbitrairement une proprićté, comme le fait le service des Contributions directes, el considerent comme propriété ayant son individualité et en quelque sorte son état civil, tout domaine connu sous un nom et dont l'ensemble, plutòt juridique que cultural, représente en réalité la propriété, l'héritage.

M. de Foville a donc raison, pour élucider les éléments d'appréciation du morcellement et en déterminer la valeur relative, de poser les deux formules : $1^{\circ} \mathrm{Il}$ y a en France plus

1. Statistique agricole décennale de 185:, Introduction, p. 276 et 277.

2. Stalistique agricole décennale de 189\%, Introduction, p. 346. 
de propriétés que de cotes foncières, puisque la même cote doit comprendre les diverses propriétés qu'une seule personne, ou un mème ménage, a dans la mème commune ${ }^{1}$; $2^{\circ}$ Il y a en France plus de cotes foncières que de propriétaires, puisque la même personne est souvent propriétaire dans plusieurs communes et anmule ainsi plusieurs cotes. En attendant qu'on se décide à surveiller le service des mutations, en souffrance depuis 1830 , el à créer, en fait, l'unité de cote par contribuable dans chaque commune ${ }^{2}$, les économistes devront se résigner à raisonner sur des à-peuprès.

Aussi M. Levasseur dit-il avee raison que les statistiques ne sont pas toujours l'expression exacte des faits dont elles cherchent à déterminer la quantité et que le devoir des statisticiens, qui ont pour mission d'éclairer le public et non de le tromper par de fausses apparences, est de présenter leurs chiffres comme des approximations on mème comme de simples vraisemblances numériques, quand ils nont pas assez d'éléments sérieux pour être phus alfirmatifs ${ }^{3}$.

Si l'on ne peut déterminer que d'une manière conjecturale le nombre des propriétaires fonciers, urbains el ruraux ${ }^{4}$, on ne peut pas non plus arriver à une précision même relative quant à limportance des diflérentes catégories de propriétés.

1. Se reporter aux prescriptions lu titre IV de la loi clu 3 frimaire an VIl relatives à la répartition, à l'assietle et all recouvrement de la contribution foncière, prescriptions si ètrangenent méconnues depuis par l'irresponsabilité où a glissé la routine administrative.

$\therefore$. L'inexactitude des statistiques du service des Contributions directes tient à la négligence du contrôle; à la non-exéculion du service des mulations malgré l'énorme crédil qui figure, chaque année, au hudget pour cet objet; á la mauraise gestion des percepteurs qui, ayant unc retribution par cote, les multiplient au lieu de les fusionner quand il y a lieu, comme cest leur devoir. Tout ceci est de notoriété publique, et pas un ministre n'a jaru le savoir.

3. Note sur la valeur de la production agricole, présentée à la Socićté nationale l'Agriculture de France, dans la séance du 15 avril 1891.

4. Il serait aisé de dresser avec une précision absolue, avec une unise à jour automatique, le terrier général de la France si l'ou se décidait ¿. voter le projet de loi sur la réforme hypotlécaire déposé par le ministre de la Justice au Sénat, le 27 octobre 1896, en attendant la creation des Livres fonciers. 
Ladministration a fait à cinq reprises, dans le courant du $\mathrm{xix}^{\mathrm{e}}$ siècle, le classement des coles foncières d'aprís leur chiffre, en 1816, en 1826, en 183\%, en 1812, en 1838; en 1876, nouvelle enquète; les mêmes recherches ont été faites, non plus d'après le chiffre de l'impòt mais d'après la contenance des cotes du cadastre primilif, en 1831, en 1861, en 1871, en 1881; les enquètes pour le recensement des propriétés bâties et des propriétés non bàlies, fort discutées d'ailleurs quant à l'exactitude de leur exécution et à la sincérité de leurs résultats ', n’ont pas donné les certitudes qu'on en attendait.

On y peut trouver cependant des renseignements intéressants; ainsi la commune du Vésinet (Seine-et-Oise) qui, en 182', couverte par une forèt depuis allotie et non pas défrichée mais bâtie, ne comptait que 11 heetares imposables avec son unique proprićtaire, possède, d'après le cadastre de 1883, 392 hectares cotisés, 1766 parcelles et 7\%9 propriétaires ${ }^{2}$.

L'arrondissement d'Avesnes (Nord) présente les changements ci-après, si l'on compare le cadastre primitif au nouveau :

\begin{tabular}{|c|c|c|c|}
\hline & \multicolumn{3}{|c|}{ NOMBRE } \\
\hline CADASTRE & $\begin{array}{l}\text { d'hectares } \\
\text { imposables } \\
\text { - }\end{array}$ & $\begin{array}{c}\text { de } \\
\text { parcelles }\end{array}$ & propriétaires \\
\hline Primitif le $1 \$ 26 \ldots . .$. & 35616 & 75664 & 931 t \\
\hline Nouveau de 1862 à 1883. & 36683 & 77393 & 11502 \\
\hline
\end{tabular}

En cinquante-sept ans, le morcellement a donc produit, dans les 41 communes recadastrées, 1729 parcelles de plus, et une augmentation de 2188 dans le nombre des propriétaires inscrits au ròle de l'impòt foncier.

Le morcellement, dans le langage ordinaire, s'entend de la division de la propriété, de sa répartition entre un nombre

1. Dans la commune que j'habite, j'ai 5 cotes pour une somme d'impóts de 369 fr. 71, portant sur une propriété très dispersée de 40 hectares seulement.

2. Bulletin de statistique du ministère des Finances, dẻcembre 1883, p. 669 et 681 . 
de propriétaires plus ou moins considérable. Ce mouvement est la conséquence des érolutions économiques; nul ne peut aroir la prétention de le régler, el sa mesure, toujours variable, peut être considérée comme l'expression de circonstances dont Yincidence se produit dans tel ou Lel sens. Nos préférences n'hésitent pas entre la grande et la petile propriélé, car il faut voir dans les progrès de celle-ci une influence morale dont l'effel social est infiniment supérieur aux avantages matériels qui découlent de lextension de la culture intensive.

La question du fractionnement parcellaire est aussi distincte de celle de la division de la propriété que de celle de la dispersion des propriétés. La parcelle n'est pas l'unité foncière. Son existence administrative ${ }^{1}$ et cadastrale n“implique pas nécessairement une discontinuité dans la propriété du sol; le domaine le plus compact, aggloméré avec méthode, peut fournir au cadastre cent unités distinctes, et, dans la pratique des bureaux chaque propriété bàtie fournit deux pareclles, une pour la construction, une pour le sol qui la porte, ce qui est déjà en contradiction avec la définition du règlement. On admet actuellement pour la France une moyenne de 1 140 millions de parcelles de 27 à 34 ares et même moins. Cet émiettement apparent n'il rien d'inguiétant et il suffirait de quelques mesures administratives pour fusionner les parcelles parasites et en réduire le nombre au chiffre normal; on voit déjà, par la comparaison de l'ancien et du noureau cadastre que, là oủ le nombre des proprićtaires a augmente de 10 p. 100 (Eure, Seine-et-Marne, llauteMarne, ete.), de 182:; à 1880 , celui des parcelles a diminué de 7 is 8 p. 100.

Le probleme de la dispersion des parcelles est tout autre; ses origrines historiques sont fort anciennes ${ }^{2}$, on est manime à en déplorer les inconvénients et l'on n’y peut porter remèle

1. Recueil méthodìue des lois, décrets, règlements, instructions et décisions sur le cadastre de la livance.

2. Hémoire sur la propriété rurale en Bourgogne au moyen áge (Bulletin du Comité des travaux historiques, 1896). 
que par l'organisation de syndicats de remembrement. Le nombre est infini de ees domaines discontinus dont les parcelles petites ou grandes sont éparses comme des îlots sur le territoire d'une commune. Cet état de choses quasi universel crée it l'exploitation agricole des embarras sans nombre, difficultés de voisinage et d'accès, difficultés de clòture, d'aménagement et de culture, difficultés de surveillance et de récolte. Franģois de Neufehâteau écrivait en 1806 :

Avec les territoires hachés, cisallés, sans chemins pour arriver aux lambeaux qui les constituent, l'igriculture, froissée par les entraves que le parcour's et la vaine pâture multiplient autour d'elle, ne peut pas plus gran!lir qu'un enfant qu'on garrotterait au berceau avec des liens de fer 1.

On évalue à 40 p. 100 la moins-value pour la eulture des proprićtés disséminées, par comparaison aux domaines eompacts oì le laboureur peut se retourner librement. Le mal ne s'étend pas, comme on l'a dit après un examen superficiel, et il n'a point pour origine la loi des partagres.

Dans notre société démocratique issue de 1789, affirmait un député dans une proposition de loi sur les échanges ${ }^{2}$, la division des héritages a fini par en amener la dispersion. Ils se sont fractionnés, enchevètrés parfois, en damiers irrégulièrement découpés, bizarrement déchiquetés. C'est ın mal qui est sorti d'un bien.

Le mal ne vient pas du Corle civil : il est plus ancien; on le trouve ì l'étranger comme en France, et le législateur, chez nous comme ailleurs, s'est efforcé de faciliter les groupements ${ }^{3}$. Bien avant 1789 , les propriétaires ruraux de certaines provinces se sont syndiqués pour procéder à des remaniements collectifs, et l'on y trouve le témoignage du développement de la très petite propriété autant que de la dispersion excessive des parcelles et de leur enchevêtrement.

On a surtout cité ceux de Rouvres en Bourgogne (de 1697

1. Voyage agronomique dans la sénatorerie de Dijon.

2. Séance de la Chambre des députés du 1 ł janvier $18 S 2$.

3. M. de Foville, Le morcellement, chap. xv. 
à 1701), ceux de Lorraine (de 1763 à 1768), ete. Les règles de ces remaniements territoriaux, appropriées à nos lois actuelles et basées sur la pratique el l'expérience, ont été formulées par M. Freyssinaud ${ }^{1}$.

L'étude de ces nuances du morcellement est nécessaire parce qu'elle fournit la réponse aux pessimistes et aux avocats de l'utopie sociale. Les programmes collectivistes, tout en flatlant les paysans propriétaires de la dislocation et du partage des grands domaines, ne cachent point l'espérance que la marche plus ou moins rapide des circonstances amènera dans l'élat de la propriété agraire la mème unité que dans le régime industriel, el qu'alor's il sera facile de faire intervenir dans toute son intégrité la solution communiste.

Il reste donc vrai que la plus solide barrière à opposer à cette évolution, c'est le maintien et l'extension de la petite propriété. Le morcellement, c'est-ì-dire la décentralisation agricole, est la meilleure façon de ralentir l'intrusion de l'État, d'arrèter le pouroir envahissant et déprimant qui se manifeste par l'autocratie anonyme et irresponsable d'un réseau de plus en plus serré de fonetionnaires, qui décourage les initiatives et démoralise lindividu.

La France est merreilleusement préparée à être la ciladelle de celte résistance légale au bouleversement social, an renversement caleulé des vraies conditions du travail. Ses mours qui sont celles de la finmille el n'ont pas de goùt pour le phalanstère, ses traditions qui associent l'individualité romaine à l'indépendance celtique, son climat qui favorise les productions privilégiées de la petite culture, toul, chez elle, contredit les principes de cette fausse démoeratie qui met au sommet une apparence de liberlé et la servitude it la hase, transformant les eiloyens en administrés serviles et en contribuables doeiles. La configuralion même de notre sol, avec peu de vastes plaines el de plateaux étendus, mais des vallons arrosés et des coleaux abrités, réunit toutes les conditions physiques, ethniques, historiques qui font de la France

\section{Le bornage cadastral par l'initiative des propriétaires ruvaux, 1899.}


le patrimoine de la petite propriété et de la petite culture, gage des libertés civiles et du progrès moral. Ce n'est pas aux pouvoirs publies qu'il faut demander la sauregarde de cette organisation tutélaire, dont la tradition est une des forces, c'est à nous-mêmes.

\section{I1. - La distribution inégale du sol dépend moins des lois que des mœurs.}

L'Agriculture est une école de travail, ce doit être aussi une école de liberté; or, on ne défend bien que ce qu'on possède.

L’état actuel de la division de la propriété atteste que le nombre des très petits propriétaires est infiniment plus grand que le nombre des capitalistes, des financiers et des spéculateurs qui possèdent de vastes domaines et mème des héritages d'une valeur moyenne. Les statistiques officielles font l'areu, par surcroît, qu'on néglige d'y compter ces infiniment petits oì, entre quatre murs ou quatre haies, l'ouvrier des banlieues cultive des légumes, des fleurs, des fruils, ces minuscules potagers, vergers, le courtil d'autrefois, contigus à la maison et qui se perdent, faute d'être classés, dans la masse des propriétés urbaines. En 189\%, sur 8 millions de maisons, on en trouve 4 millions n'ayant qu'un rezde-chaussée, ce sont les maisons rurales par excellence, et 2 millions 1/2 à un étage; en 1892, sur 22330 405 exploitations de moins d'un hectare embrassant une superficie de 1327 milliers d'hectares, près du tiers (environ 4330 millions d'hectares) est cultivé en jardins maraìchers, potagers et fleuristes, d'un rapport de plus de 272 millions de franes. Point n'est besoin de légiférer, de changer les habitudes et les mœurs, pour que chaque salarié rêve de posséder quelque jour sa maisonnette et son jardin; l'instinct national l'y mène, il suffit, pour rendre plus rapide son accession à la propriété, de mettre à sa portée les moyens de garantir son 
épargne et de s'assurer lui-même contre les accidents du travail et les risques de la vie ${ }^{1}$.

Il ne faut pas croire, d'ailleurs, que la France ait le monopole de la petite propriété; la Belgique, la Suisse, les Provinces rhénanes, se rapprochent du type territorial français; la Hollande, les États scandinares, certaines régions de l'Allemagne occidentale abondent en pelits héritages dont le maitre peut chanter, comme nos paysans de Bretagne, la chanson de Botrel :

Dans le mitan de mon verger

Je possède un fameux pommier!

L'Angleterre s'efforce de multiplier les petits tènements qu'elle possède ${ }^{2}$; l'Autriche et la Hongrie, malgré leurs vastes étendues, comptent une quantité d'héritages exploités par leurs propriétaires, et les États-Unis eux-mèmes commencent à morceler les concessions primitives ${ }^{3}$.

Dans tous ces pays, comme en France, le morcellement des terres entre le plus grand nombre possible de détenteurs pratiquant la culture direcle, a été l'un des facleurs principaux de la richesse publique et de l'indépendance nationale.

Partout où la population se fait dense et jouit de la liberté, où la terre est affranchie des liens d'une législation restrictive, d'une procédure obscure et coûteuse, de droits de transfert élevés, elle se subdivise, elle change de mains, elle se mobilise, se met à l'enchìre, revèt successivement, suivant les variations des circonslances économiques, les formes qui conviennent le mieux au service essentiel qu'on réclame d'elle : fournir au consommateur la plus grande somme de produits et de jouissances. Ce rôle de matérialité pure, en apparence, s'associe étroitement à l'inlluence qui lui permet de consolider la famille et de moraliser l'mdividu.

1. Vais par d'autres principes et par d'autres moyens que ceux imaginés avec une regrettable précipitation dans la loi sur la responsabilité des accirlents du travail, du 9 avril 1898.

2. Bulletin de statistique du ministire des Finances, 1886, 402; 1887, 198 , etc.

3. II. Levasseur, L'agricullure aux Etals-Unis, 189 '. 
Lhistoire contemporaine de la propriété en Allemagne, n'est pas moins documentaire pour le sujet qui est l'objet de cette étude, que le mouvement de fractionnement des héritages anglais en allotments et en cottages, ou la crise agraire à allures tragiques de la Sicile el de l'Italie méridionale. Le monde moderne tend à s'uniformiser; il obéit à des lois instinctives d'évolution; nos vertus et nos vices, nos qualités el nos défauts, remarquait M. Luzzatti à propos des mutualités ombardes et florentines, dans l'ordre économique comme dans l'ordre intellectuel, pour les nations comme pour les ndividus, ne nous sont pas absolument personnels et nous restent communs avec l'humanité tout entière.

Ce qui les dirersifie, en effet, ce qui les caractérise, ce qui les fait plus ou moins profitables, c'est aussi bien l'accident que la race. et notre histoire économique est encore trop mal connue pour qu'on puisse en tirer des conclusions absolues.

Les agrariens de Prusse réclament la protection à outrance pour défendre la production nationale agricole sur les marchés de l'intérieur contre la concurrence étrangère; à cet expédient, ils en ajoutent un autre, la reconstitution des grands domaines nobles menacés par le système politique qu'intronisa Manteuffel et dont la formule se traduisit par les lois agraires de 18:30. De 1816 à 1848, le système de Hardenberg voulant créer une classe intermédiaire de gros tenanciers ', n'avait abouti qu'ì l'cxtension exorbitante du domaine noble qui s'accrut de milliers de petites tenures abandonnées par les émigrants d'Amérique ${ }^{2}$. Les lois de 1816 facilitèrent l'absorption des petits héritages quasi serviles par la grande propriété et aidèrent au groupement de la moyenne $^{3}$; celles de 1850 ne ralentirent pas le mourement

1. Ceux qui exploitent un domaine d'environ 15 hectares et qui entretiennent des attelages de labours.

2. On sait que la population d'origine allemande déversée chaque année sur le territoire des États-Unis de l'Amérique du Nord est levenue si nombreuse et si influente que les Anglo-Saxons en prennent ombrage.

3. On évalue à 70000 gros tenanciers à titre précaire, exploitant 3 mil- 
d’émigration. Tout en essayant pour les petits tenanciers, mais trop tard, ce que Hardenberg lit pour les gros, Manteuffel ne dégagea guère plus de douze mille chefs de famille, en les cantonnant, en déclarant les corvées et les services rachetables, en leur concédant $1 / 3$ de leur tenure en toute propriété moyennant l'abandon aux hobereaux des 2/3 du sol qu'ils détenaient à titre précaire. C'est l'un des exemples les plus frappants de linfluence de la politique sur la division de la propriété. L'intervalle transactionnel de $\mathbf{1 8 1 6}$ à 1800 , mis à profit par la noblesse féodale, a escompté le libéralisme qui naquit de 1830 , et les principes semi-autoritaires, semi-démocratiques qui ont prévalu depuis 1892 dans les lois sur les assurances obligatoires sont désormais jmpuissants à transformer en propriétaires les prolétaires ruraux dépossédés par une sorte de métayage dont la précarité fait la dépendance.

Il a fallu près d'un siècle à la loi allemande pour produire dans des sens alternatifs un mouvement accentué vers la division de la propriété rurale; combien de temps faudra-l-il aux lois anglaises de 1887 et de 1892 sur les allotments (cultures ouvrières) et les small holdings (petits domaines ruraux) pour créer de toutes pièces celte nouvelle classe de très pelits propriélaires qu'on juge i propos de superposer par des procédés empiriques à celle qui existe déjà el qui s'est constituée d'elle-mème, par la force naturelle des choses?

Lingleterre avait longtemps condamné, avec une sévérilé égale, la petitc culture, la petite propriété, et l'intervention des pouvoirs publics dans la sphère des intérèts privés. Mais les idées changent avee le temps et cet essai n’est que le résullat d'une manœuvre exchisivement politique. A la veille des élections qui allaient déplacer la majorité dans la Chambre des communes, tous les partis se sont trouvés d'accord pour voter une innovation que jadis ils auraicnt tous repoussée, et M. Gladstone lui-même, dans son

lions et demi dhectares dont ils retinrent $1 / 3$ comme propriétaires définitifs, ceux qui profitèrent des lois de rachats de 1826 , à plus d'un million les petits, qui, cessant d'être protégés, furent expulsés. 
manifeste électoral, se bornait à exprimer le regret qu'on n'eùt pas fait davantage pour accélérer la multiplication des petits domaines ruraux.

$\mathrm{Si}$, au point de vue politique, le législateur intervient dans certains pays, pour modifier la division traditionnelle de la propriété, soit en faveur de la concentration, soit au profit du morcellement, il est des cas où, plus préoccupé de l'intérèt économique exclusif, il se borne à laciliter les échanges, la cohésion des domaines, les mesures d'ordre collectif ou privé qui peuvent remédier au défaut de la dispersion des parcelles, défaut qui n'est pas particulier à la France mais qui se retrouve dans tous les pays à populations denses et à cultures variées. En Suisse, dès 1591 , se pratique le système des échanges forcés, à Berne d'abord, puis dans les autres Cantons. En Écosse, dès 1695, le parlement autorise l'autorité judiciaire, sur la réclamation d'un seul propriétaire, à reviser la distribution des parcelles et des enclaves si elle est préjudiciable à la bonne exploitation des héritages.

Tandis qu'en France le gouvernement laisse à l'initiative privée le soin de réaliser des remaniements collectifs, nos voisins de l'Est et du Nord, moins respectueux du droit de propriété, manifestent par des mesures radicales leur sollicitude pour les intérêts agricoles.

Frédéric II se vantait de refaire la carte des domaines dont les confins étaient mal dessinés; en Allemagne, dès 1778, le paysan demeure exposé à voir son bien changer de place ou de forme, pour peu qu'il plaise à ses voisins de se croire mal délimités. Les divers États eurent leurs lois particulières, ne diflérant que par des nuances et visant à la fois (de 1821 à 1867) : le rachat des servitudes, le partage des biens indivis et les réunions parcellaires dans le sens des principes de l'économie rurale. Une loi du 18 mars 1883 met un autre expédient à la disposition des propriétaires de parcelles enchevêtrées, c'est l'exploitation en commun, si elle est requise par le quart des intéressés ${ }^{1}$.

1. Bulletin du ministère de l'Agriculture, 1883̈, vir. 
En Autriche, la loi du 7 juin 1883 règle le remaniement des propriétés, à la demande de la moitié plus un des exploitants, pour supprimer les inconvénients de la dissémination des parcelles cultivées par le même exploitant ${ }^{1}$. Il faut admettre que ces expédients, disculables si l'on se place à l'unique point de vue de la liberté du propriétaire, ont de sérieux avantages puisqu'ils se sont généralisés depuis près de deux siècles dans l'Europe centrale 2. M. Léon Say, M. Tisserand, M. de Foville, considèrent que les résultats pratiques en ont été excellents.

Malgré ces exemples, on ne peut dire que la répartition inégale du sol dépende plus des lois que des mœurs. La distribution de la propriété entre les divers occupants du sol, les formes diverses par lesquelles s'exerce le droit de propriété et se manifeste la jouissance de la terre par le profit que le propriétaire en retire, soit de la culture directe, soit du travail salarié des intermédiaires ${ }^{3}$ sont les éléments constitutifs de l'ordre social, et c'est de leur équilibre que découle la prospérité publique. Mais, si cet équilibre est rompu, sous l'effet des circonslances, par le hasard des événements, il s'ensuit une gène momentanée soit par arrêt de la production, soit faute de débouchés, soit vilité des prix; si cette gêne se prolonge, elle impressionne à son lour les rapports des propriétaires et des exploitants, puis ceux des exploitants ou producteurs avec les consommateurs, et ce sont ces embarras, privés on collectifs, dont l'ensemble s'appelle une crise. Un changement dans la distribution du sol ne peut pas empêcher les crises; c'est l'état individuel

1. Bulletin de statistique du ministére des Finances, novembre 1883, p. 607 .

2. Dès 1774, l'Angleterre imila l'exemple de l'Écosse; le Danemark depuis 1758 , la Suède depuis 1765 adoptìrent le principe de la suppression des enclaves el des écarts par roie d'ćchanges obligatoires.

3. Olivier de Serres disait que le métayer n'est qu'un ouvrier qu'on paie en nature et que le fermier lui-meme, payant partie en argent, partie en nature, redevances, services ou corvées, n'était rémunéré à vrai dire que par les produits de sa culture dont il rachetait une portion, à forfait, an maître de la ferre. 
de chaque propriétaire pris dans son groupe qu il faut améliorer afin de lui assurer la foree de résistance qui permet d'attendre le beau temps après l'orage.

\section{III. - La Dépopulation.}

La dépopulation est l'un des fléaux de la France contemporaine. Pour la matière de cette étude, elle exerce une influence des plus nuisibles, non pas seulement sur l'activité de l'exploitation agricole mais sur la répartition de la propriété, moyenne el petite, et sur les progrès de la constitution de la très petite, et deviendra, si l'on n'y prend garde, l'un des facteurs négatifs les plus puissants de notre décomposition sociale.

11. Levasseur a épuisé la question ${ }^{1}$; je n'ai d'autre but, dans ce rapide aperçu, que d'en signaler l'importance et de montrer le ròle de la population dans le maintien ou la dislocation de l'équilibre foncier', quant à la division du sol.

Le dénombrement de 1891 a été un coup de cloche d'alarme. Les décès excèdent les naissances. Et si, contrairement à ce qui se passe dans les autres parties de l'Europe et du monde, le produit de la vitalité normale ne produit plus, en France, ses effets habituels, c'est que les Français deviennent de moins en moins prolifiques. La proportion des unions radicalement stériles est, à Paris, de 22 p. 100, en province de 12 p. 100 . Notre pays manque d'enfants et dans dix ans il manquera d'hommes. Il y a des causes accidentelles à cette pénurie ${ }^{2}$; il y a surtout une cause permanente, déplorable, la grève des pères de famille. La stérilité

1. La population française. - Histoire de la population avant 1 is 89 et démographie de la France comparée à celle des autres nations au XIXe siècle, 3 vol. 1859-1892.

2. Les misères de 1870 et 1871 ont aggravé toutes les formes de la mortalité en même temps que ralenti la natalité : la périodicité de l'influenza, les Expositions de 1878 et 1889 ont exercé une influence déprimante. (Revue des Deux Mondes, 1892, vi, 319.) 
relative des familles françaises réjouissait déjà nos envieux et nos ennemis quand il n'en résultail pour nous qu'une trop lente multiplicalion à còté de l'accroissement continu, régulier, des nations voisines; aujourd'hui, le mal semble s'être subitement aggravé et l'on redoute que le ralentissement aboutisse, à bref délaj, à un arrêt, puis à un recul. Il semble que ce terme fatal soit atteint. La balance des naissances et des décès pour l'année 1900 se solde par un excédent de 230988 décès ${ }^{1}$.

Le mal, il faut y insister, est moins dans la stérilité ellemême que dans la préméditation de la stérilité. La discrétion systématique des ménages français, remarque M. de Foville, a souvent pour cause la difficulté de concilier autrement les intérêts de l'héritier et ceux de l'héritage; et M. Paul Leroy-Beaulien ajoute: il reste loujours un moyen de faire un aîné, là où il n'y a plus de droit d'aînesse, c'est de n'avoir qu'un fils.

La distinction établie par la statistique, depuis 1846 , entre la population urbaine (qui comprend les agglomérations de plus de 2000 habitants) et la population rurale, permet d'apprécier la marche progressive de l'élan qui pousse les habitants des campagnes ì quitter leur village pour aller se fixer à la ville ${ }^{2}$.

\begin{tabular}{|c|c|c|}
\hline & \multicolumn{2}{|c|}{ PROPORTION POUR 100} \\
\hline ANNÉES & Population urbainc. & Population rurale. \\
\hline $1846 \ldots \ldots \ldots \ldots \ldots \ldots$ & $24, \mathfrak{k}$ & 75,6 \\
\hline $1851 \ldots \ldots \ldots \ldots \ldots \ldots \ldots$ & 25,3 & 74,5 \\
\hline $1856 \ldots \ldots \ldots \ldots \ldots \ldots \ldots$ & 27,3 & 72,7 \\
\hline $1861 \ldots \ldots \ldots \ldots \ldots \ldots \ldots$ & 28,9 & 71,1 \\
\hline $1866 \ldots \ldots \ldots \ldots \ldots \ldots \ldots$ & $30, \ddot{3}$ & 69,5 \\
\hline $1872 \ldots \ldots \ldots \ldots \ldots \ldots$ & 31,1 & 68,9 \\
\hline $1876 \ldots \ldots \ldots \ldots \ldots \ldots \ldots$ & 32,4 & 67,6 \\
\hline $1881 \ldots \ldots \ldots \ldots \ldots \ldots \ldots$ & $3 /, 8$ & 65,2 \\
\hline $1886 \ldots \ldots \ldots \ldots \ldots \ldots \ldots$ & 35,9 & $6 t, 1$ \\
\hline $1891 \ldots \ldots \ldots \ldots \ldots \ldots \ldots$ & $37, \mathbf{t}$ & 62,6 \\
\hline
\end{tabular}

1. Journal officiel slu 2 décembre 1901, p. 7489.

2. Le résultat de l'émigration l'urale n'a pas seulement pour effet la diminution de la population municipale, mais l'abandon des villages. 11 est quelquefois imprudent de juger par les chilfres seuls de l'étendue d'une plaie sociale, la visite personnelle des régions contaninées est 
Les tableaux par départements font ressortir qu'il y en a très peu où la population urbaine n'ait pas auģmenté par immigration et la population rurale diminué par émigration. II serait eurieux de suivre pour le dénombrement de 1896, le mouvement plus ou moins intense de ce double phénomène en sens inverse mais constituant le mème fait économique et social. En 1887, il n'y eut que quatre départements dont la population rurale eùt augmenté, et dans une bien faible proportion, variant de 0,1 p. 100 (Vendée) à 0,4 p. 100 (Hautes-Alpes et Lot). En 1891, l'émigration rurale semble perdre un peu de son intensité, et le ralentissement s'est aceentué légèrement en 1896, du fait des grèves et des mécomptes de l'industrie.

Les centres les plus aceentués de l'émigration rurale sont, au nord, la Seine-Inférieure et le Nord; à l'ouest, les Côtesdu-Nord et la Nayenne; au centre, l'Indre et la Corrèze; au midi, les Landes, l'Ariège et l'Aveyron; à I'est, la Loire, le Rhòne et la Ilaute-Saône ${ }^{1}$.

Si l'on rapproche cette carte de celle qui donne la proportion des communes renfermant des familles de plus de sept enfants ${ }^{2}$, on est surpris de n'y pas voir figurer, sauf pour les Còtes-du-Nord, l'Aveyron et la Loire, les départements cotés comme centres d'émigration. Faudrait-il en conclure qu'une famille nombreuse trouve toujours à occuper tous

souvent une révélation. En Normandie, dans un des plus riches territoires de notre pays, on trouve des champs en friche et des maisons écroulées. Le canton de Beaumont-Hague (Nanche) a perdu, depuis cinquante ans, le tiers de sa population, autrefois si prolifique. La commune de Diguleville a reculè de 781 habitants en 1870 à 353 en 1898; à Omonville, suivant un proverbe local, le poisson meurt de vieillesse, faute de pêcheurs pour le prendre; à Vauville, qui de 613 habitants en 1855 n'en compte plus que 172, le mètre carré de bonne terre arable se vend un centime. A Auderville, commune à 2 bi kilomètres de Cherbourg, peuplée de petits propriétaires cultivateurs, la natalité n'est plus aussi forte qu'autrefois, mais on n'émigre pas, l'aisance est générale, il n'y a pas un mendiant.

1. Carte de la dépopulation des campagnes pendant la période de $\mathbf{1 8 7 6}$ à 1881, dressée par M. Victor Turquan. En 1891, la proportion n'avait pas sensiblement changé.

2. Bulletin du Comilé des travaux historiques, 1890, p. 96 et suiv. 
les bras dont elle dispose el que l'émigration se manifeste surtout dans celles où, le personnel familial ne se trouvant pas assez nombreux, les besoins de la culture obligent à s'aider d'auxiliaires salariés? Le gain des enfants à la ville devrait alors, dans les calculs du chef d'exploitation, compenser et au delà la dépense des ralets ou servantes de ferme; mais, combien de déceptions pour une réussite!

Les conclusions des rédacteurs de l'enquête spéciale sur les résultats de l'application de l'article 3 de la loi du 47 juillet 1889, exemptant de la contribution personnelle-molilière les père et mère de sept enfants vivants, ne sont pas lout à fait conformes aux prévisions du législateur. Sur l'ensemble des communes, 77 p. 100 possèdent des familles de sept enfants, soit en nombre 148808 familles; mais les familles d'indigents qui, en principe ne sont pas cotisées à l'impôt, échappent à cette statistique. C'est l'explication de l'écart énorme (102830 familles) qui existe entre les chiffres de l'enquête de 1890 et ceux du dénombrement de 1891 (9231638 familles). Les familles indigentes seraient donc particulièrement farorisées par la natalité, et les régions où vivent les plus nombreuses familles sont les pays au nord de la Somme, la Bretagne, le Béarn, la Creuse, les $\mathrm{Al}_{\text {pes el la }}$ Corse. Sur l'ensemble des familles dégrevées, les aisées ou riches sont aux peu fortunćes dans la proportion de 23,68 p. 100 contre 76,32 p. 100.

Une loi récente a limité le bénéfice de la loi de 1889 aux familles dont lia cote est inférieure à 10 francs; ce recul est une maladresse ${ }^{1}$. Ce n'est pas d'ailleurs la famille de sept, de huit ou de neuf enfants, déclare l'ençuête, qui caractérise la forte natalité; mille familles de cinq enfants valent mieux pour l'accroissement el surtoul pour la richesse d'un dépar-

1. La cote moyenne, avant la revision, était de $13 \mathrm{fr}$. 45 , ce qui n'était pas un allégement très apprécialłe. Ce sera toujours l'inconvénient des lemi-mesures; il en est arrive de mêne pour le soi-disant dégrèvement de l'impôt foncier, pen sensible peur l'intéressé et consilérable pour le Tresor obligé de compenser la perte par une surchage ailleurs, simple déplacement de taxe. 
tement que denx eents ou trois cents familles de livit ou de neuf enfants.

En dehors de la stérilité voulue, la eause la plus active et la plus effrayante de la dépopulation, e'est la mortalité infantile, à laquelle on peut remédier par des lecons d'hygiène pratique et une sérieuse organisation de l'assistance médicale. A ee chiffre des pelits enfants morts dès la première année, il faut ajouter celui des mort-nés qui est en moyenne de 41 p. too. Il laut également tenir compte des ravages eausés tous les ans par l'alcoolisme et la tuberculose, et l'on sera étonné du vaste ehamp ouvert à la surveillance et à la préroyance de l'administration si elle remplissait avec intelligence son rôle de tutrice au lieu d'irriter les populations et de se diseréditer elle-même par des niaiseries ${ }^{1}$,

Les mariages tendent à augmenter; mais il existe, dans l'état démographique de la France, un facteur autre que la fréquence des mariages, qu'il importe de ne pas perdre de vue: le degré de vitalité de l'habilant. Aussi est-il naturel de rencontrer les plus fortes proportions de gens mariés dans les régions oì la vie moyenne est plus longue; sous ce rapport, la Bourgogne et la Gascogne sont privilégiées. La Normandie a perdu eet arantage depuis que la consommation de l'aleool et du labae $y$ a pris de si regreltables proportions. Il serait à souhaiter qu'un économiste dressàt une carte des départements où seraient combinés ces divers éléments de la dépopulation : nalalité, mortalité, consommations nuisibles. L'allongement incontesté de la vie humaine cn France devrait avoir pour corollaires la protection de l'enfance et la suppression des poisons dont la fisealité de l'État provoque le débit.

Un des signes de la dépopulation des campagnes est le nombre eroissant des maisons racantes. Je ne parle pas des logements, car le nombre varie suivant la saison où s'est

1. A ce point de rue particulicr la lecture des rapports soumis aux Conseils généraux dans leur session annuelle d'août est douloureusement instructive. 
effectué le recensement, et s'explique par les habitudes de villégiature, aux bords de la mer, et, à Paris, par les déplacements continuels de la population flottante. Toutefois, on ne voit pas pourquoi le Lot-et-Garonne est, de tous les départements, celui qui présente la proportion la plus forte, 8,88 p. 100 pour les maisons, 10,95 p. 100 pour les logements, chiffres qui ne se retrouvent que dans les AlpesMaritimes (7,25 et 10,02 p. 100), puis dans le Calvados ( 7,503 et 9,84 p. 100).

L'influence de la dépopulation ou de l'accrøissement du nombre des habitants se fait sentir très inégalement dans les diverses régions de la France et souvent en contradiction arec les déductions qui sembleraient découler naturellement du constat de certains autres faits. A cet égard, la Normandie se détache nettement de la Bretagne, la Bourgogne el la Champagne diffèrent également des provinces du Nord et de la banlieve de Paris '.

Autant qu'il est possible de le voir malgré les lacunes des statistiques, la dépopulation du territoire rural atteint plutòt les bourgs et les petites villes que les villages et les hameaux. Les trois quarts de la population vivent à l'état d'agglomération et un peu plus d'un quart habite des hameaux ou des maisons isolées dans la campagne, condition que les spécialistes considèrent comme éminemment favorable aux soins de la culture el au progrès personnel de l'exploitant agricole.

L'éparpillement des habitations est intimement associé, en faisant exception pour la banlieue des villes qui reste assujettie ì des influences spéciales, à l'accroissement de la petite propriété. Le paysan se sent d'autant mienx chez lui qu'il est plus isole des autres.

Les départements de lEst sont remarquables par la

1. Pour ne point risquer de s'égarer dans les hypothèses, il faudrait soumettre chaque département à une étude particulière. M. Paul Heuriot vient de donner un exemple excellent de ces monographies par les chiffres en examinant les causes el les résultats de la dépopulation rurale dans le département de la Somme. (Journal de la Société de statistique de Paris, aont 1900, p. 265.) 
constitution historique de leurs eommunes, presque tout entières groupées, dans un but de défense, en une seule agglomération. Aussi, compte-t-on fort peu de hameaux dans cette partie de la France. La même particularité se retrouve, mais à un degré moindre, dans le Sud-Est et le Sud de la France, principalement sur les côtes de la Méditerranće, el surtout en Corse. Les régions dans lesquelles, au contraire, la plus grande partie de la population est éparse dans la eampagne et vit dans des hameaux jsolés, ou diversement groupés sur le territoire des communes, sont la Bretagne et les provinees voisines, le Massif Central, mais surtout la Creuse, les Côtes-du-Nord et les Landes.

La proportion de la population éparse, - écarts, fermes isolées, - varie par rapport à la population tolale ramenée à 100 habitants, de ö p. 100 dans la Meuse, à 72 p. 100 dans la Creuse el 73 p. 100 dans les Còtes-du-Nord ${ }^{1}$.

Les départements qui oflrent le plus d'agglomérations distinctes, dont la réunion constitue les municipalités, sont ceux de l'Ouest et du Centre, depuis la Manche, qui compte 18926 villages, hameaux et sections de commune, avec כ̋13 813 habitants (en 1891), jusqu’à la Marne, qui n’en a que 1376 avec 434734 habitants.

Le dénombrement de 1896 constate une légère augmentation sur 1891 ( 173027 tètes). Il continue, comme les précédents, à démontrer que la natalité reste stationnaire et que l'accroissement de population des grandes agglomérations se produit encore au détriment des campagnes par un déplacement de la population rurale vers les centres industriels et commerciaux ${ }^{2}$.

\section{IV. - L’Absentéisme.}

L'absentéisme est le fléau de la grande propriété comme l'hypothèque est le fléau de la petite.

1. Viennent ensuite la Corrèze, la Dorlogne, les Landes.

2. Bulletin de statistique de l'Enregistrement, 1897, 226. 
Il n'existe pas de statistiques établissant avec précision le nombre des propriétaires ruraux qui n'exploitent pas et ne résident mème pas. On ne peut en évaluer l'ensemble que très approximativement, sans même pouvoir s'aider des cotes foncières foraines, car on peut ne pas habiter la commune ou l'une des communes où l'on est propriétaire sans être pour cela un absentéiste. Le défaut d'unité de la cote foncière en fail une probabilité et non une certitude, et comme la contenance des cotes, étant donnée par des moyennes, ne peut servir de contròle, les éléments qui pourraient être les plus probants nous échappent. On a fait pour Paris, lors du dénombrement de 1891, un essai de monographie numérique fort curieuse. Ce n'est que par des constats analogues que le service des Contributions directes arriverait à combler cette lacune. Il conviendrait de faire pour les propriétaires fonciers, en les distinguant en résidants et forains, ce qu'on fit pour savoir combien il y a de Parisiens en France et de Provinciaux à Paris '. Il y a, par exemple, pour 100 natifs des Alpes-Maritimes à Paris, 138 Parisiens à Nice; il y a pour 100 Corréziens dans la Seine, ॐ̈ Parisiens dans la Corrèze.

L'enquète agricole de 1892 constate qu'il y a 30 p. 100 d'exploitants ì titre intermédiaire pour 70 p. 100 de propriétaires exploitant directement et personnellement leurs biens héréditaires. Ces $140 \$ 569$ fermiers et mélayer's, représentant chacun une exploitation rurale ${ }^{2}$, représentent-ils également autant de propriétaires non résidants? Ce n'est pas probable. Leur nombre ne doit pas être aussi considérable.

Beaucoup de propriétaires, en effet, possèdent plusieurs fermes et métairies; d'autres, plus nombreux, habitent leurs terres, sans les exploiter, et pourraient, si nos mours

1. La population du département de la Seine, abstraction faite des étrangers et des Français nès hors de France, était, en 1891, de $2 \$ 58102$, dont 1653390 provinciaux immigrés. W'autre part, le nombre des originaires du département de la Seine recensés en France était de 1514438 dont 315726 se trouvaient en province, surtout en Seine-et-Oise.

2. Enquéte, p. 369 : Modes d'exploitation. 
actuelles s'en accommodaient mieux, rendre une partie des services que l'économie sociale exige, théoriquement, des propriétaires fonciers.

Les fermiers et métayers sont............ 1405569

Les exploitations de la grande culture. 138671 )

Celles de la moyenne.............. 711118

Difference.........

555780

Or, comme les détenteurs de la pelite propriété, sauf peut-ìtre quelques milliers d'exploitations de 6 à 10 hectares, cultivées à métayago, ne valent que par la culture directe et ne peuvent ètre portés au passif des absentéistes, on peut almettre que ceux-ci, au pis aller, ne dépassent pas un million d'individıs, presque tous ayant d'autres ressources que celles de la terre.

Comme je l'ai indiqué dans les chapitres précédents, il faut se rappeler que le nombre des exploitations de la grande propriété ne cesse de décroître :

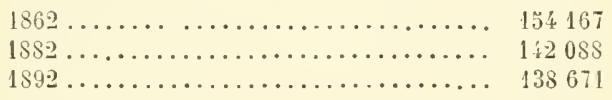

et que le nombre des exploitations de la moyenne est descendu de 727 222 en 1882 à 711118 en 1892.

On ne fait guère le procès aux grands propriétaires qui ne résident pas sans invoquer le triste exemple de l'Irlande; mais les deux pays ne sont pas comparables; les fermiers el les mélayers de France, en grande partie propriétaires euxmêmes, ne sont pas des misérables et des déshérités comme les pauvres tenanciers de la verte Erin, menacés par l'Insecurity of tenure, exploités par l'odieux procédé des Rackrents, et toujours sous le coup d'une éviction brutale.

On compare souvent l'étal des populations rurales en Franee et en Angleterre et l'on serait disposé à donner à la Grande-Bretagne la préférence sur notre pays. Il ne convient pas d'en décider ainsi d'une façon trop absolue, car l'ouvrier agricole anglais ne vaut notre paysan de France 
ni comme intelligence ni comme dignité morale; ce n'est qu'une vaillante bète de somme, a dit Taine, ce ne sera jamais un citoyen. Mais la bourgeoisic anglaise a sur la nôtre un avantage incomparable; elle préfère l'industrie agricole à la carrière maussade du ionctionnarisme.

Les rangs supérieurs de la société rurale sont honorés, en Angleterre, par les plus grandes individualités de la naissance et de la fortune; la vie agricole est aussi développée chez nos voisins qu'elle semble dédaignée chez nous. C'est une carrière, c'est presque une fonclion. Le fermier français est un homme de pratique; il paie de sa personne, travaille, laboure, charrie, moissonne; le fermier anglais fait travailler, et surveille. C'est plutôt un régisseur madré qu'un fin laboureur; il ne s'occupe ni du bien-être ni de l'avenir de ses ouvriers, qu'il paie le moins cher possible et quill exploite à merci. Le propriélaire anglais s'absente, voyage, dépense son revenu sur loutes les routes des deux mondes et vient, de temps à autre, se refaire, se reposer, reprendre haleine dans son Home. Comme il a lui mème d'autres revenus que ceux de sa terre, et que son fermicr aussi est riche, la culture ne souflre pas; mais le mouvement ascensionnel n'existe pas, ce progrès indéfini qui fait de notre journalier le plus ignorant un métayer, un fermier, puis un propriélaire. Et puisque le fermier français réussil si sourent, sans avances, sans crédit, par la seule puissance de son travail et de sa ténacité, pourquoi ce million de propriélaires forains qui restent indifférents el routiniers ne s'efforceraient-ils pas de rivaliser avec les Landlords et de tripler la valeur de leurs héritages?

Les propriétaires forains possèdent tous une certaine aisance, qu'ils soient dans l'industrie et le commerce, on qu'ils occupent des fonclions publiques; ils ont, avec l'intérêt immérlial d'améliorer leurs terres, les ressources qui le leur permeltent. La propriété donne des droits, mais elle impose des devoirs ${ }^{1}$. Le premier devoir d'un propriétaire

1. V. de Tracy, Lelles sur l'agricullure, Paris, 1857. 
foncier est de réscrver une partic de son temps et de ses capitaux à l'amélioration de sa culture; il augmente ses revenus et contribue au progrès social.

Au lieu de lois impuissantes et d'institutions coûteuses, rénovons nos mœurs rurales. L'absentéisme a été la plaie des deux avant-derniers siècles, il a été la mode du xix ${ }^{\mathrm{e}}$, que sa suppression devienne le salut de celui qui commence.

Vers 1680, Bussy-Rabulin écrivait à Mme de Sérigné qui se plaignait de ne rien tirer de ses terres : Faites-vous exiler, belle cousine, vous y gagnerez.

Depuis trop longtemps les cliamps sont abandonnés par le propriétaire riche, par celui qui, possédant des capilaux libres, est dégagé des trois fléaux de l'Agriculture : la fiscalité, la dette agraire et la procédure. La vic à la campagne passait pour une vie monotonc, inélégante; la culture paraissait être un métier rustique, inférieur; une réaction favorable commence, on s'aperçoit quil est au moins aussi honorable et certaincment plus lucratif d'être agriculteur que chef de bureau.

A force de répéter, d'affirmer ces vérités élémentaires, peut-ĉtre réussira-t-on à ramener vers le travail salubre de la terre tant d'intelligences qui s'étiolent à la poursuite de diplòmes chimériques, tant d'aptitudes qui s’ignoraient elles-mêmes. Si le goût de la vie rurale s'empare de nouveau de nos mœurs, la nation tout entière y gagnera en vigueur, en dignité, cn richesse et en moralité.

Le paysan se laisse plutôt convaincre par la lente pénétration de l'exemple et de la réussite qu'entraîner par des discours. Ce n'est pas seulement au point de vuc agricole que le séjour prolongé des propriétaires à la campagne semble désirable, c'est au point de vue moral, si négligé depuis trente ans, et dont la criminalité croissante des très jeunes gens donne chaque annéc, dans les statistiques du ministèrc de la Justice, l'éliage inquiétant. C'est aussi au point de vue politique, pour l'exereice du suffrage universel, fiction dangereuse d'une soi-disant consultation populaire tant qu'il ne sera pas rendu obligatoire et ramené à une 
mesure mieux proportionnée à lignorance sociale des électeurs.

La révolution de 1789 a porté la liberté dans les droits civils, la révolution de 1848 a introduit l'égalité dans les droits politiques, mais les lois électorales de 1875 et de 1880 n'ont pas réglé avec prévoyance un mécanisme dont la simplicité est d'autant plus dangereuse qu'elle n'est pas comprise el que les partis en exploitent l'insulfisance.

Habituée au silence par les traditions despotiques de l'Aneien Régime, a-t-on dit souvent, la population des campagnes s'est déshabituée de ses propres affaires et s'en remet à l'administration qui la bride et la tient en lisières jusque dans les plus minees détails de l'existence. Le paysan se tait, travaille et demeure insouciant de ce qui n“intéresse pas directement sa condition matérielle. Il sinquiète fort peu, malgré ses droits acquis, de la manière plus ou moins libérale, plus ou moins morale dont les affaires du pays sont conduites, et ne demande qu'à s'enrichir; tout au plus se passionne-t-il quelquefois pour les élections municipales à cause des coteries qui l'entrainent ou des ambitions locales qui s'y mêlent; mais il se préoccupe infiniment moins du triomphe de ses idées que de la satisfaction de ses intérèts.

Pourquoi en serait-il autrement? Ne voit-il pas autour de lui une pareille indifférence, le mème détachement de tout ce qui ne se paie pas complant?

Pourquoi le grand propriétaire se jetterait-il dans l'embarras des luttes électorales, alors qu'il ne peut profiter de sa situation, de sa fortune, de sa compétence, qu’après avoir sollicité el obtenu l'investiture administrative? La cenl.ralisation excessive, l'arbitraire de la continuelle intervention des agents du pouvoir eentral nuisent au développement de la vic rurale, en isolant les intérèts, en écartant les gens honnêtes et capables, en laissant à des agités on à des exprits serviles les emplois et la direction. Le niveau baiste te plus en plus, dans les municipalités comme ailleurs; la conluite des affaires s'en ressent parce que la ilignité, la molalité, l'idéal, sont choses désormais inconnues ou gênantes. 
Le petit propriétaire, le fermier, le métayer, l'ouvrier de ferme, l'artisan de village, se contentent du présent, prennent leur part plus ou moins négative de cette apparente puissance électorale que dirigent des comités anonymes et dont ils ne sont que les instruments, et se rassasient à peu près des arantages incertains d'une démocratie qui n'a que le nom de la chose. Arec une décentralisation effective, en imposant aux cabinets éphémères une neutralité qui corrigerait peut-ètre par la liberté d’agir l'affaiblissement de lesprit public, en ramenant tous les propriétaires forains dans les communes où ils possèdent, on restituerait à la nation celte classe intermédiaire qu'on cherche à faire disparaitre au profit du quatrième état, on utiliserait pour le bien commun des ressources, des capitaux et des talents dont il est odieux de priver, de parti pris, le pays en les molestant ou en les dédaignant.

Quand la situation sociale d'un peuple est compromise par quelque vice capital, on dit, pour se disculper de sa propre indifférence, qu'il est impossible d'y porter remède à moins d'un changement complet, paree que toutes les mesures prises pour atténuer l'effet du mal ne font d'habitude que laggraver. Voici pourtant une solution simpliste du problème de l'absentéisme : toujours la liberté.

Que de bien-ètre moral et matériel serait apporté à la province laborieuse et économe par le retour, sinon définitif, tout ou moins s'affirmant par des séjours prolongés des propriétaires dans leurs domaines petits ou grands : moins de préjugés par des points de contact plus fréquents, échanges de services, bons conseils, bons exemples, commandite du cultivateur par le capitaliste en économisant le courtage des intermédiaires et les risques de la procédure, progrès dans la culture, création de syndicats plus nombreux ${ }^{1}$, consommation sur place de produits qu'il faut

1. Ce qui a fait, depuis 1884, la fortune des syndicats agricoles, c'est leur caractère mixte qui les transforme en terrain neutre de transaction, d'entente; ce qui a ralenti, au contraire, le développement des syndicats industriels, c'est que très peu sont mixtes; ce sont des groupements 
exposer sans cela à la mévente des marchés, que de profits pour le cultivateur à voir vivre de sa vie le propriélaire qu'il ne connaissait pas. Les progrès agricoles de la Bretagne, en particulier, n'ont-ils pas pour origine la mauvaise humeur de la petile noblesse, déconcertée par la révolution de 1830, et désertant la ville, les places, les emplois, pour se réfugier dans le paisible labeur des champs et meltre en valeur l'héritage paternel? La bourgcoisie du xx siècle peut rendre à toute la France, par son énergie et sa volonté, les mèmes services que la noblesse boudeuse de 1830 rendit à la Bretagne, à l'Anjou, au Poitou, sans s'en douter.

Que de questions délicates se résoudraient d'elle-mêmes par la suppression de l'absentéisme, à commencer par les trois capitales : le crédit agricole, le débat entre le métayage et le fermage, l'emploi des machines!

Lorsque les hommes politiques, les économistes, les publicistes, déplorent la dépopulation de la France el l'émigration rurale vers les villes, lorsquils reprochend avec quelque sévérité aux paysans l'abandon de la culture, ne craignent-ils pas qu'on leur réplique par le mot de l'Évangile? Que celui d'entre vous qui est sans péché leur jette la première pierre!

Quel est le plus coupable, du paysan qui s'expatric ou du propriétaire qui déscrte?

Ceux qui s'absentent, sont-ils moins infidèles à la terre que ceux qui se déclassent; ne sont-ils pas eux-mêmes des déclassés de l'agrriculture, ces propriétaires qui ne voient dans la possession d'un domaine qu'une occasion de villégiature, surfout au lemps de la chasse, ou qu'un moyen d'influence d'à côté pour les élections? Ces capitalistes, ecs bourgeois, dont beaucoup n'ont eu que la peine de naître, suivant le mol cinglant de $M$. Thiers, ont recherché avec excès les fonctions publiques, l'industrie, les aflaires, le luxe et les plaisirs des grandes villes, de même que les

l'onvriers ou de patrons beaucoup mieux armés pour la lutte que pour la conciliation. 
métayers et les ouvriers ruraux allaient aux métiers urbains, aux chanticrs de travaux publics, aux emplois des chemins de fer, au petit commerce, aux places de burean et aux déprimantes distractions de Paris ${ }^{1}$. Le mélayage a diminué en raison des progrès du fonctionnarisme, de lindustrie et de la spéculation; le fermagge, qui dispense de surveillance, croit-on à tort, s'est accru d'autant, et il suffit de comparer les chiflres pour s'apercevoir que si la grande propriété semble, par son prestigc, ses ressources, rendre son délachement du sol plus regrettable et plus fitcheux, la moyenne propriété y prend en nombre, et par consécuent en dispersion sur tout le territoire, c'est-à-dire en influence immédiate et effective, une part plus onéreuse encore puisciu'elle représente les $4 / \not ̈$ de l'ensemble.

Peut-ètre n'est-il pas chimérique de croire que l'universel élan du xix ${ }^{\mathrm{e}}$ siècle vers l'industrie et la spéculation tend à se calmer, que le goût des diplòmes et des études stériles passera, quion se lassera de la vie médiocre et assujettie du fonctionnaire, el que le retour à la vie rurale apparaîtra ce qu’il est en réalité, l'appropriation des facultés les plus variées de l'homme à un travail qui rémunère et qui moralise. Rien ne serait plus favorable à une division de la terre dans le sens qu’indique le mouvement économique, rien ne serait plus propice à l'harmonie des classes et à la liberté du travail.

\section{V. - La Fiscalité.}

La fiscalité est une des lormes de l'absentéisme, et la plus détestable. Elle crée des embarras sans nombre à la constitution du droit de propriélé et à la facilité de ses trans-

1. D'après le dénombrement de 1891, dans la journée du 12 avril, sur les 6216660 Français qui se trouvaient en déplacement hor's de leur commune d'origine, il s'en est recensé 1659390 dans le seul département de la Seine. $A$ Paris, le nombre des hôtes de passage varie, suivant les saisons, de 40000 à 70000 . 
ferts; elle est un obstacle invincible, au dire des spécialistes, à la division de la propriété et à la sécurité des titres destinés à perpétuer l'héritage dans les mains de la famille rurale.

Et, tout d'abord, l'impôt prélevé sur les produits de la terre et même sur ses dettes ${ }^{1}$ ne lui profite pas et on l'emploie ailleurs. Les voies de transport elles-mêmes, cet élément indispensable de l'outillage agricole, sont entretenues par les habitants, à leurs frais exclusifs, sans que l'impôt prélevé par l'État pour les besoins généraux du pays y contribue pour une part appréciable. Les chemins ruraux et de grande communication sont payés par les centimes additionnels des communes et des départements, et par les prestations; les chemins de fer ont pour bailleurs de fonds les particuliers qui souserivent à leurs émissions.

Il est opportun de signaler, à ce propos, l'erreur de ceux qui rapprochent sans cesse l'Angleterre de la Franee, comparant leurs conditions agricoles si peu semblables, et s'ingéniant à découvrir des analogies là où il ne peut y en avoir, aussi bien pour la division de la propriété entre les divers occupants que pour la mesure de l'intervention de l'État sur notre territoire ou dans les Iles Britanniques.

L'impòt, qui est, en France, pour les campagnes, une machine à épuisement, n'a pas du tout, en Angleterre, le même caractère. Tout l'impôt direct s'y dépense sur les lieux mêmes où il est payé, el cetle appropriation n'est que naturelle. La taxe des paurres, la dîme de l'église, sont à peine sorties des mains du cultivateur qu'elles y rentrent par l'achat de ses denrées; les autres taxes servent uniquement à des travaux d'intérêt local. La moitié des impôts indirects élant absorbée par le paiement des arrérages de la dette publique, dont les titres appartiennent en grande partie aux propriélaires du sol, il en revient une grosse somme à la vie rurale. Quand un tiers au moins du budget

1. Article $15, \S$ VII de la loi du 22 frimaire an VII et article 60 de la mème loi (Annales de l'Enregistrement 1899, 218). 
français se condense à Paris et un autre tiers dans les grandes villes de province, en Angleterre, les trois quarts des dépenses publiques se dispersent sur les populations rurales et contribuent avec les revenus des propriétaires et des fermiers, à y répandre l'abondance et la vie par une intelligente décentralisation.

Il serait difficile de détourner le courant qui porte l'emploi des produits de l'impot vers les grands centres; mais si quelque chose peut ralentir l'eflort de cette pompe aspirante toujours avide, c'est la résidence sur leurs terres, au milieu de leurs intérêts ruraux, de propriétaires influents qui défendraient mieux leurs roisins et leurs compatriotes s'ils les royaient habituellement de plus près ${ }^{1}$.

Novicow, dans la sévérité de sa doctrine ${ }^{2}$, a fait le procès des gouvernements contemporains qui, imprégnés pour la plupart des fausses doctrines de l'État touche à tout, semblent rivaliser pour ouvrir les voies toutes larges aux appétits collectivistes.

Le démon fiscal, dit-il, apporte le trouble dans la société et se plaît à rompre les harmonies préparées avec art. Si les sociétés modernes s'enrichissent, ce n'est pas grâce à l'intervention du gouvernement dans le domaine économique, e'est malgré cette intervention. Le gouvernement peut étouffer sans remède possible une nationalité par l'accumulation méthodique et raffinée de ses exigences fiscales.

Le gouvernement dont la sérieuse intention est de favoriser le développement national, ajoute-t-il, doit en premier lieu ne point prélever de salaires excessifs pour prix des services qu'il rend; en second lieu, il doit confier à l'initiative privée quantité de services dont il croit avantageux de se réserver le monopole et que cette initiative accomplirait mieux et à meilleur marché.

Par malheur, les gouvernements contemporains s'obstinent à l'opposé et accomplissent leurs fonctions normales

1. C'est ce que disait déjà M. Léonce de Lavergne, en 1853, dans une étude sur l'économie rurale en Angleterre.

2. Les luttes entre les sociélés humaines, Paris, 1893 (p. 346 et suiv.). 
de la façon la plus détestable. Ils procurent aux citoyens le minimum de sécurité arec le maximum de dépense et compliquent tout en se mèlant de besognes qui sont absolument hors de leur compétence. En réalité, sous le régime quasi général actuel, un grand nombre de citoyens, en Europe, sont réduits à la condition de serls de l'Étal.

Proudhon avait dit l'équivalent avec plus de force et de précision : L'État, la police (ou l'administration), c'est le nom officiel de la classe qu'on désigne en économie politique sous le nom d'improductifs, en un mol de la domesticité sociale, et qui a pour moyen d'existence l'impòt prélevé sur le travail des producteurs. Le pouvoir qui par essence est, comme le capital, l'auxiliaire et le subordonné du travail, devient, par l'antagonisme de la sociélé, l'espion, le juge el le tyran des fonctions productrices; le pouvoir, simple mandataire, à qui son infériorité originelle commande l'obéissance, s'est fait prince et souverain.

L'impôt d'après J.-B. Say, est une privation qu'il faut chercher à diminuer le plus possible, en le linitant jusqu'à concurrence des besoins de la société. L'impôt, a vail dit Turgot, est la contribution que la société entière se doit à elle-mème pour subvenir aux dépenses publiques. L'impôt est l'abus du prince, proclamait avant eux Machiavel; e'est une violente atteinte au droil de propriété, avouent les économistes contemporains, mais une atteinte nécessaire, puisqu'il y a contrat entre la société el les citoyens, ceux-ci payant celle-là pour ètre administrés et défendus par elle. L'impôt est un mal nécrssaire, concluait Léon Say, et il en résumait ainsi les règles: L'idéal, en matière d'impôt, consiste à demander le moins possible, par les procédés les moins coûteux, les moins désagréables, les moins gènants pour la production.

La fiscalité est l'abus de l'impòt, 'qu'il s'agisse de son assietle, de sa répartition ou de sa perception.

Or, à l'extrême limite du Xix siècle, en France, l'impôt est devenu, par l'arbitraire de la répartition el l'exagération des tarifs, un élément démoralisateur par excellence.

Il excite à la fraude, aussi bien le contribuable qui se 
dirobe it la perception que le ministre qui maquille son budget ${ }^{*}$; il est inégal et déconcerte toute idée de justice ${ }^{2}$; très lourd pour les ciloyens payés à l'heure, parce qu'il s'accroit pour eux de loutes sortes de frais accessoires et vexatoires, il reste à peine appréciable pour les grosses fortunes, les sociétés de crédit, les syndicats, pour tous ceux dont la puissance capitaliste, centuplée par le groupement, exploite les individus.

La propriété rurale, plus que toute autre, est la matière imposable qui a toujonrs soulfert des raffinements de la fiscalité. Moins écrasée qu on ne le dit d'ordinaire sous le poids des impòts directs, elle souffre surtout des formalités, des procédures, des lenteurs que crée l'impôt par le désir qu'on a de multiplier les faits ou les actes pour multiplier les taxes.

L'esprit fiscal poussé à l'excès, plıs que la fraude et la mobilité de la jurisprudenee, remarque un des organes les plus autorisés du Notariat ${ }^{3}$, contribue à perpétuer le désordre qui tourmente la pereeption des droits d'enregistrement; il importe de réagir contre un mal qui empire chaque jour et de barrer résolument la route aux audaces d'une fiscalité qui bientôt n'aurait plus de bornes.

Cette fiscalité s'étend à tous les impòts, et il faudrait un volume, seulement avee les aveux des propositions officielles de réforme, pour en analyser les graves ineonvénients. Je ne retiendrai que eeux de ces abus qui affligent directement le régime de la propriété foneière et les conditions de sa division.

Je passerai rapidement sur les critiques adressées, non pas au principe de l'impôt foncier, mais aux interprétations de plus en plus arbitraires qui dirigent son application. Nul n'ignore que, chaque année, la cole cles contribuables augmente d'une petite quantité el que les bureaux tiennent

1. Documents parlemenlaires. - Rapport général de M. Boudenoot sur le budget de 1900 ( 4 juillet $1899, n^{\circ} 1158, p$. 41 et 61 , etc.).

2. Chambre des députés, séance du 4 mars 1898 (Discours de M. Gamard sur la fiscalité). - Annales de l'Enregistrement (1898, 361, Les iniquités légales).

3. Revue du Nolariat, $41^{\circ}$ année, mars $1900, n^{\circ} 10433$, p. 161. 
en particulière estime les contrôleurs assez habiles pour accrôitre le produit de l'impôt sans provoquer trop de réclamations. Les sociétés d'agriculture ont protesté a vec énergie contre les procédés et les résultats de l'enquète sur les nouvelles évaluations de la propriété rurale '; les mêmes réclamations sont formulées contre les évaluations de la propriété bâtie actuellement en cours et qui touchent à leur achèvement ${ }^{2}$. Parmi les prétentions administratives les plus contestées, est celle qui, en exécution de l'article 8 de la loi du 8 août 1890 ordonnant la revision décennale des évaluations, considère conme immeubles par destination de nature à motiver la plus-value de la valeur locative servant de base à l'impôt, non seulement les moteurs et les transmissions, mais uniformément toutes les machines fixées au sol, ne serait-ce que par un simple boulon, et celles mème n'adhérant que par leur propre poids aix installations spécialement destinées à les recevoir. Cet excès de zèle, vouloir faire payer limpôt foncier sur le matériel d'exploitation, équivaut à une seconde patente de superposition ${ }^{3}$.

Ce procédé a sa répercussion sur l'industrie rurale, car il n'affecte pas seulement les usines et locaux industriels, au nombre de 137019 en 1891, mais toutes les fermes de la grande et de la moyenne culture où sont installés des appareils élévatoires pour l'eau, des machines à vapeur mobiles ou fixes d'un emploi temporaire, des distilleries, des barattes mécaniques, etc. Plus encore que l'industrie proprement dite l'exploitation agricole serait entravée dans son essor par l'évaluation fiscale de tout son matériel au moment où il lui est plus nécessaire que jamais de se munir d'un outillage

1. Les résultats de cette enquête, ordonnée par la loi du 9 aoû 1879 , ont été condensés dans le magnifique Atlas publié par les soins de M. Boutin, superbe travail de statistique économique en 82 planches in-folio mais hors de proportion avec son objet.

2. Les résultals généraux du recensement des propriétés bàties et de leur valeur locative, preserit par l'article 3 ' de la loi du 8 août 1885 , n'ont pas ité publiés dans leur intégralité conme eeux du recensement rural. Leur exactitude est contestée partout.

3. Protestation de la Chambre de commerce du Havre (13 mai 1900) 
coùteux et perfectionné pour soutenir la lutte contre la concurrence étrangère. Ce serait reprendre d'une main ce qu'on a l’air de donner de l'autre par des dégrèrements fictifs.

Les taxes indirectes sont toutcfois infiniment plus pesantes pour la propriété foncière que ne peuvent l'êtrc les impôts directs, dont le chiffre est connu d'avance, au moins pour l'annéc en cours, et qui ne déconcertent pas le contribuable par de brusques surprises et des exigences inattendues, comme les autres.

De tous les fléaux qui menacent l'agriculture, écrivait en 1893, M. Georges Michel dans l'Économiste Français, le plus redoutable est, sans contredit, le Code civil combiné avec le Code de procédure.

Il ne faut pas perdre de vue, en effet, que notre systèmc d'impôts date d'un temps où l'on ne connaissait guère d'autres formes de la richesse acquise que la terre et les prêts hypothécaires qui la mobilisent; les grandes sociétés de crédit, les compagnies de chemins de fer, les compagnies d'assurances, les commandites industrielles n'existaient pas. Depuis l'an XI, les rapports entre la fortune territoriale et la richesse mobilière se sont renversćs; les besoins de l'État ont grandi et le chiffre de l'impòt a suivi leur accroissement; les intermédiaires, courtiers, fonctionnaires, officiers ministériels, se sont multipliés et leurs exigences ont crû avec leur nombre. L'équilibre est détruit et, malgré les ingénieuses démonstrations de quelques publicistes, tout porte à croire qu'il le sera de plus en plus au détriment de la propriété agricole.

La fiscalité frappe la propriété foncière dans les manifestations les plus naturelles de sa constitution et de son activité : l'hérilage, l'acquisition, l'emprunt. Ce dernicr cas ayant une importance particulière dans l'étude de la division de la propriété, je lui réscrve un paragraphe spécial et, pour abréger, je ne donnerai qu'une analyse rapide des conditions déplorables qui sont imposées par la fiscalité contemporaine à l'héritage et à l'acquisition, c'est-à-dire aux deux moyens par lesquels le droit de propriété se transmet de 
mains en mains, soit à titre gratuit par la dévolution successorale, soit à titre onéreux par la vente, volontaire ou forcée.

La véritable unité sociale, ce n'est pas l'individu mais la famille, de mème que l'unité cadastrale et foncière ne doit pas être la parcelle mais l'exploitation, le domaine dont le dossier juridique forme le titre constitutif, l'état civil, tandis que le plan parcellaire n'en est que la photographie plus ou moins exacte. Tout ce qui touche à la famille, e'est-à-dire à l’héritage, doit être particulièrement éludié.

Le droit de mutation par décès est la juste compensation, d'après les jurisconsultes, de la protection que l'Étal accorde aux transmissions héréditaires, de la garantie donnée à la conservation et à l'égale réparlition des biens de la famille, ainsi qu'à la possession tranquille des fruits du travail el de l'aclivité domestiques, origine de la propriélé patrimoniale. Les économistes lui accordent cet avantage de frapper le transfert de la lortune aequise dans d'autres mains que celles yui l’ont eréée, augmentée ou seulement conservée, sans que celles-ci aient fait aucun effort pour la produire. Par des larifs gradués suivant le degré de parenté el croissant à mesure que l'héritier s'éloigne de l'auteur commun, en même temps que proportionnels aux valeurs transmises, l'Etat manileste la faveur dont il entoure la famille; mais ces principes ne sont pas toujours applirfués avec un égal discernement et le droit civil ne se lrouve que trop souvent contredit par le droit fiscal ${ }^{1}$.

Certains prineipes généraux de la fiscalité ont motivé sans sueces, depuis un siècle, des propositions de réforme qui demeurent encore en suspens, l'équilibre budgétaire exigreaut le maintien de règles qui ne sont d'aceord ni avec le Jon sens ni avee l'équité. Telles élaient la nou-déduclion des dettes de l'actif successoral ${ }^{2}$, qui laisail payer l’impôt sur des

1. Annales de l'Enregistrement, 1895, p. 346 .

2. La réparation de cette iniquite est contenue dans la loi sur le tarif successoral volée nar la Chambre des dépulés le 22 novembre 1895 , lemeurée en suspens au Sénal depuis le y juillet 1896 , date du dépôt du rapport $n^{\circ} 171$, et enfin devenue définitive par son insertion dans la loi de finances du 23 février 1901. 
valeurs qui n'existent pas; la non-restitution des droits sur un acte déclaré nul postérieurement en justice ${ }^{1}$; la laculté d'exiger la taxe de mutation non pas sur le prix réel, valeur vénale ou déclaration ², mais sur une évalualion arbitraire à élablir par voie d'expertise, elc.

Mème en ne tenant pas compte de ces énormités de la jurisprudence fiscale ${ }^{3}$, si l'on considère uniquement le résultat de lapplication régulière des tarifs en malière de succession en ligne directe, catégorie de mutations particulièrement intéressante pour la division de la propriété ${ }^{4}$, on est effrayé des conséquences économiques de l'incidence fiscale. Le père de famille meurl, il laisse à sa reuve, avec une exploitation agricole, industrielle ou commerciale sans chef et qui va péricliter pendant un temps plus ou moins long, la lourde charge de nomrir et d'élever plusieurs enfants en bas âge ${ }^{5}$. Les frais de maladie, les frais funéraires, ont entamé sinon épuisé les ressources d’épargne; l'Élat vient à son tour prélever 1,20 p. 100 sur l'actif brut, sans déduire les dettes qui, dans toute industrie, sont un élément nécessaire d'exploitation. On ne sail que trop la mortalilé qui décime les enfants; l'un d'eux meurt, c'est souvent un enfant de quelques jours dont la mort a pu ètre amenée par la maladie d'une mère affaiblie par le chagrin et les latigues, quand ce n'est pas cette mère elle-mème qui meurt en le meltant au jour. Il disparaìt, nanti sans qu'il s'en doute de sa part indivise dans l'hérédilé paternelle ou maternelle. Sa succession est dévolue pour 1/4 à son père ou à sa mère survivant, pour $3 / 4$ à ses frères et sours. Sur le premier lot, le trésor prélève de nouveau 1,20; p. 100 comme il l'a fait, quel-

1. Article 60 de la loi du 22 frimaire an VII.

2. Articles 17 de la loi de l'an VIl et 13 de celle đlu 23 août 1871.

3. ‥ Bisseuil, La fiscalité (Nouvelle Revue ru Ler février 1898).

4. En 1898, sur 430810 déclarations de successions, celles en ligne directe figurent pour presque les $2 / 3$ (exactement 281353). (Bulletin de statistique de l'Enregistrement, $1899, \mathrm{p}$ 18.2 et 191.)

5. En 1898, sur 281333 successions en ligne directe, on en trouve plus du quart, soit 62592 concernant des familles de 4 enfants et au-dessus (Loco citato.) 
ques jours ou quelques mois plus tôt, sur l'intégralité de l'hoirie; mais, sur les $3 / 4$ attribués, le plus souvent sans qu'ils s'en doutent, aux petits frères et aux petites sœurs, le taux s'élève subitement à 8,13 p. 100 . La mort frappe rarement des coups isolés, un autre des enfants, deux peut-être, meurent; et le receveur de diviser et de taxer derechef, ici à 1,20 p. 100, là à 8,13 p. 100, n'oubliant pas, dans sa liquidation aveugle, muette et sourde, qui nivelle automatiquement, que le second enfant transmet, outre sa part virile, la portion de celle dont il a hérité de son frère, et que le troisième petit défunt avait déjà, à l'àge de quinze mois peutêtre, réalisé trois héritages dont les droits accumulés ont fait une forte brèche à son capital souvent plus nominal que réel.

Supposez un paysan laissant un petit domaine évalué 12000 francs et six jeunes enfants ${ }^{1}$. Sa succession donne ouverture à 1000 francs de droits (abstraction faite des frais accessoires plus considérables encore de notaire, greffier, inventaire, tutelle, etc.); celle du premier enfant qui décède après lui, nanti d'une part légale de 2000 franes à $128 \mathrm{fr} .13$; celle du second, nanti de 2300 franes à $1 / 48$ fr. 63 ; celle du troisième, nanti de 2731 franes à 176 franes; au tolal, 602 fr. 76. La mulation est réelle, la perception est logique; mais on ne peut s'empêcher de penser qu'avant $1789 \mathrm{ct}$ même pendant les premières années de la Révolution, les mutations par décès en ligne directe étaient affranchies de toute taxe fiscale ${ }^{2}$.

J'ai noté la taxe en ligne collatérale de 8,13 p. 100 dans l'hypothèse d'un actif non grevé de dettes; mais si l'on suppose le cas infiniment plus fréquent où le patrimoine du petit propriétaire est enchainé par l'hypothèque, soit pour des prix d'acquisition encore dus, soil dans les pays à domaines agglomérés et à famille fixe (Béarn, Auvergne,

1. En 1898, il s'est trouvé 9275 successions dans ces conditions, pour des valcurs dépassant 90 millions de francs.

2. liéforme sociale du 16 novembre 1890 . 
Provence, Savoie) par res soultes de partage ${ }^{1}$ ou des prix de cession de droits successifs, le droit de 8,13 p. 100 se transformait, en raison de la non-déduction du passif, en un droit eflectif de 12 ou de 16 p. 100. Que de fois mème le droit de mort, suivant l'énergique expression des paysans, est-il perçu sur une valeur absolument fictive, telle la dot de la mère employéc à dégrever l’immeuble paternel qui n’en sera pas moins un jour taxće sur sa valeur entière.

Ce n'est pas tout. Les conséquences de la mort du père ou de la mère propriétaire de biens fonciers sont radicalement destructives du droit de propriété, par la combinaison du droit civil, du droit fiscal el de la procédure, si le défunt laisse des enfants mineurs.

Les articles 826 (partage forcé en nature) et 827 (vente par licitation) du Code civil ont souleré de violentes critiques; mais les articles 838 et 839 qui prescrivent, lorsqu'un ou plusieurs des héritiers sont mineurs, le partage ou la vente en justice, sont autrement dangereux pour les familles car ils ont pour effet immédiat la dislocation et l'évanouissement du petit patrimoine rural.

Le petit domaine d'une valeur vénale de ö00 francs laissé par un paysan à ses enfants mineurs ne peut pas être confié à la mère qui en continuerait l'exploitation; il doit forcément ètre mis en adjudication devant le tribunal, exposé aux enchères et vendu dans les plus mauvaises conditions; même, par un heureux concour's de circonstances, se vendrait-il bien, il est établi par les statistiques du ministère de la justice que les frais non seulement absorbent mais dépassent le prix de la vente, de telle sorte que l'héritier, dépossédé de son héritage, reste le débileur des intermédiaires chargés juridiquement de le ruiner.

Le nombre des licitations et soultes de partage varie de 99404 (en 1880) à 70091 (en 1890)² ; celui des ventes forcées

1. D'après la loi de l'an VII, le droit sur les retours de lot est perçu au taux réglé pour les ventes; s'il s'agit d'immeubles, il est aujourd'hui de 5 p. 100 y compris les 2 décimes et demi. (Dictionnaire des Rédacteurs, iv, 550.)

2. Bulletin de statistique de l'Enregistrement, 1898, p. 166. 
de biens de mineurs de 12140 (en 186ă) à 17152 (en 1891) ${ }^{1}$ et les héritages estimés d'une valeur de moins de 500 francs $\mathrm{y}$ figurent pour 8 à 10 p. 100.

La loi du $2 \%$ octobre 1884 a affranchi des taxes fiscales les ventes judiciaires au-dessous de 2000 francs et a voulu réduire les frais; elle n'y a pas réussi dans une proportion appréciable et les rapports de la Chancellerie en dénoncent la cause. Les officiers ministériels coalisés ont multiplié les incidents et les formalités à ce point que la moyenne des frais pour les ventes de 5000 francs et au-dessous, qui était de 146,72 p. 100 avant 1884 est encore de 123,72 p. 100 en 189\%. Les incidents soulevés sont de 95 p. 100 devant les notaires commis et de 69 p. 100 à la barre; cel écart donne un certain intérèt au choix des deux procédés puisque le premier est infiniment moins coûteux que le second. L'usage de confier aux officiers publics les adjudications d'immeubles est beaucoup plus répandu dans le nord de la France que dans le sud. Ainsi, pendant la période antérieure à 1887 , les tribunaux des 13 ressorts de la région nord out renvoyé les $3 / 3$ des veutes ( 60 p. 100 ) devant des notaires, tandis que, pour les 13 ressorts du Midi, la proporlion des renvois n'atteint pas $14 \mathrm{p} .100^{2}$. La siluation n'a pas sensiblement cliangé de 1887 à 1897. Il faut noter, à la charge des lenleurs traditionnelles des tribunaux, que le nombre des affaires terminées dans le délai de trois mois est de 69 p. 100 à la barre, de 83 p. 100 devant notaire ${ }^{3}$. La moyenne de la proportion des frais est de 132 p. 100 pour les ventes au-lessous de 500 francs, de 53 p. 100 au-dessous de 1000 franes, el de seulement 2 p. 100 au-dessus de 10000 francs. Cela seul est la condamnation du système.

Anx mineurs ruinés par la loi, remarquail à ce propos

1. Bulletin des sciences ćconomiques el sociales du Comilé des lravaux historiques, 1893 (Mémoire sur le rlroil de saisie).

2. Bulletin de stalistique du ministère des Finances, 1887, 1. 179.

3. 11 y aurait un rapprochement topique a faire entre ces trois étapes le la vente judiciaire : estimation on valeur vénale le l'immeuble mis en vente, mise à prix, prix d'adjudication. 
II. Georges Michel, il ne reste qu'une vaine consolation, c'est de savoir que les débiteurs hypothécaires, les victimes de la saisie immobilière, sont encore plus malheureux qu'eux.

N'est-il pas déplorable de voir la classe des très petits proprićtaires, dont les familles sont les plus riches d'enfants" si rudement atteinte par l'impôt? La propriété n'est plus la récompense du travail, de la patience et de l'épargne : c'est le tonneau des Danaïdes, un vase que le paysan remplit à la sueur de son front et que le Fisc vide dès qu'il est plein.

N'est-elle pas admirable, cette race de travailleurs qui, en dépit des obstacles, des déceptions, persiste, grandit, sc perpétue, semblable à ces ruches dont on coupe périodiquement le meilleur du miel et qui ne se lassent pas de produire pour autrui.

Si l'héritage est fort maltraité par la fiscalité, et surtout par la procédure, les acquisitions volontaires, les mutations entre vifs à titre onéreux, pour parler le langage fiscal, ne le sont pas moins.

Supposons quatre actes d'acquisition de menues parcelles de terre, du prix de 20 francs, 100 francs, 300 francs et 500 francs. Dans les quatre cas, les honoraires du notaire pour la rédaction du contrat seront de ö francs, minimum fixé par le tarif de 1898 (non compris les frais d'expédition); le Trésor percevra le droit proportionnel sur le prix, plus une série de droits fixes frappant aveuglément la convention quelle que soit son importance (timbre de la minute, de l'expédition, du dépôt, du récipissé, du registre, droits fixes de transcription, salaires du conservateur); tout cela se solde par 8 fr. 74 qu'il faut ajouter au droit proportionnel de vente sur 20 francs et à l'honoraire du notaire, 5 francs; total $15 \mathrm{fr} .12$ pour une vente de 20 francs, soit plus de $700 \mathrm{p} .100$ de frais ${ }^{2}$.

1. Bulletin du Comité des travaux historiques, 1890, x, p. 95.

2. La loi du 27 juillet 1900 sur les taxes hypothécaires, d'ailleurs incomplète et mal préparée, semble avoir diminué ces proportions; il n'en est rien, la charge est seulement déplacée; comme pour la loi successorale de 1901, ce ne sont pas les particuliers qui profiteront du changement, mais les notaires.

LA PROPRIÉTÉ RURALE EN FRANCE. 
Pour 100 francs on paiera $20 \mathrm{fr}$. 62; la proportion tombera à $11,0 ّ 5$ pour 300 francs et à 9,62 pour 500 francs. A partir de ce chiffre, le poids des droits fixes va naturellement en s'atténuant; à mesure que le prix grossit, (ute le droit proportionnel augmente, la somme invariable de $8 \mathrm{fr} .74 \mathrm{se}$ perd, en quelque sorte, dans le total général. Ne retenons finalement que deux chiffres, négligeons pour un instant les honoraires de l'officier ministériel, nous constatons que lorsqu'un paysan achète à son voisin un lopin de terre de $20 \mathrm{fr}$., il paie un impôt de 50,60 p. 100, tandis que l'acquéreur d'un domaine de 100000 francs ne paie pas 7 p. 100. C'est la proportionnalité à rebours. On conçoil l'horreur du paysan pour le Fise et l'empressement qu'il met à se dérober à l'impòt, dès qu’il croil pouvoir s'y soustraire sans payer l'amende.

Il serait pourtant si facile, si l'on voulait avoir le courage de se dégager des coteries el de la politique mesquine, de mieux répartir l'impòt sur les actes et le droit de mutation, afin de supprimer de notre législation fiscale les iniquités qui la discréditent. Tout a élé dil, tout a été étudié; les projets pratiques de refonte sont rares, car la simplicité est le dernier terme de l'art, en finanees comme pour le reste, mais il y en a qui ont subi la double épreuve de la crilique et de l'expérience; il suffit de faire un choix.

Un homme d'esprit ${ }^{1}$ a récemment publié sous ce titre suggestif: Lobsession fiscale, le résumé des différentes propositions offertes au Parlement pour reviser notre système d'impòts ${ }^{2}$. Il pense que lorsque les taxes sont produclives et d'un recouvrement facile, il n'y faut toucher qu'avec précaution, il affirme que l'établissement de limpôt sur le revenu ne donnerait que des dégrèvements illusoires; qu'avec de légères retouclıes ${ }^{3}$ il fant maintenir l'ensemble des contri-

1. II. A. Neymarck, janvier 1900.

2. M. Guillemet en 1898 (Impót sur les revenus), M. Jacob (Impót progressif sur le revenu), M. Klotz. (Imprit global sur te revenu), M. Rose (Impit sur le capital), M. Ménier (Impôt sur les capitaux fixes), M. Massabuau (Impôt progressif sur le revenu aggloméré), etc.

3. Projet de M. Ribot, du 22 octohre $189 \%$, sur la transformation de la contribution personnelle-mobilière. 
butions directes, mais dégrever la propriété rurale des taxes indirectes qui en entravent la jouissance ou en gènent la circulation. Ses conclusions sonl fortement motivées.

Il n'est pas juste, avouons-le, que par l'eflei combiné de la contribution personnelle-mobilière et de celle des portes et fenêlres, pour un mème loyer de 500 francs, le contribuable parisien paie $23 \mathrm{fr}$. $10 ّ$ el le contribuable rural $40 \mathrm{fr}$. 7 ŏ. En rétablissant l'égalité fiscale entre les différentes sources de la fortune publique on aidera puissamment à la répartition économique de la propriété foncière.

Les arbres portent des fruits plus abondants lorsqu'ils sont taillés par une main compétente, ils n’en portent plus lorsqu une main maladroite a fait tomber les pousses qui en contenaient les germes. Or, en tous sens, disait récemment un économiste, le législateur contemporain a méconnu le caractère, la portée el l'incidence des impôts; il a dépassé la mesure, aussi bien dans les taxes directes que dans les indirectes, aussi bien au dedans dı pays qu’à la frontière.

La diminution graduelle (relative) de la richesse de la France a été la conséquence de ces erreurs. Plus l'impôt est arbitraire, plus la confiance diminue, et le mauvais emploi des ressources communes finit toujours par influencer les contribuables; ils protestent, ils réclament, ils s"indignent, puis, de guerre lasse, ils finissent par imiter dans la gestion de leurs affaires privées le mauvais exemple que leur donne l'Etat.

Bref, l'impôt, par le chiffre excessif des frais dont il empoisonne les formalités élémentaires de la vie civile ${ }^{1}$, arrète le développement de la famille, entrave les transactions, frappe les besoigneux dans des proportions inoües, transforme l'administration de la justice en un laminoir inconscient qui crée un niveau de misère. C'est un ferment d'iniquités, et

1. Soit qu'il les provoque, soit qu'il les tolère. L'exemple le plus récent est l'établissement du tarif légal des notaires qui, décrété en principe dans un but d'économie par la loi du 20 juin 1896, est devenu, par les décrets du $2 \ddot{3}$ août 1898 , une surtaxe injustifiée et une charge écrasante pour la propriété foncière. urbaine ou rurale. 
si l'on recherche les causes premières de ce vent de socialisme qui souffle plus que jamais en tempète sur le monde, la plus apparente, la plus acluelle, sera l'excès d'impòts.

D'une part, en eflet, il froisse l'instinct d'équité du citoyen; de l'autre, il surexcite chez les utopistes l'illusion de l'État se substituant à l'individu au détriment de la liberté.

\section{VI. - La dette agraire.}

La question foncière reste la plus délıcate et la plus obscure de toutes nos équivoques économiques et sociales; en lui cherchant une solution pratique, on peut espérer une accalmie dans les esprits et une amélioration dans l'acuité des conflits matériels qui exaspèrent les capitalistes autant que les industriels et les ourriers, et menacent la fortune en voie de formation autant que la fortune accuise.

C'est le pelit propriétaire rural qui dénouera, en Franec, le nœud gordien; mais à la condition qu'au lieu de l'entraver et de le ruiner, la législation le dégagera du filet qui l'enserre el rendra son élasticité à sa naturelle expansion, si vivace el si féconde. La fiscalité el la dette agraire, celle-ci engendrée par celle-là, voilà les ennemies irréconciliables de la propriété foncière, parce qu'clles l'exploitent, parce qu'elles en vivent, et qu'elle-mème ne peut renaître qu'en les jetant hors de sa route.

Le poids de l'impôt sur les propriétés rurales est tel qu'on a la penséc, sitól que les populations agricoles sont éprouvées par quelque sinistre d'une étendue régionale, ou de demander le dégrèvement, ou de réclamer des secours. Ces deux remèdes, aussi inefficaces l'un que l'autre, ont de plus l'inconvénient de déprimer les initiatives el d'engourdir les énergies; une solide mutualité vaut mieux que le recours ì l'Etal.

Le propriétaire rural n'a point de crédit? Pourquoi? Quel est l'obstacle à la véritable et définitive libération du terri- 
toire? La dette agraire. Il est enfermé daus ce double cercle de l'impòt et de la dette d'où les parasites de l'État l'empêchent de sortir paree que c'est ee prisonnier qui les nourrit.

Si 1790 l'a délivré des servitudes féodales, nous le voyons, en 1900, tout entier courert d'hypothèques, et retomber sous des chaìnes financières qui, pour ne sembler que volontairement acceptées, n'en sont pas moins pesantes et irréductibles.

En 1900, sur 100 propriétaires terriens, il n'en est pas 4 qui soient leurs maîtres et dont les profits puissent grossir l'épargne patrimoniale; aux servitudes féodales abolies en 1789 , la spéculation el la procédure ont substitué une autocratie financière inexorable. La plupart des propriétaires ruraux, a dit un spécialiste ${ }^{1}$, ne travaillent plus pour eux, ils sont passés à l'élat de simples exploitants pour le compte d'autrui; ce ne sont mème plus les métayers du xviı siècle, ils sont relombés dans l'esclavage irrémédiable des colons du Bas-Empire, ce sont les salariés du capital, avec cette aggravation qu'ils ne sont pas libres de faire grève.

Ce sombre tableau, vrai pour certaines régions de la France, ne serait pas tout à fait exact si on l'appliquait à l'ensemble du territoire; il suffit de quelques bonues récoltes successives pour permettre aux paysans de se libérer, au moins en partie, surtout dans les pays de vignobles (les vallées du Rhône el de la Garonne, les Charentes) ou d'élevage (la Normandie, la Nièvre). La vérité ne sera tout à fait connue que lorsque le service de l'Enregistrement se décidera à recueillir et à publier les statistiques hypothécaires; c'est un des còtés essentiels de notre état économique sur lesquels on est le moins renseigné, et la Commission extra-parlementaire du cadastre, elle-même, depuis tantôt dix ans qu'elle existe, n’a pu obtenir satisfaction sur ce point.

La situation hypothécaire de la France, si imparfaitement connue qu'elle soit ${ }^{2}$, ne paraît pas brillante. Dans la seule

1. M. de Saint-Genis, La dette agraire et l'héritage foncier, 1894 .

2. Bulletin de statistique de l'Enregistrement, III, 1899, 212 
année 1898, il a été pris 770494 inseriptions nouvelles et il n'en a disparu, à la suite de radiations tolales ou partielies (les relevés ne marquant pas la différence) que moins de 380957 ; il y aurait done ehaque annéc, dans la dette hypothécaire, un accroissement régulier d'environ 400000 créances, 4 millions en nombre de deltes nouvelles pour la période décennale qui est leur vie juridique, prélevant le produit le plus clair de la culture pour le paiement des iniérêts et des frais. Il est impossible de distinguer avec précision dans celte masse passive la part de la propriété rurale et celle de la propriété urbaine ${ }^{1}$.

On ne peut mème pas en évaluer le chifire. Pour les 770494 inscriptions de l'année 1898, les hypothèques conventionnelles et judiciaires, au nombre de $46 \ddot{4} 473$, ont leur montant déterminé par la perception de la taxe de 1,23 p. 1000 ; clles garantissent une dette nouvelle de 2 milliards de francs; mais il en reste 303021 dont le chiffre est indéterminé el, parmi celles-ci, 293786 inscriptions doffice représentant le tolal inconnu des prix d'acquisition payables à des ćchéances plus ou moins éloignées. Le nombre des ventes de 1898 na pas encore été publié; mais, en $189 \%$, on en comptait 71360 . dont 571170 d'immeubles ruraux; si l'on remarque que sur ce nombre 544902 sont inférieures à 5000 francs, que, sur celle catégorie, la moyenne des prix payés comptant ne dépasse guère 20 p. 100, el que la valeur de ces acquisitions monte à 378 millions de franes, on peut admettre que la detle rurale s'accroît, de ee chef, de 93 a 100 millions de plus en capital.

Faut-il une révolulion agraire pour lirer le paysan, le fermier, le propriétaire foncier de cetle voie, sans issue puisque son capital n'acquiert pas, cluaque aunée, une plus-value égale ì sa dette ni son revenu net une majoration de 5 p. 100 de celle même defte? Non. Faut-il bouleverser nos codes, exproprier les créanciers de la terre, déposséder les capita-

1. Celles des statistiques dı Crédit foncier yui sont publiées ne permettent pas une étude approfondie et complète de la question. 
lisles? Nullement. Le remède est plus simple, il est à notre portéc, on le connait depuis nombre d'années, il est préconisé par les esprits les plus pondérés, par les politiques les moins aventureux. Mais où est le Ministre qui osera porter le premier coup de hache dans cette forêt enchantée des abus? Qui possède le talisman au contact duquel s'évanouiront les mauvais génies de la terre?

La Révolution, d'une part, a consolidé le droit de la propriété acquise par la suppression des droits féodaux; d'autre part, elle a ouvert à tous le libre accès de la propriété par la réforme des lois successorales. La résultante généralc des principes et des actes de la Révolution a donc été l'affranchissement et l'extension du droit de propriété, dans le sens de la propriété individuelle. Mais, depuis 1798, il semble que la réaction qui suit les grands mouvements ait accumulé les obstacles devant cet entraînement libéral. Les contradictions d'un code de transition, les subtilités de la doctrine, les chicanes de la jurisprudence ont, petit à petit, reconstitué au tour de l'individu le domaine obscur des équivoques juridiques, el la sécurité du titre foncier est devenue aussi douteuse que son acquisition paraîl facile.

En France, répète-t-on avec un certain orgueil, le respect de la liberté individuelle est tel que, en matière de droits immobiliers, le seul consentement suffit à rendre les conventions parfaites. Le plus misérable des hommes, s'il est laborieux, économe, peut, en France, c'est vrai, devenir propriétaire foncier. Mais le droit qu'il achète lui est-il à tout jamais garanti? Est-il à l'abri des revendications el des procès sur cette parcelle de terre qu'il a loyalement payée? J'affirme que non.

Depuis 1798, rien n'a été fait pour les quatre millions de petits propriétaires fonciers émancipés par la Révolution.

Rien n'a été essayé sérieusement pour consolider dans leurs mains ce titre foncier qu'ils poursuivaient avec une impatience légitime et dont l'accession légale leur était désormais assurée. Le Code civil, considérant la richesse territoriale comme le patrimoine par excellence, mais comme un patri- 
moine à laisser intact entre les mains des riches et ne voyant dans l'emprunt hypothécaire que le préliminaire de la vente forcée, n’a favorisé ni les mutations, ni les prèts. Il a, au contraire, accumulé les restrictions el les entraves, plutòt par prétérition qu'autrement ", de telle façon que l'exercice du droit de propriété est redevenu une sorte de privilège accessible aux capitalistes seulement, et dont le paysan, l'artisan, l'homme vivant de son travail, le prolétaire, ne peurent utiliser les libertés apparentes qu'en courant les risques - faute dargent pour payer la purge de l'éviction ou de la forclusion ${ }^{2}$.

Que réclame le crédit territorial pour jour de son élasticité théorique, et que manque-t-il au propriétaire foncier, pour se dégager des exigences des marchands d'argent? La simplification de la procédure et l'abaissement des tarifs.

La solution de la crise agraire, erise dont la répercussion se fait rudement sentir dans le commeree el l'industric et dont la complexité permet, suirant le point de vue auquel on se place, de la nier ou de l'affirmer, ne se trouvera ni dans des palliatifs ni dans des expédients; il ne faut pas surtout, comme en Angleterre el en Allemagne, créer des classes ennemies dans le quatrième état et opposer les paysans aux ouvriers. Tous sortent de la même souche, et ces classifications arbitraires sont pleines de dangers. Le quatrième élat se compose des travailleurs, dira-t-on; mais où est l'homme qui ne travaille pas? Et, abstraction faite de quelques familles qui dépensent le fruit des travaux de leur chef disparu, quel est le chef de famille assez aveugle pour

1. La simple promesse de vente par acte sous seing privé (art. 15: 2 , C. civ.) équivaut à une vente parfaite (Cassation, 23 août 18.3); mais le droit de suite (art. 211', C. civ.) maintient l'immeuble vendu comme le gage des tiers sans que la publicité de leurs droits avertisse l'acquéreur (art. 2135. C. civ.) el le tiers létenteur, même s'il a payé son prix, même si la liberté de l'immeublèa déé régulièrement certifiẻe (art. 2197), ne peut se considérer comme propriétaire définitif qu'après avoir rempli les lentes el coûteuses formalités de la double purge (art. 2167, 2181 et 2193, C. civ.).

2. H. de Saint-Genis, Le Crédit territorial en France et la Réforme hypothécaire, $2^{\circ}$ edition, 1889, p. 8 de l'Introduction. 
ne pas comprendre qu’à bref délai ce qu’on appelle le capitaliste ne pourra plus virre de ses rentes et retombera de nouveau sous le joug salulaire du travail. En ce moment mème, le patron n'est-il pas presque toujours le premier ourrier de l'atelier? Et qui saurait dire où commence le bourgeois, où s'arrète l'ourrier? Si le malaise foncier, père de la erise économique, n'avait pas jeté les ouvriers agricoles dans les centres industriels, verriez-rous les variations du salaire, l'abaissement de la main-d'œurre, le fléau des gréres?

La Nation n'a que faire des étiquetles de la politique; les hommes de France se partagent uniquement en deux grandes calégories : ceux qui possèdent, ceux qui ne possèdent pas.

Liidéal d’un gourernement démocratique n’est pas de recommencer la propriété eollective et de nationaliser les propriétés privées, sclon l'expression à la mode, mais de rendre la propriété individuelle accessible à tous, et d'y maintenir en possession les acquéreurs ou les héritiers, sans gèner leur liberté, mais en les protégeant à la fois contre leur propre imprudence et contre les surprises d'autrui. La division de la propriété, son fractionnement en un grand nombre de mains, cette concurrence pour l'acquisilion qui fait les petits domaines relativement plus chers que les grands, sont des tendances quimarquent la réritable voie du progrès économique.

A toutes les époques, le tenancier, le métayer, le laboureur ont élé le nombre; jamais ils ne sont devenus la force, parce que le fléau de la dette s'est toujours abattu sur eux, el qu'en exigeant d'eux des devoirs innombrables, on leur a sans cesse refusé ou disputé les droits les plus élémentaires.

Le droit de mutation qui frappe les transferts de droits réels, soit entre vifs, soit par décès, est trop élevé, il devient surtout onéreux par les accessoires qui s'y ajoutent, ainsi que je l'ai noté à propos de la fiscalité pour la matière des successions. Le droit de mutation sur les acquisitions fon- 
cières à litre onéreux est de 6,88 p. 100 qui se décomposent ainsi :

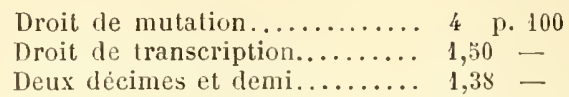

Et, chose bizarre, ce droit proportionnel de transeription qui taxe à l'avance la formalité facultative de la publicité des contrats translatifs de droits réels, prévue par l'article 2181 du Code civil et les articles 1 el 2 de la loi du 23 mars 185๊, ne dispense pas des divers droits dus lors du dépôt au bureau des hypothèques. Quant aux honoraires des notaires, ils sont de 1 p. 100 jusqu'à 100000 francs (ventes volontaires) et de 2 p. 100 jusqu'à 20000 franes (ventes judiciaires el adjudications volontaires) avec réduction graduée pour les sommes plus fortes ${ }^{1}$. Dans certaines études, le prix à forfait varie de $\mathbf{1 1}$ à $\mathbf{1 4}$ p. 100.

Mème si le petit propriétaire a fait la dépense d'un titre devenu incontestable, ec pelit domaine qu'il a créé à la sueur de son front, trop pelit pour être divisé, ne va-t-il pas sombrer, disparaitre entre ees deux fléaux de nos codes : la saisie (Art. 2071, 2092, 2095 du Code civil; art. 673 du Code de procédure civile), la licitation (Art. 813,826 et 827 du Code civil; art. 953 du Code de procédure civile)?

Qu'il s'endette, ce qui est probable, qu'il meure, ce qui est certain, le chef de famille est impuissant à transmettre intact à ses enfants le champ sur lequel il a vécu, le toil qu'il a bâti. Souvent, il ne leur lègue que des charges, el lc petit hérilagge, source de bien-être s'il vit, devient, quand il meurt, l'origine de la dette agraire qui pèsera peut-être sur cette famille durant plusieurs grénérations.

Les récents débats sur la preuve juridique du droit de propriété, sur le cadastre, le bornage, si approfondis, si prolongés qu'ils aient été, n'ont rien conclu de pratique ni d'efficace pour attribucr aux petits propriétaires un titre

1. Tarif légal du 25 aoùt 1898 pour les ressorts des Cours d'appel de la région de l'Ouest (Angers, Bourges, Limoges, Orléans, Poitiers, Rennes). 
régulier et définitif qui dégage le passé, les exonère des réclamations fiscales et les libère des dangers d'éviction 1.

Dans certaines régions, on possècle de père en fils, sans titres notariés, et sans mème que la loi des partages ait contrarié en fait les combinaisons de famille traditionnelles en Champagne, en Savoie, en Languedoc, dans le Pays Basque. Si la terre provient d'héritages, elle se transmet sans complications, sauf le cas toujours à craindre d'un procès provoqué par des héritiers mécontents; si elle a été achetée ou échangée, c'est neuf lois sur dix au moyen d'un sous-seing privé irrégulier ou adiré, et le fail d'ètre en possession supplée au titre d'aequisition. L'absence de titres rend les emprunts difficiles; le Crédit foncier, particulièrement, ne prête que sur des origines de propriété justifiées pour une période d'au moins trente ans, ce qui lui permet de rebuter dédaigneusement tous les petils prêts ruraux.

Le prêt hypothécaire par lui-même, même dans les conditions les plus lavorables, est très onéreux. Non seulement, pour la très grande majorité des cas, la sécurité du titre foncier est équivoque, mais l'emploi du crédit qui découle de la propriété est discuté. Qu'on me permelte de signaler ici un danger réel pour l'avenir de la petite propriété, erreur causée par l'espril de généralisation trop absolu qui veut comparer des situations dissemblables. Dans les pays neufs et mal peuplés, au Far West des États-Unis, en Tunisie, à la Nouvelle-Calédonie, à Madagascar, la vaste étendue des concessions à exploiter permet d'utiliser le droit de propriété comme un moyen de crédit, et la dette hypothécaire est la condition mème du peuplement el de la culture de ces déserts. Dans ces contrées, l'hypothèque, ainsi que l'a dit II. Levasseur avec un rare bonheur d'expression, est le pont qui conduit du prolétariat à la propriété.

Il n'en est pas de même en Europe, et surtout en France

1. Rapport sur les privilèges et hypothèques présentẻ avec un projet de loi à la commission du cadastre par M. Challamel en janvier 1900. Rapport sur le projet d'institution de Livres fonciers présenté à la mème date par M. Massigli. 
en ce qui concerne la petite propriété, celle qui n’a point de tilres authentiques el qui, par sa valeur médiocre, ne peut devenir utilement un gage hypothécaire. L'hypothèque n'est plus l'auxiliaire du droit de propriété, e'est une brèche à la propriété elle-même, et les expédients qu'on oflre comme remède ne peuvent qu'aggraver le mal. Sous prétexte de créer le crédit, on excile à l'emprunt. C'est un immense danger économique; le mot spirituel de Law et de Calonne: La dette prouve le crédit, a causé bien des déceptions et, dans un pays comme le nôtre surtout, il parail plus sage de conseiller au très petit propriétaire le travail, l'économie, l’épargne, que de le solliciler par les séduisantes tentations d’un crédit éphémère.

Létude de la delte agraire dans les différents pays serait des plus instructives mais dépasserait le cadre limité de cette étude. Il me suffira de signaler l'état économique, sur ce point spécial, de deux contrées dont la comparaison conslitue a priori une antinomie : l'Italie, qu'on représente comme acherant sa propre ruine, les Étals-Unis, dont la richesse est plus envahissante que jamais. En Ilalie, l'ensemble des prèts sur hypothèque a passé de 12 milliards 1/2 en 1871, à 17 milliaris en 1893, à 22 milliards en 1898; les $2 / 3$ de ces prêts ont pour gage des biens ruraux, et sur ces 2/3 ils ont pour origine, dans la proportion d'environ 60 p. 100, l'acquisition de parts indivises ou des soultes de partage. On eslime à 100 millions l'accroissement annuel de la delle agraire '.

Aux Élats-Unis, cest l'inverse. M. Levasseur estimait que la dette hypothécaire, en 1891, y dépassait 26 milliarls, dont 17 milliards de franes à la cliarge de la propriété urbaine (Lots) et 9 milliards à la charge de la propriété rurale (Acres). La eharge de la propriété urbaine est donc, en bloc, double

1. Indications fournies par les dèlegués italiens au Congrès international de la propriété fonciere en 1900. En 1893, sur 136321 contrats de prêts, s'élevant à près de 8301 millions, les créances inscrites sur des biens ruraux (terrains et bâtiments) dépassaient le elị̂re de 402 millions de francs. 
de celle de la propriété rurale; mais celle-ci est endetlée jusqu'au tiers de sa valeur vénale ${ }^{1}$. La delte agraire y a été contractée, dans la proportion de 68 p. 100, pour l'acquisition de la terre et l'achat du cheptel; sur 100 fermes, on en trouve 32 qui sont lourdement grevées el 68 qui ne le sont pas. Un doeument officiel de 1889 avouait que, de notoriélé publique, l'hypothèque était effroyablement étendue el dangereusement onéreuse; et M. Levasseur ajoule, d'après l'expérience de einq années de plus : L'hypothèque est une ombre qui se projette sur le tableau de la prospérité agricole de ce pays.

Tous les témoignages sont unanimes à déclarer que la detle agraire est le rer rongeur du droil de propriélé, el que, toutes les fois que l'emprunt n'a pas pour unique emploi l'amélioration de la cullure, mieux vaut dissuader le propriétaire d'user de son erédit que de l'y provoquer.

La mise en gage d'un immeuble pour garantir un emprunt se caractérise par l'hypothèque. Le droit d'hypothèque se décompose lui-même en deux droils dislinets : $1^{\circ}$ le droit de suite, c'est-à-dire le droit pour le eréancier de réelamer son dù de quiconque détient, à litre de propriétaire, l'immeuble donné en garantie; $2^{\circ}$ le droit de priorité, e'est-à-dire le droit de se faire payer sur le prix de l'immeuble grevé d'après le rang quassigne à la créance, la date, la loi ou le titre.

Mais l'hypothèque à se défendre elle-même contre d'autres causes de préférence, les hypothèques légales et les privilèges; parmi ceux-ci, le plus favorisé est celui du vendeur, dégagé de l'inseription, puisque la transcription y supplée, grevant indéfiniment la propriété d'une manière latente, el forcé seulement de se produire à la clôture de la purge.

La dette a pour sanction l'exéeution sur les biens donnés en gage, et même sur lous autres si les biens hypothéqués sont insuffisants (Article 2092 du Code civil), par la saisie.

1. L'agriculture aux Etats-Unis, p. 303. - Bulletin de statistique du ministère des Finances, 189', p. 623 . 
Il n'est peut-ètre pas de pays où l'individu soit moins protégé qu'en France contre l'âpreté d'un créancier et les surprises de la procédure.

Dans nos codes, qui ont une certaine prétention à la mansuétude, à la protection, à côté d'immunités inouïes, comme celles qui laissent indemnes l'escroquerie foncière et le crime de Stellionat, on ne trouve que rigueurs inexorables contre le débiteur simplement gèné qui ne peut payer à l'échéance. Le plus honnête homme, s'il est en voyage et qu'il oublie le percepteur, peut apprendre, au retour, que son domicile a été envahi, ses portes ouvertes, son mobilier saisi, bouleversé, vendu, pour une cote de quelques francs.

En matière d'immeubles, on ra moins vite et avec moins de sans-gêne, car il faut le temps d'espacer les formalités et de les multiplier, mais les délais et les frais n’en sont que plus oppressifs et plus ruineux. Si l'on s'en tient au texte de la loi, la saisie érfuivaut à une véritable éviction el peut entraîner, par le désarroi qu’elle apporte dans la vie de famille, les plus graves conséquences sociales, surtout s'il s'agit de propriétés rurales, celles à qui l'on clevrait le plus de ménagements. Ainsi, l'article 5092 du Code de procédure civile protège contre la saisie les meubles et les vêtements indispensables, ainsi que les outils de toute nature nécessaires à l'exercice de la profession; mais, par une étrange inconséquence, le législateur moderne permet qu'on expulse le débiteur du toit familial, de l'atelier où il emploie ses outils, du sol qu'il exploite pour vivre.

Si les immeubles saisis ne sont pas lonés ou affermés à des tiers, par actes ayant acquis date certaine avant le début te la procédure, ou si le saisi les habite ou les exploite, il restera en possession jusfu 'à la vente, comme serpuestre judiciaire, à moins qu'il v'en soit autrement ordonné par le président du tribunal. Pendant ce temps, les créanciers pourront néanmoins faire procéder à la coupe et ì la vente des fruits pendants par les racines (Art. 681 du Code de procédure civile). Quant aux loyers et fermages, ils seront immobilisés, ainsi que les fruits naturels et industriels, etc. (Ibidem, 
art. 682 à 683). En d'autres termes, la saisie coupe les virres au débiteur et lui arrache les aliments.

Ce n'est pas tout, le droit au travail lui est interdit; il n'a plus la liberté d'achever son labourage ou sa fenaison; tout au plus peut-il louerses bras à son voisin, pour ne pas mourir de faim. En cas de saisie d'animaux ou d'ustensiles servant à l'exploitation des terres, le juge de paix, sur la demande du saisissant, peut, en effet, établir un gérant à l'exploitation, indépendamment du gardien (Ibid., art. 59.4 et 596 ).

Les rentes d'immeubles sur saisie étaient au nombre de כ̆ 338 en 1863 , de 6267 en 1869 , de 9303 en 1872, de 14278 en 1889 , de 11753 en $1891^{1}$. En 1894, elles avaient augmenté de $\$ 4$ p. 100 depuis $187 \%$, et les $7 / 10$ des ordres sont ouverts sur des propriétés d'une valeur vénale minime ${ }^{2}$; en 1896 , il a été transcrit aux hypothèques 20390 procès-verbaux de saisie immobilière.

Il est fàcheux que les statistiques du service de l'Enregistrement, quoique plus détaillées et plus précises que celles de la Chancellerie, ne fassent pas connaitre la nature des biens saisis et vendus et de quels capitaux prêtés à l'origine les immeubles étaient le gage. Ce serait une précieuse contribution à l'histoire de la division du sol. On y a suppléé en une certaine mesure par des travaux personnels ${ }^{3}$ auxquels j'emprunte les chiffres qui suivent et qui sont les derniers connus. Il faut en conclure, remarque l'auteur, ou que l'appréciation de la valeur du gage au moment du prêt est faite par les notaires avec une constante légèreté, ce qui est peu probable; ou que l'on ne prête qu'à des gens à demi-ruinés avec l'arrière-pensée de les évincer, ce qui cst possible; ou que le seul fait des poursuites déprécie instantanément le gage, ce qui n'est que trop exact.

1. La distinction n'est plus faite dans les statistiques entre les ventes judiciaires ordinaires et les ventes sur saisie, au total 26193 en 1894. (Bulletin de statistique du ministère des Finances, mars 1897. p. 246.)

2. 11. de Saint-Genis, Mémoire sur le droit de saisie en France, 1895 (tableaux A et D).

3. La dette agrcire, 1894 (loco cilato). 
L'écart entre les productions faites par les créanciers et l'actif à distribuer augmente à chaque période, ainsi que le nombre des créanciers. En 1881, la perte pour 57352 créanciers, après la réalisation du gage, dépasse 104 millions et demi de franes; en 1891, celte perte, pour 93217 produisants, arrive à près de 247 millions. Des renseignements que nous arons des motifs particuliers de croire exacts font supposer que de 1892 à 1899 la situation ne s'est pas sensiblement améliorée; on peut calculer la brèche que fait la saisie dans le capilal des prèts fonciers en rappelant que le chiffre moyen des eréances hypothécaires déclarées annuellement dans l'actif des successions ${ }^{1}$ dépasse 800 millions de francs, ce qui porterait la perte à plus de 30 p. 100.

Le Midi, le Sud-Onest, le Nord et le Centre sont les régrions les plus éprouvées; les grandes villes le sont très peu; tout le poids de la procédure porte sur la propriété rurale, et èest dans les départements où la propriété présente l'accroissement le plus marqué que sévit avec le plus d’intensité le fléau de la saisie, conséquence quasi inévitable de la dette agraire. Le moreellement est-il, dans ce cas particulier, une cause ou un effet? Des statistiques bien établies pourraient seules fixer sur ce point essentiel.

Les départements les plus alteints sont ceux où la population est la plus nombreuse; densité de la population et grand nombre des très petits propriétaires sont des faits corrélatifs. De 1876 à 1891 , le nombre des saisies suivies d'exécution a triplé dans le Gers, les Hautes-Pyrénées, le Pas-deCalais, la Creuse, la Haute-Vienne, la Haute-Loire, le Puy-deDôme; il a décuplé dans l'Aude, y passant de 30 en 1876, à 60 en 1881, ̀̀ 260 en 1886, à 336 en 1891. En 1898, le nombre des saisies transerites au bureau des hypothèques a été de $17002^{2}$.

Le nombre des affaires au-dessous de 1000 franes, quoique toujours considérable, reste stationnaire; celui des ordres

1. Bulletin de statistique de l'Enregistrement, 1899, p. 188.

2. Ibid., 1899, p. 212. 
de 1000 à $ّ 000$ franes a presque doublé et les distributions de $\ddot{3} 000$ à 10000 franes ont augmenté des deux cinquièmes; les grosses affaires se sont accrues d'un tiers seulement. L'exagrération des frais ${ }^{1}$, en delıors des abus de la procédure proprement dite ${ }^{2}$ conduit à un impôt sur la gêne inversement proportionnel à la valeur imposée, c'est l'impòt progressif à rebours, avec la ruine pour résultat ${ }^{3}$. Je note, pour mémoire, que dans la masse des frais la part de l'impòt est de un tiers, eelle des officiers ministériels des deux tiers.

La dette agraire ne mord que rarement sur la très grande propriété qui a d’autres ressources que les produits du sol; elle est parfois inquiétante pour la grande (telle que la définit la terminologie officielle, de 40 à 100 hectares), surtout lorsqu'il y a partage et liquidation; toujours menaçante, sauf dans des cas exceptionnels, pour la moyenne propriété, elle est absolument destructive de la petite.

En 1889, sur 736000 ventes immobilières portant sur plus de un milliard deux tiers, on trouve 579000 contrais infé-

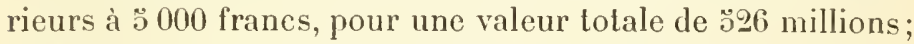
en 1894, on en compte $647500 ั$ (sur un total de 713604), en augmentation quoique le total général ait diminué, et qui se répartissent ainsi :

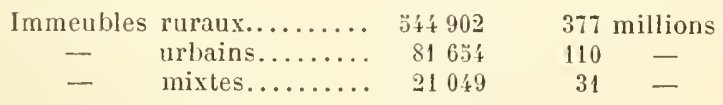

1. Je refrouve dans le Réveil des campagnes dı 3 avril 1892 (publié ả Ilelle, département des Deux-Sèvres) l'analyse d'un procès oủ, pour avoir paiement d'une créance de 1000 francs on fit vendre 7000 francs un immeuble qui en valait 30000 ; les frais dépassèrent 6000 francs. Le crẻancier fut condamné ả 3000 francs de dommages-in térêts, les avoués et les huissiers réprimandés.

2. Journal du Notarial, janvier 1897. - Circulaires du ministre de la Justice des $1^{\text {er }}$ mars 1898 et 7 decembre 1899.

3. S'il s'agit d'un petit bien rural de 300 francs de valeur vénale, les frais s'élevent à 126 p. 100 ; si l'immeuble se vend 2000 francs les frais tombent à 50 p. 100 ; s'il se vend 5000 franes à 16 p. 100. (Voir Stollon et Henricet, Les victimes des lois de l'Enregistrement, 1892, Giard et Brière, à Paris.)

La PROPRiÉté RURALE EN FraNCE. 
Les petites ventes rurales sont donc très nombreuses et leur masse compacte ne fait que s'accroître. Est-ce à dire que ce déplacement incessant de propriétaires sur des exploitations où la continuité des méthodes et la persévérance du labeur sont une condition de succès doive être considéré comme une preuve absolue de prospérité? Il serait bien aventuré de le prétendre. Les économistes feront ressortir la contre-partie de ce fait en parlant d'acquisitions au lieu de ventes; mais il faudrait prouver que ces acquisitions ont été faites par d'autres que des capitalistes ou des spéculateurs.

Je crois pouvoir affirmer, sauf la preuve contraire que jai vainement cherchée, que la majorité de ces vendeurs sont de petits propriétaires désormais dépossédés, réduits à devenir ou à redevenir fermiers ou simples salariés ou à émigrer dans les villes. Ce résultat de la delle agraire stérilisant la fortune acquise est, il est vrai, compensé par le nombre des aequisitions, puisque l'étendue de la petite propriété augmente avec le chiffre des exploitants, preuve que non seulement il y a échange entre petits terriens, les uns ruinés, les autres à l'aise, mais que les petits mordent sur les moyens et sur' les gros. Le rève serail, tout en s'applaudissant de la marée montante des très petites propriétés, de les fixer toutes, de maintenir le plus possible la permanence de l'héritage, de mettre un frein à eette extrème mobilité, de telle sorte que les acquisitions nouvelles ne se fassent pas au détriment des propriétés minuseules mais par le morcellement des grandes plutôt que des moyennes, et surtout par la mise en valeur des six millions d'hectares qui restent encore stériles sur notre territoire continental el dont les deux tiers appartiennent à des particuliers. 


\section{CHAPITRE IV}

Conditions économiques et juridiques particulières à chaque groupe de propriétés et qui entravent ou facilitent leur exploitation.

Si l'on a coutume de distinguer trois sortes de propriétés: la grande, la moyenne, la petite, il est malaisé de les elasser avec précision dans chaque catégorie. De l'une à l'autre, sauf pour la très grande, les limites sont indécises, et la science de la statistique voudrait des tranches plus nombreuses, car il est certain que les lois générales qui règlent la condition de la propriété territoriale ne l'affeetent pas uniformément et que leurs incidences sont inégales d'après les caractères qui distinguent chaque nature de propriété.

Les lois eiviles et fiscales édictées pour l'ensemble d'une nation, ne peuvent mesurer leur aetion probable sur des cas particuliers, et l'effet des lois écononiques vient souvent contrarier leurs prévisions. Elles font, bon gré mal gré, des conditions différentes à chaque catégorie de propriétés, et il en résulte que ce qu'on offrait à celle-ci à titre de protection ou d'encouragement risque de devenir pour les autres une gêne et une entrave. Le véritable esprit économique doit moins se préoccuper, à cet égard, de rechercher à des maux avérés des palliatifs ou des expédients, que d'établir ou plutòt de permettre l'équilibre par la pratique de la liberté el la discrétion de l’État. 
Le morcellement, la fiscalité, la detle agraire sont les causes qui entravent le développement de la richesse agricole; elles le font à des degrés inégaux, dans des mesures différentes, suivant qu'il s'agit de la pelite, de la moyenne ou de la grande propriété.

On peut, à ce point de vue spécial seulement, je me lâte de le dire, se désintéresser de la grande propriété qui, représentant l'une des formes les plus enviées de la fortune capitaliste apparaît, en somme, comme une exploitation industrielle avec les chances et les risques habitucls de toute industrie. Les deltes des millionnaires ne sont qu'un des éléments de l'activité sociale.

La moyenne propriété comporte à peine $20 \mathrm{p} .100$ de propriétaires exploitant personnellement leur propriété; dans la proportion de $80 \mathrm{p} .100$, elie est cullivée par des intermédiaires, fermiers, métayers, colons partiaires, el appartient ou à des bourgeois (rentiers, industriels, commerçants) qui ont trouvé quelque satisfaction de vanité ou quelque profit à placer en terres une parlie de leurs capilaux, ou à des héritiers qui ne peuvent ou ne veulent se dessaisir de biens quils risqueraient de vendre il perte. Bien rares sont les domaines (sauf de 10 ì 30 hectares peut-être) appartenant à des agriculteurs de profession, soit qu'ils aient été hérilés, soit qu'ils représentent des proprićtés peu importantes à l'origine et qui se sont successivement agrandies par la prudence, le savoir-faire et l'industrie te leurs détenteurs.

Il s'agit donc ici, pour la majorité, de propriétaires fonciers qui n'ont que l'apparence de vérilables terriens et qui restent, en réalité, des capitalistes ou des possesseurs accidentels, sans esprit de possession durable. Cetle sorte d'aristocratie territoriale, à laquelle songeait sans doute le comte de Falloux quand il souhailail de la voir habiter ses domaines, au moins une partic de l'année, est relativement riclıe; elle a les moyens de se proléger el de rendre ses titres définitif's. C'est moins son élément le plus à l'aise, celui que j'appellerai l'élément forain, que le plus modeste, celui du faire-valoir, qui utilise le gage foncier et fait usage 
du erédit hypothécaire. Mais, à n'envisager que l'ensemble et à ne raisonner que d'après l'état actuel de notre législation foncière, e'est à la moyenne propriété que profitera la réforme hypothécaire, lorsqu’on se décidera à la réaliser, car e'est elle qui désire, non seulement un titre bien assis et la possibilité de rendre les mutations rapides, mais pardessus tout la quasi mobilisation de son erédit par la facilité des emprunts et plus de simplicité dans la réalisation du gage.

Il y aurait beaucoup à dire sur ce courant d'idées, car si la dette agraire pèse d'un poids si lourd sur la propriété territoriale, moyenne, petite ou grande, ce ne seront ni la rélorme hypothécaire ni mème l'institution des Livres fonciers et le retour aux cédules de messidor qui supprimeront l'hypothèque et la balayeront du sol où elle joue le rôle de livraie.

Quels sont les trois cas où le propriétaire foncier ou celui qui veut le devenir a besoin d'argent? Quand il hérite, et qu'il doit payer en même temps des droits de succession fort lourds ${ }^{1}$ et des soultes de partage ${ }^{2}$; quand il achète un domaine sans pouvoir en payer intégralement le prix ${ }^{3}$; enfin, quand il manque des capitaux suffisants pour exploiter sa terre dans de bonnes conditions, par l'achat de cheptels, d'un train de culture, etc ${ }^{4}$.

Chacune de ces catégories d'emprunt répond à des besoins déterminés el l'on comprend que les garanties qu'exige le créancier soient calculées en raison des risques à courir. Ce

1. En 1898, le droit de mutation par décès sur les immeubles ruraux-a porté sur une valeur de 1 milliard 520 millions; en 1897 , la masse successorale a dépassé 6 milliards $1 / 2$ sur lesquels le Trẻsor a prélevé 186 millions de droits.

2. En 1897, les soultes de partage entre cohéritiers et copropriètaires ont dépassé 221 millions de franes auxquels il faut ajouter, sans compter les frais, 11 millions de droits perçus au profit du Trésor.

3. En 1898, on a pris l'inseription d'office dans les bureaux d'hypothèques (Art. 2108 du Code eivil) pour 293786 prix d'aequisition non payés.

4. Les statistiques de l'Enregistrement ne distinguent pas les baux à cheptel, ni les autres conventions spéciales à l'agriculture. 
qui n'est pas douteux, c'est que les banquiers qui consentent de petits prèts hypothécaires ont tout à perdre à réaliser judiciairement leur gage. Pendant l'année 1890, près de 3000 immeubles d'une valeur inférieure à 500 francs ont été exéculés; les frais ont toujours dépassé le prix obtenu; la moyenne des ventes se soldait par 293 francs de frais déboursés contre 260 franes de prix réalisé. Le prèteur a perdu sa eréance et le débiteur a été dépossédé à vil prix de son patrimoine. Cet état de choses durera tant que les officiers ministériels et les courtiers d'affaires ne vivront que de ce qưils prélèvent sur les procès des autres.

La prospérité des classes rurales est en raison directe des progrès de la culture; la division de la propriété est éminemment favorable à ce progrès, mais celte division ne serait plus qu'mn danger au lieu d'ètre un ferment d'initiative el d'activité si, d'une part, le propriétaire qui n'a point payé son prix d'acquisition demeurait indéfiniment le débiteur de la rente, le fermier à vrai dire du précédent détenteur et si, d'autre part, le nourel exploitant ne pouvait, sans risquer la saisie, c'est-à-dire l'éviction et la ruine, se procurer les capitaux nécessaires pour la mise en train de son entreprise.

Ce ne sont pas les lois mais les mœurs, ce n'est pas la spéculation des banquiers ou la protection toujours pesante de l'État mais la mutualité par les syndicats qui peuvent résoudre le problème.

Syndicats et mulualité, cela veut dire association, mais variétí. Les moyens de guérir un mal dont les symptòmes paraissent semblables mais dont les causes sont différentes doivent être appropriés à l'objet et aussi diversifiés que le sont les caractères du malaise.

Pour s’en convainere, à défaut d'expérienee personnelle, il suffit de lire l'enquête que fit M. H. Baudrillart, de 1881 a $188 \%$, sur les indications de l'Académic des sciences morales et politiques, porr étudier l'état économique et moral des populations rurales de la France. On y voil les différences profondes qui séparent les paysans de Normandie on de Bretagne de ceux du Languedoc ou de la Provence, el 
combien on serait dupe de ses propres utopies si l'on essayait de généraliser, si l'on avait la prétention de tout centraliser, d'assujettir à la mème règle, écrite dans un bureau de Paris, le Lorrain ou le Basque, de condamner au lit de Procuste des exigences qui ne sont ni caprieieuses ni arbitraires mais qui, nées du sol, des tradilions, des habitudes, dégagent d'elles-mèmes la loi qui leur convient.

Rien, par exemple, de plus démonstratif, quand on lui compare celui de la Bourgogne ou du Dauphiné, que le tableau que fait M. Baudrillart de la division du sol en Artois, après 1789.

Lorsque la liberté fut rendue aux achats et aux ventes, dit-il 1 et qu'une grande quantité du sol fut mise à la disposition des acquéreurs, lessor vers la propriété se fit tantòt dans le sens de la petite, qui d'ailleurs existait đéjà, tantôt, et dans des proportions plus étendues, dans le sens de la moyenne, ce qui fut alors dans le reste de la France un fait beaucoup plus rare. Toute une population de moyens propriétaires sembla sortir du sol dans les circonscriptions d'Arras, de Saint-Pol, de Saint-0mer. Le capital était prêt; l'esprit qui le mettait en jeu fit voir qu'il ne l'était pas moins.

Ces derniers mots donnent en quelque sorte la formule de cette étude. Ce n'est pas la division du sol qui vaut par elle-mème; ce qui la rend efficace et productive, quelle que soit sa mesure, c'est le eapital qu'on y met, c'est l'esprit avec lequel on l'exploite.

I. - La grande propriété peut multiplier sa production par la combinaison de la science et du crédit.

Il n'est pas aisé d'évaluer la fortune de la France, les plus compétents ne sont pas d'accord, à quelques milliards près; mais le pays qui paie sans effort, sans poursuiles ni saisies fiscales, sur le simple avis de quelques papiers multicolores, près de cinq milliards par an d'impòts de toutes sortes (à I'État, aux départements et aux communes), sans 
parler des honoraires des officiers ministériels, impôt de surtaxe qui est le plus lourd, n'est pas un pays pauvre.

Est-il plus facile de déterminer quel est le nombre des riches sur une population de plus de 38 millions 1/2 d'habitants (dénombrement de 1893)? Est-il possible, ensuite, d'établir quels sont les détenteurs actuels de la grande propriété territoriale?

M. Levasseur pour les différentes classes de la population, M. de Foville pour le morcellement de la propriété foncière, M. Neymarck pour le fractionnement plus moléculaire encore des valeurs mobilières, ont fourni pour celte étude des matériaux précieux et qui en rendent la synthèse d'autant plus délicate. La France, en résumé, compte très peu de gens riches, beaucoup de gens à l'aise, un nombre infiniment plus grand de gens gênés, surtout dans la classe moyenne et dans celle des fonctionnaires, et une infinité de familles qui ne vivent que de leur travail salarié par autrui.

A ne considérer que les valeurs successorales, comme l'ont fait quelques économistes, on pourrait croire au bien-être universel, et la répartition de ces valeurs par nature de biens marque dans les combinaisons de l'épargne française une méthode bien raisonnée; mais cette fortune se disperse en tant de mains qu'elle apparaît comme une nappe d'eau très étendue mais peu profonde.

En 1898, les mutations par décès en ligne directe seulement ont porté sur un ensemble de capitaux dépassant 3 milliards 469 millions, mais répartis entre 692883 héritiers, ce qui fait ì peine $\$ 000$ francs par tête. Si l'on calcule sur l'ensemble, pour la même année, et qu'au lieu du nombre des héritiers directs, collatéraux et étrangers, qui n'est pas connu, on sarrête au nombre des successions ${ }^{1}$, on trouve que la moyenne de chacune d'elles atteint à peine 10362 francs.

1. Les valeurs successorales de 1898 dépassent 6621 millions de franes, et le nombre des successions déclarées a été de 430810 . (Bulletin de statistique de l'Enregistrement, 1899; p. 181 à 196.) 
Il faut noter que quantilé de très minimes successions, indépendamment de celles des indigents notoires, ne sont pas déclarées et que cetle proportion serait vraisemblablement réduile de moitié si l'on prenait pour l'un des termes de comparaison le nombre des décès (8\%1 986 en 189̋̈) au lieu du nombre des déclarations. Les valeurs successorales de 1898 se répartissent ainsi par nature :

Valeerrs Mobiliėres :

En millions de franes.

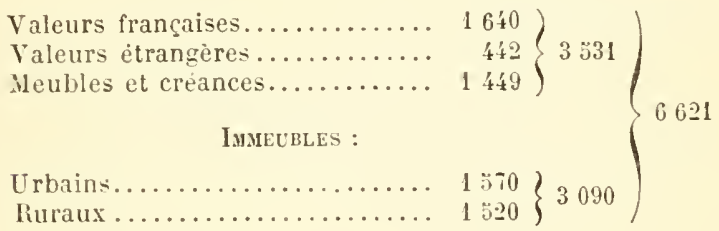

Combien de riches se partagent ces 6 milliards $1 / 2$ qui composent l'annuité successorale de 1898? Combien de grands propriétaires peut-on compter parmi les héritiers du capital rural de 1 milliard $1 / 2$ dont la propriélé se déplace?

A défaut de statistiques précises, on ne peut raisonner que sur des hypothèses.

Le dénombrement de 1891 a compté 907699 personnes vivant exclusivement de leurs revenus, soit à titre de propriétaires d'immeubles ou de valeurs mobilières, soit à titre de pensionnaires ou retrailés. L'ensemble des personnes vivant exclusivement de lenrs revenus, y compris leurs familles et leurs domestiques, comporte 21697000 individus et constitue près de 6 p. 100 seulement du total de la population classée. Les départements dans lesquels on rencontre plus de rentiers (au sens vulgaire du mot) sont ceux de Seine-el-Oise, 13 p. 100, Seine-et-Marne, 10,3 p. 100, AlpesMaritimes, 10 p. 100, Bouches-du-Rhône, 9,8 p. 100, Eureet-Loir, 9,3 p. 100. La Seine ne figure que pour 10 p. 100 ; l'impôt des loyers révèle qu'à Paris, sur 100 familles (en 189.4), 68 ont un budget inférieur à 2400 francs, 23 possèdent un revenu de 2400 à $7 \% 00$ francs et 9 seulement 
dépassent ce dernier chiffre. La statistique suceessorale de 1893, en province, établit que 00 p. 100 du nombre des héritages ne dépassent pas 1000 franes, que sur 300000 successions on en trouve 7000 de plus de 100000 franes et une moyenne de 2000 à 300 atteignant le capital brut d'un million (dettes non déduites).

Le nombre des riches est done très peu considérable, et l'argument tiré de l'énormité des impôts prouve uniquement que la charge est disséminée sur la masse de la nation et non sur une classe de privilégiés.

Quant aux propriétaires des grandes exploitations territotoriales, on ne peut en connaître le nombre que par approximation. L'enquête agricole de 1892 accusait comme suit la répartition de la grande propriété par catégorie de superficie.

Catégories

de superficie.
Nombre

les exploitations.

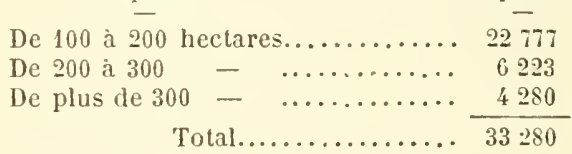

Le relevé, exécuté en 188', par département, des cotes foncières de plus de 100 hectares, donnait le résultat suivant : 49243 cotes d'une contenance totale de 123303782 hectares. La répartition par nombre et par contenance en était fort inégale suivant les régions ${ }^{1}$.

\begin{tabular}{|c|c|c|}
\hline DÉPARTEMENTS & $\begin{array}{l}\text { Nombre de cotes } \\
\text { de plus } \\
\text { de } 100 \text { hectares. }\end{array}$ & $\begin{array}{c}\text { Contenance } \\
\text { moyenne } \\
\text { par cote. }\end{array}$ \\
\hline Allier....... & $\ldots \quad 1336$ & 225 \\
\hline Ilautes- $\Lambda$ lpes. & $2 \nmid 2$ & 1176 \\
\hline Cher........ & 1423 & 257 \\
\hline Landes.... & 1558 & 280 \\
\hline Nièvre...... & 1168 & 257 \\
\hline Var........ & 718 & 328 \\
\hline
\end{tabular}

Étant donné le nombre relativement restreint des détenteurs de la grande propriété, examinons ce qu'est leur

1. Bulletin de statistique du ministère des Finances, octobre 1884. 
influence et ce que pourrait être leur rôle, aux points de vue multiples de la science agricole, de l'outillage, de l'industrie et de la commandite financière.

I. Li Scıexce. - Il ne l'aut pas médire du xrin ${ }^{0}$ siècle, car c'est avant 1789 que de bons Français ont donné l'exemple que réclamaient Vauban, Letrosne, Quesnay, Turgot, et appliqué les méthodes scientifiques au développement de l'industrie agricole. Le progrès matériel se résume dans la manière nouvelle dont une population se loge, s'habille et se nourrit; à ce point de vue, le xvil ${ }^{e}$ siècle ne peut se comparer au xix el celui-ci n'a avancé aussi vite que parce que la France n’a plus dans son sein que des citoyens libres de vendre, d'acheter, de travailler, de cultiver, égaux devant les lois civiles, politiques et fiscales. Mais, avant et depuis 1789, hàtons-nous de le reconnaitre, c'est aux grands propriétaires qu'on doit la lutte contre les préjugés, contre la routine, l'essai des machines, l'acclimatation de races productives, les tentatives coûteuses qui ont permis des expériences, combattu les fléaux, ouvert la voie à la moyenne et à la pelite culture. Nos grands proprićtaires, pareils aux moines défricheurs et initiateurs du moyen âge, ont repris et continué leur rôle, dans la mesure qu'autorisait la différence des temps; ils furent, surtout depuis la Restauration, les pionnicrs de la civilisation agricole.

Les Dailly, les Béhague, les Tracy, les Bugeaud, les Dupin, les Falloux, les Lavergne, et mille autres, à des degrés divers, par des moyens différents, ont consacré leur talent et leur fortune à restaurer en province le goût de la culture el à ramener vers la terre toutes ces énergies sans but qui se consumaient dans l'indolence el la paresse. Et, tout d'abord, leur principal mérile a été de démontrer qu’il fallait être intelligent, hardi, instruil, pour derenir bon agriculteur el que l'industrie agricole concentrait toutes les sciences. En rehaussant dans l'opinion le travail de la culture, trop longtemps considéré comme de pur labeur matériel, en y utilisant depuis la chimic jusqu'à l'économie sociale, ces hommes ont bien mérité du pays car ils ont pris 
le moyen le plus efficace de recruter des successeurs dignes d'eux.

Ce n'est plus la qualité de la personne ou de la terre qui fixe les rangs de la population agricole, c'est l'influence naturelle du capital et du travail. Ce qu'on peut attendre de l'association volontaire de ces deux forces apparaît dans les syndicats agricoles, lypes du vrai syndicat mixte, où le patron coudoie l'ouvrier, où le grand éleveur et le modeste métayer délibérent en commun sur des intérêts qu'ils ne croyaient pas aussi solidarisés par la nature même des choses.

Le jour où la classe éclairée s'occupera réellement de l'agriculture, disait-on il y a trente ans, les progrès ne tarderont pas à se manifester dans toutes les directions; on verra les cultures micux réparties, les montagnes reboisées, les prairies irriguées el la production des céréales restreinte aux localités où elle est arantageuse, tandis qu'on lui substituera, là où elle produit peu ou mal, d'autres cultures ef d'autres industries.

L'enseignement agricole est le corollaire de la direction qu'un grand propriétaire peut utilement donner dans sa région, par ses conseils et par son exemple. Pour la masse des petits propriélaires, des fermiers, des mélayers, la grande propriété devient une école permanente de leçons de choses, un raste champ d'essai, un terrain d'expériences gratuiles. La vue des machines en action, la compréhension de certains procédés, l'étude de l'hygiène rurale, la comparaison des semences, des engrais, les procédés d'exploitation, la propagation des espèces utiles, animales et végétales, tout cela rentre dans le ròle de la grande propriété qui, seule, peut utiliser avec profit les déeouvertes de la science à l'amélioration de l'exploitation rurale et faire participer ses voisins aux avantages de ce qu'elle sail, de ce qu'elle apprend et de ce qu'elle possède ${ }^{~}$.

1. L'école de Le Play pense que le devoir de patronage qui existail à la charge des classes riches, daus les régimes du travail oỉ elles avaient la puissance politique entre les mains, et oủ leur supériorité 
Frédéric Bastiat disait que les forces naturelles sont toujours gratuites el qu'il n'y a d'autre valeur que celle produite, sous n'importe quelle forme, par le travail. A ce titre, la grande propriété restera l'un des facteurs importants de la richesse publique, car elle est seule en état de faire les avanees et les sacrifices d'où dépendent les découvertes et les innorations rémunératrices. La terre doit appartenir à ceux qui l'ont rendue productive, déclarait le droit coutumier; e'est la justification de l'existence de la grande propriélé.

II. L'Outillage. - Le problème agricole, dit-on, consiste dans l'abaissement indéfini des prix de revient.

C'est rrai surtout pour la grande culture où les vastes espaces dont on dispose permettent l'emploi d'un outillage perfectionné et de machines coûteuses que les petits propriétaires ne peuvent se procurer qu'en s'associant et dont la plupart, en dehors des charrues et des balteuses, ne sont pas utilisables par eux en raison de l'exiguïté de leurs domaines ou du morcellement de leurs pièces de terre.

Réduire la main-d'œurre à sa plus simple expression, telle est la préoceupation de ceux qui, dans tous les métiers, peuvent substituer au travail manuel le travail plus régulier et plus expéditif des machines. La lendance à éliminer le plus possible les frais de la main-d'œurre est très accentuée chez les cultivateurs auxquels la science de l'ingénieur permet désormais d'abattre beaucoup d'ouvrage en économisant les forces. On a cru longtemps que la machine agricole resterait le monopole de la grande propriété; mais les inventeurs ont compris que cette clientèle restreinte ne valait pas celle de millions de travailleurs, et ils se sont ingéniés à approprier leurs engins à toutes les mesures de la propriété

sociale était sanctionnée par les lois, n’a point disparı et qu'il doit s'exercer librement sous les régimes qui donnent à l'ouvrier la liberté civile et politique.

La tàche des classes riches, des grands propriétaires fonciers, est rendue plus facile par les immenses progrés qu'a réalisés la science dans ses innombrables applications à tous les détails de l'industrie agricole. 
et du erédit. Aussi est-on frappé, dans les concours agricoles et les expositions, du développement croissant qu’il faut donner aux emplacements réservés pour les machines qu'on destine à faciliter et à abréger le travail des champs.

Aujourd'hui, avec la machinerie moderne, le cultivateur qui ne se tirait autrefois d'affaire qu'avec les bandes d'ouvriers nomades ou trois ou quatre auxiliaires permanents à son service, arrive à exploiter son héritage avec la seule assistance de sa famille, et il l'exploite beaucoup mieux qu'avec l'aide d'un personnel salarié. Là est certainement une des causes de la diminution du nombre des journaliers et de la continuité du mouvement d'émigration des campagnes vers les villes. Mais, qu'adviendrait-il si, ne profitant pas de la science du constructeur, nous revenions à l'ancienne routine et à l'outillage primitif? Nos prix de revient sont encore trop élevés puisque nous fléchissons, malgré nos progrès, sous la concurrence des Américains, un recul ou même un simple arrêt nous forcerait à vendre à perte ou nous réduirait à ne plus vendre. Tout ce que la machine peut faire plus économiquement que l'homme, il faut que la machine le fasse. La grande propriété a montré l'utilité des machines et, peu à peu, son exemple s'est propagé autour d'elle, dans la moyenne propriété d'abord puis mème dans la petite pour tout ce qu'elle a pu approprier à ses besoins ou se proeurer par l'association. Cependant, on trouve encore dans la plupart des départements une certaine obstination à ne rien changer à des procédés qui, théoriquement, doivent èlre rayés lu programme des travaux réservés à la force musculaire. En moins d'une heure, certaines machines exécutent un travail que la main de l'homme n'achìverait pas en plusieurs mois. Lorsque l'outillage agricole aura atteint sa perfection et que le probleme de la transmission de la force motrice sera résolu, il deviendra possible aux familles rurales d'abréger assez la durée des travaux agricoles pour consacrer plusieurs heures par jour à des travaux industriels aujourd'hui centralisés dans les usines.

La question du machinisme el de ses avantages économi- 
ques a été l'objet de vifs débals; elle paraît désormais résolue en faveur du progrès.

M. Levasseur en a récemment fourni les conclusions à propos d'une enquête sur le travail à la main et le travail à la machine, confirmant le théorème que depuis longtemps il a démontré sous le nom de paradoxe économique, et d'où il résulte qu'arec un outillage perfectionné par la science il est possible de livrer le produit à meilleur marché, tout en payant des salaires plus élerés à l'ouvrier et en réservant plus de profit à l'entrepreneur.

Ce qui est rrai de la mécanique industrielle l'est également, réserve faite des nuances que comporte la différence des sujets, de la mécanique agricole. La grande propriété n'a donc sur ce point qu'à continuer son rôle d'initiatrice et d'éducatrice, en vulgarisant clans ses fermes el ses métairies tous les appareils de nature à simplifier le travail manuel et à substituer, par la conduite de machines, petites ou grandes, depuis la moissonneuse à vapeur jusqu’à la baratte articulée, l'intelligence et le raisonnement à la force musculaire. M. Levasseur a démontré que, étant donné que le prix de vente d'un produit se compose du salaire des ouvriers, du prix des matières premières, de l'intérêt des capitaux et du profit de l'entrepreneur, il est possible, grâce à la science, de créer un produit à meilleur marché, avec des matières plus chères, des salaires plus élevés, plus d'intérêt pour le capital et, en définitive, avec un profit plus considérable pour l'entrepreneur. Grâce à l'emploi des machines, l'ouvrier agricole aussi bien que l'ouvrier industriel bénéficie doublement du progrès scientifique, parce qu'il vend son travail plus cher et parce qu'il achète moins cher certains objets de consommation courante. Les chiffres qu'il a produits à l'appui de son affirmation sont des plus suggestifs et des plus curieux.

En ce qui concerne spécialement l'agriculture, si l'on compare la fabrication de 10 charrues par les deux procédés, les charrues faites à la main étant en bois et les charrues faites à la machine étant en fonte, on trouve : à la main 
1180 heures de travail pour le prix de 5̆4 dollars 46, à la machine, 37 heures 28 minutes pour 7 dollars 90. Avee la machine, la division du travail a été plus grande; il a fallu ว̊2 ouvriers et 97 opérations au lieu de 2 ouvriers faisant 11 opérations; cependant, la dépense de temps a été trente et une fois moindre el la dépense en salaires sept fois moindre; on a employé 32 ouvriers au lieu de 2 , et le taux du prix de l'heure à la machine est de 21 cents au lieu de $\ddot{3}$ à la main.

Pour la culture d'un acre de blé, depuis le labour jusqu'au battage et à la mise en sac du grain, on économise 57 heures et 3 dollars; on fabrique 500 livres de fromage en 5 heures an lieu de $7 \%$, pour 830 cents au lieu de 7 dollars 50 . Si l'on objecte la dépense de l'outillage et le capital qu'elle exige, on répond que le prix des machines diminue à mesure qu'elles se perfectionnent, que l'outillage scientifique est un cheptel comme le bétail, et que l'économie réalisée couvre vite l'avance faite. Le produit à bon marché est peut-être inférieur, dira-t-on; mais il n’en résulte pas moins un progrès économique considérable, et surtout un progrès social, parce que l'économie de temps et de dépense musculairc profite à l'intelligence et par suite à la moralité.

III. L'Industrie Agricole. - La grande propriété, dont les ressources el les conditions de volonté et de pouvoir sont si favorables à l'application de la science aux procédés et à l'outillage agricoles, est aussi le terrain sur lequel peuvent le mieux naìtre el se dérelopper les industries variées qui se greffent aujourd'hui sur la culture proprement dite, telle qu'on la comprenait à ses origines primitives. L'homme n'est plus un simple laboureur qui moissonne des récoltes en quelque sorte spontanées; il provoque la terre, il lui impose des productions nouvelles, il en corrige la pauvreté, il en modifie les éléments, mais le paysan isolé ne peut ni inventer ni appliquer ces nouveautés; c'est encore, là comme ailleurs, le grand propriétaire qui ouvrira la voie ct déblaiera la route.

Le droil de douane n'est qu'un expédient dont la protection, en dehors même de ses défauts économiques, est une 
pure illusion. L'unique moyen de résister à la concurrence étrangère et au déclin continu des prix est l'usage scientifique des engrais et la vulgarisation des meilleurs modes de culture. Le perfectionnement de nos méthodes agricoles est une question d'intérêt national.

La grande propriété a provoqué l'établissement des droits protecteurs $^{1}$; son ròle est aujourd'hui d'y suppléer en augmentant le rendement du sol. Fortune oblige, comme autrefois noblesse. Le droit commun, e'est-à-dire la liberté pour tous, tel doit être le mot de ralliement des agriculteurs, quelle que soit l'étendue de leurs domaines; quant au législateur, on ne saurait admettre qu'il pût sacrifier le bien-être du consommateur, c'est-à-dire de la masse de la nation, à l'intérêt de telle ou telle industrie, à la protection de telle ou telle catégorie de producteurs. Le blé comme produit alimentaire intéresse toutes les régions de la France, car la consommation du pain est plus considérable chez nous que dans n’importe quelle partie de l'Europe; le blé comme objet d'échange doit être considéré tout autrement. Les gros fermiers de la Beauce, de la Bric, de la Picardie concentrent à peu près tout le commerce des céréales; la très nombreuse classe des petits propriétaires qui cultivent eux-mêmes et qui consomment personnellement leurs récoltes ou les échangent contre d'autres denrées dans un rayon limité, est hors de cause, et l'on a affirmé qu'en général il lui importe peu que le blé soit cher ou bon marehé, puisqu'clle ne le vend pas et le réserve pour son usage.

Les industries agricoles de la France sont, par rang d'importance, le blé, le vin, l'élevage. La désertion de la cam-

1. Ce fut en 1819 que, pour donner satisfaction aux réclamations des grands propriétaires, on imagina de frapper de droits à l'entrée les blés étrangers et d'établir ce qu'on appela l'échelle mobile, dont le mécanisme, très compliqué, consistait à faire varier le droit de façon à ce que les blés du dehors pussent entrer quand les prix sur les marchés intérieurs s'élevaient et fussent écartés lorsque les prix s'abaissaient.

La loi de 1861 supprima l'échelle mobile et le régime du libre échange n'a été modifiẻ que par des lois rẻcentes qui n'ont pas donné les résultats promis.

LA PROPRIÉTÉ RURALE EN FRANCE. 
pagne, disait-on en 1894', a été aecrue par l'invasion du phylloxéra dans la vallée du Rhône, et par la transformation des eultures en Normandie; tel serait, sur une bien autre échelle, le résultat de l'abandon mème partiel de nos deux grandes cultures, ear si nos agriculteurs cessaient de produire du blé et du vin, dix millions de paysans quitteraient les ehamps et, si les eentres industriels ne pouvaient les utiliser et les retenir, sortiraient de Franee.

Les eéréales ont de tout temps été en France la base de la nourriture de la population, et par conséquent la eulture principale du pays; à mesure que l'aisance se répand, les grains inférieurs sont remplaeés par le froment. Le rendement du blé à thectare a fait de sérieux progrès grâce aux mille formes que prend aujourd'hui l'enseignement agricole. La moyenne a passé de 10 hectolitres en 1830 à 14 en 1881 , à 15 en 1886, à 18 en 1897. Supérieur au rendement des pays jeunes, quoique le produit des défrichements dans les États de l'Amérique du Nord, la République Argentine, l'Inde, la Russie, ne cesse de eroître, il est bien inférieur à ceux des pays d'Europe où la culture est perfectionnée : 21 hectolitres et plus pour la Belgique, la Hollande, le Danemark et jusqu'à 29 pour l'Angleterre. On eite exceptionnellement en France, dans certaines régions à culture intensive, et surtout dans certaines exploitations où l'on a fait emploi, à grands frais, des méthodes fertilisantes, des rendements de $2 \%$ et de 30 heclolitres, mais on peut les assimiler, eomme pour quelques résultats industriels étonnants mais trop eoûteux, à des expériences de laboratoire ${ }^{2}$, hors de la portée de la pratique eourante.

Si le rendement en blé du sol français atteignait seulement le rendement belge, il en résulterait un prodigicux accrois-

1. Congrès des Syndicats agricoles à Lyon, en août 1894.

2. En 1895, M. Grandeau obtenait, dans ses essais au pare des Princes, une récolte moyenne de 45 hectolitres, presque le double de la récolte moyenne de la région du Nord, mais il se défendait de toute illusion en déclarant que de pareils résultats ne pouvaient être atteints qu'à titre exceptionnel, même dans la grande culture. 
sement de richesse dont l'ineidence économique ne se peut calculer. Les résultats immédiats seraient un gros bénéfice pour l'agriculteur puisque, avec des labours moins étendus et de moindres frais de main-d'œuvre, il recerrait, à prix égal, par hectolitre, une somme plus forte de 20 à 40 p. 100 que celle qu'il obtient aujourd'hui. L'étendue emblavée de nos terres, gràce à la culture intensive et au meilleur choix des semences, pourrait ètre réduite, soit au profit de l'élevage pour la grande propriété, soit au profit de l'industrie maraìchère pour la petite, et un mourement de reflux se produirait inévitablement des villes dans les campagnes, surtont à la suite des crises industrielles dont il est aisé de préroir dès à présent la future acuité. Ce n'est pas seulement pour le froment, que les rendements pourraient ètre élevés dans une forte proportion, mais pour toutes les autres cultures : avoine, pommes de terres, raves, maïs, lentilles, légumes, plantes industrielles, fruits et primeurs.

La consommation générale en blé, viande, légumes, s'accroîtrait d'autant au grand profit des qualités de vigueur et d'endurance de la race, que débilitent chaque jour davantage l'alcool, le tabac et la tuberculose, et nous économiserions chaque année le milliard que coûte l'importation des denrées alimentaires.

La supériorité de la France pour la production des vins est incontestable; nul pays au monde n'en donne de meilleurs ni plus abondamment. La culture de la vigne, depuis la reconstitution des vignobles dans le Midi, le Sud-Est et l'Ouest, fournit de nouveau des profits abondants; mais ce n'est pas contre la concurrence étrangère que nos viticulteurs auraient le plus besoin d'ètre protégés, c'est principalement contre le mélange et la falsification des spiritueux de toute nature qui discréditent les producteurs et avilissent les prix, en ruinant la santé publique.

D'autres industries précieuses ont disparu, comme la culture de la garance qui fit la fortune du Vaucluse et s'éranouit par la concurrence des produits chimiques tirés de la houille, l'élevage des vers à soie qui enrichissait la vallée du Rhòne 
et depuis 180̋3, malgré les découvertes de Pasteur, a perdu $7 \ddot{p}$ p. 100, elc. On les a remplacées; le travail de l'homme possède une souplesse incomparable et la science, pareille à la lance d'Achille, sail guérir les blessures qu'elle fait.

L'élevage paraît ètre le monopole naturel de la grande propriété, possédant de vastes espaces, qui peut clore de grandes étendues de prés, capter les eaux, diriger les irrigations, repeupler les déboisements. Le bétail n'a cessé, depuis trente années surlout, d'augmenter en France; les bœufs, les moutons, les porcs, ont crù en nombre et en qualité; malheureusement, la race chevaline, sous la coalition de diverses causes, a perdu du terrain, el ce n'est pas l'expédient immoral du Pari Mutuel qui aidera à son relèvement. La viande, la laine et les peaux, indépendamment du travail des transports et du labourage, sont les produits immédiats de l'élerage. Les grands propriétaires ont beaucoup aidé à l'augmentation des cheptels de culture et surtout à l'amélioration des races précoces et rustiques qui alimentent nos boucheries.

L'espace est une des conditions nécessaires de l'industrie pastorale; cependant, si la petite propriété est moins propre que la grande à l'élevage du bétail, il ne faut pas supposer qu'elle soit privée de celte ressource. Sur le sol le plus morcelé, remarque 11. de Foville, le bétail trouve sa place. Il y est même plus nécessaire qu'ailleurs, car sa présence est l'une des causes de l'extrême fécondité relative de la petite culture; si le bétail prend sa large part des récoltes, ce n'est qu'une restitution, et les moindres champs sont souvent ceux qui recoivent le plus de fumier. L'élevage du porc est la ressource des plus humbles ménages, et l'utilisation pour la pâture raisonnée des milliers d'hectares que la grande propriété laisse sans emploi procurerait aux ouvriers agricoles de la viande à bon marché, meilleure pour l'hygriène des familles paysannes que les importations des viandles et des poissons salés ou fumés.

Le mouton peut être considéré comme l'élevage de la moyenne propriété, tandis que le porc est celui de la très 
petite et le cheval et le bœuf' les apanages de la grande. On faisait remarquer dès $1833^{1}$ que les fermiers anglais en avaient tiré un merveilleux parti, même dans les exploitations d'une étendue médiocre, sachant que, de tous les animaux, le mouton est plus facile à nourrir, celui qui tire le meilleur parti des aliments qu'il consomme, et en mème temps celui qui donne, pour entretenir la fertilité de la terre, le fumier le plus actif et le plus chaud. Il y a, dans la Grande-Bretagne, d'immenses fermes qui n'ont pas d'autre bétail, et, tandis que nos agriculteurs, petits et grands, divisent leur attention et se laissent distraire par la variété de leur's essais, l'élève du mouton est apprécié par nos voisins d'outre Manche comme la plus rémunératrice des industries agricoles. Depuis le moyen âge, le chancelier d'Angleterre préside la Chambre des Lords assis sur un sac de laine, symbole pittoresque de cette étroite association de l'agriculture et du commerce qui fit la richesse et la fierté de la nation.

La grande propriété est également mieux préparée que les autres à prendre l'initiative des industries accessoires qui dérivent de l'exploitation du sol par la culture ou de I'élevage, et dont les principales sont les laiteries, les fromag eries, les distilleries, les scieries, etc. Et c'est à ce point de vue que se révèlent avec le plus de force les inconvénients de l'absentéisme et les avantages d'une classe moyenne de propriétaires, instruits et aisés, venant habiter leur domaine et y dépenser leur revenu.

Que de facilités locales immédiates apporte dans une commune la résidence habituelle d'un propriétaire foncier riche ou influent! Il veut arriver facilement chez lui et recevoir régulièrement sa correspondance, il fera en sorte par ses relations et ses subventions en argent qu'il s'établisse de bonnes routes, un service rapide de la poste, un bureau de télégraphe au besoin; voilà le canton sorti de son isolement et relié au reste du monde. Il fait venir des outils et

1. Lẻonce de Lavergne, L'économie rurale en Angleterre, 1853. 
des machines de la meilleure marque; ils sont imités et se répandent autour de lui. Pour les entretenir, il faut un charron habile; les autres besoins du château et du domaine exigent la présence d'artisans, d'ouvriers de métier; le voìsinage de ces ouvriers crée des besoins qu'on ignorail, dont on ne s'apercevail pas faute de pouvoir les salisfaire. Le village se déreloppe, le bien-être s'y accuse d'abord sous ses formes élémentaires, l'hygiène, la propreté, le confort. Le goût du luxe utile, celui de l'intérieur, que la mère de famille crée à peu de frais, gagne de proche en proche et le village est transformé. Le paysan, en effet, imite plus volontier's ce que fera l'un de ses pareils que les innovations d'un homme riche, dont les habiludes ne sont pas les siennes et qui peut dépenser sans compter.

IT. Les Sociétés fixaxcières. - La question d'argent, de l'aris unanime des publicistes, domine la question agraire.

La tendance à la concentration qui se manifeste dans toutes les branches de l'industrie et du commerce est en voie de pénétrer dans l'agriculture el, si elle dépassait une certaine limite, ce pourrait être un grand malheur.

La grande propriété, si elle veut remplir son rôle ou seulement mettre en valeur le capital terrien dont elle dispose, capital relativement improductif si on ne l'alimente pas, a besoin, comme toute industrie, d'un fonds de ronlement, d'un capital additionnel incessamment renouvelé. On a institué dans ce but des sociétés financières qui généralement ont avorté, entrainant dans leur ruine les spéculateurs qui leur avaient confié leurs fonds; celles qui ont résisté ne l'ont pu que par l'intervention directe de l'État et l'oetroi de privilegres exeeptionnels.

Depuis sa création en 1853, le Crédit Foncier a réalisé 4 milliards 1/2 de prêts fonciers, particulièrement sur des immeubles urbains, el près de 3 milliards de prèts communaux. Malheureusement, la plupart de ces prèts à longue échéance, surtout en ce qui concerne les propriétés rurales, devenus trop onéreux par le poids des annuités, ont provoqué de très nombreux remboursements par anticipa- 
tion ', effectués par la vente forcée des immeubles donnés en garantie ou par un délaissement qui a constitué au profit de l'établissement un domaine de mainmorte dont l'accroissement devient un embarras économique ${ }^{2}$. En raison des conditions particulières dans lesquclles le Crédit Foncier exerce son monopole, on a pu dire, sans être démenti, que le Crédit Foncier ne prête qu'aux riches et qu'il achève de les ruiner; c'est d'ailleurs, dans l'état actuel de notre législation foncière, le résultat presque toujours inévitable de l'emprunt hypothécaire.

D’autres sociétés se sont formées avec le dessein de participer d'une manière effective à l'exploitation de la grande propriété, non plus par un simple prêt sur hypothèque, non pas mème par une commandite, mais par une ingérence active et personnelle dans la conduite de la culture et des industries qui en dérivent.

Partout, en effet, sous l'influence de la spéculation à outrance qui tend à remplacer le travail par le jeu, l'épargne laborieuse par les gains faciles, l'action collective cherche à se substituer à l'action individuelle, et l'anonymal à la responsabilité. Partout le capital tend à se grouper pour acquérir une force plus grande, et les groupements de capitaux domnent nécessairement lieu à des émissions de papier et, par roic de conséquence, à l'agio.

On a vu, dès 1889, des capitalistes se réunir pour posséder en commun des lots d'immeubles au lieu de rester isolés et de posséder individuellement un seul immeuble. Les compagnies immobilières se sont multipliées dans les villes, toutes n'ont pas eu un égal succès. Ce système d'exploitation s'est étendu à la province et s'est exercé sur les propriétés rurales. Les arguments des promoteurs de ces entreprises ne sont ni variés ni péremptoires ${ }^{3}$.

1. De 113 millions en 1890 , les remboursements anticipés se sont élevés à 134 millions $1 / 2$ en 1895 , pour retomber à 91 millions en 1899 .

2. Rapport aux actionnaires du Crédit Foncier dans l'assemblée gẻnérale du 11 avril 1900 .

3. Avoir une part dans un groupe d'immeubles est une situation moins aléatoire que celle que l'on se crée en opérant sur un immeuble unique 
Tout cela serait acceptable si l'on trouvait des formules offrant toutes garanties contre l'esprit de spéculation; mais le problème est encore à résoudre. Les pratiques de l'association immobilière ne sont pas encore entrées dans nos mœurs, malgré les valeurs à lots, el l'image de cette propriété collective est trop en contradiction avec le principe de l'héritage, avee la conception de son unité, de sa fixité.

L'idée de l'exploitation agricole sous la forme industrielle ne peut être pratique que dans des cas très rares, tels qu'on en rencontre dans les départements du Nord où des fabricants de sucre et des raffineurs, pour se passer d'intermédiaires, entreprennent dans de grandes proportions la culture des betteraves; encore ces associations constituent-elles des groupes isolés en commandite plutôt que des sociétés anonymes ${ }^{1}$.

L'utopie qui suppose que le succès des entreprises agricoles réside uniquement dans la puissance des capitaux engagés ${ }^{2}$ et ne roil dans l'unité de direction, la prévoyance, la patience et le travail, que des éléments accessoires, rappelle, par l'étroitesse du raisonnement, la théorie qui fait de l'influence des changes la cause unique des crises économiques et particulièrement de ce qu'on appelle la crise agricole. Particulariser n'est pas simplifier.

Certes, la question des prix joue le plus grand rôle dans la plupart des questions économiques, mais il n'y faut pas tout ramener, et faire dépendre le progrès agricole et le

qui peut causer des mécomptes. Avoir une participation dans une vaste exploitation agricole, cela ne vaut-il pas mieux que d'exploiter directement une petite propriété, et n'en acquiert-on pas plus de loisir et plus de sẻcurité?

1. En 1836, de grands banquiers mirent en commandite la terre de Beauny-Saint-Hippolyte, dans le Midi, et répartirent ses 3550 arpents en 31 fermes. Le capital social se divisait en 4000 actions de 4000 franes. On s'aperçut que pour payer seulement l'intérêt de ce capital il fallait industrialiser l'exploitation pour profiter du bénéfice des interınédiaires qui font metier de transformer les produits; on bâtit des moulins, des fẻculeries, des distilleries, des fours à chaux, etc. On liquida au bout de trois ans d'essais.

2. Loi sur les sociétés du 24 juillet 1867. 
sort de la propriété rurale de l'issue de la querelle entre le métal blanc et le métal jaune. C'est pure fantaisie.

La mise en société des grands domaines n'offre que des inconvénients sans compensation et serait un péril économique et social de plus si elle se généralisait. C'est une erreur de dire qu'en divisant la propriété territoriale en actions, et surtoul en coupures accessibles aux plus petites épargnes, comme les 1/ö de titres du Crédit Foncier, on multiplierait avec profit le nombre des propriétaires fonciers, car la division de la propriété n'entraînerait plus Je moreellement du sol ${ }^{1}$. Ce système est aussi utopique qu'est peu sérieuse l'opinion que rien ne serait changé à I'harmonie de l'exploilation, soit que les titres fussent dispersés entre des milliers de détenteurs, soit qu’ils fussent réunis dans trois ou quatre gros portefeuilles. Ce n'est pas la grande propriété qu'il faut diviser et réduire à d'infiniment petits lots en papier; c'est la pelite qu'il est utile d'associer et de syndiquer, non pas pour l'absorber dans une unité fictive, mais pour la fortifier par la mutualité sans lui faire perdre sa personnalité ni sa responsabilité.

Ce sont les financiers et les spéculateurs qui ont discrédité le crédit agricole et le crédit foncier en en faisant la matière de leurs combinaisons, presque toujours trop ingénieuses, et visant moins le bien des agriculteurs que la poursuite des primes et des dividendes. C'est le vice originel des théories exposées et des tentatives faites. Il en coûterait trop cher d'exproprier la grande propriété au profit d'un syndicat de banquiers, on a imaginé de syndiquer les petits propriétaires et de constituer par l'agglomération de leurs parcelles une autre grande propriété, rivale de la première; les promoteurs de ces banques d'accaparement appellent ironiquement nos paysans les forçats du morcellement.

A cette association des petits sous la main des financiers,

1. La doctrine saint-simonienne qui mit ces idées dans la circulation de 1834 à 1840 , a pour émules les différentes sectes socialistes, communistes, collectivistes, etc., qui rêvent de nationaliser le sol et la production en supprimant, de fait ou fictivement, la propriété individuelle. 
nous opposons l'association des petits entre eux, dans la plénitude de leur liberté, sans autre lien que celui de la mutualité, sans autre pression que celle de l'intérêt commun, abstraction faite de toute idée de lucre au profit de bailleurs de fonds inconnus.

Les forces anonymes sont loujours suspectes; la saine politique sociale, comme la vraie philosophie, a son plus ferme fondement dans la compréhension complète de tous les éléments de la vie, et de tous ces éléments, le plus vivace est le sentiment du Moi.

\section{II. - La moyenne propriété végète parce qu'elle} souffre d'institutions mal appropriées à ses besoins.

La France a toujours été et demeure encore, malgré l'envahissement des entreprises industrielles, une nation agricole. Rien ne prouve mieux la richesse de son territoire, la solidité de son fonds social, que l’aceroissement indéfini des valeurs mobilières qui n'ont d'autre gage, en somme, que la propriété territoriale et qui ne sont appréciées, qui n’ont de prix d'échange, que parce qu'clles représentent de la patience, du travail, et la continuité de l'eflort ${ }^{1}$.

C'est aux races énergiques et fécondes de ses campagnes qu'elle a demandé de tout temps ses défenseurs et ses colons. C'est le sang pur et jeune du paysan qui, par l'émigration régulière des foyers ruraux vers les villes, est venu sans cesse renouveler la vitalité et l'énergie des populations urbaines.

Si cette source, jusqu'ici inépuisable, de la vie nationale venait à se tarir, ce serait un désastre plus grand que toutes les défaites. Il faut que les villages se repeuplent, il convient qu'ils ne cessent pas d'alimenter les villes qui s'étiolent et s’appauvrissent par les fléaux qui désolent les familles

1. Voir les chiffres publiés par le Journal de la Société de statistique de Paris, décembre 1890, p. 416. 
ouvrières ${ }^{1}$, mais à la condition de ne point se vider euxmèmes et d'y fixer, par un universel retour aux champs, les souches sociales.

On le peut par la famille, en reconstituant le patrimoine de famille, en revisant une législation incohérente parce qu'elle a été maintes fois remaniée, au cours de ce siècle, arec des vues différentes. La propriété constitue la base sociale par excellence, la division de la propriété traduit par ses évolutions les exigenees variables de chaque époque historique, e’est la moyenne propriété, trop négligée entre les infatuations de la grande et les légilimes ambitions de la petite, qui dans l'avenir servira de frein, de régulateur el d'excitant.

I. L'Héritage foxcier. - Qu'on veuille s'en défendre ou non, qu'on ferme les yeux pour ne pas la voir, il y a une question agraire. En France, elle se résout lentement, pacifiquement, par l'effel même de la division de la proprićté; ce n'est qu'une des faces de la question sociale qui partout grossit, s'étend et s'avance sur nous avec une formidable rapidilé, e'est l'éternelle lutte des riches et des pauvres, de ceux qui possèdent el de ceux qui ne possèdent point. Elle se traduit dans l'industrie par le conflit des salaires, les grèves, l'arrèt du travail qui ruine à la fois ouvriers et patrons; elle se manifeste chez les travailleurs agricoles, quand ils ne parviennent pas à devenir propriétaires, par la désertion. Le vide se fait daus les villages, et le nombre croissant des prolétaires des centres industriels y réduit d'autant le salaire.

A Rome, aux temps des Gracques, en Prusse de 1820 à $18 \% 0$, en Irlande et en Sicile, sous nos yeux, et avec des incidents tragiques, c'est la terre qui est en jeu; el, qui ne peut en avoir, tue ou émigre. En France, depuis 1789, il n'est pas besoin de recourir à la violence; le jeu des lois et des mœurs permel l'accès des plus pauvres à la propriété,

1. Les trois fléaux de la classe outrière, par Mgr Turinaz, évêque de Nancy, 1899. 
pourvu qu'ils soient laborieux et économes, et l'économiste n'a qu'à rechercher les moyens de rendre l'évolution plus rapide el ses résultats plus permanents.

Pour l'ensemble du pays, la propriété immobilière urbaine est à la propriété immobilière rurale comme $47,5 \%$ est à 52,45 .

Limitant mon étude à la propriété rurale, je vois que la proportion de la propriété agricole par rapport à la valeur totale de la propriété immobilière de chaque département est de 87,73 p. 100 dans la Lozère, de 87,30 p. 100 dans les Côtes-du-Nord, de 89,03 dans le Cantal, et seulement de 26,70 p. 100 dans le Rhòne, de 2,39 p. 100 dans la Seine.

Sur cette masse foncière (urbaine et rurale) évaluée 107247 millions 4 par les enquètes de l'Enregistrement, à la date de 1898, la spéculation on le crédit, comme on voudra, a greffé ou gagé, suivant le point de vue, des valeurs de papier que M. Neymarck estime à 123 milliards ${ }^{1}$.

Il ne serait pas impossible d'évaluer la part de la propriété rurale dans cet ensemble de valeurs représentatives dont le fonds primitif est la terre, mise en valeur par toutes les formes de l'activité humaine, ce qui en a décuplé, et parfois centuplé, le prix intrinsèque; mais ce travail, qui exigerait la précision patiente d'un actuaire et les larges vues d'un économiste, n’a pas été fait. Ces données générales suffisent pour apprécier le rôle de l'héritage foncier dans la division de la propriété, c'est-à-dire dans l'état social d'un peuple.

La civilisation, à mesure qu'elle se complique et se raffine, nous ramène aux origines primitives de l'humanité. Là est l'explication de l'émotion économique et philosophique qui secoue la vieille Europe à la fin du $\mathrm{xix}^{\mathrm{e}}$ siècle. Le mouvement social qui tend à dégager la propriété du sol des liens publics ou privés qui en gênent la liberté, et qui rêve d'assurer au propriétaire foncier, petil ou grand, l'absolue sécu-

1. Voir les publications du bureau dı Congrès international des valeurs mobilières de 1900 , tome $I^{\text {or }}$ de la Statistique, page 5 , note 2. Le revenu des valeurs mobilières francaises taxées par l'Enregistrement, en 189?, pour l'impôt sur le revenu, a dépassé 1 nilliard 658 millions. (Bulletin gris, 1901, page 200.) 
rité de sa possession, ne date pas de 1789. II a des racines profondes clans notre histoire et, si la formule de revendication est devenue plus nette, si l'on précise, cela prouve uniquement que la secousse produite dans le monde moral par la Révolution française a eu sur les intérêts matériels une répercussion immédiate.

La terre, pour peu qu'on interroge l'histoire du monde, a toujours été le signe de la puissance, cn même temps que le but de l'épargne. Ces deux idées ne sont-elles pas, en effet, solidaires l'une de l'autre? On désire ce qui domine, et le groupe des miséreux cherche fatalement à s'introduire par l'adresse ou la force dans le groupe des riches. Comme on le remarquait récemment dans l'une des études les plus documentées qui aient été faites, gràce à l'appoint de nombreux chercheur's, sur' la condition de la terreà travers les âges, c'est moins la valeur intrinsèque du sol qu'on recherche que son prix relatif et la puissance apparente qu'apporte sa possession.

Il y a dans notre pays certains territoires, parmi les moins productifs, qui ont trois fois passé de l'état brut à l'état civilisé el de l'état civilisé à l'état brut; que l'homme a successivement pris, quittés et repris, qu'il s'est disputés avec acharnement pour les abandonner plus tard avec insouciance.

Aujourd'hui, la terre ne classant plus l'individu, l'homme ne cherche en elle que le produit net, le revenu, puisque c'est uniquement l'argent et non la condition qui donne les moyens d'agir, c'est-à-dire qui constitue la suprématie de l'homme riche sur celui qui n'a rien.

Le domaine rural, pour être cultivé avec profit, doit être stable dans les mains de celui qui l'exploite, il doit devenir sa propriété, son bien, son héritage. Il laut la paix du présent et la sécurité de l'avenir pour continuer avec lenteur et méthode les travaux ingrats qui préparent la production, pour relever les récoltes, pour en tirer profit par l'échange.

Il faut la certitude d'une possession durable pour entrainer l'homme à ces travaux de longue haleine dont les résultats lointains sont exposés à mille chances mauvaises; il faut un intérêt personnel de gain, d'avenir, d'épargne, pour que l'ou- 
vrier agricole se passionne pour son œurre et y dépense les efforts nécessaires.

Si le domaine rural ne se reconstitue pas, quelle que soit la mesure de son étendue, il n'y aura bientôt plus, dans notre société contemporaine, que des individus sans colı́sion, des parcelles sans groupement. Arec la division actuelle du sol, et dans les conditions économiques où s'ouvre le $\mathrm{xx}^{\mathrm{e}}$ siècle, la moyenne propriété, c'est-à-dire le point intermédiaire où la fortune acquise prend un caractère relatif de fixité et permet ce commencement de loisir qui suppose la possibilité de s'instruire, semble un terrain à souhait pour immobiliser dans l'action des aptitudes et des énergies qui se perdent faute d'objet. Le còté moral de la vie rurale est éminemment favorable à ce retour aux saines doctrines; la fixité des contrats, la stabilité de la vie sont les choses auxquelles le paysan est le plus attaché.

Sans tourner à lidylle, on ne peut nier que les conditions habituelles de l'existence, à la campagne, ne soient meilleures, plus salubres, plus saines qu'à la ville. L'air pur, la vie à découvert, l'activité des champs évitent aux paysans les effets d'une fermentation que développent les occupations sédentaires, les lieux renfermés, le contact des voisins, les mauraises lectures. Si, au lieu de pousser machinalement leurs enfants, les fils vers les fonctions publiques où ils deviennent presque tous serviles et routiniers, les filles vers la mode des diplômes et les éléments des arts dit d'agrément, les pères et les mères de famille les attiraient vers la vie rurale, les intéressaient par un enseignement professionnel approprié, ils rivaliseraient avec ces fermiers anglais, ces petits propriétaires de Belgique, de Hollande, de Suisse, chez qui l'on trouve tant de confort, de bonne humeur et d'instruction, s'accommodant à une parfaite entente du métier et avec le souci du labeur personnel.

En 1764, La Chalotais disait déjà comme nous:

II n'y a pas assezde laboureurs dans ce pays où il y a des terres en friches. Trop d'éerivains, trop d'académies, trop de collèges. II n'y a jamais eu tant d'étudiants dans un royaume où toutle monde se plaint de la dépopulation. 
Le président Rolland ajoutait, en citant les doléances de la municipalité de Thouars:

Une ambition mal entendue des pères dle famille enlève à l'agriculture et au commerce d'excellents laboureurs et des maitres ouvriers.

La lourde faute sociale qu'on a commise en provoquant au déclassement, en ourrant trop largement un enseignement secondaire mal raisonné ct qui reste en dehors des besoins réguliers et pratiques du pays, a été nettement et unanimement constatée par l'enquète que vient de faire la Chambre des députés à propos de la réorganisation de l'enseignement secondaire. Les Facultés de droit et de médecine sont tcllement engorgées ${ }^{1}$, dit un professeur, que la multitude des arocats et des médecins sans cmploi est devenue un réritable danger social ${ }^{2}$.

Nous n'avons pas seulement créé un prolétariat de bacheliers, selon l'expression de M. A. Leroy-Beaulieu, nous avons fait pis, remarquent M. Buisson et M. Hanotaux. Les déclassés ne sont pas les bacheliers, ce sont ceux qui, ayant prétendu au baccalauréat, ayant passé toute leur enfance et toute leur jeunesse à s'y préparer, finalement ne s'y présentent pas ou n'y réussissent pas; c'est de ceux-là que le nombre est énorme et inquiétant; c'est ce nombre-là qu'il faut absolument diminuer. La vanité des pères et des mères des classes populaires et bourgeoises, voulant donner à leurs fils l'instruction la plus élevée, vanité excusable mais dangereuse, est la cause unique du nombre croissant des ratés.

Que ne leur donne-t-on l'instruction professionnelle et l'éducation rurale!

Supposez ces enfants de fonctionnaires, de commerçants, d'industriels, de cultivateurs, de propriétaires, parmi ceux qui ne veulent pas suivre la carrière de leur père, habitués dès l'enfance, au lieu de s'étioler dans ces collèges et ces

1. L'Annuaire de la France accuse pour 1897 un total de 26819 étudiants, dont 8147 dans les Facultés de droit, 11638 dans celles de médecine.

2. Enquête, 1899, tome I, p. 214; 11, 581, etc. 
lycées où l'on n'apprend rien ${ }^{1}$, à vivre aux champs, à en prendre le goût, à en saisir le charme, à en comprendre l'avenir. Qu'ils vivent sur le domaine familial, qu'ils l'exploitent, y dépensant leurs revenus, y utilisant leurs aptitudes et leurs initiatives, profitant de leur instruction et de leur expérience pour enseigner autour d'eux les bonnes méthodes et les idées saines. Ne serait-ce pas là de précieuses recrues pour la pratique de la science agricole, et tous ces forains déclassés jusqu'ici ne remplaceraient-ils pas, au profit commun, les fermiers et les métayers auxquels ils confient actuellement le soin de gérer leurs biens?

La réforme de l'enseignement serait le point de départ rationnel du relèvement de l'agriculture; au lieu de programmes uniformes surchargés de banalités, ne conviendrait-il pas de diversifier l'enseignement suivant les aptitudes et la condition des élèves, suivant l'avenir auquel ces aptitudes et cette condition les destinent, et aussi suivant les régions? L'essai en a été fait avec un plein succès ${ }^{2}$.

Ce qui manque le plus à nos campagnes, ce ne sont ni les capitaux, ni mème les bras, c'est la science, non pas celle des livres mais celle de la pratique et du savoir-faire. Celle qui apprendrait aux paysans à ne pas laisser perdre leurs fumiers, à utiliser les eaux, à diriger leurs efforts vers la production du bétail, à convertir en pàturages et en bois les terres incultes, à trier les semences, à tirer parti de toutes les ressources qu'ils négligent faute d'avoir appris à les utiliser. C'est un progrès que d'enseigner dans les écoles primaires les notions élémentaires de l'agriculture, mais c'est insuffisant. Ce n'est pas seulement sur les paysans et sur les

1. Élevé par l'Université, je sais par mon expérience personnelle et par ce que je vois aujourd'hui qu'avec des professeurs très distingués (trop, peut-être) il n'y a pas dans chaque classe 10 élèves sur 30 dont on s'oceupe utilement.

2. L'enquèle de 1899 révèle que, d'après les statistiques de 30 établissements où les Frères des Eeoles chrétiennes distribuent l'enseignement secondaire en l'approprianl aux lesoins des diverses régions, les carrières choisies se répartissent ainsi : commerce, 35 p. 100; agriculture, 33 p. 100 ; industrie, 15 p. 100 ; administration, 7 p. 100; marine, 5 p. 100 ; études, 5 p. 100. 
ouvriers agricoles qu'il faut agir, c'est aussi et surtout sur les propriétaires.

Il est temps que les détenteurs du sol se mettent en mesure de le cultiver eux-mêmes ou tout au moins d'intervenir personnellement dans la direction de l'exploitation.

L'habitude de se désintéresser de leurs propriétés autrement que pour en toucher les fermages les a éloignés de la terre; il faut quils s'en rapprochent et qu'ils l'étudient. Ceux qui n'auront pas le courage de violenter leur insouciance en s'occupant directement de la gestion de leurs domaines, comme tant d'hommes éminents leur en donnent l'exemple ${ }^{1}$, iront en s'appauvissant jusqu'au jour où ils seront forcés de vendre leurs biens à ceux qui sauront les mettre en valeur. Quel appoint considérable apporteraient à la fortune de la France les parents qui, au lieu de peupler les bureaux de leurs enfants, enverraient ceux-ci apprendre la vie en administrant leur futur héritage. C'est pour les jeunes gens de cette classe qu'ont été créées les Écoles pratiques de Grignon, de Grand-Jouan, de Montpellier, et l'Institut agronomique de Paris.

Le jour où la classe éclairée s'occupera avec méthode et persévérance de l'agriculture, les progrès ne tarderont pas à se manifester dans toutes les directions; c'est alors que les sociétés départementales d'agriculture pour la théorie, les syndicats agricoles pour la praticue, les groupements de cultivateurs pour des cas particuliers de desséchements, d'irrigations, de remembrements, prendraient un rôle prépondérant et exerceraient une action salutaire et vivifiante sur l'existence rurale.

Les propriétaires de la moyenne propriété sont naturellement désignés pour provoquer et diriger ce mouvement.

Et comme résultats, du bas en haut de l'échelle administrative et de la hiérarchie gouvernementale, depuis le maire de village jusqu'au ministre, n'y aurait-il pas un avantage

1. Il suffit de consulter les listes de la Société des Agriculteurs de France et de la Société nationale d'agriculture et de remarquer la constitution de leurs bureaux et comités. 
social de premier ordre, dans un pays agricole et laborieux comme est la France, à ètre gouverné surtout par les propriétaires fonciers au lieu de l'être presque exclusivement par des déracinés?

II. Le Fermage. - Les forains, détenteurs de plusieurs millions d'hectares dont ils ne se préoceupent pas et que certains n'ont même jamais vus, exploitent leurs terres par intermédiaires, généralement sous la forme du fermage, plus rarement, à cause des difficultés de la surveillance, sous la forme du métayage.

Le fermage est le mode d'exploitation le plus répandu dans le nord et le nord-ouest de la France; ainsi, dans la Somme, il y a 27430 fermiers contre seulement 3516 propriétaires faisant valoir personnellement leur bien et 901 métayers; dans la Côte-d'Or, le seul département des autres régions où le fermage prédomine, l'écart n'est pas aussi considérable; 16434 fermes contre 11454 faire-valoir.

Si l'on considère la France daus son ensemble, on y apercoit deux îlots de mélayage : les Landes au sud, l'Allier au centre; les pays à fermiers forment une masse compacte et dont la Côte-d'Or n'est séparée que par l'Aube où les fermiers sont aux exploilants directs comme 1 est à 3 .

Aux conditions que l'on exige aujourd'hui d'un bon fermier, il serait infiniment préférable pour les propriétaires de confier cette mission à leurs enfants, quand ils le peuvent.

Tandis qu'autrefois la culture de la terre était abandonnée à des paysans grossiers, ignorants, dépourvus de ressources personnelles, il faut maintenant, pour exploiter une ferme d'une certaine étendue, disposer d'un capilal relalivement considérable et avoir une instruclion qui suppose des études el un esprit ouvert. Mais, ne trailant ici que le problème de la moyenne culture el me conformant à la définition officielle, d'ailleurs très discutable (de 10 à 10 hectares), j'écarle les grosses fermes dont les conditions sont lout autres el où il faut des chefs d'exploitalion riches et instruits, qui sont quelquefois, par leur mérite el leur condilion sociale antérieure ou présente, les égaux du propriétaire. 
La siluation du petit fermier, de celui qui exploite les domaines de la moyenne propriété est plus que médiocre, malgré les efforts récents lentés par le législateur en sa faveur. Le fermier, sans action légale sur ses ouvriers qui peuvent le ruiner par une grève subite en pleine moisson ou en pleine rendange, est à la merci du propriétaire par la courte durée des baux ' ; s'il améliore sa terre, c'est un prétexte, en fin de bail, pour l'augmentation du prix de ferme; s'il la laisse dans l'état où il la trouve, il perd le bénéfice immédiat que lui donneraient une culture plus soignée et des efforts plus attentifs, mais dont le résultat complet ne lui serail acquis qu'après plusieurs années d'attente.

Il est naturel, dès lors, qu'un homme actif, pouvant disposer de quelques capitaux, hésite à cultiver la propriété d'autrui, qui peut lui être enlevée au bout de quelques années par le caprice du propriétaire, et qu'il préfère acheter et cultiver pour son propre compte une terre dont la valeur s'accroitra en proportion des sacrifices qu'il fera pour l'améliorer.

Il est difficile, faute de précision dans les statistiques et d'uniformité de méthode chez les différents services, ce qui empêche qu'elles soient comparables, de savoir quel est le nombre des baux à ferme. L'embarras est devenu plus grand depuis que, en subordonnant tout à l'idée fiscale, on a confondu sous la même rubrique les baux écrits et les locations verbales, même quand celles-ci ont pour objet une chambre dans une maison de ville ou une minuscule parcelle de jardin ${ }^{2}$. De 1878 à 1883 , le nombre des baux et locations

1. D'après les statistiques agricoles qu'il faudrait contrôler par celles de l'Enregistrement, la proportion par 1000 baux est la suivante :

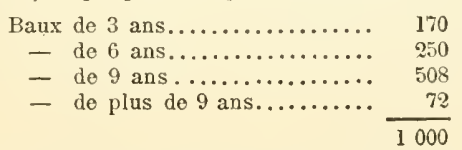

2. La loi du 23 août 1871 a assujetti au droit de bai! les locations verbales de toute nature désormais soumises à l'obligation de la déclaration. 
aurait augmenté de 34 p. 100, passant de 1877819 à $2630130^{1}$ d'après le relevé des Contributions directes; mais, si l'on ajoute foi à celui de l'Administration de l'Enregistrement $^{2}$, ce nombre se serait accru, puisqu'il s'élève à 33341003 en 1896, tout en s'abaissant à 3129231 en 1897. Ce qu'on ignore, et ce qui ôte toute valeur probante à ces comparaisons, c'est le contingent qu'apporte à ces chiffres la masse nouvelle des locations verbales.

Il y a vingt ans, un observateur compétent signalait le fait de l'accroissement régulier du nombre des exploitations soumises au régime du faire-valoir direct ${ }^{3}$. En 1900, la situation s'accentue dans le mème sens; dans les régions où domine la moyenne propriété, sur 1000 agriculteurs on en compte כ̋2 travaillant pour eux-mêmes, et 476 pour autrui ${ }^{4}$; le nombre des propriétaires terriens louant leurs services parce que leur propre héritage ne suffit pas à les occuper est toujours considérable, ce sont les auxiliaires naturels du faire-valoir direct qui s'installerait au milieu d'eux. La grande et la moyenne propriété ont un intérêt de premier ordre à maintenir et à développer celte classe particulière de salariés qui tiennent au sol par les altaches les plus solides et les plus moralisantes, et ne cherchent pas à se déplacer.

Il ne faut pas se lasser de le répéter, la fonction de propriétaire rentier a fait son temps, et celui qui veut vivre de la terre devra désormais la cultiver lui-même, à ses risques et périls.

La difficulté de trouver des fermiers devient de plus en

1. Bulletin bleu, 188', p. 150.

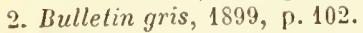

3. M. Ileuzé, La France agricole.

4. Ce dernier chifrre se décompose ainsi :

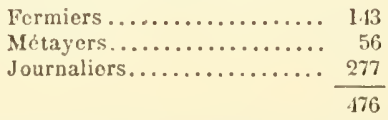

Ces proportions ne sont qu'une indication, elles varient par région, par département, et même dans chaque département par canton rural. 
plus générale, on ne peut plus exiger d'eux, avec les nouvelles mours, qu'ils reprennent les habitudes d'antan, la blouse bleue et la soupe au lard; le capital et l'intelligence réclament une équitable rémunération, aussi bien dans la classe agricole que dans toute autre. Il faut done réduire les prix de ferme, améliorer ou reconstruire les bâtiments d'habitation, créer des abris pour un bétail plus nombreux. Quantité de propriétaires hésitent à diminuer ainsi leur revenu et sont tentés de vendre; leurs acquéreurs seront sans doute beaucoup des fermiers actuels, et ce morcellement serait appréciable puisqu'il remplacerait les périls économiques de l'absentéisme par les bienfaits du faire-valoir direet.

En France, les départements où l'on trouve le plus grand nombre de fermiers sont les Côtes-du-Nord (47470 en 1892), l'Ille-et-Vilaine $(40029)$, le Finistère $(39539)$, le Nord (38 932), le Pas-de-Calais (34 480), le Morbihan (34 001) et la Manche (33 779). Le groupe le plus compact se trouve done à l'extrême Ouest et se rattache au groupe du Nord par une zone intermédiaire, Calvados, Seine-Inférieure, Somme, où la population fermière est sensiblement plus élevée que sur le reste du territoire. L'ensemble des terres affermées, en France, atteint 13 millions d'hectares, mais il y aurait erreur à raisonner sur la moyenne de 12 hectares par ferme qu'accepte l'enquête de 1892.

A ce point de vue, il est intéressant de voir ee qu'est derenu le fermage en Prusse ${ }^{1}$. Sur le chiffre global des exploitations rurales dont vivaient leurs propriétaires, il y en avait 66 p. 100 livrées au faire-valoir direct et 34 p. 100 au fermage. Parmi les exploitations totalement prises à ferme, la moyenne propriété ne figurait que pour une très faible proportion, 2 à 4 p. 100, tandis que les petites exploitations y contribuaient pour 16 p. 100 et les grandes pour 18 p. 100 , par comparaison au nombre total des domaines de celte catégorie. Comme étendue, le fermage des petites parcelles de

1. Voir le Bulletin de statistique du ministère des Finances, janvier 1885 , p. 87 , et février 1888, p. 204. 
moins de 2 hectares englobe seulement 1,52 p. 100 contre $3 \check{,}, 79$ p. 100 pour les domaines de plus de 100 hectares.

Cette variété de condition se constate avec des écarts considérables suivant les provinces; ce qui se produit en Saxe diffère du tout au tout de ce qui se passe dans un rayon de trente lieues de Berlin; autant il y a peu de grandes propriétés à exploitation directe dans le Hanovre, la Westphalie et la Prusse rhénane, autant il s'en trouve en Poméranie et dans la Prusse de l'Ouest.

IIJ. Le Métayage. - Si tous les propriétaires forains ne veulent ou ne peuvent exploiter leurs domaines par la culture directe, il leur est loisible tout au moins de s'en rapprocher le plus possible en substituant le métayage au fermage à prix d'argent. Le métayage, si usité autrefois, longtemps dédaigné du fait de la plaie de l'absentéisme, et qui redevient à la mode, grâce à l'exemple de quelques hommes intelligents, repose sur le principe du partage de la récolte; il représente l'association aussi étroite que possible entre le propriétaire et le fermier, el correspond au régime de la commandite dans l'industrie.

Il n'existait guère autrefois que dans les régions pauvres où les cultivateurs, manquant de capitaux, étaient obligés d'avoir recours au propriétaire pour les avances les plus modiques et les améliorations les plus indispensables. Il deviendra peut-être, surtoul dans certaines régions et pour certaines cultures, le mode d'exploitation de l'avenir, parce qu'en réalité il est le seul équitable el fail une part proportionnelle aux intérêts engagés qu'il associe au lieu de les mettre en conflit. Aujourd'hui, il s'impose dans les pays d'où il avait disparu, pourvu que le proprićtaire puisse le contrôler ou le faire surveiller par un régisseur, ce qui est moins sûr. Tantôt, en effet, en dehors de la pauvreté du métayer, des difficultés de transport el l'éloignement des débouchés, tantôt, surtout pour certains produits, les eonditions elimatériques ou eulturales de la contrée multiplient dans une telle proportion les risques que nul cultivateur ne veut s'exposer seul aux chances de l'entreprise. La Corse, la 
Corrèze, les Bouches-du-Rhône, la Gironde, comptent beaucoup de métairies où le système de la participation a été choisi pour l'un des motifs que je viens d'énumérer.

Les départements qui comptent encore plus de métayers que de fermiers ou de propriétaires cultivant exclusivement leurs biens sont l'Allier et les Landes; viennent ensuite la Dordogne, la Gironde, etc., en tout 13 départements où leur nombre dépasse 10000 . Dans les Landes, on trouve 19 วั92 métairies contre 19032 faire-valoir directs; dans l'Allier, 17073 contre 12191 ; dans la Mayenne, 6094 contre 6780 faire-valoir et 18329 fermes.

Dans certaines régions, le métayage n'est pas choisi pour les convenances des intéressés, il est imposé par la nature même des choses; il y persiste, et ne peut s'y transformer que lentement, par le dévcloppement des voies de communication, des moyens de crédit et de la division du sol permettan $\mathbf{a}$ des métayers de morceler une métairie pour devenir propriétaires chacun d'une portion qui, grâce à la culture intensive, deviendra bientôt plus féconde à elle seule que l'ancienne métairie tout entière.

Les avantages économiques du métayage ont été discutés; certains publicistes l'ont critiqué, la plupart y ont vu un parfait exemple de la solidarité qui unit les intérêts du cultivateur aux droits du propriétaire sans les subordonner arbitrairement les uns aux autres.

Le métayage résultant principalement de la difficulté de la location pure et simple provoque moins que le fermage les changements de personnes; ce sont d'ordinaire les mêmes familles qui possèdent et les mêmes familles qui cultivent, de temps immémorial, les mêmes terres, et cette continuité de relations, cette permanence d'intimités, ne laissent pas que de produire d'excellents résultats ${ }^{1}$.

1. Au point de vue moral seulement, font remarquer les spécialistes, car il s'en suit une routine fàchcuse et qui accentue le reproche qu'on fait habituellement au métayage de retarder les perfectionnements et d'affaiblir les travaux aussi bien que les avances en prévision du partage des bénéfices; nouvel argument en faveur du faire-valoir direct et de l'unité de direction et de responsabilité. 
Le métayage n'est pas rigoureusement une exploitation à moitié fruits; il peut varier à l'infini dans ses combinaisons; c'est, en somme, une exploitation de la tcrre basée sur le partage proportionnel des charges et des récoltes. Tel il se produit dans les régions où domine la culture des oliviers, des mûriers, de la vigne, des arbres à fruits, là où les récoltes exigent des soins multipliés el si minutieux que ni propriétaires ni fermiers ne veulent en courir isolément les risques. C'est le premier type de la mutualité agricole.

La loi du 18 juillet 1889, en donnant au métayage le nom de Bail à colonat partiaire, a déterminé sa nature juridique, complété les articles 1763 et 1764 du Code civil et légalisé toutes les modalités dont ce contrat est susceptible en les subordonnant à la convention, à la loi, à l'usage des lieux.

Malheureusement, le législateur a laissé échapper l'occasion d'alténuer un des abus de la fiscalité en matière rurale en mettant ici le droit fiscal en harmonie avec le droit civil; et ce n'est qu'à titre gracieux que le Fisc n'applique plus, depuis une décision du 19 juillet 1872, les dispositions formelles de larticle $1 \ddot{5}, \mathrm{n}^{\circ} 4$ de la loi du 22 frimaire an VII.

Le bail à colonage ou à moitić fruits n'est qu'un contrat de société, disent Cujas, Troplong et les Parlementaires de 1871; c'est un bail à ferme, soutient Duvergier, c'est un contrat mixte, dit la jurisprudence (Limoges en 1848, Nîmes en 1830). En substituant à la perception séculaire du droit proportionnel de bail celle du droit gradué de société, l'Enregistrement a judicieusement décidé en principe, mais, en fait, la perception du droil fixe pour l'universalité des petits métaynges est plus onércuse que ne l'était l'autre. Ce résultat est alténué par l'exonération de la taxe pour les baux partiaires consentis verbalement; tous devraient bénéficier d'une inmunité absolue.

IV. Le Crédit. - Le sol français, s'il était cultivé comme il doit l'être, c'est-à-dire en utilisant les découvertes de la science et ses procédés, aussi bien pour la fertilisation de la terre que pour la lutte contre les parasites qui attaquent végétaux et animaux, donnerait des milliards de plus-value. 
Quels sont les obstacles à ce mouvement en avant? La routine et le défaut d'argent.

Quels sont les remèdes? La diffusion de l'enseignement agricole et la commandite.

Gràce aux expositions régionales el à l'action des syndicats, le paysan, mème dans les contrées les plus reculées, a pu se rendre compte de l'utilité de tel ou tel instrument, de sa forme, de son emploi, de la supériorité de telle méthode de culture, de la préférence à donner à telle race de bétail; son esprit s'est ouvert au progrès et les conversations, les conférences, les images, ct même les journaux, lui ont appris beaucoup de choses qu'il ignorait. Ces notions restent confuses; elles le préparent à comprendre, elles le disposent à accepter des noureautés contre lesquelles il eût protesté jadis, mais elles ne lui feront pas renoncer à ses habitudes et à ses préjugés. C'est à l'école primaire qu'il faut prendre le petit paysan en herbe et l'engraisser, selon un mot de II. Tisserant, de leçons de choses. Ce qu'on a essayé dans ce sens est insuffisant et reste inefficace.

Il est indispensable que les enfants nés au village, en pleine exploitation rurale, soient initiés de bonne heure aux notions de la science agricole et qu'ils y prennent goût. En fait, nous ne possédons pas encore, en France, l'enseignement agricole non pas classique mais pratique et expérimental, donné dans la famille, à l'école, au collège, dans le milieu où l'enfant est appelé à vivre, avec les particularités spéciales à la région.

On évitera ainsi, par un apprentissage substantiel, les erreurs communes à tant de gens qui ont, avec les meilleures intentions du monde, retardé la diffusion de la science agronomique en abusant de la science. Là est l'écueil pour les propriétaires qui, sans préparation raisonnée, ont voulu aborder le faire-valoir direct. Lorsqu'un homme qui s'est peu occupé d'agriculture pratique prend enfin la résolution de diriger la culture de ses terres, il obéit trop souvent, si ce n'est à une idée fixe, du moins à des préférences que ses lectures ou sa tournure d'esprit lui ont inspirées d'une façon abstraite. Au lieu de consulter le 
climat, le sol, le milieu, les habitudes, les voisins, la terre elle-même, il ouvre ses livres, achète, construit, transforme, et n'aboutit le plus souvent qu'à de cruels mécomptes. Le paysan raille ces entreprises, le propriétaire voisin s'en effraie, el voilà un canton où la science est discréditée, où le progrès est ajourné pour une ou deux générations.

Rien ne s'improvise, surtout en agriculture : Natura non facit saltus. On le remarque en étudiant les rapports des concours agricoles et des syndicats. Où rencontre-t-on le succès? où admire-t-on le profit? N'est-ce pas sur les domaines que leurs propriétaires ont successivement amenés d'un état médiocre à un état passable, d'un état passable à un état fertile, en se contentant tout d'abord de faire du fromage dans le Cantal, du blé dans la Beauce et du vin dans le Nédoc? La ferme, a dit un maître en l'art agricole, ne doit ètre ni un muséum ni un laboratoire expérimental; c'est une fabrique, el quiconque l'oublie paie cher sa méprise. Le savant, s'il veut tenir la charrue ou greffer un arbre, doit s'effacer devant le praticien, et l'homme à systèmes devant l'observateur modeste et patient.

Le vrai crédit agricole, c'est l'instruction professionnelle, l'entente des choses, qui permet de voir où la dépense sera profitable et où clle sera perdue.

En multipliant les écoles pratiques où se distribuera l'esprit de discernement, on donnera à la propriété foncière la commandite dont elle a besoin avant tout. En matière agricole, les écoles feront plus de bien que les banques ".

1. Le $1^{\text {or }}$ mars 1805 (Correspondance, $\mathbf{X}, 179$ ), Napoléon dictait une note sur les encouragements à donner à l'agriculture dont il est opportun de détacher les passages suivants :

"S. M. a fait disparaitre l'École d'agriculture qui n'aurait donné que de la dépense et un vain fruit. Dans nombre de départements, la culture est entre les mains de colons et de métayers qui ne songent qu’a vivre et n'améliorent rien. Ce ne sont pas ces gens-là qu'il faut encourager.

"Il faut aviser là où par suite de la vente des biens nationaux les terres se trouveront dans les mains des verritables cultivatezers.

"Il faut exciter les riches par dles éloges et des distinctions, les autres par des comparaisons et des exemples; on ne ferait rien en ce genre avec des dons d'argent. " 
Certes, en agriculture comme à la guerre, l'argent est devenu le nerf des choses; mais il est aussi maladroit de dépenser mal à propos que de reculer devant une dépense utile, et ce n'est pas un compte courant dans une société de crédit qui enseigne cela. Le crédit agricole, d'ailleurs, a ses conditions propres qui découlent de la nature elle-même; on ne peut, comme dans l'industrie ou le commerce, ni lui fixer un ehiffre absolu, ni lui assigner un emploi immédiat. Toutes les prévisions peuvent ètre déeoncertées, en dépit des assurances, par un aléa aussi incertain que sont multiples les risques, par la maladie, les épidémies, la grêle, la sécheresse, l'inondation; on ue doil dépenser que peu à peu, par échelons, en proportion des pertes qu'il faut couvrir ou des profits qu'on doit préparer. La semence germe lentement; pas à pas, prudemment, le chef d'exploitation observe son bétail, ses labours, ses vignes. Ce n'est pas un emprunt dont il a besoin, sitôt dépensé qu'encaissé, c'est d'un compte courant ${ }^{1}$.

Les détenteurs de la moyenne propriété sont dans les meilleures conditions pour procéder ainsi et pour donner le bon exemple autour d'eux. Ils sont à cet égard, pour leurs voisins, par comparaison avec les grands propriétaires, ce que Grand-Jouan ou La Saulsaie sont à l'Institut agronomique : plus rapprochés des paysans, ils sont mieux écoutés et plus souvent compris. Ils ont, relativement tout au moins, l'argent qui permet d'agir et l'instruction qui conseille l'emploi de l'argent. Ils peuvent, par l'exemple du raisonnement, du calcul, de la modération, empêcher les emprunts immodérés, ce qu'on appelle la spéculation à l'hypothèque.

Ce qui a été jusqu'ici l'écueil de toutes les tentatives faites pour organiser le crédit à l'agriculture, c'est qu'on a voulu créer un crédit spécial et uniforme, alors qu'il se

1. Sans compte-courant, le cultivateur qui n'a pas des capitaux à lui est incapable d'améliorer sa propriété scientifiquement, par des chaulages, des amendements, des engrais. (Société nationale d'agriculture, mai 1900.) 
diversifie à l̈̈nfini, et que le problème est insoluble par l'excellente raison que, posé comme il l'est, il n'existe pas. Il n'y a pas plus de crédit agricole qu'il n'y a de crise agricole. A vouloir serrer de près les deux questions, on voit qu'elles échappent et s'évanouissent. Le crédit qu'on peut faire ${ }^{1}$, et que fait depuis trente ans la Banque de France aux éleveurs de la Nièvre et du Cher, ne ressemble pas à celui qu'offre le Crédit Foncier aux grands propriétaires ruraux, pas plus qu'à celui que réclame le petit vigneron du Beaujolais ou le puissant viticulteur de Cette ou de Montpellier, pas plus que la modique avance négociée par le maraîcher du Vaucluse ou le rosiériste du Var n'est pareille à l'emprunt du laboureur de Bourgogne ou de Savoie. Chez tous, les besoins, les échéances, le chiffre, sont différents; la centralisation du crédit agricole a été essayée, elle a avorté; la centralisation régionale a été décidée ${ }^{2}$, on hésite à la traduire en fait, et l'on fera bien de s'abstenir pour ne point courir à un échec certain; il faut laisser l'initiative de ces prèts aux individualités locales. Le besoin crée l'organe, a-t-on dit; rien n'est plus exact. Partout oì le propriétaire exploitant son héritage ${ }^{3}$ aura besoin de capitaux, il les trouvera par l'intermédiaire des syndicats, des caisses d'épargne, de la mutualité.

La loi du 18 juillet 1898 sur les warrants agricoles a créé, en France, le gage sans déplacement, au profit exclusif des agriculteurs. C'est une tentative dont jusqu'ici les résultats sont négatifs " et qui, vraisemblablement, comme toutes les lois d'exception, ne sera utilisée que par les gros propriétaires ou fermiers dont les greniers et les celliers sont

1. Nos législateurs ignorent sans doute la loi belge du 20 décembre 1882 organisant les préts agricoles par les caisses d'épargne, et le gage à domicile.

2. Loi du 31 mars 1899 , ayant pour but l'institution des caisses régionales de crédit agricole mutuel et les encouragements à leur donner ainsi qu'aux sociétés et aux banques locales de crédit agricole mutuel.

3. Je ne parle pas, bien entendu, du propriétaire non exploitant, du forain, qui n'emprunte pas pour la culture et dont la propriété n'est, pour lui, qu'un gage banal.

4. M. H. Pascaud, Le warranlage des produits agricoles, 1899. 
encombrés; elle ne peut servir à la petite ni même à la moyenne culture.

Elle mérite cependant une attention particulière en ce sens qu'elle s'écarte du principe fàcheux de la tutelle de l'État pour incliner vers le droit commun, vers l'égale liberté.

En jetant les yeux sur les marchés financiers ou commerciaux, on est surpris de l'extension du crédit mobilier, qu'il s'effectue sous la forme du nantissement sur titres ou du dépòt de marchandises dans les magasins généraux. Jusqu'ici, l'agriculteur français ne voyait aucun de ces moyens s'offrir à lui, en raison des règles étroites du Code civil qui exige la remise du gage entre les mains du créancier, et aussi à cause du privilège du propriétaire sur tous les produits de la ferme et sur ce qui sert à son exploitation. Cependant, au moment de la récolte, l'agriculteur se trouve souvent pressé par le besoin d'argent de vendre rapidement ses produits à la grande joie de la spéculation qui le guette et profite de sa pénurie pour déprécier les cours. Depuis 1880, dans les pays qui se sont inspirés de notre Code civil, lequel, à l'étranger, passe pour avoir créé dans le monde moderne le discrédit agricole, un mouvement s'est produit pour libérer l'agriculture des liens qui l'enchainent et lui restituer le droit commun de toute industrie. La Roumanie, le Portugal, l'Italie, la Belgique ont permis le prêt sur gage aux cultivateurs. De tout temps, en Angleterre, en Écosse, aux Élats-Unis, les agriculteurs ont trouvé du crédit parce que les fermiers y sont assimilés aux commerçants, ce qui n'est que justice puisque leur métier est de produire, de vendre et d'acheter ${ }^{1}$.

1. C'est cependant la France qui a donné l'impulsion aux États régis par notre Code civil; en fait, ces derniers n'ont fait que s'approprier nos travaux et prendre les devants. La loi du 14 juillet 1851, ratifièe par la loi organique du 24 juin 1874, établit dans les colonies le gage à domicile sur récoltes même pendantes; la question, posée législativement en 1856 et reprise en 1878 , n'aboutit qu'après vingt ans de délais, en 1898, grâce aux lenteurs et aux incohérences de notre système parlementaire. 
En admettant que le paysan s'habitue au jeu des warrants, il ne pourra les escompter qu'auprès d'institutions de crédit qui n'existent pas encore, et jusqu'ici, comme l'avoue M. Pascaud, les notaires el les escompteurs ignorent ce genre de prêt ${ }^{1}$.

\section{III. - La propriété, à peine constituée, succombe sous l'étreinte de trois fléaux.}

La petite propriété a trois ennemis : l'insécurité de son titre, l'hypothèque, le partage. Le dernier lui est imposé par la loi; les autres naissent de la liberté même qu'on lui donne d'acquérir et d'emprunter trop facilement.

Parmi les questions sociales qui agileront les premières années $\mathrm{du} \mathrm{xx}^{\mathrm{e}}$ siècle, il en est une dont on a pris en réalité peu de souci malgré toul le bruit qu'on a fail autour d'elle ${ }^{2}$, cest la protection de la propriété foncière et particulièrement de la propriélé rurale.

Ainsi que j’ai été amené à le démontrer dans les premiers chapitres de cette élude, l'exercice du droit de proprićté a torjours été le pirot des érolutions politiques el sociales.

Quand on suit, à travers l'histoire, le développemenl, les crises, les variations de la division de la terre, on voit que les lultes de l'humanité se concentrent, si différentes qu'en soient les formes, entre cenx qui possèdent cette lerre sans la cultiver et ceux qui la cultivent sans la posséder.

Depuis soixante ans, la multiplication des valeurs industrielles et des papiers de crédil, malgré les déceptions qu'a causées cetle merveilleuse extension de la fortune mobilic̀re, a fail perdre à la propriété foncière son prestige tradilionnel.

1. La question fiscale, à elle seule, suffira pour empêcher l'application de la loi. La réalisation du gage affecté à un warrant agricole de 50000 francs couterait 1250 francs de droits d'enregistrement; celle d'un warrant commercial ne conte que 62 fr. 50. Pauvre législateur!

2. Témoin la Commission extra-parlementaire du carlastre qui, instituée en mars 1891, n'a point cncore formulé ses résolutions définitives en décembre 1901, malgré de savantes et laborieuses discussions. 
Déjà, en voulant la protéger, le Code civil l'avait garottée; les lois fiscales l'ont écrasée d'impôts; la spéculation des syndicats de capitalistes a achevé son discrédit. La Juslice elle-mème ne peut la défendre qu'en la ruinant, et les plus longues prescriptions, interrompues par les subtilités de la jurisprudence, sont également impuissantes à lui créer un titre définitif.

La plus mauvaise hypocrisic, c'est de condamner tout le monde sous couleur de prolcction, a dit Bossuet ${ }^{1}$; c'est précisément le caractère de nos lois foncières.

I. La Sécurité du titre foncier. - A toutes les époques, la sécurité du titre foncier fut le privilège de la richesse; mais, aujourd'hui, l'on sent plus que jamais notre société étreinte par la double servitude de la fiscalité el du formalisme. Ce vice, qui n'est pas une nouveauté, car il a accompagné l'humanité dans toutes ses évolutions, du simple au composé, est peut-être plus agressif, plus obstiné, plus impertinent en France que partout ailleurs. Il est d'autant plus visible quand il affecte le problème de la division du sol à son étape la plus intéressante, au début de la constitution de l'héritage par l'homme de labeur, à ce point précis de l'existence du prolétaire où celui qui n'a jamais travaillé que pour autrui se sent la légitime ambition de travailler enfin pour lui-mème. Il est d'autant plus inquiétant qu'il moleste des hommes la plupart sans instruction, ignorants des exigences légales, et qui ne peuvent se douter qu'un délai qui passe, une forme juridique qu'on néglige, risquent de compromettre le fruit de leurs travaux et de leurs efforts.

Il faut qu'il y ait au cœur de notre peuple un fonds inaltérable de franchise, de loyauté, pour qu'on essaie si rarement d'exploiter la candeur des honnêtes gens, la simplicité relative des paysans, en utilisant les moyens qu'offrent aux habiles les détours de la chicane et les surprises de la procédure ${ }^{2}$.

1. Méditations sur l'Évangile, $1^{\text {re }}$ partie, $S$ XXXVII.

2. Les statistiques du ministère de la Justice publiées en 189t (tableau XVII) accusent pour les tribunaux civils, à propos de contesta- 
Presque toutes les nations d'Europe ont adopté nos lois; mais elles ont toutes revisé et simplifié nos formalités. Les lois de 1790 avaient libéré le propriétaire foncier et émancipé la terre; la fiscalité de l'an VII, de 1816, de 1824 el de 1872, exagérant par ses contingenees les formules étroites ou timides du Code de 1804, a, de nouveau, enchaîné l'un et monopolisé l'autre.

Cependant, tout en signalant le petit nombre relatif des procès pour cause de fraude et de lésion dont l'existence est juridiquement constatée, il faut reconnaître que l'acquisition et les transferts du droit de propriété donnent lieu à d'innombrables instances, les unes forcées (ventes judiciaires, licitations), les autres volontaires, et que, tout en pouvant être plus nombreuses encore, sans l'honnêteté native de la masse, tant la procédure prête à la chieane, elles n’en constiluent pas moins un danger permanent et une cause légale d'affaiblissement et de ruine, affectant spécialement la petite propriété, c'est-à-dire celle qui offre le moins de résistance en raison de sa mobilité et de sa fragilité.

Si les statistiques étaient assez détaillées ${ }^{1}$ pour permettre de distinguer la propriété rurale de la propriété urbaine et, pour la première, la valeur par catégories des immeubles objets du litige, la preuve serait faite. Elle l'est, par la pratique des ehoses, aux yeux des spécialistes et des professionnels qui savent combien il est difficile aux travailleurs d'accepter les transactions qui exigent un paiement immédiat, ce qui a pour conséquence de les obliger à subir les frais autrement onéreux d'un procès; les autres, au contraire, préfèrent transiger, même ayant le droit pour eux, pour peu qu'ils aient des ressourees suffisantes pour lenr permettre un sacrifice. Si l'on eonnaissait, par les registres

tions en matière de vente, 164 ' jugements, et, en matière d'échange, 30 , sur 76263 relatifs à l'ensemble des conflits sur la propriété foncière, dont seulement 94 pour éviction et $9 \dot{4} 0$ pour résolution des conventions.

1. Comptes généraux de l'administration de la justice civile et commerciale, présentés au chef de l'État par le ministre de la Justice. 
de caisse des officiers ministériels, le nombre des réclamations en justice, on serait effrayé de la quantité de compromis et de désistements dont les courtiers d'affaires profitent largement; la seule indication de l'écart normal entre les affaires portées devant les tribunaux civils, celles qui sont inscrites au rôle, et celles qui sont terminées par une décision judiciaire, montre quel est le mouvement incessant et menaçant de la procédure civile :

Affaires portées devant le tribunal et qui n'ont pas èté inscrites au rôle général.......... $67 \dot{497}$

Affaires inscrites au rôle............... 185713

Affaires terminées par jugement............ 109 71't

Sur les affaires notées par le compte-rendu de 1894 comme inscrites au rôle, 34171 ont été radiées à la demande des parties, à la suite de désistement ou de transaction, indépendamment des 67497 qui sont restées sans suite, soit 5o̊ p. 100 du total.

$\mathrm{Si}$, pour la même année, on recherche le nombre par catégorie des jugements des seuls tribunaux civils (en dehors des appels) qui ont trait à la propriété foncière, on trouve des indications qui pourraient suggérer de longs commentaires.

I. - Des biens et des différentes hodifications de la propriété.

Revendication de biens fonciers............ 1276

Restitution de fruits..................... 70

Questions d'usufruit.................... $\quad 255$

Règlements de servitudes.................. 1826

Actions en bornage...................... $\quad 482$

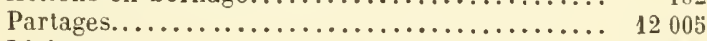

Licitations......................... 5342

Homologation des partages de mineurs........ 5484

Séparation de patrimoine.................. 68

Rescision pour cause de lésion.............. 93

II. - Des contrats d'Acquisition.

Exẻcution de vente...................... $\quad 379$

Eviction................................

Rẻduction de prix....................... 97

Résolution de vente...................... 910

Ėchanges............................ 30

LA PROPRIÉTÉ RURALE EN FRANCE. 
III. - Du contrat de louage des biens ruraux.

Résolution, expulsion, elc.............. 6533

IV. - Des PRIVILĖges ET HYPOTHE்QUes.

Nullité, réduction, main-Jevée, etc.......... 1924

Devant les juges de paix, sur 1538969 billets d'avertissement ayant donné lieu à 1500744 arrangements, et sur 30103 citations, on estime que les conflits à propos de propriétés rurales figurent pour 47 p. 100 dans les ressorts du Nord, pour 54 p. 100 dans ceux du Midi.

Malgré le dégrèvement apparent de la loi du 26 janvier 1892, les droits d'enregistrement sur les actes judiciaires (non compris les droits de limbre el les honoraires des officiers ministériels) ont produit 20 millions $1 / 2$ au Trésor en 1894, 23 millions $1 / 3$ en 189.3. Les droits d'hypothèques (y compris les salaires des conservateurs dont on a le chiffre exact) ajoutent à ces frais une moyenne de 6 millions par an.

C'est la propriélé rurale qui paie la plus grosse part de ce prélèvement.

A ce prix, la propriélé est-clle protégée, constituée, garantie? Nullement.

Il existe en France, d'après les statistiques les mieux étudiées, environ 4 millions de pelits propriétaires fonciers; ils n'ont pas de titres, ou ces titres, sanf de bien rares exceptions, sont précaires, irréguliers, attaquables. En raison même du peu de garanties que ces droits de propriété mal assis et insuffisamment constatés offrent à l'aequéreur ou au prêteur sur hypothèque, le crédit du propriélaire est quasi nul; quand il est aecepté, ce n'est qu'au prix de l'usure ${ }^{1}$.

L'épargne la plus intéressante puisqu'elle a pour objet

1. La récente loi sur le taux de l'intérêt légal (7 avril 1900), en le réduisant de 5 à 4 p. 100 en matière civile et de 6 à 5 p. 100 en malière de commerce, ne modifie pas le droil de fixer l'intérêt conventionnel au taux qu'acceptent les débiteurs. (Loi du 12 janvier 1886, sauf la réserve en matière civile que contredil la jurisprudence actuelle.) 
d'offrir un aliment au travail, celle du paysan, celle de l'ourrier éeonome, n’est donc pas protégée. Le prolétaire même, sil a l'espoir de devenir propriétaire à son tour, sait que ce foyer domestique qu'il désire ardemment, sujet à éviction, éerasé d'impòts, deviendra pour lui une gêne plutôt qu'un abri.

Ne serait-il pas digne de la démoeratie française contemporaine de supprimer ces injustices, d'effacer de nos mœurs les iniquités légales qui réservent tout à l'argent? Mais qu'elle se hàte si, après un siècle d'hésitations et de tâtonnements, elle se décide à compléter 1789, car, an dehors, nos voisins plus avisés nous devancent et, après nous avoir emprunté les grandes clartés de nos philosophes et de nos révolutionnaires, ils marehent plus hardiment que nous dans la large voie des libertés eiviles.

Une société tire sa foree de l'ordre moral, a dit F. Le Play, beaucoup plus que de l'ordre matériel; de telle sorte que le travail est plus utile que la richesse. Mais il est des cas où le travail se solidarise tellement avec une richesse relative qu'il est impossible de les séparer et que, se prêtant un mutuel appui, ils sont néeessaires l'un à l'autre. Tel est le caractère du travail rural qui ne se révèle avec toutes ses ressources et ne se développe à l'aise, se retrempant à chaque effort, que lorsqu'il agit ehez soi, pour soi, dans la séeurité du présent et dans la certitude de créer l'avenir de la famille.

Il faut donc encourager l'acquisition de la propriété, et surtout maintenir la propriété dans les familles qui l'ont acquise par le travail et l'épargne et qui s'appliqueront d'autant plus à la rendre productive qu'elle leur a eoûté plus de labeur en réalisant leur rêve.

Le fait de l'acquisition est aisé ; la loi du 27 ventôse an IX autorise même les mutations verbales; mais s'il s'agit de régulariser le titre et de défendre son bien eontre les entreprises des voisins ou les revendications des ayants-droit des précédents détenteurs, les difficultés commencent. Il faut d'abord rédiger l'acte, puis le faire enregistrer pour lui 
donner date certaine s'il n'est pas passé devant notaire. La seule évaluation des charges fiscales frappant dans une proportion à rebours les ventes de minime importance serait pour effrayer les amateurs de terre si on leur expliquait l'engrenage dans lequel ils vont s'engager.

En supposant le prix payé comptant, une vente notariée de 100 francs coûtera à l'acquéreur, pour les seuls droits du Trésor, $17 \mathrm{fr} .37$; sous signatures privées, $14 \mathrm{fr} .97$; une vente de 500 francs, $44 \mathrm{fr} .87$ ou $42 \mathrm{fr}$. 47. L'écart est minime; mais il faut ajouter aux frais de la première les honoraires du notaire qui sont de 1 p. 100 , au minimum de $\breve{5}$ francs, soit 22 fr. 37 pour une minuscule acquisition de 100 francs, tandis que pour un prix de 5000 franes la proportion tombera à $7,20 \mathrm{p} .100^{1}$.

Si le nouvel acquéreur veut consolider son droit de propriété pour l'avenir vis-à-vis des tiers, en utilisant le privilège facultatif de la publicité hypothécaire, il doit faire transcrire son titre au bureau des hypothèques, ce qui, surtout s'il emploie, comme on le fait d'habitude, l'intermédiaire du notaire ${ }^{2}$, rapprochera sensiblement le taux des frais de 30 à 33 p. 100.

Si, enfin, il veut liquider le passé et dégager eette parcelle des charges occultes qui peuvent pendant trente ans et plus (Art. 2231 et suivants du Code civil) provoquer son éviction, il doit remplir les formalités de la double purge des privilèges et hypothèques (Art. 2181, Code civil) et des hypothèques légales (Ibid., art. 2193), ce qui coûte en moyenne de 300 à 400 francs.

Avant de se trouver en possession définitive, le nouvel acquéreur peut donc avoir à payer, s'il veut obtenir un titre régulier, quatre à cinq fois la valeur de l'immeuble. Si le prix n'est pas payé complant, l'opération motive d'autres formalités et des frais additionnels. II est juste d'ajouter que ces frais, constitués en majeure partic par des droits fixes,

1. Bulletin de statistique de l'Enregistrement, 1997, page 218.

2. Tarif légal du 25 août 1898. 
ront en diminuant à mesure que le prix d'acquisition s'élève, et quion les évalue de 10 à 12 p. 100 seulement, contrat en mains, pour les mutations à titre onéreux de plus de §ั 000 francs.

Mais on peut dire que, au-dessous de ce chiffre, c'est-àdire pour la quasi généralité des cas ${ }^{1}$, l'immeuble acquis ne supporte pas les frais de la purge, à peine ceux préliminaires; et qu'il n'est pas étomnant, dès lors, que tant de petits acquéreurs refusent de faire la dépense d'un titre régulier et se dispensent mème de l'enregistrement ${ }^{2}$, attendant d'y ètre contraints par un cas de force majeure ${ }^{3}$, et comptant que, plus tard, la déclaration de leur succession par leurs héritiers suffira pour servir de titre et couvrir l'absence de papier's en règle.

Les essais pour simplifier la procédure et supprimer les formalités inutiles ont avorté devant la résistance passive des intérèts corporatifs; les travaux préparatoires qui peuvent conduire à une réforme radicale sont abondants, on n'y peut rien ajouter, que la volonté d'aboutir. Peut-être parviendrait-on à suppléer à İinsuffisance des titres de la petite propriété par des procès-verbaux de bornage et leur inscription sur les futurs livres fonciers".

II. L'Hүротнѐque. - L'hypothèque, telle qu'elle est réglée par notre législation et comprise par la pratique, est le ver rongeur de la propriété foncière.

Malgré la cherté des emprunts hypothécaires, en dépit du peu de solidité juridique de la plupart des gages offerts, surtout pour les petites affaires, la dette agraire ne cesse

\section{Bulletin de statistique de l'Enregistrement, 1897, p. 213.}

2. Pendant l'année 1897 , les pénalités en matière de mutations immobilières ont produit une surtaxe de 2 millions $1 / 2$ de francs. (Ibid., 1899, p. 113.) M. Garnier, conseiller-maître à la Cour des comptes, rédacteur en chef du Répertoire périodique de l'Enregistrement, affirmait, en 1873, avec chiffres à l'appui, que la réduction des droits dans la proportion de 50 p. 100 procurerait aussitôt une plus-value de recettes de $100 \mathrm{mil}$ lions par la suppression de la fraude.

3. Articles 41 à 47 de la loi du 22 frimaire an VII.

4. Freyssinaud, Le bornage cadastral, 1899. 
d'augmenter; ses créances sont monopolisées pour la propriété urbaine par les sociétés de crédit et particulièrement le Crédit Foncier, pour la propriété rurale par les capitalistes de province. En 1898, il y a eu 465473 prêts hypothécaires pour 2 milliards 7 millions de franes ${ }^{1}$.

L'Administration de l'Enregistrement établit en ce moment, pour la première fois, des statistiques hypothécaires suffisamment détaillées pour qu'on en puisse dégager la portée économique; la question ne pourra être élucidéc avec toute son ampleur que lorsqu'elles auront été publiées. Mais on sait déjà, de l'aveu des professionnels, que la proportion des très petits prêts, dans la masse du passif hypothécaire, n'a jamais cessé d'ètre dans une très forte mesure la plus considérable, et que l'écart, au lieu de diminuer, n'a fait que croître. La seule statistique officielle sur ce point a été fournie par l'Enregistrement en 1841, à propos de l'enquête judiciaire sur l'urgence de la réforme hypothécaire; elle se résumait en quelques chiffres : QUOTITÉ DES PRÉTS
de l'année 1811

De 400 fr. et au dessous..... 1505220

De 400 à $1000 \mathrm{fr} \ldots \ldots \ldots \ldots . . . . \quad 89803$

Au-dessus de $1000 \mathrm{fr} . \ldots . . .84533$
329576

PRĖTS HYPOTHÉCAIRES

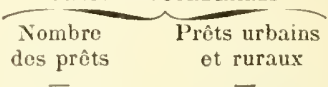

36640948 .

62421267

302513625

401575840

De l'année 1841 à l'année 1898, le nombre des prèts a donc augmenté de $133 ̈ 897$ et leur valeur, fait inouï, de plus de 1605 millions de franes; elle a quintuplé. Ce fait matériel est l'une des preuves de l'énorme plus-value acquise depuis cinquante ans par la propriété foncière.

En 1892, parmi les documents fournis au Congrès de la

1. Dans son dernier compte-rendu (avril 1900) le Crédit Foncier donne en bloc le montant total de sés prêts en cours, pour les $3 / 4$ sur immeubles urbains, et qui vont en croissant :

\begin{tabular}{|c|c|}
\hline 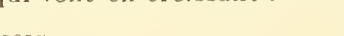 & En millions de franes. \\
\hline ............ & . $\quad 1771,6$ \\
\hline $1898, \ldots \ldots \ldots \ldots \ldots \ldots \ldots \ldots \ldots$ & 1789,9 \\
\hline $1899 \ldots \ldots \ldots \ldots \ldots \ldots \ldots \ldots \ldots \ldots \ldots \ldots \ldots$ & 1848,9 \\
\hline
\end{tabular}


propriété foncière ', il a été expliqué qu'en Normandie, dans l'arrondissement du Havre, pays de moyenne propriété, où les cotes de 100 hectares sont de $100 \mathrm{p}$. 100 à peine de l'ensemble, malgré l'appoint d'une des huit plus grandes villes de France, les prèts ruraux sont dans la proportion des trois cinquièmes. La répartition est à peu de chose près celle de 1841, sauf l'augmentation en nombre et en valeur. Le plus grand nombre des opérations de prèts porte sur de faibles capitaux mais le total des prêts dépassant 2000 francs l'emporte sur celui des petits. Ceux au-dessous sont les trois quarts en nombre et un quart en capital; d'où l'impossibilité d'établir une moyenne pour le poids de l'intérêt et des frais, variant de 7 à 13 p. 100, sans diviser les prèts par catégories.

La terre ne rapportant net que $21 / 2$ et rarement 3 p. 100, le propriétaire non exploitant est vite obéré, les intérèts qu'il paie le ruinent en cinq ou six ans, durée ordinaire des prèts. S'il exploite lui-mème, il vit sur la terre, tout lui est profit, et il peut encore réussir, s'il est patient et s'il modère ses besoins, à se libérer. Mais, qu'une mauvaise année survienne, que la grêle emporte la récolte, qu'une épidémie décime le bétail, diminuant à la fois le cheptel, le travail et l'engrais, voilà l'emprunteur ruiné, s'il a épuisé son gage, et hors d'état de rétablir assez vite sa situation pour faire honneur à ses engagements. Le prêteur, de son còté, n'a pas de chances meilleures, et l'on ne s'explique que par la fàcheuse activité des officiers ministériels ce phénomène qui engage le créancier à saisir, alors qu'il sait que cette procédure déprécie le gage de 30 p. 100 et qu'il court le risque, neuf fois sur dix, de ruiner son débiteur sans rentrer dans l'intégralité de sa créanice.

Ainsi que j'ai été amené plusieurs fois à le signaler au cours de cette étude, la cause la plus fréquente de la dette agraire est le non-paiement au comptant du prix d'achat, et,par suite, l'inscription de l'hypothèque jusqu'à concurrence

1. Statistique des prêts hypothécaires et des saisies dans l'arrondissement du Havre, par M. de Saint-Genis, conservateur des hypothèques. 
du prix d'acquisition stipulé payable à terme, qu'il s'agisse du privilège du vendeur (Art. 2103 et 2100 du Code civil) ou de celui du co-partageant (Ibid., art. 2103, n³, et 2109).

La différence est grande, sous le régime du Code civil, entre le privilège du vendeur et celui du co-partageant quoique le but final de la transaction soit, dans les deux cas, l'aequisition de la terre. Le privilège du vendeur se conserve par la seule transcription du titre de vente, celui du co-parlageant exige une inscription. Cette différence s'explique, sans se justifier, par le fait qu'avec notre régime incomplet de publicité le parlage n'est pas sujet à être transerit. Il faut souhaiter que le futur Code foncier assimile les divers modes d'acquisition et les assujettisse à la même large publicité, car il est illogique d'entraîner les tiers acquéreurs dans l'erreur sous le prétexte spécieux que l'acte de vente constitue, à l'égard des intéressés el du public, un état de choses nouveau, tandis que l'acte de partage consacre un état ancien qui date, en réalité, de l'ouverture de la suceession ou de la dissolution de la communauté .

Pour tout ce qui touche, de près ou de loin, à la division de la terre, le côté juridique reste en contact permanent avec le côté économique, celui-ei presque toujours contrarié et entravé par l'autre qui, au lieu le le tyranniser, ne devrait être que son très humble serviteur.

III. Le Partage. - Après l'insécurité du titre foncier, qui est la faute des lois fiscales et de la cherté des procédures, après l'hypothèque, qui est une plaie économique, vient le partage qui ôte à tout petit propriétaire qui réfléchit l'espoir de faire ceuvre durable en constituant un patrimoine à sa famille.

Le partage est, après l'hypothèque, un des agents les plus actifs de la division du sol, agent indépendant de la volonté de l'homme, et dontl'incidence est d'une appréciation d'autant plus délicateque son application lui écliappe et que le niveau brutal de la loi ne considère ni les conditions ni les nuances.

1. Commission extra-parlementaire du cadastre. - Rapport de M. Challamel sur les Privilèges et Hypothèques, 1897, p. 15. 
Sans discuter, après tant d'autres, les théories socialistes qui nient la légitimité de l'héritage, prétendant que l'appropriation primitive du sol a toujours commencé par une usurpation et que le travailleur seul a droit de posséder, tant qu'il travaille, on ne peut nier que le partage forcé ne soit une atteinte à la liberté individuelle et au droit de propriété. El n'est-ce pas une des plus étranges contradictions de notre époque de voir, simultanément appliquées, les lois qui interdisent l'indivision dans la famille et les lois qui invitent les étrangers à s’associer en étroites mutualités?

Pour concilier ces antinomies, ne serait-il pas à propos de tout ramener au droit commun et de laisser à chacun la liberté de ses contrats?

Examinons, en dehors des théories et par les chiffres, quelle est l'influence du partage sur la division de la propriété.

D’après les statistiques de l'Enregistrement, auxquelles il faudra toujours se reporter quand on cherchera des indications exactes, parce que ces statistiques, reposant sur des articles de recettes, ne sont ni arbitraires, ni arrangées, ni calculées avec des moyennes, il y a eu, en 1896, 83877 actes de partage simple et 830161 en 1897, auxquels il faut ajouter, pour les mèmes époques, 8.1552 ou 805016 licitations avec soulte entre cohéritiers ou co-propriétaires et environ autant de donations à titre de partage anticipé.

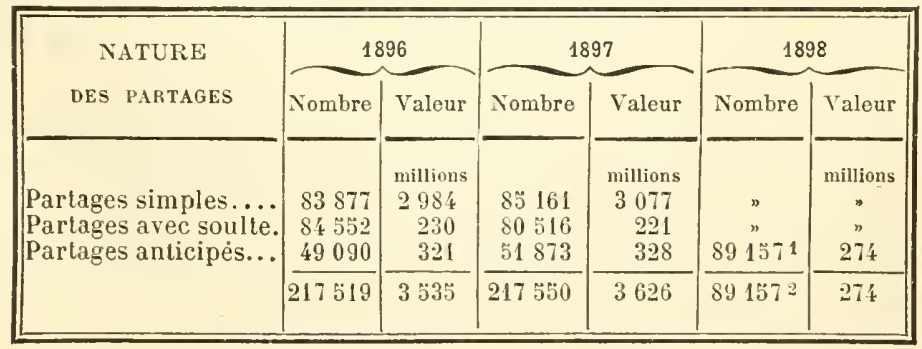

1. Ce chiffre parail excessif et doit être plutôt ramenẻ au chiffre du nombre des actes de l'espèce qui était de 27732 seulement en 1898. (Bulletin, 1899, p. 174.)

2. Bulletin de 1899, p. 96,10 et $17 \%$. Sous toutes réserves pour les partages anticipés dont le détail n'est donné qu'à partir de 1898. 
En prenant pour terme de comparaison le nombre des successions déclarées (438 810 en 1898), on voit qu'il y aurait un peu plus d'un acte de partage pour deux successions.

Pour la même année, le nombre des héritiers en ligne directe seulement a été de 692833 , pour 281333 déclarations de succession. Il existait des immeubles ruraux dans $2465 \% 5 \%$ déclarations. Enfin, si l'on décompose le résultat des 27732 contrats de donation à titre de partage anticipé enregistrés au cours de l'année 1898, on voit que pour cette seule catégorie de mutations qui n'est guère usitée que dans la population agricole el surtout parmi les détenteurs de la petite propriété, des immeubles ruraux estimés 223 millions de francs, ont été répartis entre 76257 bénéficiaires, soit une moyenne de 2662 francs par lot; pour les immeubles urbains, 51 millions de francs se subdivisent en 12900 parts. On roit dès lors quel est le résultat de l'article 815 du Code civil qui interdit l'indivision mais permet de suspendre le partage pour des périodes de cinq ans renouvelables (entre majeurs seulement), et surtout de l'article 826 ordonnant le partage en nature, sans même aller jusqu'à la vente forcéc prescrite par les articles 827 et 838 . Les propriétés se fractionnent en un très grand nombre de lots, et même, si l'héritage est très petit et ne peut se partager, il disparait par la vente et n'est plus représenté que par une somme d'argent singulièrement ébréchée par les frais de toutes sortes qui écrasent la famille, riche ou pauvre, à la mort de son chef.

Ce n'est pas tout; même dans le cas le plus favorable celui où les héritiers sont majeurs, où le chiffre de la dette hypothécaire n'oblige pas à lirquider d'office, où les lots sont assez importants pour servir de centre à une exploitation ou les soultes assez modérées pour ne point écraser le nouveau possesseur sous le poids des intérêts dus aux colicitants, le partage ne crée pas; ipso faclo, un titre définitif de propriété, el lous ces frais, toutes ces charges, peuvent ètre en pure perte.

11 arrive assez souvent, en effet, surtout dans les familles 
rurales, habituellement nombreuses et dont plusieurs membres ont pu émigrer à l'intérieur, à l'élranger, aux colonies, que lorsqu'une succession vient de s'ouvrir, les héritiers apparents, les héritiers connus au premier moment ne sont pas les véritables héritiers ou ne sont pas les seuls héritiers ayant droit à la dévolution successorale. Ils procèdent toutefois à la liquidalion et au partage avec la plus parfaite bonne loi. Plus tard, survient un véritable héritier qui justifie de ses droits et évince en tout ou en partie les héritiers apparents co-partageants. Le droit à la revendication, la pétition d'hérédité, ne prescrit que par trente ans et l'hérilier oublié ou inconnu a toujours le droit jusqu'à cette lointaine échéance de réclamer l'intégralité, en nature, de la part qui lui était légalement dévolue, à lui ou à l'héritier qu'il représente '.

La petite propriété est donc menacée à chacun de ses transferts; et il n'est pas une seule de ses modifications mème légales, qui ne soit pour elle un risque de mort.

La moyenne propriété, dont l'étendue se prète d'autant mieux à la subdivision intégrale, n'est pas moins atteinte.

On discutait récemment, à la Société nationale d'agriculture ${ }^{2}$, les révélations des cartes agronomiques; on y citait le canton de Redon(Ille-et-Vilaine) oủ certains propriétaires possèdent, pour constituer des domaines de 20 à 30 hectares, jusqu'à 100 et 230 parcelles, la plupart éparses, et l'on déclarait que, dans de telles conditions, aucune amélioration agricole méthodique et scientifique n'était possible, mème à l'état d'essai.

En faut-il conclure, d'une manière absolue, que la loi du partage est un fléau économique? Et ne doil-on pas plutôt attribuer certains de ses résullats fâcheux, en France, aux formalités coûteuses de notre procédure civile et à l'ingérence obligatoire d'intermédiaires officiels dans nos règlements de famille?

1. On a proposé de rendre définitifs les partages faits de bonne foi et dans les formes légales, en complétant l'article 840 du Code civil. (Sénat, séance du $1^{\text {er }}$ avril 1892. Rapport de II. Thézard, $\left.\mathrm{n}^{\circ} 36.\right)$

2. Journal officiel du 7 mai 1960, p. 2863 . 
Nous avons dans une de nos provinees encore séparées de la métropole par la politique, dans nos îles normandes, un plaidoyer vivant et plusieurs fois séculaire en faveur de l'innocuité du partage forcé. Les lois de la vieille Normandie, qui prescrivent le partage égal des terres entre les enfants, n'ont pas cessé d'ètre en vigueur dans l'île de Jersey et ses annexes. L'effet inévitable de cette coutume, remarque le jurisconsulte David Low, agissant depuis plus de neuf cents ans dans les étroites limites de cette petite île, a été de réduire tout le sol du pays en petites possessions. A peine pourrait-on trouver dans l'île entière une propriété de 10 hectares, beaucoup varient de 5 à 130 acres, el le plus grand nombre ne dépasse pas cette étendue (15 acres ou 6 hectares). L'agriculture n'en est pas plus pauvre, la terre ainsi divisée est cultivée comme un jardin, et il n'y en a pas pour tout le monde puisqu'elle s'afferme dans les environs de Saint-Hélier jusqu’à 12 livres sterling par acre, ce qui équivaut à $7 \check{0} 0$ francs l'hectare.

En France, les mariages refont par la dot des filles ce que la loi de succession défait ${ }^{1}$; à Jersey, et l'on peut en citer d'autres exemples, le morcellement du sol se limite de luimême en vertu d'arrangements que prennent les familles pour l'arrêter aussitôt qu'il devient nuisible aux intérêts de l'exploitation rurale.

Les mœurs sont plus forles que les lois, le jeu des nécessités économiques rétablit toujours l'équilibre qui paraît un instant le plus compromis; l'essentiel est de ne point entraver par des mesures fiscales le mouvement des mutations et l'élasticité de l'initiative el du travail de l'homme.

1. Le désir de proportionner entre eux les dilférents chapitres de cette étude m'empêche de développer des points suggestifs el dont je ne puis que signaler l'intérêt.

En 1898, les apports des futurs époux constatés dans les contrats de mariage se sont élevés à la somme đe 1 I66 857800 francs. Les donations

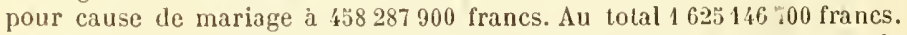

Sur le chiffre des donations faites aux futurs époux par le contrat de mariage, on trouve 12358 immeubles ruraux estimés 51 millions 1/2. Le nombre total des contrats s'élevant à 82346 . (Bulletin de slatist. de l'Enregistrement, 1899, 149 et 161.) 


\title{
DEUXIÈME PARTIE
}

\author{
AVENIR DE LA PROPRIÉTÉ TERRITORIALE \\ EN FRANCE, D'APRÈS LES TENDANCES \\ QUE MARQUENT LES MEURS, LES LOIS \\ ET L'ESPRIT DE LA PHILOSOPHIE SOCIALE
}

\section{CHAPITRE I}

L'avenir de la propriété rurale a pour termes corrélatifs la coexistence de la grande et de la petite propriété ef l'exploitation intensive de la moyenne.

La grande propriété est répartie entre environ 138671 propriétaires dont les vastes domaines couvrent 23 millions d'hectares; la petite propriété compte 2617558 détenteurs exploitant 11 millions d'hectares.

Tout le problème foncier est dans la comparaison de ces deux groupes; sa solution, qui ne sera jamais qüimparfaite et sujette à variations, consisterait à diminuer l'étendue de la grande propriété et à augmenter celle de l'unité rurale minuscule; bref, à constituer un groupe intermédiaire solide et vivace, empruntant à la grande propriété de la terre, à la petite des hommes. L'idéal serait, en même temps, en diminuant le groupe négatif des forains, de ramener vers le fairevaloir direct les oisifs, les désœuvrés, les déracinés de la classe moyenne, et d'utiliser à l'accroissement de la plus- 
value territoriale les aptitudes et les ressources qu'ils gaspillent dans les villes, au grand dommage des harmonies économiques. Ce programme ne revient-il pas à la formule que j'énonçais au début de ce livre : la moyenne propriété est une région délaissée, l'avenir de l'industrie agricole est dans l'exploitation directe de cet héritage national, qui n'a qu'à exiger de ses détenteurs négligents la terre, les capitaux et les bras dont ils disposent?

Ce vœu ne tend pas, loin de là, à la suppression de la grande propriété, mais à son appropriation aux exigences contemporaines. La grande propriété et la petite sont également nécessaires à l'équilibre économique qui naît de la division du sol sous l'effet des lois naturelles; j'en ai donné les raisons. Ce sont les deux terrains extrêmes et corrélatifs de l'exploitation agricole du territoire.

Les publicistes qui condamnent les vastes domaines et ceux qui s'effraient de la multiplication des très petits héritages sont également dans l'erreur; le préjugé, la passion, l'intérêt politique sont pour beaucoup dans les manières d'apprécier les conséquences lu groupement ou de la dislocation possessive des 8068409 parcelles qui forment le territoire imposable, c'est-à-dire cultivé, de notre pays. Il ne faut s'en rapporter qu'aux chiffres, et ne conclure quavec discrétion.

Tandis que les uns voudraient recommeneer une sorte de vente de biens nationaux et démembrer les grandes propriétés en les expropriant pour les vendre aux enchères, théorie analogue à celle qui attribuerait à l'État les successions collatérales pour les liquider comme on fail des concessions aux colonies ", d'autres déraisonnent en sens inverse, se plaignent des frais, des pertes de temps, des servitudes d'une culture trop morcelée el, réclamant comme les premiers le concours de l'État, le convient à ordonner le remembrement d'office des parcelles éparses.

Pour mieux élucider la question, il conviendrait, peut-

1. Journal officiel. Documents parlem., 1891, Chambre, p. $2 \$ 15$. 
être, de modifier le classement administratif de la division du sol et de lui substituer une répartition plus conforme à la réalité des choses, qu'on pourrait appeler le classement économique.

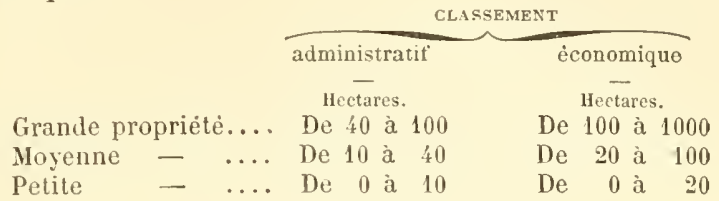

La grande propriété compte 33280 exploitations de plus de 100 hectares et, parmi celles-ci, seulement 4280 de plus de 300 hectares.

Sans l'utile accompagnement de petites et même de très petites propriétés qui lui fait unc ceinture, d'autant plus efficace qu'elle est plus épaisse, a dit M. de Foville ${ }^{1}$, la grande languit, elle n'a plus de main-d'œuvre sur qui elle puisse compler, de débouchés immédiats pour certains de ses produits; elle voit le vide se faire aulour d'elle, elle est obligée de faire venir de loin ses ouvriers et d'envoyer au loin ses récoltes.

La grande culture n'a besoin qu'à certaines époques d'un nombreux personnel, elle le trouve autour d'elle si la population est dense, elle est obligée d'avoir recours à des nomades ou à des étrangers si les villages ne possèdent pas ces groupes de cultivateurs qui, tout en exploitant leur propre héritage, s'offrent volontiers à travailler pour autrui.

La grande propriété, pour satisfaire à ses besoins propres, prend l'initiative de travaux d'intérêt public dont profite toute la région dont elle est devenue le centre d'aclivité. Les routes, les ponts, les véhicules, la circulation qu'ellc provoque, lc mouvement vers les marchés, tout cet ensemble d'avantages dont seraient privés les petits propriétaires sils demeuraient dans leur isolement, constitue une plus-value ambiante qui n'a rien coûté.

1. Le morcellement, 1885. - La France économique, 1859. 
La question des transports présente une double face, et il convient de l'examiner à deux points de vue différents : les débouchés, les frais de transports. Petits et grands y sont intéressés.

Le problème des débouchés conduit à celui de la concurrence. Les progrès de la science, qui, en abrégeant les distances, ont diminué dans une forte proportion les frais de transport, ont modifié du tout au tout les conditions économiques des échanges internationaux. Notre vieille terre française, disent les agronomes, parce qu'elle nourrit depuis plusieurs siècles les populations très denses accumulées sur son sol privilégié, ne rapporte plus qu'à proportion de ce qu'on lui donne. Il faut désormais la nourrir pour qu'elle nourrisse à son tour. Elle se trouve exposée à la concurrence de terres moins épuisées ou de terres vierges qui, à de moindres frais, donnent des rendements triples ou décuples. Les océans rapprochent les marchés, après avoir si longtemps séparé les peuples; et cette nouveauté économique est une de ces forces majeures qu'on ne peut ni discuter ni enrayer ${ }^{1}$.

L'existence des vastes domaines incultes comme on en voyait tant avant 1789, qu'on laissait stériles pour le seul plaisir de la chasse, serail une calamité; ce n'est plus le cas. La plupart des grandes propriétés sont de véritables écoles d'enseignement agricole par l'exemple, et les $3914 \mathrm{mil}$ liers d'hectares de landes qu'elles détiennent ne représentent pas une main-morte absolue mais plutôt une réserve dont l'ouvrier agricole, le prolétaire rural réclame sa part dès qu'il a économisé sur son salaire de quoi faire l'acquisilion d'un lopin de terre, jardin d'abord, puis champ labour, métairie, à mesure qu'il s'est élargi par de petits

1. C'est ici qu'apparait la lutte entre les deux systèmes de la protection et du libre éclıange, et qu'on pourrait discuter les combinaisons mixtes de l'échelle molile et de la loi du cadenas. Mais la thèse qui, par exemple, en taxant le sucre à 60 francs les 100 kilogr., porte l'impôt au double de la valeur commerciale de cet aliment, n'est-clle pas condamnée d'avance? 
achats successifs. Le grand propriétaire a tout intérèt à faciliter cette mise en train de valeurs inertes que féconde le travail. Le mot de M. Paul Leroy-Beaulieu est très juste : L'accroissement de valcur de la petite propriété qui confine et enveloppe la grande crée en quelque sorte le maintien de valeur de celle-ci. Les pelits domaines autour des grands ressemblent, au point de vue économique, à ce que sont, en politique, les petits États neutralisés qui séparent les autres. Quand ils disparaissent, on s'apercoit mieux de la place qu'ils tenaient, des services permanents qu'ils rendaient; la Prusse en a fait la rude expérience lorsque les lois de 1807 et de 1811 permirent aux hobereaux d'aequérir à vil prix les terres paysannes et de transformer les tenancier's en nomades.

Le cri de douleur de Pline : Latifundia perdidere Italiam, retentit encore à travers les siècles comme un lugubre avertissement ${ }^{1}$; mais ce danger a disparu en France. C'est la grande propriété, disent les historiens, qui a perdu les Empires de l'Orient et l'Empire romain lui-même; pourquoi? Parce que c'était l'accaparement, l'immobilisation de la propriété et la stérilisation de la culture, el que le contrepoids de la petite propriété était insuffisant, en raison de l'esclavage et de la fiscalité. C'est la grande propriété qui arrêtait le développement économique de l'Irlande, de l'Espagne, de la Sicile, de la Russie et de l'Autriche. Soit, mais paree qu'elle y était démesurée et que le paysan s'y trouvait réduit à un salaire trop minime pour lui permettre l'épargne et par suite l'acquisition.

Dans tous ces pays, l'évolution économique traduite par des lois favorise et même provoque, comme en Angleterre et en Russie, la formation de cette classe de petits propriétaires qui fit, à toutes les époques, la force de résistance et de production de la France. Là est la preuve de l'utilité, de l'efficacité sociale de la coexistence de ces deux termes extrêmes, la grande et la petite propriété. Leurs conditions

1. Em. de Laveleye (Revue des Deux Mondes, 1867, III, 900). 
de vie et leurs procédés d'exploitation se complètent par leur opposition même; l'une s'accroîl et l'autre se morcelle, toutes deux en profitent sans se nuire.

C'est pour des raisons analogues qu'il ne faut point déplorer comme un phénomène calamiteux le déplacement des populations rurales et leur tendance à se grouper dans les centres industriels, là où les salaires sont plus élevés et le travail plus abondant. Tant que ce n'est que le trop-plein de la population rui s'écoule, on ne peut dire qu’il y ait péril. Il n'apparaîtrait que si le dépeuplement avait pour résultat l'abandon de la culture. D'ailleurs, le seul moyen d'arrêter l'exode, c'est de fixer le paysan au sol par l'acquisition de la terre et de retenir au village celui qui est déjà propriétaire. Les autres, en émigrant, ne rendent-ils pas des services d'un autre genre?

Les agriculteurs tomberaient dans la misère, a-t-on dit ${ }^{1}$, s'il n'y arait plus de grandes villes. Les centres d'activité, les agglomérations industrielles ne sont-ils pas les foyers de consommation des produits agricoles, depuis les œufs, les légumes, les poules, les moutons, les bœufs, jusqu'aux vins et aux fruits? N'esl-cc pas là qu'on tronve le débit assuré d'une quantité de produits destinés à une consommation immédiate et qui cesseraient d'être rémunérateurs s'il fallait chercher des débouchés au loin? Dans les villes, se forment par le mourement incessant des entreprises industrielles el commerciales ces groupements de capitaux qui refluent à un moment donné sur la propriété et sur la culture. S'il n'y avait pas tant d'ouvriers employés dans les fabriques, tant de commis dans le commerce, la banque, les transports, que deviendraient les produits de la terre? Et les résultats de la mévente ne seraient-ils pas destructifs des initiatives agricoles? Cliacun serait réduit à consommer sa propre récolte et à se suffire à lui-même.

Quel profit aurait le cultivateur à voir autour de lui une population plus dense, peu occupée, gagnant peu et con-

1. Roscher, Economie rurale. 
sommant peu? Ce qu'il lui faut à lui, comme à tout producteur, ce sont des débouchés, des marchés aussi rapprochés que possible, et son grand client est, en premier lieu, la population urbaine.

Cetle thèse peut se soutenir, alors surtout que l'emploi des machines, dont l'ingéniosité des constructeurs augmente chaque jour le nombre par quelque variété, supplée à la pénuric de la main-d'œuvre et parfois provoque l'émigration, partout où le travail trouve intérêt à se transformer ou à se déplacer.

On ne peut que méditer à ce propos les récentes paroles de M. Levasseur.

La machine est un puissant générateur de richesse, quand on l'emploie à propos. Propriètaires fonciers, industriels, ingénieurs, vous avez à calculer cet à-propos, et sachez en profiter. Pour vous, ouvriers, la machine est un libérateur; appréciez le rude labeur qu'elle vous épargne et l'accroissement de bien-être qu'elle vous procure ${ }^{1}$.

Il n'est pas de thèse qui n'ait deux faces, pas de fait économique dont les incidences ne puissent devenir détestables après avoir élé avantagouses, et réciproquement. Ce qui ne peut être mis en doute, c'est que la grande propriété est favorable à la grande culture, à l'élevage, à l'emploi de procédés perfectionnés, el qu'au lieu de nuire à la petite propriété, qu'elle n'absorbe pas et qu'elle alimente, au contraire, elle demeure son souticn, sa patronne et son associée naturelle. Les opposer l'une à l'autre serait une faute économique; elles s'entr'aident, et l'une ou l'autre ne pourrait disparaitre sans rompre l'équilibre social.

Les collectivistes, tantôt condamnent la grande propriété parce qu'elle est individuelle, tantôt la prônent, pour le cas où ils la nationaliseraient, suivant leur expression. Mais il ne faut pas confondre la concentration de la culture avec l'agglomération de la propriété. Que 10 à 30 propriétaires contigus, si l'exploitation exige l'emploi d'un outillage

1. Académie des Sciences morales et politiques (Journal officiel du 13 avril 1900, p. 2297). 
coùteux et perfectionné, afferment leurs terres à un seul et mème fermier qui cultivera dès lors 100 ou 300 hectares, cette culture concentrée n'en laissera pas moins intacts les droits individuels des propriétaires; leur abdication n'est que temporaire; elle n’a ni la gravité ni les dangers de celle qui les déposséderait en fait au profit d'une société financière, et ce ne sera pas une forme de la grande propriété mais une simple combinaison de mise en valeur.

\section{I. - Rôle pondérateur et initiateur de la moyenne propriété dans la démocratie française.}

La moyenne propriété, au premier abord, paraît n'être qu'un état intermédiaire entre la grande et la petite propriété, une de ces situations équivoques, effacées, sans relief, qui, ayant les défauts de l'une sans les mérites de lautre, constitue une position d'attente, un objet neutre, sans individualité, sans caractère dans le présent, sans ròle dans l'arenir.

A tout bien considérer, il semble, au contraire, que la moyenne propriété, qu'on prétend inexorablement vouée au triste sort d'être absorbée par la grande et dévorée par la petite, ne soit le centre normal du mouvement foncier, le régulateur de la division du sol, le pivot de l'avenir rural.

Elle ne cesse de s'accroître ${ }^{1}$.

C'est à la moyenne propriété que l'absentéisme a fait le plus de tort; elle appartient pour les trois quarts à des propriétaires forains qui la font exploiter par l'intermédiaire des métayers et des fermiers, et la maintiennent par conséquent, du fait de leur abstention voulue ou forcée, dans un état d'infériorité manifeste, tant vis-à-vis de la grande

1. Le nombre des exploitations de la moyenne culture a suivi le mouvement progressif de hausse ci-après :

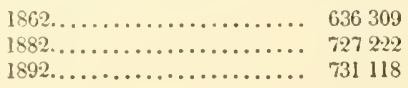


propriété, qui abonde en moyens d'action, que vis-à-vis de la petite, où l'intensité passionnée du travail supplée au manque de capitaux. Et cependant c'est là, parmi les détenteurs de la moyenne propriété, que se rencontrent habituellement à la fois et le eapital, qui fait trop souvent défaut aux propriétaires inférieurs, et le goùt des améliorations agricoles, l'intelligence des intérèts ruraux, que n'ont pas toujours les très grands propriétaires, faute de contact suffisant avec les populations agricoles dont ils ignorent les habitudes, les besoins et le tour d'esprit.

Attribuer à la grande propriété un rôle exclusif serait aussi imprudent que de nier l'influence de la petite propriété parce qu'elle manque de direction et de commandites. Les mobiles du progrès agricole sont variés et complexes; ils se retrouvent tous, à des degrés inégaux, dans les conditions où se erée, se développe ou avorte la propriété moyenne; point n'est besoin d'une révolution sociale pour résoudre le problème.

Malheureusement c'est sur la moyenne propriété que le fardeau fiscal pèse le plus, c'est aussi celle que les filets de la procédure emmaillottent le plus étroitement; elle se débat dans un cercle vicieux. Les entraves légales la gênent dans son expansion, exploitée à demi elle se stérilise elle-même faute de pouvoir produire tout ce qu'elle contient.

Il est une vérité qu'on ne saurait trop proclamer, malgré son apparence paradoxale, e'est qu'en France le droit de propriété, mal assis dans la pratique, livré sans défense aux subtilités de la chicane, est demeuré, comme sous le régime du dernier siècle, le privilège de l'homme riche.

Le propriétaire est exposé à des charges, à des revendications et à des responsabilités qui, déjà très lourdes quand il exploite lui-mème son domaine, le deviennent plus encore quand il se sert d'intermédiaires, et c'est là le cas à peu près général dans la moyenne propriété. N'est-ce pas un argument irréfutable pour décider le moyen propriétaire à tirer parti de son lot territorial par le faire-valoir direct, ou s’il ne le peut, à vendre son bien à de véritables cultivateurs 
plutôt que de le laisser végéter et dépérir entre des mains ignorantes et mercenaires?

Déjà, le propriétaire se voit préférer sur le produit de la récolte, dans les années où la récolte peut couvrir le prix de ferme ou ses propres frais, le fournisseur de semences parce que la récolte n'existerait pas sans les semences (Art. 2102, $\$ 4$, du Code civil); il est question d'étendre ce privilège aux marchands d'engrais, sous le prétexte de moraliser ce genre de commerce et de relever le crédit agricole en lui fournissant de nouveaux gages ${ }^{1}$. On a mème demandé que le fermier participât au droit de propriété et pût réclamer en justice, au propriétaire, à l'expiration de chacune des périodes cle son bail, une indemnité pour ceux de ses travaux de culture qui auraient amélioré le fonds ${ }^{2}$. On a même fixé cette indemnité, sans souci des conditions d'exploitation qui varient comme le sol et comme les individus, aux deux tiers de la plus-ralue qui serait constatée par les experts ${ }^{3}$.

Certes, les dispositions de l'article 1766 du Code civil, relativement aux droits du fermier qui construit ou qui plante sont des plus équitables, mais ne serait-il pas excessif de les étendre à toutes les améliorations que peut imaginer le fermier pour augmenter le rendement de la terre. Et, arec cette porte ouverte à la fantaisie et à la fraude, ne peut-il pas arriver que le fermier ruine son propriétaire sous prétexte de l'enrichir? D'ailleurs, ce serait eréer une nouvelle matière à procès; les sources de conflits ruraux ne sont déjà que trop abondantes; la suppression des occasions de litiges servirait mieux la cause agricole que la généralisation légrale de contestations aujourd'hui très rares.

L'inégalité que la loi crée, entre les propriétaires, suivant

1. Proposition de loi déposée ả la Chambre des députés par M. Míax Lecomte, le 2 décembre 1883.

2. Proposition de loi déposée à la Chambre des députés par M. de Poncheville le 28 novembre 1889.

3. De 1890 à 1899 , il y a eu au Parlement 27 propositions déposées (et restées sans suite) concernant les rapports entre propriétaires, fermiers, métayers et ouvriers agricoles. 
qu'ils sont riches ou qu'ils sont pauvres, a sa répercussion dans les entreprises, compagnies et syndicats autorisés à exercer une industric d'intérêt public au mieux des profits particuliers de leurs actionnaires. Les primes d'assurances contre l'incendie étant, par nécessité, proportionnelles aux risques, sont plus élevées dans les villages que dans les villes; le labourcur, le vigneron, le pêcheur, pour sa masure couverte en chaume, paie 60 fois plus que le rentier de Paris ou de Bordeaux; le meunier de Bourgogne ou des Vosges paie 80 fois plus que le chàtelain de Seinc-et-Oise.

Cela se comprend à la rigueur, car l'incendic au village est moins vite el moins bien combattu qu'à la ville, bien que le résultat soit toujours pareil et que la protection des voisins soit le seul bénéfice sérieux des citadins sinistrés; mais, ce qui est inique, c'est que l'impôt ait pour base la prime, c'est-à-dire le risque. De telle sorte que la taxe établie par la loi de 1871, au lieu d'imposer la richesse frappe le danger, et que deux propriétés, l'une à la ville, assurée pour 200000 franes, l'autre à la campagne, assurée pour 000 franes, paient au taux d'environ 10 p. 100 le même chiffre d'impôts ${ }^{1}$.

L'un des inconvénients de la moyenne propriélé, d'après les agronomes et les spécialistes, c'est moins le fractionnement illimité des parcelles que leur excessive dispersion. Sans recourir à l'expropriation comme en Allemagne, on sait que l'entente des propriétailes peut amener, sans contrainte, des résultats analogues et provoquer avee des frais minimes des groupements rapides et fort avantageux; la loi sur les syndicats permet de procéder juridiquement par des bornages contradictoires et des remembrements généraux dont la portée serait pratique et infiniment préférable à ce que l'utopie administrative cherche vainement à réaliser par la revision cadastrale ${ }^{2}$.

1. La situation est ainsi présentée dans les débats parlementaircs (Journal officiel, mars 1890 , p. 251). De récents remaniements de tarifs l'ont améliorée en une certaine mesure.

2. Pour faciliter le bornage collectif, il suffit d'invoquer l'article 646 
La constitution intégrale du groupe de la moyenne propriété et son développement par l'extension du faire-valoir direct auraient des conséquences considérables au double point de vue économique et social.

S'il arrivait qu'un jour, par suite du perfectionnement des machines agricoles et de leur baisse de prix et, amélioration qui faciliterait l'emploi des machines, par la substitution de domaines d'un seul tenant aux domaines morcelés qui multiplient les pertes de temps et compliquent la main-d'œuvre, l'on économisât un quart du temps actuellement consacré aux travaux agricoles, on aurait augmenté, de ce seul fait, la production nationale de plusieurs milliards de francs ${ }^{1}$.

Le plus large emploi possible de l'outillage agricole est, avec le faire-valoir direct, la condition des plus-values de la moyenne propriété sur ses produits actuels. Tout se tient en éeonomie rurale, ainsi que l'occasion s'est maintes fois retrouvée de le rappeler, de même que l'emploi de certaines machines n'est efficace que sur de vastes étendues, ce qui suppose la suppression préalable par voie d'échange des enclaves et des éearts, ainsi la renaissance du faire-valoir obligera à des constructions, à des achats de cheplel, à des travaux d'ensemble qui exigeront la mise en dehor's de nombreux capitaux. Les syndicals, les banques de crédit mutuel local y pourvoieront.

Il ne faut pas croire que la grande propriété ait le mono-

du Code civil et d'utiliser la loi du 22 décembre 1888. Un cadastre formé de la réunion des procès-verbaux de bornage aurait un parfait caractère juridique et toute la force probante; de plus, il fournirait des références exactes aux titres de propriété et serait comme une souche matérielle du Livre foncier.

1. L'emploi régulier des machines représente au bas mot une économie de 90 journées de travail, par au, pour 10 nillions d'ouvriers ruraux, soit 900 millions de journées à 3 franes l'une qui, employées dans une autre branche de production, donneraient à la population rurale un revenu supplémentaire de 2700 millions. Est-ce qu'ul semblable résultat ne serait pas autrement intéressant que celui qu'on poursuit en demandant pour la propriété rurale des dégrèvements qui, si larges qu'ils soient, atteindraient difficilement 30 ou 40 inillions et ne profiteraient à chaque contribuable que pour une quantité négligeable? (La Finance nouvelle, 21 février 1895.) 
pole de l'élevage, en raison de l'étendue et du capital dont elle dispose. M. de Foville a démontré, dans l'un de ses chapitres les plus piquants ${ }^{1}$ que la très petite propriété ellemème n'était pas exclusive de l'élève du bétail. La moyenne propriété serait appropriée, mieux que la grande et la petite, à ce genre d'industrie par les soins qu'elle y peut apporter et les produits accessoires qu'elle en peut tirer, en procédant toutefois sur une échelle restreinte et sans s'aventurer dans la spéculation.

Il n'y a en Angleterre aucune espèce de vaches qui dépasse sensiblement nos vaches flamandes, normandes, bretonnes, pour l'abondance et la qualité du lait, non plus que pour la proportion du rendement en lait à la quantité de nourriture consommée. L'industrie du lait et des fromages, si ingénieusement conduite en Angleterre où le lait et le thé remplacent le vin, semble naturellement réservée à la moyenne propriété française, tandis que la grande conserverait pour la plus grosse part le monopole de l'élevage des races de boucherie ${ }^{2}$.

Une autre condition de progrès serait, pour notre moyenne propriété réorganisée, la suppression pour le gros bétail du travail de labour el de charroi. Nous avons, en France, deux régions, l'une où le gros bétail travaille, l'autre où il ne travaille pas; sa valeur par tète est bien plus élevée dans la seconde que dans la première.

La substitution des races de laiterie et de boucherie aux races de travail ne peut s'improviser et exige un certain apprentissage. Je ne fais aucun reproche aux cultivateurs qui font traîner par des bœufs et même par des vaches leurs charrues et leurs chariots, disait un de nos éminents agronomes, je ne conseille aucune transformation brusque et

1. Le Morcellement, chapitre IX (La petite propriété et le bétail).

2. M. L. de Lavergne ]e conseillait déjả en 1833, prouvant que, dans des conditions semblables, mais avec des soins mieux compris, la vache anglaise donnait par an 1000 litres de lait, dont $1 / 3$ pour les veaux, et la vache française seulement 500 litres, dont $1 / 2$ pour les veaux; d'où, perte sur l'élevage et perte sur la rente. 
irréfléchie; je me borne à constater ce qui est; par le seul fait de l'abandon complet du travail par les boufs, le sol britannique, mème y compris l'Écosse et l'Irlande, est arrivé à un produit double du nôtre pour le gros bétail. Telle est en agriculture la puissance d'une idée juste, quand il est possible de l'appliquer.

La substitution des chevaux ou des mulets aux bœufs pour le travail aurait de plus cet avantage, inappréciable au point de vue de la défense nationale, de repeupler nos provinces et de reconstituer nos races chevalines trop négligées depuis les chemins de fer et les cycles, en même temps que nos jeunes gars se réhabitueraient à l'exercice quotidien de l'équitation. Nos Percherons, nos Limousins, nos Bretons, nos Béarnais, nos Barbes d'Afrique sont des races précieuses et qu'il serait aisé de multiplier.

Voilà donc quelques-uns des horizons nouveaux qui s'ouvriraient à l'initiative des propriétaires de la nouvelle propriété moyenne s'ils se décidaient à utiliser les ressources qu'ils laissent sommeiller dans son sein. Il en est beaucoup d'autres. C'est comme une immense et féconde colonie dont on viendrait de faire la découverte, et quelle entreprise serait plus facile et plus rémunératrice que celle-là, quelle spéculation plus sûre? En est-il de plus honnête, de plus légitime, que d'acheter pour améliorer el d'améliorer pour revendre?

Quant à ceux qui, déjà propriétaires, ne cherchent pas à faire le commerce des terres mais à exploiter leur héritage, à accroître son revenu, à enrichir le foyer domestique pour qu'il reste l'abri de la famille et le centre d'où ses rejetons germeront, combien leur tâche est plus intéressante et plus enviable!

En somme, la fixité de l'héritage est la règle, ou du moins devrait l'être, car si profitable que paraisse, à un examen superficiel, l'activité du marché foncier, rien ne vaudrait le maintien de la propriété dans les mêmes mains. C'est parfait, surtout en ce qui concerne la très petite propriété, si l'on ne considère que les acquisitions; mais ces acquisitions ont une contre-partie, les ventes, et l'accession de tel ou tel 
prolétaire au premier échelon de la propriété correspond trop souvent à la dégringolade, qu'on me permelte ce mot, de tel ou tel qui a trop compté sur ses forces ou que la mauvaise chance à poursuivi.

L'obligation de vendre, dans la propriété moyenne, est une conséquence normale de la dette agraire; c'est l'impôt de mutation qui, bien souvent, contribue à former la lourde dette de la propriété foncière, parce que, afin d'éviter une perte, on préfèrc emprunter que de vendre, et c'est ainsi que les deux maux conduisent au déboire final. Malgré tout, la passion de la propriété est si naturelle aux Français, et l'effort qui, dans les plus néfastes conjonctures, les fait se cramponner au sol ct résister à l'orage, est si passionné que les mulations sont moins fréquentes qu'on ne le croit.

D’après un document fourni par le service des Contributions directes à la Commission du cadastre ${ }^{1}$, on trouve que, pour une période de vingt années, de 1876 à 1896, sur l'ensemble des propriétés foncières rurales, le mouvement des mutations se proportionnalise comme suit :

Propriétés n'ayant pas changé de mains...... 34 p. 100

Ayant été l'objet d'une seule mutation...... 48 -

Ayant été l'objet de 2 mutations.......... 12 -

Ayant été divisées à la suite de partages ou

fractionnées par lotissement 2 .............

La durée, la sécurité sont les conditions premières du progrès agricole, qui s'alimente de travaux à long terme, de préparations à échéances lointaines. La création de domaines nouveaux, si petits soient-ils, est chose excellente; mais, pour les anciens, sauf des cas exceptionnels, moins ils changent de mains et mieux ils profitent.

Au point de vue social, le maintien et mème l'accroissement indéfini d'un groupe intermédiaire de propriétés

1. Séance du 12 janvier 1898.

2. D'après un relevé officiel fourni le 18 février 1895 à la Commission, on n'aurait guère, dans la plupart des communes rurales, que 5 à 6 fractionnements d'îlots par année. - Voir Annales de l'Enregistrement 1898, p. 274 . 
n'est pas moins désirable, en raison des conséquences multiples qui s'en dégagent. Un homme d'Élat anglais ${ }^{1}$ s'en est expliqué en termes quil faut retenir, car, tout en s'applaudissant de la multiplication des petites fortunes et en laissant pressentir les lois qui suivirent pour créer la classe des très petits propriétaires ${ }^{2}$, M. Goschen insiste sur la puissance économique el sociale que représente la classe intermédiaire.

Je me propose, dit l'éminent orateur, de vous entretenir de la multiplication, en Angleterre, depuis quelques années, des fortunes moyennes (moderate fortunes) et des petits capitalistes (small investors), en même temps que des symptômes qui semblent indiquer qu'il y a moins qu'autrefois tendance à l'accumulation de richesses considérables dans un petit nombre de mains.

Je voudrais montrer combien s'élargissent les rangs inférieurs de la classe moyenne dans notre état de clioses économique, social et même politique. Sans insister sur ce dernier point, je crois quaucun parti ne se formalisera si je dis qu'un Etat ne peut que gagner à voir la masse centrale de la nation se développer numériquement; et je vondrais, ici, rechercher dans quelle mesure ce développement s'effectue chez nous à l'époque actuelle.

Qu'il y ait ou non accroissement dans le montant total des profits, il est certain que la diffusion en devient de plus en plus large entre tous ceux qui se livrent au commerce, à l'industrie, et même à l'agriculture, et que si les grands eapitalistes voient leurs revenus fléchir, ceux qui gagnent de l'argent, fût-ce à petite dose, se sont considérablement multipliès.

Les statistiques produites par MI. Goschen sont des plus suggrestives, je n'en retiendrai qu'un exemple tiré du nombre croissant des maisons d'habitation taxées (au-dessus d'une valeur locative de 20 livres ou 500 franes).

De 1873 à 1886 , le nombre des maisons d'une valeur localive de 30 à $\check{00}$ livres (de 750 à $12 \% 0$ francs) a passé de 393881 à 5̃9' 787 ; les catégories au-dessus ont peu varié, et de moins en moins à mesure que le prix locatif s'élevait. Quant aux petites maisons non imposées, l'augmentation

1. Discours de II. Goschen, chancelier de l'Échíquier, en prenant possession de la présidence de la Société de statistique de Londres, le 6 décembre 1887.

2. Loi du 27 juin 1892 sur la constitution de petits domaines agricoles (small holdings). 
proportionnelle, de 1873 à 1886 , a été de 58 p. 100 pour celles de 10 à 10 livres, de 50 p. 100 pour celles de 13 à 20 . Pendant la mème période, la cote de l'income taxe a augmenté de 21 p. 100 pour les fortunes moyennes, diun revenu de 1000 à 000 livres sterling, et a baissé pour toutes les autres catégories supérieures de la cédule D. M. Goschen conclut ainsi :

Alors que certaines gens réclament à grands cris la reconstitution artificielle de la société sur une base socialiste, une sorte de socialisme latent fait son chemin. La richesse tend à se répandre en surface, et, à quelque point de vue qu'on se place, on ne peut qu'en éprouver une joie patriotique. On n'a pas employé pour créer ce mouvement des spécifiques violents. Le travail constant des lois économiques, sous un régime de liberté commercialc et industrielle. amène le résultat que j'ai décrit. Nous le constatons de la façon la plus claire dans la classe moyenne, et aussi, bien qu'à un degré moindre, dans le haut de la classe ouvrière.

Cette influence pénètre la société en suivant un mouvement descendant. Espérons que ce progrès continuera et finira par arriver à cette grande masse des populations ouvrières qu'il n'a pas encore atteinte et qui, si sa position s'est quelque peu adoucie, grâce à la baisse des prix de ce qu'elle achète, n'a cependant pas obtenu sa part de la richesse accumulée. Le grand avantage de ce socialisme automatique, c'est qu'il opère même en temps de crise.

Il en est de mème en France, on se plaint du chômage, de la rigueur des temps, de la peine qu'on a à gagner sa vie et, malgré cela, la masse centrale de la nation, selon l'expression de M. Goschen, voit sa situation économique s'affermir de jour en jour.

Si les statistiques des mutations foncières et celles des hypothèques étaient établies par le ministère des Finances de manière à permettre de faire pour la répartition des propriétés rurales ce que M. Neymarck a réalisé pour se rendre compte du morcellement des valeurs mobilières et de la condition sociale des groupes qui les possèdent, on verrait que notre classe moyenne française est déjà fort riche mais que, surtout en ee qui concerne sa part dans la fortune territoriale du pays, elle n'utilise pas toutes ses ressourees. Se bornât-elle à mettre en valeur les 1367 milliers d'hectares enclavés dans ses héritages et qu'elle laisse en friche, 
elle augmenterait sensiblement son capital foncier et n'aurait rien à envier aux bourgeoisies rurales d'Angleterre, de Belgique, de Hollande, de Suisse qui font la force de ces contrées comme notre peuple de travailleurs économes fait la puissance de résistance de la France.

En s'engageant dans un ordre d'idées parallèle et dont l'étude est plus étroitement liée à notre sujet qu'on ne le croit communément, il serait facile d'établir que notre pays, plus et mieux que tous les autres, possède les capitaux nécessaires à l'exploitation scientifique et intégrale de son territoire ${ }^{1}$.

Il est acquis que la possession de la terre, la création d'un chez-soi, est le dernier terme des combinaisons de l'homme qui a conscience de sa dignité personnelle, qui se sent, se sait et se veut libre. Pour les riches, c'est un luxe; pour les autres, c'est une nécessité, si tel est leur métier, ou le but de leur's efforts à travers les labeurs de l'atelier ou du bureau, assujettis à la traversée lente, pénible et trop souvent infructueuse du salariat.

La propriété en soi, terme unique de la plupart des ambitions humaines, lorsqu'elles raisonnent et, en vue de la sécurité ou du progrès ascendant de la famille, cherchent à fonder un héritage stable, est l'indispensable base sociale. Les Physiocrates du xvine siècle, véritables fondateurs de l'économie politique, avaient raison de faire de la terre la source principale de la richesse, ils eurent tort de nier la plus-value qu'y apportent l'industrie et le commerce. La théorie de Quesnay ne vaut que par le commentaire de Turgot; elle ne se développe que par les contingents qu'y apportent Bastiat, Proudhon, Le Play.

Mais la forme que prend la propriété suivant l'air ambiant et les conditions qui la restreignent ou l'élargissent, dépend de la rolonté de celui qui l'acquiert ou la met en valeur, et surtout de ses possibilités d'action, c'est-à-dire des res-

1. A. Neymarck, La Statistique internationale des valeurs mobilières, 1897. 
sources dont il dispose. L'utilité pratique de la propriété, toujours relative, est subordonnée à ses procédés d'exploitation, à son appropriation plus ou moins étendue à des besoins immédiats ou contingents, suivant que le cultivateur lui demande seulement le pain quotidien ou cherche, par surcroît, à en tirer un gain qu'il puisse épargner pour en faire la source d'acquisitions nouvelles et, par elles, de profits accrus. De là cette répartition de la propriété en lots plus ou moins vastes, appropriés aux réserves du possesseur ou à sa puissance de travail et qui sont assujettis à l'imprévu d'une perpétuelle mobilité.

De là des groupes qui, sous l'influence de nécessités semblables, obéissant à des exigences pareilles, finissent par prendre un air de famille, tout en se différenciant par des particularités sans nombre; de là cette classification arbitraire que la méthode scientifique impose et qui donne à ces groupements naturels les noms de grande, moyenne et petite propriété.

De là encore cette conséquence qu'il faut multiplier les distinctions pour établir, avec l'approximation la plus rapprochée de la vérité qu ill soit possible, le rôle particulier de chacune des grandes catégories de la division de la propriété dans l'harmonie sociale. Ce n'est qu'en les étudiant sous toutes leurs faces, en s'arrètant à tous les points de vue, qu'on peut déterminer le caractère des entraves qui en gênent le développement régulier.

Ces entraves elles-mêmes varient à l'infini, non seulement pour la grande, la moyenne et la petite propriété, mais dans chacune de ces catégories, suivant les conditions différentes qui en modifient l'état, en raison des particularités de fait ou d'opinion, des influences morales et des courants économiques de nature à affecter, dans tel ou tel sens, telle région plutôt que telle autre, à ralentir ici le progrès et à le précipiter là-bas, sans qu'il en apparaisse de raisons immédiatetement appréciables.

Et ici, c'est moins la qualité productive de la terre, ou l'atmosphère économique, ou le nombre des débouchés qui 
pèseront sur le produit du sol et fixeront le bénéfice net du propriétaire cultivateur, que son revenu personnel, que sa condition relative de riche ou de pauvre, tant est vrai le proverbe agricole dans sa mélancolique résignation : L'eau va toujours à la rivière.

Un exemple suffira pour éclaircir ma pensée. Le coût de la vie matérielle est la grosse dépense des petits budgets. D'après les slatistiques les moins pessimistes, il absorbe environ les trois cinquièmes des ressources dans les foyers où l'on a pour vivre moins de 2500 francs par an, c'est-àdire dans quatre familles françaises sur cinq.

Au lieu d'absorber pour sa nourriture 60 p. 100 de son salaire ou de son revenu, comme le ménage ouvrier ou le ménage paysan, le ménage bourgeois, rentier, industriel, commerçant, qui possède 10000 livres de rentes, ne consacre à cet objet que $3 \check{\text { ou }} 40 \mathrm{p}$. 100 de sa dépense. Plus on s'élève parmi les couches aisées ou riches de la population, plus l'écart augmente entre le coùt de la vie matérielle el la part du salaire ou du revenu disponible pour d'autres emplois. Les plus favorisés de la fortune n'ont qu'un estomac comme le rouleur des quais, disait l'Amérieain George; quels que puissent ètre son luxe et sa prodigalité, il ne dépensera pas pour vivre plus de $1: 3$ p. 100 de son revenu, et tout le reste vient grossir le chiffre de sa richesse acquise et accumuler dans ses mains de nouveaux instruments de fortune.

Toutes proportions gardées, de deux propriétaires cultivateurs, dont l'un dispose sur son revenu de 40 p. 100 et l'autre de $80 ̈$ p. 100, quel est celui qui pourra le mieux accroìtre son cheptel, fumer ses terres, élargir son effort?

Les considérations économiques et philosophiques sur l'état agricole, sur la division du sol, sur les allénuations qu'il serait désirable d'apporter à certains malaises dont l'acuité n'est pas contestable, ne peuvent done s'appliquer uniformément ni à un pays, ni à une région, ni à l'ensemble des propriétés rurales. Ce qui est exact dans les Flandres ne l'est plus en Provence, ce qui est vrai dans les plaines du Languedoc ne l'est plus dans les vallées des Alpes ou sur 
les plateaux du Larzac et de la Margeride; ce que désire le grand éleveur de la Nièvre n'est pas davantage ce que souhaite le gros fermier de la Beauce, le maraîcher du Vaucluse et le vigneron du Beaujolais. Mais, si le détail échappe à une appréciation synthétique, il est permis de critiquer ce qui frappe, inversement à leur valeur, les sources du travail agricole, et d'examiner les iniquités légales ct les abus de la fiscalité.

I. Le Travail et le Capital. - Le travail est, après la religion, le mobile qui élève le plus sûrement l'humanité vers l'ordre moral. C'est un moyen d'éducation en même temps que la source unique du bien-être matériel ; et si je dis unique c'est que l'époque approche où non seulement l'oisiveté sera considérée comme un vice troublant l'harmonie sociale mais où les nouvelles conditions économiques feront que nul désormais n'aura les moyens de vivre s'il ne cherche pas sa subsistance dans un salaire.

Quant au eapital, Frédéric Bastiat en a donné la définition la plus exacte dans sa concision : c'est le blé du travail. Cela signifie, en mème temps, que le capital alimente le travail et qu'il en est le produit. M. Frédéric Passy, à son tour, a condensé en quelques mots ${ }^{1}$ les définitions variées que les économistes ont données de ce mot: Le capital ne consiste pas uniquement, ni principalement en espèces. Il ne vaut qu'autant qu'il produit. C'est l'arbre qui donne des fruits et qui n'est bon qu’à brûler sill n'en donne plus. C'est le champ qui porte des récoltes; c'est la charrue qui ouvre le sein de la terre; e'est le grain que le laboureur confie à cette terre pour qu'elle le lui rende l'année suivante avec accroissement; e'est tout ce qui, sur les résultats du travail d'hier, a été réservé pour rendre plus facile et plus fructueux le travail de demain. C'est le produit net de la vie humaine, l'excédent de son compte de doit et avoir. Et Cobden avait dit, vingt-deux ans plus tôt ${ }^{2}$ : Le capital n'est autre chose que clu travail accumulé; le travail est la semence du capital.

1. Journal des Économistes, juillet 1883.

2. Discours à Rochdale, 26 juin 1861.

LA PROPRIÉTÉ RURALE EN FRANCE. 
Ils sont donc bien ignorants ou bien coupables les hommes néfastes qui désorganisent les conditions de la liberté du travail en supposant entre le travail et le capital un antagonisme d'opinion qui, sül existait dans la réalité, serait en mème temps la négation de l'un et de l'autre.

Le capital et le travail ne peuvent se faire concurrence ni se nuire parce que, quoiqu'ils soient solidaires l'un de l'autre, ce ne sont pas des facteurs du même ordre, el, s'engendrant alternativement l'un l'autre, ils s'entr'aident au lieu de se contrarier. Le travail est un effort, le capital est un résultat.

Puis, capitaliser, c'est utiliser des épargnes pour la production ${ }^{1}$. Le capital est le produit de l'effort antérieur, et le travail, qui représente l'effort actuel, serait le plus souvent désarmé s`il ne pouvait pas faire appel aux capitaux d'autrui, à défaut des siens.

Cela est vrai pour toutes les industries, et l'industrie agricole est la première, la plus répandue et la plus néccssaire de toutes.

Le problème à résoudre consiste donc à rapprocher le capital, ou la richesse acquise, du travail, c'est-à-dire de la richesse en roie de formation, et rien ne devrait être plus aisé que dans l'industrie agricole où le capital et le travail se côtoient sans cesse, sous leur forme la plus condensée, soit qu'ils s'échangent à tout instant, soit qüils existent dans les mêmes mains. En les opposant l'un à l'autre sous le prétexte qu'ils n'ont pas le même champ d'action, on commet la même erreur que lorsqu'on compare les campagnes aux villes, en reprochant à celles-ci de faire déserter celles-là. La pratique de la vie, le déplacement démographique, la désertion rurale si l'on veut, ne comportent pas des situations aussi tranchées; en réalité, beaucoup de gens cessent d'appartenir à la classe agricole sans devenir des citadins; de même qu'il y a des groupes industriels dans des villages, ainsi l'on trouve dans toutes les villes de province, même les plus peuplées,

1. Levasseur, Précis d'économie politique, 1886, p. 67. 
de véritables paysans, vivant de la culture du sol. Beaucoup de propriétaires fonciers sont en même temps des capitalistes; n'est-ce pas à eux, plutôt qu'à des syndicats de spéculation, sans attaches avec le fonds territorial, qu'il conviendrait de commanditer le laboureur ou le vigneron qui vit autour de lui et travaille souvent à ses gages?

Le phénomène qui permel aux villes de drainer les campagnes n'est point particulier à la France, on le signale en tous pays, et les causes de la fascination de la ville sur la naïre ambition paysanne sont multiples. En dehors des causes générales, vraies il y a cent ou deux cents ans comme elles le sont aujourd'hui et qui expliquent en partie le goût pour le changement de profession, d'où résultent la désertion des métiers agricoles et l'affluence dans les centres, il est d'autres facteurs dont la simultanéité a précipité l'évolution qu'on déplore. Ces éléments nouveaux d'activité, ferments d'une société en fusion, sont la diffusion de l'instruction, l'état d'âme particulier à la démocratie qui en est la conséquence logique, c'est-à-dire l'instinct de progression professionnelle, la multiplication des points de contact et de comparaison par la rapidité et le bas prix des transports, la science appliquée ou l'emploi des machines, la vulgarisation du bien-être, enfin la rupture de lisolement économique se traduisant par des concurrences lointaines qui aboutissent à la mévente des produits.

Depuis $187 \%$, le prix du blé a baissé de 40 p. 100; il est vrai que l'agriculture n'est pas vonée fatalement à la production d'une denrée unique et que, le blé coûtant plus qu'il ne rapporte, il lui reste d'autres emplois de sa terre et de son travail. C'est toutefois un changement considérable, pour les terres à labour, que de voir tomber à rien le blé, considéré depuis des siècles, en France, comme le rendement type d'une ferme, et la moins-value subite qui en résulte dans la valeur vénale du sol provoque une crise dont les effets réflexes sont nombreux. La routine ou le respect des traditions et des usages, souvent synonymes, ne permettent pas d'évoluer brusquement en matière agricole; il faut pour 
se résigner à changer la barre de droite à gauche, l'expérience, la réflexion, le temps, une compréhension plus vive de l'imprévu contemporain, une certaine hardiesse d'esprit que nos paysans n'ont pas encore, pas plus d'ailleurs que les paysans de Saxe ou d'Italie.

En Allemagne, depuis dix ans, le prix du sol a baissé de 200 p. 100 pour les grands domaines, de 150 p. 100 pour les petits; dans la régence d'Erfurt, la moins-value a atteint 40 p. 100 et le prix des gros fermages a diminué de 50 p. 100 avec une moyenne de 30 à 33 p. 100 pour les autres ${ }^{1}$. N'avonsnous pas vu une crise aussi violente atteindre de 1880 à 1890 , nos grandes fermes du Nord, du Pas-de-Calais et de la Somme? La crise du phylloxéra n'a-t-clle pas, un instant, réduit à rien, de 1882 à 1894, nos riches vignobles du Midi et de l'Ouest? cela seul ne suffirait-il pas à motiver la désertion des campagnes, le peuplement industriel des villes aux dépens du travail agricole qui se raréfie quoique, par une incidence dont la contradiction est rassurante, il se paie plus cher ${ }^{2}$.

En Belgique, la proportion de la population agricole à la population totale est tombée de 23 p. 100 en 1846 , à 22 p. 100 en 1880 , à 16 p. 100 en 1898. En Suisse, de $ّ 0$ p. 100 en 1848, à 42 p. 100 en 1888. En Angleterre, de 49 p. 100 en 183̈1, à 40 p. 100 en 1880, à 37 p. 100 en 1890. En Allemagne, où le développement industriel est si remarquable depuis trente ans, elle décline de 64 p. 100 en 1872 , à 56 p. 100 en 1885 , à 53 p. 100 en 1890, à 49 p. 100 en 1898.

Certes, l'équilibre est rompu entre les centres urbains et les groupements ruraux, mais on aperçoit un temps d'arrêt dans le mouvement; il semble qu'il y ait flottement; certains indices font supposer quil se dessine un mourement con-

1. G. Blondel, Enquêle sur les populations rurales de l'Allemagne, 1897.

2. En soi, le phẻnomène de lıausse des salaires agricoles, qui s'accentue depuis plusieurs années, n'a rien que de normal et on peut mème s'en féliciter, il fait partie d'un mouvement plus ample qui tend à l'amélioration de la condition des travailleurs dans leur ensemble. (L. Wuarin, Revue des Deux Mondes, 1900, juin, 873.) 
traire, et cette tendance de retour aux champs, également sensible chez les paurres et chez les riches, provoquée par l'encombrement des carrières, par la cherté des villes, ne pourra que s'accentuer après la crise de désorganisation du travail que nous traversons, crise due à l'exploitation par les politiciens de la loi du 21 mars 1884. L'abus des grèves en effet, si maladroitement employé au cours des années 1899 et 1900, ne peut que décourager les capitalistes par la ruine des industries qu'ils commanditaient; nos ateliers seront forcés de fermer, leurs profits passeront à l'étranger, les ouvriers sans ourrage se rejetteront sur l'agriculture et lui infligeront, pour d'autres causes, une crise nouvelle et plus aiguë.

Cette réaction probable, ce flot énorme de revenants dont il est permis de préroir l'intrusion à bref délai dans les conditions actuelles de la vie agricole qu'ils troubleront en y rentrant beaucoup plus profondément, peut-être, qu'ils ne le firent par leur émigration, ne constituerait un danger réel que s'il nous surprenait sans qu'on l'eût prévu. Un des moyens de l'empêcher de nuire serait de le diriger sur notre empire colonial; mais est-ce possible avec nos procédés de colonisation?

C'est à l'occasion de ce problème qu'apparaît le vice initial de vouloir tout réglementer d'après les mêmes principes et avec une fâcheuse uniformité, lit de Procuste qui tasse ou écartèle, toujours à l'inverse de ce qu'il faudrait, l'IndoChine n'ayant pas les mœurs de la Martinique, ni la Tunisie les préjugés du Soudan. Ce vice a particulièrement influencé, en France, le régime de la propriété. Il est imprudent, dans une vieille nation, de substituer en toutes choses les prescriptions d'une loi radicale aux coutumes traditionnelles résultante logique de besoins et d'habitudes, et de contraindre les intérèts les plus opposés à subir l'uniformité et la symétrie. Si nécessaire que soit l'unité de législation, il y a, en matière économique, des compromis auxqucls il faut se résigner. L'une des méprises administratives qui ont le plus retardé le progrès agricole est celle, notamment, d'avoir 
assimilé les communes rurales et les agglomérations urbaines et d'avoir confondu en les assujettissant à des règles identiques des intérêts qui, chez tous les peuples et dans notre propre tradition, ont toujours été non seulement différents, mais indépendants l'un de l'autre.

Parmi les faits qui exercent l'influence la plus immédiate sur le mouvement foncier, sur la répartition de la propriété et le déplacement incessant des fortunes agricoles, surtout dans les catégories de la moyenne et de la petite propriété, il faut signaler l'exercice des monopoles et l'emploi des machines.

La division du travail est une des lois générales de l'humanité laborieuse. Chaque homme, s'il était réduit à son champ et à son travail pour se pourvoir de tout, a dit Turgot, consumerait beaucoup de temps el de peines pour être très mal équipé à tous égards, et cultiverait très mal son terrain. 11 faut, en effet, des laboureurs, des charpentiers, des sabotiers, des forgerons, etc. Mais les progrès de l'industric ont introduit dans chaque métier la division infinie du travail, pour fabriquer chaque pièce mieux et plus vite, de telle sorte que l'ouvrier de précision, par exemple, ne fait plus une montre mais un des rouages de cette montre; il tombe de l'état d'horloger à la condition de simple auxiliaire. Cette distribution entre ouvriers spéciaux des diverses parties d'un même ouvrage a celle conséfuence commerciale d'abaisser le prix de revient, chaque collaborateur arrivant ainsi à produire en se spécialisant des résultats de beaucoup supérieurs à ceux qu'il obtenail avec la même somme d'efforts répartis au lieu d'être concentrés. Mais le travail parcellaire est une occupation d'esclave; l'ouvrier enchaîné à un labeur spécial ne créc plus, il devient un instrument, aveugle et sourd. Comment résoudre le problème? Trop peu divisé, le travail reste improduclif; trop divisé, il abrutit l'homme.

La science intervient et le résout par la machine. La voltige industrielle, selon le mot de Proudhon, faisait de l'ouvrier un artiste mais diminuait son salaire; l'emploi de la 
machine supplée à l'effort continu et toujours identique qui rétrécit l'intelligence en l'emprisonnant dans l'étroitesse d'un cercle de plus en plus dininué; la machine est le symbole de la liberté humaine, de la domination de l'esprit sur la matière. Maudire les machines à cause du trouble qu'elles apportent dans les habitudes, disait Bastiat, c'est maudire l'esprit humain. M. Levasseur a démontré comment elles diminuent l'effort musculaire, abrègent le travail, réduisent l'effectif des ateliers, augmentent la production, élèvent le salaire en exigeant pour les conduire des ouvriers plus instruits, ayant l'esprit ouvert et de l'initiative. La science des ingénieurs commence à transformer les industries agricoles comme elle l'a fait pour l'industrie proprement dite.

L'atténuation du gros travail, de celui qui exige surtout l'emploi de la force physique, labour, fenaison, moisson, vendange, battage, coupe des bois et scierie, aura sa répercussion immédiate sur le retour aux champs et une répartition nouvelle de la propriété, en rendant le travail rural abordable pour quantité de jeunes hommes instruits, à l'aise, actifs, qui n'avaient ni la santé ni l'endurance qu'exigeaient, en toute saison, les rudes el lents labeurs de la campagne, singulièrement abrégés et allégés par lingréniosité pratique des constructeurs.

Le perfectionnement des appareils, l'économie qu'ils apportent, leur vulgarisation par les syndicats, leur appropriation aux plus petites exploitations par les combinaisons de la mutualité, apporteront un rapide changement dans la condition des ouvriers agricoles et des propriétaires cultivateurs, et par voic de conséquence dans laccession du prolétaire rural à la propriété. Les considérations philosophiques sont d'accord avec les considérations économiques pour voir l'avenir de l'Agriculture, c'est-à-dire l'attrait croissant de la propriété rurale, intimement associé au progrès intelleetuel et moral des cultivateurs, quelle que soit leur place dans l'échelle sociale. Petits et grands ont tous à s'améliorer, et c'est de leur entente de plus en plus éprouvée que sortira laccord définitif du capital et du travail; l'écart est 
trop tranché dans les villes, et l'on y perpétuera longtemps encore, pour l'exploiter, une équivoque qui disparaîtra dans le commun effort de culture lorsque le propriétaire, au milieu des populations rurales, trouvera plus honorable et plus profitable de se mêler à la vie des champs que de toucher son revenu par intermédiaire.

C'est l'apathie des classes laborieuses qui engendre leur misère; l'élévation du taux des salaires ne suffit point pour rompre le cercle fatal où se traîne leur vie. Le bien-être sans éducation abrutit le peuple et le rend insolent, a dit la sagesse antique: Incrassatus est, et recalcitravit. Il faut que le développemeut successif de leurs facultés intellectuelles et morales, par une saine instruction, leur inspire l'intelligence, l'énergie, le vouloir du mieux d'où naissent ensuite, simultanément, l'émulation et la dignité de soi.

C'est à ce rôle d'éducateurs par l'exemple, par les leçons de choses, par le contact personnel, que semblent destinés les détenteurs de la moyenne propriété, plus efficacement que ceux de la grande.

Les monopoles, non moins que la misère dans l'ordre matériel et l'ignorance dans l'ordre moral, figurent parmi les entraves qui gènent le développement régulier de la propriété et le jeu libre de sa répartition normale entre les différentes catégories de propriétaires fonciers. Il y a, dans l'industrie agricole, des monopoles de toutes sortes, depuis celui du crédit jusqu'à ceux des transports et de la vente.

Au début des civilisations, le monopole a été le pivot autour duquel s'agitent et circulent les divers éléments économiques ${ }^{1}$. C'est un moyen ; son caractère est essentiellement d'être précaire; et, à moins que l'État ne s'en cmpare et ne le rende participant de sa propre fixité, relative ellemême, il est censé ne durer qu'aulant que son titulaire sait l'exploiter el le défendre ${ }^{2}$. Le privilège de gain qui est l'essence du monopole lui ôte tout caractère personnel et

1. Proudhon, Contradictions économiques, II, chap. xl (La propricté).

2. Kant, Fondements de la métaphysigue des mœurs, 1785. 
c'est l'un des traits qui ont contribué à discréditer le eapital aux yeux des travailleurs. Les monopoleurs, disait-on hier ${ }^{1}$, dévorent la substance des enfants des hommes sous prétexte de leur donner du travail; ils exigent des autres l'effort et s'attribuent le profit; c'est la mise en œuvre universelle du Sic vos, non vobis. Mais le bénéfice de l'usurpation est éphéinère et contient le germe de son propre anéantissement.

Le premier effet du monopole est le renehérissement des objets. Il faut des courtiers, dans la vie éeonomique, il est indispensable que des intermédiaires avisés et actifs rapprochent le consommateur du producteur, il est juste qu'ils prélèvent un honnête courtage; mais l'abus derient insupportable lorsque le courtier abuse de sa situation et se fait accapareur, s'attribuant un bénéfice léonin au lieu de le débattre loyalement, et fixant les prix à son caprice au lieu de les laisser varier suivant la loi de l"offre et de la demande. On a ru les fàcheux résultats de eette tyrannie de la spéeulation dans le syndicat des métaux, dans le syndicat des raffineurs, etc., qui écrasent toute concurrence par le groupement irrésistible de leurs capitaux. C'est une des plaies de l'agriculture.

LAmérique est la terre privilégiée de ces Trusts ${ }^{2}$. En France, la question des tarifs de transport exerce une influence de premier ordre, et, malgré le contrôle de l'État, l'interprétation des compagnies peut ourrir ou fermer des débouchés pendant assez de temps pour favoriser un coup de bourse sur les blés, les vins, le bétail ${ }^{3}$. C'est surtout en matière de crédit que le monopole devient onéreux. Lorsque des compagnies puissantes, gràce à des privilèges juridiques exorbitants, drainent les eapitaux de la petite épargne pour

1. Ernest Brelay, Revue économique de Bordeaux, novembre 1897.

2. E. Lerasseur, L'agriculture aux États-Unis, p. 361.

3. Le Journal des Chambres de commerce (1899) rappelait récemment que l'ennemi du travailleur n'était pas le capitaliste mais l'intermédiaire. Lorsque le capitaliste obtient à peine une rémunẻration de 3 à 4 p. 100 , le parasitisme industriel prélève de 50 à 100 p. 100 sur le producteur et le consommateur. 
les prèter aux propriétaires fonciers, dans des conditions dont le long terme n'est qu'une fiction ${ }^{1}$, elles ne font pas œuvre salutaire; le résultat de leurs combinaisons, au lieu d'aider de leurs capitaux le véritable propriétaire-cultivateur, est de provoquer la spéculation et de surexciter les appétits par l'appât des valeurs à loís et du gain facile. Étreint par les forces convergentes de monopoles qui s'engendrent les uns par les autres et offrent, par leur coalition intéressée, une puissance de résistance inébranlable à tous les essais de réforme, le propriétaire rural est voué à la ruine. Il est écrasé, vidé, anéanti, entre le monopole du crédit qui ferme devant lui loutes les caisses sauf une, et le monopole de la procédure et des formalités que lui impose le monopole du crédit par l'absolutisme de ses règlements ${ }^{2}$, lesquels l'épuisent par les frais accessoires.

Le crédit hypothécaire exploité par les collectivités de spéculateur's n'est plus qu'un enjeu anonyme. L'homme ne tient plus au sol, il ignore quel est son créancier comme celui-ci n'a nul souci de ce que peut être son débiteur. Le gage lui-mème n'est qu'une fiction.

Sa valeur intrinsèque et sa garantie morale disparaissent également dans cel anonymat universel qui transforme l'ensemble de milliers de contrats individuels en une vaste assurance mutuelle garantie, non plus par les immeubles des emprunteurs, mais par les capitaux qu'ont fournis des particuliers, actionnaires ou obligataires, dont bien peu sont propriétaires fonciers ${ }^{3}$. La seule restriclion à cette

1. Les statistiques du Crédit Foncier ètablissent que les prêts à long terme (trente, quarante et même soixante ans), remboursables par annuilés, se liquident dans le délai moyen de cinq ans, par la vente forcée ou par le délaissement du gage. (Voir les publications du Congrès international des valeurs mobilières de $1900,2^{\circ}$ volume, Les Crédits fonciers en Europe.)

2. L'obligation de procéder uniquement par actes notariés. Une procuration pure et simple par deux époux, pour empruntcr, rédigée par un notaire de petite ville (mai 1900), a cốté 33 fr. 30.

3. La loi du 6 juillet 1860 autorise le Crédit Foncier à prêter méme sans affectation hypolhécaire (article 2). En représentation des prêts, c'est-ä-dire pour se procurer les fonds d'emprunt, le Crédit Foncier est 
expansion du monopole est que le montant des émissions ne peut pas dépasser vingt fois le capital-action.

Un écrivain qui, malgré la légende, a été un défenseur éloquent et convaincu du droit de propriété, Proudhon, a formulé la conséquence philosophique d'un état de fait qu'il voyait naître sous ses yeux et dont il pressentait l'exagération prochaine :

e crédit, à force de dégager le capital, a fini par dégager l'homme lui-même de la Société et de la Nature. La terre n'est plus possédée de personne. Elle n'a plus que des maitres qui la dédaignent et des serfs qui la haïssent; car ils ne la cultivent pas pour eux, mais pour un por. teur de coupons que nul ne connaît, qu'ils ne verront jamais, qui peutètre passera sur cette terre sans la regarder, sans se douter qu'elle est à lui 1 .

Quant au propriétaire réel de ces domaines, le détenteur exploitant par lui ou par des métayers ou des fermiers n'étant qu'un régisseur de biens pour autrui, quant au porteur de coupons, il est si détaché de cette forme de richesse qu'il en cédera volontiers le papier représentatif pour quelques cenlimes de hausse. Tel est le résultat, encore localisé, que voudraient imposer à la généralité du territoire les utopistes qui rêvent l'émiettement et la mobilisation du sol.

Contre l'abus des monopoles et surtoul contre l'absorption de la propriété privée par les syndicats, qu'ils prennent la marque de l'État avec les socialistes ou la forme de sociétés financières avec les spéculateurs, il y a des remèdes simples et souverains.

D'abord, la consolidation de la propriété entre les mains de ses détenteurs par la sécurité du titre foncier et l'extinetion de la detle agraire ${ }^{2}$; plus on immobilisera l'héritage et plus on y attachera l'homme, en solidarisant la terre et la famille dans une même harmonie productrice.

autorisẻ à faire, jusqu'à concurrence, des émissions d'obligations (article 5). Chaque catégorie de prêts est gagée sur le titre de créance ou sur le gage, suivant qu'il y a eu hypothèque ou non (article 6).

1. Contradictions, II, chap. xI, 193.

2. Fẻlix Hecht, Le désendeltement de la propriété rurale (MI. Block, Comptes rendus de l'Académie des sciences morales et politiques, juillet 1899, p. 104). 
Ensuite, en opposant à la coalition des spéculateurs, qui exploitent la propriété en faisant des propriétaires leurs fermiers, l'entente des travailleurs, c'est-à-dire des propriétaires qui cultivent directement, catégorie dont il faut accroître à tout prix le nombre et les ressources. On y peut arriver, contre les divers monopoles, par l'action des syndicats agricoles, et contre le monopole spécial du crédit par l'assurance et la mutualité. C'est cncore ici, par la concentration des ressources locales, que le monopole absorbant de la richesse de spéculation, celle qui ne possède ni ne commandite mais qui agite et accapare, verra sa force contrebalancée par le groupement des petits, l'emploi des machines, l'association tellement étroite du travail et du capital qu'on ne puisse plus distinguer ces éléments l'un de l'autre ni les séparer sans les détruire.

La famille et la propriété marchent de front, appuyées l'une sur l'autre, n'ayant l'une et l'autre de signification et de valeur que par le rapport qui les unit. L'histoire de I'humanité l'atteste; clle alteste aussi que leur solidité réciproque el que leur expansion dérivent du plus ou moins de liberté avec lequel elles se meuvent dans la spirale socialc. Comme l'a dit avec autorité M. Maurice Block ', les faits, influencés par la moyenne des opinions, finissent par constituer un arbitrage que tous acceptent. L'individu peut avoir un penchant arbitraire, mais dans la vic sociale el économique les arbitraires individuels se combattent, se neutralisent et aident les lois naturelles à triompher.

II. La Famile et la Lor. - Dans toutes les civilisations, la loi a fait du droil de propriété le fondement de la famille.

Le droit à l'héritage est le pivot de l'histoire sociale. En France, depuis plus d'un siècle, celle question, dont l'incidence pèse sur tous les problèmes économiques, est livrée aux équivoques et devient, un argument de parti au lieu de rester un principe que nul n'aurait à discuter.

Dès le vir siecle, l'esprit du clıristianisme inspirait, sous 
la plume de Marculfe, la formule de succession ${ }^{2}$; mais la Féodalité, substituant le principe matériel de la force à l'idée spiritualiste, erée le droit d'aînesse, l'exclusion des femmes, la prédominance de la qualité des biens sur la parenté des personnes. La Révolution décréta l'égalité des partages dans les familles ${ }^{2}$. Le problème n'était qu’à moitié résolu, car le droit de tester demeurait intact, l'Assemblée Constituante ne pourait le proscrire ou le limiter sans contredire à ses doctrines spiritualistes ${ }^{3}$, elle ajourna la loi à intervenir, se bornant à assurer la liberté des héritiers institués et des légataires que pouvait entraver la volonté d'un testateur imbu des principes ${ }^{4}$.

Les rédacteurs du Code civil furent fidèles à ces indications. Notre objet, disent-ils ${ }^{5}$, a été de lier les mœurs aux lois et de propager l'esprit de famille, qui est si favorable, quoi qu'on en dise, à l'esprit de cité. Les sentiments s'afłaiblissent en se généralisant; il faut une prise naturelle pour pouvoir former des liens de conventions. Les vertus privées peuvent seules garantir les vertus publiques; et c'est par la petite patrie, qui est la famille, qu'on s'altache à la grande. Ce sont les bons maris, les bons fils qui font les bons citoyens. Il appartient aux institutions civiles de sanctionner les lois naturelles.

De même que, de 1791 à 180', il a paru que les règles de l'hérédité, $a b$ intestat ou testamentaire, constituaient l'œuvre sociale par excellence et que toutes les autres lois civiles

1. Comme Dieu a donné également au père tous ses enfants, ils doivent avoir une part égale dans les biens du père. (Edition de Jérôme Bignon, 1613.)

2. Le décret du 15 mars 1790 abolit en premier lieu les droits d'ainesse et de masculinité qui représentaient au sein de la famille le principe féodal dans toute son énergie; celui du 8 avril 1791 appliqua l'égalité à toutes les successions.

3. Le testament, dit Quintilien, e'est la volonté de l'homme qui se prolonge au delà de son existence terrestre : voluntas ultra mortem; et Leibniz insiste : Testamenta vero, mero jure nullius essent momenti nisi anima esset immortalis.

4. Décret du 7 avril 1791.

5. Discours préliminaire, rédigé par Portalis pour servir d'exposé des motifs au projet du Code civil. 
n'en étaient que les accessoires ou les conséquences, ainsi, à notre époque, alors que la richesse territoriale, morcelée et mobilisée pour les trois quarts par le crédit ${ }^{1}$ s'émiette en valeurs industrielles, il semble que la répartition de la propriété foncière en lots plus ou moins étendus, en cxploitations plus ou moins rémunératrices, et dont le propriétaire demeure plus ou moins indépendant d'autrui, plus ou moins dégagé des servitudes contemporaines, doit être la principale préoccupation du législateur ${ }^{2}$.

En constituant la famille par rapport aux biens, et en prohibant les substitutions, sauf une execption, le Code civil compléta et réglementa l'œuvre démocratique inaugurée par la Révolution; toutefois, il la laissa imparfaite en voulant en mème temps, par un parti pris trop accusé, enchaîner et libérer le contrat matrimonial.

Le Code a posé comme règle fondamentale de l'hérédité ab) intestat la division des biens entre les deux lignes paternelle et maternelle, sans tenir compte de l'origine des biens héréditaires; quant à la succession par délégation, il a limité le droit de tester en fixant la réserve, la quotité disponible, le chiffre des libéralités entre époux, etc.

Le principe du partage forcé a été critiqué, el les arguments les plus vifs sont ainsi résumés par F. Le Play ${ }^{3}$ :

Comment un grand propriétaire se décidera-t-il à créer une rraic résidence rurale sill doit y mourir dans l'isolement, après la dispersion de ses enfants, si d'ailleurs cette création doit être rendue après sa mort à un étranger ou détruite par les dépeceurs de la bande noire? Pourquoi s'épuiser à ébaucher une ouvre qui restera inachevée puisqu'elle ne pourrait être fécondée que par la succession de plusicurs générations animées lu même esprit?

1. Mines, Chemins de fer, Crédit Foncier, Sociétés financières de construction, de culture, Compagnies d'assurances, ete.

2. Cela est si vrai, qu'il faudrait plusieurs pages pour dresser la liste des projets de loi déposés par le Gouvernement et des propositions dues a l'initiative parlementaire, visant le régime de la propriété rurale, sa constitution, sa consolidation, sa division, ses charges, elc.

3. La réforme sociate, 1, chap. II, \$20. 
Quant aux moyens-propriétaires et aux petits, les suites légales de la mort du chef de famille troublent leur vie privée el, pour se rendre compte de ce désordre, il suffit d'apprécier les pertes de temps, les frais, les froissements, les chances de procès qu'impose à chaque génération la désobligeante et coùteuse procédure de la transmission des biens ${ }^{1}$.

En France, on a obéi à la préoccupation impérieuse d'eflacer tous les vestiges du passé et de créer un ordre de choses nouveau; les Anglo-Saxons, moins détournés des rues économiques par l'obstiné souci de la politique, s'inspirent surtout, dans leur régime successoral, de la pensée que la propriété privée est le principal fondement de l'indépendance individuelle, et que, en conséquence, le droit absolu de transmettre ses propres biens à qui bon lui semble est l'un de ceux que chaque ciloyen a le plus d'intérèt à exercer. Aussi, chez les Anglais comme chez les Américains du Nord, le propriétaire a, de son vivant comme à l'heure de sa mort, la liberté sans réserve de donner et de tester.

Entre les deux systèmes en présence, laisser le père de famille libre de disposer de son bien comme il l'entendrait, ou confier à l'État centralisateur le droit de faire cette répartition, et par conséquent celui de procéder par voie inquisitoriale dans lintimité des familles, le législateur de 1791 avait hésité. Mais, lorsqu'on réclama, l'expérience une fois acquise, contre les inconvénients du partage forcé, que Thuriot et Cambacérès montrèrent les héritages morcelés, la petite propriété rurale dissoute à chaque décès , la Convention coupa court à tout retour en arrière en votant, le I mars 1793, l'abolition du droit de tester.

1. Les exigences légales, interprétées à l'extrême, décident presque toujours les héritiers honnêtes et scrupuleux à s'abstenir de toute intervention personnelle, en sorte que, par la force des choses, le plus intime intérêt des familles se trouve abandonné à la discrétion des officiers ministériels; de là, pour ces derniers, des profits excessifs et une prépondérance anormale (La réforme sociale, p. 186).

2. Monileur du 28 décembre 1793. 
En 1803, lorsque Portalis essaya de faire prévaloir le principe de la liberté testamentaire sur celui du partage forcé, il ne craignait pas de s'exprimer ainsi :

Le droit de disposer est un droit d'arbitrage. Là où le pére est législateur dans sa famille, la Société se tronve déchargée d'une partie de cette sollicitude. Qu'on ne dise pas que c'est là un droit aristocratique. 11 est tellement fondé sur la raison, que c'est dans les classes inférieures que le pouvoir du père est le plus nécessaire. Un laboureur, par exemple, a eu d'abord un fils qui, se trouvant le premier élevé, est devenu le compagnon de ses travaux. Les enfants nés depuis, étant moins nécessaires au père, se sont répandus dans les villes et $y$ ont poussè leur fortune. Lorsque le père mourra, sera-t-il juste que l'ainé partage également le champ amélioré par ses labeurs avec les frères qui déjà sont plus riches que lui 1 ?

Le chef de famille pourrait, de son vivant, remédier aux inconvénients du partage forcé par les donations entre vifs et les attributions de dot dans les contrats de mariage; mais il y a été pourvu par l'article 843 du Code civil.

Ce n'est pas seulement en France que la question se pose. Les différents modes de répartition de la propriété rurale ont inquiété les législateurs étrangers par l'accentuation de la tendance au morcellement. En Allemagne, les lois de succession, depuis le commencement du groupement agrarien jusquien 187\%, ont eu pour objectif de permettre à la propriété rurale de lutter contre la concurrence des pays neufs. Linstitution du Ileferolle garantit la transmission intégrale des domaines dont la division compromettrait la bonne exploitation; cctte combinaison s'accorde sans tronbler les habitudes aux conditions économiques de la société moderne; aussi le partage forcé a-t-il été aboli en Autriche.

Je dirai, dans le paragraphe suivant, quels sont les rapports de la propriété, c'est-à-dire de la famille rurale, avec l'impòt el quelles lourdes entraves, sous la double incidence de nos lois civiles el de nos conditions économiques, pèsent sur elles de ce côté.

Il en est d'autres encore, en dehors de la fiscalité, qui,

1. Locré, Discussion au Conseil d'Élat pour la préparation du Code civil. 
avec la prétention d'être une sauvegarde, arrêtent son essor, gênent ses mouvements et la détournent souvent de sa pente naturelle.

Le caractère agricole de la France est le plus ancien et le plus grand de ses intérêts ${ }^{1}$; depuis un demi-siècle, la législation, obéissant aux vues de la philosophie du droit qui domine les lois positives du présent et, par leur eritique, prépare celles de l'avenir, a apporté à nos Codes des modifications, des additions qui les mettent mieux en harmonie avec la situation économique, tout en ne réalisant qu'une faible partie des vœux des réformistes. Le législateur de 1804, ne pourant prévoir l'essor des valeurs de crédit, essor proroqué par les impedimenta des mutations foncières, avait multiplié les règles qui devaient, dans son esprit, créer la sécurité du droit de propriété. Par l'effet du temps et du déplacement des valeurs, e'est l'inverse qui s'est produit; de simples expédients ne suffisent plus, il faut faire table rase des vieux principes pour rétablir sur ce point l'équilibre nécessaire.

La fortune immobilière, en 1804, fut la préoecupation exclusive du Code civil. Mais, en voulant consolider la propriété foncière, il l'avait surchargée de préeautions gênantes, de garanties superflues, de formes multipliées, et son système incomplet et hésitant sur les hypothèques, sur la réalisation du gage, sur la sûreté des transferts de droits immobiliers provoquait les doléances des jurisconsultes et des économistes. Dès 1827, Casimir Périer faisait appel aux juristes pour réaliser la réforme hypothécaire; en 1828, Decourdemanche proposait une combinaison de livres fonciers que l'Angleterre adopta pour ses colonies; en 1838, Rossi dénoncait l'urgence d'approprier les lois civiles à l'évolution d'un pays agricole qui, prenant dans le sol son point d'appui pour s'élancer vivement vers l'industrie et les échanges, erée la richesse mobilière, si variable, si capricieuse, dont l'cxpansion va modifier les conditions économiques de la possession

1. Laferrière, Essai sur l'histoire du Droit francais, II, p. 415 . 
de la terre ${ }^{1}$; de $\mathbf{1 8 4 1}$ à 1814 , une vaste enquête judiciaire constate les imperfections de la législation foncière; la loi du 23 mars $180 ̃$ sur les transferts fonciers, le développement du Crédit Foncier, appuyés d'essais parliels et infructueux pour simplifier la procédure et réduire les frais, n'apportent à la crise agricole aucun allègement appréciable; les Congrès fonciers de 1889, 1892 el 1900 révèlent l'étendue du mal et affirment, en même temps, l'opposition des officiers ministériels à toute mesure qui, en supprimant des formalités inutiles, risquerait de diminuer leurs profits; les travaux laborieusement étudiés de la Commission extra-parlementaire du cadastre, quantité de projets de loi, déposés par les différents cabinets, des propositions, plus nombreuses encore, émanées de l'initialive parlementaire, tout cela se heurtant, se contredisant, se discutant à demi, faute de méthode, et s'ajournant, de crainte de faire fausse route en s'avançant à l'aventure, sans vues générales, sans plan d'ensemble, sonl la preuve du désarroi des esprits, des incertitudes de la jurisprudence, du défaut de faire marcher les réformes sur des lignes parallèles, isolément, au lieu de les pénétrer l'une par l'autre et de les associer clans une participation efficace et raisonnée vers un but commun.

Rien ne marque mieux l'éearl, né de la politique, qui sépare les courants actuels du parlementarisme que l'état d'opposition des plus importants projets en discussion. D’un côté, abondent les formules cherchant à simplifier les formalités; à réduire les honoraires excessils des intermédiaires, le plus souvent inutiles, dont la loi impose les services et qui, pour gagner davantage, multiplient les incidents ${ }^{2}$ et

1. Némoires de l'Académie des sciences morales, $2{ }^{\circ}$ sẻrie, $\mathrm{I}, 278$.

Louvrier agricole doit pouvoir fonder sur une base indestructible cette famille rurale qui est J'une des plus grandes forces de la nation. (G. Siegfried, Proposition de lol sur la petite propriété rurale, du 5 juillet 1897.)

2. Nombreuses circulaires de la Chancellerie, dont l'une des plus récentes est celle du 3 mai 1900, sur les rémunérations d'à côté, faisant suite à celle du 29 décembre 1899 sur l'exagération des frais. 
épuisent la matière jusqu'au fond '; à défendre le petit propriétaire contre le fléau de l'hypothèque; à faciliter la réalisation du gage de façon à ce qu'il ne soil pas dévoré par la procédure en ruinant à la fois le prêteur et l'emprunteur ${ }^{2}$; à protéger l'intégrité de l'héritage contre sa dislocation juridique; à eréer l'insaisissabilité du patrimoine de famille; à préparer l'accession du travailleur salarié à la propriété par l'épargne, l'assurance et la mutualité; à punir l'escroquerie foncière ou stellionat dont nos lois et nos règlements se font les complices inconscients; à écarter la fortune des femmes mariées des pièges du régime dotal; à supprimer l'obligation du partage en nature ${ }^{3}$. A l'inverse, et, détail caractéristique, sur l'initiative non pas de novateurs imprudents mais du gouvernement établi, on exagère la centralisation, on crée de nouveaux services publics, on multiplie les emplois, on grossit le tarif des frais ${ }^{4}$, on surtaxe jusqu'à 10 p. 100 les frais de la publicité hypothécaire ${ }^{5}$; on dégrève à rebours, d'où la nécessité de eréer des taxes de remplacement; on cherche à substituer à une partie de l'impôt foncier l'impôt global sur le revenu; on frappe les successions du tarif progressif; on viole à tout instant, par des mesures arbitraires, des exceptions, des exemptions et des privilèges, le principe

1. Parmi les abus criants qui, même dans la pratique du Notariat parisien, accumulent les frais qu'on peut éviter, je citerai un seul exemple que me suggère l'examen d'un ètat de frais que j'ai sous les yeux. I1 s'agit d'un prêt de 20000 francs; les frais du notaire s'élèvent à $773 \mathrm{fr}$. 4 3̆. Parmi ces frais, on relève ceux de notification à la Compagnie d'assurances pour 36 fr. 90 ; or il est de jurisprudence que l'intervention de l'huissier est inutile; une simple lettre recommandée suffit; l'accusé de réception de la Compagnie établit un lien de droit entre elle et le créancier. (Loi du 19 février 1889. - Revue des clercs de notaires, 10 octobre $1899, \mathrm{n}^{\circ} 6817$.) Le notaire a donc fait payer à son client 36 fr. 90 pour une précaution qui ne devait con̂ter que 40 centimes.

2. D'après les comptes rendus annuels du ministre de la Justice, les frais des ventes judiciaires dépassent 100 p. 100 pour les petites ventes, c'est-à-dire absorbent le prix; pour les autres, la perte des créanciers a été de 983 millions en 1876 , de 247 en 1891 sur 414 millions de créances, soit en moyenne de 50 à 56 p. 100.

3. Article 826 du Code civil.

4. Décrets du 25 août 1898 réglant le tarif légal des notaires.

5. Loi du 27 juillet 1900 sur la taxe hypothècaire. 
dominant de la proportionnalité dans l'impôt, l'une des plus précieuses conquêtes de 1789.

La loi, au lieu de protéger la sécurité de la famille par la consolidation du foyer domestique, l'ébranle et la compromet. Qui veut des couvées, sauve le nid. Pourquoi s'étonner de la dépopulation croissante quand on chasse les enfants du toit paternel? Les déracinés ne font pas souche, ils se disséminent et s'évanouissent.

Tandis que les races germaniques et anglo-saxonnes rendent insaisissable et permanent le manoir familial, même sous sa forme la plus modeste, le foyer privé, le home stead, le chez-soi, il semble que nous prenions à tâche d'ôter au père la joie de penser que le nid qu'il bàtit sera durable. Rien de plus anti-social, de plus anti-démocratique. Les hommes d'État qui ont la prétention d'asseoir sur des bases nouvelles une société inquiète et dont l'impatience risque de faire avorter, en les préparant mal, les réformes les plus légitimes, ont tort de marquer tant de dédain pour le passé. Les nouveautés sont rares. On vit surtout de souvenirs, de précédents, de traditions qu'on ne renie pas sans ingratitude et qu'on n'écarterait pas sans danger. Il est toujours délicat de toucher à ce qui constitue l'essence mème de la famille, à ce qui est, en même temps, son point de départ et son but final.

Ili. La Propriété et l'Iupòt. - Si certaines dispositions de la loi civile apportent des cntraves au jeu normal de la diffusion du droit de propriété et de sa répartition plus ou moins étendue aux mains des exploitants du sol, la loi fiscale, à son tour, avec plus de brutalité et des ingérences plus fréquentes, gêne et parfois arrête son développement régrulier et transforme ses lois naturelles en accidents.

L'ouvre de 1789, en ce qui touche à la propriété foncière, est restée inachevée. La fiscalité d'État en a aggravé les conditions provisoires de telle sorte qu'elle semble vouée à une infériorité sans remède en face de l'ordre économique naturel et de l'arenir des démocraties européennes.

Tout ce que la Révolution de 1789 a fait pour la proprićté 
se résume dans l'article $\breve{3} 37$ du Code eivil : Les particuliers ont la libre disposition des biens qui leur appartiennent.

La Révolution, d'une part a consolidé le droit de la propriété acquise par la suppression des droits féodaux; d'autre part, elle a ouvert à tous le libre accès de la propriété, par la réforme des lois successorales. La résultante générale des principes et cles actes de la Révolution a donc été l'affranchissement et l'extension du droit de propriété, dans le sens de la propriété individuelle. Mais, depuis 1793 et surtout 1798, il semble que la réaction qui suit les grands mouvements ait accumulé les obstacles devant cet entraìnement libéral. Les contradictions de la loi, les subtilités de la doctrine, les chicanes de la jurisprudence ont, petit à petit, reconstitué autour de l'individu le domaine obscur des équivoques juridiques, et la sécurité du titre foncier est devenue aussi douteuse que son acquisition paraît facile. Le défaut de sécurité est le premier obstacle à la mobilité du droit de propriété, et par mobilité je n’entends pas ici la fréquence des mutations d'un domaine rural d'une main dans l'autre, mais la possibilité d'acquérir vite et sûrement.

J'ai signalé, au cours de cette étude, les points faibles de notre législation foncière; ils peuvent se grouper en trois catégories : $1^{\circ}$ défaut de précision, d'uniformité et d'authenticité dans la constitution du titre de propriété; $2^{\circ}$ frais énormes qui grèvent le propriétaire, d'abord sous forme d'impôt, ensuite et surtout par les profits arbitraires que prélèvent les intermédiaires dont la loi oblige à réclamer le concours $^{1} ; 3^{\circ}$ défectuosité de la publicité hypothécaire, qui est coûteuse, partielle et facultative.

De cet ensemble de charges très lourdes et dont le poids n'est nullement compensé par l'utilité du service rendu, ensemble dont l'étude critique exigerait à elle seule un

1. Pour le prêt de 20000 francs que je cite à la note 1 de la page 371 , l'Enregistrement a perçu 250 francs, les Hypothèques $244 \mathrm{fr}$. 55 y compris des honoraires de notaire dont le ditail n'est jamais fourni, et le Notaire prélève pour ses légaux honoraires 200 francs et pour frais et profits accessoires d'expédition, etc., $78 \mathrm{fr} .80$. 
volume, je détacherai l'impôt, parce qu'il constitue un élément distinct qu'on peut plus facilement distraire des autres pour en spécifier la valeur exacte.

La part contributive de chaque citoyen dans les charges publiques ne cesse d'augmenter. De $\mathbf{5 6}$ francs par tête en 1869 , elle a monté à 67 francs en 1872, à 86 francs en 1877, à 88 francs en 1884, à 93 franes en 1896, à 103 francs en 1900 .

Mais ee n'est là qu'une partie des charges du contribuable; aux impòts d'État, il faut ajouter les centimes additionnels communaux et départementaux qui ont augmenté, depuis 1872, de 40 p. 100 pour les centimes spéciaux, de 40 à 70 p. 100 suivant les localités, pour les autres; dans quantité de communes, l'accessoire dépasse le principal. On estime à 140 francs par tête au minimum, la laxe annuelle du Français, et, pour amortir la dette publique, chacun de nous devrait verser un capital de 1000 francs.

Quelle est, dans le total énorme de nos impôts, la part proportionnelle de la propriété foncière? L'évaluation ne peut en être quapproximative et il serait inopportun de disculer ici les statistiques plus ou moins capricieuses, les calculs plus ou moins arbitraires qui servent aux financiers, aux économistes et aux publicistes à justifier leurs thèses réciproques, ear il y a deux écoles en présence, celle des mobilisateurs à outrance qui font du crédit une valeur effective et voudraient tout convertir en papier; celle des traditionncls qui ne voient pas des rivales dans l'agriculture et l'industrie, mais des sœurs dont la cadette est aventureuse et ne peut se passer du concours de l'aînée. De ces deux écoles, c'està celle qui seplaindra le plus fort d'êtresacrifiée à l'au tre.

Au récent Congrès des valeurs mobilières, on a évalué à 100 milliards le capital rural de la France ${ }^{1}$ et à un minimum de 83 milliards le capital de ses valeurs mobilières ${ }^{2}$; on a

i. Se décomposant ainsi, d'après le Bulletin du ministère de l'Agriculture d'août 1889 : capital foncier, 91584 millions; capital d'exploitation 8545 ; ensemble, 100129 millions.

2. D'après les évaluations combinées de MM. Coste (en 1895), Neymarck (en 1896) et Théry (en 1897). 
fixé à 2,89 p. 100 le revenu net du premier ${ }^{1}$ et à 3,38 p. 100 celui du second. L'écart d'une richesse à l'autre s'explique par la sécurité relative de la fortune territoriale et les risques des valeurs de crédit.

Est-il préférable, se dit-on, de posséder 100 hectares de bonnes terres, à 3000 francs l'hectare, qu'un capital de 300000 francs représenté par de la rente française? La terre est le plus solide élément de la propriété. Cette solidité fait son avantage et son inconvénient; car la terre ne peut se dérober et, mème à l'heure des crises, lorsque les valeurs de crédit se déprécient et qu'il suffit d'un retour d'opinion pour les réduire à néant, c'est toujour's à elle qu'on s'adresse, c'est elle qu'on taxe et qu'on surtaxe. La propriété foncière n'est pas, comme l'autre, une participation temporaire aux bénéfices d'une industrie qui peut disparaitre; c'est une possession certaine, indéfinie; c'est pour ainsi dire, et sauf les lois générales de la société, un petit État dans l'État. Est-ce une raison pour limposer par des séries d'impòts qui atteignent toutes les manifestations de sa vitalité? Ne serait-il pas sage, au contraire, de la respecter comme une source intarissable de production? La molester outre mesure, n'estce pas risquer de tuer la poule aux œufs d'or?

Les financiers ì rues courtes qui voudraient qu'on dégrevât les valeurs de papier afin d'accroître leurs gains, non seulement prétendent que la propriété rurale n'est pas surtaxée, mais équivoquant sur les chiffres et les ajustant à leur caprice, ils écrivent qu'on pourrait prétendre, d'une façon générale, que la propriété non bâtie agricole ne supporte aucun mpôt ${ }^{2}$. Ces naïvetés n'ont pas besoin qu'on les réfute, mais il est nécessaire de protester contre de pareilles assertions lorsqu'on leur permet de prendre place dans un recueil quasi officiel ${ }^{3}$.

1. Ce calcul n'est pas exact, car M. G, Nanchez, qui l'établit, déduit du capital rural la valeur de l'outillage d'exploitation qui en est inséparable.

2. Publications du Congrès des valeurs mobilières, t. II, Les valeurs mobilières et la terre devant l'impôt. p. 10.

3. Le même publiciste prétend aussi que le revenu du propriétaire 
Aussi ne peut-on accorder qu'une créance relative aux calculs qui établissent comme suit la proportion des charges qui pèsent sur le revenu respectif de la propriété agricole et de quelques-unes des valeurs mobilières :

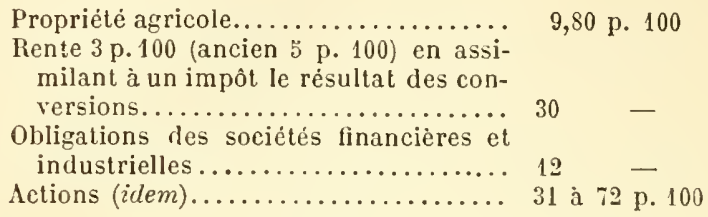

Par contre, tout en exagérant la charge de la fortune mobilière, on diminue dans une proportion invraisemblable celle que supporte, en réalité, la fortune territoriale. Celle-ci est évaluée à seulement 259 millions de francs par an ', parce qu'on en déduit les droits de mutation, d'hypothèques, etc.: tandis qu'elle s'élève, année moyenne, au chiffre de 1198 millions de francs ${ }^{2}$.

D'après les statistiques officielles les plus exactes puisqu'elles sont tirćes d'opérations de comptabilité soumises à un triple contrôle, le seul droit de mutation sur les transferts de droits immoliliers (année 1896) s'élève à.

Les droits d'hypothèques à.................

Les droils de timbre a environ................

Le droit de bail a........................

Les honoraires, taxes et droits des notaires que la Chancellerie évalue, d'habitude, aux $4 / 33$ des Iroits payés au Trésor...................

Sans tenir compte, à moins d'entrer dans un détail infini, des droits sur les actes et $A$ reporter...................

$230694468 \mathrm{fr}$.

23250000

12750000

8212000

Mémoire

$274906468 \mathrm{fr}$.

foncier non exploitant est net d'impôt parce que l'impôt foncier est à la charge du fermier. Il ignore que eette avanee s'impute sur le prix de ferme ou qu'il en est tenu compte lorsqu'on lixe le fermage; en se reportant a la jurisprudlence administrative qui a réglementé l'application du $n^{\circ} 1$ de l'article 15 de la Ioi du 22 frimaire an VII sur les baux, il apprendrait que le paiement de l'impôt foncier par le fermiel est une clarge qui s'évalue d'office, le cas échéant, ì 25 p. 100 du revenu net, et qui retombe toujours sur le propriétaire.

1. Manchez, loco citalo, page 10 (pour l'année 1896).

2. Le crédit terrilorial en France et la réforme hypothécaire, 20 èdition, Introduction, p. Ix. 
Report.....................

274906468

des frais de réalisation du gage en matière hypothécaire, on peut évaluer, avec II. de Foville, les Intérêts de la dette agraire à..... A quoi il faut ajouter l'impót direct : contribu500000000 tion foncière 1 :

Propriétés bàties

Propriétés non bàties.............
Contribution personnelle et nobi-

lière.....................

Contribution des portes et fenêtres.

Centimes additionnels............

Prestations...................

149813125
245611367
138256615
89873789
Mémoire
59205700

Total

$1477667114 \mathrm{fr}$.

702760646

Ce total formidable d'impôts est à prélever sur un revenu net agricole dont la détermination exacte, à quelques centaines de millions près, est impossible ${ }^{2}$, mais que les spécialistes les plus autorisés évaluent de 2093 à 2640 millions ${ }^{3}$.

On a récemment publié des chiffres plus précis indiquant la répartition par département de la propriété urbaine et de la propriété rurale d'après l'enquête administrative de $1898^{*}$.

Ces indications sur la quotité de la fortune privée, par tête, dans chaque circonseription, pourront être utilement rapprochées des charges fiscales qui y atteignent les capitaux ou les revenus. A ce titre, elles prennent une imporlance particulière en derenant la preuve du poids dont l'impôt grève avec des inégalités choquantes la principale source de a fortune publique, celle d'où toutes les autres découlent.

Pour l'impôt foncier, le département des Hautes-Alpes est taxé à 7,20 p. 100, celui de la Corse à 0,9 ' p. 100 ; le canton d'Archiac (Charente-Inférieure) à 14,70 p.100, celui de Bastia à 0,37 p. 100. On répond à ces écarts que l'inégalité devient, en raison des différences de culture et de débouchés, une

1. Bulletin de statistique de l'Enregistrement, 1898.

2. Annuaire statistique de la France, 1896, p. 469 et 478.

3. D'après les travaux de la Commission extra-parlementaire de l'impôt sur les revenus (1897) et les documents produits au Sénat lors de la discussion de la nouvelle loi d'èvaluation du revenu des propriétés non bálies.

4. Journal de la Société de statistique de Paris, octobre et décembre 1899. 
preuve de proportionnalité; la valeur taxée n'en est pas moins identique. Les dégrèvements partiels opérés depuis 1819 n'ont apporté aux propriétaires fonciers qu'un allègement négligeable ${ }^{1}$, témoin celui qui fut voté en décembre 1898, qui n'a fait qu'augmenter le déficit budgétaire sans améliorer la situation des débiteurs de petites cotes. Quand on rappelle la série de ces dégrèvements, en ajoutant que c'est la fortune mobilière qui en a fait les frais par des taxes de remplacement, on oublie que le chiffre global de limpòt direct n'a cessé de s'accroître. De 387 millions qu'il était en 1838 , le produit des quatre contributions s'est élevé à 822 en 1893.

Mais ce n'est point de ce côté que le bàt le blesse, disait Léon Say quand il comparait le paysan à la bète de somme du budget. Mieux vaut ne point toncher à cette nature d'impôt à laquelle on est habitué et qui rentre sans frais appréciables ${ }^{2}$; il faut réformer ailleurs.

Ce sont les droits de mulation et les abus de la procédure, conséquence directe de l'emploi forcé d'intermédiaires officiels, toujours coûteux et le plus souvent inutiles, qui pèsent le plus lourdement sur la propriété rurale.

Le seul arantage des valeur's industrielles sur la propriété foncière, avantage qui n'a pas peu contribué à leur vulgarisation, à leur classement rapide, malgré leurs inconvénients et leurs risques, c'est la facilité de leur réalisation, si aisée grâce à l'absence de formalités accessoires au transfert, si peu coûteuse par la modicité des frais de mutation que représentent le courtage de l'agent de change et l'impôt sur les opérations de bourse.

Seule de toutes les représentations actuelles de la fortune

1. De 1797 à 1805, ả mesure qu'on décréta les droits sur les actes, l'impôt foncier fut dégrevé de 69 millions de franes, plus de 100 de notre monnaie actuelle.

2. On sait que la moitié tout au moins des frais de poursuites dépensés par les percepteurs ne sont pas motivés et seraient évités si l'arbitraire administratif se dirigeait d'après les leçons de la vieille équité latine : Summum jus, summa injuria. (Lettre du ministre des Finances du 9 août $1900, n^{\circ}$ :788.) 
a cquise, la terre se transmet avec les procédés, les lenteurs et les taxations d'un autre siècle, procédés que leur imperfection rend trompeurs, taxations qui sont onéreuses, lenteurs qui sont préjudiciables. Les mutations sont de deux sortes : entre vifs et par décès. Chacune de ces deux catégories est assujettie depuis 1798 à une iniquité légale d'un caractère particulier.

Les mutations entre vifs ${ }^{1}$, acquisitions ou échanges de droits immobiliers, sont frappées de la taxe fiscale non point sur leur prix réel, sur les sommes inscrites au contrat et qui font la loi des parties, conformément à l'article 15083 du Code civil, mais sur leur valeur vénale présumée, calculée par les agents du fise comparativement avec les fonds voisins de même nature ${ }^{2}$. Pour la seule année 1897, l'application de cet arbitraire fiscal a ajouté au produit normal du droit de mutaíon, sous le titre de Pénalités, une surtaxe additionnelle et accidentelle de 2703167 franes.

Les mutations par décès sont assujetties, pour leur part, à l'impôt, non point sur l'actif successoral net, mais sur l'actif brut, c'est-à-dire sans déduction des dettes et charges, de telle sorte que l'héritier paie le transfert sur une valeur fictive dont une portion plus ou moins considérable ne lui appartiendra jamais ${ }^{3}$. Un projet de loi, destiné à supprimer cette flagrante injustice, pendant plusieurs années en discussion au Parlement, adopté par le Sénat, n'a enfin été voté par la Chambre que grâce à son intercalation dans la loi de finances de $\mathbf{1 9 0 1 .}$

Malheureusement, comme dans toutes les réformes où les exigences du budget viennent contrarier et transformer les

1. Je ne fais point la distinction habituelle mais purement fiscale entre les mutations entre-vifs à titre onéreux el celles à titre gratuit, parce que ces dernières rentrent forcément, à un moment donné, sous les règles du droit successoral (Articles 843 à 869 du Code civil).

2. Article 17 de la loi du 22 frimaire an VII, modifié, dans le sens de l'aggravation, par les articles 12 et 13 de la loi du 23 aout 1871 et $8, \$ 3$, de celle du us février 1872 .

3. Numéro 7 de l'article 15 de la loi du 22 frimaire an VII modifiè par l'article 3 de la loi de finances du 25 février 1901. 
meilleures intentions, la déduction des dettes fera dans l'équilibre des recettes un tcl vide, vide dont la mesure est elle-mème inconnue, faute de statistiques, qu'il a fallu y pourvoir un peu à l'aventure par une taxe de remplacement, et, de peur que cette compensation ne fùt insuffisante, on en a exagéré le taux. Le résultat sera une détaxe à gauche par la déduction des dettes, ct une surtaxe à droite par l'élévation du tarif; le projet reprend d'une main ce qu'il abandonne de l'autre; l'assiette de l'impôt sera plus équitable, mais l'impòt lui-même sera plus lourd, et il est vraisemblable que, le tarif nouveau une fois appliqué, le contribuable sera plus directement atteint que par le passé.

Les conséquences en seront particulièrement déplorables pour la propriété foncière; la propriété ruralc sera mème plus rudement frappée que la propriété urbaine paree que les deux éléments qui en permettent l'exploitation, le sol d'abord, puis l'outillage agricole, le matériel de culture, sont immobilisés, visibles, connus, et ne peuvent ni disparaître ni s'amoindrir ou se dissimuler comme les éléments fuyants de la richesse mobilière, le numéraire, le mobilier, les créances, les valeurs au porteur. L'enquête de l'Enregistrement a vite raison des dissimulations tentées dans ces conditions ${ }^{1}$.

M. Emile Faguet, examinant les ineidences du nouveau régime successoral et du tarif progressif qui est son earaetère saillant, disait qu'il serait bientôt ruineux d'hériter. Ce mot ironique est l'expression de la vérité. Le futur barême dégrève légèrcment les petits héritages, mais il accroît énormément la eharge des autres. L'héritier de 2000 francs paie aujourd'hui 20 francs de droits pour la ligne directe; demain, il ne paiera plus que 20 franes. En revanche, en ligne directe, le prélèvement de l'État sur l'hérilage, aujourd'hui uniformément de 1 p. 100 (plus les déeimes), pourra s'élever, suivant le capital hérité, jusqu'à $21 / 2$ p. 100. Entre époux, on ne paiera plus 3,73 p. 100 (décimes compris) mais 7,50 p. 100, ce

1. Article 18 de la loi du 22 frimaire an VIl; articles 5 de la loi du 27 ventose an IX, 11 et 15 de celle du 23 aoùt 1871 , etc. 
qui absorbera à peu près trois ans du revenu. Entre collatéraux, on arrivera à $181 / 2$ p. 100 , ce qui fera perdre sept ans de revenu.

Est-ce un régime normal que celui qui oblige l'héritier, fùt-il le neveu du défunt, à vendre l'héritage pour payer les droits, ou, s'il est déjà riche, à faire des avances sur le revenu futur en se résignant à ne jouir des bénéfices de sa nouvelle fortune que sept ans après en avoir hérité? C'est un essai de collectivisme. C'est une maladresse.

Cette loi, très onéreuse au point de vue de la propriété rurale, n'offre pas moins de dangers en matière économique pure, que la fortune soit immobilière ou non. La matière imposable se dissimulera. Les capitaux se déplaceront plus encore, ils abandonneront de plus en plus la terre pour se porter sur les valeurs de crédit; on choisira de préférence les titres au porteur, richesse fluide, fuyante, insaisissable, et, à l'avantage des titres nominatifs, diminuant les risques de vol et de destruction, se substitue déjà l'assurance; le développement de cette industrie neuve, visant un point spécial, doit avertir le législateur. On le pressentait déjà en 189\%, lorsqu'un économiste s'exprimait ainsi : Les valeurs mobilières offrent aujourd'hui de si grandes facilités pour la dissimulation des fortunes que, le jour où les fortunes seront forcées de se dissimuler pour échapper à la confiscation partielle dont on les menace par l'impòt, chacune choisira dans le groupe des valeurs étrangères celles qui risqueront le moins de subir un traitement tiscal excessif; et alors, si au lieu d'employer nos capitaux à commanditer le travail national, nous les portons à nos voisins et à nos concurrents, il adviendra que le salaire lui-même fera vite défa ut à l'ouvrier.

Tout s'enchaîne. L'écrasement de la propriété rurale par l'impôt, alors que sa sécurité et sa perpétuité sociale sont déjà compromises par les lacunes de nos lois civiles, amène l'appauvrissement de la culture, la clépopulation, le discrédit du métier agricole, l'émigration des hommes et de l'argent à l'étranger. Instruits à la fois par nos propres études et par les 
exemples d'antrui, nous ne pouvons tarder davantage à faire pénétrer dans notre législation fiscale une conception plus démocratique de l’impôt, et à engager la France dans la voie d'un progrès où l'Europe tout entière l'a devancée ${ }^{1}$.

La politique de l'impòt n'a pas que des incidences privées, elle a des effets publics. Elle peut ou raffermir ou désagréger les deux soutiens de l'ordre social : la propriété, fruit légitime du travail et de l'épargne, et l'héritage qui, reliant intimement le père aux fils, est bien la première, la plus sainte et la plus féconde des solidarités humaines.

1. Expression du rapport de M. Trouillot sur le projet de rẻformation de l'impôt (Chambre des députés, 12 mai 1899). 


\section{CHAPITRE II}

Réformes nécessaires, dans le droit civil et le droit fiscal, pour que l'État n'entrave point le libre jeu de l'équilibre économique en matière de proprièté foncière.

L'état d'infériorité dans lequel l'Agriculture est retenue par l'ensemble de nos institutions politiques et sociales est mis en relief par un trait caractéristique des mours actuelles, par la tendance qu'ont, en France, les agriculteurs à quitter leur profession.

Et pourtant, l'agriculture est la plus sûre, la plus féconde, la plus rémunératrice et la plus honorable des industries; elle fournit aux hommes, directement ou par l'échange, tous les objets nécessaires à l'existence et leur assure ainsi la véritable indépendance. Malgré les maux dont elle souffre, maux qui ne sont pas inhérents à sa nature mais dérivent de préjugés et d'intérêts qui l'exploitent à outrance au lieu de s'associer avec désintéressement à son avenir, l'évolution actuelle des conditions du travail provoque une réaction dont on sent les tendances et l'esprit si l'on n'en voit pas encore les effets matériels. Déjà l'émigration des ouvriers agricoles vers les villes s'est ralentie; il s'établit une sorte de niveau entre le net des salaires de la culture et celui des salaires industriels; l'isolement des campagnes a disparu 
par le bon marché des transports et la rapidité des communications; parallèlement à l'hésitation qui retient le paysan au village, même au sortir du régiment, il se produit, parmi les jeunes gens de famille, un goût inattendu de retour aux champs, dù peut-être moins à une saine appréciation des choses qu'à l'encombrement des carrières, au nombre croissant des déclassés, à la difficulté toujours plus grande de trouver dans les villes et dans les services publics l'emploi de ses aptitudes et de son instruction. Ce sont d'heureuses tendances, qu'il faut développer dans l'intérêt du pays, et auxquelles les mouvements économiques dont la brusque intensité nous surprend peuvent apporter un appoint décisif.

Il est certain, en effet, que l'habitude des grèves, la légèreté avec laquelle on les accepte et la duplicité qui les provoque troublent la liberté du travail et découragent le patronat dont les sacrifices et le zèle sont méconnus. Il n'y a plus de sécurité dans l'industrie; les contrats, les marchés ne sont plus seulement menacés par des causes de force majeure, prévues et rares, ils sont à la discrétion du caprice d'un agitateur. Les capitaux se trouvent engagés dans un nouveau risque devenu permanent, et le rude métier de patron sera désormais une carrière où il y aura de moins en moins de concurrents. Des millions de jeunes gens, ingénieurs, dessinateurs, ouvriers d'art, contremaîtres, sortis des écoles spéciales ou professionnelles, se verront fermer à bref délai les débouchés sur lesquels ils comptaient, soit que les fabriques, les usines, les atcliers soient forcés de liqquider, soit que le personnel dirigeant en soit réduit, soil qu'on renonce à s'aventurer dans des entreprises dont, à tout instant, le profit peut être compromis et l'existence brisée.

Celte masse d'intelligences, d'énergies, d'activités, va refluer sur l'industrie agricole qui manque précisément, dit-on partout, des deux éléments de fécondation, la science technique, les capitaux, que la faillite de l'industrie urbaine, du fait des grèves, va déverser sur elle.

Le fait économique, si grave, dont les préliminaires s'accomplissent sous nos yeux, n'est-il pas la preuve décisive 
des incidences réciproques des mours sur les lois et des lois sur les conditions du travail. Les deux actes législatifs sur lesquels on comptait pour pacifier les esprits, transiger avec les opinions extrèmes, réaliser l'aceord définitif du travail el du capital, sont précisément devenus, par l'intransigeance des uns et la coupable timidité des autres, des instruments de désordre, des ferments de dissolution sociale. Les articles $1^{\text {er }}$ el $\ddot{~}$ de la loi du 21 mars 1884 sur les syndicats professionnels el le droit de coalition, les articles $1^{\mathrm{er}}$ à 10 de la loi du 9 arril 1898 sur la responsabilité des aceidents du travail, interprétés et appliqués comme ils le sont, restent destructifs de toute sérieuse organisation du travail industriel.

Qu'on en profite au moins dans l'intérêt de la propriété rurale el que les travailleurs instruits, chassés du travail industriel, au lieu de s'expatrier, s'attachent plus étroilement au sol natal et se réfugient dans le travail agricole.

Comment pourrait-on aicler cette migration à l'intérieur, de telle sorte qu'elle profitat en son entier à l'exploitation agricole et qu'elle consolidât, en l'élargissant et en la fécondant, cette répartition du sol français en petite, moyenne et grande propriété, dont les proportions relatives, dans leur mobilité incessante, témoignent du plus ou moins de vitalité de tel ou tel groupe de propriétaires? On le peut en combinant l'eflet d'habitudes nouvelles avec l'action de lois raisonnées el prévoyantes.

Les traditions des siècles passés, mal comprises, ont discrédité l'agriculture; ceux mèmes qui s'y étaient enriehis s'en évadaient avec orgueil et engageaient leurs fils dans les emplois de bureaux, les professions bourgeoises qui continuent l'ancien régime des charges vénales, les situations dites libérales parce qu'elles supposent l'étude du droit ou de la médecine, et mème la littérature et les arts, devenus des métiers lucratifs. L'opinion courante, en fait, classait l'agriculture au dernier rang, éétail une occupation quasi servile.

L'exemple de quelques familles anciennes, les leçons personnelles données par de nobles esprits, la voie de progrès 
ouverte et continuée par des gentilshommes campagruards ', l'engouement de certains parvenus, quelques fortunes rapidement rétablies, l'imitation des mours anglaises, la mode enfin, relevèrent la profession agricole de son discrédit, et l'on se targua d'y revenir uniquement peut-être parce qu'une légende se forma affirmant quil fallait être riche pour y réussir.

Depuis trente ans surtout, le mélange des classes, l’appoint électoral des ruraux, les discussions économiques à la tribune et dans la presse, le mouvement syndical, ont accentué ce refour à une plus saine apprécialion des choses.

Les propriétaires fonciers se sont honorés de vivre des champs, la spéculation leur apporte son appoint el le simple cultivateur lui-même a profité de ce revirement. Nul ne rougit plus d'avoir viré la charrue ou touché les boufs.

Ce que le service militaire obligatoire a fait pour rehausser le soldat dans l'opinion se produit, et se produira plus largement avec le temps, pour la profession d'agriculteur. On serait surpris, n'est-il pas vrai, qu'un banquier fît gérer sa banque par un commis, qu'un industricl abandonnct la direction de ses ateliers à un contre-maître, se contentant de passer tous les six mois à la caisse? pourcpuoi en serail-il autrement en matière agricole? Pourquoi le propriélaire foncier ne ferait-il pas valoir lui-mème sa terre? Les mocurs peuvent beaucoup en pareil cas, el c'est aux classes dirigeantes à donner le ton.

Jadis, il y a de cela une génération à peine, le loourgeois lıésitait à marier sa fille à un officier ou à un artisle. C'est bien changé. Depuis que le paysan coudoie au régiment le docten en droit ou l'oisif titré, depuis que le peintre et le musicien sont comblés des plus haules distinctions, les préjugés d'antan ont disparu. 11 en sera de même pour ceux 'fui, pouvant ne pas le faire surtout, se décideront à cultiver

I. H. Baudrillart, Gentilshommes ruraux de la France, faisant suite aux pénétrantes études sur l'État moral et matériel des populations agricoles de la France (18\$3 à 1889 et 1893). 
leur héritage. Tout homme dislingué, s'il est avocat, médecin, député ou simple bureaucrate, peut aspirer aux plus brillantes alliances; pourquoi refuserait-on à l'homme qui réside sur un domaine rural ce qu'on accorde au snobisme et à la prétention? Et à quoi servirait d'avoir des lycées de filles si I'on ne corrigeait chez elles le goût des préjugés et l'abus des superfluités? Les tendances des jeunes filles de ce temps exerceront sur notre constitution sociale unc influence décisire, soit qu'elles se détachent joyeusement des plaisirs compliqués et négatifs des villes, soit qu'elles s'étourdissent dans lcur stérilité.

Les groupes, d'origine et de destination différentes, que constitue la division normale du sol exploitable en grande, moyenne et petite propriété, exigent, pour le développement in légral de leur utilisation sociale, des moyens de conservation appropriés à leur caraclère particulier. Les traiter uniformément serait une faute, leurs besoins sont aussi différents que leur nature.

La pelite propriété s'accroîtrait indéfiniment, aux dépens des terres incultes et de l'excédent inutilisé de la grande, si on empèchait son moreellement ou sa vente forcée à la mort du chef de lamille, et si on la protégeait contre l'appât de l'emprunt hypothécaire.

La moyenne propriété doublerait sa valeur intrinsèquc par la généralisation des bornages collectifs et la suppression des enclaves. L'abondance des parcelles détachées des domaines à demi agglomérés et cnclavées au milieu des domaines voisins, est une des circonstances de fait qui mettent le micux en relief l'impuissance des cultivateurs à capitaux restreints à réformer l'assiette de leur propriété sans inconvénients essentiels pour les vignobles et les petites cultures des banlieues urbaines; les enclaves, ct surtout celles de dimensions médiocres, sont un obstacle permanent à toute exploitation perfectionnée des bestiaux, des céréales, des plantes fourragères et industrielles, elles empèchent les irrigations et l'emploi des machines.

Quant à la réforme de la grande propriété, elle se réclame 
moins de l'ordre matériel que de l'ordre moral. Pour donner à ses détenteurs les habitudes de résidenee sinon permanente au moins fréquente, il faudrait réagir contre le nivellement à outrance qui assure le pouvoir aux médiocres ${ }^{1}$ et les encourager à diriger les affaires locales, à s'associer à l'exereice des hautes fonctions, en vertu du principe qui fait la force politique des États européens les plus solidement constitués.

\section{I. - La liberté.}

L'état d'infériorité de l'Agriculture explique la fièvre de doléances qui tourmente les propriétaires ruraux et l'urgence des réformes que réclament les trois groupes principaux de propriétés, la grande, la petite et la moyenne, réformes dont certaines pourraient paraître contradictoires si l'on ne se reportait invariablement, avant toute discussion, à ce principe que les conditions d'origine el les procédés d'exploitation de chacun des trois groupes n'étant pas semblables, chacun a des besoins qui lui sont propres et reut des remèdes particuliers.

Mais il est deux points principaux, et ce sont les seuls en réalité pour lesquels l'uniformité d'application soit possible, où l'on s'accorde en réclamations communes et où l'entente est unanime : ce sont les deux servitudes de la procédure et de la fiscalité, celle-ci moins pesante et moins àpre que l'autre, car l'impôt peut se modérer ou se déplacer, tandis que les privileges accordés aux intermédiaires créés par la loi pour servir et protéger les intérêts privés en sont devenus les exploiteurs intraitables et que tout essai de simplification et d'économie passe pour une atteinte aux droits aequis.

1. L'Empire et Ia Restauration ont voulu développer, au moyen de privilèges, l'influence dle la grande propriété; les gouvernements révolutionnaires se sont, au contraire, appliqués à l'amoindrir; tous se sont abusés en croyant trouver un point d'appui dans la classe qu'ils prétendaient favoriser, dans un esprit exclusif aux dépens de la justice et de la liberté. (La réforme sociale, $\mathrm{I}, \& 34$.) 
L'un des plus puissants mobiles de l'humanité, l'intérêt personnel, est constamment mis en jeu dans les entreprises individuelles, mais si l'énergie et l'esprit d'initiative se heurtent, dans chacune des manifestations de leur activité, à l'intérèt collectif de corporations professionnelles ou à la main-mise de l'État, elles se découragent et se dérobent. Dans un état démocratique tel qu'est, théoriquement, celui de la société française contemporaine, l'effort du législateur, au lieu de multiplier les entraves autour de l'individu, doit être de lui ouvrir toute les voies de la liberté.

Plus que loute autre industrie, l'industrie agricole a besoin d'aroir les coudées franches. Précisément à cause de la complexité de ses travaux, de la variété de ses entreprises, des changements qu'elle peut être amenée à apporter, d'une saison à l'autre, dans ses procédés d'exploitation et dans le choix de ses cultures ou de ses débouchés, c'est nuire à son essor, c'est la condamner à végéter sans s'épanouir, que de l'emprisonner dans des règlements ou la contraindre à se heurter sans cesse à des formalités.

La répartition utile, normale, du sol entre les catégories de propriétaires qui l'exploitent de leur personne ou par intermédiaires, ne peut ètre avantageuse, au sens économique, que si elle s'opère librement, sous la seule influence des intérêts en jeu, en dehors de toute pression gouvernementale, de toute ingérence doctrinale, de toute prime fictive ou intermitlente. 11 convient donc, avant tout autre essai, d'alléger l'ensemble des impôts qui pèsent directement sur le propriétaire rural, notamment en ce qui concerne le droit de mutation et ses accessoires, et surtout d'abréger les formalités de la procédure en matière civile de façon à ne point metire à la charge quasi exclusive de la propriété rurale les 25000 officiers ministériels et les 30000 rabatteurs de procès ou courtiers d'affaires qu'entretient la France.

C'est à l'État à donner l'exemple en réduisant le nombre des emplois rétribués par le budget et en faisant brèche dans cette centralisation, vieille de deux siècles et demi, dont l'excès est clevenu la pire cause du désordre social. 
Quel est le mal dont nous souffrons le plus, celui qui entrave avec le plus d'évidence le développement de l'agriculture industrielle et qui, par conséquent, retarde indéfiniment l'expansion du rôle spécial que doit jouer, dans l'ensemble harmonique de notre société, chacun des groupes de propriétaires fonciers? N'est-ce pas l'absorption de plus en plus entreprenante des forces individuelles, des personnalités actives par l'anonymat collectif? N'est-ce pas la substitution de plus en plus envahissante du gouvernement au citoyen pour tous les acles de la vie sociale et son intrusion dans la vie privée?

Qu'en résulte-t-il, sinon l'affaiblissement progressif de toute initiative personnelle sous la tutelle dissolvante d'une centralisation administrative qui devient insupportable par son ingérence universelle ${ }^{1}$ ?

Que faut-il désirer? Que les citoyens, cessant de solliciter à tout propos l'intervention, l'aide et les faveurs de l'État, mettent quelque fierté à se suffire à eux-mèmes et fondent sur leur propre énergie le succès de leurs entreprises. Peutêtre conviendrait-il de parler ici du régime municipal, du rôle des assemblées locales, de l'utilité des chambres consultalives de paysans, des avantages électoraux el sociaux que mériteraient les propriétaires résidant sur leurs terres et exploitant leur héritage; mais ces considérations touchent de trop près à la politique, il suffit d'en indiquer la valeur.

Certes, notre unité nationale est le fondement incontesté de la puissance du pays, mais si, en se dégageant de la servitude des mots et des formes, on savait joindre it l'unité sociale cette force qui naît du concours spontané des individus et des associations libres, on aurait résolu du mème coup le problème posé par les réformateurs de 1789.

1. Grâce à l'appareil réglementaire que nous a légué le passé, on ne peut pas remuer une pierre, creuser un puits, exploiter une mine, élever une usine, s'associer, et, pour ainsi dire, nser et abuser de son bien, sans la permission ou le contrôle du pouvoir central; et de grands intérêts se trouvent ainsi retartés ou sacrifiés inconsciemment, dans les degrés inférieurs de l'éclelle administrative. (Moniteur du 30 aoùt 1838.) 
Sous quelles formes pourrait se manifester celle liberté d'agir, sans autres entraves que celles qui dérivent, en droit privé, du respect des droils d'autrui, en droit public, de l'obéissance au pouroir dominant de l'État? Par quelques réformes d'ordre civil, fiscal et économique, d'une extrème simplicité pratique, ne troublant point nos mœurs juridiques, ne substiluant pas la tyramnie de la forme à une prétendue faculté dans le choix, adoptant des combinaisons déjà éprourées ailleurs, el autorisant lïndividu, sans l'y contraindre, à échapper aux monopoles et aux sujétions qui, dans notre hiérarchie de burcaucrates irresponsables, brident totalement son initiatire et émasculent sa volonté.

Tout d'abord, au lieu de glisser sur cette pente méprisable qui, depuis que Pilate s'en est lavé les mains, mène chacun à se désintéresser des choses et à rejeter sur autrui les conséquences de ce qu'il fait, il faudrait restituer à quiconque agit ou décide l'honneur et la responsabilité de ses actes et de ses opinions. En Angleterre, en Allemagne, en Italie, les autorités supérieures n'attirent point à elles, pour les absorber dans leur personnalité, toutes les branches d'activité placées lans leur dépendance; clles les subdivisenl, au contraire, en services distincts el pour ainsi dire autonomes dans le ressort de leurs attributions, avec des chefs en rapport direct avec les administrés et personnellement responsables de lunité quils dirigent. Le gouvernement et le public ont done aflaire à des hommes dont on sail le nom au lieu de se heurter, comme en France, à l'inertie, au mauvais vouloir, au parti pris de bureaux anonymes.

Cela fait, le contrôle des services publics deviendrail facile, aussi bien dans l'exercice de la justice el dans l'assielte el le recouvrement de l'impôt que dans les actes quotidiens de l'administration proprement dite. Les juges se résigneraient à véritier les états de frais ${ }^{1}$ et les officiers ministériels, assurés d'un examen sérieux, hésiteraient peul-ètre à forcer

1. Voir la circulaire du ministre de la Justice du 3 décembre $1899 \mathrm{et}$ les discussions sur le projet de loi portant réforme du notariat, adopté par le Sénat et transmis à la Chambre des députès le 2 mai 1899. 
la note ${ }^{1}$; quant aux percepteurs de l'impôt, ils seraient sans doute arrêtés dans l'exagération de cette fiscalité outrancière dont ils se font un titre à l'avancement et qu'amnistie trop facilement l'autorité supérieure, préoccupée de la plus-value des recettes beaucoup plus que du souci d'empêcher les contribuables d'être molestés.

Rien n'empêcherait alors d'aborder les grandes réformes juridiques dont le but serait de dégager lindividu du recours obligatoire aux courtiers d'affaires, et la propriété foncière du réseau deformalités lentes et coûteuses sous lequel elle agonise.

La formule vient d'en être donnée sous la forme parlementaire ${ }^{2}$. Elle se résume dans la Réforme hypothécaire, en utilisant le mécanisme juridique et administratif existant, en créant la publicité absolue et obligatoire, en faisant passer dans le droit commun les procédures simplifiées de purge et de réalisation du gage dont le privilège est réservé à la société du Crédit Foncier.

Cette réforme aurait le triple avantage : $1^{\circ}$ de créer, sans frais, le grand livre de la propriété foncière, rève depuis si longtemps caressé par les économistes et toujours éconduit gràce à la coalition des professionnels qui vivent des procès des autres; $2^{\circ}$ d'uliliser l'instruction obligatoire el gratuite établie par la loi du 16 juin 1881 pour l'exercice intégral et pratique de l'article 113 i du Code civil, de telle sorte que chaque citoyen, sauf les cas réservés par la loi, puisse gérer personnellement ses affaires privées, en dehors de tout emploi obligatoire d'intermédiaires; $3^{\circ}$ de procéder automatiquement, et sans imposer à la propriété rurale une dépense considérable et inutile ${ }^{3}$, à la réfection du cadastre ${ }^{4}$ et à sa con-

1. Voir la lettre du 13 juin 1900 adressée sur ce sujet par le président du tribunal civil de la Seine au président de la Chambre des notaires de Paris. (Journal du Notariat, septembre 1900, p. 576.)

2. Proposition de loi sur la sécurité du titre foncier déposée à la Chambre des députés, le 22 juin $1900, n^{\circ} 1741$, par M. Chastenet.

3. D'après les flocuments publiés par la Commission extra-parlementaire du cadastre, la réfection générale du cadastre coùterait, au bas mot, 600 millions et durerait vingt ans.

4. Par le jeu des bornages généraux et des plans de lotissement, sur 
servation ${ }^{1}$, résultat inappréciable pour la très petite propriété, et mème pour la petite et la moyenne ${ }^{2}$.

Subsidiairement, et comme conséquence logique d'une réforme qui aurait pour résultats immédiats d'assujettir au contròle fiscal tous les contrats de transferts immobiliers, de supprimer la fraude et d'anéantir le stellionat légal, la matière imposable s'étant accrue d'autant, il deviendrait possible d'alléger pour la propriété rurale la charge du droit de mutation, soit en le réduisant à 2 p. 100 par un retour pur et simple au tarif des 5ั-19 décembre $1790^{3}$, soit en le transformant.

La transformation du droit de mutation (entre vifs et par décès) et de l'impòt foncier en une taxe unique d'abonnement, a été proposée par un spécialiste dès $1884^{4}$; depuis, l'idée a été reprise par l'initiative parlementaire, mais avec limitation aux transferts de la propriété rurale à titre onéreux, en laissant de côté les donations entre vifs et les mutations par décès ${ }^{5}$. A mon avis, la réforme, pour être efficace, doil être complète et englober toutes les mutations foncières, quelles que soient leur nature et leur origine.

Ce ne serait que l'extension aux transferts foneiers du système de taxation adopté depuis la loi du 20 février 1849

l'initiative des propriétaires eux-mêmes. (Freyssinaud, Le cadastre et les bornages généraux, 1899.)

1. En réorganisant le service des mutations foncières qui, dans son état actuel, ne se fait pas et coùte 6300000 francs par an.

2. Le cadastre fiscal se trouvant ainsi remplacé par un cadastre contradictoire, e'est-à-dire juridique et opposable aux tiers. Voir la loi du 17 mars 1898 sur le cadastre municipal et les articles 19 à 22 de la loi de finances du 13 avril 1900.

3. La loi du 9 pluviôse an $I V$, rendue à l'époque de la démonétisation des assignats et de la création des mandats territorianx, doubla le tarif en le portant à 4 p. 100, taux maintenu par la loi du 22 frimaire an ViI et qui fut accru de $1,0 \ddot{0}$ p. 100 , à titre de droit de transcription, par la loi du 28 avril 1816.

4. Annales de l'Enregistrement, 1897, p. 438.

5. " Proposition de loi concernant la transformation de l'impôt foncier et du droit de mutation à titre onẻreux des propriétés non bâties en une taxe annuelle, dénommée taxe foncière et de transmission, basée sur leur valeur en cap̣ital, déposée à la Chambre des députés, par M. Malzac, le 9 février 1897. 
afin de soumettre les biens de main-morte à l'impôt de mutation ", et l'équivalent, comme procédés d'assiette et de perception, du droit de transmission établi sur les valeurs mobilières (titres nominatifs et titres au porteur), sous forme de taxe d'abonnement, par l'article 6 de la loi du 23 juin $18: 7$ et l'article 3 de celle du 29 juin 1872 .

Dès 1896, M. Méline exprimait le vou de voir établir pour les mutations de la terre un régime analogue à celui qui régit les transmissions de valeurs mobilières. La mesure que je propose ne changerait pas les habitudes des populations rurales puisque ce ne serait, en somme, qu'une légère addition au principal de l'impôt foncier, avec cette seule différence, arantageuse pour la propriété agricole, que le droit fusionné ne serait plus assis, partic sur le revenu, partie sur le capital, mais uniformément sur la valeur vénale, ce qui supprime l'arbitraire par la fixité de la matière imposable et le bénéfice de la fraude par la modicité de la prime. D'autres, plus autorisés, l'ont dit avant moi. Ce n'est pas la suppression de l'impòt foncier, si inégal qu'on le suppose, qui peut améliorer d'une façon sensible les conditions d'exploitation de la terre, c'est l'atténuation du droit de mutation. Cette taxe varie, dans les divers États européens, de 1 à 3 p. 100; n'estil pas inouï de la voir alteindre, en France, 6,88 p. 100 et 10 p. 100 avec le timbre?

De là, à n’en point douter, l'état d'infériorité de notre agriculture vis-à-vis des pays voisins; la cherté des acquisilions et des échanges en éloigne les capilaux, et ceux qui tentent d'échapper à l'impôt ne possèdent que des titres incomplets et discutables, qu'ils ne peuvent produire en justice sans payer de fortes amendes et qui, en raison de la longueur des délais de prescription ², restent indéfiniment exposés à la revanche fiscale.

1. Le taux de la taxe d'abonnement a été augmenté depuis et va l'ètre de nouveau par un projet de loi déposé le 12 avril 1900.

2. Le droit simple d'une mutation étant un droit principal reste soumis a la prescription de trente ans (cinq arrêts de Cassation du 2 décembre 1873). Les droits en sus et les amendes bénéfieient seuls des 
Le paiement d'une taxe annuelle serait moins onéreux par sa modicité relative que le déboursé immédiat d'une forte somme; cn se répartissant sur des périodes étendues, limpòt atteindrait plus équitablement les détenteurs successif's de la propriété, et ne brusquerait pas tout d'un coup, comme il le fait avec les lois actuelles, les convenances et les intérêts d'un héritier ou d’un acquéreur. Il ne faut pas se dissimuler, d'ailleurs, que les frais sont dans le prix et que, en dernière analyse, c'est le vendeur qui paie l'impôt, de même rque l'emprunteur voit l'argent dont il a besoin diminué de 10 à 13 p. 100 par le prélèvement des frais, hono. raires et accessoires de l'acte.

Telles sont les mesures principales que les propriétaires peuvent réclamer, en matière administrative et économique, de l'intervention de l'Etat ${ }^{1}$. En principe, il faut s'en passer; malheureusement, ici, elle est indispensable et ce quion lui demande surtout e'est de laisser le plus de liberté possible à l'activité du propriélaire. Les conditions de la culture, dans notre pays, sauf de rares exceptions, ne sont pas favorables aux grères; toutefois, il est des circonstances où l'exercice du droit de coalition deviendrait nuisible aux exploitations industrielles de l'agriculture. Il faut souhaiter que le gouvernement s'attache particulièrement à assurer la liberté du travail aux ouvriers qui ne veulent pas faire grève, et qu'il n'étende pas aux risques agricoles les expériences de la loi de 1898 sur les accidents du travail et les utopies allemandes sur l'assurance obligatoire contre la vieillesse, l'invalidité eî le chômage.

Au droit civil, le propriétaire rural peut demander des libertés d'un autre ordre, d'une intimité non moins pratique, et qui visent notamment le partage forcé, le droit de tester, la perpétuité de l'héritage.

prescriptions spéciales établies par l'article 61 de la loi du 22 frimaire an VII, l'article 14 de celle du 16 juin $18 z$ 't, etc.

1. F. Le Play, La réforme sociale. Comte de Butenval, Les lois de succession appréciées dans leurs effets économiques, $4^{\circ}$ édition en 1884. Claudio Jannet, Le Code civil et les réformes indispensables à la liberté des familles, $3^{\text {e }}$ édition, en 188 '. 
L'article 826 du Code civil autorise chaque copartageant à demander sa part en nature de chacune des catégories de biens formant l'actif; à défaut d'entente sur les conditions du partage forcé, la vente forcée ${ }^{1}$.

L'article 832 corrigerait ce que ce principe a de trop brutal; malheureusement, l'esprit de compromis provisoire qui caractérise les titres les plus importants du code de 1804 s'y manifeste par une contradiction flagrante. Le premier alinéa prescrit au notaire, dans la formation et la composition des lots, d'éviter de morceler les héritages et de diviser les exploitations. C'est un conseil très sage mais qui ne vise que les grosses successions comprenant plusieurs domaines ou plusieurs exploitations qu'on peut répartir entre les héritiers sans avoir besoin d'en rompre l'unité: on ne semble pas avoir préru le cas du modeste héritage qui disparait si on le morcèle. Puis, le deuxième alinéa, corroborant l'article 826 et contredisant l'avis donné au notaire, autorise expressément chaque cohéritier à réclamer pour chaque lot la mème quantité de meubles, dimmeubles, de droits ou de créances de mème nature et valeur.

C'est à ces stipulations trop absolues, trop géométriques, remarque $\mathrm{M}$. de Foville ${ }^{2}$, et non à la simple limitation des quotités disponibles, qu'incombe d'ordinaire la responsabilité des liquidations brutales qui désorganisent un héritage et en détruisent la valeur. Il conviendrait que le père de famille eût la liberté de désigner celui de ses enfants qu'il jugge le plus apte à continuer son ouvre et à diriger l'exploitation de l'héritage, sauf à payer les parts de ses frères ou sours par des soultes en argent, conformément aux articles 1073 et suivants du Code civil.

Il conviendrait tout au moins, à défaut de cette désignation, que l'article 813 fùt modifié dans un sens moins impératif, de façon à ce que la nécessité du partage ne restât pas à la discrétion d'un seul des intéressés, ou que, si le partage

1. La réforme sociale au Congrès de 1889 (1890).

2. Le morcellement, p. 201. 
est décidé d'un commun accord, la vente judiciaire pùt être évitée et l'un ou même plusieurs des héritiers autorisés à maintenir l'exploitation rurale dans son intégrité, même en indivision (art. 1832 du Code civil), sauf à remplir leurs cohéritiers de leurs droits en autres valeurs ou par des soultes.

La liberté de tester est toujours l'objet de controverses très délicates. Certes, le droit de tester est reconnu au père de famille, mais l'usacre en est périlleux à cause des restrictions dont il est enveloppé : insuffisance de la quotilé disponible, qui varie de la moitié au quart suirant le nombre des enfants, obligation d'attribuer à chaque cohéritier la mème quantité de meubles et d'immeubles et, par suite, impossibilité de composer les lots au mieux des convenances et des intérêts communs. Les prévisions du testateur sont à tout instant déconcertées, mème dans les partages d'ascendants les plus minutieusement étudiés, par les risques de l'action en nullité et de l'action en rescision et les entraves discrètes qu'amoncèlent les articles $913,826,832$ et 1079 .

Quelles furent les conséquences de cet état de choses? Le paysan, a-t-on dit avec espril ${ }^{2}$, mécontent de ne pouvoir plus avantager le fils de son choix, prit le parti de faire des aînés en supprimant les cadets. Ce moyen fàcheux d'éviter le morcellement des héritages coùte cher à la France et, depuis 1889, le mal n'a fait qu'empirer.

Nul ne peut penser à rétablir le droit absolu de tester, le principe de l'égalité des partages est entré si profondément dans nos mours qu'il fait désormais partie intégrante de notre droit public. Mais, ne pourrait-on pas réduire la réserve, sans tenir compte en aucun eas du nombre des enfants, afin de ne point faire de cette réserve une prime à la dépopulation?

Le Code civil espagnol du $1^{e^{r}}$ mai 1889 nous fournit une solution ingénieuse, sorte de compromis entre une liberté qui peut devenir dangereuse et une réserve trop forte. La

1. M. Boyenval, Les réformes successorales, 1889. 
réserve légale est d'un tiers, le père peut disposer des deux autres tiers; mais, de ces deux tiers, il ne peut disposer que du tiers au profit d'étrangers, quel que soit le nombre de ses enfants; l'autre tiers n'est répartissable qu'entre ses enfants, en faveur de l'un ou de l'autre desquels, d'ailleurs, il peut faire emploi intégral des deux tiers qui constituent la quotité disponible.

Il y a, dans certains esprits, une tendance marquée sinon à réduire le nombre des degrés successibles dans les hérédités $a b$ intestat ${ }^{2}$, tout au moins à surtaxer la dévolution en ligne collatérale. Cette manière de voir peut se discuter, car avec les mœurs actuelles, le peu de fixité du foyer domestique, les déplacements continuels et la dispersion des familles, le cercle de la vraie famille se rétrécit de plus en plus, et les liens se relàchent singulièrement sitôt qu'on n'est plus entre frères el sœurs. Ce doit être un motif de plus pour consolider entre les mains des enfants l'héritage que le chef de famille a reçu de son père, ou qu'il a créé de ses mains et dont il veut perpétuer l'existence au profit des siens.

Mème en dehors de l'intérêt agricole, el au point de vue national le plus élevé, la fixité du foyer rural doit ètre le but constant des efforts du législateur. La liberté demeure létat le plus farorable à l'exercice des aptitudes individuelles, et la valeur de l'effort est doublée quand le proprićtaire, en cultivant sa terre ou en bàtissant sa maison, se sent indépendant el possède la certitude que son labeur et ses privations profiteront à lui el aux siens. Cette conviction est l'un des puissants mobiles du travail. Mais la continuité dans l'effort est une condition non moins essentielle du succès.

Celte continuité ne peut c̀tre obtenue que par la confiance du propriétaire rural dans la sécurité de l'avenir. On espère

1. Voir l'exposé des motifs du projet de loi : $1^{\circ}$ portant modification du régime fiscal en matiere de transmission d'usufruit et de nue-propriété; $2^{\circ}$ portant modification de l'article 755 du Code civil sur la vocation héréditaire (février 1889). 
la donner à la très petite propriété tout au moins en établissant linsaisissabilité du bien de famille, de l'hérilage patrimonial, du toit qui abrite les enfants, de l'atelier rural qui les fait vivre, de même que l'article 592 du Code de procédure civile prolège contre la saisic les meubles et les rêtements indispensables, ainsi que les outils de toute nature nécessaires à l'exercice de la profession.

La création du chez-soi, en Franee, au profil des classes laborieuses, serait la plus solide barrière qu'on pût opposer aux utopies dissolvantes des soeialistes-collectivisies. Quant aux elasses bourgeoises, qui sont considérées comme détenant la moyenne propriété, elles possèdent des ressources suffisantes pour se garantir de tout aceident futur par les combinaisons variées de l'assurance.

Celle liberté permise par la loi au père de famille, dans une limite fixée et à certaines conditions, de meltre en quelque sorte hors du commerce une portion déterminée de son patrimoine, n'est pas une nouveauté. Les Américains du Nord, qui l'ont emprunté à la vieille Europe, l'ont vulgarisé sous le nom de home stead ${ }^{1}$; la Suisse, l'Autriche el bientôt l'Allemagne en ont aceepté ou en préparent l'appropriation pratique aux exploitations rurales ${ }^{2}$.

La question est soumise au Parlement; des propositions émanées de linitiative parlementaire ${ }^{3}$ ont essayé de donner la formule de linstitution nouvelle. Leur ajournement tient, peut-être, à ce que ces projets ne se sont pas assez inspirés des textes similaires, en paraissant innover au lieu de s'adapter à des exceptions juridiques déjà pratiquées et connues.

Voici comment il serait possible de présenter le problème en l'étudiant sous son aspect véritable, qui est double: $1^{\circ}$ création, à peu de frais, d'un titre pour la petite propriété

1. Bulletin du Comité des travaux historiques, section d'économie sociale. 1893,177 à 238 .

2. H. Levasseur a expliqué (L'agriculture aux Élats-Lnis, p. 464) comment il y avait deux sortes de home stead, le home stead de peuplement (loi du 20 mai 1862) el le home stead exemption ou privilège du foyer.

3. Notamment, en 1894, celles de M. Lemire et de M. Léveillé. 
qui, lorsquielle en a, n'en possède que de défectueux; $2^{\circ}$ consolidation de ce titre au nom de la famille, devenant une unité collective.

Les discussions de la Commission extra-parlementaire du Cadastre ont montré comment il était possible de convertir en droit le fait de la possession s'il existait des livres fonciers $^{1}$. Le petit propriétaire rural, même dépourvu des preures de son droit, pourrait ainsi être nanti d'un titre gratuit qui, soumis préalablement à la discussion des tiers par la publicité, deviendrait définilif et inattaquable dans le délai de deux ans, courte prescription remplaçant équitablement les prescriptions de dix, vingt et trente ans que la chicane peut rendre indéfinies. (Art. 2242, 2262, 2263 et 2266 du Code civil.)

Cela ne suffirait pas. Il faudrait que ce domaine, constitué par l'héritage, l'épargne ou le travail, devînt un patrimoine qui, sans perdre son caractère individıel, fùt maintenu à titre collectif dans la famille, et se transformât en une propriété d'une nature spéciale, immatriculée en quelque sorte au nom du foyer domestique comme le serait un titre de rente nominatif indivis grevé de substitution ${ }^{2}$. Nos lois autorisent ces exceptions, et la fixité de l'héritage rural est aussi respectable, tout au moins, que le principe des majorats ${ }^{3}$ ou le régrime faeultatif de la dotalité ${ }^{4}$.

Par quel moyen pratique retenir cet héritage aux mains de la famille, et empècher qu'il ne soit détruit, du virant du chef de famille, par son imprudence, par l'hypothèue et la saisie, après sa mort, par l'obligation du partage ou le caprice de ses héritiers? On le trouve aussi dans le Code.

Il suffirait de le placer sous le régime absolu du bien dotal

1. Conformément aux articles 39 et 63 du projet sur l'institution des Livres fonciers. Le fait de la possession et de sa durée peut être constaté par une certification du juge de paix, sur enquête sommaire.

2. Articles 10 is et suivants du Code civil; loi du 17 mai 1826 abrogée par celle du 7 mai 1849 .

3. Article $896, n^{\circ} 3$, du Code civil.

4. Article 1554 du Code civil. 
mais en le préservant des modalités équivoques qui, dans la plupart des cas, grâce à des interprétations subtiles, au lieu de protéger la femme, la ruinent. En combinant ainsi l'appropriance de la coutume de Bretagne avec l'inaliénabilité de la dot, on obtiendrait l'immobilisation du foyer domestique et, par roie de conséquence, on ferait revivre la puissance d'expansion que l'histoire reconnaît à nos familles rurales d'autrefois. La liberté du propriétaire ne serait limitée que par ses obligations de chef de famille et sa volonté n'en deviendrait que plus autorisée en réclamant l'assentiment de l'épouse et des enfants. Tant il est vrai que toutes les réformes économiques reposent sur la solidarité des libertés.

\section{II. - Le crédit personnel.}

Notre procédure civile et notre législation fiscale, a dit un maître ès sciences économiques ${ }^{1}$, produisent, en se combinant, ce résultat extraordinaire que la Justice, c'est-à-dire l'autorité chargée d'assurer l'exécution des conventions privées ruine fatalement ceux dont les intérèts lui ont été confiés d'une manière spéciale et auxquels la société devait effectivement une sollicitude particulière.

Cette conséquence de règlements mal faits et qu'on n'a pas coordonnés, se constate avec la plus fàcheuse évidence en matière de crédit.

C'est à l'ensemble de ces causes néfastes qu'il faut attribuer la désertion des campagnes à l'intérieur ${ }^{2}$ et l'émigration à l'étranger ${ }^{3}$. Le déplacement des ouvriers agricoles s’accentue par la réunion de trois circonstances: l'esprit

1. M. de Foville, Le morcellement, 102.

2. L'enquête agricole de 1892 constate un déplacement de près d'un million d'individus affectant 69 departements.

3. Variant de 18809 en 18577, à 3133 é en 18\$9, de 20560 en 1890, à seulement 5300 en 1893.

J.A PROPRIÉTÉ RURALE EN FRANCE. 
d'initiative des partants, l'attrait de réussites lointaines ' ou la proximité de centres de travail 2 .

Le peu de rémunération que trouve le paysan dans la culture d'une terre ingrate, et le manque de capitaux pour améliorer ou transformer les exploitations défaillantes sont acceptés comme les motifs déterminants de l'abandon du sol. Il est une autre cause qu'on néglige, et dont l'influence est peut-être plus déeisive, c'est la déchéance lente mais continue de la plupart des petites industries locales associées au travail des ehamps. A l'heurc actuelle, pour eiter l'une des plus précieuses, l'élevage des vers à soie, entravé par les concurrenees exotiques, ne donne plus que des profits dérisoires, après avoir enriehi le bassin du bas Rhòne et les vallées cévenoles. Le tissage de la toile, la saboterie, les outils élémentaires, supplantés par la grande industrie, n’apportent plus dans la famille paysanne ce contingent de travaux aecessoires qui occupaient l'époque du chômage des champs, eet appoint de gains qui dédommageait d'une mauvaise récolte ou, dans les bonnes années, accroissait le profit et l'épargne. La plupart des ouvriers de métier qui, tout en eultirant leur héritage ou en louant leurs journées d'agriculteurs, trouvaient à exercer leur profession au village, ne le pouvant plus à eause de la coneurrence des ateliers urbains plaçant à meilleur marehé le produit de leur fabrieation, ont émigré dans les villes, eroyant y rencontrer un plus fruetueux emploi de leur état. L'agriculture y a perdu du même coup l'homme et le métier. Bref, beaucoup de menus travaux intermiltents on d'ocension, qui faisaient, il y a quelques années, de l'argent dans le milieu rural ne se demandent plus, quantité d'objets d'une fabrication primi-

1. En 1894, sur près de 600000 français résidant à l’étranger, le tiers avail émiqré dans l'Anérique du Sud. Certaines communes de Savoie dans les environs de Chambéry, la vallée de Barcelonnette dans les Alpes. certaines paroisses du Béarn dans les Pyrénées. se sont dépeuplées a liappel de compatriotes qui firent fortune à Buenos Ayres ou à La Plata.

2. Tels les maçons de la Creuse et les manouvres du Cantal et dı puy-de-Dôme émigrant à Paris et à Lyon pour la campagne d'été. 
tive ne se rendent plus; il en résulte une déperdition de forees regrettable, une brèehe dans le budget campagnard, et cela à un moment où, dans certaines régions et aux heures de presse, la main-d'œurre rurale est hors de prix.

Nais, au lieu de chercher à remédier aux inconvénients de cette transformation par des expédients connus, dont le plus efficace serait la reconstitution des ateliers de famille par le transport de la force motrice à distance, on s'est uniquement préoccupé, sous la pression de spéculateurs toujours en quête d'émissions financières, de la création d'un erédit agrieole.

Pour quiconque a vécu à la campagne et s'est mêlé à l'industrie agricole, les thèses sur le Crédit foncier, le Crédit agricole, le Crédit rural, sont des fietions d'imagination ou des combinaisons d'agio; elles ne peuvent servir que dans des occasions très rares, dans des espèces particulières, aux progrès de l'agriculture ou aux besoins terriens du propriétaire rural. Tous les essais tentés dans cette roie ont arorté. Les projets les plus récents, même amendés dans le sens de l'effort local et mulualiste, sont ajournés ${ }^{1}$, parce qu'on s'est heurté, dès qu'on a voulu en étudier de près les possibilités d'exécution, à de telles difficultés qu'on a eu le bon esprit de s'arrêter plutòt que de s'aventurer dans une entreprise mort-née; et eependant, ce n'étaient pas les capitaux qui faisaient défaut, l'entreprise était largement dotée ${ }^{2}$, mais, dans le retour d'opinion qui se produit, et dont il convient de féliciter l'esprit public, les institutions dont l'État se réserve le contrôle et la direction sont frappées de

1. Loi du 31 mars 1899, ayant pour but l'institution des Caisses régionales de crédit agricole mutuel et les encouragements á leur donner ainsi qu'aux sociétés et aux banques locales de crédit agricole mutuel.

2. La loi de 1899 met à la disposition des Caisses régionales, destinées à servir de trait d'union naturel entre les caisses locales et l'État, non seulement 40 millions avancés à titre gratuit par la Banque de France (loi du 17 novembre 1897) et une rente annuelle de 2 a 3 millions, mais un fonds de roulement que les opérations de banque autorisées par la loi porteront au chiffre de plus de 200 millions, le jour où les mutualistes auront eux-mémes souscrit des parts suffisantes pour avoir droit au maximum des avances. 
discrédit et on leur préférera, avec raison, les sociétés créées par l'initiative locale, avec des vues d'indépendance et de décentralisation.

La loi du 31 mars 1899 est une erreur législative et économique, parce qu'elle restreint et atténue l'esprit libéral qui avait inspiré la loi du 5 novembre 189', favorisant la création de sociétés locales de crédit agricole mutuel en les exonérant, notamment, de la patente et des droits sur les valeurs mobilières; la loi de 1900 a corrigé en partie cette erreur; mais pourquoi mettre les sociétés agricoles en dehors de la loi organique du 24 juillet 1867 en ce qu'elle a de libéral, et pourquoi ne pas commercialiser, purement et simplement, la condition juridique des cultivateurs?

L'erreur génératrice de toutes les déceptions qui ont accompagné les mesures prises jusqu'à ce jour soi-disant au profit de l'agriculture, a été de croire que l'État seul pouvait intervenir, directement ou par des sociétés interposées; et de prétendre, surtout, que la propriété rurale avait besoin d'un crédit spécial exclusivement destiné à des améliorations agricoles.

La propriété territoriale ne meurt pas du défaut de erédit, au contraire, elle meurt de sa dette.

Comment, affirme-t-on dans les documents parlementaires, obtenir de nos petits cultivateurs qu'ils donnent à leurs terres les améliorations reconnues nécessaires par la science, si on ne met entre leurs mains les capitaux indispensables pour effectuer ces opérations? Ce qui a si longtemps paralysé les efforts de l'agriculture, n'est-ce pas en effet le manque d'argent, l'insuffisance du crédit dont elle jouit pour se procurer des capitaux? Sans le crédit, c'est en vain que la science découvre chaque jour de nouveaux éléments de fertilisalion, c'est en vain que la mécanique invente des engins qui suppléent au défaut des bras et accélèrent la rapidité du travail.

Ces réflexions sont judicieuses. Mais on oublie d'ajouter que si la propriété ne trouve pas le crédit quasi illimité dont elle aurait besoin pour faire de la culture intensive, de 
l'exploitation industrielle, c'est qu'elle est déjà obérée à un tel point qu'elle n'offre plus de gage suffisant pour contracter de nouveaux emprunts. Le propriélaire rural cultivant son hérilage exclusivement ou, avec le sien, le bien d'autruit, entre pour 63 p. 100 environ dans le nombre global des propriétaires fonciers; ceux de ces propriétaires qui n'exploitent pas sont tous très largement endettés; les autres le sont dans des proportions qui varient de 1 ’̀ à 50 p. 100 et davantage, de la valeur vénale de leurs biens ${ }^{1}$. C'est le propriétaire foncier et non pas le cultivateur proprement dit qui a créé la dette agraire.

Les causes de la dette, les spécialistes le savent, sont exceptionnellement les avances faites à l'exploitation agricole; d'une manière générale, leur origine est tout autre. Les propriétaires forains, ceux qui ne résident ni n'exploitent, ne considérant leur héritage que comme un gage, l'utilisent jusqu'à épuisement et empruntent pour leurs besoins personnels, qui n'ont aucun lien avec les exigences de l'exploitation; certains, qui résident à intervalles ou en permanence, empruntent pour construire, se réduisant ainsi à la condition de simples locataires. Quant aux propriétaires cultivateurs, ceux-là tiennent à la terre, ils ont la passion de l'acquisition, et les deux uniques sources de leur dette hypothécaire sont, d'une part, des prix d'achat impayés ", de l'autre, des soultes de partage dues à des cohéritiers ${ }^{3}$. Cette situation n'est pas particulière à la France; l'endettement de la propriété petite et moyenne, en Autriche, a les mèmes sources, et dès 1889, le législateur y a pris des mesures radicales en faveur de la

1. Il est regrettable, à tous les points de vue, que l'Administration des finances s'obstine à ne point établir les statistiques des hypothèques; si l'on connaissait avec certitude la répartition de la dette agraire entre les propriétaires exploitants et ceux qui n'exploitent pas, le problème foncier serait aux trois quarts résolu.

2. En 1898, on a inscrit 293786 privilèges du vendeur pour des prix d'acquisition impayès dont le chiffre n'a pas été relevé, sur une moyenne de 703000 mutations (715 913 en 1895 ).

3. En 1890̈, les statistiques de l'Enregistrement accusent 88510 soultes dont le montant dépasse $23 \dot{z}$ millions de francs. 
transmission intégrale de l'atelier agricole, le $H_{0}$; les races ont été affaiblies par la brisure du foyer rural, disait V. Kaempfe, fortifiées par sa reconstitution. Nos lois civiles et fiscales, remarque M. Alfred Fouillée pour la France ${ }^{1}$, dévorent et émieltent la substance du sol. La terre qui changerait de mains tous les trois ans rapporterait zéro et deviendrait pour ses possesseurs l'équivalent d'un verger planté d'arbres morts.

On avoue qu'il y aurait imprudence à ouvrir inconsidérément du crédit à l'agriculteur si l'argent prêté devait être employé à autre chose qu'à l'amélioration de la culture ou à l'accroissement raisonné de l'outillage agricole. Ce qui se dépense ailleurs, en effet, coùte de 7 à 10 p. 100 et rapporte de $21 / 2$ à 3 p. 100; si, au lieu d'acheter d'autre terre, on jette cet argent, sous forme d'engrais, de semences, de cheptels, sur la terre qu'on a, on peut en tirer 10 à 12 p. 100, et l'on y gagne au lieu d'y perdre.

Cette considération met sur la roie des mesures à prendre pour ne point courir ce risque : celles qui permettent de ne faire bénéficier des prêts d'argent que les agriculteurs qui en font un bon usage et d'assurer au prêteur le remboursement de sa créance sans avoir à réaliser le gage. Autrement dit, il faut, ainsi que le fait la Banque de Russie arec ses nouveaux statuts, exiger la déclaration de la destination des fonds et en surveiller l'emploi au fur et à mesure des acomptes fournis sur l'ouverture de crédit. C'est pour avoir méconnu la sagesse de cette précaution que le Crédit agricole de $\mathbf{1 8 6 0}$ a liquidé en $\mathbf{1 8 7 6}$, que la Banque de crédit au travail de 1863, la Caisse d'escompte de $1860 ̈$, la Caisse centrale de l'epargne et du travail de 1851, etc., ont successivement sombré. En disparaissant, toutes ces sociétés ont dû reconnaître que la raison de leur insuccès était dans leur centralisation excessive, dans leur éloignement de la clientèle, dans leur impuissance de se procurer des renseignements précis sur les aptitudes et la moralité de leurs emprunteurs.

\section{La propriété sociale et la démocralie.}


Ce qui revient à dire qu'en matière de crédit rural, il est indispensable de se pénétrer de la connaissance locale des choses et des gens et que la solvabilité morale est une garantie plus sủre que le gage, mème immobilier.

Il est donc superflu de chercher, après tant d'essais infructueux, quelle pourrait ètre une organisation efficace du crédit agricole; la formule est trouvée, elle est simple et il faut s'y résigner, c’est le crédil personnel.

Le crédit personnel est essentiellement différent du crédit réel. Celui-ci ne se base point sur la moralité du débiteur mais sur la valeur et la solidité du gage. Or, la dette agraire étant déjà si absorbante qu'elle écrase la propriété territoriale sous le poids des intérèts, il serait logique de l'en dégager avant de lui superposer une dette nouvelle d'un caractère plus spécial.

Les procédés empiriques que l'on a essayés, soit par les lois limitatives de l'emprunt, soit par des amortissements à long terme demeurent inefficaces. Cependant, le système des annuités représente des avantages de plus en plus accentués, à mesure, bien entendu, qu'on en relève le taux, el à la condition de ne point annihiler le système par des remboursements anticipés. Si les intérêts sont à 4 p. 100 et qu'on amortisse à $1 / 2$ p. 100, la detle est éteinte en cinquante-cinq ans parce que les annuités travaillent à intérêts composés. Le vrai principe économique est : d'abord que chaque génération doit liquider ses dettes immobilières, ensuite que la dette agraire doit non pas se rembourser mais s’amortir. Dès 1893, en Allemagne, on a inauguré cette ingénieuse combinaison en garıntissant l'amortissement par une assurance sur la vie, ce qui permet de ne point dépasser pour la prime annuelle plus de 4,90 à 3,73 p. 100, l'équivalent de l'intérèt simple des emprunts les moins onéreux ${ }^{1}$.

1. Rapport de M. Block sur le système de désendettement de la propriété rurale proposé par M. Félix Hecht, à llannheim (Comptes-rendus de l'Académie des sciences morales, juillet 1899, p. 10't). 
Mais, si l'on parvient à supprimer ainsi la dette hypothécaire qui grève la propriété rurale on n'aura résolu qu'une partie du problème, on aura dégagé le sol et le propriétaire de charges très lourdes et d'entraves fort gènantes, mais on n'aura pas mis à la disposition du cultivateur les ressources dont il a besoin pour améliorer son exploitation et tirer du sol toul ce qu'il peut produire. Lorsque le proprićlaire foncier réunit en sa personne le double caractère de propriétaire et d'exploitant, ce qui est le cas le plus fréquent et le plus digne de sollicitude, il se trouve gêné aussitôt qu'il a hypothéqué son bien, il s'est créé à lui-mème des embarras dont il aura quelque peine à se débarrasser et qui, par la force des choses, diminueront le prestige de sa personnalité, aflecteront son crédil moral. Un propriétaire exploilant, dont la situation hypothécaire est libre, trourera plus facilement du crédit sur sa simple signature qu'en offrant ses biens en gage. Il se passe en cette occurrence, à tort ou à raison, quelque chose d'analogue a l'anomalie qui fera confier à un négociant cent mille francs de marchandises payables à diverses échéances, tandis qu'on ne lui prêterait pas 10000 francs d'argent ì 90 jours.

Le Crédit personnel restera toujours la véritable source de la confiance. Un gagre minime ou déja entamé ne peut pas ajouter grand'chose a la solidité du prèl, tandis que ce mème gage, ou libre ou insaisissable, donne une assielle plus sùre au crédit personnel dontil est, en quelque sorle, la réserve.

Si done l'emprunt hypothécaire est facile au propriétaire rural qui n'est que propriélaire, celui qui est en mène lemps cultivateur doit l'éviter le plus possible et se contenter du crédit personnel que lui méritent, à des degrés inégaux selon l'opinion qu'on a de lui, sa situation privée, ses aptitudes, ses précédents, sa moralité. L'oi la conséquence logique que, pour s'exercer utilement, le crédit personnel implique le crédit local, la décentralisation, le rapprochement du prêteur et du débiteur.

Le paysan n'a échappé au servage féodal, à la servitude 
légale de sa terre et de sa personne, que pour tomber sous la servitude de l'argent, servitude plus dure et plus envahissante parce qu'elle est anonyme, et c'est iei qu'on se heurte à l'une des plus dangereuses équivoques de l'état social contemporain.

Sous prélexte de créer le crédit, on excite à l'emprunt. C'est un péril économique, et, dans un pays comme le nòtre surtout, il parait plus sage de conseiller au chef de famille, au pelit et mème au moyen propriétaire, le travail, l'économie, l'épargne, que de le solliciter par les séduisantes tentations d'un crédit éphémère qui le mène droit à la saisie.

Il ne suffit pas, en effet, qu'on puisse devenir propriétaire; il faut, surtout, qu'on puisse le rester.

Plus on ofirira au travailleur mal instruit des vaines doctrines économiques la facilité de s'engager dans les roies néfastes de l'emprunt, plus on le proroquera à transformer en gage sa maison, ses champs, son bétail, ses récoltes, et moins on consolidera sa situation qui, si modeste fùt-elle, vaudra toujours mieux que la précarité et le souci de la condition de débiteur. Il faut distinguer entre les différentes appropriations du crédit; ce qui est fécond pour les grosses affaires devient désastreux pour les petites, et c'est un danger social que d'habituer l'homme de travail à dépenser l'argent qu'il n'a pas vu s'amasser lentement et péniblement dans ses mains. Le numéraire sous forme de crédit n’est plus, à ses yeux, qu'une valeur fietire, comme le jeton sous les doigts du joueur.

En voulant tout régler par le même niveau et offrir des expédients semblables à des situations qui n'ont rien de commun, on s'est aventuré dans des expériences stériles.

Le mourement d'opinion qui a cherché le crédit agricole dans les modifications partielles d'une législation qu'on a accusée de perpétuer le discrédit agricole, n'a pas été heureux dans ses manilestations. Telle la loi du 18 juillet 1898 sur les warrants agricoles, qui tombe en désuétude avant 
d'ètre appliquée ${ }^{1}$ et qui, même dans les cas où on pourrait l'utiliser avec avantage, ne peut profiter qu'aux très gros producteurs de blés ou de vins.

Les vues du législateur étaient excellentes; mais on oublie souvent qu'il y a loin de la théorie à la pratique et que, si favorables que certaines combinaisons financières ou juridiques puissent paraître à ceux qui les imaginent, il n'est pas aisé de les imposer aux habitudes de ceux qui auraient à s'en servir.

Si l'on considère, en effet, les centres les plus actifs de l'initiative industriclle, on est surpris de l'extension croissante du erédit mobilier, qu'il s'effectue sous la forme du nantissement par un dépôt de titres ou de marchandises, ou qu'il se réalise par d'autres formules de l'ingéniosité des courtiers et spéculateurs financiers.

Jusqu'ici, l'agriculteur, propriétaire, métayer, ou fermier, ne voyait, en France ${ }^{2}$, aucun de ces moyens s'offrir à lui, en raison des règles étroites du Code civil (Article 2076) qui exige la remise effective du gage entre les mains du créancier, et aussi à cause du privilège du propriétaire (Article 2102, $\mathrm{n}^{\circ}$ 1) sur tous les produits de la ferme et sur son matériel d'exploitation. Sans doute, il arait la faculté de transporter ses récoltes dans les Magasins généraux, mais les distances et les frais rendaient cet expédient impraticable 99 fois sur 100, el le producteur, pressé d'argent, demeurait à la discrétion de la spéculation qui le guette et profite de la précipitation des ventes pour céprécier les cours. D'où l'idée assez naturelle de constituer à domicile le warrantage des produits en ítablissant des règles, forcément trop compliquées, pour créer la publicité, assurer l'identité du gagae et punir la fraude.

1. II. Pascaud, Le warrantage agrieole, 1899.

2. La loi dı 1 k juillet 183̈, maintenue par la loi organique du $2 ;$ juin 187\%, a etabli clans les colonies françaises le gage à domicile, uncme sur récoltes pendantes. Depuis, la Roumanie, le Portugal, l'Italie, la Belgique, ont organise le privilige agricole sur les cheptels et les rẻcoltes. 
La loi de 1898 soulèvera de nombreux conflits dont on a remis l'arbitrage au juge de paix. Son application, restreinte aux grandes exploitations qui ont besoin, a courte échéance, du renouvellement incessant de leurs fonds de roulement, restera en suspens jusqu'à ce que se soient établies des banques locales pour tournir des capitaux; ce n'est qu'un palliatif, une pierre d'attente. Au lieu de ces réformes partielles du Code civil, mieux vaudrait accepter franchement la situation telle qu'elle est et imiter plus simplement qu'en greflant des lois stériles sur des lois vieillies, l'exemple, autorisé par une expérience séculaire, de l'Angleterre, de l'Écosse, des États-Lnis, où les propriétaires fonciers et les agriculteurs trouvent aisément du crédit, parce qüils sont assimilés aux commerçants et peuvent s'engager comme eux.

C'est par un étrange abus d'interprétation par l'absurde que notre jurisprudence refuse aux cultivateurs, quils soient on non propriétaires, le droit d'utiliser la procédure commerciale. Rien n'est à changer dans nos Codes. Il suffirait seulement de conserver à l'article premier du Code de commerce son acception normale et grammaticale :

Sont commercants, ceux qui exercent des actes de commerce et en font leur profession habituelle.

Or, le commerce consistant dans l'éehange des produits et se résumant dans la pratique d'acheter pour revendre, on ne comprend pas que le cultivateur soit exclu, au point de vue juridique, du groupe commercial, lui dont la vic se passe à acheter, à façonner, à transformer et à revendre des objets et des produits de toutes sortes, ce qui lui donne le double et incontestable caractère d'industriel en même temps que de commerçant ${ }^{3}$.

Si done la logique dominait et écartait les préjugés qui, en discréditant par une fausse interprétation historique la

1. Un arrèt de Cassation du 5 novembre 1812 classe dans la catégorie des commerçants les artisans ou industriels qui achètent des matières premières et les revendent après les avoir façonnées. 
condition de l'exploitant de la terre, semble refuser au propriétaire rural le droit de jouir de son héritage autrement que par des intermédiaires (métayage et fermage); si l’on restituait à l'agriculteur le privilège d'agir et de contracter comme un simple commerçant; si, en dégageant le sol de la dette agraire, on faisait de la liberté du fonds et de la moralité de l'homme les gages véritables du crédit personnel, on aurait fait table rase de tous les obstacles qui gênent le libre développement de la propriété rurale, sa répartition et son groupement.

Linitiative individuelle, sous la direction des syndicats agricoles, pourrait alors utiliser les lois du 5 novembre 189. sur les Caisses mutuelles de crédit agricole, du 31 mars 1899 sur les encouragements à donner aux Sociétés el aux Banques locales de crédit mutuel, et du 4 juillet 1900 relative à la constitution des Sociétés ou Caisses d'assurances mutuelles agricoles.

\section{III. - L'assurance.}

Le crédit hypothécaire, qui con̂te au moins כ̈ p. 100 alors que la terre rapporte au plus 3 p. 100, conduit à bref délai le propriélaire foncier à la ruine, à moins que celui-ci ne l'emploie exclusivement à des améliorations agricoles et ne le rembourse sur la plus-value.

Le crédit personnel, gagé sur le travail et la moralité, est le senl qui puisse satisfaire aux exigences de l'exploitation foncière, si tant est qu'il n'y ait pas imprudence à solliciter, même dans les meilleures conditions, le concours des capiLalistes au lieu de limiter son ambition à l'emploi de sa propre épargne. Mais le crédit personnel, s'il reste isolé, est lui-même impuissant; trois éléments solidaires sont nécessaires à sa libre et complète expansion : la coopération, la mutualité, l'assurance ${ }^{1}$.

1. Aussi ne peut-on qu'applaudir aux mesures de faveur prises par la loi du \& juillet 1900 en vue de faciliter la création et le fonctionnement 
L'histoire de nos syndicats agricoles atteste à quels merveilleux résultats pourrait atteindre l'initiative privée, si l'État ne l'appauvrissait pas par la fisealité et s'il ne l'entrarait pas par la protection. En Angleterre, en Allemagne, en Italie, la coopération et la mutualité ont réussi au delà de toute espérance. Un observateur perspicace ${ }^{1}$ a recueilli des preuves remarquables de ce que peut obtenir le plus modeste propriétaire rural, s'il s'inspire des bonnes méthodes et s'il s'associe à des groupes bien conduits.

Ainsi, il existe à Glogau, en Silésie, une suererie qui est alimentée par les propriétaires cultivateurs du roisinage, réunis en société. Sur 216 adhérents, 98 sont engagés pour 2 hectares de culture en betteraves et 32 pour seulement $3 / 4$ d'hectare. La cotisation pour la participation des sociétaires aux frais généraux étant de 1000 franes à l'hectare, voilà donc 32 petits propriétaires qui ont versé chacun 750 franes et qui touchent un dividende variant de 10 à 16 p. 100. L'assolement étant de quatre ars, l'engagement pour $3 / 4$ d'heetare suppose la possession de 3 hectares. C'est par l'association seule que d'aussi petits propriétaires peuvent profiter de la grosse entreprise industrielle d'une sucrerie, qui coùte fort cher et immobilise un capital important tout en exigeant pour la eulture et pour la fabrication l'emploi des procédés scientifiques les plus perfectionnés.

En dehors du bénéfice direct que retirent les associés des produits de leur culture (achetés non au poids mais d'après leur valeur saccharine), eette combinaison leur assure des avantages indirects fort appréciables, surtout au point de vue de l'avancement économique de la région. Elle associe des propriétaires très modestes à un progrès industriel remarquable; elle les amène, par persuasion ou par contrainte, à une pratique raisonnée; elle fait leur éducation,

de sociètés ou caisses d'assurances mutuelles agricoles, gérées gratuitement et qui, en fait, ne produisent aucun bénéfice destiné à être réparti à des actionnaires ou à des commanditaires.

1. Paul de Rousiers, La puissance commerciale de l'Allemagne; ses causes économiques et sociales, 1900. 
les arrache à la routine, leur donne confiance dans la culture scientifique 1 .

C'est sur ce principe d'insuffisances isolées qui réunies en faisceau deviennent une force, que Schulze-Delitzsch fonda les Caisses rurales qui portent son nom et que garantit la responsabilité solidaire illimilée des membres ${ }^{2}$.

Bref, lidéal serait que toul individu qui en a besoin pût profiter du crédit, mais que personne n'en abusàt ${ }^{3}$. Le contròle exercé dans un cercle étroit par des mutualistes qui se connaissent peut pourvoir au risque de la faillite du débiteur, l'assurance garantit par surcroîl le débiteur et ses associés contre les accidents qui ne sont pas de son fait.

L'assurance peut se définir par la mulualité, et réciproquement. Au sens économique du mot, et en ne considérant que le principe, sans s'arrèter aux nuances de détail des différents modes d'assurance contre tel ou tel risque déterminé, on peut dire que c'est une association ayant pour objet la répartition de certains risques sur le plus grand nombre possible d'associés, afin de rendre presque insensible l'elfet de ces risques sur chacun d'eux ${ }^{4}$.

Jamais les applications de l'assurance ne peuvent être plus nombreuses qu'en matière d'exploitation rurale, car son principe s'applique à tout et peut tout prévoir. Il consolide et cautionne toul, depuis l'acquisition de la propriété

1. Les Syndicats agricoles peuvent lutter surtout contre l'intrusion des intermédiaires qui, en raison de l'abondance de leurs capitaux. deviennent les maitres des marchés. Consommateurs et producteurs subissent le joug de quelques grompes de spéculateurs que guide seul J'intérèt personnel. Les prix ne varient plus sclon l'abondancc ou l'insuffisance de la récolte, devenues des éléments négligeables, mais suivant que s'accroissent ou que diminuent les stocks du commerce el de l'industrie.

2. Les caisses Raiffeisen sont, au contraire, fondées sur le sentinient et dérivent de l'idée d'une solidarité charitable qui n'a point les racines solides de la réciprocité des services.

3. Naurice Block, Une crise de la propriété rurale en Allemagne, 1898.

4. La mutualité est l’association combinée ou conciliée avec la liberté de chacun et l'égalité de tous, quelles que soient les incompatibilités des caractères et la différence des interêts; c'est le principe de l'assurance sous ses diverses formes. ( . Neymarck, Vocabulaire-Manuel d'économie politique, 286.) 
jusquà sa mise en valeur, il s’approprie à l'extinction de la dette agraire comme à la grarantie du crédit personnel, à la vulgarisation des habitations à bon marché ${ }^{1}$ comme à la protection de l'héritage contre tous les risques qui peurent latteindre, incendie, grèle, contagion du bétail, accidents du travail, mort prématurée 2 .

La répartition du territoire entre les trois groupes économiques de ses détenteurs assigne à l'assurance, et spécialement à lassurance mutuelle, qui tend à absorber les autres, un rôle particulier suivant le groupe qui sollicite son concours. Liexploitation de la petite propriété a pour base le travail : louvrier agricole aura pour protection fraternelle et efficace l'association tant qu'il sera valide, l'assurance dès qu'il cessera de l'ètre. La grande et la moyenne culture ont pour moyens d'action et pour élément d'activité fécondante le crédit, c'est encore à l'assurance que les possesseurs du sol demanderont une double caution : contre les risques qui menacent leur production, contre la dette qui compromet leur héritage.

A ce point de vue particulier, l'expérience d'assurance obligatoire qui se poursuit en Allemagne et en Autriche est pleine d'intérêt. La théorie qui refuse à l'assurance contre les risques communs dont lincidence affecte la collectivilé le caractère de contrat privé est soutenue par de bons esprits.

Si la prime d'assurance, prise comme précaution d'ordre public, était assimilée à la vaccine, ne deviendrait-elle pas une simple forme de l'impôt? La compétence absolue de l'État serait-elle alors discutable?

1. Lois du 30 novembre 189 ; et du 31 mars 1896.

2. L'assurance agricole peut même devenir internationale comme l'essaie le comité de Fribourg en Suisse (1899). On peut opposer trust à trust, comme le font les agrariens d'Amérique. On combine les efforts, en France, vers la production intensive mais on ne s'organise pas pour la vente des produits en cherchant des dẻbouchés assurés. 


\section{CHAPITRE III}

\section{Conclusions.}

Il serait présomptueux de vouloir indiquer ce que sera en France l'avenir de la grande, de la petite et de la moyenne propriété ; tout au plus est-il permis de dire ee qu'elles pourraient être pour remplir le rôle auquel chacun de ees groupes paraît destiné dans les conditions sociales et économiques où se trouve le pays.

La recherche de l'exactitude dans l'état présent est déjà fort dilficile, car les statistiques, en admettant leur sincérité, ne sont pas toujours l'expression complète des faits dont clles cherchent à déterminer la quantité; d'ailleurs le risque de se tromper sur leurs incidenees est fréquent. On ne peut mème pas se mettre d'accord sur les constatations les plus essentielles et, suivant les auteurs et leur point de vue, l'évaluation des forces productives agricoles de la France varie du simple au double ${ }^{1}$, ce qui interdit toute affirmation absolue.

La matière en discussion n'en est pas moins du plus haut intérêt et jamais problème plus délieat que celui de la répartition normale de ces sources de richesse n'a retenu l'attention de l'historien social et la sollicitude du législateur.

1. E. Levasseur, Note sur la valeur de la production agracole de la France, 1891 . 
Il s'agit, en effet, d'un territoire de כ̋2 millions d'hectares, dont plus de moitié en terres labourables, qui produit de 10 à 12 milliards de franes en revenu brut ${ }^{1}$ et qui est habité par 38 millions d'individus. Il faut éviter les exagérations des publicistes qui évaluent le revenu agricole brut à 24 milliards comme le pessimisme des sectaires qui résument ainsi l'état rural de notre pays : Un tiers de la surface de la France n'est pas cultivé, un tiers est mal cultivé, et le reste, qui l'est mieux, coûte trop cher pour nourrir son homme?

Il est donc utile de rechercher si la répartition actuelle de la propriété entre les différentes mains qui la détiennent est en harmonie avec les besoins économiques du pays, si les conditions du groupement peuvent se modifier dans un sens progressif et si, soit par un changement dans la distribution des exploitations, soit par une extension de l'intensité culturale, on peut accroitre dans une forte proportion ce revenu brut de 10 à 12 milliards qui se réduit à un revenu net de 3 milliards au plus, c'est-à-dire qui con̂te 70 à 73 p. 100 de prix de revient, ce qui serait exorbitant si l'on ne tenait pas compte de la consommation par le producteur lui-même de la plus grande partic de ce qu'il produit. Sans la dette agraire, ne pourrait-on pas admettre, en accueillant cette théorie, que le revenu net agricole représente l'épargne de l'exploitant? Il est certain, dans tous les cas, qu'un peuple comme le nòtre, apte à vite lout comprendre, condensateur d'idées, prodigue de ses forces, assimilateur par excellence et qui se fait un sang bien ì lui de chaque nourriture, ayant dans les mains un aussi puissant instrument de production que l'est notre sol ${ }^{3}$, peut en tirer, pour peu qu'on lui laisse la liberté de le faire et quion ne stérilise pas son énergie par de mauvaises lois, des sources intarissables de richesse.

Il faut examiner la question de haut ct fixer les lignes générales pour ne pas brouiller le point de vue par des

1. Le territoire agricole, l'aprés l'enquête de 1892, est de 50467909 hectares dont 2371419 en terres lahouralies.

2. Congris de la riforme agraire en 1889.

3. Toir le Tableau de la France de Michelet (Ilistoinc, livre III). 
détails accessoires et relativement négligeables. Comme j’a cherché ì le faire au cours de cette étude, il est nécessaire de distinguer l'action différente des lois et des habitudes suivant qu'elles intéressent tel ou tel groupe de propriétés, car si la réglementation paraît uniforme clle n'en pèse pas moins sur les choses à des degrés très différents. L'équivoque la plus fertile en erreurs est celle qui, faisant de la moyenne propriété une sorte de terrain vague, de grève qu'abandonne et que recouvre le flot, lui conteste une existence personnelle, un rôle, un avenir, lui permetlant à peine d'être le rêve de la petite et la victime résignée de la grande, dans une mobilité incessante de groupements éphémères et de dislocations subites. De là l'opinion qu'il ne faut sérieusement tenir compte que de la petite el de la grande propriété, d'une part les petits propriétaires cultivant leur héritage de leurs propres mains, de l'autre les grands propriétaires faisant exploiter leur domaines par des intermédiaires ou des salariés, comme si, entre ces leux termes extrèmes, il n'y arait pas une infinité de situations aussi vivaces, aussi fécondes.

Quelques publicistes ont profité de celte classification trop absolue pour créer une sorte d'antagonisme social entre la petite propriété et la grande, de même qu'on l'a fail entre le capial et le travail, et liilentité des deux situations s"impose. Plusieurs écoles politiques et sociales ont cru qu'il élait possible de fonder exclusivement la puissance de notre pays sui le bien-être des classes ouvrières et des petits propriétaires ruraux, et leurs adhérents s'accorderaient à considérer comme un progrès la destruction de la grande culture au profit de la petite. C'est une crreur radicale. Ces deux catégories d'exploilations sont aussi nécessaires l'une à l'autre que le capital au travail el le travail au capital; elles pâtiraient loutes deux de lenr isolement ou de lenr servage si rlles ne pouvaient, librement, se prêter un muluel appui.

Leur rôle actuel est défini par les conditions de temps et de lieu; il pourra se modifier dans l'avenir, mais sans troubler les profits de relation qui conslituent la puissance 
sociale et économique de diversités associées dont les contrastes mèmes deviennent féconds. C'est la coexistence de la grande, de la petite el de la moyenne propriété qui fait un faisceau de nos ressourees nationales. Le dessein de l'économiste doit être de définir la part de chaque élément dans la résultante, celui du législateur d'en favoriser l'action productive, par abstention plutôt que par intervention.

Mais la loi qui régit impérieusement la formation et l'évolution des trois groupes de propriétés, le principe qui leur est indispensable à tous trois, le seul peut-être qui puisse leur ètre appliqué uniformément malgré la diversité de leur constitution et de leur rôle, e'est l'idée de permanence, de prolongation pourrait-on dire si l'on jugeait trop ambitieuse l'expression de perpétuité.

La propriété d'une entreprise, en effet, résulte à la fois, a-t-on dit avec justesse ${ }^{1}$, d'une accumulation de valeurs matérielles et de leur parfaite adaptation au producteur et à la production. Mais il faut que cette adaptation soit continue.

Ainsi la terre, aux mains de cultivateurs intelligents qui l'exploitent de père en fils, vaut plus qu'aux mains d'un fermier étranger obligé de faire son apprentissage dans la connaissance du sol, du climat, des habitudes, des débouchés.

Pourquoi en serait-il autrement pour la terre, où tout est variable, intermittent, compliqué, dont l'exploitation exige tant d'observations et d'application patiente, que pour l'usine, lorsqu'elle est conduite par celui qui l'a outillée ou qui en a formé le personnel, que pour le commerce mème ou le métier queleonque exeré par des industriels qui ont l'expérience consommée du pays où ils vivent et de la clientèle qu'ils ont à servir?

Dans ces conditions, et toutes choses égales d'ailleurs, le fonds productif vaut plus pour l'homme qui en a la pratique journalière que pour l'étranger qui l'acquiert par caprice ou spéculation et n'en connaîtra que par son expérience personnclle les ressources et les défauts. Aussi ai-je toujours été 
étonné d'entendre certains économistes vanter comme un signe de prospérité le mouvement plus vif des mutations immobilières à titre onéreux. Si le fiscal peut se réjouir du nombre croissant des transactions qui apportent une plusvalue aux receltes du Trésor, l'ćconomiste doit déplorer l'instabilité de la propriété rurale dans les mêmes mains et cette déperdition de la valeur effective qui, à chaque transfert hors de la famille, disperse un peu du capital de combinaison el d'expérience et réduit en fumée la plus précieuse partie de l'outillage rural, celle quion ne voit pas, celle qui réside dans la tradition. dans le culte du foyer, dans l'identification de l'homme et de la terre.

Les récentes statistiques de l'impôt ${ }^{1}$, si on les examine avec soin, établissent que les plus fortes recettes du droit de mutation sur les ventes ne correspondent pas à des périodes prospères mais suivent des époques de crise. Ce que les administrateurs des finances représentent comme un signe de bien-être et d'activité ne peut être, pour un économiste, que le résultat d'une liquidation laborieuse dégageant des fortunes compromises.

C'est ce (pui s'est produit de 1832 à 1836 , après la révolution de 1830 , de 1842 à 1840 après la crise de 18.0-1841, de de 1833 ì $18 \% 6$, après la période révolutionnaire de 18.48 à 18\%2, se complétant par la crise de $18: 3$ et la liquidation de 1839 , de 1870 à 1880 , après la gruerre de 1870-1871, de 1889 à 1891, après l'Exposition de 1889 el le krach, de 1897 à 1898, après la crise des valeurs de mines en 189\%. Il ne faut pas perdre de vue, en effet, que e'est envisager la question sous une seule de ses faces que de faire de la multiplicité des acquisitions une preuve de la prospérilé publique; chaque acquisition est la conséquence diune rente, el si l'acheteur, au lendemain des crises commerciales et industrielles dont le contre-coup se résout en crises agricoles,

1. Le mouvement des mutations immobilières à titre onéreux, de 1826 à $189 \%$, d'après les comptes définitifs des finances (Bulletin de statistique de l'Enregistrement, 1898, p. 164 et suiv.). 
peut avoir fait une bonne affaire, il n'en est pas de même du rendeur.

Lor'sque, pendant la seule année 1894, on constate 324902 actes de vente de petites propriétés rurales d'une valeur inférieure à ว 000 francs, pour une somme totale de 368 millions ${ }^{1}$, peut-on s'en applaudir? Et ne deviait-on pas regrelter, au contraire, que les familles de 544902 petits propriétaires aient élé contraintes d'abandonner à vil prix l'œurre de leur laborieuse épargne, de disséminer à la criée le modeste matéricl si patiemment et si joycusement amassé. D'aulres ouvriers agricoles, dira-t-on, d'autres prolétaires montant d'un échelon dans l'échelle sociale, auront remplacé ceux-ci; ce n'est pas sûr, car la crise du travail, dans les familles laborieuses, alleint aussi généralement la très petite forlune acquise que la très petite fortune en voie de formation, et les liquidations foncières ne profitent d'habitude qu'à des capitalistes et à des spéculateurs possédant assez de ressources pour altendre, sans se hàter, le moment propice à la revente. Dans tous les cas, il est préférable, au double point de vue social et économique, d'assurer le maintien de $\$ 44902$ petits héritages dans les mains de leurs détenteurs que de favoriser, à leurs dépens, l'accession à la propriété d'un même nombre de propriétaires, voués au bout de quelques années à des destinées semblables.

C'est la vérité qu'affirmait le baron de Stein, l'un des restaurateurs de l'autonomie prussienne après 1813, lorsqu'il écrivait :

La divisibilité sans limites des grands ou des petits domaines dissout la classe des paysans et en fait un ramassis de traineurs de brouette et de gens sans aveu, et, quant aux nobles, les transforme de propriétaires indépendants en oisifs de service et de cour. Du maintien des fermes de laboureurs et des domaines nobles, répartis en masses bien proportionnées, dépend la conservation de la classe la plus solide, la mieux douée, la plus militaire, qui a d'instinct les qualités de travail et de moralité. 
Roscher, qui cite ce passage des Mémoires de Stein ${ }^{2}$, ajoute qu'à la fin de sa vie l'objet principal des méditations de Stein était d'empêcher que les paysans ne fussent réduits, par l'application de théories néfastes, à retomber, après avoir été propriétaires, dans la condition des journaliers el qu'à leur indépendance relative sous la domination des seigneurs terriens ne s'en substituàt une pire qui les mit à la merci de l'usure.

La vitalité d'une institution dépend des garanties de sa durée; ce qui est livré à de trop fréquents changements n'a que la valeur d'un feu de paille ct, chez ceux que la chance a mal servis, la lassitude et l'aigreur succèdent vite à l'effort.

Dans chacune des trois calégories de propriétés, mais à des degrés différents, la fréquence habituelle des mutations est un danger social.

Résumons, afin de conclure, ou est le péril, pour la grande propriété, pour la petite, pour la moyenne; el quels sont, pour chacune d'elles, l'idée de fixité et de permanence étant posée comme point de départ, les moyens de développer le plus pratiquement leur rôle social dans l'avenir.

La grande propriété, en France, ne paraît avoir besoin ni d'aide ni de protection; il lui suffira de bénéficier, par contrecoup, des réformes juridiques et financières qui sont en projel, mais il ne faudrait pas qu'on l'atteignît et qu'on la désagrégeât par l'impôt progressif et la spoliation déguiséc.

Elle n'est pas, comme en Angleterre, le vaste fleuve où les minces rivières viennent se confondre; elle a cessé d'ètre encombrante et absorbante. Elle fournit de bons exemples ct de gros salaires. Son avenir est de développer plus encore ce rôle d'initiatrice et d'éducatrice où elle pourrait s'avancer davantage, pour le profit commun, sans la faute politique

1. Économie politique rurale, 1889 , p. $\$ 68$. 
qui l'écarte de l'action administrative locale dont elle est, en temps normal, la directrice et le soutien.

Une enquête récente sur les causes du progrès déconeertant de l'Allemagne dans ces dernières années, fournit assez d'indications pour qu'il ne reste plus de doutes sur l'impulsion décisive que l'effort des grands propriétaires fonciers peut donner à l'arancement d'une nation. Les forces productrices agricoles de l'Allemagne, à la faveur de vues d'ensemble poursuivies avec une persérérante unité, se sont développées par l'initiative individuelle de riches propriétaires, actifs, et scientifiquement préparés à leur tâche. Le déreloppement industriel et commercial a suivi, venant à son tour exciter et enrichir la production rurale.

En Saxe, en Silésic, rapporte M. Paul de Rousiers ${ }^{1}$, les plus importants domaines sont exploités directement par de riches propriétaires. C'est là qu'a commencé le progrès. Les personnes compétentes qui en ont suivi la marche sont très affirmatives sur ce point. Aujourd'hui que de modestes domaines de moins de cent hectares sont eultivés avec tous les perfectionnements modernes, on a oublié que leurs maîtres ont suivi un exemple venu des grands propriétaires. Ce sont ceux-ci qui ont donné l’impulsion, commencé l'éducation agricole et provoqué l'exploitation scientifique des petits domaines et des terres médiocres.

Si l'on a, en Allemagne comme en Angleterre et en Autriche, l'impression très nette d'une exploitation agricole servie par l'intelligence et fécondée par les capitaux de ceux qui détiennent les grandes propriétés, l'Allemagne fournit de plus la preuve de l'entraînement passionné avec lequel les classes intermédiaires ont suivi l'exemple. Il faut de grosses fortunes ou de solides associalions de capitaux pour bâtir des écuries, des distilleries, organiser la force motrice, acheter des bêtes de race, créer des haras, se servir de machines coùteuses, diriger un nombreux personnel et $y$ spécialiser les emplois; mais la science professionnelle est à

1. Revue de Paris, $1^{\text {er }}$ août $1900,510$. 
la portée de tout individu et c'est par la voie intellectuelle que les grands propriétaires sont arrivés à inculquer autour d'eux le goût des nouveautés utiles et la recherche des meilleures méthodes scientifiques appliquées à l'industrie.

C'est là le rôle que la grande propriété a à remplir en France, par l'exemple qui devient une mode et, par ce véhicule d'apparence légère, se transforme en devoir social. Le champ d'expériences ne lui manque pas puisque, en dehors de 18 millions $1 / 2$ d'hectares cultivés, elle en détient près de 4 millions à l'état de landes et de friches.

La petite propriété, je l'ai dit, malgré des progrès apparents qui marquent plutôt une énergie et une patience admirables dans la continuité de l'effort que la réalisation définitive de ses espérances, se troure dans une situation précaire, et tout ce qu'on a tenté jusqu'ici pour l'améliorer a passé autour d'elle sans lui apporter d'allègement appréciable et sans créer sa sécurité.

Sans aller jusqu'à dire que l'œuvre de destruction commencée en 1793 a produit la désorganisation simultanée des races de nos grands propriétaires et de nos paysans ${ }^{1}$, et surtout sans vouloir porter atteinte au principe de l'égalité des partages, il est permis de rechercher les moyens de conserver sans détruire.

Au milieu de conditions géographiques, climatériques, ethnographiques et économiques variant à l'infini, les petits propriétaires ruraux forment, en France, delux classes principales, entre lesquelles il existe beaucoup de contrastes. D'abord, le paysan à famille souche, dont F. Le Play a célébré les vertus coutumières, possédant un domaine transmis intégralement de génération en génération, cultirant eet héritage avec le concours d'un personnel nombreux, et dispensé à la fois de prendre des salariés à son service et de chercher, pour lui-même ou pour les siens, du travail au dehors. Cetle classe de cultirateurs se trouvait autrefois dans toutes les provinees, ayant conservé jus- 
qu’après 1789 la force de cohésion et de résistance dont les coutumes du moyen âge l'avaient dotée; on ne la rencontre plus aujourd'hui que çà et là, dans les Alpes, dans les Cérennes, dans le Béarn, en Bretagne. Mais, sous l'influence des lois modernes, la famille s'est dispersée, les industries accessoires créées autour du foyer domestique ont disparu, le nombre des enfants a diminué dans une telle proportion que leur collaboration si précieuse s'étant évanouie il a fallu faire appel à des salariés.

L'autre type de paysan est le déraciné, celui que Le Play appelle le chef de la famille instable. Il se multiplie de plus en plus et sil arrive quelquefois au bien-être, il est rare qu'il puisse en transmettre les bienfaits à ses enfants, la famille ne se reconstituant par le mariage que pour se dissoudre à la mort du père et épuiser ainsi dans ces brusques ruptures d'intérêts tout l'efiort accumulé que les générations d'autrefois se léguaient avec une sorte de piété attendrie.

Dans certaines régions, les conséquences du partage forcé en nature ont été éludées par l'expédient du partage anticipé (Art. 107ö et 1076 du Code civil), usité dans les Alpes, le Plateau Central, l'Ouest, les Cévennes ${ }^{1}$; dans la plupart des départements, la dislocation du patrimoine s'est opérée, la petite propriété et la petite culture ont disparu en même temps, de propriétaires les paysans sont devenus fermiers et les meix, les masures, les métairies d'autrefois se sont agglomérés en vastes exploitations dans les mains d'un propriétaire forain. Le pays de Caux offre l'un des exemples les plus complets de cette transformation; l'ancienne race y tient à ferme les terres qu'elle possédait autrefois et qu'ont

1. D'après les statistiques de l'Enregistrement pour l'année 1898 , le Puy-de-Dôme tient la tête avec 998 actes, puis viennent l'lsère, avec 690, Saône-et-Loire avec 665, la Gironde avec 759, la Dordogne avec 629, les deux Charentes avec 1161, le Finistère avec 358, les Còtes-duNord avec 385, la Loire-Inférieure avec 524; le Gard en a 364 et l'Yonne 393 . Les départements viticoles sont les plus partisans de cette combinaison qui se traduit, en 1898, par 27732 actes portant sur 390 millions de valeurs dont 223 en immeubles ruraux. 
achetées les armateurs et les industriels du Havre, de Dieppe, de Rouen.

A vrai dire, ces deux types de familles paysannes peuvent aujourd'hui être considérés comme également instables, et la stérilité voulue des ménages n'a heureusement pas encore assez pénétré les mœurs villageoises ${ }^{1}$ pour remédier aux spoliations de la procédure. Bien plus âpre et dévorante que la fiscalité elle-même, la procédure s'insinue par toutes les fissures du code. La loi écrite triomphe des traditions, du bon vouloir des intéressés, de l'entente des familles les plus unies.

Les gens d'afaires, a-t-on dit, ont un intérèt direet à détruire, en cette matière. l'accord des familles; ils s'ingénient à faire naitre, chez les héritiers peu intelligents ou peu scrupuleux, des sentiments de eupidité, et à s’assurer ainsi le profit des expertises contradictoires, des licitations, et surtout des procès que provoque le partage et qu'ils savent rendre interminables.

La préoccupation du législateur, depuis 1789 et jusqu'à l'heure où j'écris ces lignes, a été uniquement de faciliter à tout individu, même ignorant et même pauvre, l'accès au droit de propriété.

Aujourd'hui, on cherche à le léfendre contre le péché de pauvreté et on s'évertue à lui offrir des moyens de erédit, complexes et variés, alors qu'il n'y en a qu'un de pratique : le crédit personnel, cautionné des centaines ou des milliers de fois par la mutualité.

La loi sur les habitations à bon marché est un procédé ingénieux pour permettre aux familles des salariés, qui n’ont d'autres ressources que le travail chez autrui, l'acquisition d'un foyer, d'un toit; mais ce n'est que l'amorce d'un généreux mouvement, déjà plus efficace s'il se combine avec l'assurance sur la vie, mais qui ne se propage pas assez vite?

1. En 1599, les successions ouvertes en ligne directe descendante dans des familles complant \& cnfants el au-dessus ont été au nombre de 52592 sur un chilfre global de 281353 déclarations. (Bulletin gris, 18.9, 191.)

2. D'après les statistiques de l'Enregistrement, il n'y avait en 1897 que 287 maisons collectives ou logements compris dans ces maisons 
et qui d'ailleurs exige, pour sa réalisation, des conditions qui ne sc trouvent réunies que dans les villes importantes ou les centres industriels : philanthropes désintéressés pour organiser les comités, capitaux résignés à courir les risques d'une œuvre humanitaire, ouvriers consentant à se soumettre à une discipline relative 1 .

Ce qu'il faut souhaiter par-dessus tout, ce qu'il faut obtenir sans entreprendre sur la liberté des contrats, c'est que le nouvel acquéreur d'un bien rural puisse conscrver ce qu'il a acheté; que l'héritier ne soit pas forcé de vendre ses droits pour payer limpôt de mutation ou les frais de procédure; que le domaine patrimonial, sur lequel ont pciné tant de générations, de père en fils, ou que le chef de famille a créé au prix de toutc unc existence de labeur, ne reste pas virtuellement exposé à la dislocation, ni voué à la vente forcée, et qu'il puisse avec certitude se transmettre indéfiniment des enfants aux petits-cnfants. Les procédés d'exécution sont connus.

La Réforme hypothécaire et l'organisation des Livres fonciers permettront d'établir la sécurité du titre foncier; la faculté de pouvoir rendre insaisissable et inengageable le bien de famille, assimilera l'atelier rural à l'atelier industriel et rendra la famille stable et féconde en lui assurant un minimum de revenu et en la délivrant de la terreur de l'éviction; la suppression du partage forcé en nature consacrera la permanence de l'héritage et sa transmission intégrale, à moins que tous les intéressés ne soient d'accord pour en décider autrement; enfin, le crédit est assuré à tout propriétaire par la coopération se combinant avec l'assurance ${ }^{2}$.

ayant profité des immunités fiscales accordées aux habitations à bon marché par les lois des 30 novembre 1894 et 31 mars 1896 . 11 n'en a pas été publié d'autres, sur ce sujet, en 1899 ct 1900.

1. L'un des promoteurs de la loi sur les habitations ouvrières à bon marché, M. Jules Siegfried, a proposé de faciliter à l'ouvrier agricole les moyens d'acquérir une petite propriété, et, pour y arriver, il demande au législateur d'accorder à la petite propriété rurale les mêmes avantages que ceux attribués à la petite propriété urbaine par les lois de 1894 et 1896 . (Rapport au Sénat, 5 juillet 1898 .)

2. Je laisse intentionnellement en dehors de cette ćtude, comme 
L'avenir de la petite propriété, à la condition qu'on en rende non seulement l'acquisition facile, ce qui est, mais la possession durable, ce qui n'est pas, est considérable. C'est le plus solide contingent de la civilisation économique, l'appoint décisif lu progrès social. Fixer au sol les nomades du travail contemporain, les ramener par le bien-être au goùt des familles nombreuses, les provoquer à l'épargne, les exciter à l'avancement intellectuel par l'enseignement que le loisir permet, n'est-ce pas une cuvre de haute moralité?

On lc comprend en Europe, et l'on nous envie ces millions de paysans propriétaires dont-il faut conserver et raffiner la race à tout prix. L'Angleterre, qui fonde sur cette nouveauté l'espoir de son futur recrutement militaire, crée et multiplie les allotments et les small holdings; l'Allemagne reconstitue ses anciennes tenures rurales jadis serviles, aujourd'hui libres, sous le nom de Bauerhof ou domaine paysan; l'Autriche réorganise ses $H$ of ferolle, la Suisse ses manoirs héréditaires. En France, nous possédons en germe cette sève de vitalité, et nous derons d'autant mieux la respecter qu'clle constitue, on même temps, l'affirmation visible de la première et plus laboricuse étape de l'épargne vers l'aisance.

La crise économique et la crise sociale sont solidaires l'une de l'autre, disait naguère un spécialiste à la Commission du cadastre ${ }^{1}$; la France, avec son immense majorité de petites fortunes, n'a rien à envier aux pays oủ l'équilibre est rompu par l'exagération de l'opulence, d'une part, de la misère, de l'autre. C'est pourquoi les Français émigrent peu.

Il faut, pour nous conserver cet avantage relatif, multiplier les attaches de l'homme au sol et maintenir le droit de propriété comme la raison d'être de la famillc. La possession de la terre est le but certain de l'épargne; favorisons cet

n'ajpartenant pas à mon sujet. la question de l'aide qu'apporte aux petits propriétaires ruraux l'affiliation aux sociètés de secours mutuels et aux syndicats agricoles. Cet appoint considérable va toujours en augmentant.

1. Procés-verbaux sténographiés, séance du 26 novembre 1891. 
entraînement. Tout petit propriétaire foncier est libéral, paree que la eonseienee de son droit lui donne celle de son indépendance; il est patriote, parce qu'il détient une parcelle de la patrie.

Mais il faut échapper à un double péril. Il faut éviter que les syndicats des capitaux de spéculation, sous forme de sociétés foncières ou de banques centrales agricoles, ne rachètent ou ne transforment la dette agraire pour ramener à la servitude ou au colonage d'autrefois les paysans, en les tenant sous l'alternative du remboursement ou de l'évietion; il faut empêeher aussi que le titre de propriété, s'il était trop aisément mobilisable et cessible sans contrôle ', ne puisse être aceaparé à vil prix par des spéculateurs associés, comme on le vit en Russie, après le rachat des serfs et le partage des terres communes.

On préviendrait ce danger en imitant l'institution du Bauerhof hanovrien et du Hoefrecht d'Autriche ${ }^{2}$, c'est-à-dire en définissant l'héritage foncier, restreint à une valeur vénale correspondant à ce qui peut assurer la subsistance d'une famille rurale ct son habitation, et immobilisé au profit eollectif de la famille par l'application de l'article $45 \% 3$ civil.

La répartition de la propriété rurale entre les trois catégories d'exploitations qui en résument les conditions diverses est étroitement liée au plus ou moins de stabilité que la législation accorde à chacune d'elles. La France, déjà distancée par toutes les législations étrangères, sans exception, quant à la revision du régime hypothécaire, res-

1. C'est ainsi qu'il y aurait danger à accepter tel quel l'article 86 du projet élaboré par la commission du cadastre sur la transmission des droits hypothécaires et qui est libellé comme suit : La transmission des créances hypothécaires revêtues de la clause à ordre on au porteur, comme celle des bons hypothécaires (créés par l'article 43 pour mobiliser l'immeuble jusqu'à concurrence de la dette inscrite), s'opère indèpendamment de toute mention au Livre foncier.

2. Dans ce pays, le Codle civil de $1 \$ 12$ fixe la quotité disponible à la moitié du patrimoine et stipule que le droit de légitime se règle en valeur et non en nature. 
tera-t-elle également indifférente à l'une des questions les plus inquiétantes de son état économique?

La force des choses est plus puissante que toutes les théories et toutes les préventions; quelles que soient les controverses pour déterminer normalement ce que devrait être la répartition de la propriété entre les mains de ses détenteurs, puisque l'égalité économique est une chimère comme toutes les autres, il est prudent de s'arrêter uniquement aux possibilités. L'expérience de la vie pratique ne nous enseigne-t-elle pas que l'harmonie ne peut ètre faite que d'inégalités? La conclusion de tous les débats, c'est que la productivité du capital est une nécessité qu'il a toujours fallu se résoudre à accepter, même quand on la niait. La terre doit produire. L'économiste et le législateur ont pour mission d'assurer au travailleur toutes les libertés de nature à favoriser l'exploitation du capital foncier; el la plus excellente répartition du sol sera celle qui donnera le plus d'essor à la production.

C'est dire que toute préférence accordée à la grande propriété sur la petite ou à la petite sur la grande serait une erreur; elles sont toutes deux nécessaires l'une à l'autre, et leur solidarité effective est rendue apparente par le rôle de ce groupe intermédiaire, participant à la fois des caractères de l'une et de l'autre, leur empruntant les ressources qui leur sont propres, qu'on appelle la moyenne propriété.

C'est là qu'est l'avenir. La moyenne propriélé a toujours été, dans l'histoire politique ct sociale, la réserve, la sauvegarde des nations; son rôle est pareil dans leurs annales économiques, et c'est à cette source intarissable, alimentée d'en haut el d'en bas, qu'on ne cessera de puiser sans craindre de la voir tarir. Les grosses fortunes ne sont plus aujourd'hui que de rares exceptions, la très petite propriété reste la porte élroite, d'un accès difficile, par où le travail s"installe dans le capital; la moyenne propriété, qui, à tant d'échelons et à des hauteur's si inégales, est la représentation par excellence de ces fortunes intermédiaires dont la 
possession ne dispense pas de travailler mais, au contraire, provoque à travailler davantage.

Comme dans la petite propriété, dans la moyenne l'homme ne peut être séparé de la famille. Dans toutes les conditions de la vie, mais surtout dans l'isolement relatif du proprićtaire rural qui habite son domaine, la famille crée un centre naturel d'effort ou de résistance dans le rayon duquel les vieillarls et les infirmes trouvent le repos et la sécurité, tandis que les valides y développent la plénitude de leur énergie et la variété de leurs aptitudes. L'influence des divers membres de la famille associés dans une action commune, et surtout eelle des femmes, s'y exerce avec plus d'efficacité que dans le modeste ménage des ouvriers industriels et agricoles, paree que le champ d'activité est plus vaste et qu’il embrasse quantité de détails dont la valeur productive ou les risques de déperdition sont en raison du plus ou moins de minutie et de régularité de la surveillance. Plus le niveau d'instruction el d'éducation s'élève, d'ailleurs, el plus l'élément moral prend une place prépondérante et jette de semences fécondes.

Les détenteurs actuels de la moyenne propriété sont en mesure par leur initiative personnelle et le libre choix de leur volonté, mieux que ne pourront le faire les lois, de ramener aux champs les travailleurs agricoles et les capitaux qui doirent féconder leur labeur. Comme je l'ai indiqué plus haut, le personnel des propriétaires de cette catégorie de domaines en général possède assez d'instruction et assez d'argent pour révolutionner l'industrie agricole. Ceux qui habitent déjà sur le domaine, qu'ils exercent le faire-valoir direet ou qu'ils exploitent par intermédiaires ${ }^{1}$, n'ont que peu à faire pour donner à leur ròle toute l'amplitude qu'il comporte. Quant aux propriétaires forains, beaucoup plus nombreux, surtout dans l'aristocratic de la moyenne propriété, celle qui possède de 30 à 100 hectares (en élargissant un peu

1. Rappelons qu'il y a 806494 fermiers et métayers non propriẻtaires cultivant la terre d'autrui. (Enquête de 1892, p. 386.) 
la classification officielle), c'est dans leurs rangs, c'est en faisant appel à leur bon sens, à leur expérience des affaires, à leurs vues d'avenir pour l'établissement de leurs enfants, qu'on pourrait repeupler la France.

La bourgeoisie, en effet, trouverait dans la transformation en faire-valoir direct ou en exploitation par le métayage ${ }^{1}$ de la culture par fermiers de ses propriétés rurales, des débouchés illimités pour ses fils et une destination rémunératrice pour ses capitaux. En présence de l'encombrement des carrières libérales, du discrédit où tombe le fonctionnarisme mal rétribué el sans issue, du peu de sćcurité que présente l'industric où l'on désorganise les conditions du travail, de la baisse constante du prix de l'argent, les pères de famille prévoyants ont lout intérêt à diriger vers la science agricole les aptitudes el les goùts des jeunes générations. La récento enquête sur les résultats de l'enseignement secondaire a ouvert les yeux des plus optimistes; la chasse aux diplòmes n’a pour but que de gagner la prime d'exonération du service militaire; quant à ce qu'on leur a enseigné et à ce qu'ils savent, nos fils sont dans un état d'infériorité manifeste vis-ä-vis de leurs contemporains de mème condition de n'importe quel pays. Au point de vue de la défense nationale et mème de la réduction du service militaire, cette évolution de l'enseignement serait éminemment profitable 2 .

Imitons les Allemands; chez eux, l'aristocratie territoriale

1. D'après des expériences prolongées et des comparaisons très docnmentées, le metayage conserve l'avantage sur le fermage. Lors de la crise agricole de 1883 à $185 \%$. la perte sur les prix de ferme a varié de 23 p. 100 (Allier) à 50 p. 100 (Nord), tandis que la moins-value du métayage n’a pas dépassé 7 p. 100. (Némoire de M. (le Garidel sur le metayagre dans l'Allier, 1889.) L'enquête de 1892 constate l'augmentation du nombre les métayers si l'on corrige l'erreur commise en englobant le chifre des locations verbales dans celui des baux proprement dits.

2. Je préfère au régiment un paysan dur á la marche, sachant manier un cheval et creuser rapidement une tranchée, à tous ces demi-diplômés, carabins, potards, clercs d'avoue ou surnuméraires, étiolés, avariés ou surmenés, a lit un de nos lrillants généraux. Le chasseur de village, ajoutait-il, toujours un peu braconnier, sachant marcher et voir la nuit, a le coup-d'cil plus sûr qu'un docteur en droit. 
a donné le branle, les propriétaires de domaines d'étendue moyenne ont suivi, puis les autres, si bien que les voyageurs sémerveillent de la direction scientifique qui préside à l'exploitation des plus modestes Bauerhôf.

11 n'est pas rare de rencontrer des fils de paysans-propriétaires, disposant de ressources restreintes, mais consacrant deux, trois et quatre années à suivre des cours de chimie agricole, d'agronomie, etc., pour appliquer ensuite les connaissances ainsi acquises à l'exploitation de leur patrimoine. Luniversité de llalle, en particulier, compte beaucoup de ces étudiants; ils forment une pépinière précieuse d'Inspektoren (régisseurs) pour les grandes terres et de propriétaires instruits pour les petites ${ }^{1}$.

En France, où notre moyenne propriélé se classerait comme superficic par unité parmi la petite d'Allemagne (sauf certaines provinces el en particulier la vallée du Rhin), les ressources qui pourraient être consacrées à l'enseignement technique sont plus considérables. Il suffirait d'y appliquer le prix des sept années coùteuses et presque toujours stériles que nos fils perdent dans les lycées et les collèges à la poursuite de diplômes dont la valeur probante est contestable. Au bout de peu de temps, il serait aussi honorable d'avoir été diplômé par l'École de Grignon que d'être entré à l'École polytechnique ${ }^{2}$, et i'Instilut agronomique serait aussi couru que l'École des ponts et chaussécs ou l'École des mines.

Il est véritablement étrange que, dans un pays essentiellement agricole comme le nôtre, on se livre à la direction des travaux de l'élevage et de la culture sans préparation préalable, comme si la science agronomique n'existait pas et que le premicr venu cùt eette science infuse que le paysan le plus retors n'atteint qu'après une longue carrière d'expériences. C'est l'ignorance présomptueuse qui perpétuc la routine el qui discrédite les nouvelles méthodes en les appliquant sans discernement.

1. Paul de Rousiers, Conférences du Musée social, 1898.

2. Depuis sa fondation en $1 \$ 26$, l'École de Grignon n’a délivré que 807 diplômes sur 2232 élèves. (Annuaire stat., 189', p. 302.) 
On estime que le nombre des chefs d'exploitations agricoles, en France, dépassait de beaucoup, en 1893, 3 millions et demi; or, cette mème année, les cinq écoles nationales ${ }^{1}$ ne comptaient que $\ddot{3} 9 \ddot{3}$ élèves, et les $3 \ddot{a}$ écoles pratiques d'agriculture un effectif variant pour chacune de 12 à 40 .

Parallèlement, 2479̋̈ étudiants étaient inscrits dans les différentes facultés de l'enseignement supérieur. Celle simple comparaison suffit à montrer quel est le rôle social des propriétaires fonciers el ce que leur réserve l'avenir s'ils se dégagent des préjugés pour entreprendre avee entrain la mise en valeur de la moyenne propriété.

Un autre élément d'appréciation est la comparaison entre l'étendue moyenne d'une ferme et celle d'une exploitation directe; cette derniere, variant de 9 hect. 11 dans l'Aube ì 2 hect. 61 (Côtes-du-Nord) est de 4 hect. 37 contre 11 heet. 71 (dont le maximum est de 42 heel. 28 dans l'Indre el le minimum de 4 hect. 78 dans le Vaucluse). Cel écart des deux tiers au profit de la culture directe représente, d'après les professionnels de la science agronomique, le bénéfice du fermier s'il est habile, diminué de son manque à gagner s'il est négligent. Si la moyenne propriété était tout entière exploitée par le faire-valoir direct, elle ajouterait cette énorme plus-value à son revenu actuel.

Après l'instruction scientifigue qui, ì part de très rares exceptions, fait absolument délaut à cette catégorie de proprićtaires, pourtant les mieux placés pour la rechercher et la recevoir, ce qui lui manque, a-t-on l'habitude de dire, ce sont les capitaux. Ce raisonnement tourne dans un cercle vicicux, ear si le personnel exploitant ehangeait d'allures, s'il se recrutait lésormais parmi les fils des propriétaires, instruits et averlis, les capitanx se détourneneraient aussi des placements plus ou moins aventurés où les retient l'engouement pour les valeurs mobilières, même

1. Cirignon, Crand-Jouan, Montpellier, Versailles (Horticulture) et Mamirolle, qui date de $1 \$ \$ 8$. Depuis leur fondation, ces 5 établissements n'ont formé que 4726 èlèves. 
exotiques, et viendraient se faire concurrence pour commanditer la reprise de l'industrie agrieole.

Supposez, par impossible, que cet afflux inévitable de capitaux à bon marché ne se réalisât pas, la moyenne propriété aurait à sa disposition les combinaisons de la mutualité qui, puisqu'elles réalisent de tels suceès en Allemagne el en Italie sur la seule garantie de la solidarité de prolétaires, les réaliseraient d'autant plus sûrement en cette occurrence où elles s’appuieraient sur la triple garantie de la moralité, de la science et de la propriété.

Je fais abstraction de la dette agraire, que pourrail supprimer un vaste syndicat d'amortissement groupant les ressources que les banques de crédit mutuel local destineraient à cel emploi. Il ne faut pas se le dissimuler, le pauvre, dans notre société contemporaine, est moins celui qui n'a rien que celui qui n'a pas assez. La France, où l'on comple un propriélaire foncier sur deux chefs de famille, est peuplée d'hommes laborieux, mais gênés. C'est de celte gêne, qui entrave leurs efforts, qui stérilise leurs sacrifices incessants, qu'il est urgent de les délivrer.

Un proverbe du Nord dit que paysan endelté est paysan ruiné. C'est aussi vrai pour le propriétaire foncier que pour le simple paysan ayant ou non du bien; l'hypothèque, si l'on n'y prend garde, conduit droit à la saisie. Ici encore, l'avenir de la moyene propriété, dans son ròle futur et très prochain, comme il faut le souhaiter, sera, après avoir pris de la grande propriélé des leçons de culture intensive et progressive, de distribuer à son tour, à la petite propriété, des leçons de morale appliquée.

Le paysan a cessé d'être économe, il faut qu’il le redevienne. La vanité de paraître l'a saisi, le bien-être ne lui suffit plus, il se lanee dans des superfluités coûteuses qui n’ajoutent rien à son confort et rognent son épargne. La simplicité voulue des propriétaires instruits el bien élevés avec lesquels il va se trouver en contact le ramènera par l'heureuse contagion du bon exemple à des habitudes plus saines et plus discrètes. 
Le paysan s'imprégnera, petit à petit, de cet esprit de méthode et de calcul anquel le négociant ou l'industriel s'habitue dès les premiers temps de son apprentissage et qui se traduit soit par la discussion d'un prix de revient, soit par l'ajournement de certaines ventes ou de certains achats, soit par la prévision des variations de cours, et surtout par le sentiment des échéances. Le paysan ne gaspille pas encore, il est retenu par l'atavisme, mais il est sur la pente de la dépense improductive et celte pente est glissante. Il ne faudrait pas qu'on pût dire de lui comme des vignerons de l'Hérault et des Charentes avant 1882, comme des fermiers américains en 1879: Grisés par le succès, ils sont devenus déréglés dans leurs mœurs, extravagants dans leurs idées et dans leur dépense; quand survient une bonne récolte, au lieu de créer une réserve pour les vaches maigres, ils se bâtissent une maison neuve et, d'échéance en échéance, de billets en hypothèques, se ruinent par leur inconscience.

L'exemple des détenteurs de la moyenne propriété, en collaboration constante arec les détenteurs de la petite, pour des échanges de services, des ventes, des achats, des rencontres dans les syndicats, des intérêts mis en commun, ne sera pas l'un des résultats les moins rémunérateurs de ce changement dans le mode d'exploitation. L'inflinence s'en fera sentir dans tous les ordres de faits. Les particuliers se dégageront peu à peu de l'engrenage oì les attirent les courtiers d'aflaires el les escompteurs de village; ils apprendront à marcher sans lisières. L'initiative des citoyens remplacera, comme dans toute nation où le progrès est réel, lintervention des parasites et des fonctionnaires. Tout cela naîtra du sentiment de la responsabilité personnelle el de la compréhension de ce que peut la volonté de l'homme, quand elle est bien conduite.

Que pourrait la loi pour mettre les détenteurs de la moyenne propriété en mesure de remplir pratiquement ce grand rôle, qui n'est pas surfait, d'éducaleurs, de conciliateurs et de producteurs? Peu de chose, car l'avenir que je pressens 
dépend uniquement de l'initiative, de l'énergic ct de la persévérance des individus.

La moyenne propriété devant bénéficier, comme la grande d'ailleurs, des réformes civiles, fiscales et économiques qu'on est d'accord de réaliser dans l'intérêt de la propriété foncière en général et de la petite propriété rurale en particulier, il est peu de mesures spéciales à cette catégorie d'exploitations et dont elle puisse espérer un profit exclusif.

Les lois actuelles lui permettent de se mouvoir librement dans toutes les voies ouvertes à l'association par les syndicats et la mutualité; si le partage en nature, d'obligatoire devenait facultatif, si la réforme hypothécaire se réalisait à bref délai el que la transformation du droit de mutation allégeât le poids de l'impôt, il ne resterait qu'à abréger les procédures et à supprimer les deux tiers des intermédiaires officiels pour restituer à la propriété foncière sa force créatrice, sa puissance expansive, sa souplesse et son crédit.

Toutefois, il est deux questions que de bons esprits croient intimement liées à la prospérité de la moyenne propriété el qu'il convient d'indiquer.

La première est celle des enclaves. La loi du 27 juillet 1870 , modifiée par celle du 3 novembre 1884, a modéré le droit de mutation en faveur des échanges de parcelles contiguës, mais cette légère faveur, souvent contrariée par des interprétations arbitraires ${ }^{1}$, n'a pas donné les résultats qu'on en attendait. La difficulté vient moins de la gène de l'impôt que de l'àpreté des propriétaires spéculant sur le désir d'acquérir et exagérant outre mesure ce qu'on appelle par euphémisme le prix de convenance?

1. L'historique du tarif fiscal des échanges est assez curieux. L'article 2 de la loi du 16 juin 1824 accorde aux échanges d'immeubles contigus des immunités considérables que supprima l'article 16 de la loi du 24 mai 183 í. Mais, après l'enquête agricole de 1868, l'article 4 de la loi du 27 juillet 1870 créa un tarif réduit, maintenu par l'article 4 de la loi du 21 juin 1875 , moyennant six conditions rigoureuses dont l'une est de ne s'appliquer qu'aux parcelles de moins de 50 ares.

2. La loi de 1870, remarque le Dictionn. des droits d'Enregistrement, est 
La solution qu'on attend de l'exécution des remembrements ou des abornements généraux, semblables à ceux du XvIII $^{e}$ siècle en Bourgogne et en Franche-Comté, et à d'autres plus récents affectués en Meurthe-et-Moselle, se fera longtemps désirer dans la plupart des cas et reste dépendante de la concordance de tant de conditions locales qu'il n'y faut compter que comme sur un expédient accidentel. Il faudrait donc organiser la possibilité de l'acquisition.

C'est ce qu'a prévu le Code civil allemand, mis en vigueur récemment, et c'est un emprunt que la législation francaise aurait tout intérêt à lui faire, car l'agglomération des parcelles, l'homogénéité des cultures, la liberté de passage, sont indispensables à l'unité d'exploitation, et la moyenne culture est infiniment plus gènée que la petite par cet embarras topographique.

Le Code allemand, qui n'a pas notre timidité sentimentale à propos de soi-disant libertés, pose en principe que l’intérèt de tous est plus respectable que le caprice d'un seul. Il ne se borne pas à prévenir la dislocation du domaine rural en autorisant le père de famille à l'attribuer à l'héritier de son choix sauf à dédommagrer par des soultes ses frères et sœurs, il favorise son extension et la suppression des enclaves par le groupement. Au cas de vente d'une propriété rurale, d'un seul tenant ou en écarts, qui n'excède pas 2 hectares, il permet aux propriétaires limitrophes d'exercer le droit de préemption et de se substituer au tiers acquéreur ${ }^{1}$.

La seconde question vise la donation entre-vifs à titre de partage anticipé, telle qu’elle cst réglée par les articles $107 \ddot{\text { e }} 1076$ du Code civil français.

une faveur dont il est bien rarement possible de revendiquer le bénéfice (édition de $18 \$ 1$. III, p. 67).

On ne constate que 827 échanges d'immenbles contigus en 1895 . et 7.45 en 1896. (Bulletin de statist. de l'Enregistrement, 189s, p. 60.)

1. Ce droit ne peut être exercé que pendant les neuf jours qui suivent la notification faite aux riverains par l'acquéreur. S'il y a plusieurs concurrents, la préférence est donnée à celui dont la propriété est la moins étendue; à sunerficies égales, à celui qui a manifesté le premier l'intention d'exercer le retrait. 
Les partisans de ce compromis accepté par le législateur de 180. comme devant atténuer les défauts du partage après décès cntraînant la division el le morcellement de chacun des éléments de l'actif successoral, raisonnent ainsi, à l'heure actuelle.

La dette agraire écrase la propriété, l̇attrait des valeurs de papier accapare et disperse le capital héréditaire ${ }^{1}$, le tarif successoral et le partage forcé en nature détruisent le foyer domestique à peine constitué, à ce point que le grand nombre des enfants, considéré jadis comme une bénédiction, est devenu un dissolvant et un danger. Dans de semblables conditions, tout doit converger vers un but unique, la consolidation de la famille, la permanence et la fixité du centre patrimonial. Non seulement il faut aider l'homme laborieux à devenir propriétaire, mais surtout il faut lui assurer la sécurité de sa propriété; il faut aussi qu'après lui cet héritage qu'il a créé ne se dissolve pas et que sa volonté se continue dans un autre lui-mème. Pour la très pelite propriété, on y peut pourvoir par l'insaisissabilité du foyer héréditaire, mais cet expédient ne garantit plus la propriété dès qu'elle dépasse des limites étroites et c'est précisément la moyenne propriété, celle dont la cohésion et la fécondilé, si elles étaient mieux défendıes, assureraient par leur expansion le triomphe de notre état économique vis-à-ris de la concurrence élrangère, qui se trouve le plus menacée par la coalition néfaste de la procédure et de la fiscalité.

D'où la conclusion, qu'il faudrait vulgariser l'usage du partage anticipé, le dégager de formalités gênantes, exonérer I'hérilier associé de charges trop pesantes en augmentant pour ce cas unique la quotité disponible, et dispenser de tout impòt supéricur à 1 p. 100 l'acte réalisant l'ouverture de celte succession fictive. Serait-ce vraiment un progrès?

1. D'après les calculs de M. Neymarck, les 20 milliarıls qui forment le capital en circulation des six grandes compagnies de chemins de fer, sont répartis entre plus de 700000 familles. Les Caisses d'épargne détiennent près de 4 milliards de francs répartis entre $S$ millions $1 / 2$ de livrets, etc. 
A l'heure actuelle, le partage anticipé ne porte pas exclusivement sur des biens ruraux ${ }^{1}$, il embrasse des valeurs de Loutes sortes; pour répondre à l'intention de ceux qui recherchent le maintien intégral du domaine rural dans les mains du successeur désigné de son vivant par le père de famille, le privilège des immunités fiscales et de l'exception juridique devait être exclusivement réservé à la dévolution de l'exploitation rurale.

Mais il y a, peut-être, un danger sérieux dans cette tendance à encourager outre mesure la démission des biens, le partage anticipé. Pourquoi favoriser ces contrats par un tarif de faveur? Lors de la discussion au Sénat du projet de loi sur les successions l'intervention du notariat rural a impressionné le législateur dans un sens contraire à l'opinion émise. Sauf des cas particuliers assez rares, on ne voit ni le profit social ni le profit économique qu'on tire d'une succession ouverte prématurément dans une famille de laborieux, eussent-ils à exploiter de 10 à 40 hectares. Cet expédient peut satisfaire l'ambition, l'égoïsme des enfants, mais il compromet singulièrement l'autorité paternelle.

Cetie rupture anticipée du lien familial n'existe pas chez les nations prolectrices du foyer domestique el où le respect des traditions est un gage de repos et de progrès; même si l'âge empèche un vieillard de conduire la charrue ou d'abattre les arbres, ne conserve-t-il pas le prestige de l'expérience et du conseil? Le maintien du père comme chef d'exploitation est une condition de durée pour l'héritage; sa dépossession est une déperdition morale et matérielle

1. Ainsi, pour l'année 1898 , les 27732 contrats de démission de biens à titre de partage anticipé comprennent les valeurs en capital dont le létail suit, en millions de francs :

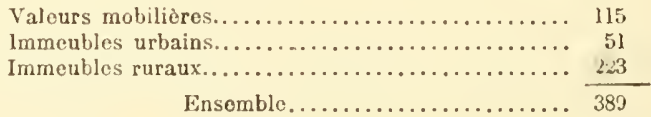

D'après les chiffres détaillés du Bulletin de statislique de l'Enregistrement, 1899, p. 177. 
rappelant trop les barbaries primitives où l'on rejette au néant l'lomme qui a cessé d'être le plus fort. A peine justifiable dans les très petites exploitations où le travail matériel et personnel est une nécessité, ce procédé brutal devient moins excusable encore à mesure que le domaine s'accroit en étendue et exige l'emploi d'auxiliaires salariés; la foree musculaire n'est plus en jeu et la valeur intellectuelle la domine.

Telles sont, du moins à mon humble avis, les conditions d'avenir de la grande, de la petite et de la moyenne propriété en France.

Le ròle prépondérant et civilisateur, dans l'évolution économique qui se continue, est réservé à la moyenne propriété; la petite servira de pont entre la fortune en voie de formation et la fortune acquise, entre le travail et le capital; la grande, privilège des gros capitaux et des hommes de loisir qui ont hérité de leur père ou se sont faits eux-mêmes, restera comme un but pour les ambitieux, comme un modèle pour les professionnels de la science agricole, comme un instrument de progrès social pour les braves gens.

Et c'est, en eflet, ce progrès, fait de bien-être et de moralité, celui-ci conduisant à celle-là, qu'il faut rechercher et réaliser sous toutes ses formes, par tous les moyens. Le travail est son instrument, et comme il résume dans son activité patiente les plus nobles sentiments de l'humanité, il a droit à tous les respects.

Les civilisations avancées le tirent de la servitude, le défendent contre l'âpreté et l'arbitraire. Je ne sais pas d'image plus saisissante de la protection du travail que le souvenir des laboureurs et des charpentiers du moyen âge, raffinés dans leur rudesse, préservant par des gants l'outil délicat qu'est la main. Ce ne sont pas, en effet, les mains calleuses 
qui réussissent les meilleurs ouvrages, pas plus que l'endurcissement de la conscience ne fait la force du caractère. C'est une marque de faiblesse d'être brutal, disait Pascal, et il faut que l'homme s'estime son prix. La raison du travail moderne est enfermée entre ces deux termes, el cela suffit pour qu'il achève la conquète pacifique de la terre.

FIN 


\section{TABLE DES MATIËRES}

Avant-Propos. - Rapport de I. A. de Foville à l'Acadèmie des sciences morales et politiques, dans la séance du 3 août 1901. v à xvıl

\section{PREMIÉRE PARTIE}

\section{ORIGINES HISTORIQUES ET SOCIALES}

DE LA DISTRIBUTION DE LA PROPRIÉTÉ DU SOL ENTRE LES FAMILLES FRANÇAISES

\section{CHAPITRE I}

La puissance productive du sol dépend moins des proportions de sa distribution cadastrale que de son appropriation sociale.

I. - Pourquoi la valeur intrinsèque du proprietaire rural est supérieure à celle du citadin.....................

II. - Importance comparée à l'époque contemporaine des propriètés urbaine et rurale d'après les qualités de leurs détenteurs.............................. 23

III. - Statistiques de la propriété rurale en France d'après les chiffres combinés des publications officielles...........

\section{CHAPITRE II}

Classement du territoire agricole de la France, à la date de 1898, en grande, moyenne et petite propriété.

I. - Répartition proportionnelle de la grande propriété dans les diffẻrentes régions de la France................. 
11. - Origines historiques de la grande propriẻté territoriale..

III. - Rôle économique de la grande proprièté par son morcelle-

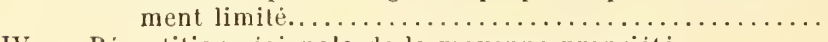

IV. - Répartition rẻgionale de la moyenne propriètè......... 117

i. - Origines historiques de la moyenne proprièté.......... 124

VI. - Rôle économique de la moyenne propriété par sa fixité relative................................ 129

VII. - Répartition régionale de la petite proprièté............ 14ä

VIII. - Origines historiques des mouvements alternatifs de disloeation, de concentration et de morcellement qui ont, tour à tour, favorisé ou entravé la constitution de la petite propriété...........................

\section{CHAPITRE III}

L'effort constant des populations rurales pour arriver à l'acquisition de la terre atteste l'importance sociale du droit de propriété.

I. - Pourquoi il faut distinguer, en matière économique, entre le morcellement, le fractionnement et la dispersion de

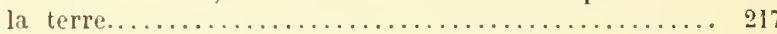

II. - La distribution inégale du sol dépend moins des lois que des mours ............................. 228

III. - La dépopulation............................. 231

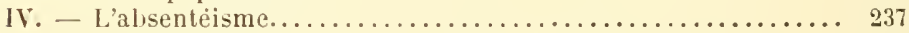

V. - La fiscalitẻ..................................... 245

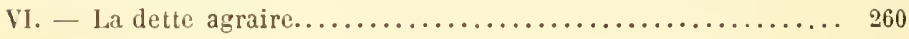

\section{CIIAPITRE IV}

Conditions économiques et juridiques particulières à chaque groupe de propriétés et qui entravent ou facilitent leur exploitation.

I. - La grande propriété peut multiplier sa production par la combinaison de la science et du crédit.............. 279

1. - Culture scientifique................... 283

11. - Outillage perfeetionne................. 285

III. - Industries agricoles greffées sur la culture.... 288

Iv. - Syndicats financiers................. 294

11. - La moyenne propriété vėgète parce qu'elle souffre d'institutions inal appropriées à ses besoins............. 298

1. - L'hèritage foncier....................... 299

II. - Le fermage....................... 306

111. - Le métayage......................... 310

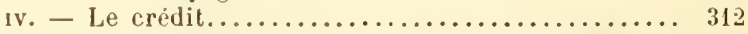

111. - La petite propriété, à peine constituée, succombe sous l'étreinte de trois fléaux..................... 318

I. - l'insécurité du titre foncier.............. 319

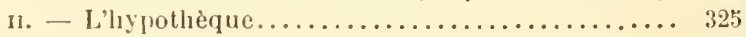

11. - Le partage......................... 328 


\section{DEUXIEME PARTIE}

\section{AVENIR DE LA PROPRIÉTÉ TERRITORIALE} EN FRANCE, D'APRĖS LES TENDANCES QUE MARQUENT LES LOIS ET L'ESPRIT DE LA PHILOSOPHIE SOCIALE

\section{CHAPITRE I}

L'avenir de la propriété rurale a pour termes corrélatifs la coexistence de la grande et de la petite propriété et l'exploitation intensive de la moyenne.

1. - Rôle pondèrateur et initiateur de la moyenne propriété dans la démocratie francaise........................ $34^{\prime}$

I. - Le travail......................... 353

ı. - La famille......................... 364

nII. - L’impòt.......................... 372

\section{GHAPITRE 11}

Réformes nécessaires dans le droit civil et le droit fiscal, pour que l'État n'entrave point le libre jeu de l'équilibre économique en matière de propriété foncière.

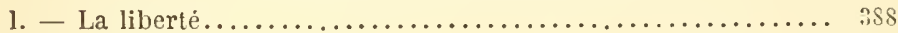

11. - Le crédit personnel............................ 401

IIl. - L'assurance .................................... 412

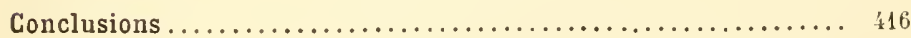





\section{CAPTES}

I. - Carte de la répartition géographique de la grande propriété, donnant la proportion par département du nombre des cotes de plus de 100 hectares à la date de $1898 . . . \ldots \ldots$.

II. - Carte de la moỵenne propriété. - Départements où la superficie des exploitations de 1 à 40 hectares dépasse 200000 hec-

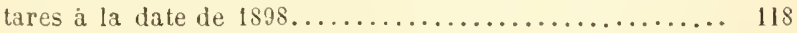

III. - Carte de la répartition proportionnelle par département, en 1898 , des trois modes d'exploitation de la terre : fairevaloir direct, métayage, fermage................ 192

Coulommiers. - Imp. PAUL BRODARD. - 1121-1901. 





\section{PLEASE DO NOT REMOVE CARDS OR SLIPS FROM THIS POCKET}

\section{UNIVERSITY OF TORONTO LIBRARY}

HD

645

F6
Flour de Saint Genis, Victor Benigne

La propriéte rurale en France 
